\title{
Journal of Radioanalytical and Nuclear Chemistry, 2005-2009: a citation-based bibliography and impact analysis using Hirsch-type statistics
}

\author{
Tibor Braun • András Schubert
}

Received: 7 April 2010/Published online: 29 April 2010

(C) Akadémiai Kiadó, Budapest, Hungary 2010

\begin{abstract}
All papers published in the Journal of Radioanalytical and Nuclear Chemistry (JRNC) in the period 2005-2009 (source dataset) and all papers citing these papers and published in other journals (target dataset) have been compiled. A scientometric analysis of the datasets has been performed using Hirsch-type statistics. A comprehensive bibliography of the citing papers is presented.
\end{abstract}

\section{Introduction}

In an information leaflet [1] published in 2004, a comprehensive overview has been given on the history of the Journal of Radioanalytical and Nuclear Chemistry (JRNC) from its launching in 1968 until the publication year, 2004, as reflected in publication and citation statistics. In the present study, the subsequent five years are analysed in a similar spirit.

Data sources and data processing

Source and citation data have been retrieved from the Web of Science (WoS) database of Thomson-Reuters (Philadelphia, PA, USA). JRNC data have been checked and, if necessary, supplemented and corrected using the Editorial Office files. The WoS "Analyze Results" option was utilized to gain primary statistical surveys; for more detailed or thorough analysis, the necessary bibliographic data have been downloaded, and analyzed by suitable software tools.

T. Braun · A. Schubert $(\bowtie)$

Editorial Office, Journal of Radioanalytical and Nuclear

Chemistry, Budapest, Hungary

e-mail: schuba@iif.hu
Hirsch-type statistics- the h-core

In his groundbreaking paper [2], Hirsch defined what he called the "h-index" (a scientist has index $\mathrm{h}$ if $\mathrm{h}$ of his/her $\mathrm{N}$ papers have at least $\mathrm{h}$ citations each, and the other $(\mathrm{N}-\mathrm{h})$ papers have fewer than $\mathrm{h}$ citations each) to quantify an individual's scientific output. The apparently innocent paper generated a flood of literature (it was cited more that 500 times until the publication of the present study) ranging from the rudely rejective to the glorifying. Apart from original purpose (evaluation of individuals), it proved to be useful in various other scientometric exercises (see, e.g., [3-8]), and even far beyond the realms of scientometrics $[9,10]$.

Among others, the h-index defines a "natural" top class of ranked objects or, at least, a somewhat more selfadjusting one than the usual "top 20 " or "top $1 \%$-s". The so-called "h-core" includes all items scoring at and above the $h$-index value. It contains exactly $h$ elements if there is no tie at the h-index value, may contain more if all tied items are included.

In the top lists presented in this paper, the h-core concept is used to cut the lists at their "natural" limits.

\section{Results}

In the 5-year period, 2005-2009, JRNC published 2,310 papers. Until the date of the present study (April, 2010), 1,145 of them have been cited at least once. They received 2,923 citations (1.27 citations per item; 2.55 citations per cited item) from 2,095 citing papers. 601 citing papers were published in JRNC itself, 1,494 in 494 other source titles. These two datasets, the 2,310 papers published in JRNC (source dataset) and the 1,494 papers citing them in 
other journals (target dataset), form the object of the present analysis.

Analysis of countries

Table 1 presents the h-cores of the countries assigned to the papers of the source and the target datasets, respectively, on the basis of the authors' institutional affiliation indicated in the by-line of the publication. Here and in the following tables core elements occurring in both the source and the target lists are highlighted in italics.

As seen, there is a great overlap among the two core lists, and even the distributions are rather similar. USA leads both lists with a share of about $20 \%$ followed by the

Table 1 The h-core of source and target countries

\begin{tabular}{|c|c|c|c|c|c|c|c|}
\hline \multicolumn{4}{|c|}{ Source countries } & \multicolumn{4}{|c|}{ Target countries } \\
\hline Rank & Country & Count & $\%$ & Rank & Country & Count & $\%$ \\
\hline 1 & $U S A$ & 493 & 21.34 & 1 & $U S A$ & 281 & 18.81 \\
\hline 2 & Japan & 239 & 10.35 & 2 & $\begin{array}{c}\text { Peoples } R \\
\text { China }\end{array}$ & 152 & 10.17 \\
\hline 3 & $\begin{array}{l}\text { Peoples } R \\
\text { China }\end{array}$ & 174 & 7.53 & 3 & Japan & 141 & 9.44 \\
\hline 4 & Brazil & 157 & 6.80 & 4 & Germany & 108 & 7.23 \\
\hline 5 & India & 148 & 6.41 & 5 & India & 95 & 6.36 \\
\hline 6 & $\begin{array}{l}\text { Czech } \\
\quad \text { Republic }\end{array}$ & 100 & 4.33 & 6 & Turkey & 73 & 4.89 \\
\hline 7 & Hungary & 77 & 3.33 & 7 & Italy & 71 & 4.75 \\
\hline 8 & Germany & 73 & 3.16 & 8 & England & 67 & 4.48 \\
\hline 9 & Italy & 70 & 3.03 & 9 & Hungary & 65 & 4.35 \\
\hline 10 & $\begin{array}{l}\text { South } \\
\quad \text { Korea }\end{array}$ & 68 & 2.94 & 10 & France & 63 & 4.22 \\
\hline 11 & Egypt & 57 & 2.47 & 11 & Iran & 58 & 3.88 \\
\hline 12 & Turkey & 57 & 2.47 & 12 & Brazil & 57 & 3.82 \\
\hline 13 & Russia & 53 & 2.29 & 13 & Canada & 53 & 3.55 \\
\hline 14 & Canada & 48 & 2.08 & 14 & $\begin{array}{l}\text { South } \\
\quad \text { Korea }\end{array}$ & 47 & 3.15 \\
\hline 15 & France & 48 & 2.08 & 15 & Spain & 45 & 3.01 \\
\hline 16 & Portugal & 47 & 2.03 & 16 & Poland & 42 & 2.81 \\
\hline 17 & Poland & 46 & 1.99 & 17 & Austria & 36 & 2.41 \\
\hline 18 & England & 45 & 1.95 & 18 & Belgium & 36 & 2.41 \\
\hline 19 & Mexico & 45 & 1.95 & 19 & Australia & 35 & 2.34 \\
\hline 20 & Netherlands & 43 & 1.86 & 20 & Russia & 34 & 2.28 \\
\hline 21 & Pakistan & 42 & 1.82 & 21 & $\begin{array}{l}\text { Czech } \\
\quad \text { Republic }\end{array}$ & 33 & 2.21 \\
\hline 22 & Iran & 40 & 1.73 & 22 & Egypt & 29 & 1.94 \\
\hline 23 & Austria & 39 & 1.69 & 23 & Romania & 28 & 1.87 \\
\hline 24 & Belgium & 30 & 1.30 & 24 & Portugal & 25 & 1.67 \\
\hline 25 & Romania & 29 & 1.26 & & & & \\
\hline 26 & Greece & 26 & 1.13 & & & & \\
\hline
\end{tabular}

two Asian giants, Japan and China. Asian and Latin American countries are very strongly represented in both lists; as to the EU, the new member states are preeminent.

\section{Analysis of institutions}

Table 2 shows the h-cores of the institutions the source and target datasets came from. In the identification of the institutions we completely relied upon the "Analyze Records by Institution Name" option of WoS. We are well aware of the fact that the cleaning of institution names is far from perfect in WoS, particularly, as less developed and non-English speaking countries are concerned. Since, however, we did not want to use this feature for any evaluative purposes, just to have a rough overview on the main sources and targets of JRNC papers, the information provided by WoS seemed to be appropriate.

It can be seen that the two lists are less overlapping than in the case of the countries. There are typically "producer" and typically "consumer" institutes. From an editorial point of view, it is particularly informative to find institutions (such as Florida State Univ and IAEA) extensively using JRNC papers as references, but less active in publication in the journal. Such institutions can be considered potential future contributors of the journal.

Analysis of authors

Table 3 contains the h-cores of source and target authors. The overlap here has been reduced to three authors; the editors may consider ways to motivate the other authors in the lists to a more "balanced" publication behavior.

\section{Analysis of citing journals}

The h-core of the 494 journals citing 2005-2009 JRNC papers is given in Table 4. The list contains no odd item, neither any expected title is missing. In a recent paper [11], a similarity measure named h-similarity for journals has been advised on the basis of common references and using Hirsch-type statistics. Reassuringly, the top three elements of the list in Table 4 are exactly the three journals with highest h-similarity to JRNC, and one more title in the list belongs to the most similar journals (they are highlighted in italics).

\section{Analysis of citing subject areas}

In the WoS database all journals are assigned to one or more subject areas. Using this assignment, a statistics has been made on the subject area classification of the citing papers. The h-core is shown in Table 5. 
Table 2 The h-core of source and target institutions

\begin{tabular}{|c|c|c|c|c|c|c|c|}
\hline \multicolumn{4}{|c|}{ Source institutions } & \multicolumn{4}{|c|}{ Target institutions } \\
\hline Rank & Institution & Count & $\%$ & Rank & Institution & Count & $\%$ \\
\hline 1 & Bhabha Atom Res Ctr & 75 & 3.25 & 1 & Chinese Acad Sci & 52 & 3.48 \\
\hline 2 & Los Alamos Natl Lab & 70 & 3.03 & 2 & Hungarian Acad Sci & 40 & 2.68 \\
\hline 3 & Chinese Acad Sci & 66 & 2.86 & 3 & Bhabha Atom Res Ctr & 36 & 2.41 \\
\hline 4 & Pacific NW Natl Lab & 65 & 2.81 & 4 & Natl Inst Radiol Sci & 30 & 2.01 \\
\hline 5 & Univ Sao Paulo & 56 & 2.42 & 5 & Japan Atom Energy Agcy & 27 & 1.81 \\
\hline 6 & Univ W Bohemia & 51 & 2.21 & 6 & Florida State Univ & 26 & 1.74 \\
\hline 7 & Hungarian Acad Sci & 48 & 2.08 & 7 & IAEA & 21 & 1.41 \\
\hline 8 & Korea Atom Energy Res Inst & 47 & 2.03 & 8 & Korea Atom Energy Res Inst & 21 & 1.41 \\
\hline 9 & Atom Energy Author & 43 & 1.86 & 9 & Atom Energy Author & 18 & 1.20 \\
\hline 10 & Prague Inst Chem Technol & 42 & 1.82 & 10 & Indira Gandhi Ctr Atom Res & 18 & 1.20 \\
\hline 11 & Natl Inst Radiol Sci & 38 & 1.65 & 11 & Zhejiang Univ & 18 & 1.20 \\
\hline 12 & Delft Univ Technol & 35 & 1.52 & 12 & Univ Missouri & 16 & 1.07 \\
\hline 13 & Ege Univ & 31 & 1.34 & 13 & Acad Sci Czech Republic & 15 & 1.00 \\
\hline 14 & Japan Atom Energy Agcy & 31 & 1.34 & 14 & CNR & 15 & 1.00 \\
\hline 15 & Univ Texas Austin & 31 & 1.34 & 15 & Ege Univ & 15 & 1.00 \\
\hline 16 & Natl Inst Stand \& Technol & 28 & 1.21 & 16 & Univ Sao Paulo & 15 & 1.00 \\
\hline 17 & Nucl Res Inst & 27 & 1.17 & 17 & Univ W Bohemia & 15 & 1.00 \\
\hline 18 & Univ Nacl Autonoma Mexico & 27 & 1.17 & & & & \\
\hline 19 & IPEN & 26 & 1.13 & & & & \\
\hline 20 & Lawrence Livermore Natl Lab & 25 & 1.08 & & & & \\
\hline 21 & Pakistan Inst Nucl Sci \& Technol & 25 & 1.08 & & & & \\
\hline 22 & IPEN CNEN SP & 24 & 1.04 & & & & \\
\hline 23 & Saha Inst Nucl Phys & 24 & 1.04 & & & & \\
\hline 24 & Univ Tokyo & 24 & 1.04 & & & & \\
\hline
\end{tabular}

Table 3 The h-core of source and target authors

\begin{tabular}{|c|c|c|c|c|c|c|c|}
\hline \multicolumn{4}{|c|}{ Source authors } & \multicolumn{4}{|c|}{ Target authors } \\
\hline Rank & Author & Count & $\%$ & Rank & Author & Count & $\%$ \\
\hline 1 & Makrlik, $E$ & 52 & 2.25 & 1 & Jalilian, AR & 24 & 1.61 \\
\hline 2 & Vanura, $P$ & 51 & 2.21 & 2 & Zhang, AY & 16 & 1.07 \\
\hline 3 & Freitas, MC & 31 & 1.34 & 3 & Burnett, WC & 15 & 1.00 \\
\hline 4 & Saiki, M & 28 & 1.21 & 4 & Vanura, $P$ & 15 & 1.00 \\
\hline 5 & Bacchi, MA & 26 & 1.13 & 5 & Makrlik, E & 14 & 0.94 \\
\hline 6 & Landsberger, S & 25 & 1.08 & 6 & Uchida, S & 14 & 0.94 \\
\hline 7 & Revay, Z & 23 & 1.00 & 7 & Chettle, DR & 13 & 0.87 \\
\hline 8 & Spyrou, NM & 23 & 1.00 & 8 & Pejovic-Milic, A & 13 & 0.87 \\
\hline 9 & Chai, ZF & 21 & 0.91 & 9 & Tagami, K & 13 & 0.87 \\
\hline 10 & Lahiri, S & 21 & 0.91 & 10 & Srinivasan, TG & 12 & 0.80 \\
\hline 11 & Selucky, P & 21 & 0.91 & 11 & Chai, $Z F$ & 11 & 0.74 \\
\hline 12 & Pacheco, AMG & 19 & 0.82 & 12 & Povinec, PP & 11 & 0.74 \\
\hline 13 & Belgya, T & 17 & 0.74 & 13 & Rao, PRV & 11 & 0.74 \\
\hline 14 & Tagliaferro, FS & 16 & 0.69 & 14 & Soylak, M & 11 & 0.74 \\
\hline 15 & Bode, $\mathrm{P}$ & 15 & 0.65 & 15 & Venkatesan, KA & 11 & 0.74 \\
\hline 16 & Chung, YS & 15 & 0.65 & & & & \\
\hline 17 & Ebihara, M & 15 & 0.65 & & & & \\
\hline 18 & Fernandes, EAD & 15 & 0.65 & & & & \\
\hline 19 & Unak, $\mathrm{P}$ & 15 & 0.65 & & & & \\
\hline 20 & Vasconcellos, MBA & 15 & 0.65 & & & & \\
\hline
\end{tabular}


Table 4 The h-core of journals citing JRNC

\begin{tabular}{llll}
\hline Rank & Title & Count & $\%$ \\
\hline 1 & Applied Radiation and Isotopes & 92 & 6.16 \\
2 & Radiochimica Acta & 56 & 3.75 \\
3 & Journal of Environmental Radioactivity & 54 & 3.61 \\
4 & Journal of Hazardous Materials & 41 & 2.74 \\
5 & Journal of Analytical Atomic Spectrometry & 39 & 2.61 \\
6 & Nuclear Instruments \& Methods in Physics Research Section A: & 38 & 2.54 \\
& Accelerators Spectrometers Detectors and Associated Equipment & & \\
7 & Nuclear Instruments \& Methods in Physics Research Section B: & 36 & 2.41 \\
& Beam Interactions with Materials and Atoms & & \\
8 & Radiation Protection Dosimetry & 22 & 1.47 \\
9 & Analytical Chemistry & 21 & 1.41 \\
10 & Analytica Chimica Acta & 20 & 1.34 \\
11 & Nukleonika & 16 & 1.07 \\
12 & Talanta & 15 & 1.00 \\
13 & Environmental Science \& Technology & 14 & 0.94 \\
14 & Science of the Total Environment & 14 & 0.94 \\
\hline
\end{tabular}

Table 5 The h-core of subject areas of the journals citing JRNC

\begin{tabular}{|c|c|c|c|}
\hline Rank & Subject area & Count & $\%$ \\
\hline 1 & Nuclear Science \& Technology & 342 & 22.89 \\
\hline 2 & Environmental Sciences & 264 & 17.67 \\
\hline 3 & Chemistry, Inorganic \& Nuclear & 207 & 13.86 \\
\hline 4 & Chemistry, Analytical & 172 & 11.51 \\
\hline 5 & $\begin{array}{l}\text { Radiology, Nuclear Medicine \& Medical } \\
\text { Imaging }\end{array}$ & 166 & 11.11 \\
\hline 6 & Chemistry, Multidisciplinary & 130 & 8.70 \\
\hline 7 & Spectroscopy & 124 & 8.30 \\
\hline 8 & Chemistry, Physical & 88 & 5.89 \\
\hline 9 & Physics, Nuclear & 87 & 5.82 \\
\hline 10 & Instruments \& Instrumentation & 80 & 5.35 \\
\hline 11 & Physics, Atomic, Molecular \& Chemical & 78 & 5.22 \\
\hline 12 & Engineering, Chemical & 70 & 4.69 \\
\hline 13 & Engineering, Environmental & 70 & 4.69 \\
\hline 14 & Materials Science, Multidisciplinary & 58 & 3.88 \\
\hline 15 & Physics, Particles \& Fields & 50 & 3.35 \\
\hline 16 & Geosciences, Multidisciplinary & 46 & 3.08 \\
\hline 17 & $\begin{array}{l}\text { Public, Environmental \& Occupational } \\
\text { Health }\end{array}$ & 45 & 3.01 \\
\hline 18 & Engineering, Civil & 44 & 2.95 \\
\hline 19 & Chemistry, Applied & 39 & 2.61 \\
\hline 20 & Oceanography & 34 & 2.28 \\
\hline 21 & Biochemical Research Methods & 32 & 2.14 \\
\hline 22 & Geochemistry \& Geophysics & 32 & 2.14 \\
\hline 23 & Water Resources & 31 & 2.08 \\
\hline 24 & Pharmacology \& Pharmacy & 30 & 2.01 \\
\hline 25 & Physics, Multidisciplinary & 27 & 1.81 \\
\hline
\end{tabular}

Obviously, physical and chemical areas are dominating the scene and, as already could be seen in the journal list of Table 4, JRNC information is utilized preeminently by environmental sciences (with related areas of earth and water sciences), as well. A negative lesson of the list is the striking lack of biological-biomedical sciences among the target fields - a lesson that should be seriously considered in the editorial policy of the journal.

Analysis of citations

The h-index of JRNC for the period under study was 13, i.e., there were 13 papers in the source dataset receiving at least 13 citations. These papers are listed in Table 6.

The target dataset received a total of 4,590 citations (3.07 citations per item). The h-index of the target dataset was 25 , i.e., there were 25 items among the papers citing at least one JRNC paper from the period under study receiving at least 25 citations. Short descriptions of these papers are listed in Table 7. A more detailed description of these papers can be found in the bibliography in the Appendix; for reference, the item \# is given in the last column.

The fact that the papers citing JRNC are apparently higher cited than JRNC papers themselves (whether measured by the average or the h-index) may be first thought to be an alarming sign, but it is not necessarily the case. It may also be regarded as the effect of the attention (manifested in the form of citations) to JRNC by papers in highprestige, high-impact journals (see the Nature, Chem. Rev., 
Table 6 The h-core of the most cited papers in the source dataset

\begin{tabular}{|c|c|c|c|c|}
\hline Rank & Authors & Title & Bibliographic data & $\begin{array}{l}\text { Times } \\
\text { cited }\end{array}$ \\
\hline 1 & $\begin{array}{l}\text { Dulaiova, H; Peterson, R; } \\
\text { Burnett, WC; et al. }\end{array}$ & $\begin{array}{l}\text { A multi-detector continuous monitor } \\
\text { for assessment of } \mathrm{Rn}-222 \text { in the coastal ocean }\end{array}$ & 263 (2005) 361-365 & 29 \\
\hline 2 & $\begin{array}{l}\text { Giridhar, P; Venkatesan, KA; } \\
\text { Srinivasan, TG; et al. }\end{array}$ & $\begin{array}{l}\text { Extraction of uranium(VI) from nitric acid medium } \\
\text { by } 1.1 \mathrm{M} \text { tri- } n \text {-butylphosphate in ionic liquid diluent }\end{array}$ & 265 (2005) 31-38 & 26 \\
\hline 3 & $\begin{array}{l}\text { James, WD; Hirsch, LR; } \\
\text { West, JL; et al. }\end{array}$ & $\begin{array}{l}\text { Application of INAA to the build-up and clearance } \\
\text { of gold nanoshells in clinical studies in mice }\end{array}$ & 271 (2007) 455-459 & 25 \\
\hline 4 & $\begin{array}{l}\text { Lee, SH; La Rosa, J; } \\
\text { Gastaud, J; et al. }\end{array}$ & $\begin{array}{l}\text { The development of sequential separation methods for the } \\
\text { analysis of actinides in sediments and biological materials } \\
\text { using anion-exchange resins and extraction chromatography }\end{array}$ & 263 (2005) 419-425 & 22 \\
\hline 5 & $\begin{array}{l}\text { Zhang, A; Wei, Y; } \\
\text { Kumagai, M }\end{array}$ & $\begin{array}{l}\text { Bleeding evaluation of the stationary phase from a few } \\
\text { novel macroporous silica-substrate polymeric } \\
\text { materials used for radionuclide partitioning } \\
\text { from HLLW in MAREC process }\end{array}$ & 265 (2005) 409-417 & 19 \\
\hline 6 & $\begin{array}{l}\text { Pike, SM; Buesseler, KO; } \\
\text { Andrews, J; et al. }\end{array}$ & $\begin{array}{l}\text { Quantification of Th- } 234 \text { recovery in small volume sea } \\
\text { water samples by inductively coupled plasma-mass } \\
\text { spectrometry }\end{array}$ & 263 (2005) 355-360 & 17 \\
\hline 7 & $\begin{array}{l}\text { Revay, Z; Belgya, T; } \\
\text { Molnar, GL }\end{array}$ & Application of Hypermet-PC in PGAA & 265 (2005) 261-265 & 15 \\
\hline 8 & $\begin{array}{l}\text { Freitas, MC; Farinha, MM; } \\
\text { Pacheco, AMG; et al. }\end{array}$ & $\begin{array}{l}\text { Atmospheric selenium in an industrialized } \\
\text { area of Portugal }\end{array}$ & 263 (2005) 711-719 & 15 \\
\hline 9 & Kilincarslan, A; Akyil, S & $\begin{array}{l}\text { Uranium adsorption characteristic and thermodynamic } \\
\text { behavior of clinoptilolite zeolite }\end{array}$ & 264 (2005) 541-548 & 14 \\
\hline 10 & Povinec, PP & $\begin{array}{l}\text { Ultra-sensitive radionuclide spectrometry: } \\
\text { Radiometrics and mass spectrometry synergy }\end{array}$ & 263 (2005) 413-417 & 14 \\
\hline 11 & $\begin{array}{l}\text { La Rosa, J; Gastaud, J; } \\
\text { Lagan, L; et al. }\end{array}$ & $\begin{array}{l}\text { Recent developments in the analysis } \\
\text { of transuranics }(\mathrm{Np}, \mathrm{Pu}, \mathrm{Am}) \text { in seawater }\end{array}$ & 263 (2005) 427-436 & 14 \\
\hline 12 & $\begin{array}{l}\text { Gmeling, K; Harangi, S; } \\
\text { Kasztovszky, Z }\end{array}$ & $\begin{array}{l}\text { Boron and chlorine concentration of volcanic rocks: } \\
\text { An application of prompt gamma activation analysis }\end{array}$ & 265 (2005) 201-212 & 13 \\
\hline 13 & $\begin{array}{l}\text { Szelecsényi, F; Kovács, Z; } \\
\text { Suzuki, K; et al. }\end{array}$ & $\begin{array}{l}\text { Production possibility of } \mathrm{Cu}-61 \text { using proton induced } \\
\text { nuclear reactions on zinc for PET studies }\end{array}$ & 263 (2005) 539-546 & 13 \\
\hline
\end{tabular}

Table 7 The h-core of the most cited papers in the target dataset

\begin{tabular}{|c|c|c|c|}
\hline Rank & Short bibliographic description & Times cited & Item \# in the Appendix \\
\hline 1 & LEWINSKI-N, SMALL, 4 (2008) 26-49 & 164 & $\# 1400$ \\
\hline 2 & QIAN-XM, NAT BIOTECHNOL, 26 (2008) 83-90 & 143 & $\# 1049$ \\
\hline 3 & BLAIN-S, NATURE, 446 (2007) 1070-U1 & 92 & \#1051 \\
\hline 4 & BINNEMANS-K, CHEM REV, 107 (2007) 2592-2614 & 84 & \#326 \\
\hline 5 & LANZ-VA, ATMOS CHEM PHYS, 7 (2007) 1503-1522 & 72 & \#230 \\
\hline 6 & ARRUEBO-M, NANO TODAY, 2 (2007) 22-32 & 65 & \#1043 \\
\hline 7 & BURNETT-WC, SCI TOTAL ENVIR, 367 (2006) 498-543 & 62 & \#1367 \\
\hline 8 & CHAN-CK, ATMOS ENVIRON, 42 (2008) 1-42 & 61 & \#232 \\
\hline 9 & DUMONT-E, ANAL BIOANAL CHEM, 385 (2006) 1304-1323 & 53 & \#42 \\
\hline 10 & FISCHER-HC, CURR OPIN BIOTECHNOL, 18 (2007) 565-571 & 43 & \#384 \\
\hline 11 & DIETZ-ML, SEPAR SCI TECHNOL, 41 (2006) 2047-2063 & 43 & \#1387 \\
\hline 12 & BACON-JR, ANALYST, 133 (2008) 25-46 & 40 & \#98 \\
\hline 13 & HERMAN-M, NUCL DATA SHEETS, 108 (2007) 2655-2715 & 38 & \#1056 \\
\hline 14 & SOYLAK-M, J HAZARD MATER, 137 (2006) 1496-1501 & 38 & \#821 \\
\hline 15 & WANG-JH, ANAL CHEM, 79 (2007) 620-625 & 37 & \#56 \\
\hline 16 & VAN DER LOEFF-MR, MAR CHEM, 100 (2006) 190-212 & 37 & \#986 \\
\hline 17 & YANG-XM, NANO LETT, 7 (2007) 3798-3802 & 35 & \#1041 \\
\hline
\end{tabular}


Table 7 continued

\begin{tabular}{llrr}
\hline Rank & Short bibliographic description & Times cited & Item \# in the Appendix \\
\hline 18 & DURRANT-SF, J ANAL ATOM SPECTROM, 20 (2005) 821-829 & 35 & $\# 642$ \\
19 & CAI-LS, EUR J ORG CHEM, (2008) 2853-2873 & 34 & \#473 \\
20 & LAL-S, ACCOUNT CHEM RES, 41 (2008) 1842-1851 & 33 & $\# 1$ \\
21 & KOESTER-CJ, ANAL CHEM, 77 (2005) 3737-3754 & 33 & 31 \\
22 & PIETRALLA-N, PROG PART NUCL PHYS, 60 (2008) 225-282 & 30 & $\# 1229$ \\
23 & BUTLER-OT, J ANAL ATOM SPECTROM, 21 (2006) 217-243 & 25 & $\# 645$ \\
24 & DU-Z, CHEM-EUR J, 13 (2007) 2130-2137 & 25 & $\# 329$ \\
25 & TUZEN-M, ANAL CHIM ACTA, 581 (2007) 241-246 & $\# 75$ \\
\hline
\end{tabular}

etc. papers in Table 7). In a recent paper [12], it was shown that the h-index of a set of citing papers (to a single paper or a set of papers, e.g., a journal) characterizes the "indirect citation impact" of the source set, i.e., its influence exerted through the presence in reference lists. Researchers frequently get acquainted with publications by finding them cited in other papers, and it is no secret, either, that "second-hand" informations are often cited without even consulting the original source. The set of citing papers, the target dataset, is in this sense the clue to a wider impact beyond direct visibility.

This significance of target dataset motivated us to compile a comprehensive bibliography of all papers citing JRNC papers published in the period 2005-2009 as a natural extension of the source dataset, the JRNC papers themselves.

\section{Appendix: A citation-based bibliography}

The bibliography presented here contains all papers citing at least one JRNC paper published in the period 20052009. Papers published in JRNC itself have been exluded from the analysis. The citation period ends at the date of the compilation, April 2010.

The items in the bibliography is sorted in the alphabetical order of the publishing journals; within a journal, items are ordered by publication date.

An identification number (\#) is assigned to each bibliographic item, which is used as reference in the indexes.

The bibliographic description of each item is followed by a $>>$ mark and the short bibliographic identifier (first author, volume, year, page number) of the cited JRNC paper(s).

The bibliography is supplemented with an Author Index and a Keyword Index. For the latter, author keywords indicated in the original paper have been used in reasonably standardized form. For about $25 \%$ of the papers in the bibliography no author keywords were provided. For these items relevant title words have been used as keywords.

\section{A1 Bibliography}

\#1 ACCOUNTS CHEM. RES. 41(2008)1842-1851

Lal, S; Clare, SE; Halas, NJ: Nanoshell-Enabled Photothermal Cancer Therapy: Impending Clinical Impact $>>$ JAMES WD 271(2007)455

\#2 ACCREDIT. QUAL. ASSUR. 13(2008)531-535

Korun, M; Vidmar, T; Vodenik, B: Improving the reliability of peak-evaluation results in gamma-ray spectrometry >> GLAVICCINDRO D 271(2007)467

\#3 ACS NANO 2(2008)2075-2084

Altinoglu, EI; Russin, TJ; Kaiser, JM; Barth, BM; Eklund, PC; Kester, M; Adair, JH: Near-Infrared Emitting Fluorophore-Doped Calcium Phosphate Nanoparticles for In Vivo Imaging of Human Breast Cancer $>>$ JAMES WD 271(2007)455

\#4 ACTA BIOMATER. 5(2009)1389-1398

Leibner, ES; Barnthip, N; Chen, WN; Baumrucker, CR; Badding, JV; Pishko, M; Vogler, EA: Superhydrophobic effect on the adsorption of human serum albumin $>>$ EFIMOVA YM 264(2005)91

\#5 ACTA CHIM. SLOV. 54(2007)273-283

Osterc, A; Jacimovic, R; Stibilj, V: Development of a method for I-129 determination using radiochemical neutron activation analysis >> KUCERA J 269(2006)251 \#6 ACTA CHIM. SLOV. 54(2007)375-377

Makrlik, E; Vanura, P: Extraction of zinc from water into nitrobenzene using strontium dicarbollylcobaltate in the presence of valinomycin $>$ MAKRLIK E 268(2006)155

\#7 ACTA CHIM. SLOV. 55(2008)111-119

Pomme, S; Sibbens, G: Alpha-particle counting and spectrometry in a primary standardisation laboratory $>>$ POMME S 277(2008)207

\#8 ACTA CHIM. SLOV. 55(2008)209-218

Sheikhan, N; Mirjalili, BF; Hajipour, A; Bamoniri, A: Ruthenium(III) chloride as an efficient catalyst for conversion of aldehydes to 1,1-diacetates under mild conditions >> DING HJ 268(2006)433 
\#9 ACTA CHIM. SLOV. 55(2008)430-433

Makrlik, E; Vanura, P; Selucky, P: Solvent extraction of $\mathrm{Ba} 2+, \mathrm{Pb} 2+$ and $\mathrm{Cd} 2+$ into nitrobenzene by using strontium dicarbollylcobaltate in the presence of tetraethyl p-tert-butylcalix[4]arene tetraacetate $>$ MAKRLIK E 268(2006)155

\#10 ACTA CHIM. SLOV. 55(2008)653-659

Repinc, U; Benedik, L: Development of the Procedure for Simultaneous Determination of Vanadium, Uranium and Manganese in Biological Materials Using RNAA >> REPINC U 264(2005)39

\#11 ACTA CHIM. SLOV. 56(2009)278-281

Makrlik, E; Budka, J; Vanura, P: Stability Constants of Some Univalent Cation Complexes of Tetra-tert-butyl p-tert-Butylcalix[4]arene Tetraacetate in Nitrobenzene Saturated with Water $>>$ MAKRLIK E 268(2006)155; MAKRLIK E 275(2008)217

\#12 ACTA CRYSTALLOGR. SECT. E.-STRUCT REP. ONLINE 63(2007)O4072-U3264

Liu, YH; Zhao, Y; Liu, XL; Tong, BW; Ye, J: (1S)-Benzyl 3-methyl-5-oxo-4-phenyl-hydrazono4,5-dihydro-1H-pyrazole1-dithiocarboxylate $>>$ BOSE R 265(2005)115 \#13 ACTA CRYSTALLOGR. SECT. E.-STRUCT REP. ONLINE 64(2008)M788-U524

Kawasaki, T; Kitazawa, T: Dioxidobis(pentane-2,4-dionato-kappa O-2, $\mathrm{O}^{\prime}$ )(pyridine-4-carbaldehyde oxime-kappa N-1)uranium(VI) >> SAEKI M 270(2006)379

\#14 ACTA CRYSTALLOGR. SECT. E.-STRUCT REP. ONLINE 64(2008)O1472-U2117

Li, SX; Li, HM; Lu, ZL; Fun, HK; Chantrapromma, S: O-(tert-butyldimethylsilyl)tris(O-4-methylphenylsulfonyl) pentaerythritol >> ABDELJALIL RJ 267(2006)557 \#15 ACTA CRYSTALLOGR. SECT. E.-STRUCT REP. ONLINE 64(2008)O152-U3874

Liu, XL; Zhao, Y; Li, ZG; Liu, YH: Methyl 3-methyl-5oxo-4-(phenylhydrazono)-4,5-dihydro-1H-pyrazole-1-carbodithioate $>>$ BOSE R 265(2005)115

\#16 ACTA CRYSTALLOGR. SECT. E.-STRUCT REP. ONLINE 64(2008)O1690-U1344

Li, SX; Zhu, L; Li, HM; Fun, HK; Chantrapromma, S: 1,2bis[2-(2-nitro-1H-imidazol-1-yl)ethoxy]ethane $>>$ ABDELJALIL RJ 267(2006)557

\#17 ACTA CRYSTALLOGR. SECT. E.-STRUCT REP. ONLINE 65(2009)O3167-U1248

Yang, Y; Zhu, L; Zhang, HB: Di-tert-butyl 2,2'-[(2hydroxyethyl)azanediyl]diacetate $>>$ YANG Y 273(2007) 31

\#18 ACTA CRYSTALLOGR. SECT. E.-STRUCT REP. ONLINE 66(2010)O709-U5797

Ding, YJ; Zhao, CX: 1-(4-Chlorophenyl)-3-phenyl-1Hpyrazol-5(4H)-one $>>$ BOSE R 265(2005)115
\#19 ACTA OCEANOL. SIN. 26(2007)73-83

Zeng, XZ; Qiu, MH; Yin, MD; Zeng, WY; He, JH; Wan, XG; Chen, HL; Li, J; Tan, BZ: An in-situ analysis and measurement of thorium-234, uranium isotopes in seawater $>>$ PIKE SM 263(2005)355

\#20 ACTA PHARM. 59(2009)45-55

Jalilian, AR; Rostampour, N; Rowshanfarzad, P; Shafaii, K; Kamali-Dehghan, M; Akhlaghi, M: Preclinical studies of [Cu-61]ATSM as a PET radiopharmaceutical for fibrosarcoma imaging >> JALILIAN AR 268(2006)605; JALILIAN AR 269(2006)147

\#21 ACTA PHARM. 59(2009)421-429

Jalilian, AR; Khoshdel, MR; Garousi, J; Yousefnia, H; Hosseini, MA; Rajabifar, S; Sardari, D: Development of a radiolabeled beta-human chorionic gonadotropin $>>$ JALILIAN AR 274(2007)175

\#22 ACTA PHYS. SLOVACA 58(2008)1-

Povinec, PP; Betti, M; Jull, AJT; Vojtyla, P: New isotope technologies in environmental physics $>>$ BRODZINSKI RL 264(2005)139; OSVATH I 263(2005)437; PERNA L 263(2005)367; POVINEC PP 263(2005)413; POVINEC PP 273(2007)383

\#23 ADSORPT. SCI. TECHNOL. 23(2005)721-737

Zhang, AY; Wei, YZ; Hoshi, H; Kumagai, M; Koma, Y: Leakage of octyl(phenyl)-N,N-di-isobutylcarbamoylmethylphosphine oxide from a macroporous silica-based chelating polymeric adsorption material and its recovery by some selected porous adsorbents $>>$ ZHANG A 265(2005)409 \#24 ADSORPT. SCI. TECHNOL. 25(2007)257-272

Zhang, AY; Mei, CM; Wei, YZ; Kumagai, M: Preparation of a novel macroporous silica-based diglycolamide derivative-impregnated polymeric composite and its adsorption mechanism for rare earth metal ions $>>$ ZHANG A 265(2005)409

\#25 ADSORPT. SCI. TECHNOL. 25(2007)285-293

Chen, L; Yu, XJ: Sorption of radiocaesium onto powdered and compacted rectorite studied by batch and capillary methods >> XU D 267(2006)357

\#26 ADSORPT. SCI. TECHNOL. 26(2008)705-720

Zhang, AY; Chen, CM; Chat, ZF; Kumagai, M: SPEC Process III. Synthesis of a Macroporous Silica-based Crown Ether-impregnated Polymeric Composite Modified with 1-Octanol and its Adsorption Capacity for Sr(II) Ions and Some Typical Co-existent Metal Ions $>>$ ZHANG A 265(2005)409

\#27 ADSORPT. SCI. TECHNOL. 27(2009)337-347

Yehia, A; Ezzat, K: Fluoride Ion Uptake by Synthetic Apatites $>>$ BADILLOALMARAZ VE 271(2007)741 \#28 ADSORPT.-J. INT. ADSORPT. SOC. 13(2007)61-71

Borai, EH; El-Dessouky, SI; Hassan, HS: Mixed silica and alumina hosted carboxylate oxide for removal of 
chromium species from wastewater $>>$ HASSAN SSM 269(2006)135

\#29 ADV. COLLOID INTERFACE SCI. 152(2009)2-13

Plazinski, W; Rudzinski, W; Plazinska, A: Theoretical models of sorption kinetics including a surface reaction mechanism: A review $>$ KAMEIISHIKAWA N 274(2007)555

\#30 ADV. INORGANIC CHEM. 61(2009)131-178

Pascu, SI; Waghorn, PA; Conry, T; Lin, B; James, C; Zayed, JM: DESIGN CONSIDERATIONS TOWARDS SIMULTANEOUSLY RADIOLABELED AND FLUORESCENT IMAGING PROBES INCORPORATING METALLIC SPECIES >> LI YG 265(2005)127

\#31 ADV. MATER. 21(2009)2334-

Troutman, TS; Leung, SJ; Romanowski, M: LightInduced Content Release from Plasmon-Resonant Liposomes $>>$ JAMES WD 271(2007)455

\#32 ADV. ORGANOMETALLIC CHEM. 57(2008)353447

Nagy, L; Pellerito, L; Fiore, T; Nagy, E; Pellerito, C; Szorcsik, A; Scopelliti, M: Equilibrium, structural and biological activity studies on [organotin(IV)] $(\mathrm{n}+)$ complexes >> NAGY L 275(2008)193

\#33 ADV. SYNTH. CATAL. 349(2007)841-845

Liegault, B; Renaud, JL; Bruneau, C: Palladium-catalyzed synthesis of functional tetralins via benzylic activation $>>$ LASETER AG 264(2005)723

\#34 ADV. SYNTH. CATAL. 350(2008)1544-1556

Polo, E; Forlini, F; Bertolasi, V; Boccia, AC; Sacchi, MC: Self-immobilizing precatalysts: Norbornene-bridged zirconium ansa-metallocenes $>>$ BERGAMASCHI L 263(2005)745; GIAVERI G 263(2005)725

\#35 AFR. J. BIOTECHNOL. 8(2009)1520-1527

Demirkeser, TH; Kaplankiran, M; Toplu, C; Agca, N; Yildiz, E; Serce, S: Comparison of several plant nutrient elements in conventionally and organically grown citrus orchards $>>$ TURRA C 270(2006)203

\#36 AGRIC. ECOSYST. ENVIRON. 136(2010)16-27

Pandey, VC; Singh, N: Impact of fly ash incorporation in soil systems >> PAPASTEFANOU C 275(2008)29

\#37 AGROCHIMICA 53(2009)405-417

Fraenzle, S; Hoffmann, V; Panaiotu, C; Jordanova, D; Jordanova, N; Djingova, R; Wuenschmann, S; Markert, B: Formation and determination of magnetite particles in biological samples for biomonitoring inputs of $\mathrm{Fe}$ and other heavy metals $>>$ FREITAS MC 270(2006)21

\#38 AM. FERN J. 99(2009)207-216

Fiori, CCL; Santos, M; Randi, AM: Aspects of Gametophyte Development of Dicksonia sellowiana Hook (Dicksoniaceae): an Endangered Tree Fern Indigenous to South and Central America $>$ DEFRANCA EJ 276(2008)221
\#39 AM. J. CLIN. NUTR. 85(2007)269S-276S

Yetley, EA: Multivitamin and multimineral dietary supplements: definitions, characterization, bioavailability, and drug interactions $>>$ VEATCH AE 264(2005)33 \#40 AM. J. IND. MED. 52(2009)742-750

Pejovic-Milic, A; Aslam; Chettle, DR; Oudyk, J; Pysklywec, MW; Haines, T: Bone Manganese as a Biomarker of Manganese Exposure: A Feasibility Study $>>$ BYUN SH 269(2006)615; PEJOVICMILIC A 269(2006)417

\#41 AM. J. PHYS. ANTHROPOL. 140(2009)399-409

Dolphin, AE; Goodman, AH: Maternal Diets, Nutritional Status, and Zinc in Contemporary Mexican Infants' Teeth: Implications for Reconstructing Paleodiets >> GHARIB AG 270(2006)209

\#42 ANAL. BIOANAL. CHEM. 385(2006)1304-1323

Dumont, E; Vanhaecke, F; Cornelis, R: Selenium speciation from food source to metabolites: a critical review >> VEATCH AE 264(2005)33

\#43 ANAL. BIOANAL. CHEM. 386(2006)1137-1151

Zeisler, R; Murphy, KE; Becker, DA; Davis, WC; Kelly, WR; Long, SE; Sieber, JR: Standard Reference Materials (R) (SRMs) for measurement of inorganic environmental contaminants >> LINDSTROM RM 263(2005)787; ZEISLER R 263(2005)315

\#44 ANAL. BIOANAL. CHEM. 388(2007)385-389

Merchel, S; Berger, A: Determination of nitrogen in boron carbide by instrumental photon activation analysis >> GORNER W 263(2005)791

\#45 ANAL. BIOANAL. CHEM. 389(2007)37-46

Dwyer, JT; Holden, J; Andrews, K; Roseland, J; Zhao, C; Schweitzer, A; Perry, CR; Harnly, J; Wolf, WR; Picciano, MF; Fisher, KD; Saldanha, LG; Yetley, EA; Betz, JM; Coates, PM; Milner, JA; Whitted, J; Burt, V; Radimer, K; Wilger, J; Sharpless, KE; Hardy, CJ: Measuring vitamins and minerals in dietary supplements for nutrition studies in the USA >> VEATCH AE 264(2005)33 \#46 ANAL. BIOANAL. CHEM. 390(2008)503-510

Gunther-Leopold, I; Kivel, N; Waldis, JK; Wernli, B: Characterization of nuclear fuels by ICP mass-spectrometric techniques $>>$ WOLF SF 263(2005)575; WOLF SF 263(2005)581

\#47 ANAL. BIOANAL. CHEM. 390(2008)1653-1658

Bode, P; Bueno, MIMS; Bortoleto, GG; Hoffmann, G; van den Ingh, TSGAM; Rothuizen, J: Neutron activation analysis and X-ray Rayleigh and Raman scattering of hair and nail clippings as noninvasive bioindicators for $\mathrm{Cu}$ liver status in Labrador Retrievers $>>$ FAVARO PC 264(2005)61

\#48 ANAL. BIOANAL. CHEM. 393(2009)809-833

Nerin, C; Salafranca, J; Aznar, M; Batlle, R: Critical review on recent developments in solventless techniques for extraction of analytes >> JINXIN G 275(2008)365 
\#49 ANAL. BIOANAL. CHEM. 394(2009)759-782

Ojeda, CB; Rojas, FS: Separation and preconcentration by a cloud point extraction procedure for determination of metals: an overview >> PEREZGRAMATGES A 269(2006)491 \#50 ANAL. BIOANAL. CHEM. 396(2010)1105-1114

Wang, LM; Li, YF; Zhou, LJ; Liu, Y; Meng, L; Zhang, $\mathrm{K}$; Wu, XC; Zhang, LL; Li, B; Chen, CY: Characterization of gold nanorods in vivo by integrated analytical techniques: their uptake, retention, and chemical forms $>>$ JAMES WD 271(2007)455

\#51 ANAL. CHEM. 77(2005)3737-3754

Koester, CJ; Moulik, A: Trends in environmental analysis $>>$ MARCHETTI AA 263(2005)483

\#52 ANAL. CHEM. 78(2006)2254-2261

Hughes, LD; DeVol, TA: Evaluation of flow cell detector configurations combining simultaneous preconcentration and scintillation detection for monitoring of pertechnetate in aqueous media $>$ EGOROV O 264(2005)495

\#53 ANAL. CHEM. 78(2006)4069-4096

Szaloki, I; Osan, J; Van Grieken, RE: X-ray spectrometry >> BEASLEY D 264(2005)289

\#54 ANAL. CHEM. 78(2006)5480-5490

Egorov, OB; O'Hara, MJ; Grate, JW: Equilibrationbased preconcentrating minicolumn sensors for trace level monitoring of radionuclides and metal ions in water without consumable reagents $>>$ ROANE JE 263(2005)51

\#55 ANAL. CHEM. 78(2006)5805-5812

Nishi, N; Murakami, H; Imakura, S; Kakiuchi, T: Facilitated transfer of alkali-metal cations by dibenzo18-crown-6 across the electrochemically polarized interface between an aqueous solution and a hydrophobic room-temperature ionic liquid $>>$ GIRIDHAR P 265 (2005) 31

\section{\#56 ANAL. CHEM. 79(2007)620-625}

Wang, JH; Cheng, DH; Chen, XW; Du, Z; Fang, ZL: Direct extraction of double-stranded DNA into ionic liquid 1-butyl-3-methylimidazolium hexafluorophosphate and its quantification $>>$ GIRIDHAR P 265(2005)31

\#57 ANAL. CHEM. 79(2007)3126-3134

Hou, XL; Ostergaard, LF; Nielsen, SP: Determination of Cl-36 in nuclear waste from reactor decommissioning $>>$ FRECHOU C 263(2005)333

\#58 ANAL. CHEM. 79(2007)4365-4384

Brettell, TA; Butler, JM; Almirall, JR: Forensic science $>$ BHADKAMBEKAR CA 266(2005)113; TAKATA MK 264(2005)5

\#59 ANAL. CHEM. 79(2007)8016-8023

Ikeda, A; Yaita, T; Okamoto, Y; Shiwaku, H; Suzuki, S; Suzuki, T; Fujii, Y: Extended X-ray absorption fine structure investigation of adsorption and separation phenomena of metal ions in organic resin $>>$ IKEDA A 263(2005)605; SUZUKI T 272(2007)257
\#60 ANAL. CHEM. 80(2008)801-807

Pandey, U; Dhami, PS; Jagesia, P; Venkatesh, M; Pillai, MRA: Extraction paper chromatography technique for the radionuclidic purity evaluation of Y-90 for clinical use $>>$ VANURA P 267(2006)501

\#61 ANAL. CHEM. 80(2008)4253-4267

Sherma, J: Planar chromatography $>$ ROOHI S 267(2006)561

\#62 ANAL. CHEM. 80(2008)4421-4454

Tsuji, K; Nakano, K; Hayashi, H; Hayashi, K; Ro, CU: X-ray spectrometry $>>$ KHUDER A 273(2007)435; YUE WS 274(2007)115

\#63 ANAL. CHEM. 80(2008)6066-6071

Revay, Z; Belgya, T; Szentmiklosi, L; Kis, Z; Wootsch, A; Teschner, D; Swoboda, M; Schlogl, R; Borsodi, J; Zepernick, R: In situ determination of hydrogen inside a catalytic reactor using prompt gamma activation analysis >> REVAY Z 265(2005)261; REVAY Z 276(2008)825

\#64 ANAL. CHEM. 81(2009)1068-1078

O'Hara, MJ; Burge, SR; Grate, JW: Quantification of Technetium-99 in Complex Groundwater Matrixes Using a Radiometric Preconcentrating Minicolumn Sensor in an Equilibration-Based Sensing Approach $>>$ EGOROV O 264(2005)495

\#65 ANAL. CHEM. 81(2009)1228-1237

O'Hara, MJ; Burge, SR; Grate, JW: Automated Radioanalytical System for the Determination of Sr-90 in Environmental Water Samples by Y-90 Cherenkov Radiation Counting >> BRODZINSKI RL 277(2008)235

\#66 ANAL. CHEM. 81(2009)1297-1306

Schwantes, JM; Douglas, M; Bonde, SE; Briggs, JD; Farmer, OT; Greenwood, LR; Lepel, EA; Orton, CR; Wacker, JF; Luksic, AT: Nuclear Archeology in a Bottle: Evidence of Pre-Trinity US Weapons Activities from a Waste Burial Site >> FARMER OT 276(2008)489

\#67 ANAL. CHEM. 81(2009)4695-4711

Brettell, TA; Butler, JM; Almirall, JR: Forensic Science >> DUFF MC 275(2008)579; HATZISTAVROS VS 277(2008)399

\#68 ANAL. CHEM. 81(2009)6851-6859

Revay, Z: Determining Elemental Composition Using Prompt, gamma Activation Analysis >> KASZTOVSZKY Z 278(2008)293; MARSCHALL HR 265(2005)339; PERRY DL 276(2008)273; REVAY Z 265(2005)261; REVAY Z 276(2008)825; SZENTMIKLOSI L 271(2007)447

\#69 ANAL. CHEM. 81(2009)8061-8067

Bhandari, D; Wells, SM; Retterer, ST; Sepaniak, MJ: Characterization and Detection of Uranyl Ion Sorption on Silver Surfaces Using Surface Enhanced Raman Spectroscopy $>>$ ORTIZOLIVEROS HB 279(2009)601

\#70 ANAL. CHEM. 81(2009)8185-8192

Qiao, JX; Hou, XL; Roos, P; Miro, M: Rapid Determination of Plutonium Isotopes in Environmental Samples 
Using Sequential Injection Extraction Chromatography and Detection by Inductively Coupled Plasma Mass Spectrometry >> HANG W 263(2005)467; JERNSTROM J 274(2007)95; LEE SH 263(2005)419; MAXWELL SL 275(2008)395; MAXWELL SL 275(2008)605; VARGA Z 274(2007)87

\#71 ANAL. CHEM. 81(2009)8327-8334

Varga, Z; Wallenius, M; Mayer, K; Keegan, E; Millett, S: Application of Lead and Strontium Isotope Ratio Measurements for the Origin Assessment of Uranium Ore Concentrates $>>$ BADAUT V 280(2009)57; SVEDKAUSKAITELEGORE J 278(2008)201

\#72 ANAL. CHIM. ACTA 570(2006)88-92

Tagami, K; Uchida, S; Hirai, I; Tsukada, H; Takeda, H: Determination of chlorine, bromine and iodine in plant samples by inductively coupled plasma-mass spectrometry after leaching with tetramethyl ammonium hydroxide under a mild temperature condition $>$ TSUKADA $\mathrm{H}$ 263(2005)773

\#73 ANAL. CHIM. ACTA 573(2006)445-452

Kamnev, AA; Tugarova, AV; Antonyuk, LP; Tarantilis, PA; Kulikov, LA; Perfiliev, YD; Polissiou, MG; Gardiner, PHE: Instrumental analysis of bacterial cells using vibrational and emission Mossbauer spectroscopic techniques >> PERFILIEV YD 266(2005)557

\#74 ANAL. CHIM. ACTA 579(2006)68-73

Jamali, MR; Assadi, Y; Shemirani, F; Hosseini, MRM; Kozani, RR; Masteri-Farahani, M; Salavati-Niasari, M: Synthesis of salicylaldehyde-modified mesoporous silica and its application as a new sorbent for separation, preconcentration and determination of uranium by inductively coupled plasma atomic emission spectrometry $>>$ KRISHNA PG 266(2005)251

\#75 ANAL. CHIM. ACTA 581(2007)241-246

Tuzen, M; Uluozlu, OD; Usta, C; Soylak, M: Biosorption of copper(II), lead(II), iron(III) and cobalt(II) on Bacillus sphaericus-loaded Diaion SP-850 resin >> OZEROGLU C 268(2006) 211

\#76 ANAL. CHIM. ACTA 587(2007)165-169

Varga, Z: Application of inductively coupled plasma sector field mass spectrometry for low-level environmental americium-241 analysis >> LEE SH 263(2005)419

\#77 ANAL. CHIM. ACTA 587(2007)263-271

Metilda, P; Gladis, JM; Venkateswaran, G; Rao, TP: Investigation of the role of chelating ligand in the synthesis of ion-imprinted polymeric resins on the selective enrichment of uranium(VI) >> PREETHA CR 267(2006)265 \#78 ANAL. CHIM. ACTA 590(2007)232-238

Tarancon, A; Garcia, JF; Rauret, G: First approach to radionuclide mixtures quantification by using plastic scintillators-Influence of the diameter of the plastic beads $>>$ BROWN SA 264(2005)505
\#79 ANAL. CHIM. ACTA 592(2007)101-105

Tagami, K; Uchida, S: Rapid uranium preconcentration and separation method from fresh water samples for total $\mathrm{U}$ and U-235/U-238 isotope ratio measurements by ICP-MS $>>$ LEE SH 263(2005)419; ROANE JE 263(2005)51 \#80 ANAL. CHIM. ACTA 599(2007)16-23

Varga, Z; Suranyi, G: Production date determination of uranium-oxide materials by inductively coupled plasma mass spectrometry $>>$ LAMONT SP 264(2005)423; VARGA Z 274(2007)87

\#81 ANAL. CHIM. ACTA 608(2008)105-139

Hou, XL; Roos, P: Critical comparison of radiometric and mass spectrometric methods for the determination of radionuclides in environmental, biological and nuclear waste samples $>$ ARGINELLI D 263(2005)275; BROWN SA 264(2005)505; EBAID YY 270(2006)609; EGOROV OB 263(2005)629; ELLIOT NL 267(2006)637; FRECHOU C 263(2005)333; GONZALES ER 263(2005) 457; JERSTROM J 274(2007)95; JIA GG 267(2006)505; KIESER WE 263(2005)375; LEE SH 263(2005)419; NORISUYE K 267(2005)183; POPOV L 269(2006)161; VARGA Z 274(2007)87

\#82 ANAL. CHIM. ACTA 619(2008)137-142

Moon, JH; Kim, SH; Chung, YS; Lee, YN; Kim, HJ; Kim, YE: Determination of the elemental contents in stream sediments collected from Cheongju city by instrumental neutron activation analysis $>$ CHUNG YS 267(2006)35; CHUNG YS 271(2007)339

\#83 ANAL. CHIM. ACTA 627(2008)184-197

Ouyang, G; Pawliszyn, J: A critical review in calibration methods for solid-phase microextraction $>>$ DUFF MC 275(2008)579

\#84 ANAL. CHIM. ACTA 631(2009)69-73

Memon, JR; Hallam, KR; Bhanger, MI; El-Turki, A; Allen, GC: Evaluation of sorption of uranium onto metakaolin using X-ray photoelectron and Raman spectroscopies $>$ DONAT R 265(2005)107; KONSTANTINOU M 273(2007)549; MAHRAMANLIOGLU M 273(2007)621 \#85 ANAL. CHIM. ACTA 633(2009)1-18

Szoboszlai, N; Polgari, Z; Mihucz, VG; Zaray, G: Recent trends in total reflection X-ray fluorescence spectrometry for biological applications $>$ KHUDER A 273(2007)435; VIVES AES 270(2006)231 \#86 ANAL. CHIM. ACTA 634(2009)135-152

Pedrero, Z; Madrid, Y: Novel approaches for selenium speciation in foodstuffs and biological specimens: A review $>>$ VEATCH AE 264(2005)33

\#87 ANAL. CHIM. ACTA 635(2009)6-21

Giussani, B; Monticelli, D; Rampazzi, L: Role of laser ablation-inductively coupled plasma-mass spectrometry in cultural heritage research: A review $>>$ JAMES WD 263(2005)697 
\#88 ANAL. CHIM. ACTA 635(2009)22-44

Rojas, FS; Ojeda, CB: Recent development in derivative ultraviolet/visible absorption spectrophotometry: 2004$2008>>$ ELSAYED AA 270(2006)629

\#89 ANAL. CHIM. ACTA 652(2009)66-84

Qiao, JX; Hou, XL; Miro, M; Roos, P: Determination of plutonium isotopes in waters and environmental solids: A review >> EFURD DW 263(2005)387; JERNSTROM J 274(2007)95; LEE SH 263(2005)419; NORISUYE K 267(2006)183; VARGA Z 274(2007)87

\#90 ANAL. CHIM. ACTA 652(2009)148-153

Alcalde-Molina, M; Ruiz-Jimenez, J; de Castro, MDL: Automated determination of mercury and arsenic in extracts from ancient papers by integration of solid-phase extraction and energy dispersive X-ray fluorescence detection using a lab-on-valve system $>>$ GREENBERG RR 278(2008)231

\#91 ANAL. CHIM. ACTA 658(2010)107-119

Dadfarnia, S; Shabani, AMH: Recent development in liquid phase microextraction for determination of trace level concentration of metals - a review $>>$ MALLAH MH 278(2008)97

\#92 ANAL. LETT. 41(2008)1494-1546

Ferreira, SLC; Pereira, PAD; Nobrega, JAN; Fatibello, O; Feres, MA; Reis, BF; Bruns, RE; de Aquino, FR: A glimpse of recent developments in Brazilian analytical chemistry $>$ ALMEIDA KCS 270(2006)93; BUCHMANN JH 270(2006)291; FAVARO DIT 269(2006)719; FIGUEIREDO AMG 269(2006)383; FLUES M 270(2006) 597; FRANCA EJ 271(2007)405; JUNIOR JAS 269(2006) 451; JUSTO J 269(2006)733; KFOURI PBP 265(2005) 459; MOREIRA S 270(2006)87; RIZZUTTO MA 269(2006)727; SAUEIA CH 264(2005)445; SILVA PSC 264(2005)449; SILVA PSC 269(2006)739; VIVES AES 270(2006) 231

\#93 ANAL. LETT. 41(2008)3074-3087

El-Ashgar, N; Abdel-Latif, M: Synthesis and Applications of a New Polysiloxane-Immobilized Macrocyclic Ligand System >> PREETHA CR 267(2006)265

\#94 ANAL. LETT. 43(2010)644-657

Satpati, AK; Sahoo, S; Ravindran, PV; Venkateswaran, G: Stripping voltammetric determination of uranium traces in sea water samples: effect of surfactants on the measurements >> ANDREOTTI E 277(2008)413

\#95 ANAL. METHODS 2(2010)49-53

Nomura, CS; Oliveira, PV: Method for cadmium and lead longitudinal profiles determination in hair by solid sampling graphite furnace atomic absorption spectrometry $>>$ SAIKI M 276(2008)53

\#96 ANAL. SCI. 23(2007)611-615

Zheng, J; Yamada, M: Precise determination of put isotopes in a Seawater reference material using ID-SF-ICP-MS combined with two-stage anion-exchange chromatography
>> LAROSA J 263(2005)427; NORISUYE K 267(2006) 183; PERNA L 263(2005)367

\#97 ANAL. SCI. 25(2009)881-885

Miura, T; Matsue, H; Kuroiwa, T; Chiba, K: Precise Determination of $\mathrm{Cr}$ and $\mathrm{Co}$ in Certified Reference Material of Silicon Nitride by Neutron Activation Analysis Using Internal Standardization $>>$ GREENBERG RR 278(2008)231

\#98 ANALYST 133(2008)25-46

Bacon, JR; Davidson, CM: Is there a future for sequential chemical extraction? >> DESIDERI D 267(2006)551 \#99 ANALYST 134(2009)966-972

Ahmed, MU; Saito, M; Hossain, MM; Rao, SR; Furui, S; Hino, A; Takamura, Y; Takagi, M; Tamiya, E: Electrochemical genosensor for the rapid detection of GMO using loop-mediated isothermal amplification $>>$ SANTOS LS 269(2006)505

\#100 ANN. CHIM. 96(2006)301-308

Bakraji, EH; Romeie, M; Issa, H: Radioisotope x-ray fluorescence analysis of ancient pottery from Tel Kouzama site in Damascus, Syria >> BAKRAJI EH 264(2005)645 \#101 ANN. NUCL. ENERGY 34(2007)953-957

Jonah, SA; Liaw, JR; Matos, JE: Monte Carlo simulation of core physics parameters of the Nigeria Research Reactor-1 (NIRR-1) >> JONAH SA 266(2005)83 \#102 ANN. NUCL. ENERGY 36(2009)1285-1286

Jonah, SA; Ibikunle, K; Li, Y: A feasibility study of LEU enrichment uranium fuels for MNSR conversion using MCNP >> JONAH SA 266(2005)83

\#103 ANN. NUCL. ENERGY 37(2010)241-247

Zamani-Valasiadou, M; Fragopoulou, M; Manolopoulou, M; Stoulos, S; Jokic, S; Sosnin, AN; Krivopustov, MI: Performance of a $\mathrm{Pb}$-spallation target surrounded by a Ublanket during irradiations with 1.6 and $2.5 \mathrm{GeV}$ deuteron beams: Comparison with relativistic proton beams $>>$ KRIVOPUSTOV MI 279(2009)567

\#104 ANN. NUCL. MED. 23(2009)843-848

Ogawa, K; Kawashima, H; Kinuya, S; Shiba, K; Onoguchi, M; Kimura, H; Hashimoto, K; Odani, A; Saji, H: Preparation and evaluation of Re-186/188-labeled antibody (A7) for radioimmunotherapy with rhenium(I) tricarbonyl core as a chelate site $>>$ XIA JY 279(2009)245 \#105 ANNU. REV. MED. 61(2010)359-373

Singhal, S; Nie, SM; Wang, MD: Nanotechnology Applications in Surgical Oncology $>$ JAMES WD 271(2007)455

\#106 ANTONIE VAN LEEUWENHOEK 96(2009)537-544

Dunkley, KD; Callaway, TR; O’Bryan, C; Kundinger, MM; Dunkley, CS; Anderson, RC; Nisbet, DJ; Crandall, PG; Ricke, SC: Cell yields and fermentation responses of a Salmonella Typhimurium poultry isolate at different dilution rates in an anaerobic steady state continuous culture >> DUNKLEY CS 276(2008)35 
\#107 APPL. CLAY SCI. 32(2006)172-178

Nemes, Z; Nagy, NM; Komlosi, A; Konya, J: The effect of mineral composition on the interaction of strontium ions with geological formations >> NEMES Z 266(2005)289 \#108 APPL. CLAY SCI. 38(2008)219-226

Yu, SM; Chen, CL; Chang, PP; Wang, TT; Lu, SS; Wang, XK: Adsorption of Th(IV) onto Al-pillared rectorite: Effect of $\mathrm{pH}$, ionic strength, temperature, soil humic acid and fulvic acid >> CHEN CL 273(2007)227; XU D 266(2005)419; XU D 267(2006)357; YU S 273(2007)129

\#109 APPL. CLAY SCI. 41(2008)37-46

Xu, D; Tan, XL; Chen, CL; Wang, XK: Adsorption of $\mathrm{Pb}$ (II) from aqueous solution to MX-80 bentonite: Effect of $\mathrm{pH}$, ionic strength, foreign ions and temperature $>>$ PATHAK PN 270(2006)299; XU D 267(2006)43

\#110 APPL. CLAY SCI. 42(2008)292-299

Hong, HL; Jiang, WT; Zhang, XL; Tie, LY; Li, ZH: Adsorption of $\mathrm{Cr}(\mathrm{VI})$ on STAC-modified rectorite $>>$ CHANG P 274(2007)153

\#111 APPL. CLAY SCI. 44(2009)21-26

Omar, H; Arida, H; Daifullah, A: Adsorption of Co-60 radionuclides from aqueous solution by raw and modified bentonite $>>$ ABDELRAHMAN KM 268(2006)221; DONAT R 265(2005)107; FILIPSKA H 270(2006)531; PATHAK PN 270(2006)299; TSAI S 258(2005)101

\#112 APPL. CLAY SCI. 44(2009)83-94

Tateo, F; Ravaglioli, A; Andreoli, C; Bonina, F; Coiro, V; Degetto, S; Giaretta, A; Orsini, AM; Puglia, C; Summa, $\mathrm{V}$ : The in-vitro percutaneous migration of chemical elements from a thermal mud for healing use $>>$ PARR RM 270(2006) 155

\#113 APPL. GEOCHEM. 21(2006)715-723

Sheppard, PR; Speakman, RJ; Ridenour, G; Witten, ML: Reply to comment on "Elevated tungsten and cobalt in airborne particulates in Fallon, Nevada: Possible implications for the childhood leukemia cluster", by R. Seiler $>>$ RAJPUT MU 266(2005)343

\#114 APPL. GEOCHEM. 21(2006)1362-1375

Steinhauser, G; Sterba, JH; Bichler, M; Huber, H: Neutron activation analysis of Mediterranean volcanic rocks-An analytical database for archaeological stratigraphy >> STEINHAUSER G 267(2005)3

\#115 APPL. GEOCHEM. 21(2006)1681-1691

Pearson, CL; Manning, SW; Coleman, M; Jarvis, K: A dendrochemical study of Pinus sylvestris from Siljansfors Experimental Forest, central Sweden >> UNLU K 264 (2005) 21

\#116 APPL. GEOCHEM. 23(2008)1127-1139

Geraedts, K; Maes, A: Determination of the conditional interaction constant between colloidal technetium(IV) and Gorleben humic substances >> LIU DJ 264(2005)691
\#117 APPL. GEOCHEM. 23(2008)3545-3560

Neculita, CM; Zagury, GJ; Bussiere, B: Effectiveness of sulfate-reducing passive bioreactors for treating highly contaminated acid mine drainage: II. Metal removal mechanisms and potential mobility $>$ PALAGYI S 269(2006)103

\#118 APPL. GEOCHEM. 23(2008)3589-3596

Steinberg, SM; Buck, B; Morton, J; Dorman, J: The speciation of iodine in the salt impacted Black Butte soil series along the Virgin river, Nevada, USA $>>$ STEINBERG SM 277(2008)175; STEINBERG SM 277(2008)185 \#119 APPL. GEOCHEM. 25(2010)345-356

Le Druillennec, T; Lelsch, G; Bour, O; Tarits, C; Tymen, G; Alcalde, G; Aquilina, L: Hydrogeological and geochemical control of the variations of Rn-222 concentrations in a hard rock aquifer: Insights into the possible role of fracture-matrix exchanges >> XINWEI L 267(2006)669 \#120 APPL. OPTICS 48(2009)D96-D108

Kah, JCY; Chow, TH; Ng, BK; Razul, SG; Olivo, M; Sheppard, CJR: Concentration dependence of gold nanoshells on the enhancement of optical coherence tomography images: a quantitative study >> JAMES WD 271(2007)455 \#121 APPL. PHYS. A-MATER. SCI. PROCESS. 97(2009) $11-18$

Mayoral, A; Vazquez-Duran, A; Barron, H; Jose-Yacaman, M: Polyhedral shaped gold nanoparticles with outstanding near-infrared light absorption >> JAMES WD 271(2007)455 \#122 APPL. PHYS. LETT. 93(2008)94104-

Zitnik, M; Pelicon, P; Grlj, N; Karydas, AG; Sokaras, D; Schutz, R; Kanngiesser, B: Three-dimensional imaging of aerosol particles with scanning proton microprobe in a confocal arrangement >> ZITNIK M 275(2008)17 \#123 APPL. PHYS. LETT. 95(2009)233701-

DasGupta, D; von Maltzahn, G; Ghosh, S; Bhatia, SN; Das, SK; Chakraborty, S: Probing nanoantenna-directed photothermal destruction of tumors using noninvasive laser irradiation $>>$ JAMES WD 271(2007)455

\#124 APPL. RADIAT. ISOT. 63(2005)37-40

Alfassi, ZB; Groppi, F; Bonardi, ML; de Goeij, JJM: On the "artificial" nature of Tc and the "carrier-free" nature of Tc-99m from Mo-99/Tc-99m generators. >> DEGOEIJ JJM 263(2005)13 \#125 APPL. RADIAT. ISOT. 63(2005)705-710

Martinez, T; Martinez, G; Mendoza, D; Juarez, F; Cabrera, L: Electronic microscopy and EDX characterization of teotihuacan prehispanic mortar from the cave under the sun pyramid $>>$ MARTINEZ T 264(2005)511 \#126 APPL. RADIAT. ISOT. 64(2006)337-341

Jalilian, AR; Rowshanfarzad, P; Sabet, M; Shafiee, A: Preparation of [Cu-61]-2-acetylpyridine thiosemicarbazone complex as a possible PET tracer for malignancies $>>$ SZELECSENYI F 263(2005)539 
\#127 APPL. RADIAT. ISOT. 64(2006)778-788

Biber, FZ; Unak, P; Ertay, T; Medine, EI; Zihnioglu, F; Tasci, C; Durak, H: Synthesis of an estradiol glucuronide derivative and investigation of its radiopharmaceutical potential >> BIBER FZ 266(2005)445

\#128 APPL. RADIAT. ISOT. 64(2006)789-791

Szelecsenyi, F; Steyn, GF; Kovacs, Z; van der Walt, TN; Suzuki, K: Comments on the feasibilty of $\mathrm{Cu}-61$ production by proton irradiation of (na)tZn on a medical cyclotron $>>$ SZELECSENYI F 263(2005)539

\#129 APPL. RADIAT. ISOT. 64(2006)812-814

Zeevaart, JR; Olsen, S: Recent trends in the concept of specific activity: Impact on radiochemical and radiopharmaceutical producers $>>$ DEGOEIJ JJM 263(2005)13

\#130 APPL. RADIAT. ISOT. 64(2006)818-822

Jonah, SA; Umar, IM; Adipo, MOA; Balogun, GI; Adeyemo, DJ: Standardization of NIRR-1 irradiation and counting facilities for instrumental neutron activation analysis $>>$ JONAH SA 266(2005)83

\#131 APPL. RADIAT. ISOT. 64(2006)1563-1573

Rowshanfarzad, P; Sabet, M; Jahlian, AR; Kamalidehghan, M: An overview of copper radionuclides and production of $\mathrm{Cu}-61$ by proton irradiation of Zn-nat at a medical cyclotron $>>$ SZELECSENYI F 263(2005)539

\#132 APPL. RADIAT. ISOT. 65(2007)1-8

Jia, GG; Torri, G: Determination of Pb-210 and Po-210 in soil or rock samples containing refractory matrices $>>$ BROWN SA 264(2005)505

\#133 APPL. RADIAT. ISOT. 65(2007)155-163

Chen, CL; Wang, XK: Sorption of Th (IV) to silica as a function of $\mathrm{pH}$, humic/fulvic acid, ionic strength, electrolyte type >> XU D 266(2005)419

\#134 APPL. RADIAT. ISOT. 65(2007)234-238

Pelled, O; Bonardi, ML; German, U; Groppi, F; Alfassi, ZB: Improved determination of the position and activity of a radioactive point source inside a bulky medium using several detectors. The special case of a lung counter-A general view $>>$ ALFASSI ZB 266(2005)245

\#135 APPL. RADIAT. ISOT. 65(2007)267-279

Matthews, KM; Kim, CK; Martin, P: Determination of Po-210 in environmental materials: A review of analytical methodology >> BROWN SA 264(2005)505

\#136 APPL. RADIAT. ISOT. 65(2007)280-286

Mishra, SP; Prasad, SK; Dubey, RS; Mishra, M; Tiwari, D; Lee, SM: Biosorptive behaviour of rice hulls for Cs-134 from aqueous solutions: A radiotracer study $>>$ MISHRA SP 268(2006)191

\#137 APPL. RADIAT. ISOT. 65(2007)413-418

Koarashi, J; Saito, F; Akiyama, K; Rahman, NM; Iida, T: A new digital autoradiographical method for identification of $\mathrm{Pu}$ particles using an imaging plate $>>$ KERKAPOLY A 265(2005)423
\#138 APPL. RADIAT. ISOT. 65(2007)488-503

Steinhauser, G; Sterba, JH; Bichler, M: Chemical fingerprints of pumice from Cappadocia (Turkey) and Kos (Greece) for archaeological applications $>>$ STEINHAUSER G 267(2005)3

\#139 APPL. RADIAT. ISOT. 65(2007)764-768

Csikai, J; Doczi, R: A comparison of the neutron thermalization and reflection methods used for bulk hydrogen analysis >> DOCZI R 266(2005)11

\#140 APPL. RADIAT. ISOT. 65(2007)849-857

Jia, GG; Torri, G: Estimation of radiation doses to members of the public in Italy from intakes of some important naturally occurring radionuclides (U-238, U-234, U-235, Ra-226, Ra-228, Ra-224 and Po-210) in drinking water $>>$ JIA GG 267(2006)505

\#141 APPL. RADIAT. ISOT. 65(2007)1095-1100

Varga, Z: Preparation and characterization of manganese dioxide impregnated resin for radionuclide pre-concentration $>>$ MAXWELL SL 270(2006)651

\#142 APPL. RADIAT. ISOT. 65(2007)1125-1133

Chichester, DL; Pierce, GD: Analysis of a shield design for a DT neutron generator test facility $>>$ SHYPALIO RJ 263(2005)759

\#143 APPL. RADIAT. ISOT. 65(2007)1134-1139

Lu, J; Kong, DJ; Yang, Z; Yang, SY; Fan, WW; Jia, HM; Wang, XB: Preparation of Tc-99m-nitrido asymmetrical heterocomplex with 4-(cyclohexylpiperazin-1-yl)dithioformate and its biological evaluation as a potential myocardial imaging agent $>$ CHU JF 269(2006) 175

\#144 APPL. RADIAT. ISOT. 65(2007)1193-1201

Fawdry, RM: Radiolysis of 2-[F-18]fluoro-2-deoxy-Dglucose (FDG) and the role of reductant stabilisers $>>$ BURIOVA E 264(2005)595

\#145 APPL. RADIAT. ISOT. 65(2007)1227-1231

Shen, B; Loffler, D; Zeller, KP; Ubele, M; Reischl, G; Machulla, HJ: Decarbonylation of multi-substituted [F18]benzaldehydes for modelling syntheses of F-18-labelled aromatic amino acids >> ALLABADI A 270(2006)313 \#146 APPL. RADIAT. ISOT. 65(2007)1249-1264

Broeders, CHM; Konobeyev, AY: Systematics of (p,alpha) (p,n alpha), and (p,np) reaction cross-sections $>>$ SZELECSENYI F 263(2005)539

\#147 APPL. RADIAT. ISOT. 66(2008)1-8

Steinhauser, G; Bichler, M: Adsorption of ions onto high silica volcanic glass $>>$ STEINHAUSER G 267(2005)3

\#148 APPL. RADIAT. ISOT. 66(2008)103-107

Yamada, M; Zheng, H: Determination of Pu-240/Pu-239 atom ratio in coastal surface seawaters from the western North Pacific Ocean and Japan Sea $>>$ NORISUYE K 267(2006)183; ZHENG J 267(2005)73 
\#149 APPL. RADIAT. ISOT. 66(2008)178-187

Muftuler, FZB; Unak, P; Teksoz, S; Acar, C; Yolcular, S; Yurekli, Y: I-131 labeling of tamoxifen and biodistribution studies in rats $>>$ ENGINAR H 264(2005)535 \#150 APPL. RADIAT. ISOT. 66(2008)236-246

Kulahci, F; Sen, Z: Multivariate statistical analyses of artificial radionuclides and heavy metals contaminations in deep mud of Keban Dam Lake, Turkey $>>$ KULAHCI F 268(2006)517

\#151 APPL. RADIAT. ISOT. 66(2008)653-667

Skakun, Y; Qaim, SM: Measurement of excitation functions of helion-induced reactions on enriched $\mathrm{Ru}$ targets for production of medically important $\mathrm{Pd}-103$ and $\mathrm{Rh}$ $101 \mathrm{~m}$ and some other radionuclides $>$ DITROI $\mathrm{F}$ 272(2007)231

\#152 APPL. RADIAT. ISOT. 66(2008)705-710

Sima, O; Arnold, D: A tool for processing decay scheme data that encompasses coincidence summing calculations $>>$ BERLIZOV AN 264(2005)169

\#153 APPL. RADIAT. ISOT. 66(2008)823-828

Lee, SH; Povinec, PP; Wyse, E; Hotchkis, MAC: Ultralow-level determination of U-236 in IAEA marine reference materials by ICPMS and AMS $>$ LEE SH 263(2005)419; POVINEC PP 273(2007)383

\#154 APPL. RADIAT. ISOT. 66(2008)835-840

Inn, KGW; Kurosaki, H; Frechou, C; Gilligan, C; Jones, R; LaMont, S; Leggitt, J; Li, C; McCroan, K; Swatski, R: A blueprint for radioanalytical metrology CRMs, intercomparisons, and PE >> INN KGW 269(2006)351

\#155 APPL. RADIAT. ISOT. 66(2008)860-864

Klouda, GA: Investigating the background of a 1-cm(3) quartz gas proportional counter $>$ BAND $\mathrm{AH}$ 276(2008)657

\#156 APPL. RADIAT. ISOT. 66(2008)1138-1145

Liao, HQ; Zheng, J; Wu, FC; Yamada, M; Tan, MG; Chen, JM: Determination of plutonium isotopes in freshwater lake sediments by sector-field ICP-MS after separation using ion-exchange chromatography $>>$ ELLIOT NL 267(2006)637; ZHENG J 267(2005)73

\#157 APPL. RADIAT. ISOT. 66(2008)1183-1189

Wang, TH; Li, MH; Teng, SP: Cs diffusion in local Taiwan laterite with different solution concentration, $\mathrm{pH}$ and packing density $>>$ XU D 267(2006)357

\#158 APPL. RADIAT. ISOT. 66(2008)1300-1306

Medvedev, DG; Mausner, LF; Greene, GA; Hanson, $\mathrm{AL}$ : Activation of natural $\mathrm{Hf}$ and $\mathrm{Ta}$ in relation to the production of Lu-177 >> KNAPP FF 263(2005)503 \#159 APPL. RADIAT. ISOT. 66(2008)1321-1324

Takamiya, K; Ota, Y; Akamine, M; Shibata, S; Shibata, T; Ito, Y; Imamura, M; Uwamino, Y; Nogawa, N; Baba, $\mathrm{M}$; Iwasaki, S; Matsuyama, S: Excitation function for $\mathrm{Cu}-$ $63(n, p) N i-63$ reaction in neutron energy range up to $15 \mathrm{MeV}>>$ SHIBATA S 273(2007)517
\#160 APPL. RADIAT. ISOT. 66(2008)1325-1336

Daga, R; Guevara, SR; Sanchez, ML; Arribere, M: Source identification of volcanic ashes by geochemical analysis of well preserved lacustrine tephras in Nahuel Huapi National Park $>$ DAGA R 270(2006)677; GUEVARA R 265(2005)481

\#161 APPL. RADIAT. ISOT. 66(2008)1359-1362

O'Dwyer, JN; Tickner, JR: Quantitative mineral phase analysis of dry powders using energy-dispersive X-ray diffraction $>>$ LIM CS 264(2005)15

\#162 APPL. RADIAT. ISOT. 66(2008)1377-1380

Jonah, SA; Ibrahim, YV; Akaho, EHK: The determination of reactor neutron spectrum-averaged cross-sections in miniature neutron source reactor facility $>>$ JONAH SA 266(2005)83

\#163 APPL. RADIAT. ISOT. 66(2008)1396-1402

Guo, N; Alagille, D; Tamagnan, G; Price, RR; Baldwin, RM: Microwave-induced nucleophilic [F-18]fluorination on aromatic rings: Synthesis and effect of halogen on [F18]fluoride substitution of meta-halo ( $\mathrm{F}, \mathrm{Cl}, \mathrm{Br}, \mathrm{I})$-benzonitrile derivatives $>>$ ALLABADI A 270(2006)313 \#164 APPL. RADIAT. ISOT. 66(2008)1419-1426

Tsabaris, C; Bagatelas, C; Dakladas, T; Papadopoulos, CT; Vlastou, R; Chronis, GT: An autonomous in situ detection system for radioactivity measurements in the marine environment $>>$ OSVATH I 263(2005)437 \#165 APPL. RADIAT. ISOT. 66(2008)1478-1487

Jia, GG; Torri, G; Ocone, R; Di Lullo, A; De Angelis, A; Boschetto, R: Determination of thorium isotopes in mineral and environmental water and soil samples by alpha-spectrometry and the fate of thorium in water $>>$ HOLLRIEGL V 266(2005)441

\#166 APPL. RADIAT. ISOT. 66(2008)1623-1626

Talha, SA; Lindsay, R; Newman, RT; de Meijer, RJ; Maleka, PP; Hlatshwayo, IN; Mlwilo, NA; Mohanty, AK: gamma-Ray spectrometry of radon in water and the role of radon to representatively sample aquifers $>>$ DULAIOVA H 263(2005)361

\#167 APPL. RADIAT. ISOT. 66(2008)1675-1678

Hirose, K; Igarashi, Y; Aoyama, M: Analysis of the 50year records of the atmospheric deposition of long-lived radionuclides in Japan $>>$ HIROSE K 273(2007)115 \#168 APPL. RADIAT. ISOT. 66(2008)1711-1717

Pham, MK; Sanchez-Cabeza, JA; Povinec, PR; Andor, K; Arnold, D; Benmansour, M; Bikit, I; Carvalho, FP; Dimitrova, K; Edrev, ZH; Engeler, C; Fouche, FJ; GarciaOrellana, J; Gasco, C; Gastaud, J; Gudelis, A; Hancock, G; Holm, E; Legarda, F; Ikaheimonen, TK; Ilchmann, C; Jenkinson, AV; Kanisch, G; Kis-Benedek, G; Kleinschmidt, R; Koukouliou, V; Kuhar, B; LaRosa, J; Lee, SH; LePetit, G; Levy-Palomo, I; Kwong, LLW; Llaurado, M; Maringer, FJ; Meyer, M; Michalik, B; Michel, H; Nies, H; Nour, S; Oh, JS; Oregioni, B; Palomares, J; Pantelic, G; Pfitzner, J; 
Pilvio, R; Puskeiler, L; Satake, H; Schikowski, J; Vitorovic, G; Woodhead, D; Wyse, E: A new Certified Reference Material for radionuclides in Irish sea sediment (IAEA-385) >> POVINEC PP 273(2007)383

\#169 APPL. RADIAT. ISOT. 66(2008)1810-1813

Sadeghi, M; Shirazi, B: Extraction separation of no-carrier-added Pd-103 from irradiated $\mathrm{Rh}$ target, $\mathrm{Cu}$ and $\mathrm{Zn}$ using alpha-furyldioxime, dimethylglyoxime and alphabenzildioxime $>>$ SADEGHI M 265(2005)455

\#170 APPL. RADIAT. ISOT. 66(2008)1870-1872

Doczi, R; Csikai, J: An improved method for bulk hydrogen analysis using epithermal neutrons $>>$ DOCZI R 266(2005)11

\#171 APPL. RADIAT. ISOT. 66(2008)1901-1904

Lahiri, S; Roy, K; Sen, S: Complexation study on no-carrier-added astatine with insulin: A candidate radiopharmaceutical $>>$ LIU N 272(2007)85

\#172 APPL. RADIAT. ISOT. 66(2008)1913-1915

Park, K; Kang, N; Cho, K; Lee, J: Determination of Cd and $\mathrm{Cr}$ in an ABS candidate reference material by instrumental neutron activation analysis $>$ ZEISLER $\mathrm{R}$ 263(2005)315

\#173 APPL. RADIAT. ISOT. 66(2008)1916-1919

Mokobia, CE; Ogundare, FO; Inyang, EP; Balogun, FA; Jonah, SA: Determination of the elemental constituents of a natural dolerite using NIRR-1 $>$ JONAH SA 266(2005)83

\#174 APPL. RADIAT. ISOT. 66(2008)1945-1947

Sawant, PD; Prabhu, SP; Kalsi, PC: Application of alpha track registration technique for plutonium estimation in bioassay samples $>>$ SAWANT PD 274(2007)67

\#175 APPL. RADIAT. ISOT. 67(2009)7-10

Sadeghi, M; Aboudzadeh, M; Zali, A; Mirzaii, M; Bolourinovin, F: Radiochemical studies relevant to Y-86 production via $\mathrm{Sr}-86(\mathrm{p}, \mathrm{n}) \mathrm{Y}-86$ for $\mathrm{PET}$ imaging $>>$ AARDANEH K 270(2006)641

\#176 APPL. RADIAT. ISOT. 67(2009)14-20

Plionis, AA; Gonzales, ER; Landsberger, S; Peterson, DS: Evaluation of flow scintillation analysis for the determination of Sr-90 in bioassay samples >> FJELD RA 263(2005)635; GRATE J 263(2005)629

\#177 APPL. RADIAT. ISOT. 67(2009)21-29

Fosterova, M; Petrik, M; Laznickova, A; Laznicek, M; Hermann, P; Lukes, I; Melichar, F: Complexation and biodistribution study of In-111 and Y-90 complexes of bifunctional phosphinic acid analogs of $\mathrm{H}(4)$ dota $>>$ LAZNICKOVA A 273(2007)583

\#178 APPL. RADIAT. ISOT. 67(2009)70-72

Csikai, J; Doczi, R: Optimization of source-sampledetector geometries for bulk hydrogen analysis using epithermal neutrons >> DOCZI R 266(2005)11
\#179 APPL. RADIAT. ISOT. 67(2009)79-82

Zhang, JB; Lin, Y; Sheng, X; Wang, XB: Synthesis of a novel Tc-99m nitrido radiopharmaceutical with isopropyl xanthate, showing brain uptake >> GUO HX 275(2008)121 \#180 APPL. RADIAT. ISOT. 67(2009)212-219

Kulahci, F; Inceoz, M; Dogru, M; Aksoy, E; Baykara, $\mathrm{O}$ : Artificial neural network model for earthquake prediction with radon monitoring $>>$ KULAHCI $\mathrm{F}$ 269(2006)63

\#181 APPL. RADIAT. ISOT. 67(2009)378-386

Kacperek, A: Protontherapy of eye tumours in the UK: A review of treatment at Clatterbridge $>>$ KACPEREK A 271(2007)731

\#182 APPL. RADIAT. ISOT. 67(2009)650-653

Chao, JH; Chuang, CY; Yeh, SA; Wu, JM: Relationship between radioactivity of radium and concentrations of barium and lead in hokutolite $>>$ SAITO T 272(2007)443; TOMITA J 270(2006)567

\#183 APPL. RADIAT. ISOT. 67(2009)696-700

Zhu, H; Venkataraman, R; Mueller, W; Lamontagne, J; Bronson, F; Morris, K; Berlizov, A: X-ray true coincidence summing correction in Genie $2000>>$ RUSS W 264(2005)193

\#184 APPL. RADIAT. ISOT. 67(2009)701-705

Sima, O; Arnold, D: On the Monte Carlo simulation of HPGe gamma-spectrometry systems $>>$ BERLIZOV AN 276(2008)663

\#185 APPL. RADIAT. ISOT. 67(2009)786-793

Kim, CK; Al-Hamwi, A; Torvenyi, A; Kis-Benedek, G; Sansone, U: Validation of rapid methods for the determination of radiostrontium in milk $>$ CHOBOLA $R$ 267(2006)297

\#186 APPL. RADIAT. ISOT. 67(2009)990-995

Jacobson, MS; Dankwart, HR; Mahoney, DW: Radiolysis of 2-[F-18]fluoro-2-deoxy-D-glucose ([F-18]FDG) and the role of ethanol and radioactive concentration $>>$ BURIOVA E 264(2005)595

\#187 APPL. RADIAT. ISOT. 67(2009)1007-1012

Song, XP; Wang, SW; Chen, L; Zhang, ML; Dong, YH: Effect of $\mathrm{pH}$, ionic strength and temperature on the sorption of radionickel on Na-montmorillonite $>>$ CHEN CL 273(2007)227

\#188 APPL. RADIAT. ISOT. 67(2009)1013-1022

Chichester, DL; Seabury, EH; Zabriskie, JM; Wharton, J; Caffrey, AJ: Dose profile modeling of Idaho National Laboratory's active neutron interrogation laboratory $>>$ SHYPALIO RJ 263(2005)759

\#189 APPL. RADIAT. ISOT. 67(2009)1084-1088

Brockman, JD; Robertson, JD: Analysis of k(0) neutron activation analysis at the University of Missouri Research Reactor >> VERMAERCKE P 276(2008)235 
\#190 APPL. RADIAT. ISOT. 67(2009)1121-1126

Chao, JH; Liu, MT; Yeh, SA; Huang, SS; Wu, JM; Chang, YL; Hsu, FY; Chuang, CY; Liu, HY; Sun, YC: Using medical accelerators and photon activation to determine $\mathrm{Sr} / \mathrm{Ca}$ concentration ratios in teeth $>>$ RANDA Z 271(2007)589 \#191 APPL. RADIAT. ISOT. 67(2009)1259-1268

Mourad, NM; Sharshar, T; Elnimr, T; Mousa, MA: Radioactivity and fluoride contamination derived from a phosphate fertilizer plant in Egypt >> SAUEIA CH 264(2005)445

\#192 APPL. RADIAT. ISOT. 67(2009)1320-1323

van der Meulen, NP; van der Walt, TN; Steyn, GF; Szelecsenyi, F; Kovacs, Z; Perrang, CM; Raubenheimer, HG: The production of Y-88 in the proton bombardment of Sr-nat: New excitation and separation studies $>>$ AARDANEH K 270(2006)641

\#193 APPL. RADIAT. ISOT. 67(2009)1392-1396

Sadeghi, M; Aboudzadeh, M; Zali, A; Zeinali, B: Y-86 production via $\mathrm{Sr}-86(\mathrm{p}, \mathrm{n})$ for PET imaging at a cyclotron $>$ AARDANEH K 270(2006)641

\#194 APPL. RADIAT. ISOT. 67(2009)1405-1411

Huang, FY; Huang, LK; Lin, WY; Luo, TY; Tsai, CS; Hsieh, BT: Development of a thermosensitive hydrogel system for local delivery of Re-188 colloid drugs $>>$ HSIEH BT 274(2007)569

\#195 APPL. RADIAT. ISOT. 67(2009)1559-1569

Beesley, AM; Crespo, MT; Weiher, N; Tsapatsaris, N; Cozar, JS; Esparza, H; Mendez, CG; Hill, P; Schroeder, SLM; Montero-Cabrera, ME: Evolution of chemical species during electrodeposition of uranium for alpha spectrometry by the Hallstadius method $>$ ALVAREZ A 265(2005)383

\#196 APPL. RADIAT. ISOT. 67(2009)1609-1615

Pant, HJ; Sharma, VK; Kamudu, MV; Prakash, SG; Krishanamoorthy, S; Anandam, G; Rao, PS; Ramani, NVS; Singh, G; Sonde, RR: Investigation of flow behaviour of coal particles in a pilot-scale fluidized bed gasifier (FBG) using radiotracer technique $>>$ PANT HJ 280(2009)47 \#197 APPL. RADIAT. ISOT. 67(2009)1676-1682

Park, SD; Kim, JS; Han, SH; Ha, YK; Song, KS; Jee, KY: The measurement of I-129 for the cement and the paraffin solidified low and intermediate level wastes (LILWs), spent resin or evaporated bottom from the pressurized water reactor (PWR) nuclear power plants $>>$ HOU X 277(2007)503; PARK SD 270(2006)507; PARK SD 277(2008)503

\#198 APPL. RADIAT. ISOT. 67(2009)1707-1710

Naqvi, AA; Garwan, MA; Maslehuddin, M; Nagadi, MM; Al-Amoudi, OSB; Khateeb-ur-Rehman; Raashid, M: Prompt gamma analysis of fly ash, silica fume and $\mathrm{Su}-$ perpozz blended cement concrete specimen $>>$ NAQVI AA 271(2007)151
\#199 APPL. RADIAT. ISOT. 67(2009)1748-1750

Chattopadhyay, S; Das, SS: A simple and rapid technique for radiochemical separation of iodine radionuclides from irradiated tellurium using an activated charcoal column $>>$ ELAZONY KM 275(2008)275 \#200 APPL. RADIAT. ISOT. 67(2009)1874-1877

Bonardi, ML; Martano, L; Groppi, F; Chinol, M: Rapid determination of Sr-90 impurities in freshly "generator eluted" Y-90 for radiopharmaceutical preparation $>>$ GROPPI F 263(2005)521

\#201 APPL. RADIAT. ISOT. 67(2009)1952-1956

Kanisch, G; Vidmar, J; Sima, O: Testing the equivalence of several algorithms for calculation of coincidence summing corrections $>>$ BERLIZOV AN 264(2005)169 \#202 APPL. RADIAT. ISOT. 67(2009)1957-1963

Mekarski, P; Zhang, W; Ungar, K; Bean, M; Korpach, E: Monte Carlo simulation of a PhosWatch detector using Geant4 for xenon isotope beta-gamma coincidence spectrum profile and detection efficiency calculations $>>E L Y$ JH 263(2005)245; FOLTZBIEGALSKI KM 276(2008)407 \#203 APPL. RADIAT. ISOT. 67(2009)1965-1973

Karir, T; Samuel, G; Sivaprasad, N; Venkatesh, M: Comparative evaluation of various solid phases for the development of coated tube assays for the estimation of progesterone in human serum, bovine serum and bovine milk >> KARIR T 267(2006)321

\#204 APPL. RADIAT. ISOT. 67(2009)2013-2018

Mou, TT; Yang, WJ; Peng, C; Zhang, XZ; Ma, YC: [F18]-labeled 2-methoxyphenylpiperazine derivative as a potential brain positron emission tomography imaging agent $>>$ YANG SY 278(2008)165

\#205 APPL. RADIAT. ISOT. 67(2009)2062-2067

Kolarz, PM; Filipovic, DM; Marinkovic, BP: Daily variations of indoor air-ion and radon concentrations $>>$ KITTO ME 264(2005)381

\#206 APPL. RADIAT. ISOT. 67(2009)2070-2074

Canella, L; Kudejova, P; Schulze, R; Turler, A; Jolie, J: PGAA, PGAI and NT with cold neutrons: Test measurement on a meteorite sample $>>$ BELGYA T 278(2008) 713; BELGYA T 278(2008)751; KUDEJOVA P 278 (2008)691

\#207 APPL. RADIAT. ISOT. 67(2009)2075-2078

Tomlin, BE; Zeisler, R: Improvements in determinations using the Cu-64 annihilation gamma rays $>>$ JACKMAN KR 279(2009)355

\#208 APPL. RADIAT. ISOT. 67(2009)2097-2099

Kapsimalis, R; Landsberger, S; Ahmed, YA: The determination of uranium in food samples by Compton suppression epithermal neutron activation analysis $>>$ ANDERSON DL 276(2008)23; FREITAS MC 276(2008)149; ZIKOVSKY L 267(2006)695 
\#209 APPL. RADIAT. ISOT. 67(2009)2104-2109

Landsberger, S; Kapsimalis, R: An evaluation of Compton suppression neutron activation analysis for determination of trace elements in some geological samples $>$ ANDERSON D 267(2008)23; FREITAS MC 276(2008)149; STEINNES E 278(2008)313

\#210 APPL. RADIAT. ISOT. 67(2009)2110-2112

Pavelka, S; Vobecky, M; Babicky, A: Ascertainment of variations in the biological half-lives of bromide and sodium ions in the rat by in vivo gamma-ray measurement of $\mathrm{Br}-82$ and Na-24 radionuclides $>>$ PAVELKA S 278(2008)571 \#211 APPL. RADIAT. ISOT. 67(2009)2117-2122

Adlassnig, W; Steinhauser, G; Peroutka, M; Musilek, A; Sterba, JH; Lichtscheidl, IK; Bichler, M: Expanding the menu for carnivorous plants: Uptake of potassium, iron and manganese by carnivorous pitcher plants $>>$ STEINHAUSER G 274(2007)403

\#212 APPL. RADIAT. ISOT. 67(2009)2133-2136

Cincu, E; Craciun, L; Manea-Grigore, I; Cazan, IL; Manu, V; Barbos, D; Cocis, A: Application of the INAA technique for elemental analysis of metallic biomaterials used in dentistry $>>$ CINCU E 271(2007)655; CINCU E 274(2007)199

\#213 APPL. RADIAT. ISOT. 67(2009)2137-2141

Farinha, MM; Almeida, SM; Freitas, MC; Verburg, TG; Wolterbeek, HT: Local and regional sources of air pollutants at Northern Lisbon area, Portugal $>>$ FREITAS MC 263(2005)711; FREITAS MC 278(2008)381

\#214 APPL. RADIAT. ISOT. 68(2010)444-449

Saha, P; Sen Raychaudhuri, S; Chakraborty, A; Sudarshan, M: PIXE analysis of trace elements in relation to chlorophyll concentration in Plantago ovata Forsk $>>$ LAMARI Z 276(2008)95

\#215 APPL. RADIAT. ISOT. 68(2010)505-510

Joe, K; Jeon, YS; Song, BC; Han, SH; Jung, EC; Song, $\mathrm{K}$ : Isotope correlations for determining the isotopic composition of plutonium in high burnup pressurized water reactor (PWR) samples >> KUMAR P 278(2008)137 \#216 APPL. SPECTROSC. REV. 44(2009)317-334

Im, HJ; Song, K: Applications of Prompt Gamma Ray Neutron Activation Analysis: Detection of Illicit Materials $>$ AGHARA SK 265(2005)321; ANDERSON DL 263(2005)683; BIRO KT 265(2005)235

\#217 APPL. SURF. SCI. 253(2007)4348-4355

Chisholm, C; Kumann, E; El-Sharif, M; Doyle, O; Stichleutner, S; Solymos, K; Homonnay, Z; Vertes, A: Preparation and characterisation of electrodeposited amorphous Sn-Co-Fe ternary alloys $>>$ CHISHOLM C 266(2005)533

\#218 AQUAT. CONSERV.-MAR. FRESHW. ECOSYST. 18(2008)891-895

Linde, AR; Izquierdo, JI; Moreira, JC; Garcia-Vazquez, E: Invasive tilapia juveniles are associated with degraded river habitats $>>$ PEREIRA MO 269(2006)707
\#219 AQUAT. GEOCHEM. 14(2008)133-146

Santos, IR; Machado, MI; Niencheski, LF; Burnett, W; Milani, IB; Andrade, CFF; Peterson, RN; Chanton, J; Baisch, P: Major ion chemistry in a freshwater coastal lagoon from southern Brazil (Mangueira Lagoon): Influence of groundwater inputs >> OLIVEIRA J 269(2006)689 \#220 AQUAT. GEOCHEM. 15(2009)443-456

Huber, F; Lutzenkirchen, J: Uranyl Retention on QuartzNew Experimental Data and Blind Prediction Using an Existing Surface Complexation Model $>>$ PATHAK PN 272(2007)37

\#221 ARCH. ANIM. NUTR. 63(2009)104-111

Tayo, GO; Tang, SX; Tan, ZL; Sun, ZH; Wang, M; Zhou, CS; Han, XF: Determination of endogenous faecal phosphorus loss in goats $>>$ DIAS RS 269(2006)661 \#222 ARCH. BIOCHEM. BIOPHYS. 484(2009)94-99

Ward, WC; Zucca, FA; Bellei, C; Zecca, L; Simon, JD: Neuromelanins in various regions of human brain are associated with native and oxidized isoprenoid lipids $>>$ ZECCA L 263(2005)733

\#223 ARCH. MED. RES. 39(2008)768-774

Vallejo, E; Martinez, I; Tejero, A; Hernandez, S; Jimenez, L; Bialostozky, D; Sanchez, G; Ilarraza, H; FerroFlores, G: Clinical Utility of Tc-99m-Labeled Ubiquicidin 29-41 Antimicrobial Peptide for the Scintigraphic Detection of Mediastinitis after Cardiac Surgery $>>$ FERROFLORES G 266(2005)307

\#224 ARCHAEOMETRY 49(2007)395-402

James, WD; Raulerson, MR; Johnson, PR: Archaeometry at Texas A and M University: Characterization of Samoan basalts $>>$ JAMES WD 263(2005)697

\#225 ARCHAEOMETRY 50(2008)12-29

Kasztovszky, Z; Biro, KT; Marko, A; Dobosi, V: Cold neutron prompt gamma activation analysis-A non-destructive method for characterization of high silica content chipped stone tools and raw materials $>>$ KASZTOVSZKY Z 265(2005)193; KUDEJOVA P 265 (2005) 221 \#226 ARCHAEOMETRY 51(2009)231-251

Iriarte, E; Foyo, A; Sanchez, MA; Tomillo, C; Setien, J: The origin and geochemical characterization of red ochres from the Tito Bustillo and Monte Castillo caves (Northern Spain) $>>$ POPELKAFILCOFF RS 272(2007)17 \#227 ASIAN J. CHEM. 21(2009)635-643

Mahramanlioglu, M; Ozgen, O: Adsorption of alphaPicoline and gamma-Picoline on the Adsorbent Produced from Spent Bleaching Earth $>>$ MAHRAMANLIOGLU M 273(2007)621

\#228 ASIAN J. CHEM. 22(2010)3122-3126

Amoli, HS; Barker, J: Analytical Advantages of Electrodeposition for Determination of Cadmium by Atomic Absorption Spectroscopy >> AMOLI HS 268(2006)497; AMOLI HS 273(2007)281 


\section{\#229 ASTROPART PHYS. 32(2010)286-293}

Nayak, PK; Gupta, SK; Jain, A; Mazumdar, I; Raha, S; Saha, SK; Bobrov, AV; Osipov, A; Shwartz, B: A study of the gamma-ray flux during the total solar eclipse of 1 August 2008 at Novosibirsk, Russia $>$ LIU CL 274(2007)507

\#230 ATMOS. CHEM. PHYS. 7(2007)1503-1522

Lanz, VA; Alfarra, MR; Baltensperger, U; Buchmann, B; Hueglin, C; Prevot, ASH: Source apportionment of submicron organic aerosols at an urban site by factor analytical modelling of aerosol mass spectra $>$ CHUNG YS 267(2005)35

\#231 ATMOS. ENVIRON. 40(2006)2058-2067

Almeida, SM; Pio, CA; Freitas, MC; Reis, MA; Trancoso, MA: Source apportionment of atmospheric urban aerosol based on weekdays/weekend variability: evaluation of road re-suspended dust contribution $>>$ FREITAS MC 263(2005)711

\#232 ATMOS. ENVIRON. 42(2008)1-42

Chan, CK; Yao, X: Air pollution in mega cities in China $>>$ LI X 266(2005)141; ZHANG YX 267(2006)497

\#233 ATMOS. ENVIRON. 42(2008)7775-7785

Biswas, KF; Ghauri, BM; Husain, L: Gaseous and aerosol pollutants during fog and clear episodes in South Asian urban atmosphere $>>$ QADIR MA 267(2006)545 \#234 ATMOS. ENVIRON. 43(2009)2971-2980

Igarashi, Y; Inomata, Y; Aoyama, M; Hirose, K; Takahashi, H; Shinoda, Y; Sugimoto, N; Shimizu, A; Chiba, M: Possible change in Asian dust source suggested by atmospheric anthropogenic radionuclides during the 2000s >> HIROSE K 273(2007)115

\#235 ATMOS. ENVIRON. 43(2009)4754-4765

Canepari, S; Pietrodangelo, A; Perrino, C; Astolfi, ML; Marzo, ML: Enhancement of source traceability of atmospheric PM by elemental chemical fractionation $>>$ BERGAMASCHI L 263(2005)745; GIAVERI G 263 (2005)725; KOLTAY E 267(2006)449

\#236 ATMOS. ENVIRON. 44(2010)1116-1125

Hellebust, S; Allanic, A; O'Connor, IP; Wenger, JC; Sodeau, JR: The use of real-time monitoring data to evaluate major sources of airborne particulate matter $>>$ CHUNG YS 267(2005)35

\#237 ATMOS. RES. 95(2010)88-100

Lim, JM; Lee, JH; Moon, JH; Chung, YS; Kim, KH: Source apportionment of PM10 at a small industrial area using Positive Matrix Factorization $>$ LEE JH 263(2005)667

\#238 ATOM. ENERGY 100(2006)359-364

Berlizov, AN; Danilenko, VN; Solov'eva, SL; Kazimirov, AS: Calculations of the corrections for the true summation of cascade gamma rays on the basis of statistical simulation using evaluated nuclear data $>$ BERLIZOV AN 264(2005)169
\#239 ATOM. SPECTROSC. 26(2005)209-214

Tagami, K; Uchida, S: Sample storage conditions and holding times for the determination of total iodine in natural water samples by ICP-MS $>$ TSUKADA $\mathrm{H}$ 263(2005)773

\#240 ATOM. SPECTROSC. 30(2009)47-53

Longas, N; Arrona, RG; Ostra, M; Millan, E: Application of Experimental Design in Acid Digestion Optimization for $\mathrm{Cd}, \mathrm{Cr}$, and $\mathrm{Pb}$ Determination in Plastic Materials by Atomic Absorption Spectrometry >> SOARES EP 264(2005)9 \#241 AUST. J. CHEM. 63(2010)38-46

Manickam, E; Sdraulig, S; O’Brien, R: An Improved and Rapid Radiochemical Method for the Determination of Polonium-210 in Urine >> HOLGYE Z 274(2007)647 \#242 BALTICA 22(2009)37-50

Klavins, M; Silamikele, I; Nikodemus, O; Kalnina, L; Kuske, E; Rodinov, V; Purmalis, O: Peat properties, major and trace element accumulation in bog peat in Latvia $>>$ FRONTASYEVA MV 265(2005)11

\#243 BIOCHEM. ENG. J. 45(2009)82-85

Roy, K; Ghosh, K; Banerjee, A; Mukhopadhyay, D; Lahiri, S: Biomolecule-metal interactions: Applications in extraction and separation techniques $>$ GHOSH K 274(2007)233; NAYAK D 271(2007)387 \#244 BIOCONJUGATE CHEM. 19(2008)958-965

Pruszynski, M; Bilewicz, A; Zalutsky, MR: Preparation of Rh[16aneS(4)-diol]At-211 and $\operatorname{Ir}[16$ aneS(4)-diol]At-211 complexes as potential precursors for astatine radiopharmaceuticals. Part I: Synthesis $>$ PRUSZYNSKI M 268(2006)91

\#245 BIOELECTROCHEMISTRY 71(2007)60-65

Andrzej, K; Malgorzata, R; Maria, W; Witold, W: Heavy metal sorption in the lichen cationactive layer $>>$ BERGAMASHI L 263(2005)721

\#246 BIOGEOSCIENCES 2(2005)141-157

Crusius, J; Koopmans, D; Bratton, JF; Charette, MA; Kroeger, K; Henderson, P; Ryckman, L; Halloran, K; Colman, JA: Submarine groundwater discharge to a small estuary estimated from radon and salinity measurements and a box model $>>$ DULAIOVA H 263(2005)361 \#247 BIOINORG. CHEM. APPL. (2009)702730-

Yang, Y; Zhang, JX; Zhu, L; Zhang, HB: Synthesis, Novel Crystal Structure, and beta-Amyloid Binding Property of $\operatorname{Re}(\mathrm{I})$ (tricarbonyl)(+) EHIDA Analogue $>>$ YANG Y 273(2007)31

\#248 BIOL. TRACE ELEM. RES. 111(2006)71-95

Arribere, MA; Guevara, SR; Bubach, DF; Vigliano, PH: Trace elements as fingerprint of lake of provenance and of species of some native and exotic fish of northern Patagonian lakes $>>$ GUEVARA R 265(2005)481

\#249 BIOL. TRACE ELEM. RES. 120(2007)148-162

Choudhury, RP; Reddy, AVR; Garg, AN: Availability of essential elements in nutrient supplements used as 
antidiabetic herbal formulations $>>$ CHOUDHURY RP 274(2007)411; GARG AN 263(2005)39; GARG AN 271(2007)481

\#250 BIOL. TRACE ELEM. RES. 122(2008)1-18

Kazi, TG; Afridi, HI; Kazi, N; Jamali, MK; Arain, MB; Jalbani, N; Kandhro, GA: Copper, chromium, manganese, iron, nickel, and zinc levels in biological samples of diabetes mellitus patients $>>$ GARG AN 263(2005)39 \#251 BIOL. TRACE ELEM. RES. 122(2008)42-63

Arribere, MA; Guevara, SR; Bubach, DF; Arcagni, M; Vigliano, PH: Selenium and mercury in native and introduced fish species of patagonian lakes, Argentina >> GUEVARA R 265(2005)481

\#252 BIOL. TRACE ELEM. RES. 129(2009)107-115

Zaichick, V; Zaichick, S; Karandashev, V; Nosenko, S: The Effect of Age and Gender on $\mathrm{Al}, \mathrm{B}, \mathrm{Ba}, \mathrm{Ca}, \mathrm{Cu}, \mathrm{Fe}, \mathrm{K}$, $\mathrm{Li}, \mathrm{Mg}, \mathrm{Mn}, \mathrm{Na}, \mathrm{P}, \mathrm{S}, \mathrm{Sr}, \mathrm{V}$, and Zn Contents in Rib Bone of Healthy Humans >> TAKATA MK 264(2005)5 \#253 BIOL. TRACE ELEM. RES. 133(2010)357-363

Gowrishankar, R; Kumar, M; Menon, V; Divi, SM; Saravanan, M; Magudapathy, P; Panigrahi, BK; Nair, KGM; Venkataramaniah, K: Trace Element Studies on Tinospora cordifolia (Menispermaceae), Ocimum sanctum (Lamiaceae), Moringa oleifera (Moringaceae), and Phyllanthus niruri (Euphorbiaceae) Using PIXE $>>$ OLABANJI SO 270(2006)515

\#254 BIOL. TRACE ELEM. RES. 134(2010)41-54

Zaichick, S; Zaichick, V: The Effect of Age and Gender on 37 Chemical Element Contents in Scalp Hair of Healthy Humans >> ZAICHICK V 269(2006)303

\#255 BIOMATERIALS 29(2008)4709-4718

Lee, H; Lee, K; Kim, IK; Park, TG: Synthesis, characterization, and in vivo diagnostic applications of hyaluronic acid immobilized gold nanoprobes $>>$ JAMES WD 271(2007)455

\#256 BIOMATERIALS 30(2009)1928-1936

Zhang, GD; Yang, Z; Lu, W; Zhang, R; Huang, Q; Tian, M; Li, L; Liang, D; Li, C: Influence of anchoring ligands and particle size on the colloidal stability and in vivo biodistribution of polyethylene glycol-coated gold nanoparticles in tumor-xenografted mice $>>$ JAMES WD 271(2007)455

\#257 BIOMETALS 21(2008)219-228

Moll, H; Johnsson, A; Schafer, M; Pedersen, K; Budzikiewicz, H; Bernhard, G: Curium(III) complexation with pyoverdins secreted by a groundwater strain of Pseudomonas fluorescens $>>$ MOLL H 274(2007)603

\#258 BIOORG. MED. CHEM. 17(2009)1118-1124

Sun, YH; Liu, GF; Zhang, YQ; Zhu, H; Ren, YF; Shen, YM: Synthesis and in vitro anti-proliferative activity of beta-elemene monosubstituted derivatives in HeLa cells mediated through arrest of cell cycle at the G1 phase $>>$ XIA JY 266(2005)313
\#259 BIOPHARM. DRUG DISPOS. 28(2007)511-516

Tan, B; Ma, YM; Shi, R; Wang, TM: Simultaneous quantification of three alkaloids of Coptidis Rhizoma in rat urine by high-performance liquid chromatography: Application to pharmacokinetic study >> LI ZJ 265(2005)355 \#260 BIOPOLYMERS 85(2007)264-273

Efimova, YM; Haemers, S; Wierczinski, B; Norde, W; van Well, AA: Stability of globular proteins in $\mathrm{H} 2 \mathrm{O}$ and D2O >> EFIMOVA YM 264(2005)271

\#261 BIORESOUR. TECHNOL. 100(2009)603-608

Mashkani, SG; Ghazvini, PTM; Aligol, DA: Uptake of $\operatorname{Re}$ (VII) from aqueous solutions by Bacillus sp GT-83-23 $>>$ LIU N 275(2008)173

\#262 BIORESOUR. TECHNOL. 100(2009)1915-1921

Mashkani, SG; Ghazvini, PTM: Biotechnological potential of Azolla filiculoides for biosorption of Cs and Sr: Application of micro-PIXE for measurement of biosorption $>>$ LIU N 275(2008)173

\#263 BIORESOUR. TECHNOL. 100(2009)2361-2368

Ghazvini, PTM; Mashkani, SG: Effect of salinity on vanadate biosorption by Halomonas sp GT-83: Preliminary investigation on biosorption by micro-PIXE technique $>>$ LIU N 275(2008)173

\#264 BRAIN PATHOL. 19(2009)586-595

Baurle, J; Kucera, J; Frischmuth, S; Lambertz, M; Kranda, K: Dynamics of Trace Element Concentration During Development and Excitotoxic Cell Death in the Cerebellum of Lurcher Mutant Mice $>>$ KRANDA K 269(2006)555

\#265 BRAZ. ARCH. BIOL. TECHNOL. 51(2008)31-37

Unak, P: Imaging and Therapy with Radionuclide Labeled Magnetic Nanoparticles >> DAGDEVIREN K 273(2007)635

\#266 BRAZ. J. OCEANOGR. 55(2007)265-279

Costa, OS: Anthropogenic nutrient pollution of coral reefs in Southern Bahia, Brazil $>$ OLIVEIRA J 269(2006)689

\#267 BRAZ. J. OCEANOGR. 56(2008)115-131

Santos, IR; Burnett, WC; Godoy, JM: Radionuclides as tracers of coastal processes in Brazil: Review, synthesis, and perspectives >> DULAIOVA H 263(2005)361; OLIVEIRA J 269(2006)689; SAUEIA CH 264(2005) 445; SILVA PSC 264(2005)449; SILVA PSC 269 (2006)739

\#268 BRAZ. J. PHYS. 39(2009)260-263

Zangirolami, DM; Ferreira, AV; Oliveira, AH: Specific Induced Activity Profile at the Rotary Specimen Rack of IPR-R1 TRIGA Reactor $>>$ VEADO MAR 272(2007)511 \#269 BULL. CHEM. SOC. JPN. 81(2008)857-862

Moll, H; Glorius, M; Bernhard, G: Curium(III) complexation with desferrioxamine $\mathrm{B}$ (DFO) investigated using fluorescence spectroscopy >> MOLL H 274(2007)603; MULLEN L 273(2007)683 
\#270 BULL. ENG. GEOL. ENVIRON. 68(2009)331-338

Kilic, AM; Aykamis, AS: The natural radioactivity levels and radiation hazard of pumice from the East Mediterranean Region of Turkey $>>$ PAPASTEFANOU C 266(2005)367

\#271 BULL. ENVIRON. CONTAM. TOXICOL. 80(2008)475-479

Yazici, K; Ertugral, B; Damla, N; Apaydin, G: Radioactive contamination in lichens collected from Trabzon and Rize in the Eastern Black Sea Region, Turkey, and a comparison with that of $1995>>$ LABRECQUE JJ 273(2007)401; PAPASTEFANOU C 264(2005)699

\#272 BULL. ENVIRON. CONTAM. TOXICOL. 83(2009) 668-673

Carvalho, FP; Oliveira, JM; Faria, I: Alpha Emitting Radionuclides in Drainage from Quinta do Bispo and Cunha Baixa Uranium Mines (Portugal) and Associated Radiotoxicological Risk >> CARVALHO FP 274(2007) 167

\#273 BULL. GEOSCI. 83(2008)175-206

Hladil, J; Strnad, L; Salek, M; Jankovska, V; Simandl, P; Schwarz, J; Smolik, J; Lisa, L; Koptikova, L; Rohovec, J; Bohmova, V; Langrova, A; Kocianova, M; Melichar, R; Adamovic, J: An anomalous atmospheric dust deposition event over Central Europe, 24 March 2007, and fingerprinting of the SE Ukrainian source $>>$ KOLTAY E 267(2006)449

\#274 BULL. KOREAN CHEM. SOC. 27(2006)1194-1198

Choi, KH; Hong, YD; Pyun, MS; Choi, SJ: Preparation of an amino acid based DTPA as a BFCA for radioimmunotherapy $>>$ YANG JQ 265(2005)467

\#275 BULL. KOREAN CHEM. SOC. 29(2008)211-213

Yeo, HM; Kang, SY; Ryu, BJ; Ko, SW; Nam, KC: Cesium selective calix[6]arene bisbridged receptor $>>$ KHANCHI AR 273(2007)141

\#276 C. R. CHIM. 12(2009)762-771

Benguella, B; Yacouta-Nour, A: Removal of acid dyes from aqueous solutions by bentonite and kaolin $>>A B-$ DELRAHMAN KM 268(2006)221

\#277 C. R. PALEVOL 7(2008)419-427

Bellot-Gurlet, L; Pelon, O; Seferiades, ML: Provenance study of some obsidians from the Malia Minoan palace (Crete) $>>$ ARIAS A 268(2006)371

\#278 CAD. SAUDE PUBLICA 24(2008)2151-2159

Moreira, MDR; Neves, EB: Use of urine lead level as an exposure indicator and its relationship to blood lead $>>$ DOSSANTOS CR 269(2006)481

\#279 CAN. J. CHEM.-REV. CAN. CHIM. 86(2008)10631069

Green, AEC; Harrington, LE; Valliant, JF: Carboranecarbohydrate derivatives-Versatile platforms for developing targeted radiopharmaceuticals $>$ UNAK T 273(2007)767
\#280 CAN. J. EARTH SCI. 44(2007)835-856

Bonny, SM; Jones, B: Barite $\left(\mathrm{BaSO}_{4}\right)$ biomineralization at Flybye Springs, a cold sulphur spring system in Canada's Northwest Territories $>$ DESIDERI D 267(2006)551

\#281 CAN. J. EARTH SCI. 45(2008)15-29

D'hulst, A; Beaudoin, G; Malo, M; Constantin, M; Pilote, P: Geochemistry of Sainte-Marguerite volcanic rocks: implications for the evolution of Silurian-Devonianvolcanism in the Gaspe Peninsula $>>$ CONSTANTIN M 267(2006)407

\#282 CAN. J. EARTH SCI. 46(2009)331-353

Sappin, AA; Constantin, M; Clark, T; van Breemen, O: Geochemistry, geochronology, and geodynamic setting of $\mathrm{Ni}-\mathrm{Cu}+/$ - PGE mineral prospects hosted by mafic and ultramafic intrusions in the Portneuf-Mauricie Domain, Grenville Province, Quebec $>>$ CONSTANTIN M 267(2006)407

\#283 CANCER BIOTHER. RADIOPHARM. 22(2007) 748-754

Acar, C; Teksoz, S; Unak, P; Muftuler, FZB; Medine, EI: Somatostatin with Tc-99m and biodistribution studies in rats >> ACAR C 273(2007)3; ERTAY T 265(2005)473 \#284 CANCER BIOTHER. RADIOPHARM. 24(2009) 707-716

Yurt, A; Muftuler, FZB; Unak, P; Yolcular, S; Acar, C; Enginar, H: Synthesis of a Novel Antiestrogen Radioligand (Tc-99m-TOR-DTPA) >> ENGINAR H 264(2005)535 \#285 CANCER CAUSES CONTROL 20(2009)1161-1171

Gill, JK; Franke, AA; Morris, JS; Cooney, RV; Wilkens, LR; Le Marchand, L; Goodman, MT; Henderson, BE; Kolonel, LN: Association of selenium, tocopherols, carotenoids, retinol, and 15-isoprostane F-2t in serum or urine with prostate cancer risk: the multiethnic cohort $>>$ MORRIS JS 276(2008)7

\#286 CANCER EPIDEMIOL. BIOMARKERS PREV. 18(2009)1962-1970

Epplein, M; Franke, AA; Cooney, RV; Morris, JS; Wilkens, LR; Goodman, MT; Murphy, SP; Henderson, BE; Kolonel, LN; Le Marchand, L: Association of Plasma Micronutrient Levels and Urinary Isoprostane with Risk of Lung Cancer: The Multiethnic Cohort Study >> MORRIS JS 276(2008)7

\#287 CANCER RES. 68(2008)2904-2911

Waibel, R; Treichler, H; Schaefer, NG; van Staveren, DR; Mundwiler, S; Kunze, S; Kuenzi, M; Alberto, R; Nuesch, J; Knuth, A; Moch, H; Schibli, R; Schubiger, PA: New derivatives of vitamin B12 show preferential targeting of tumors >> YANG JQ 265(2005)467

\#288 CANCER RES. 69(2009)1659-1667

Schwartz, JA; Shetty, AM; Price, RE; Stafford, RJ; Wang, JC; Uthamanthil, RK; Pham, K; McNichols, RJ; Coleman, CL; Payne, JD: Feasibility Study of Particle- 
Assisted Laser Ablation of Brain Tumors in Orthotopic Canine Model >> JAMES WD 271(2007)455

\#289 CANCER RES. 69(2009)3892-3900

von Maltzahn, G; Park, JH; Agrawal, A; Bandaru, NK; Das, SK; Sailor, MJ; Bhatia, SN: Computationally Guided Photothermal Tumor Therapy Using Long-Circulating Gold Nanorod Antennas >> JAMES WD 271(2007)455 \#290 CARBOHYDR. POLYM. 76(2009)622-631

Metwally, E; Elkholy, SS; Salem, HAM; Elsabee, MZ: Sorption behavior of Co-60 and Eu152+154 radionuclides onto chitosan derivatives $>>$ METWALLY E 270(2006)559 \#291 CARBON 47(2009)2014-2025

Zhu, JZ; Deng, BL; Yang, J; Gang, DC: Modifying activated carbon with hybrid ligands for enhancing aqueous mercury removal >> DEVI PSR 269(2006)217 \#292 CARBON 48(2010)1388-1396

Xu, JY; Su, YY; Cheng, JS; Li, SX; Liu, RL; Li, WX; $\mathrm{Xu}, \mathrm{GT}$; Li, QN: Protective effects of fullerenol on carbon tetrachloride-induced acute hepatotoxicity and nephrotoxicity in rats >> LI YG 265(2005)127

\#293 CATAL. LETT. 121(2008)179-188

Kuhn, JN; Ozkan, US: Effect of Co content upon the bulk structure of Sr- and Co-doped LaFeO3 $>>$ NEMETH Z 271(2007)11

\#294 CATAL. TODAY 137(2008)397-402

Gac, W; Giecko, G; Pasieuna-Patkowska, S; Borowiecki, T; Kepinski, L: The influence of silver on the properties of cryptomelane type manganese oxides in $\mathrm{N} 2 \mathrm{O}$ decomposition reaction >> BILEWICZ A 268(2006)485 \#295 CERAM. INT. 36(2010)313-322

Bianco, A; Cacciotti, I; Lombardi, M; Montanaro, L; Bemporad, E; Sebastiani, M: F-substituted hydroxyapatite nanopowders: Thermal stability, sintering behaviour and mechanical properties $>$ BADILLOALMARAZ VE 271(2007)741

\#296 CHEM ERDE-GEOCHEM. 69(2009)223-234

Stojanovic, A; Kogelnig, D; Mitteregger, B; Mader, D; Jirsa, F; Krachler, R; Krachler, R: Major and trace element geochemistry of superficial sediments and suspended particulate matter of shallow saline lakes in Eastern Austria >> RIBEIRO AP 263(2005)645

\#297 CHEM. ANAL. 51(2006)3-34

Srogi, K: Hair analysis-a tool in biomedical, environmental and forensic sciences: a review of literature published after 1989 >> MOHAGHEGHI AH 263(2005)189 \#298 CHEM. ANAL. 52(2007)361-376

Polkowska-Motrenko, H; Dybczynski, RS; Chajduk, E; Danko, B; Kulisa, K; Samczynski, Z; Sypula, M; Szopa, Z: New polish certified reference materials for inorganic trace analysis: Corn flour (INCT-CF-3) and soya bean flour (INCT-SBF-4) $>$ POLKOWSKAMOTRENKO H 269(2006)339
\#299 CHEM. ANAL. 53(2008)821-834

Mietelski, JW; Kitowski, I; Gaca, P; Frankowska, P; Tomankiewicz, E; Blazej, S; Kierepko, R: Radionuclides in Bones of Diurnal Birds of Prey and Owls from the Eastern Poland >> KOMOSA A 269(2006)195; MIETELSKI JW 270(2006)131; MIETELSKI JW 275(2008)571

\#300 CHEM. ANAL. 53(2008)835-843

Komosa, A; Madej, E; Piekarz, M: Determination of a Supported Radon Activity Concentration in Bottled Mineral Waters >> WALLNER G 274(2007)511

\#301 CHEM. ANAL. 53(2008)845-853

Kolotov, VP; Dogadkin, NN; Grozdov, DS; Korobkov, VI; Tsipenyuk, YM: Complex Analysis of Polymetallic Ores by Means of Gamma-Activation and Some Non Nuclear Methods $>>$ KOLOTOV VP 271(2007)671

\#302 CHEM. ANAL. 54(2009)841-855

Chajduk, E: Elemental Analysis of Black and Green Tea Leaves By Instrumental Neutron Activation Analysis >> TSUKADA H 263(2005)773

\#303 CHEM. COMMUN. (2007)2581-2583

McMillan, N; Smith, LV; de la Fuente, JM; Parenty, ADC; Gadegaard, N; Pitt, AR; Thomson, K; MacKenzie, C; Kelly, SM; Cronin, L: Incorporation of N-heterocyclic cations into proteins with a highly directed chemical modification >> EFIMOVA YM 264(2005)91

\#304 CHEM. COMMUN. (2009)6406-6408

Long, M; Thornthwaite, DW; Rogers, SH; Bonzi, G; Livens, FR; Rannard, SP: Utilising C-14-radiolabelled atom transfer radical polymerisation initiators $>>$ POSTOLACHE C 280(2009)251

\#305 CHEM. ENG. J. 144(2008)67-74

Ibrahim, HA; El-Kamash, AM; Hanafy, M; AbdelMonem, NM: Examination of the use of synthetic Zeolite NaA-X blend as backfill material in a radioactive waste disposal facility: Thermodynamic approach $>>$ ABDELRAHMAN KM 268(2006)221; PONG WM 245(2006)431; TSAI SC 266(2005)101 \#306 CHEM. ENG. J. 145(2008)64-68

Sharma, YC: Thermodynamics of removal of cadmium by adsorption on an indigenous clay $>>$ MISHRA SP 274(2007)265 \#307 CHEM. ENG. J. 149(2009)143-152

Rahman, ROA; Ibrahim, HA; Monem, NMA: Longterm performance of zeolite $\mathrm{Na} \mathrm{A}-\mathrm{X}$ blend as backfill material in near surface disposal vault $>>$ PATHAK PN 270(2006)299

\#308 CHEM. ENG. J. 151(2009)247-254

Lopes, CB; Otero, M; Lin, Z; Silva, CM; Rocha, J; Pereira, E; Duarte, AC: Removal of $\mathrm{Hg} 2+$ ions from aqueous solution by ETS-4 microporous titanosilicateKinetic and equilibrium studies $>$ MISHRA SP 274(2007)257 


\section{\#309 CHEM. ENG. J. 155(2009)580-585}

Ali, IM: Sorption studies of Cs-134, Co-60 and Eu152 + 154 on phosphoric acid activated silico-antimonate crystals in high acidic media $>$ PARK HS 268(2006)617

\#310 CHEM. ENG. J. 155(2009)728-735

Barreira, LD; Lito, PF; Antunes, BM; Otero, M; Lin, Z; Rocha, J; Pereira, E; Duarte, AC; Silva, CM: Effect of pH on cadmium (II) removal from aqueous solution using titanosilicate ETS-4 >> POPA K 269(2006)155

\#311 CHEM. ENG. J. 156(2010)193-199

Yooprasert, N; Pongprayoon, T; Suwanmala, P; Hemvichian, K; Tumcharern, G: Radiation-induced admicellar polymerization of isoprene on silica: Effects of surfactant's chain length $>>$ CATALDO F 275(2008)9

\#312 CHEM. ENG. J. 157(2010)18-24

Mustafa, S; Waseem, M; Naeem, A; Shah, KH; Ahmad, T; Hussain, SY: Selective sorption of cadmium by mixed oxides of iron and silicon $>>$ PATHAK PN 268(2006)467 \#313 CHEM. ENG. SCI. 63(2008)3485-3492

Park, BH; Lee, IW; Seo, CS: Electrolytic reduction behavior of $\mathrm{U} 3 \mathrm{O} 8$ in a molten $\mathrm{LiCl}-\mathrm{Li} 2 \mathrm{O}$ salt $>>$ JEONG SM 268(2006)349; PARK SB 268(2006)489

\#314 CHEM. GEOL. 255(2008)143-159

Pelletier, L; Muntener, O; Kalt, A; Vennemann, TW; Belgya, T: Emplacement of ultramafic rocks into the continental crust monitored by light and other trace elements: An example from the Geisspfad body (Swiss-Italian Alps) >> GMELING K 265(2005)201; MARSCHALL HR 265(2005)339

\#315 CHEM. LETT. 36(2007)294-295

Yamada, Y; Namiki, K: Spin orientation of iron films produced by laser deposition $>$ YOKOYAMA D 268(2006)283

\#316 CHEM. LETT. 37(2008)48-49

Mori, T; Ishii, Y; Hayashi, K; Yanaga, M; Satoh, I; Suganuma, H: Separation of Am-III from Eu-III using an improved system of flow-counterbalanced capillary electrophoresis >> MORI T 272(2007)247

\#317 CHEM. LETT. 38(2009)978-979

Akiyama, K; Haba, H; Sueki, K; Tsukada, K; Asai, M; Toyoshima, A; Nagame, Y; Katada, M: Ac-225 Metallofullerene: Toward Ac-225 Nanogenerator in Fullerene >> AKIYAMA K 280(2009)329

\#318 CHEM. LETT. 38(2009)1084-1085

Kasamatsu, Y; Toyoshima, A; Asai, M; Tsukada, K; Li, ZJ; Ishii, Y; Toume, H; Sato, TK; Kikuchi, T; Nishinaka, I; Nagame, Y; Haba, H; Kikunaga, H; Kudou, Y; Oura, Y; Akiyama, K; Sato, W; Ooe, K; Fujisawa, H; Shinohara, A; Goto, S; Hasegawa, T; Kudo, H; Nanri, T; Araki, M; Kinoshita, N; Yokoyama, A; Fan, FL; Qin, Z; Dullmann, CE; Schadel, M; Kratz, JV: Anionic Fluoro Complex of Element 105, Db >> KASAMATSU Y 279(2009)371
\#319 CHEM. PAP. 62(2008)207-214

Petrova, MA; Dukov, IL: Effect of crown ethers on the solvent extraction and separation of lanthanide(III) ions with 4-benzoyl-3-methyl-1-phenyl-2-pyrazolin-5-one >> REHMAN HU 267(2006)421

\#320 CHEM. PHARM. BULL. 58(2010)98-102

Sato, M; Toyazaki, H; Yoshioka, Y; Yokoi, N; Yamasaki, T: Structural Characteristics for Superoxide Anion Radical Scavenging and Productive Activities of Green Tea Polyphenols Including Proanthocyanidin Dimers $>>$ TAKEUCHI Y 272(2007)455

\#321 CHEM. PHYS. LETT. 423(2006)450-453

Revay, Z; Belgya, T; Molnar, GL; Rausch, H; Braun, T: The analysis of C-60 and C-70 fullerenes by prompt gamma neutron activation >> REVAY Z 265(2005)261 \#322 CHEM. PHYS. LETT. 453(2008)233-237

Smirnova, TI; Smirnov, AI; Chadwick, TG; Walker, KL: Characterization of magnetic and electronic properties of trimetallic nitride endohedral fullerenes by SQUID magnetometry and electron paramagnetic resonance >> ZHANG J 272(2007)605

\#323 CHEM. PHYS. LETT. 466(2008)155-158

Tiwari, A; Dantelle, G; Porfyrakis, K; Watt, AAR; Ardavan, A; Briggs, GAD: Magnetic properties of ErSc2N@C-80, Er2ScN@C-80 and Er3N@C-80 fullerenes $>>$ ZHANG J 272(2007)605

\#324 CHEM. PHYS. LETT. 475(2009)135-140

Aylikci, NK; Tirasoglu, E; Apaydin, G; Cengiz, E; Aylikci, V; Bakkaloglu, OF: Influence of alloying effect on $\mathrm{X}$-ray fluorescence parameters of $\mathrm{Co}$ and $\mathrm{Cu}$ in $\mathrm{CoCuAg}$ alloy films $>>$ CENGIZ E 278(2008)89

\#325 CHEM. REV. 107(2007)663-674

Swarzenski, PW: U/Th series radionuclides as coastal groundwater tracers $>>$ DULAIOVA H 263(2005)361 \#326 CHEM. REV. 107(2007)2592-2614

Binnemans, K: Lanthanides and actinides in ionic liquids $>>$ GIRIDHAR P 265(2005)31

\#327 CHEM. REV. 108(2008)543-562

Grate, JW; Egorov, OB; O'Hara, MJ; Devol, TA: Radionuclide sensors for environmental monitoring: From flow injection solid-phase absorptiometry to equilibrationbased preconcentrating minicolumn sensors with radiometric detection $>>$ ADDLEMAN RS 263(2005)291; EGOROV O 264(2005)495; EGOROV OB 263(2005)629; HUGHES LD 267(2006)287; ROANE JE 263(2005)51 \#328 CHEM. SOC. REV. 36(2007)881-892

Rao, LF: Thermodynamics of actinide complexation in solution at elevated temperatures: application of variabletemperature titration calorimetry $>>$ XIA Y 268(2006)445 \#329 CHEM.-EUR. J. 13(2007)2130-2137

$\mathrm{Du}, \mathrm{Z}$; Yu, YL; Wang, JH: Extraction of proteins from biological fluids by use of an ionic liquid/aqueous twophase system >> GIRIDHAR P 265(2005)31 
\#330 CHEM.-EUR. J. 15(2009)4458-4463

Wang, JS; Sheaff, CN; Yoon, B; Addleman, RS; Wai, CM: Extraction of Uranium from Aqueous Solutions by Using Ionic Liquid and Supercritical Carbon Dioxide in Conjunction >> GIRIDHAR P 265(2005)31

\#331 CHEMOSPHERE 65(2006)2358-2365

Tagami, K; Uchida, S: Concentrations of chlorine, bromine and iodine in Japanese rivers $>>$ TSUKADA H 263(2005)773

\#332 CHEMOSPHERE 70(2007)155-164

Clark, NA; Teschke, K; Rideout, K; Copes, R: Trace element levels in adults from the west coast of Canada and associations with age, gender, diet, activities, and levels of other trace elements $>>$ SHIRAISHI K 266(2005)61

\#333 CHEMOSPHERE 72(2008)578-585

Guevara, SR; Queimalinos, CP; Dieguez, MD; Arribere, M: Methylmercury production in the water column of an ultraoligotrophic lake of Northern Patagonia, Argentina >> GUEVARA R 265(2005)481

\#334 CHEMOSPHERE 72(2008)1103-1111

Schramm, KW: Hair-biomonitoring of organic pollutants >> ZHANG H 272(2007)561

\#335 CHEMOSPHERE 73(2008)261-266

Jo, HJ; Jung, JH: Quantification of differentially expressed genes in Daphnia magna exposed to rubber wastewater >> PARK EJ 277(2008)619

\#336 CHEMOSPHERE 73(2008)572-579

Poledniok, J: Speciation of scandium and gallium in soil $>$ FERRARI AA 270(2006)69; RIBEIRO AP 263(2005)645 \#337 CHEMOSPHERE 77(2009)105-114

Tagami, K; Uchida, S: Radium-226 transfer factor from soils to crops and its simple estimation method using uranium and barium concentrations $>$ JUNIOR JAS 269(2006)451

\#338 CHEMOSPHERE 77(2009)471-477

Guevara, SR; Catan, SP; Marvin-DiPasquale, M: Benthic methylmercury production in lacustrine ecosystems of Nahuel Huapi National Park, Patagonia, Argentina >> GUEVARA R 265(2005)481

\#339 CHEMPHYSCHEM 11(2010)28-41

Santos, HA; Garcia-Morales, V; Pereira, CM: Electrochemical Properties of Phospholipid Monolayers at LiquidLiquid Interfaces >> MAKRLIK E 268(2006)155; MAKRLIK E 268(2006)641

\#340 CHEMPHYSCHEM 11(2010)730-735

Zhang, F; Ali, Z; Amin, F; Feltz, A; Oheim, M; Parak, WJ: Ion and $\mathrm{pH}$ Sensing with Colloidal Nanoparticles: Influence of Surface Charge on Sensing and Colloidal Properties >> CHIBA M 269(2006)519

\#341 CHIMIA 63(2009)678-680

Bode, P; Greenberg, RR; Fernandesc, EAD: Neutron Activation Analysis: A Primary (Ratio) Method to
Determine SI-Traceable Values of Element Content in Complex Samples >> GREENBERG RR 278(2008)231 \#342 CHIN. CHEM. LETT. 19(2008)569-572

Bai, J; Wang, HY; Gao, HW: Rapid determination of Clby light-absorption ratio variation approach using $\mathrm{Cl}$-eosin$\mathrm{Y}-\mathrm{Ag}+$ adsorptive precipitation $>>$ LANDSBERGER $\mathrm{S}$ 269(2006)697

\#343 CHIN. J. ANAL. CHEM. 35(2007)1654-1656

Xiao, Y; Zheng, B; Zhang, KR: Cloud point extractiongraphite furnace atomic absorption spectrometry for the determination of thallium(III) $>>$ PEREZGRAMATGES A 269(2006)491

\#344 CHIN. J. ANAL. CHEM. 37(2009)161-168

Zang, XH; Wu, QH; Zhang, MY; Xi, GH; Wang, Z: Developments of Dispersive Liquid-Liquid Microextraction Technique $>>$ MALLAH MH 278(2008)97

\#345 CHIN. J. CHEM. 25(2007)515-520

Chen, CY; Ling, DB; Chen, WK: Elements in the sera of preschool children living in central Taiwan $>>$ CHEN CY 268(2006)83

\#346 CHIN. J. CHEM. 27(2009)915-919

Hamid, S; Syed, WH; Mohammad, GM: Synthesis and Characterization of Amino-functionalized Mesoporous Silicate MCM-41 for Removal of Toxic Metal Ions >> SEPEHRIAN H 275(2008)145

\#347 CHIN. J. CHEM. 27(2009)1079-1085

Liu, SY; Liang, ZS; Gao, F; Yu, JH; Luo, SF; Jesus, NC; Lu, GQ: A Facile Approach to the Synthesis of Gold Nanoshells with Near Infrared Responsive Properties >> JAMES WD 271(2007)455

\#348 CHIN. J. CHEM. 27(2009)2171-2174

Sepehrian, H; Waqif-Husain, S; Rakhshanderu, F; Kamel, L: Modified Mesoporous Silicate MCM-41 for Zinc Ion Adsorption: Synthesis, Characterization and Its Adsorption Behavior $>>$ SEPEHRIAN H 275(2008)145 \#349 CHIN. J. INORG. CHEM. 23(2007)833-838

Liu, JS; Cao, JM; Li, ZQ; Ji, GB; Zheng, MB: Solidstate synthesis and optical absorption properties of $\mathrm{ZnS}$ nanoparticles $>>$ EKOKO BG 265(2005)3

\#350 CHIN. J. INORG. CHEM. 25(2009)761-766

Chen, T; Tian, WY; Sun, M; Li, C; Liu, XY; Wang, LH; Wang, XY; Liu, CL: Influence of Silver Halide Additives on the Sorption of Iodine-125 on Beishan Granite Powder >> MCKINNEY DS 273(2007)289

\#351 CHIN. J. ORG. CHEM. 28(2008)1303-1312

Wang, TT; Zeng, HP: Advance in research of metallofullerene complexes $>>$ ZHANG J 272(2007)605 \#352 CHIN. PHYS. C 32(2008)259-261

Zuo, Y; Zheng, YN; Zhou, DM; Yuan, DQ; Liu, M; Fan, P; Cui, BQ; Ma, YJ; Li, LQ; Li, JZ; Zhu, SY: PAC investigation of qudropole interaction in nano-soft magnetic material Fe73.5Cu1Nb3Si13.5B9 $>$ Z ZHU SY 272(2007)615 
\#353 CHIN. PHYS. C 34(2010)143-147

Shen, HT; Jiang, S; He, M; Dong, KJ; Li, CL; Wang, XG; He, GZ; Gong, J; Lu, LY; Li, SZ; Zhang, DW; Shi, GZ; Huang, CT; Wu, SL; Wu, SY: Determination of the number of atoms of the long-lived nuclide Sn-126 by gamma-ray spectrometry $>$ CATLOW SA 263(2005)599

\#354 CHIN. SCI. BULL. 52(2007)3436-3442

Li, CL; Kang, SC; Cong, ZY: Elemental composition of aerosols collected in the glacier area on Nyainqentanglha Range, Tibetan Plateau, during summer monsoon season $>>$ GIAVERI G 263(2005)725 \#355 CHIN. SCI. BULL. 54(2009)173-182

Zhang, ZY; Zhao, YL; Chai, ZF: Applications of radiotracer techniques for the pharmacology and toxicology studies of nanomaterials $>>$ RAN TC 268(2006)599 \#356 CHROMATOGRAPHIA 69(2009)1149-1159

Ojeda, CB; Rojas, FS: Separation and Preconcentration by Dispersive Liquid-Liquid Microextraction Procedure: A Review >> MALLAH MH 278(2008)97 \#357 CHROMATOGRAPHIA 70(2009)277-280

Sepehrian, H; Waqif-Husain, S; Ghannadi-Maragheh, M: Development of Thiol-Functionalized Mesoporous Silicate MCM-41 as a Modified Sorbent and Its Use in Chromatographic Separation of Metal Ions from Aqueous Nuclear Waste $>>$ SEPEHRIAN H 275(2008)145 \#358 CIENCIA TECNOL. ALIMENT. 29(2009)189194

Favaro, DIT; Chioccola, GS; Taddei, MH; Bortoli, M; Mazzilli, BP; Cozzolino, SMF: Chemical and radiological characterization of meals served by the COSEAS (USP-SP) $>$ FARIAS LA 270(2006)217; SHIRAISHI K 266(2005)61

\#359 CLIM. CHANGE 96(2009)357-378

Lackner, KS; Brennan, S: Envisioning carbon capture and storage: expanded possibilities due to air capture, leakage insurance, and C-14 monitoring $>$ BACHELOR PP 277(2008)85

\#360 COLL. ANTHROPOL. 31(2007)209-219

Obhodas, J; Tucak-Zoric, S; Kutle, A; Valkovic, V: Indications for synergetic and antagonistic effects between trace elements in the environment to human health $>>$ KUTLE A 269(2006)605

\#361 COLLOID SURF. A-PHYSICOCHEM. ENG. ASP. 293(2007)217-223

Qadeer, R: Adsorption behavior of ruthenium ions on activated charcoal from nirtic acid medium $>>$ SHAUKAT MS 265(2005)73

\#362 COLLOID SURF. A-PHYSICOCHEM. ENG. ASP. 310(2007)46-54

Elewaut, K; Zitha, PLJ: Coupled mass transfer and reaction in water of an oil soluble chemical: A CT scan study $>>$ KUCUKONDER A 270(2006)695
\#363 COLLOID SURF. A-PHYSICOCHEM. ENG. ASP. 325(2008)115-119

Yoshida, T; Suzuki, M: Effects of humic acid on migration of montmorillonite and alumina colloid in a quartz sand column $>>$ YOSHIDA T 270(2006)363 \#364 COLLOID SURF. A-PHYSICOCHEM. ENG. ASP. 328(2008)8-14

Tan, XL; Chang, PP; Fan, QH; Zhou, X; Yu, SM; Wu, WS; Wang, XK: Sorption of Pb(II) on Na-rectorite: Effects of $\mathrm{pH}$, ionic strength, temperature, soil humic acid and fulvic acid $>>$ CHANG P 274(2007)153

\#365 COLLOID SURF. A-PHYSICOCHEM. ENG. ASP. 342(2009)76-83

Ye, XS; Wu, ZJ; Li, W; Liu, HN; Li, Q; Qing, BJ; Guo, M; Go, F: Rubidium and cesium ion adsorption by an ammonium molybdophosphate-calcium alginate composite adsorbent $>>$ KHANCHI AR 273(2007)141; PATHAK PN 270(2006)299

\#366 COLLOID SURF. A-PHYSICOCHEM. ENG. ASP. 345(2009)135-140

Alvarado-Ibarra, Y; Granados-Correa, F; Lara, VH; Bosch, P; Bulbulian, S: Nickel (II) sorption on porous $\mathrm{ZnO}$ prepared by solution combustion method $>>$ PATHAK PN 268(2006)467

\#367 COLLOID SURF. A-PHYSICOCHEM. ENG. ASP. 349(2009)195-201

Li, JX; Hu, J; Sheng, GD; Zhao, GX; Huang, Q: Effect of $\mathrm{pH}$, ionic strength, foreign ions and temperature on the adsorption of $\mathrm{Cu}(\mathrm{II})$ from aqueous solution to GMZ bentonite $>>$ HU J 279(2009)701

\#368 COMMUN. THEOR. PHYS. 52(2009)163-167

Zhao, J; Xu, XG; Wei, GZ: Phase Diagram of Transverse Spin-2 Ising Model with Longitudinal Crystal Field $>>$ DI NL 268(2006)137

\#369 COMPLEXITY 14(2008)89-99

Kalinowski, MB; Becker, A; Saey, PRJ; Tuma, MP; Wotawa, G: The complexity of CTBT verification. Taking noble gas monitoring as an example $>>$ MATTHEWS KM 263(2005)235

\#370 CONSTR. BUILD. MATER. 23(2009)3240-3247

Ajam, L; Ben Ouezdou, M; Felfoul, HS; El Mensi, R: Characterization of the Tunisian phosphogypsum and its valorization in clay bricks $>>$ REGUIGUI R 264(2005)719 \#371 CONT. SHELF RES. 26(2006)874-884

Povinec, PP; Comanducci, JF; Levy-Palomo, I; Oregioni, B: Monitoring of submarine groundwater discharge along the Donnalucata coast in the south-eastern Sicily using underwater gamma-ray spectrometry >> OSVATH I 263(2005)437

\#372 CONT. SHELF RES. 29(2009)896-902

Prego, R; Caetano, M; Vale, C; Marmolejo-Rodriguez, $\mathrm{J}$ : Rare earth elements in sediments of the Vigo Ria, NW Iberian Peninsula $>>$ ARAUJO MF 271(2007)255 


\section{\#373 CONTACT DERMATITIS 58(2008)97-100}

De Medeiros, LM; Fransway, AF; Taylor, JS; Wyman, M; Janes, J; Fowler, JF; Rietschel, RL: Complementary and alternative remedies: an additional source of potential systemic nickel exposure $>>$ OLABANJI SO 270(2006)515 \#374 COORD. CHEM. REV. 251(2007)1561-1589

Casas, JS; Garcia-Tasende, MS; Sanchez, A; Sordo, J; Touceda, A: Coordination modes of 5-pyrazolones: A solid-state overview $>>$ BOSE R 265(2005)115 \#375 COORD. CHEM. REV. 253(2009)893-962

Creaven, BS; Donlon, DF; McGinley, J: Coordination chemistry of calix[4]arene derivatives with lower rim functionalisation and their applications $>>$ YAFTIAN MR 270(2006)357

\#376 CORROSION SCI. 51(2009)2831-2839

Baja, B; Varga, K; Szabo, NA; Nemeth, Z; Kadar, P; Oravetz, D; Homonnay, Z; Kuzmann, E; Schunk, J; Patek, G: Long-term trends in the corrosion state and surface properties of the stainless steel tubes of steam generators decontaminated chemically in VVER-type nuclear reactors >> RADO K 268(2006)313

\#377 CRETAC. RES. 29(2008)217-236

Goto, K; Tada, R; Tajika, E; Iturralde-Vinent, MA; Matsui, T; Yamamoto, S; Nakano, Y; Oji, T; Kiyokawa, S; Delgado, DEG; Otero, CD; Consuegra, RR: Lateral lithological and compositional variations of the Cretaceous/ Tertiary deep-sea tsunami deposits in northwestern Cuba >> HATSUKAWA Y 272(2007)273

\#378 CRIT. REV. ANAL. CHEM. 39(2009)230-250

Michalski, R: Applications of Ion Chromatography for the Determination of Inorganic Cations $>$ HANG W 263(2005)467

\#379 CRIT. REV. ENVIRON. SCI. TECHNOL. 39(2009) 783-842

Gupta, VK; Carrott, PJM; Carrott, MMLR; Suhas: LowCost Adsorbents: Growing Approach to Wastewater Treatmenta Review >> HASSAN SSM 269(2006)135 \#380 CRIT. REV. THER. DRUG CARR. SYST. 25(2008) 347-379

Gupta, Y; Kohli, DV; Jain, SK: Vitamin B-12-mediated transport: A potential tool for tumor targeting of antineoplastic drugs and imaging agents $>>$ YANG JQ 265(2005) 467

\#381 CROAT. CHEM. ACTA 80(2007)303-311

Lyklema, J; Golub, T: Electrical double layer on silver iodide and overcharging in the presence of hydrolyzable cations $>>$ ZIELINSKA B 266(2005)339

\#382 CURR. ANAL. CHEM. 6(2010)26-36

Avino, P; Capannesi, G; Diaco, L; Rosada, A: Multivariate Analysis Applied to Trace and Ultra-Trace Elements in Italian Potable Waters Determined by INAA $>>$ SECCARONI C 278(2008)277
\#383 CURR. DRUG METAB. 9(2008)697-709

Liang, XJ; Chen, CY; Zhao, YL; Jia, L; Wang, PC: Biopharmaceutics and Therapeutic Potential of Engineered Nanomaterials $>>$ WANG JX 272(2007)527

\#384 CURR. OPIN. BIOTECHNOL. 18(2007)565-571

Fischer, HC; Chan, WCW: Nanotoxicity: the growing need for in vivo study $>>$ JAMES WD 271(2007)455 \#385 CURR. SCI. 94(2008)854-857

Krivit, SB: Low energy nuclear reaction researchGlobal scenario >> MILEY GH 263(2005)691 \#386 CURR. SCI. 94(2008)1003-1011

Shivanna, K; Tirumalesh, K; Noble, J; Joseph, TB; Singh, G; Joshi, AP; Khati, VS: Isotope techniques to identify recharge areas of springs for rainwater harvesting in the mountainous region of Gaucher area, Chamoli District, Uttarakhand $>>$ TIRUMALESH K 274(2007)307 \#387 CYTOTECHNOLOGY 58(2008)141-144

Gong, JS; Ito, Y: Peptide immobilized on gold particles enhances cell growth $>>$ JAMES WD 271(2007)455

\#388 CZECH. J. PHYS. 56(2006)D165-D175

Steinhauser, G; Sterba, JH; Poljanc, K; Bichler, M; Buchtela, K: NEUTRON ACTIVATION ANALYSIS OF SEA-, LAKE-, AND EVAPORATED SALT $>>$ WESTPHAL GP 263(2005)703

\#389 CZECH. J. PHYS. 56(2006)D177-D182

Varga, Z; Suranyi, G; Vajda, N; Stefanka, Z: Rapid methods for the determination of long-lived radionuclides in environmental samples by ICP-SFMS and radioanalytical techniques $>>$ LEE SH 263(2005)419; POVINEC PP 263(2005)413

\#390 CZECH. J. PHYS. 56(2006)D183-D194

De Corte, F; Vandenberghe, D; De Wispelaere, A; Buylaert, JP; Van den Haute, P: Radon loss from encapsulated sediments in Ge gamma-ray spectrometry for the annual radiation dose determination in luminescence dating $>>$ DECORTE F 263(2005)659

\#391 CZECH. J. PHYS. 56(2006)D195-D201

Ridone, S; Arginelli, D; Berton, G; Bortoluzzi, S; Canuto, G; Montalto, M; Nocente, M; Vegro, M: Radioanalytical determination of $\mathrm{Pu} 239+240$ and $\mathrm{Am}-241$ in bioassay samples by anion exchange and extraction chromatography: preliminary considerations about the two methods $>>$ ARGINELLI D 263(2005)275 \#392 CZECH. J. PHYS. 56(2006)D271-D277

Legin, A; Kirsanov, D; Rudnitskaya, A; Babain, V: Electronic tongue - an array of non-specific chemical sensors-for analysis of radioactive solutions $>>$ WOLF SF 263(2005)575 \#393 CZECH. J. PHYS. 56(2006)D283-D289

Sterba, JH; Steinhauser, G; Bichler, M: Application of NAA to develop a chemostratigraphy of volcanic deposits on Nisyros and Telos, Greece $>$ WESTPHAL GP 263(2005)703 
\#394 CZECH. J. PHYS. 56(2006)D315-D321

Mizera, J; Kucera, J; Randa, Z; Lucanikova, M: Advanced liquid and solid extraction procedures for ultratrace determination of rhenium by radiochemical neutron activation analysis >> KUCERA J 269(2006)251; MAS JL 265(2005)361

\#395 CZECH. J. PHYS. 56(2006)D391-D398

Ohtsuki, T; Yuki, H; Hirose, K; Mitsugashira, T: Status of the electron accelerator for radioanalytical studies at Tohoku University >> SHIKANO K 266(2005)211 \#396 CZECH. J. PHYS. 56(2006)D477-D482

Herbst, RS; Peterman, DR; Tillotson, RD: Aspects of the fundamental chemistry of cesium extraction from acidic media by HCCD >> LUTHER TA 267(2006)603 \#397 CZECH. J. PHYS. 56(2006)D483-D492

Palagyi, S; Laciok, A: Sorption, desorption and extraction of uranium from some sands under dynamic conditions $>>$ NOUBACTEP C 267(2006)591

\#398 CZECH. J. PHYS. 56(2006)D609-D615

Reich, M; Rajec, P; Gullova, M: Np-237 Separation from environmental matrices by extraction chromatography method $>>$ REICH M 266(2005)71

\#399 DALTON TRANS. (2009)3590-3598

Ray, RS; Kruger, S; Rosch, N: Uranyl monocarboxylates of aromatic acids: A density functional model study of uranyl humate complexation >> VULPIUS D 270(2006)661

\#400 DALTON TRANS. (2007)4214-4221

Billard, I; Gaillard, C; Hennig, C: Dissolution of UO2, UO3 and of some lanthanide oxides in BumimTf(2)N: effect of acid and water and formation of UO2(NO3)(3)(-) $>$ GIRIDHAR P 265(2005)31

\#401 DALTON TRANS. (2008)5083-5088

Shimojo, K; Kurahashi, K; Naganawa, H: Extraction behavior of lanthanides using a diglycolamide derivative TODGA in ionic liquids $>>$ GIRIDHAR P 265(2005)31 \#402 DALTON TRANS. (2009)5310-5318

Zhao, D; Zhang, H; Xie, Z; Zhang, WL; Yang, SL; Cheng, WD: Syntheses, crystal and electronic structures of compounds $\mathrm{AM}(\mathrm{PO} 4)(2)(\mathrm{A}=\mathrm{Sr}, \mathrm{M}=\mathrm{Ti}, \mathrm{Sn} ; \mathrm{A}=\mathrm{Ba}$, $\mathrm{M}=\mathrm{Sn})>>$ POPA K 273(2007)563

\#403 DEEP-SEA RES. PART II-TOP. STUD. OCEANOGR. 53(2006)1629-1648

Thomalla, S; Turnewitsch, R; Lucas, M; Poulton, A: Particulate organic carbon export from the North and South Atlantic gyres: The Th-234/U-238 disequilibrium approach $>>$ PIKE SM 263(2005)355

\#404 DEEP-SEA RES. PART II-TOP. STUD. OCEANOGR. 55(2008)841-855

Savoye, N; Trull, TW; Jacquet, SHM; Navez, J; Dehairs, F: Th-234-based export fluxes during a natural iron fertilization experiment in the Southern Ocean (KEOPS) $>>$ PIKE SM 263(2005)355
\#405 DEEP-SEA RES. PART II-TOP. STUD. OCEANOGR. 55(2008)1426-1444

Buesseler, KO; Lamborg, C; Cai, P; Escoube, R; Johnson, R; Pike, S; Masque, P; McGillicuddy, D; Verdeny, E: Particle fluxes associated with mesoscale eddies in the Sargasso Sea $>>$ PIKE SM 263(2005)355

\#406 DEEP-SEA RES. PART II-TOP. STUD. OCEANOGR. 55(2008)1445-1460

Maiti, K; Benitez-Nelson, CR; Rii, Y; Bidigare, R: The influence of a mature cyclonic eddy on particle export in the lee of Hawaii $>>$ PIKE SM 263(2005)355

\#407 DEEP-SEA RES. PART II-TOP. STUD. OCEANOGR. 55(2008)1473-1490

Noble, AE; Saito, MA; Maiti, K; Benitez-Nelson, CR: Cobalt, manganese, and iron near the Hawaiian Islands: A potential concentrating mechanism for cobalt within a cyclonic eddy and implications for the hybrid-type trace metals $>>$ PIKE SM 263(2005)355

\#408 DEEP-SEA RES. PART I-OCEANOGR. RES. PAP. 56(2009)875-891

Maiti, K; Benitez-Nelson, CR; Lomas, MW; Krause, JW: Biogeochemical responses to late-winter storms in the Sargasso Sea, III-Estimates of export production using Th-234:U-238 disequilibria and sediment traps $>>$ PIKE SM 263(2005)355

\#409 DEEP-SEA RES. PART I-OCEANOGR. RES. PAP. 56(2009)1143-1167

Buesseler, KO; Pike, S; Maiti, K; Lamborg, CH; Siegel, DA; Trull, TW: Thorium-234 as a tracer of spatial, temporal and vertical variability in particle flux in the North Pacific $>>$ PIKE SM 263(2005)355

\#410 DENDROCHRONOLOGIA 27(2009)213-221

Sheppard, PR; Ort, MH; Anderson, KC; Clynne, MA; May, EM: Multiple dendrochronological responses to the eruption of Cinder Cone, Lassen Volcanic National Park, California $>>$ UNLU K 264(2005)21

\#411 DESALIN. WATER TREAT. 12(2009)16-27

Anirudhan, TS; Bringle, CD; Rijith, S: Removal of uranium(VI) from aqueous solutions and nuclear industry effluents using humic acid-immobilized zirconium-pillared clay $>>$ AYCIK GA 273(2007)713

\#412 DESALINATION 232(2008)11-19

Giridhar, R; Venkatesan, KA; Srinivasan, TG; Rao, PRV: Synthesis of phosphinic acid resin using acidic 1-butyl-3-methylimidazolium chloroaluminate ionic liquid as catalyst $>>$ GIRIDHAR P 265(2005)31; VENKATESAN KA 275(2008)563 \#413 DESALINATION 232(2008)191-197

Karve, M; Rajgor, RV: Amberlite XAD-2 impregnated organophosphinic acid extractant for separation of uranium(VI) from rare earth elements $>$ KARVE M 273(2007)405 
\#414 DESALINATION 235(2009)306-318

Chegrouche, S; Mellah, A; Barkat, A: Removal of strontium from aqueous solutions by adsorption onto activated carbon: kinetic and thermodynamic studies $>>$ HUMELNICU D 270(2006)637

\#415 DESALINATION 244(2009)283-292

Li, XL; Chen, CL; Chang, PP; Yu, SM; Wu, WS; Wang, $\mathrm{XK}$ : Comparative studies of cobalt sorption and desorption on bentonite, alumina and silica: effect of $\mathrm{pH}$ and fulvic acid >> CHEN CL 273(2007)227; XU D 266(2005)419; YU S 268(2006)387

\#416 DESALINATION 248(2009)193-203

Schafer, AI; Rossiter, HMA; Owusu, PA; Richards, BS; Awuah, E: Physico-chemical water quality in Ghana: Prospects for water supply technology implementation $>>$ DAMPARE SB 265(2005)53

\#417 DESALINATION 248(2009)629-635

Harleman, C; Jacks, G; Rybeck, B: The use of a clinoptilolite-based filter in emergency situations $>>$ KHANCHI AR 273(2007)141

\#418 DESALINATION 249(2009)742-747

Otero, M; Lopes, CB; Coimbra, J; Ferreira, TR; Silva, CM; Lin, Z; Rocha, J; Pereira, E; Duarte, AC: Priority pollutants $(\mathrm{Hg} 2+$ and $\mathrm{Cd} 2+)$ removal from water by ETS4 titanosilicate $>>$ POPA K 269(2006)155

\#419 EARTH PLANET. SCI. LETT. 286(2009)131-138

Roberts, KA; Xu, C; Hung, CC; Conte, MH; Santschi, $\mathrm{PH}$ : Scavenging and fractionation of thorium vs. protactinium in the ocean, as determined from particle-water partitioning experiments with sediment trap material from the Gulf of Mexico and Sargasso Sea $>>$ CHOPPIN GR 273(2007)695

\#420 ECOL. CHEM. ENG. S-15(2008)397-423

Klos, A; Rajfur, M; Waclawek, M; Waclawek, W: Accumulation of micro- and macroelements in mosses and lichens >> PRUDENCIO MI 271(2007)431

\#421 ELECTROCHEM. SOLID STATE LETT. 9(2006) A241-A244

Choi, J; Alvarez, E; Arunkumar, TA; Manthiram, A: Proton insertion into oxide cathodes during chemical delithiation $>>$ AGHARA SK 265(2005)321

\#422 ELECTROCHEMISTRY 77(2009)370-372

Saito, M; Imaizumi, H; Kato, N; Ishii, Y; Sekiya, K: Comparison between Anode Side and Cathode Side for Tritium Enrichment of Solid Polymer Electrolysis $>>$ SAITO M 275(2008)407

\#423 ELECTROCHIM. ACTA 52(2007)3006-3012

Giridhar, P; Venkatesan, KA; Srinivasan, TG; Rao, PRV: Electrochemical behavior of uranium(VI) in 1-butyl-3-methylimidazolium chloride and thermal characterization of uranium oxide deposit $>>$ GIRIDHAR $P$ 265(2005)31
\#424 ELECTROCHIM. ACTA 52(2007)7121-7127

Jayakumar, M; Venkatesan, KA; Srinivasan, TG: Electrochemical behavior of fission palladium in 1-butyl-3methylimidazolium chloride $>>$ GIRIDHAR P 265(2005) 31; VENKATESAN KA 266(2005)431 \#425 ELECTROCHIM. ACTA 53(2007)1911-1919

Rao, CJ; Venkatesan, KA; Nagarajan, K; Srinivasan, TG; Rao, PRV: Treatment of tissue paper containing radioactive waste and electrochemical recovery of valuables using ionic liquids $>>$ GIRIDHAR P 265(2005)31

\#426 ELECTROCHIM. ACTA 55(2010)804-812

Paszternak, A; Felhosi, I; Paszti, Z; Kuzmann, E; Vertes, A; Kalman, E; Nyikos, L: Surface analytical characterization of passive iron surface modified by alkyl-phosphonic acid layers >> KUZMANN E 277(2008)699 \#427 ELECTROCHIM. ACTA 55(2010)1749-1755

Jeong, SM; Shin, HS; Hong, SS; Hur, JM; Do, JB; Lee, HS: Electrochemical reduction behavior of U3O8 powder in a LiCl molten salt $>>$ PARK BH 270(2006)575 \#428 ELECTROPHORESIS 29(2008)597-603

Kazi, MA; Khuhawar, MY; Arain, R: Determination of uranium, iron, copper, and nickel from ore samples by MEKC using N,N'-ethylene bis(salicylaidimine), as complexing reagent $>>$ MOHAGHEGHI AH 263(2005)189; ZIKOVSKY L 267(2006)695

\#429 ENERGY 34(2009)2087-2091

Principi, G; Agresti, F; Maddalena, A; Lo Russo, S: The problem of solid state hydrogen storage $>>$ PALADE P 266(2005)553

\#430 ENERGY FUELS 21(2007)3698-3701

Catharino, RR; Milagre, HMS; Saraiva, SA; Garcia, CM; Schuchardt, U; Eberlin, MN: Biodiesel typification and quality control by direct infusion electrospray ionization mass spectrometry fingerprinting $>>$ SANTOS LS 269(2006)505

\#431 ENG. LIFE SCI. 8(2008)622-630

Noubactep, C; Chen-Braucher, D; Schlothauer, T: Arsenic Release from a Natural Rock under Near-natural Oxidizing Conditions >> NOUBACTEP C 267(2006)591; NOUBACTEP C 270(2006)325

\#432 ENVIRON. CHEM. 4(2007)293-300

Wellman, DM; Pierce, EM; Valenta, MM: Efficacy of soluble sodium tripolyphosphate amendments for the insitu immobilisation of uranium $>$ MOORE RC 263(2005)97

\#433 ENVIRON. CHEM. LETT. 6(2008)189-213

Babula, P; Adam, V; Opatrilova, R; Zehnalek, J; Havel, L; Kizek, R: Uncommon heavy metals, metalloids and their plant toxicity: a review >> UNAK T 273(2007)763 \#434 ENVIRON. EARTH SCI. 59(2009)449-457

Kacmaz, H; Nakoman, ME: Hydrochemical characteristics of shallow groundwater in aquifer containing uranyl 
phosphate minerals, in the Koprubasi (Manisa) area, Turkey $>$ KAWABATA Y 270(2006)137

\#435 ENVIRON. ENG. MANAG. J. 8(2009)91-95

Hauta, OR; Macovei, A; Apostolescu, GA; Ganju, D; Cecal, A: Radiolytic output of hydrogen as environmentally friendly energy vector $>>$ YOSHIDA T 272(2007) 471

\#436 ENVIRON. ENG. MANAG. J. 8(2009)901-905

Gradinaru, R; Valu, SO; Postolache, S; Pavel, CC; Sandu, I; Popa, K: On the influence of ETS-10 porosity and surface properties in retention of some nanoions and nanomolecules $>>$ TYKVA R 279(2009)811

\#437 ENVIRON. GEOL. 55(2008)1577-1586

Ciszewski, D; Czajka, A; Blazej, S: Rapid migration of heavy metals and Cs-137 in alluvial sediments, Upper Odra River valley, Poland >> DION HM 264(2005)481

\#438 ENVIRON. GEOL. 56(2008)353-357

Lu, XW; Zhang, XL: Ra-226, Th-232 and K-40 activities in soils of Cuihua Mountain National Geological Park, China $>>$ XINWEI L 267(2006)669

\#439 ENVIRON. GEOL. 58(2009)751-760

Kljakovic-Gaspic, Z; Bogner, D; Ujevic, I: Trace metals $(\mathrm{Cd}, \mathrm{Pb}, \mathrm{Cu}, \mathrm{Zn}$ and $\mathrm{Ni})$ in sediment of the submarine pit Dragon ear (Soline Bay, Rogoznica, Croatia) >> OBHODAS J 270(2006)75

\#440 ENVIRON. INT. 33(2007)122-138

Malik, A: Environmental challenge vis a vis opportunity: The case of water hyacinth $>$ SHAWKY S 265(2005)81

\#441 ENVIRON. MONIT. ASSESS. 109(2005)81-95

Freitas, MC; Farinha, MM; Ventura, MG; Almeida, SM; Reis, MA; Pacheco, AG: Gravimetric and chemical features of airborne PM10 AND PM2.5 in mainland portugal $>>$ FREITAS MC 263(2005)711

\#442 ENVIRON. MONIT. ASSESS. 143(2008)67-74

Khuder, A; Bakir, MA; Hasan, R; Mohammad, A: Determination of nickel, copper, zinc and lead in human scalp hair in Syrian occupationally exposed workers by total reflection X-ray fluorescence $>$ KHUDER A 273(2007)435

\#443 ENVIRON. MONIT. ASSESS. 143(2008)267-272

Soylak, M; Peker, DSK; Turkoglu, O: Heavy metal contents of refined and unrefined table salts from Turkey, Egypt and Greece >> NAVARRETE JM 271(2007)599; UNAK P 273(2007)649

\#444 ENVIRON. MONIT. ASSESS. 153(2009)333-338

Ayata, S; Kaynak, I; Merdivan, M: Solid phase extractive preconcentration of silver from aqueous samples $>>$ YUSOF AM 271(2007)191

\#445 ENVIRON. MONIT. ASSESS. 160(2010)199-206

Baysal, A; Akman, S: Solid-phase extraction of Mn(II) and slurry analysis of the sorbent by electrothermal atomic absorption spectrometry >> OZEROGLU C 268(2006)211
\#446 ENVIRON. MONIT. ASSESS. 161(2010)229-236

Dorman, JW; Steinberg, SM: Analysis of iodide and iodate in Lake Mead, Nevada using a headspace derivatization gas chromatography-mass spectrometry $>>$ STEINBERG SM 277(2008)185

\#447 ENVIRON. MONIT. ASSESS. 163(2010)15-26

Belivermis, M; Kilic, O; Cotuk, Y; Topcuoglu, S: The effects of physicochemical properties on gamma emitting natural radionuclide levels in the soil profile of Istanbul $>>$ ALMASRI MS 267(2006)337

\#448 ENVIRON. MONIT. ASSESS. 163(2010)241-252

Yu, RL; Hu, GR; Wang, LJ: Speciation and ecological risk of heavy metals in intertidal sediments of Quanzhou Bay, China $>>$ GUEVARA R 265(2005)481 \#449 ENVIRON. POLLUT. 151(2008)334-340

Fuga, A; Saiki, M; Marcelli, MP; Saldiva, PHN: Atmospheric pollutants monitoring by analysis of epiphytic lichens $>>$ SAIKI M 271(2007)213

\#450 ENVIRON. POLLUT. 151(2008)559-568

Yang, H; Xing, YP; Xie, P; Ni, LY; Rong, KW: Carbon source/sink function of a subtropical, eutrophic lake determined from an overall mass balance and a gas exchange and carbon burial balance $>>$ YANG H 267(2005)205

\#451 ENVIRON. POLLUT. 154(2008)380-389

Pinho, P; Augusto, S; Martins-Loucao, MA; Pereira, MJ; Soares, A; Maguas, C; Branquinho, C: Causes of change in nitrophytic and oligotrophic lichen species in a Mediterranean climate: Impact of land cover and atmospheric pollutants $>>$ LOPES F 270(2006)43

\#452 ENVIRON. POLLUT. 156(2008)974-979

Linde-Arias, AR; Inacio, AF; Novo, LA; de Alburquerque, C; Moreira, JC: Multibiomarker approach in fish to assess the impact of pollution in a large Brazilian river, Paraiba do Sul >> PEREIRA MO 269(2006)707 \#453 ENVIRON. SCI. POLLUT. RES. 15(2008)41-50

Astel, A; Astel, K; Biziuk, M: PCA and multidimensional visualization techniques united to aid in the bioindication of elements from transplanted Sphagnum Palustre moss exposed in the Gdansk city area $>>$ SZCZEPANIAK K 270(2006)35

\#454 ENVIRON. SCI. TECHNOL. 40(2006)4103-4108

Zheng, J; Yamada, M: Plutonium isotopes in settling particles: Transport and scavenging of $\mathrm{Pu}$ in the western Northwest Pacific $>>$ ZHENG J 267(2005)73 \#455 ENVIRON. SCI. TECHNOL. 41(2007)7022-7029

Swarzenski, PW; Simonds, FW; Paulson, AJ; Kruse, S; Reich, C: Geochemical and geophysical examination of submarine groundwater discharge and associated nutrient loading estimates into Lynch Cove, Hood Canal, WA >> DULAIOVA H 263(2005)361

\#456 ENVIRON. SCI. TECHNOL. 42(2008)6532-6537

Tan, XL; Wang, XK; Geckeis, H; Rabung, T: Sorption of $\mathrm{Eu}(\mathrm{III})$ on humic acid or fulvic acid bound to hydrous 
alumina studied by SEM-EDS, XPS, TRLFS, and batch techniques >> XU D 266(2005)419

\#457 ENVIRON. SCI. TECHNOL. 42(2008)8140-8145

Yeung, LWY; Miyake, Y; Taniyasu, S; Wang, Y; Yu, HX; So, MK; Jiang, GB; Wu, YN; Li, JG; Giesy, JP; Yamashita, N; Lam, PKS: Perfluorinated Compounds and Total and Extractable Organic Fluorine in Human Blood Samples from China $>>$ KAWANO M 272(2007)501 \#458 ENVIRON. SCI. TECHNOL. 43(2009)152-156

Luo, W; Gu, B: Dissolution and Mobilization of Uranium in a Reduced Sediment by Natural Humic Substances under Anaerobic Conditions $>>$ WARWICK P 266(2005) 179

\#459 ENVIRON. SCI. TECHNOL. 43(2009)1947-1951

Mallah, MH; Shemirani, F; Maragheh, MG: Ionic Liquids for Simultaneous Preconcentration of Some Lanthanoids Using Dispersive Liquid-Liquid Microextraction Technique in Uranium Dioxide Powder >> MALLAH MH 278(2008)97

\#460 ENVIRON. SCI. TECHNOL. 43(2009)3919-3925

Peterson, SA; Ralston, NVC; Peck, DV; Van Sickle, J; Robertson, JD; Spate, VL; Morris, JS: How Might Selenium Moderate the Toxic Effects of Mercury in Stream Fish of the Western US? >> VEATCH AE 264(2005)33 \#461 ENVIRON. SCI. TECHNOL. 43(2009)4280-4286

Um, W; Wang, ZM; Serne, RJ; Williams, BD; Brown, CF; Dodge, CJ; Francis, AJ: Uranium Phases in Contaminated Sediments below Hanford's U Tank Farm >> LEHMANN S 275(2008)633

\#462 ENVIRON. SCI. TECHNOL. 43(2009)5223-5228

Wang, J; Deng, BL; Chen, H; Wang, XR; Zheng, JZ: Removal of Aqueous $\mathrm{Hg}$ (II) by Polyaniline: Sorption Characteristics and Mechanisms >> DEVI PSR 269 (2006) 217

\#463 ENVIRON. SCI. TECHNOL. 43(2009)7258-7264

Schwehr, KA; Santschi, PH; Kaplan, DI; Yeager, CM; Brinkmeyer, R: Organo-Iodine Formation in Soils and Aquifer Sediments at Ambient Concentrations >> STEINBERG SM 277(2008)185

\#464 ENVIRON. SCI. TECHNOL. 43(2009)7665-7670

Muller, K; Foerstendorf, H; Brendler, V; Bernhard, G: Sorption of $\mathrm{Np}(\mathrm{V})$ onto $\mathrm{TiO} 2, \mathrm{SiO} 2$, and $\mathrm{ZnO}$ : An in Situ ATR FT-IR Spectroscopic Study $>$ PATHAK PN 274(2007)53; SABODINA MN 270(2006)349

\#465 ENVIRON. SCI. TECHNOL. 43(2009)8599-8603

Dimova, N; Burnett, WC; Lane-Smith, D: Improved Automated Analysis of Radon (Rn-222) and Thoron (Rn220) in Natural Waters $>>$ DULAIOVA H 263(2005)361 \#466 ENVIRON. SCI. TECHNOL. 43(2009)8807-8812

Finck, N; Schlegel, ML; Bosbach, D: Sites of Lu(III) Sorbed to and Coprecipitated with Hectorite $>>$ LOPEZGONZALEZ H 274(2007)103
\#467 ENVIRON. SCI. TECHNOL. 44(2010)1610-1616

Moody, C; Green, ID: Assimilation of $\mathrm{Cd}$ and $\mathrm{Cu}$ by the Carnivorous Plant Sarracenia leucophylla Raf. fed Contaminated Prey $>>$ STEINHAUSER G 274(2007)403 \#468 ESTUAR. COAST. SHELF SCI. 76(2008)501-511

Burnett, WC; Peterson, R; Moore, WS; de Oliveira, J: Radon and radium isotopes as tracers of submarine groundwater discharge-Results from the Ubatuba Brazil SGD assessment intercomparison $>$ DULAIOVA H 263(2005)361

\#469 ESTUAR. COAST. SHELF SCI. 76(2008)522-542

Povinec, PP; de Oliveira, J; Braga, ES; Comanducci, JF; Gastaud, J; Groening, M; Levy-Palomo, I; Morgenstern, U; Top, Z: Isotopic, trace element and nutrient characterization of coastal waters from Ubatuba inner shelf area, southeastern Brazil >> ALMEIDA KCS 270(2006)93; DEOLIVEIRA J 269(2006)689

\#470 ESTUAR. COAST. SHELF SCI. 83(2009)77-89

Swarzenski, PW; Izbicki, JA: Coastal groundwater dynamics off Santa Barbara, California: Combining geochemical tracers, electromagnetic seepmeters, and electrical resistivity $>>$ DULAIOVA H 263(2005)361

\#471 ESTUAR. COAST. SHELF SCI. 86(2010)165-178

Peterson, RN; Santos, IR; Burnett, WC: Evaluating groundwater discharge to tidal rivers based on a Rn-222 time-series approach $>>$ DULAIOVA H 263(2005)361 \#472 EUR. J. INORG. CHEM. (2007)5120-5126

Servaes, K; Hennig, C; Billard, I; Gaillard, C; Binnemans, K; Gorller-Walrand, C; Van Deun, R: Speciation of uranyl nitrato complexes in acetonitrile and in the ionic liquid 1-butyl-3-methylimidazolium bis(trifluoromethylsulfonyl)imide $>>$ GIRIDHAR P 265(2005)31 \#473 EUR. J. ORG. CHEM. (2008)2853-2873

Cai, LS; Lu, SY; Pike, VW: Chemistry with [F-18] fluoride ion $>>$ ALLABADI A 270(2006)313

\#474 EUR. J. RADIOL. 68(2008)S104-S108

Pereira, GR; Rocha, HS; Anjos, MJ; Farias, PCMA; Perez, CA; Lopes, RT: Elemental distribution mapping on breast tissue samples $>>$ PEREIRA GR 269(2006)469 \#475 EUR. J. SOIL SCI. 59(2008)476-485

Moradi, AB; Oswald, SE; Massner, JA; Pruessmann, KP; Robinson, BH; Schulin, R: Magnetic resonance imaging methods to reveal the real-time distribution of nickel in porous media $>>$ NAKANISHI TM 264(2005)313 \#476 EUR. PHYS. J. A 34(2007)271-274

Goodwin, JR; Golovko, VV; Iacob, VE; Hardy, JC: The half-life of Au-198: High-precision measurement shows no temperature dependence $>>$ LINDSTROM RM 263(2005)311 \#477 EUR. PHYS. J. A 40(2009)61-64

Meierhofer, G; Kudejova, P; Canella, L; Grabmayr, P; Jochum, J; Jolie, J: Thermal neutron capture cross-section of Ge-76 >> KUDEJOVA P 278(2008)691 
\#478 EUR. PHYS. J. A 41(2009)323-334

Naik, H; Singh, S; Reddy, AVR; Manchanda, VK; Ganesan, S; Raj, D; Rahman, MS; Kim, KS; Lee, MW; Kim, G; Oh, YD; Lee, HS; Cho, MH; Ko, IS; Namkung, $\mathrm{W}$ : Measurement of photo-fission yields and photo-neutron cross-sections in Bi-209 with 50 and $65 \mathrm{MeV}$ bremsstrahlung $>>$ SINGH S 279(2009)547

\#479 EUR. PHYS. J. B 57(2007)257-263

Nemeth, Z; Homonnay, Z; Arva, F; Klencsar, Z; Kuzmann, E; Vertes, A; Hakl, J; Meszaros, S; Vad, K; de Chatel, PF; Gritzner, G; Aoki, Y; Konno, H; Greneche, M: Response of La0.8Sr0.2CoO3-delta to perturbations on the CoO3 sublattice $>>$ NEMETH Z 271(2007)11

\#480 EUR. PHYS. J. C 46(2006)107-111

Widom, A; Larsen, L: Ultra low momentum neutron catalyzed nuclear reactions on metallic hydride surfaces >> MILEY GH 263(2005)691

\#481 EUR. POLYM. J. 44(2008)3899-3907

Zhang, AY; Wang, WH; Chai, ZF; Kuraoka, E: Modification of a novel macroporous silica-based crown ether impregnated polymeric composite with 1-dodecanol and its adsorption for some fission and non-fission products contained in high level liquid waste $>$ ZHANG A 265(2005)409; ZHANG A 269(2006)119

\#482 EXPERT OPIN. DRUG DELIV. 7(2010)173-185

Alvarez-Lorenzo, C; Bucio, E; Burillo, G; Concheiro, A: Medical devices modified at the surface by gamma-ray grafting for drug loading and delivery $>$ BUCIO E 280(2009)239

\#483 EXPRESS POLYM. LETT. 3(2009)268-278

Goel, NK; Bhardwaj, YK; Manoharan, R; Kumar, V; Dubey, KA; Chaudhari, CV; Sabharwal, S: Physicochemical and electrochemical characterization of battery separator prepared by radiation induced grafting of acrylic acid onto microporous polypropylene membranes $>>$ CHAUDHARI CV 267(2006)113; CHAUDHARI CV 278(2008)47 \#484 FOOD ADDIT. CONTAM. 25(2008)51-58

Svoboda, L; Chrastny, V: Levels of eight trace elements in edible mushrooms from a rural area $>$ RANDA $\mathrm{Z}$ 264(2005)67; REPINC U 264(2005)39; SHIRAISHI K 266(2005)61

\#485 FOOD ADDIT. CONTAM. PART A-CHEM. 26(2009)839-846

Li, N; Fang, G; Zhao, L; Wang, S: Determination of arsenic in foods by flow injection on-line sorption preconcentration with hydride generation atomic fluorescence spectrometry $>>$ YUSOF AM 271(2007)191

\#486 FOOD ADDIT. CONTAM. PART A-CHEM. 27(2010)327-337

Torres-Escribano, S; Velez, D; Montoro, R: Mercury and methylmercury bioaccessibility in swordfish $>>$ FARIAS LA 270(2006)217
\#487 FOOD ADDIT. CONTAM. PART B-SURVEILL. 1(2008)114-121

Gonzalvez, A; Armenta, S; De La Guardia, M: Trace elemental composition of curry by inductively coupled plasma optical emission spectrometry (ICP-OES) $>>$ CHOUDHURY RP 270(2006)187

\#488 FOOD ANAL. METH. 2(2009)303-310

Pashkova, GV: X-ray Fluorescence Determination of Element Contents in Milk and Dairy Products $>>$ SANTOS LGC 276(2008)107

\#489 FOOD CHEM. 104(2007)1454-1463

Choudhury, RP; Garg, AN: Variation in essential, trace and toxic elemental contents in Murraya koenigii-A spice and medicinal herb from different Indian states $>>$ CHOUDHURY RP 270(2006)187; GARG AN 263(2005) 39; GARG AN 271(2007)481 \#490 FOOD CHEM. 111(2008)490-497

Mendonca, JCF; Franca, AS; Oliveira, LS; Nunes, M: Chemical characterisation of non-defective and defective green arabica and robusta coffees by electrospray ionization-mass spectrometry (ESI-MS) $>$ SANTOS LS 269(2006)505

\#491 FOOD CHEM. 113(2009)110-114

Zhou, DR; Chen, YQ; Ni, DJ: Effect of water quality on the nutritional components and antioxidant activity of green tea extracts $>>$ CHAUDHRI MA 271(2007)697 \#492 FOOD CHEM. 114(2009)212-216

Samanta, TD; Laskar, S: Chemical investigation of Erythrina variegata Linn. seed proteins $>$ NAYAK D 271(2007)387

\#493 FOOD CHEM. 115(2009)706-710

Bhagat, PR; Acharya, R; Nair, AGC; Pandey, AK; Rajurkar, NS; Reddy, AVR: Estimation of iodine in food, food products and salt using ENAA $>$ HOU XL 271(2007)133; LANDSBERGER S 269(2006)697

\#494 FOOD CHEM. 116(2009)555-560

Maidana, NL; Vanin, VR; Horii, CL; Ferreira, FA; Rajput, MU: Absolute determination of soluble potassium in tea infusion by gamma-ray spectroscopy $>>$ HARB S 274(2007)63

\#495 FRESENIUS ENVIRON. BULL. 17(2008)326-330

Kulahci, F; Sen, Z: Perturbation distribution coefficient definition $>>$ KULAHCI F 268(2006)529

\#496 FRESENIUS ENVIRON. BULL. 17(2008)1763-1772

Matijevic, S; Bogner, D; Morovic, M; Ticina, V; Grbec, B: Characteristics of the sediment along the Eastern Adriatic Coast (Croatia) > OBHODAS J 270 (2006)75

\#497 FRESENIUS ENVIRON. BULL. 18(2009)468-473

Aytekin, H; Baldik, R: An investigation on the radiological influence of an underground coal mine in Zonguldak Basin, Turkey >> DULIU OG 264(2005)525 
\#498 FUEL 86(2007)807-812

Flues, M; Camargo, IMC; Figueiredo, PM; Silva, PSC; Mazzilli, BP: Evaluation of radionuclides concentration in Brazilian coals >> FLUES M 270(2006)597

\#499 FUSION ENG. DES. 82(2007)2582-2587

Oya, Y; Miyauchi, H; Suda, T; Nishikawa, Y; Yoshikawa, A; Tanaka, S; Okuno, K: Temperature dependence of hydrogen isotope behaviors in non-He + pre-implanted $\mathrm{SiC}$ and $\mathrm{He}+$ pre-implanted $\mathrm{SiC}>>$ OYA Y 272(2007)639 \#500 FUSION SCI. TECHNOL. 52(2007)1061-1065

Nishi, T; Yoneda, K; Masuda, K; Yoshikawa, K: Development of excited helium beam injector for electric field measurement by laser-induced fluorescence method >> MILEY GH 263(2005)159

\#501 FUSION SCI. TECHNOL. 52(2007)1114-1118

Takamatsu, T; Fujimoto, T; Masuda, K; Yoshikawa, K: Spatial distribution of D-D neutrons from a compact water-cooled inertial electrostatic confinement device $>>$ MILEY GH 263(2005)159

\#502 FUSION SCI. TECHNOL. 56(2009)523-527

Masuda, K; Nakagawa, T; Kajiwara, T; Zen, H; Yoshikawa, K; Nagasaki, K: Built-in ion source for inertial electrostatic confinement in low pressure regime $>>$ MILEY GH 263(2005)159

\#503 GENET. MOL. BIOL. 33(2010)44-50

Zhao, GP; Zheng, MQ; Chen, JL; Wen, J; Wu, CM; Li, WJ; Liu, LB; Zhang, Y: Differentially expressed genes in a flock of Chinese local-breed chickens infected with a subgroup $\mathrm{J}$ avian leukosis virus using suppression subtractive hybridization $>>$ OSHTRAKH MI 269(2006)671 \#504 GEOCHEM. GEOPHYS. GEOSYST. 7(2006) Q03020-

Cai, PH; Dai, MH; Lv, DW; Chen, WF: How accurate are Th-234 measurements in seawater based on the MnO2impregnated cartridge technique? $>$ PIKE SM 263(2005)355

\#505 GEOCHEM. GEOPHYS. GEOSYST. 9(2008)Q02010-

Cai, PH; Dai, MH; Lv, DW; Chen, WF: Reply to comment by Chin-Chang Hung et al. on "How accurate are Th-234 measurements in seawater based on the $\mathrm{MnO} 2$-impregnated cartridge technique?" >> PIKE SM 263(2005)355

\#506 GEOCHEM. J. 43(2009)415-422

Osawa, T; Hatsukawa, Y; Nagao, K; Koizumi, M; Oshima, M; Toh, Y; Kimura, A; Furutaka, K: Iridium concentration and noble gas composition of CretaceousTertiary boundary clay from Stevns Klint, Denmark >> OSHIMA M 278(2008)257

\#507 GEOCHEM.-EXPLOR. ENVIRON. ANAL. 9(2009) 275-278

Carvalho, FP; Oliveira, JM; Neves, MO; Abreu, MM; Vicente, EM: Soil to plant (Solanum tuberosum L.) radionuclide transfer in the vicinity of an old uranium mine >> CARVALHO FP 274(2007)167
\#508 GEOCHIM. COSMOCHIM. ACTA 70(2006)28682884

Di Muro, A; Villemant, B; Montagnac, G; Scaillet, B; Reynard, B: Quantification of water content and speciation in natural silicic glasses (phonolite, dacite, rhyolite) by confocal microRaman spectrometry >> ARIAS A 268(2006)371 \#509 GEOCHIM. COSMOCHIM. ACTA 70(2006)47504769

Marschall, HR; Altherr, R; Ludwig, T; Kalt, A; Gmeling, K; Kasztovszky, Z: Partitioning and budget of Li, $\mathrm{Be}$ and $\mathrm{B}$ in high-pressure metamorphic rocks $>>$ GMELING K 265(2005)201; MARSCHALL HR 265(2005)339 \#510 GEOCHIM. COSMOCHIM. ACTA 71(2007)26722679

Reilly, SD; Runde, W; Neu, MP: Solubility of plutonium(VI) carbonate in saline solutions $>>$ MOORE RC 263(2005)97

\#511 GEOCHIM. COSMOCHIM. ACTA 71(2007)A906A906

Schubert, M; Schmidt, A; Scholten, J; van der Loeff, MMR; Schlueter, M: Using Rn-222 for assessing nutrient transfer into the sea via SGD $>$ DULAIOVA $\mathrm{H}$ 263(2005)361

\#512 GEOCHIM. COSMOCHIM. ACTA 72(2008)54755504

Vils, F; Pelletier, L; Kalt, A; Muntener, O; Ludwig, T: The Lithium, Boron and Beryllium content of serpentinized peridotites from ODP Leg 209 (Sites 1272A and 1274A): Implications for lithium and boron budgets of oceanic lithosphere >> GMELING K 265(2005)204; MARSCHALL HR 265(2005)339

\#513 GEOCHIM. COSMOCHIM. ACTA 73(2009)197217

Mercier, M; Di Muro, A; Giordano, D; Metrich, N; Lesne, P; Pichavant, M; Scaillet, B; Clocchiatti, R; Montagnac, G: Influence of glass polymerisation and oxidation on micro-Raman water analysis in alumino-silicate glasses >> ARIAS A 268(2006)371

\#514 GEOCHIM. COSMOCHIM. ACTA 73(2009)16661683

Lintern, MJ; Hough, RM; Ryan, CG; Watling, J; Verrall, M: Ionic gold in calcrete revealed by LA-ICP-MS, SXRF and XANES $>>$ CHAUDHRI MA 271(2007)713 \#515 GEOCHRONOMETRIA 30(2008)17-22

Takeuchi, T; Shibutani, T; Hashimoto, T: Construction of a portable mini luminescence-measurement system equipped with a miniature X-ray generator $>>$ NAKATA Y 272(2007)433 \#516 GEOCHRONOMETRIA 31(2008)53-63

Nielsen, AH; Murray, AS: The effects of holocene podzolisation on radionuclide distributions and dose rates in sandy coastal sediments $>$ KUBICA B 266(2005)3 
\#517 GEOCHRONOMETRIA 32(2008)29-35

Gasiorowski, M: Deposition rate of lake sediments under different alternative stable states $>$ GACA $P$ 267(2006)443

\#518 GEODERMA 153(2009)254-268

Tejada, M: Application of different organic wastes in a soil polluted by cadmium: Effects on soil biological properties >> PALAGYI S 269(2006)103

\#519 GEOMICROBIOL. J. 24(2007)205-210

Takenaka, Y; Saito, T; Nagasaki, S; Tanaka, S; Kozai, N; Ohnuki, T: Metal sorption to Pseudomonas fluorescens: Influence of $\mathrm{pH}$, ionic strength and metal concentrations $>>$ MIZERA J 263(2005)75 \#520 GEOMORPHOLOGY 76(2006)430-440

Kaste, JM; Heimsath, AM; Hohmann, M: Quantifying sediment transport across an undisturbed prairie landscape using cesium-137 and high resolution topography $>>$ REGUIGUI N 264(2005)469

\#521 GEOMORPHOLOGY 115(2010)11-22

Detriche, S; Rodrigues, S; Macaire, JJ; Bonte, P; Breheret, JG; Bakyono, JP; Juge, P: Caesium-137 in sandy sediments of the River Loire (France): Assessment of an alluvial island evolving over the last 50 years $>>$ DION HM 264(2005)481

\#522 GEOPHYS. RES. LETT. 33(2006)L05606-

Dulaiova, H; Burnett, WC: Radon loss across the waterair interface (Gulf of Thailand) estimated experimentally from Rn-222-Ra-224 >> DULAIOVA H 263(2005)361 \#523 GEOPHYS. RES. LETT. 33(2006)L24405-

Swarzenski, PW; Burnett, WC; Greenwood, WJ; Herut, B; Peterson, R; Dimova, N; Shalem, Y; Yechieli, Y; Weinstein, $\mathrm{Y}$ : Combined time-series resistivity and geochemical tracer techniques to examine submarine groundwater discharge at Dor Beach, Israel > > DULAIOVA H 263(2005)361 \#524 GEOPHYS. RES. LETT. 35(2008)L01604-

Aoyama, M; Hirose, K; Nemoto, K; Takatsuki, Y; Tsumune, D: Water masses labeled with global fallout Cs137 formed by subduction in the North Pacific $>>$ HIROSE K 263(2005)349

\#525 GEOSTAND. GEOANAL. RES. 30(2006)183-186

Bedard, LP: Neutron activation analysis, atomic absorption and X-ray fluorescence spectrometry review for 2004-2005 >> LUKE PN 264(2005)145

\#526 GEOSTAND. GEOANAL. RES. 30(2006)273-305

Valladon, M: Geostandards and geoanalytical research bibliographic review 2005 >> CHUNG YS 267(2005)95; DAMPARE SB 265(2005)101; DAMPARE SB 265(2005) 53; GMELING K 265(2005)201; GRANT CN 266(2005) 385; JIANG SS 264(2005)549; KFOURI PBP 265(2005)459; LAROSA J 263(2005)427; LEE SH 263 (2005)419; MACKEY EA 265(2005)273; NORISUYE K 267(2005)183; RAMOS AA 266(2005)19; REPINC U 264(2005)39; ZHENG J 267(2005)73
\#527 GEOSTAND. GEOANAL. RES. 31(2007)275-283

Randa, Z; Frana, J; Mizera, J; Kucera, J; Novak, JK; Ulrych, J; Belov, AG; Maslov, OD: Instrumental neutron and photon activation analysis in the geochemical study of phonolitic and trachytic rocks $>$ RANDA Z 264 (2005)67

\#528 GEOSTAND. GEOANAL. RES. 32(2008)399-403

Bedard, LP: Neutron Activation Analysis, Atomic Absorption and X-Ray Fluorescence Spectrometry Review for 2006-2007 >> CINCU E 271(2007)655; LINDSTROM RM 271(2007)311; RANDA Z 271(2007)589 \#529 GEOSTAND. GEOANAL. RES. 32(2008)405-452

Jochum, KP; Brueckner, SM: Reference Materials in Geoanalytical and Environmental Research-Review for 2006 and $2007>>$ ABUGASSA IO 271(2007)27; AGRAWAL YK 270(2006)453; AKAMINE AU 271(2007)607; ALRAYYES AH 274(2007)139; CHUNG YS 271(2007)339; CONSTANTIN M 267(2006)407; DEFRANCA EJ 271(2007)405; FAVARO DIT 273(2007)451; GARG AN 271(2007)481; GROSHEVA E 271(2007)565; HATSUKAWA Y 271(2007)43; HU SM 271(2007)517; JIA G 273(2007)779; JIA GG 267(2006) 505; KAFALA SI 271(2007)507; KOMOSA A 269 (2006)195; MENEZES MABC 270(2006)111; MINOWA H 272(2007)321; MOON JH 271(2007)289; OURA Y 271(2007)305; PAPAEFTHYMIOU H 274(2007)123; RAHMAN ATA 273(2007)653; RANDA Z 271(2007)589; SOLECKI J 274(2007)27; SOUPIONI MJ 268(2006)441; TAGAMI K 273(2007)147; TAKEDA M 272(2007)363; TAYLOR VF 274(2007)545; YUSOF AM 271(2007)191 \#530 GEOSTAND. GEOANAL. RES. 33(2009)115-132

Constantin, M: Trace Element Data for Gold, Iridium and Silver in Seventy Geochemical Reference Materials >> CONSTANTIN M 267(2006)407

\#531 GEOSTAND. GEOANAL. RES. 33(2009)249-259

Savard, D; Bedard, LP; Bames, SJ: Selenium Concentrations in Twenty-Six Geological Reference Materials: New Determinations and Proposed Values $>>$ CONSTANTIN M 267(2006)407

\#532 GLOB. ENVIRON. CHANGE-HUMAN POLICY DIMENS. 19(2009)292-305

Cordell, D; Drangert, JO; White, S: The story of phosphorus: Global food security and food for thought $>>$ SAUEIA CH 264(2005)445

\#533 GLOB. NEST. J. 9(2007)229-236

Konstantinou, M; Demetriou, A; Pashalidis, I: Adsorption of hexavalent uranium on dunite $>>$ DONAT R 265(2005)107; KILINCARSLAN A 264(2005)541; SHAWKY S 265(2005)81 \#534 GREEN CHEM. 9(2007)1160-1162

Ouadi, A; Klimchuk, O; Gaillard, C; Billard, I: Solvent extraction of $\mathrm{U}(\mathrm{VI})$ by task specific ionic liquids bearing phosphoryl groups $>>$ GIRIDHAR P 265(2005)31 
\#535 HEALTH PHYS. 93(2007)120-126

Alvarez, JL: Poisson-based detection limit and signal confidence intervals for few total counts $>>$ POTTER WE 263(2005)321

\#536 HEALTH PHYS. 95(2008)397-406

Ruberu, SR; Liu, YG; Perera, SK: An improved liquid scintillation counting method for the determination of gross alpha activity in groundwater wells $>$ WONG CT 264(2005)357

\#537 HEALTH PHYS. 95(2008)S110-S121

Magnusson, A; Aronsson, PO; Lundgren, K; Stenstrom, $\mathrm{K}$ : Characterization of C-14 in Swedish light water reactors $>$ MAGNUSSON A 275(2008)261 \#538 HEALTH PHYS. 96(2009)5-12

Shiraishi, K; Ko, S; Muramatsu, Y; Zamostyan, PV; Tsigankov, NY: Dietary iodine and bromine intakes in Ukrainian subjects $>>$ KO S 267(2006)575

\#539 HEALTH PHYS. 96(2009)27-36

Misdaq, MA; Karime, M: Analysis of U-238, Th-232, $\mathrm{Rn}-222$, and Rn-220 in different medical drug preparations by using CR-39 AND LR-115 Type II SSNTDs and resulting radiation doses to adult patients $>>$ MISDAQ MA 270(2006)543

\#540 HEALTH PHYS. 96(2009)636-645

Li, WB; Karpas, Z; Salonen, L; Kurttio, P; Muikku, M; Wahl, W; Hollriegl, V; Hoeschen, C; Oeh, U: A compartmental model of uranium in human hair for protracted ingestion of natural uranium in drinking water $>>$ MOHAGHEGHI AH 263(2005)189

\#541 HEALTH PHYS. 96(2009)646-654

Muikku, M; Puhakainen, M; Heikkinen, T; Ilus, T: The mean concentration of uranium in drinking water, urine, and hair of the occupationally unexposed finnish working population $>>$ AKAMINE AU 271(2007)607

\#542 HETEROATOM CHEM. 20(2009)164-171

Sene, A; Masson, S; Vazeux, M: Synthesis of alphaPhosphonylated Phosphonium and Sulfonium Ylides: Study of Their Thermal Behavior $>$ RIDONE $S$ 277(2008)117

\#543 HETEROCYCL. COMMUN. 13(2007)183-188

Li, JY; Zhang, JY; Zhou, XZ; Zhang, P: Synthesis of [H-3] quinocetone $>>$ LI JY 265(2005)17

\#544 HFSP J. 2(2008)189-204

Kiser, MR; Reid, CD; Crowell, AS; Phillips, RP; Howell, CR: Exploring the transport of plant metabolites using positron emitting radiotracers $>$ TANOI $\mathrm{K}$ 263(2005)547

\#545 HIGH ENERGY CHEM. 41(2007)370-380

Shantarovich, VP; Suzuki, T; Yampol'skii, YP; Budd, P; Gustov, VV; Kevdina, IB; Pastukhov, AV; Berdonosov, SS; Bozhevol'nov, VE: Positronium annihilation data and actual free-volume distribution in polymers $>>$ SHANTAROVICH VP 272(2007)645
\#546 HIGH ENERGY PHYS. NUCL. PHYS.-CHIN. ED. 30(2006)310-313

Ding, BG; Zhang, DL; Lu, DH: Closed shell at $\mathrm{N}=184$ for the isotope chain of neutron rich nuclei $\mathrm{Z}=96-102>>$ DING BG 265(2005)515

\#547 HUM. ECOL. RISK ASSESS. 13(2007)1359-1372

Flores-Rangel, RM; Rodriguez-Espinosa, PF; Montes de Oca-Valero, JA; Mugica-Alvarez, V; Ortiz-Romero-Vargas, ME; Navarrete-Lopez, M; Dorantes-Rosales, HJ: Metal content in air samples collected in an urban zone in Tampico, Mexico: A first survey >> LOPES F 270(2006)43 \#548 HYDROBIOLOGIA 631(2009)289-302

Guilizzoni, P; Massaferro, J; Lami, A; Piovano, EL; Guevara, SR; Formica, SM; Daga, R; Rizzo, A; Gerli, S: Palaeolimnology of Lake Hess (Patagonia, Argentina): multi-proxy analyses of short sediment cores >> DAGA R 270(2006)677 \#549 HYDROL. EARTH SYST. SCI. 11(2007)1621-1631

Kluge, T; Ilmberger, J; von Rohden, C; AeschbachHertig, W: Tracing and quantifying groundwater inflow into lakes using a simple method for radon-222 analysis $>>$ DULAIOVA H 263(2005)361

\#550 HYDROMETALLURGY 81(2006)30-39

Giridhar, P; Venkatesan, KA; Srinivasan, TG; Rao, PRV: Extraction of fission palladium by Aliquat 336 and electrochemical studies on direct recovery from ionic liquid phase $>>$ GIRIDHAR P 265(2005)31; VENKATESAN KA 266(2005)431

\#551 HYDROMETALLURGY 86(2007)221-229

Venkatesan, KA; Selvan, BR; Antony, MP; Srinivasan, TG; Rao, PRV: Extraction of palladium (II) from nitric acid medium by imidazolium nitrate immobilized resin $>>$ VENKATESAN KA 266(2005)431

\#552 HYDROMETALLURGY 92(2008)1-10

Parodi, A; Vincent, T; Pilsniak, M; Trochimczuk, AW; Guibal, E: Palladium and platinum binding on an imidazol containing resin $>$ DAKSHINAMOORTHY A 266(2005)425

\#553 HYDROMETALLURGY 95(2009)15-21

Birinci, E; Gulfen, M; Aydin, AO: Separation and recovery of palladium(II) from base metal ions by melamine-formaldehyde-thiourea (MFT) chelating resin $>>$ VENKATESAN KA 266(2005)431 \#554 HYDROMETALLURGY 96(2009)246-252

Ghazvini, PTM; Mashkani, SG: Screening of bacterial cells for biosorption of oxyanions: Application of microPIXE for measurement of biosorption $>$ LIU $\mathrm{N}$ 275(2008)173 \#555 HYPERFINE INTERACT. 165(2005)303-308

Kamnev, AA; Kulikov, LA; Perfiliev, YD; Antonyuk, LP; Kuzmann, E; Vertes, A: Application of Co-57 emission Mossbauer spectroscopy to studying biocomplexes in frozen solutions $>>$ PERFILIEV YD 266(2005) 557 
\#556 HYPERFINE INTERACT. 166(2005)429-436

Kubuki, S; Kawakami, N; Kamikawa, T; Fukagawa, M; Nishizumi, T; Nishida, T; Homonnay, Z; Kuzmann, E: Corelationship between local structure and water purifying ability of iron-containing waste glasses $>$ NISHIDA T 266(2005)171

\#557 HYPERFINE INTERACT. 181(2008)45-52

Oshtrakh, MI; Milder, OB; Semionkin, VA: A study of human liver ferritin and chicken liver and spleen using Mossbauer spectroscopy with high velocity resolution $>>$ OSHTRAKH MI 269(2006)671

\#558 HYPERFINE INTERACT. 182(2008)65-75

Yamada, Y; Kouno, K; Kato, H; Kobayashi, Y: Reaction and deposition of laser-evaporated iron $>>$ YOKOYAMA D 268(2006)283; YOKOYAMA D 272(2007)631

\#559 HYPERFINE INTERACT. 184(2008)63-68

Nemeth, Z; Klencsar, Z; Kuzmann, E; Homonnay, Z; Vertes, A; Greneche, JM; Bodogh, M: Relaxation of magnetic clusters in $\mathrm{Sr}$ and Fe doped cobaltate perovskites $>>$ NEMETH Z 271(2007)11

\#560 HYPERFINE INTERACT. 184(2008)123-128

Nomura, K; Suzuki, Y; Iio, S; Yajima, T; Yamada, Y; Hirose, Y; Hasegawa, T: Sol-gel synthesized powder and pulsed laser deposited film of amorphous indium zinc oxides doped with Fe $>>$ YOKOYAMA D 272(2007)631 \#561 HYPERFINE INTERACT. 187(2008)49-55

Kobayashi, Y; Yamada, Y; Tsuruoka, Y; Kubo, MK; Shoji, H; Watanabe, Y; Takayama, T; Sakai, Y; Sato, W; Shinohara, A; Segawa, M; Matsue, H: Neutron in-beam Mossbauer spectroscopy of iron disulfide at 298 and $78 \mathrm{~K}$ $>$ KOBAYASHI Y 272(2007)623

\#562 ICARUS 204(2009)368-380

Jorgensen, UG; Appel, PWU; Hatsukawa, Y; Frei, R; Oshima, M; Toh, Y; Kimura, A: The Earth-Moon system during the late heavy bombardment period-Geochemical support for impacts dominated by comets $>>$ HATSUKAWA Y 272(2007)273; OSHIMA M 278(2008)257 \#563 IEEE J. SEL. TOP. QUANTUM ELECTRON. 13(2007)1715-1720

Zaman, RT; Diagaradjane, P; Wang, JC; Schwartz, J; Rajaram, N; Gill-Sharp, KL; Cho, SH; Rylander, HG; Payne, JD; Krishnan, S; Tunnell, JW: In vivo detection of gold nanoshells in tumors using diffuse optical spectroscopy $>>$ JAMES WD 271(2007)455

\#564 IEEE TRANS. NUCL. SCI. 52(2005)1659-1664

Hawari, AI; Chen, JW: Computational investigation of on-line interrogation of pebble bed reactor fuel $>>$ HAWARI AI 264(2005)265

\#565 IEEE TRANS. NUCL. SCI. 53(2006)620-624

Hennig, W; Tan, H; Warburton, WK; McIntyre, JI: Single-channel beta-gamma coincidence detection of radioactive xenon using digital pulse shape analysis of phoswich detector signals $>>$ ELY JH 263(2005)245
\#566 IEEE TRANS. NUCL. SCI. 54(2007)327-332

Lu, XW; Wang, FL; Ha, XD; Wang, LQ: Radioactive analysis and radiological hazards of lime and cement fabricated in China $>>$ XINWEI L 267(2006)669

\#567 IEEE TRANS. NUCL. SCI. 56(2009)441-447

Chichester, DL; Seabury, EH: Using Electronic Neutron Generators in Active Interrogation to Detect Shielded Fissionable Material >> CHICHESTER DL 271(2007)629 \#568 IEEE TRANS. NUCL. SCI. 56(2009)1306-1311

Wu, Y; Hurley, JP; Ji, Q; Kwan, J; Leung, KN: Development of a RF-Driven Neutron Generator for Associated Particle Imaging >> CHICHESTER DL 271(2007)629 \#569 IEEE TRANS. NUCL. SCI. 56(2009)1448-1453

Lima, I; Assis, JT; Apoloni, CR; de Souza, SMFM; Duarte, MEL; Lopes, RT: Non-Destructive Imaging Materials Investigation by Microfocus 3D X-Ray Computed Tomography >> LIMA ICB 269(2006)639 \#570 IEEE TRANS. NUCL. SCI. 56(2009)2628-2635

Chen, LY; Gobar, LS; Knowles, NG; Wilson, DW; Barrett, HH: Direct Charged-Particle Imaging System Using an Ultra-Thin Phosphor: Physical Characterization and Dynamic Applications >> KANNO S 272(2007)565 \#571 IEEE TRANS. NUCL. SCI. 57(2010)309-316

Pfund, DM; Jarman, KD; Milbrath, BD; Kiff, SD; Sidor, DE: Low Count Anomaly Detection at Large Standoff Distances $>>$ ANDERSON KK 276(2008)713

\#572 IN VIVO 22(2008)93-100

Enginar, H; Unak, P; Lambrecht, FY; Muftuler, FZB; Medine, EI: Synthesis and biodistribution studies of two novel radiolabeled estrone derivatives $>$ BIBER FZ 266(2005)445; ENGINAR H 264(2005)535

\#573 IND. ENG. CHEM. RES. 46(2007)2164-2171

Zhang, AY; Xiao, CL; Kuraoka, E; Kumagai, M: Preparation of a novel silica-based DtBuCH18C6 impregnated polymeric composite modified by tri-n-butyl phosphate and its application in chromatographic partitioning of strontium from high level liquid waste $>$ ZHANG A 265(2005)409

\#574 IND. ENG. CHEM. RES. 47(2008)2349-2355

Zuo, Y; Liu, Y; Chen, J; Li, DQ: The separation of Cerium(IV) from nitric acid solutions containing Thorium(IV) and Lanthanides(III) using pure [C(8)mim]PF6 as extracting phase $>>$ GIRIDHAR P 265(2005)31 \#575 IND. ENG. CHEM. RES. 47(2008)6158-6165

Zhang, AY; Hu, QH; Wang, WH; Kuraoka, E: Application of a macroporous silica-based CMPO-impregnated polymeric composite in group partitioning of long-lived minor actinides from highly active liquid by extraction chromatography >> ZHANG A 265(2005)409; ZHANG A 269(2006)119

\#576 IND. ENG. CHEM. RES. 48(2009)2085-2092

Kim, KW; Kim, YH; Lee, SY; Lee, EH; Song, KC; Song, K: Study on Electrolytic Recoveries of Carbonate 
Salt and Uranium from a Uranyl Peroxo Carbonato Complex Solution Generated from a Carbonate-Leaching Process $>>$ KIM KW 275(2008)595

\#577 IND. ENG. CHEM. RES. 48(2009)6173-6187

Yang, YJ; Alexandratos, SD: Affinity of Polymer-Supported Reagents for Lanthanides as a Function of Donor Atom Polarizability >> NOGAMI M 273(2007)37 \#578 IND. ENG. CHEM. RES. 49(2010)2047-2054

Zhang, AY; Hu, QH; Chai, ZF: Synthesis of a Novel Macroporous Silica-Calix [4]arene-Crown Polymeric Composite and Its Adsorption for Alkali Metals and Alkaline-Earth Metals >> ZHANG A 269(2006)119 \#579 INDIAN J. CHEM. SECT A-INORG. BIO-INORG. PHYS. THEOR. ANAL. CHEM. 46(2007)1618-1620

Amoli, HS; Barker, J: Rapid analysis of americium and plutonium in environmental samples by alpha-spectrometry $>$ AMOLI HS 268(2006)497; AMOLI HS 273(2007)281 \#580 INDIAN J. CHEM. SECT A-INORG. BIO-INORG. PHYS. THEOR. ANAL. CHEM. 48(2009)30-37

Pramanik, S; Sarkar, S; Paul, H; Chattopadhyay, P: A new chelating polymer with 2-methoxy-1-imidazolylazobenzene functionality for determination of lead(II) and iron(III) >> PRAMANIK S 274(2007)237

\#581 INDIAN J. CHEM. TECHNOL. 15(2008)291-297

Shilmkar, TN; Kolekar, SS; Wadgaonkar, PP; Anuse, MA: Rapid extraction and separation of indium(III) with a high molecular weight amine $>>$ KATAGI S 274(2007)251 \#582 INDIAN J. CHEM. TECHNOL. 16(2009)84-88

Curkovic, L; Trgo, M; Mioc, AR; Medvidovic, NV: Removal of $\mathrm{Cu}$ and $\mathrm{Pb}$ ions from aqueous solutions by electric furnace slag: Kinetic and thermodynamic aspects >> ZAKARIA ES 264(2005)629

\#583 INDIAN J. PHYS. 83(2009)1129-1133

Agarwal, C; Kalsi, PC; Goswami, A: Study of fission track registration efficiency of gamma-irradiated polyester track detector in solution medium $>>$ KALSI PC 273(2007)473 \#584 INDIAN J. TRADIT. KNOWL. 8(2009)346-351

Singh, SK; Chaudhary, A; Rai, DK; Rai, SB: Preparation and characterization of a mercury based Indian traditional drug-Ras-Sindoor $>>$ GARG AN 271(2007)611; KUMAR A 270(2006)173

\#585 INDOOR BUILT ENVIRON. 17(2008)384-389

Brahmanandhan, GM; Selvasekarapandian, S; Malathi, J; Khanna, D; Meenakshisundaram, V; Santhanam, S; Balasundar, S; Dhanalakshmi, B: Seasonal variation of indoor gamma dose and radon, thoron measurement in the dwellings around Kudankulam Nuclear Power Plant (Radhapuram taluk, Tirunelveli district) $>>$ BRAHMANANDHAN GM 274(2007)361

\#586 INORG. CHEM. 45(2006)1839-1846

Reilly, SD; Neu, MP: Pu(VI) hydrolysis: Further evidence for a dimeric plutonyl hydroxide and contrasts with U(VI) chemistry >> MOORE RC 263(2005)97
\#587 INORG. CHEM. 45(2006)3657-3664

Charkoudian, LK; Franz, KJ: Fe(III)-coordination properties of neuromelanin components: 5,6-dihydroxyindole and 5,6-dihydroxyindole-2-carboxylic acid >> ZECCA L 263(2005)733

\#588 INORG. CHEM. 47(2008)1087-1095

Tesema, YT; Pham, DM; Franz, KJ: Counterions influence reactivity of metal ions with cysteinyldopa model compounds $>>$ ZECCA L 263(2005)733

\#589 INORG. CHEM. 47(2008)4691-4695

Kannan, S; Moody, MA; Barnes, CL; Duval, PB: Lanthanum(III) and uranyl(VI) diglycolamide complexes: Synthetic precursors and structural studies involving nitrate complexation $>>$ ZHANG A 265(2005)409

\#590 INORG. CHEM. 47(2008)11351-11360

Maslova, MV; Rusanova, D; Naydenov, V; Antzutkin, ON; Gerasilmova, LG: Synthesis, Characterization, and Sorption Properties of Amorphous Titanium Phosphate and Silica-Modified Titanium Phosphates >> PATHAK PN 270(2006)299

\#591 INORG. CHEM. 48(2009)5350-5360

Hennig, C; Ikeda-Ohno, A; Tsushima, S; Scheinost, AC: The Sulfate Coordination of $\mathrm{Np}(\mathrm{IV}), \mathrm{Np}(\mathrm{V})$, and $\mathrm{Np}(\mathrm{VI})$ in Aqueous Solution >> XIA Y 268(2006)445 \#592 INORG. CHEM. 48(2009)9485-9490

Vazquez, GJ; Dodge, CJ; Francis, AJ: Bioreduction of U(VI)-Phthalate to a Polymeric U(IV)-Phthalate Colloid $>>$ SINGHAL RK 265(2005)405

\#593 INORG. CHEM. 48(2009)11935-11944

Tkac, P; Precek, M; Paulenova, A: Redox Reactions of $\mathrm{Pu}(\mathrm{IV})$ and $\mathrm{Pu}(\mathrm{III})$ in the Presence of Acetohydroxamic Acid in HNO3 Solutions >> LEE MH 273(2007)375; TKAC P 280(2009)339

\#594 INORG. CHIM. ACTA 360(2007)3705-3711

Thakur, P; Singh, DK; Choppin, GR: Polymerization study of o-Si(OH)(4) and complexation with Am(III), $\mathrm{Eu}(\mathrm{III})$ and $\mathrm{Cm}(\mathrm{III})>>$ PATHAK PN 267(2006)309; PATHAK PN 267(2006)175 \#595 INORG. CHIM. ACTA 361(2008)2522-2532

Klaehn, JR; Peterman, DR; Harrup, MK; Tillotson, RD; Luther, TA; Law, JD; Daniels, LM: Synthesis of symmetric dithiophosphinic acids for "minor actinide" extraction $>>$ XU QC 273(2007)235

\#596 INORG. CHIM. ACTA 362(2009)3253-3258

Di Giandomenico, MV; Le Naour, C: Complex formation between protactinium $(\mathrm{V})$ and sulfate ions at 10 and 60 degrees C $>$ XIA Y 268(2006)445; XIA YX 274(2007)79

\#597 INORG. CHIM. ACTA 363(2010)263-269

Ray, RS; Kruger, S; Rosch, N: Ternary uranyl hydroxo acetate complexes: A computational study of structure, energetics, and stability constants $>>$ CHOPPIN GR 273(2007)695; PASHALIDIS I 273(2007)315 
\#598 INORG. MATER. 43(2007)638-641

Stolyar, SV; Bayukov, OA; Gurevich, YL; Ladygina, VP; Iskhakov, RS; Pustoshilov, PP: Mossbauer study of bacterial ferrihydrite $>>$ OSHTRAKH MI 269(2006)671 \#599 INT. J. COAL GEOL. 76(2008)227-236

Depoi, FS; Pozebon, D; Kalkreuth, WD: Chemical characterization of feed coals and combustion-by-products from Brazilian power plants $>>$ FLUES M 270(2006)597 \#600 INT. J. COAL GEOL. 78(2009)233-248

Siavalas, G; Linou, M; Chatziapostolou, A; Kalaitzidis, S; Papaefthymiou, H; Christanis, K: Palaeoenvironment of Seam I in the Marathousa Lignite Mine, Megalopolis Basin (Southern Greece) >> PAPAEFTHYMIOU H 274(2007)123 \#601 INT. J. ENVIRON. ANAL. CHEM. 88(2008)141150

Baysal, A; Tokman, N; Akman, S: The use of solid-phase extraction and direct injection of a copolymer sorbent as slurry into the graphite furnace prior to determination of cadmium by ETAAS $>>$ OZEROGLU C 268(2006)211

\#602 INT. J. ENVIRON. ANAL. CHEM. 89(2009)223231

Faghihian, H; Hajishabani, A; Dadfarnia, S; Zamani, H: Use of clinoptilolite loaded with 1-(2-pyridylazo)-2-naphthol as a sorbent for preconcentration of $\mathrm{Pb}(\mathrm{II}), \mathrm{Ni}(\mathrm{II})$, $\mathrm{Cd}(\mathrm{II})$ and $\mathrm{Cu}(\mathrm{II})$ prior to their determination by flame atomic absorption spectroscopy > > AKYIL S 266(2005)89 \#603 INT. J. ENVIRON. POLLUT. 32(2008)434-455

Freitas, MD; Marques, AP; Reis, MA; Farinha, MM: Atmospheric dispersion of pollutants in Sado estuary (Portugal) using biomonitors $>$ FREITAS MC 263 (2005)711

\#604 INT. J. ENVIRON. RES. PUBLIC HEALTH 6(2009)2470-2480

Tao, XQ; Lu, GN; Liu, JP; Li, T; Yang, LN: Rapid Degradation of Phenanthrene by Using Sphingomonas sp GY2B Immobilized in Calcium Alginate Gel Beads $>>$ CATALDO F 267(2006)679

\#605 INT. J. FOOD SCI. NUTR. 60(2009)40-50

Turker, AR; Erol, E: Optimization of selenium determination in chicken's meat and eggs by the hydride-generation atomic absorption spectrometry method $>>$ NAVARRETE JM 271(2007)599

\#606 INT. J. HYG. ENVIRON. HEALTH. 212(2009)7681

Krystek, P; Ritsema, R: An incident study about acute and chronic human exposure to uranium by high-resolution inductively coupled plasma mass spectrometry (HR-ICPMS) >> MOHAGHEGHI AH 263(2005)189

\#607 INT. J. MASS SPECTROM. 255(2006)312-327

Bandura, DR; Baranov, VI; Litherland, AE; Tanner, SD: Gas-phase ion-molecule reactions for resolution of atomic isobars: AMS and ICP-MS perspectives >> GONZALES ER 263(2005)457
\#608 INT. J. MASS SPECTROM. 257(2006)27-33

Zoriy, MV; Dehnhardt, M; Reifenberger, G; Zilles, K; Becker, JS: Imaging of $\mathrm{Cu}, \mathrm{Zn}, \mathrm{Pb}$ and $\mathrm{U}$ in human brain tumor resections by laser ablation inductively coupled plasma mass spectrometry >> ELLISTON JT 263(2005)301 \#609 INT. J. MASS SPECTROM. 264(2007)175-180

Zoriy, MV; Becker, JS: Imaging of elements in thin cross sections of human brain samples by LA-ICP-MS: A study on reproducibility $>>$ ELLISTON JT 263(2005)301 \#610 INT. J. MASS SPECTROM. 270(2008)118-126

Pitois, A; de las Heras, LA; Betti, M: Determination of fission products in nuclear samples by capillary electrophoresis-inductively coupled plasma mass spectrometry (CE-ICP-MS) >> WOLF SF 263(2005)575; WOLF SF 263(2005)581

\#611 INT. J. MASS SPECTROM. 279(2009)87-92

Jakopic, R; Richter, S; Kuhn, H; Benedik, L; Pihlar, B; Aregbe, Y: Isotope ratio measurements of pg-size plutonium samples using TIMS in combination with "multiple ion counting" and filament carburization $>>$ HRNECEK E 276(2008)789

\section{\#612 INT. J. MASS SPECTROM. 286(2009)7-10}

Watrous, MG; Delmore, JE: Metal dicarbides as intermediate species in thermal ion formation mechanisms $>>$ KUROSAKI H 269(2006)279

\#613 INT. J. MASS SPECTROM. 286(2009)70-82

Burger, S; Riciputi, LR; Bostick, DA; Turgeon, S; McBay, EH; Lavelle, M: Isotope ratio analysis of actinides, fission products, and geolocators by high-efficiency multicollector thermal ionization mass spectrometry $>>$ BUCHMANN JH 270(2006)291; BURGER S 274(2007) 491; CHAI JY 272(2007)397; EFURD DW 263(2005)387; LEE CG 272(2007)299; MCCURDY D 263(2005)447; MOHAGHEGHI AH 263(2005)189; NYGREN U 272 (2007)45; WOLF SF 263(2005)581

\#614 INT. J. MOD. PHYS. E-NUCL. PHYS. 15(2006) 911-924

Cardone, F; Mignani, R: Piezonuclear reactions and Lorentz invariance breakdown $>$ CARDONE F 265 (2005) 151

\#615 INT. J. MOL. SCI. 10(2009)2633-2661

Roques, J; Veilly, E; Simoni, E: Periodic Density Functional Theory Investigation of the Uranyl Ion Sorption on Three Mineral Surfaces: A Comparative Study $>>$ XU D 266(2005)419

\#616 INT. J. NANOMED. 2(2007)639-649

Bakry, R; Vallant, RM; Najam-Ul-Haq, M; Rainer, M; Szabo, Z; Huck, CW; Bonn, GK: Medicinal applications of fullerenes $>>$ LI JY 265(2005)17

\#617 INT. J. NANOMED. 3(2008)169-180

McBain, SC; Yiu, HHP; Dobson, J: Magnetic nanoparticles for gene and drug delivery $>$ LIANG $\mathrm{S}$ 269(2006)3 
\#618 INT. J. NUMER. METHODS FLUIDS 58(2008)319_ 353

Cleary, PW; Sinnott, MD; Morrison, RD: DEM prediction of particle flows in grinding processes $>>$ MORRIS JS 269(2006)283

\#619 INT. J. PHYS. SCI. 3(2008)22-27

Awwad, NS; Gad, HMH; Aly, HF: Extraction of Eu(III) from nitrate medium by CYANEX921 using solvent extraction technique >> AWWAD NS 264(2005)623; HASSAN SSM 269(2006)135

\#620 INT. J. PROSTHODONT. 19(2006)202-204

Sonugelen, M; Destan, UI; Lambrecht, FY; Ozturk, B; Karadeniz, S: Microbial adherence to a nonprecious alloy after plasma nitriding process $>$ SONUGELEN M 267(2006)397

\#621 INT. J. RADIAT. BIOL. 84(2008)375-387

Turtoi, A; Brown, I; Oskamp, D; Schneeweiss, FHA: Early gene expression in human lymphocytes after gammairradiation-a genetic pattern with potential for biodosimetry $>>$ TURTOI A 274(2007)435

\#622 INT. J. RADIAT. BIOL. 84(2008)959-975

Howell, RW: Auger processes in the 21st century $>>$ KNAPP FF 263(2005)503

\#623 INT. J. RADIAT. BIOL. 85(2009)281-290

Nagy, K; Istvan, B; Kovacs, T; Kavasi, N; Janos, S; Laszlo, K; Istvan, B; Bender, T: Study on endocronological effects of radon speleotherapy on respiratory diseases $>>$ SOMLAI J 273(2007)363

\#624 INT. J. RADIAT. BIOL. 85(2009)403-412

Turtoi, A; Schneeweiss, FHA: Effect of At-211 alphaparticle irradiation on expression of selected radiation responsive genes in human lymphocytes $>>$ TURTOI A 274(2007)435

\#625 IRAN. J. RADIAT. RES. 6(2008)145-150

Jalilian, AR; Hakimi, A; Garousi, J; Bolourinovin, F; Kamali-Dehghan, M; Aslani, G: Development of [T]201](III) oxinate complex for in vitro cell labeling $>>$ JALILIAN AR 275(2008) 109

\#626 IRAN. J. RADIAT. RES. 6(2009)195-200

Jalilian, AR; Mirazizi, F; Nazem, H; Garousi, J; Bolourinovin, F; Sadeghpour, H: Preparation and quality control of radiolabeled streptokinase for Thrombosis imaging $>>$ JALILIAN AR 274(2007)175

\#627 IRAN. J. SCI. TECHNOL. TRANS. A-SCI. 30(2006)259-269

Abdi, MR; Faghihian, H; Kamali, M; Mostajaboddavati, M; Hasanzadeh, A: Distribution of natural radionuclides on coasts of Bushehr, Persian Gulf, Iran $>>$ ABDI MR 270(2006)319; MOSTAJABODDAVATI M 268(2006)539 \#628 ISOT. ENVIRON. HEALTH STUD. 43(2007)105116

Popa, K; Murariu, M; Molnar, R; Schlosser, G; Cecal, A; Drochioiu, G: Effect of radioactive and non-radioactive mercury on wheat germination and the anti-toxic role of glutathione >> POPA K 269(2006)155

\#629 ISOT. ENVIRON. HEALTH STUD. 43(2007)387400

Schmidt, A; Schubert, M: Using radon-222 for tracing groundwater discharge into an open-pit lignite mining lake-a case study $>>$ TREUTLER HC 272(2007)583 \#630 J. AGRIC. FOOD CHEM. 56(2008)7750-7758

Sirk, TW; Brown, EF; Sum, AK; Friedman, M: Molecular dynamics study on the biophysical interactions of seven green tea catechins with lipid bilayers of cell membranes $>>$ TAKEUCHI Y 272(2007)455

\#631 J. AGRIC. FOOD CHEM. 56(2008)10092-10098

Ohnishi, H; Iwanaga, S; Kawazoe, K; Ishizawa, K; Orino, S; Tomita, S; Tsuchiya, K; Kanematsu, Y; Harada, $\mathrm{N}$; Mori, K; Tsuchihashi, T; Ishikawa, Y; Tamaki, T: Effect of Iron-Quercetin Complex on Reduction of Nitrite in in Vitro and in Vivo Systems $>$ SHIRAISHI K 266(2005)61

\#632 J. AGRIC. FOOD CHEM. 57(2009)6720-6728

Sirk, TW; Brown, EF; Friedman, M; Sum, AK: Molecular Binding of Catechins to Biomembranes: Relationship to Biological Activity >> KUBOTA M 272(2007)571 \#633 J. AGRIC. FOOD CHEM. 58(2010)1986-1992

Romaris-Hortas, V; Garcia-Sartal, C; Barciela-Alonso, MC; Moreda-Pineiro, A; Bermejo-Barrera, P: Characterization of Edible Seaweed Harvested on the Galician Coast (Northwestern Spain) Using Pattern Recognition Techniques and Major and Trace Element Data $>>$ SERFORARMAH Y 269(2006)711

\#634 J. AGRIC. FOOD CHEM. 58(2010)2295-2301

Cubadda, F; Aureli, F; Ciardullo, S; D'Amato, M; Raggi, A; Acharya, R; Reddy, RAV; Prakash, NT: Changes in Selenium Speciation Associated with Increasing Tissue Concentrations of Selenium in Wheat Grain >> SHARMA N 281(2009)59

\#635 J. AIR WASTE MANAGE. ASSOC. 59(2009)411418

Pacheco, AMG; Freitas, MD: Trace-Element Enrichment in Epiphytic Lichens and Tree Bark at Pico Island, Azores, Portugal >> FREITAS MC 270(2006)21; VIEIRA BJ 271(2007)377

\#636 J. ALLOY. COMPD. 440(2007)210-215

Jeong, SM; Jung, JY; Seo, CS; Park, SW: Characteristics of an electrochemical reduction of $\mathrm{Ta} 2 \mathrm{O} 5$ for the preparation of metallic tantalum in a $\mathrm{LiCl}-\mathrm{Li} 2 \mathrm{O}$ molten salt >> JEONG SM 268(2006)349

\#637 J. ALLOY. COMPD. 448(2008)104-108

Giridhar, P; Venkatesan, KA; Subramaniam, S; Srinivasan, TG; Rao, PRV: Extraction of uranium (VI) by $1.1 \mathrm{M}$ tri-n-butylphosphate/ionic liquid and the feasibility of recovery by direct electrodeposition from organic phase >> GIRIDHAR P 265(2005)31 
\#638 J. ALLOY. COMPD. 452(2008)27-31

Jeong, SM; Yoo, HY; Hur, JM; Seo, CS: Preparation of metallic niobium from niobium pentoxide by an indirect electrochemical reduction in a LiCl-Li2O molten salt $>>$ JEONG SM 268(2006)349; PARK SB 268(2006)389

\#639 J. ALLOY. COMPD. 460(2008)69-73

Oner, Y; Kamer, O; Ross, JH: Magnetic and electrical properties of NdNiSn >> PALADE P 266(2005)553 \#640 J. AM. CHEM. SOC. 129(2007)12942-

Merritt, ME; Harrison, C; Kovacs, Z; Kshirsagar, P; Malloy, CR; Sherry, AD: Hyperpolarized Y-89 offers the potential of direct imaging of metal ions in biological systems by magnetic resonance $>>$ NEVES M 266(2005)377 \#641 J. AM. SOC. MASS SPECTROM. 20(2009)19121924

Mayeux, C; Tammiku-Taul, J; Massi, L; Lohu, EL; Burk, P; Maria, PC; Gal, JF: Interaction of the Cesium Cation with Mono-, Di-, and Tricarboxylic Acids in the Gas Phase. A Cs+ Affinity Scale for Cesium Carboxylates Ion Pairs >> HUREL C 279(2009)113

\#642 J. ANAL. AT. SPECTROM. 20(2005)821-829

Durrant, SF; Ward, NI: Recent biological and environmental applications of laser ablation inductively coupled plasma mass spectrometry (LA-ICP-MS) $>>$ JAMES WD 263(2005)697

\#643 J. ANAL. AT. SPECTROM. 20(2005)1124-1154

Potts, PJ; Ellis, AT; Kregsamer, P; Streli, C; Vanhoof, C; West, M; Wobrauschek, P: Atomic spectrometry update. X-Ray fluorescence spectrometry $>$ GILES JR 264(2005)113; KADYRZHANOV KK 263(2005)197 \#644 J. ANAL. AT. SPECTROM. 20(2005)1398-1424

Fisher, AS; Goodall, PS; Hinds, MW; Penny, DM: Atomic spectrometry update. Industrial analysis: metals, chemicals and advanced materials $>$ HANG W 263(2005)467; JAMES WD 263(2005)697; LAMONT SP 263(2005)477; MARCHETTI AA 263(2005)483; MCCURDY D 263(2005)447; WOLF SF 263(2005)581 \#645 J. ANAL. AT. SPECTROM. 21(2006)217-243

Butler, OT; Cook, JM; Harrington, CF; Hill, SJ; Rieuwerts, J; Miles, DL: Atomic spectrometry update. Environmental analysis $>>$ MARCHETTI AA 263(2005)483

\#646 J. ANAL. AT. SPECTROM. 21(2006)439-491

Taylor, A; Branch, S; Day, MP; Patriarca, M; White, M: Atomic spectrometry update. Clinical and biological materials, foods and beverages $>>$ MOHAGHEGHI AH 263(2005)189

\#647 J. ANAL. AT. SPECTROM. 21(2006)785-818

Bacon, JR; Linge, KL; Parrish, RR; Van Vaeck, L: Atomic spectrometry update. Atomic mass spectrometry $>>$ CURRIE LA 263(2005)399; JIANG SS 264(2005)549; KIESER WE 263(2005)375; LAMONT SP 263(2005)477; LAMONT SP 264(2005)423; MARCHETTI AA 263(2005)483
\#648 J. ANAL. AT. SPECTROM. 21(2006)1076-1107

Potts, PJ; Ellis, AT; Kregsamer, P; Streli, C; Vanhoof, C; West, M; Wobrauschek, P: Atomic spectrometry updateX-ray fluorescence spectrometry >> GILES JR 264(2005)113 \#649 J. ANAL. AT. SPECTROM. 21(2006)1202-1208

Grinberg, P; Yang, L; Mester, Z; Willie, S; Sturgeon, RE: Comparison of laser ablation, electrothermal vaporization and solution nebulization for the determination of radionuclides in liquid samples by inductively coupled plasma mass spectrometry $>>$ POVINEC PP 263(2005)413 \#650 J. ANAL. AT. SPECTROM. 21(2006)1431-1471

Charlton, B; Fisher, AS; Goodall, PS; Hinds, MW; Lancastera, S; Salisbury, M: Atomic spectrometry update. Industrial analysis: metals, chemicals and advanced materials $>>$ ELLIOT NL 267(2006)637; NORISUYE K 267(2005)183; ZHENG J 267(2005)73

\#651 J. ANAL. AT. SPECTROM. 22(2007)187-221

Butler, OT; Cook, JM; Harrington, CF; Hill, SJ; Rieuwertsd, J; Miles, DL: Atomic spectrometry update. Environmental analysis >> AHMED M 265(2005)39; WANG CF 268(2006)15; ZHENG J 267(2005)73

\#652 J. ANAL. AT. SPECTROM. 22(2007)415-456

Taylor, A; Branch, S; Day, MP; Patriarca, M; White, M: Clinical and biological materials, foods and beverages $>>$ CHETTLE DR 268(2006)653

\#653 J. ANAL. AT. SPECTROM. 22(2007)540-546

Elish, E; Karpas, Z; Lorber, A: Determination of uranium concentration in a single hair strand by LAICPMS applying continuous and single pulse ablation $>>$ MOHAGHEGHI AH 263(2005)189

\#654 J. ANAL. AT. SPECTROM. 22(2007)827-841

Kim, CS; Kim, CK; Martin, P; Sansone, U: Determination of $\mathrm{Pu}$ isotope concentrations and isotope ratio by inductively coupled plasma mass spectrometry: a review of analytical methodology >> EFURD DW 263(2005)387; HANG W 263(2005)467; LAROSA J 263(2005)427; NORISUYE K 267(2006)183; ZHENG J 267(2005)73 \#655 J. ANAL. AT. SPECTROM. 22(2007)973-1006

Bacon, JR; Linge, KL; Parrish, RR; Van Vaeck, L: Atomic spectrometry update. Atomic mass spectrometry $>$ EFURD DW 269(2006)679; ELLIOT NL 267(2006)637; KUROSAKI H 269(2006)279

\#656 J. ANAL. AT. SPECTROM. 22(2007)1122-1130

Homazava, N; Ulrich, A; Trottmann, M; Kranhebuhl, U: Micro-capillary system coupled to ICP-MS as a novel technique for investigation of micro-corrosion processes $>>$ WOLF SF 263(2005)575 \#657 J. ANAL. AT. SPECTROM. 22(2007)1131-1137

Epov, VN; Evans, RD; Zheng, J; Donard, OFX; Yamada, M: Rapid fingerprinting of Pu-239 and Pu-240 in environmental samples with high $\mathrm{U}$ levels using on-line ion chromatography coupled with high-sensitivity quadrupole ICP-MS detection >> ZHENG J 267(2005)73 
\#658 J. ANAL. AT. SPECTROM. 22(2007)1304-1332

West, M; Ellis, AT; Kregsamer, P; Potts, PJ; Streli, C; Vanhoof, C; Wobrauschek, P: Atomic spectrometry update. $\mathrm{X}$-ray fluorescence spectrometry $>>$ CANELLAS CGL 269(2006)631; CHETTLE DR 268(2006)653; HU SM 271(2007)517; MAHAWATTE P 270(2006)657; OBHODAS J 270(2006)75; POPOVIC M 269(2006)421; VIVES AES 270(2006)231; ZAMBURLINI M 269(2006)625 \#659 J. ANAL. AT. SPECTROM. 22(2007)1501-1506

Sussulini, A; Souza, GHMF; Eberlin, MN; Arruda, MAZ: Comparative metallomics for transgenic and nontransgenic soybeans $>>$ SANTOS LS 269(2006)505 \#660 J. ANAL. AT. SPECTROM. 22(2007)1517-1560

Charlton, B; Fisher, AS; Goodall, PS; Hinds, MW; Lancaster, S; Salisbury, M: Atomic spectrometry update. Industrial analysis: metals, chemicals and advanced materials $>>$ ZHENG J 270(2006)669

\#661 J. ANAL. AT. SPECTROM. 23(2008)94-102

Pointurier, F; Hemet, P; Hubert, A: Assessment of plutonium measurement in the femtogram range by ICPMS; correction from interfering polyatomic species $>>$ NORISUYE K 267(2006)183; ZHENG J 267(2005)73 \#662 J. ANAL. AT. SPECTROM. 23(2008)249-286

Butler, OT; Cook, JM; Harrington, CF; Hill, SJ; Rieuwerts, J; Miles, DL: Atomic spectrometry update. Environmental analysis >> CHAI JY 272(2007)397; NYGREN U 272(2007)45; ZHANG YX 267(2006)497

\#663 J. ANAL. AT. SPECTROM. 23(2008)352-360

Lariviere, D; Cumming, TA; Kiser, S; Li, C; Cornett, RJ: Automated flow injection system using extraction chromatography for the determination of plutonium in urine by inductively coupled plasma mass spectrometry $>>$ ELLIOT NL 267(2006)637; HANG W 263(2005)467; MARCHETTI AA 263(2005)483

\#664 J. ANAL. AT. SPECTROM. 23(2008)521-526

Li, CS; Lariviere, D; Kiser, S; Moodie, G; Falcomer, R; Elliot, N; Burchart, L; Paterson, L; Epov, V; Evans, D; Pappas, S; Smith, J; Cornett, J: Method intercomparison for the analysis of $\mathrm{Pu}-239 / 240$ in human urine $>>$ ELLIOT NL 267(2006)637; MCCURDY D 263(2005)447

\#665 J. ANAL. AT. SPECTROM. 23(2008)527-534

Da Silva, E; Pejovic-Milic, A; Heyd, DV: The use of teeth as the site for the in vivo or ex vivo quantification of skeletal strontium by energy-dispersive X-ray fluorescence spectrometry: A feasibility study $>>$ ZAMBURLINI M 269(2006)625

\#666 J. ANAL. AT. SPECTROM. 23(2008)595-646

Taylor, A; Branch, S; Day, MP; Patriarca, M; White, M: Atomic spectrometry update. Clinical and biological materials, foods and beverages $>>$ ADMANS LL 269 (2006)619; CHAUDHRI MA 271(2007)713; EFURD DW 269(2006)679; FARIAS LA 270(2006)217; FENG LX 272(2007)333; KAWABATA Y 270(2006)137;
MAHAWATTE P 270(2006)657; OLABANJI SO 270(2006)515; POPOVIC M 269(2006)421; RIZZUTTO MA 269(2006)683; VIVES AES 270(2006)147; VIVES AES 270(2006)231; XING L 269(2006)527; ZHANG F 269(2006)535; ZHANG YX 269(2006)235

\#667 J. ANAL. AT. SPECTROM. 23(2008)792-798

Streli, C; Wobrauschek, P; Meirer, F; Pepponi, G: Synchrotron radiation induced TXRF $>$ CANELLAS CGL 269(2006)631; VIVES AES 270(2006)231

\#668 J. ANAL. AT. SPECTROM. 23(2008)1130-1162

Bacon, JR; Linge, KL; Parrish, RR; Van Vaeck, L: Atomic spectrometry update. Atomic mass spectrometry $>>$ AGGARWAL SK 273(2007)775

\#669 J. ANAL. AT. SPECTROM. 23(2008)1409-1437

West, M; Ellis, AT; Kregsamer, P; Potts, PJ; Streli, C; Vanhoof, C; Wobrauschek, P: Atomic spectrometry update. X-ray fluorescence spectrometry >> ALVAREZ AM 273(2007)427; FENG LX 272(2007)333; KHUDER A 273(2007)435; WANG YQ 272(2007)541; ZHANG F 272(2007)533

\#670 J. ANAL. AT. SPECTROM. 23(2008)1636-1692

Charlton, B; Fisher, AS; Goodall, PS; Hinds, MW; Lancaster, S; Shore, S: Atomic spectrometry update. Industrial analysis: metals, chemicals and advanced materials >> AGGARWAL SK 273(2007)775; BURGER S 274(2007)491; CINCU E 274(2007)199; GREIS C 275(2008)55; LEE CG 272(2007)299; WANG YQ 272(2007)541; YOON YY 276(2008)187; ZHENG J 275(2008)37

\#671 J. ANAL. AT. SPECTROM. 24(2009)131-177

Butler, OT; Cook, JM; Davidson, CM; Harrington, CF; Miles, DL: Atomic spectrometry update. Environmental analysis $>>$ GREIS C 275(2008)55; LARIVIERE D 273(2007)337; PACHECO AMG 276(2008)135; TAGAMI K 273(2007)147; VARGA Z 274(2007)87; WANG YS 273(2007)247; YOON YY 276(2008)187; YOSHIDA S 273(2007)211; YUE WS 274(2007)115; YUSOF AM 273 (2007)533

\#672 J. ANAL. AT. SPECTROM. 24(2009)277-287

Ranebo, Y; Hedberg, PML; Whitehouse, MJ; Ingeneri, $\mathrm{K}$; Littmann, S: Improved isotopic SIMS measurements of uranium particles for nuclear safeguard purposes $>>$ LEE CG 272(2007)299

\#673 J. ANAL. AT. SPECTROM. 24(2009)484-493

Resano, M; Aramendia, M; Vanhaecke, F: Extending the capabilities of electrothermal vaporization-inductively coupled plasma mass spectrometry (ETV-ICPMS): Coupling the graphite furnace to a sector field instrument $>>$ KUCERA J 263(2005)811

\#674 J. ANAL. AT. SPECTROM. 24(2009)508-514

Grinberg, P; Sturgeon, RE: Photochemical vapor generation of iodine for detection by ICP-MS $>>$ YOSHIDA $S$ 273(2007)211 
\#675 J. ANAL. AT. SPECTROM. 24(2009)535-579

Taylor, A; Branch, S; Day, MP; Patriarca, M; White, M: Atomic Spectrometry Update. Clinical and biological materials, foods and beverages >> BEASLEY D 276(2008)101 \#676 J. ANAL. AT. SPECTROM. 24(2009)801-807

Wellum, R; Verbruggen, A; Kessel, R: A new evaluation of the half-life of $\mathrm{Pu}-241>$ POMME $\mathrm{S}$ 276(2008)335

\#677 J. ANAL. AT. SPECTROM. 24(2009)1289-1326

West, M; Ellis, AT; Potts, PJ; Streli, C; Vanhoof, C; Wegrzynek, D; Wobrauschek, P: Atomic spectrometry update. X-Ray fluorescence spectrometry $>>$ CAKIR C 279(2009)345; DESIDERI D 279(2009)591; GUROL A 279(2009)171; HATZISTAVROS VS 277(2008)399; OZ E 279(2009)529; ZHANG HY 279(2009)307

\#678 J. ANAL. AT. SPECTROM. 24(2009)1599-1656

Carter, S; Fisher, AS; Goodall, PS; Hinds, MW; Lancaster, S; Shore, S: Atomic spectrometry update. Industrial analysis: metals, chemicals and advanced materials $>>$ BORES NE 276(2008)513; EFURD DW 276(2008)499; FARMER OT 276(2008)489; GRANIER G 279(2009)875; HATZISTAVROS VS 277(2008)399; HOPPE EW 277(2008)103; JOHNSSON A 279(2009)619; NATARAJAN V 280(2009)27; SVEDKAUSKAITELEGORE J 278(2008)201; ZHANG HY 279(2009)307

\#679 J. ANAL. AT. SPECTROM. 25(2010)103-141

Butler, OT; Cairns, WRL; Cook, JM; Davidson, CM: Atomic spectrometry update. Environmental analysis >> MIRELES A 277(2008)441; SANO J 278(2008)111; ZHENG J 280(2009)171

\#680 J. ANAL. AT. SPECTROM. 25(2010)405-407

Tanner, M: Shorter signals for improved signal to noise ratio, the influence of Poisson distribution $>>$ CURRIE LA 276(2008) 285

\#681 J. AOAC INT. 90(2007)163A-170A

Sherma, J; Larkin, JD; Larkin, FH: X-ray fluorescence Spectrometry $>>$ MAHAWATTE P 270(2006)657

\#682 J. AOAC INT. 92(2009)502-510

Anderson, DL: Determination of Bromine in Regulated Foods with a Field-Portable X-Ray Fluorescence Analyzer $>>$ ANDERSON DL 276(2008)23

\#683 J. APPL. BOT. FOOD QUAL.-ANGEW. BOT. 82(2008)90-98

Matsushima, U; Kardjilov, N; Hilger, A; Graf, W; Herppich, WB: Application potential of cold neutron radiography in plant science research $>>$ MATSUSHIMA U 264(2005)325; NAKANISHI TM 264(2005)313; YAMADA T 264(2005)329

\#684 J. APPL. CRYSTALLOGR. 41(2008)456-465

Rubinson, KA; Stanley, C; Krueger, S: Small-angle neutron scattering and the errors in protein structures that arise from uncorrected background and intermolecular interactions $>>$ EFIMOVA YM 264(2005)271
\#685 J. APPL. CRYSTALLOGR. 42(2009)329-332

Colip, LA; Koppisch, AT; Broene, RD; Berger, JA; Baldwin, SM; Harris, MN; Peterson, LJ; Warner, BP; Birnbaum, ER: A rapid method for quantifying heavy atom derivatives for multiple isomorphous replacement in protein crystallography $>>$ CHETTLE DR 268(2006)653 \#686 J. APPL. PHYCOL. 21(2009)145-152

Chakraborty, N; Banerjee, A; Lahiri, S; Panda, A; Ghosh, AN; Pal, R: Biorecovery of gold using cyanobacteria and an eukaryotic alga with special reference to nanogold formation-a novel phenomenon $>>$ CHAKRABORTY N 270(2006)645; NAYAK D 268(2006)337 \#687 J. APPL. PHYS. 102(2007)13907-

Nomura, K; Zboril, R; Tucek, J; Kosaka, W; Ohkoshi, S; Felner, I: Substitution effects of barium and calcium on magnetic properties of $\mathrm{A}(\mathrm{x}) \mathrm{Sr}(1-\mathrm{x})(\mathrm{Fe} 0.5 \mathrm{Ru} 0.5) \mathrm{O}-3$ double perovskites $(\mathrm{x}=0.05, \mathrm{~A}=\mathrm{Ba}, \mathrm{Ca})>$ NOMURA $\mathrm{K}$ 266(2005)543

\#688 J. APPL. PHYS. 104(2008)74912-

Greenfield, MB; Ito, N; Iwata, A; Kubo, K; Ishigaki, M; Komura, K: Determination of rain age via gamma rays from accreted radon progeny >> KOMURA K 269(2006)511 \#689 J. APPL. PHYS. 105(2009)64910-

Clarijs, MC; Bom, VR; van Eijk, CWE: A simple method for the analysis of neutron resonance capture spectra $>>$ POSTMA H 265(2005)297; SCHUT PAC 278(2008)151 \#690 J. APPL. PHYS. 106(2009)74909-

Festa, G; Andreani, C; De Pascale, MP; Senesi, R; Vitali, G; Porcinai, S; Giusti, AM; Schulze, R; Canella, L; Kudejova, P; Muhlbauer, M; Schillinger, B: A nondestructive stratigraphic and radiographic neutron study of Lorenzo Ghiberti's reliefs from paradise and north doors of Florence baptistery $>>$ BELGYA T 278(2008)751; BELGYA T 278(2008) UNS 713718

\#691 J. APPL. PHYS. 106(2009)113524-

Proffit, DE; Buchholz, DB; Chang, RPH; Bedzyk, MJ; Mason, TO; Ma, Q: X-ray absorption spectroscopy study of the local structures of crystalline Zn-In-Sn oxide thin films $>>$ WANG YS 273(2007)247

\#692 J. APPL. PHYS. 107(2010)44512-

Horansky, RD; Stiehl, GM; Beall, JA; Irwin, KD; Plionis, AA; Rabin, MW; Ullom, JN: Measurement of ion cascade energies through resolution degradation of alpha particle microcalorimeters $>>$ PLIONIS AA 282(2009)905 \#693 J. APPL. POLYM. SCI. 113(2009)3038-3048

Ibrahim, GM; Ahmad, MI; El-Gammal, B: Structural Development of TMMA and SSQXN-8 as Porous Chelating Resins >> MALLAH MH 278(2008)97

\#694 J. APPL. POLYM. SCI. 113(2009)3963-3971

Noto, K; Matsumoto, S; Takahashi, Y; Hirata, M; Yamada, K: Adhesion of Surface-Grafted Low-Density Polyethylene Plates with Enzymatically Modified Chitosan Solutions >> CHEN J 275(2008)81 
\#695 J. APPL. POLYM. SCI. 114(2009)3793-3801

Atun, G; Ortaboy, S: Adsorptive Removal of Uranium from Water by Sulfonated Phenol-Formaldehyde Resin $>>$ AYCIK GA 273(2007)713; DONAT R 265(2005)107; INOUE K 267(2006)435; KILINCARSLAN A 264(2005)541 \#696 J. APPL. POLYM. SCI. 116(2010)868-875

Gancarz, I; Bryjak, M; Kunicki, J; Ciszewski, A: Microwave Plasma-Initiated Grafting of Acrylic Acid on Celgard 2500 Membrane to Prepare Alkaline Battery Separators-Characteristics of Process and Product $>>$ CHEN J 275(2008)81

\#697 J. ARCHAEOL. SCI. 35(2008)752-762

Popelka-Filcoff, RS; Miksa, EJ; Robertson, JD; Glascock, MD; Wallace, H: Elemental analysis and characterization of ochre sources from Southern Arizona $>>$ POPELKAFILCOFF RS 272(2007)17

\#698 J. ARCHAEOL. SCI. 36(2009)1206-1214

Pearson, CL; Dale, DS; Brewer, PW; Kuniholm, PI; Lipton, J; Manning, SW: Dendrochemical analysis of a treering growth anomaly associated with the Late Bronze Age eruption of Thera $>>$ UNLU K 264(2005)21

\#699 J. ARCHAEOL. SCI. 37(2010)92-106

Le Bourdonnec, FX; Bontempi, JM; Marini, N; Mazet, S; Neuville, PF; Poupeau, G; Sicurani, J: SEM-EDS characterization of western Mediterranean obsidians and the Neolithic site of A Fuata (Corsica) $>>$ ARIAS A 268(2006)371

\#700 J. ATMOS. CHEM. 59(2008)199-218

Ravindra, K; Stranger, M; Van Grieken, R: Chemical characterization and multivariate analysis of atmospheric PM2.5 particles $>>$ ALMEIDA SM 276(2008)161

\#701 J. BIOMECH. ENG.-TRANS. ASME 131(2009) 74001-

Day, ES; Morton, JG; West, JL: Nanoparticles for Thermal Cancer Therapy > > JAMES WD 271(2007)455 \#702 J. BIOMED. OPT. 14(2009)24044-

Puvanakrishnan, P; Park, J; Diagaradjane, P; Schwartz, JA; Coleman, CL; Gill-Sharp, KL; Sang, KL; Payne, JD; Krishnan, S; Tunnell, JW: Near-infrared narrow-band imaging of gold/silica nanoshells in tumors $>>$ JAMES WD 271(2007)455

\#703 J. BIOMED. OPT. 14(2009)54015-

Kah, JCY; Olivo, M; Chow, TH; Song, KS; Koh, KZY; Mhaisalkar, S; Sheppard, CJR: Control of optical contrast using gold nanoshells for optical coherence tomography imaging of mouse xenograft tumor model in vivo $>>$ JAMES WD 271(2007)455

\#704 J. BRAZ. CHEM. SOC. 19(2008)380-388

Monroy-Guzman, F; Diaz-Archundia, LV; HernandezCortes, S: Mo-99/Tc-99m generators performances prepared from zirconium molybate gels $>>$ MONROYGUZMAN F 271(2007)523
\#705 J. BRAZ. CHEM. SOC. 20(2009)313-321

Amorim, ACL; Hovell, AMC; Pinto, AC; Eberlin, MN; Arruda, NP; Pereira, EJ; Bizzo, HR; Catharino, RR; Morais, ZB; Rezende, CM: Green and Roasted Arabica Coffees Differentiated by Ripeness, Process and Cup Quality via Electrospray Ionization Mass Spectrometry Fingerprinting $>>$ SANTOS LS 269(2006)505

\#706 J. BRAZ. CHEM. SOC. 20(2009)1164-1174

Guerra, DL; Viana, RR; Airoldi, C: Adsorption of Thorium(IV) on Chemically Modified Amazon Clays $>>$ NEMES Z 266(2005)289; SHARMA P 268(2006)329

\#707 J. CHEM. EDUC. 83(2006)1327-1329

Pillay, AE; Salih, FM: Solar irradiation of bilirubin: An experiment in photochemical oxidation $>$ SALIH FM 264(2005)561

\#708 J. CHEM. EDUC. 86(2009)1122-1128

Hoffman, DC: The Periodic Table Key to Past "Elemental" Discoveries-A New Role in the Future? >> HOFFMAN DC 276(2008)525

\#709 J. CHEM. ENG. JPN. 40(2007)270-274

Ogata, T; Kim, YH; Nakano, Y: Selective recovery process for gold utilizing a functional gel derived from natural condensed tannin >> INOUE K 267(2006)435

\#710 J. CHEM. ENG. JPN. 41(2008)294-297

Park, BH; Lee, IW; Seo, CS: Reduction of U3O8 in a High Temperature Molten LiCl-Li2O Salt $>>$ JEONG SM 268(2006)349

\#711 J. CHEM. PHYS. 129(2008)244704-

Veilly, E; Roques, J; Jodin-Caumon, MC; Humbert, B; Drot, R; Simoni, E: Uranyl interaction with the hydrated (001) basal face of gibbsite: A combined theoretical and spectroscopic study >> XU D 266(2005)419

\#712 J. CHEM. SOC. PAK. 31(2009)726-730

Khan, HM; Khan, AA: Identification of Radiation Treatment of Frozen Chicken and Fresh Turkey using DNA Comet Assay $>>$ KHAN AA 275(2008)337

\#713 J. CHEM. SOC. PAK. 31(2009)916-920

Siddique, N; Waheed, S: Instrumental Neutron Activation Analysis of Proposed Marine Sediment Reference Material (Iaea-158) >> SIDDIQUE N 274(2007)181 \#714 J. CHEM. TECHNOL. BIOTECHNOL. 84(2009) 949-956

Zuo, Y; Liu, Y; Chen, J; Li, DQ: Extraction and recovery of cerium(IV) along with fluorine(I) from bastnasite leaching liquor by DEHEHP in [C(8)mim]PF6 $>>$ GIRIDHAR P 265(2005)31

\#715 J. CHEM. THEORY COMPUT. 5(2009)15851596

Korona, T; Hesselmann, A; Dodziuk, H: SymmetryAdapted Perturbation Theory Applied to Endohedral Fullerene Complexes: A Stability Study of H-2@C-60 and 2H(2)@C-60 >> WATANABE S 266(2005)499 
\#716 J. CHIL. CHEM. SOC. 53(2008)1455-1463

Vanysek, P; Ramirez, LB: Interface between two immiscible liquid electrolytes: A review $>>$ MAKRLIK E 268(2006)155; MAKRLIK E 268(2006)641

\#717 J. CHIN. CHEM. SOC. 54(2007)1443-1450

Sife-Eldeen, KA; Ebraheem, S: Charge transfer complex role in the formation of chlorobenzene in the gammairradiated carbon tetrachloride-benzene system $>>$ SIFEELDEEN KA 264(2005)565

\#718 J. CHIN. CHEM. SOC. 54(2007)1529-1534

Parsa, M; Yaftian, MR; Matt, D: A new silver(I) potentiometric sensor based on a calix[4]arene substituted at the narrow rim by amide/phosphoryl groups $>>$ YAFTIAN MR 270(2006)357

\#719 J. CHIN. CHEM. SOC. 56(2009)748-754

Wang, TH; Li, MH; Wei, YY; Teng, SP: Effects of pH and Concentration on Cs Ions Sorption and Diffusion in Crushed Granite by Using Batch and Modified Capillary Method >> WANG TH 277(2008)607

\#720 J. CHROMATOGR. A 1157(2007)85-95

Zhang, A; Kuraoka, E; Kumagai, M: Development of the chromatographic partitioning of cesium and strontium utilizing two macroporous silica-based calix[4]arenecrown and amide impregnated polymeric composites: PREC partitioning process $>>$ ZHANG A 265(2005)409 \#721 J. CHROMATOGR. A 1200(2008)193-197

Niemann, RA; Anderson, DL: Determination of iodide and thiocyanate in powdered milk and infant formula by on-line enrichment ion chromatography with photodiode array detection $>>$ ANDERSON DL 276(2008)23

\#722 J. CHROMATOGR. A 1213(2008)218-223

Munoz-Munoz, AC; Grenier, AC; Gutierrez-Pulido, H; Cervantes-Martinez, J: Development and validation of a High Performance Liquid Chromatography-Diode Array Detection method for the determination of aging markers in tequila $>>$ MUNITA CS 269(2006)335

\#723 J. CLIM. 22(2009)3910-3925

Kaspari, S; Mayewski, PA; Handley, M; Kang, S; Hou, S; Sneed, S; Maasch, K; Qin, D: A High-Resolution Record of Atmospheric Dust Composition and Variability since AD 1650 from a Mount Everest Ice Core $>>$ GIAVERI G 263(2005)725

\#724 J. COAST. RES. 24(2008)15-24

Taniguchi, M; Burnett, WC; Dulaiova, H; Siringan, F; Foronda, J; Wattayakorn, G; Rungsupa, S; Kontar, EA; Ishitobi, T: Groundwater discharge as an important land-sea pathway into Manila Bay, Philippines $>>$ DULAIOVA H 263(2005)361

\#725 J. COAST. RES. (2009)851-855

Maranho, LA; Abreu, I; Santelli, R; Cordeiro, RC; Soares-Gomes, A; Moreira, LB; Morais, RD; Abessa, DMS: Sediment toxicity assessment of Guanabara Bay, Rio de Janeiro, Brazil. >> KFOURI PBP 265(2005)459
\#726 J. COLLOID INTERFACE SCI. 332(2009)158-164

Huittinen, N; Rabung, T; Lutzenkirchen, J; Mitchell, SC; Bickmore, BR; Lehto, J; Geckeis, H: Sorption of $\mathrm{Cm}(\mathrm{III})$ and $\mathrm{Gd}(\mathrm{III})$ onto gibbsite, alpha-Al(OH)(3): A batch and TRLFS study $>>$ XU D 266(2005)419; YU S 268(2006)387

\#727 J. COLLOID INTERFACE SCI. 332(2009)298-308

Sharma, P; Singh, G; Tomar, R: Synthesis and characterization of an analogue of heulandite: Sorption applications for thorium(IV), europium(III), samarium(II) and iron(III) recovery from aqueous waste $>>$ FAGHIHIAN $\mathrm{H}$ 264(2005)577; HUMELNICU D 270(2006)637; RAOUF MWA 267(2006)389

\#728 J. COLLOID INTERFACE SCI. 333(2009)448-456

Kolodynska, D; Skwarek, E; Hubicki, Z; Janusz, W: Effect of adsorption of $\mathrm{Pb}$ (II) and $\mathrm{Cd}$ (II) ions in the presence of EDTA on the characteristics of electrical double layers at the ion exchanger $/ \mathrm{NaCl}$ electrolyte solution interface $>>$ HELAL AA 267(2006)369

\#729 J. COLLOID INTERFACE SCI. 337(2009)439-448

Kosmulski, M: pH-dependent surface charging and points of zero charge. IV. Update and new approach $>>$ ORTIZOLIVEROS HB 279(2009)601

\#730 J. CONTAM. HYDROL. 106(2009)144-149

Singh, BK; Jain, A; Kumar, S; Tomar, BS; Tomar, R; Manchanda, VK; Ramanathan, S: Role of magnetite and humic acid in radionuclide migration in the environment >> PATHAK PN 270(2006)277

\#731 J. COORD. CHEM. 62(2009)2203-2211

Liu, TH; Duan, GJ; Zeng, ZZ: Synthesis and characterization of cerium, thorium, and uranyl complexes with (E)-4-(4-methoxyphenoxy)-4-oxobut-2-enoic acid >> VULPIUS D 270(2006)661

\#732 J. DRUG TARGET. 17(2009)181-193

Kah, JCY; Wong, KY; Neoh, KG; Song, JH; Fu, JWP; Mhaisalkar, S; Olivo, M; Sheppard, CJR: Critical parameters in the pegylation of gold nanoshells for biomedical applications: An in vitro macrophage study $>>$ JAMES WD 271(2007)455

\#733 J. ELECTROCHEM. SOC. 154(2007)A770-A775

Arunkumar, TA; Alvarez, E; Manthiram, A: Structural, chemical, and electrochemical characterization of layered Li[Li0.17Mn0.33Co0.5-yNiy]O-2 cathodes $>>$ AGHARA SK 265(2005)321

\#734 J. ELECTROCHEM. SOC. 155(2008)E125-E129

Allanore, A; Lavelaine, H; Valentin, G; Birat, JP; Lapicque, F: Iron metal production by bulk electrolysis of iron ore particles in aqueous media $>$ JEONG SM 268(2006)349

\#735 J. ELECTROCHEM. SOC. 156(2009)A886-A890

Whitney, S; Biegalski, SR; Huang, YH; Goodenough, JB: Neutron Depth Profiling Applications to Lithium-Ion Cell Research >> WHITNEY SM 282(2009)173 
\#736 J. ELECTRON. MATER. 38(2009)623-634

Dogra, R; Byrne, AP; Ridgway, MC: The Potential of the Perturbed Angular Correlation Technique in Characterizing Semiconductors >> ZHU SY 272(2007)615 \#737 J. ENVIRON. MANAGE. 91(2009)67-77

Mohapatra, M; Anand, S; Mishra, BK; Giles, DE; Singh, P: Review of fluoride removal from drinking water $>>$ BADILLOALMARAZ VE 271(2007)741 \#738 J. ENVIRON. MONIT. 9(2007)790-804

Krachler, M: Environmental applications of single collector high resolution ICP-MS >> ZHENG J 267(2005)73; ZHENG J 270(2006)669

\#739 J. ENVIRON. MONIT. 10(2008)1217-1225

Ma, HL; Tanner, PA: Determination of chromium in airborne particulate matter by inductively coupled plasma dynamic reaction cell mass spectrometry $>>$ ZHANG YX 267(2006)497

\#740 J. ENVIRON. MONIT. 11(2009)1782-1800

Sadik, OA; Zhou, AL; Kikandi, S; Du, N; Wang, Q; Varner, K: Sensors as tools for quantitation, nanotoxicity and nanomonitoring assessment of engineered nanomaterials $>>$ MENG H 272(2007)595

\#741 J. ENVIRON. PLAN. MANAG. 52(2009)813-832

Ioris, AAR: Water reforms in Brazil: opportunities and constraints $>>$ PEREIRA MO 269(2006)707

\#742 J. ENVIRON. QUAL. 37(2008)2243-2247

Tsukada, H; Takeda, A; Tagami, K; Uchida, S: Uptake and Distribution of Iodine in Rice Plants $>>$ TSUKADA H 263(2005)773; TSUKADA H 273(2007)199

\#743 J. ENVIRON. RADIOACT. 81(2005)221-231

Tsukada, H; Takeda, A; Takahashi, T; Hasegawa, H; Hisamatsu, S; Inaba, J: Uptake and distribution of Sr-90 and stable $\mathrm{Sr}$ in rice plants $>>$ TSUKADA H 263(2005)773 \#744 J. ENVIRON. RADIOACT. 89(2006)81-101

Povinec, PP; Aggarwal, PK; Aureli, A; Burnett, WC; Kontar, EA; Kulkarni, KM; Moore, WS; Rajar, R; Taniguchi, M; Comanducci, JF; Cusimano, G; Dulaiova, H; Gatto, L; Groening, M; Hauser, S; Levy-Palomo, I; Oregioni, B; Ozorovich, YR; Privitera, AMG; Schiavo, MA: Characterisation of submarine groundwater discharge offshore south-eastern Sicily >> OSVATH I 263(2005)437 \#745 J. ENVIRON. RADIOACT. 89(2006)219-228

Lee, JM; Kim, G: A simple and rapid method for analyzing radon in coastal and ground waters using a radon-inair monitor $>>$ DULAIOVA H 263(2005)361 \#746 J. ENVIRON. RADIOACT. 92(2007)175-182

Papastefanou, C: Chlorine-39 in rainfall at a temperate latitude $(40$ circle $\mathrm{N}$ ) $>>$ PAPASTEFANOU $\mathrm{C}$ 267(2006)315

\#747 J. ENVIRON. RADIOACT. 93(2007)170-180

Oikawa, S; Yamamoto, M: Plutonium concentration and $\mathrm{Pu}-240 / \mathrm{Pu}-239$ atomic ratio in liver of squid collected in the coastal sea areas of Japan $>>$ ZHENG J 267(2005)73
\#748 J. ENVIRON. RADIOACT. 94(2007)86-97

Desideri, D; Meli, MA; Feduzi, L; Roselli, C; Rongoni, A; Saetta, D: U-238, U-234, Ra-226, Po-210 concentrations of bottled mineral waters in Italy and their dose contribution $>>$ JIA GG 267(2006)505

\#749 J. ENVIRON. RADIOACT. 95(2007)149-160

Hoollriegl, V; Greiter, M; Giussani, A; Gerstmann, U; Michalke, B; Roth, P; Oeh, U: Observation of changes in urinary excretion of thorium in humans following ingestion of a therapeutic soil >> HOLLRIEGL V 266(2005)441 \#750 J. ENVIRON. RADIOACT. 97(2007)124-136

Godoy, MLDP; Godoy, JM; Roldao, LA: Application of ICP-QMS for the determination of plutonium in environmental samples for safeguards purposes $>>$ NORISUYE K 267(2006)183; ZHENG J 267(2005)73

\#751 J. ENVIRON. RADIOACT. 98(2007)298-314

Carvalho, FP; Oliveira, JM; Lopes, I; Batista, A: Radionuclides from past uranium mining in rivers of Portugal > > CARVALHO FP 274(2007)167

\#752 J. ENVIRON. RADIOACT. 99(2008)211-217

Saito-Kokubu, Y; Yasuda, K; Magara, M; Miyamoto, Y; Sakurai, S; Usuda, S; Yamazaki, H; Yoshikawa, S; Nagaoka, S; Mitamura, M; Inoue, J; Murakami, A: Depositional records of plutonium and $\mathrm{Cs}-137$ released from Nagasaki atomic bomb in sediment of Nishiyama reservoir at Nagasaki >> SAITOKOKUBU Y 273(2007)183

\#753 J. ENVIRON. RADIOACT. 99(2008)332-342

Turhan, S; Gunduz, L: Determination of specific activity of Ra-226, Th-232 and K-40 for assessment of radiation hazards from Turkish pumice samples $>>$ PAPASTEFANOU C 266(2005)367

\#754 J. ENVIRON. RADIOACT. 99(2008)404-414

Turhan, S: Assessment of the natural radioactivity and radiological hazards in Turkish cement and its raw materials $>>$ PAPASTEFANOU C 266(2005)367

\#755 J. ENVIRON. RADIOACT. 99(2008)415-425

Vinsova, H; Jedinakova-Krizova, V; Kolarikova, I; Adamcova, J; Prikryl, R; Zeman, J: The influence of temperature and hydration on the sorption properties of bentonite $>>$ JEDINAKOVAKRIZOVA V 269(2006)747 \#756 J. ENVIRON. RADIOACT. 99(2008)491-501

Manickam, E; Sdraulig, S; Tinker, RA: Method design and validation for the determination of uranium levels in human urine using high-resolution alpha spectrometry $>>$ AMOLI HS 268(2006)497; GABURO JC 269(2006)499 \#757 J. ENVIRON. RADIOACT. 99(2008)509-526

Ryan, B; Bollhofer, A; Martin, P: Radionuclides and metals in freshwater mussels of the upper South Alligator River, Australia >> RYAN B 264(2005)407 \#758 J. ENVIRON. RADIOACT. 99(2008)596-606

Zahringer, M; Bieringer, J; Schlosser, C: Three years of operational experience from Schauinsland CTBT monitoring station >> MATTHEWS KM 263(2005)235 
\#759 J. ENVIRON. RADIOACT. 99(2008)875-881

Tsukada, H; Takeda, A; Hisamatsu, S; Inaba, J: Concentration and specific activity of fallout Cs-137 in extracted and particle-size fractions of cultivated soils $>>$ TSUKADA H 263(2005)773

\#760 J. ENVIRON. RADIOACT. 99(2008)900-911

Takeda, A; Tsukada, H; Takaku, Y; Akata, N; Hisamatsu, S: Plant induced changes in concentrations of caesium, strontium and uranium in soil solution with reference to major ions and dissolved organic matter $>>$ TSUKADA H 263(2005)773

\#761 J. ENVIRON. RADIOACT. 99(2008)973-980

Tagami, K; Uchida, S: Determination of bioavailable rhenium fraction in agricultural soils $>$ TAGAMI $\mathrm{K}$ 267(2006)631

\#762 J. ENVIRON. RADIOACT. 99(2008)993-1002

Kamei-Ishikawa, N; Nakamaru, Y; Tagami, K; Uchida, S: Sorption behavior of selenium on humic acid under increasing selenium concentration or increasing solid/ liquid ratio $>>$ SAKURAGI T 265(2005)349

\#763 J. ENVIRON. RADIOACT. 99(2008)1011-1017

Psichoudaki, M; Papaefthymiou, H: Natural radioactivity measurements in the city of Ptolemais (Northern Greece) >> PAPAEFTHYMIOU H 274(2007)123; TSIKRITZIS LI 264(2005)651

\#764 J. ENVIRON. RADIOACT. 99(2008)1068-1074

Vaupotic, J; Gregoric, A; Kotnik, J; Horvat, M; Pirrone, $\mathrm{N}$ : Dissolved radon and gaseous mercury in the Mediterranean seawater $>>$ DESIDERI D 270(2006)523

\#765 J. ENVIRON. RADIOACT. 99(2008)1224-1232

Noureddine, A; Benkrid, M; Maoui, R; Menacer, M; Boudjenoun, R; Kadi-Hanifi, M; Lee, SH; Povinec, PP: Radionuclide tracing of water masses and processes in the water column and sediment in the Algerian Basin $>>$ POVINEC PP 273(2007)383

\#766 J. ENVIRON. RADIOACT. 99(2008)1329-1334

Machado, W; Luiz-Silva, W; Sanders, CJ; Patchineelam, SR: Coupled anthropogenic anomalies of radionuclides and major elements in estuarine sediments $>>$ SILVA PSC 269(2006)739; SILVA PSC 269(2006)767

\#767 J. ENVIRON. RADIOACT. 99(2008)1393-1407

Beresford, NA; Barnett, CL; Howard, BJ; Scott, WA; Brown, JE; Copplestone, D: Derivation of transfer parameters for use within the ERICA Tool and the default concentration ratios for terrestrial biota $>>$ HINTON TG 264(2005)417

\#768 J. ENVIRON. RADIOACT. 99(2008)1553-1557

de la Fuente, R; de Celis, B; del Canto, V; Lumbreras, JM; Alonso, BD; Martin-Martin, A; Gutierrez-Villanueva, JL: Low level radioactivity measurements with phoswich detectors using coincident techniques and digital pulse processing analysis $>>$ ELY JH 263(2005)245
\#769 J. ENVIRON. RADIOACT. 99(2008)1596-1610

Povinec, PP; Bokuniewicz, H; Burnett, WC; Cable, J; Charette, M; Comanducci, JF; Kontar, EA; Moore, WS; Oberdorfer, JA; de Oliveira, J; Peterson, R; Stieglitz, T; Taniguchi, M: Isotope tracing of submarine groundwater discharge offshore Ubatuba, Brazil: results of the IAEAUNESCO SGD project >> DULAIOVA H 263(2005)361 \#770 J. ENVIRON. RADIOACT. 99(2008)1617-1630

Hu, QH; Rose, TP; Zavarin, M; Smith, DK; Moran, JE; Zhao, PH: Assessing field-scale migration of radionuclides at the Nevada Test Site: "mobile" species $>>$ ZHAO P 276(2008)755

\#771 J. ENVIRON. RADIOACT. 99(2008)1671-1679

Koarashi, J; Davis, PA; Galeriu, D; Melintescu, A; Saito, M; Siclet, F; Uchida, S: Carbon-14 transfer into rice plants from a continuous atmospheric source: observations and model predictions $>>$ KOARASHI J 268(2006)475 \#772 J. ENVIRON. RADIOACT. 99(2008)1680-1686

Sanchez-Cabeza, JA; Pham, MK; Povinec, PP: IAEA programme on the quality of marine radioactivity data $>>$ POVINEC PP 273(2007)383

\#773 J. ENVIRON. RADIOACT. 99(2008)1764-1769

Feuerstein, J; Boulyga, SF; Galler, P; Stingeder, G; Prohaska, T: Determination of Sr-90 in soil samples using inductively coupled plasma mass spectrometry equipped with dynamic reaction cell (ICP-DRC-MS) > > SHIRAISHI K 273(2007)307

\#774 J. ENVIRON. RADIOACT. 100(2009)71-75

Zheng, J; Yamada, M; Wu, FC; Liao, HQ: Characterization of $\mathrm{Pu}$ concentration and its isotopic composition in soils of Gansu in northwestern China $>$ HIROSE K 273(2007)115

\#775 J. ENVIRON. RADIOACT. 100(2009)84-88

Meli, MA; Desideri, D; Roselli, C; Feduzi, L: Po-210 determination in urines of people living in Central Italy $>>$ ALARIFI MN 269(2006)115

\#776 J. ENVIRON. RADIOACT. 100(2009)139-143

Talip, Z; Eral, M; Hicsonmez, U: Adsorption of thorium from aqueous solutions by perlite $>$ HUMELNICU D 270(2006)637

\#777 J. ENVIRON. RADIOACT. 100(2009)144-150

Strezov, A; Nonova, T: Influence of macroalgal diversity on accumulation of radionuclides and heavy metals in Bulgarian Black Sea ecosystems $>$ NONOVA T 266(2005)411

\#778 J. ENVIRON. RADIOACT. 100(2009)361-365

Jungck, MHA; Andrey, JL; Froidevaux, P: Determination of radionuclide levels in rainwater using ion exchange resin and gamma-spectrometry >> KOMURA K 269(2006)511 \#779 J. ENVIRON. RADIOACT. 100(2009)396-406

Saey, PRJ: The influence of radiopharmaceutical isotope production on the global radioxenon background $>>$ 
MATTHEWS KM 263(2005)235; TABASI M 264(2005) 679

\#780 J. ENVIRON. RADIOACT. 100(2009)407-412

El Afifi, EM; Hilal, MA; Attallah, MF; El-Reefy, SA: Characterization of phosphogypsum wastes associated with phosphoric acid and fertilizers production $>>$ SAUEIA $\mathrm{CH}$ 264(2005)445

\#781 J. ENVIRON. RADIOACT. 100(2009)484-488

Kirchner, G; Steiner, M; Zahringer, M: A new approach to estimate nuclide ratios from measurements with activities close to background >> PADOANI F 263(2005)183 \#782 J. ENVIRON. RADIOACT. 100(2009)497-504

Cukrov, N; Mlakar, M; Cuculic, V; Barisic, D: Origin and transport of U-238 and Ra-226 in riverine, estuarine and marine sediments of the Krka River, Croatia $>>$ SILVA PSC 269(2006)767

\#783 J. ENVIRON. RADIOACT. 100(2009)509-514

Lenka, P; Jha, SK; Gothankar, S; Tripathi, RM; Puranik, VD: Suitable gamma energy for gamma-spectrometric determination of U-238 in surface soil samples of a high rainfall area in India >> REKHA AK 268(2006)453 \#784 J. ENVIRON. RADIOACT. 100(2009)605-605

Leopold, K; Gerstmann, U: A comment on "Distribution of some natural gamma-emitting radionuclides in the soils of the coastal areas of Nigeria" by Alatise, OO, Babalola, IA \& Olowofela, JA journal of Environmental Radioactivity 99 (2008) 1746-1749 >> DU JZ 278(2008)219

\#785 J. ENVIRON. RADIOACT. 100(2009)613-625

Godoy, MLDP; Godoy, JM; Roldao, LA; Tauhata, L: Determination of total content and isotopic compositions of plutonium and uranium in environmental samples for safeguards purposes by ICP-QMS $>>$ LEE SH 263(2005) 419; POVINEC PP 273(2007)383

\#786 J. ENVIRON. RADIOACT. 100(2009)704-715

Gil-Garcia, C; Tagami, K; Uchida, S; Rigol, A; Vidal, M: New best estimates for radionuclide solid-liquid distribution coefficients in soils. Part 3: miscellany of radionuclides (Cd, Co, Ni, Zn, I, Se, Sb, Pu, Am, and others) >> MU DH 267(2006)585

\#787 J. ENVIRON. RADIOACT. 100(2009)739-745

Uchida, S; Tagami, K; Shang, ZR; Choi, YH: Uptake of radionuclides and stable elements from paddy soil to rice: a review >> UCHIDA S 273(2007)205

\#788 J. ENVIRON. RADIOACT. 100(2009)835-840

Ueda, S; Ohtsuka, Y; Kondo, K; Hisamatsu, S: Inventories of Pu239 +240 , Cs-137, and excess Pb-210 in sediments from freshwater and brackish lakes in Rokkasho, Japan, adjacent to a spent nuclear fuel reprocessing plant $>>$ UEDA S 264(2005)343

\#789 J. ENVIRON. RADIOACT. 100(2009)941-949

Jia, GG; Torri, G; Magro, L: Concentrations of U-238, U-234, U-235, Th-232, Th-230, Th-228, Ra-226, Ra-228, Ra-224, Po-210, Pb-210 and Pb-212 in drinking water in
Italy: reconciling safety standards based on measurements of gross alpha and beta >> JIA GG 267(2006)505

\#790 J. ENVIRON. RADIOACT. 100(2009)970-976

Burger, S; Riciputi, LR: A rapid isotope ratio analysis protocol for nuclear solid materials using nano-second laser-ablation time-of-flight ICP-MS >> BURGER S 274(2007)491; BURGER S 279(2009)659

\#791 J. ENVIRON. RADIOACT. 100(2009)1121-1124

Dowdall, M; Sneve, M; Standring, WJF; Amundsen, I: Norway's role in international collaboration towards rehabilitation of Andreeva Bay $>>$ STANDRING WJF 279(2009) 227

\#792 J. ENVIRON. RADIOACT. 101(2010)16-21

Bondareva, L; Vlasova, I; Mogilnaya, O; Bolsunovsky, A; Kalmykov, S: Microdistribution of Am-241 in structures of submerged macrophyte Elodea canadensis growing in the Yenisei River $>>$ BOLSUNOVSKY A 277 (2008)631

\#793 J. ENVIRON. RADIOACT. 101(2010)45-50

Plastino, W; Povinec, PP; De Luca, G; Doglioni, C; Nisi, S; Ioannucci, L; Balata, M; Laubenstein, M; Bella, F; Coccia, E: Uranium groundwater anomalies and L'Aquila earthquake, 6th April 2009 (Italy) >> PLASTINO W 282(2009)809

\#794 J. ENVIRON. RADIOACT. 101(2010)106-112

Hirose, K; Igarashi, Y; Aoyama, M; Inomata, Y: Depositional behaviors of plutonium and thorium isotopes at Tsukuba and Mt. Haruna in Japan indicate the sources of atmospheric dust $>>$ HIROSE K 273(2007)115

\#795 J. ENVIRON. RADIOACT. 101(2010)148-152

Zotina, TA; Bolsunovsky, AY; Bondareva, LG: Accumulation of Am-241 by suspended matter, diatoms and aquatic weeds of the Yenisei River >> LIU N 275(2008) 173

\#796 J. ENVIRON. RADIOACT. 101(2010)153-158

Anjos, RM; Umisedo, N; da Silva, AAR; Estellita, L; Rizzotto, M; Yoshimura, EM; Velasco, H; Santos, AMA: Occupational exposure to radon and natural gamma radiation in the La Carolina, a former gold mine in San Luis Province, Argentina >> SOMLAI J 279(2009) 219

\#797 J. ENVIRON. SCI. 18(2006)610-615

Qin, YW; Meng, W; Zheng, BH; Su, YB: Heavy metal pollution in tidal zones of Bohai Bay using the dated sediment cores >> GUEVARA R 265(2005)481

\#798 J. ENVIRON. SCI. 20(2008)664-669

Yu, RL; Yuan, X; Zhao, YH; Hu, GR; Tu, XL: Heavy metal pollution in intertidal sediments from Quanzhou Bay, China >> GUEVARA R 265(2005)481

\#799 J. ENVIRON. SCI. 20(2008)814-819

Meng, W; Qin, YW; Zheng, BH; Zhang, L: Heavy metal pollution in Tianjin Bohai Bay, China $>>$ GUEVARA R 265(2005)481 
\#800 J. ENVIRON. SCI. 21(2009)1118-1124

Li, XL; Zhang, YX; Tan, MG; Liu, JF; Bao, LM; Zhang, GL; Li, Y; Iida, A: Atmospheric lead pollution in fine particulate matter in Shanghai, China $>$ ZHANG YX 267(2006)497

\#801 J. ENVIRON. SCI. HEALTH PART A-TOXIC/ HAZARD. SUBST. ENVIRON. ENG. 42(2007)16311637

Borio, R; Rongoni, A; Saetta, D; Desideri, D; Meli, MA; Feduzi, L: Natural radionuclides measurements and total dose indicative evaluation in drinking waters of an italian central region $>>$ BORIO R 266(2005)397

\#802 J. ENVIRON. SCI. HEALTH PART A-TOXIC/ HAZARD. SUBST. ENVIRON. ENG. 43(2008)335-347

Saleh, MA; Abdel-Rahman, FH; Woodard, BB; Clark, S; Wallace, C; Aboaba, A; Zhang, W; Nance, JH: Chemical, microbial and physical evaluation of commercial bottled waters in greater Houston area of Texas $>>$ SOUPIONI MJ 268(2006)441

\#803 J. ENVIRON. SCI. HEALTH PART A-TOXIC/ HAZARD. SUBST. ENVIRON. ENG. 44(2009)682-687

Orescanin, V; Mikelic, L; Tomasic, N; Medunic, G; Kampic, S; Mikulic, N; Rubcic, M; Lulic, S; Harambasic, $\mathrm{M}$ : Characterization and treatment of the phosphoric gypsum transport water $>>$ SILVA PSC 269(2006)739 \#804 J. ENVIRON. SCI. HEALTH PART A-TOXIC/ HAZARD. SUBST. ENVIRON. ENG. 45(2010)313-319

Tsakovski, SL; Zukowska, J; Bode, P; Bizuk, MK; Kowalczyk, A: Self-organizing maps classification of epidemiological data and toenail selenium content monitored on cancer and healthy patients from Poland $>>$ MORRIS JS 269(2006)283

\#805 J. ENVIRON. SCI. HEALTH PART B-PESTIC. CONTAM. AGRIC. WASTES 43(2008)588-594

Peng, X; Zhang, JS; Li, YY; Li, W; Xu, GM; Yan, YC: Biodegradation of insecticide carbofuran by Paracoccus $\mathrm{sp}$ YM3 >> LIYANAGE JA 272(2007)477

\#806 J. EUKARYOT. MICROBIOL. 55(2008)151-162

Twining, BS; Baines, SB; Vogt, S; de Jonge, MD: Exploring ocean biogeochemistry by single-cell microprobe analysis of protist elemental composition $>>$ IWATA Y 264(2005)295

\#807 J. FAC. ENG. ARCHIT. GAZI UNIV. 23(2008)119127

Unlu, A; Coban, F; Tunc, MS: Investigation of Lake Hazar water quality according to physical and inorganic chemical parameters $>>$ KULAHCI F 269(2006)63 \#808 J. FAC. ENG. ARCHIT. GAZI UNIV. 23(2008)699707

Yucel, H; Solmaz, AN; Kurt, A; Inal, T; Bor, D: Detection efficiency of CdZnTe detector in the range of 30-670 keV gamma ray energy for a disc source geometry >> HOFSTETTER KJ 263(2005)171
\#809 J. FLUOR. CHEM. 128(2007)1461-1468

Bin, S; Loffler, D; Zeller, KP; Ubele, M; Reischl, G; Machulla, HJ: Effect of aldehyde and methoxy substituents on nucleophilic aromatic substitution by [F-18]fluoride $>>$ ALLABADI A 270(2006)313

\#810 J. FLUOR. CHEM. 130(2009)216-224

Shen, B; Loffler, D; Reischl, G; Machulla, HJ; Zeller, KP: Nucleophilic substitution of nitro groups by [F-18]fluoride in methoxy-substituted ortho-nitrobenzaldehydes-A systematic study $>>$ ALLABADI A 270(2006)313

\#811 J. FOOD AGRIC. ENVIRON. 5(2007)267-273

Tayo, GO; Tan, Z: Endogenous phosphorus loss in ruminants: A review $>>$ DIAS RS 269(2006)661

\#812 J. FUSION ENERGY 29(2010)41-48

Buyukuslu, H; Kaplan, A; Tel, E; Aydin, A; Yildirim, G: Neutron Emission Spectra of Pd-104,105,106,108,110 Isotopes for $(\mathrm{p}, \mathrm{xn})$ Reactions at $21.6 \mathrm{MeV}$ Proton Incident Energy >> DITROI F 272(2007)231

\#813 J. GEODYN. 45(2008)1-17

Kovacs, I; Szabo, C: Middle Miocene volcanism in the vicinity of the Middle Hungarian zone: Evidence for an inherited enriched mantle source $>$ GMELING K 265(2005)201

\#814 J. GEOPHYS. RES.-ATMOS. 111(2006)D17306-

Saey, PRJ; Wotawa, G; De Geer, LE; Axelsson, A; Bean, M; d'Amours, R; Elmgren, K; Peterson, J; Ringbom, A; Stocki, TJ; Ungar, RK: Radioxenon background at high northern latitudes $>>$ MATTHEWS KM 263(2005)235 \#815 J. GEOPHYS. RES.-ATMOS. 112(2007)D04208

McNeary, D; Baskaran, M: Residence times and temporal variations of Po-210 in aerosols and precipitation from southeastern Michigan, United States $>>$ PAPASTEFANOU C 267(2006)315

\#816 J. GEOPHYS. RES.-ATMOS. 112(2007)D21309-

Hagler, GSW; Bergin, MH; Smith, EA; Dibb, JE: A summer time series of particulate carbon in the air and snow at Summit, Greenland >> CURRIE LA 263(2005)399 \#817 J. GEOPHYS. RES.-ATMOS. 114(2009)D06108-

Usoskin, IG; Field, CV; Schmidt, GA; Leppanen, AP; Aldahan, A; Kovaltsov, GA; Possnert, G; Ungar, RK: Short-term production and synoptic influences on atmospheric Be-7 concentrations $>>$ AARNIO PA 276(2008) 631; UNGAR K 272(2007)285

\#818 J. GEOPHYS. RES.-BIOGEOSCI. 114(2009) G01023-

Pearson, CL; Dale, DS; Brewer, PW; Salzer, MW; Lipton, J; Manning, SW: Dendrochemistry of White Mountain bristlecone pines: An investigation via Synchrotron Radiation Scanning X-Ray Fluorescence Microscopy $>>$ UNLU K 264(2005)21

\#819 J. GEOPHYS. RES.-OCEANS 111(2006)C11003-

Walsh, JJ; Jolliff, JK; Darrow, BP; Lenes, JM; Milroy, SP; Remsen, A; Dieterle, DA; Carder, KL; Chen, FR; 
Vargo, GA; Weisberg, RH; Fanning, KA; Muller-Karger, FE; Shinn, E; Steidinger, KA; Heil, CA; Tomas, CR; Prospero, JS; Lee, TN; Kirkpatrick, GJ; Whitledge, TE; Stockwell, DA; Villareal, TA; Jochens, AE; Bontempi, PS: Red tides in the Gulf of Mexico: Where, when, and why? >> BIEGALSKI SR 263(2005)767

\#820 J. GEOPHYS. RES.-OCEANS 113(2008)C04019-

Cai, PH; Chen, WF; Dai, MH; Wan, ZW; Wang, DX; Li, Q; Tang, TT; Lv, DW: A high-resolution study of particle export in the southern South China Sea based on Th-234:U-238 disequilibrium >> PIKE SM 263(2005)355 \#821 J. HAZARD. MATER. 137(2006)1496-1501

Soylak, M; Tuzen, M: Diaion SP-850 resin as a new solid phase extractor for preconcentration-separation of trace metal ions in environmental samples $>>$ DAKSHINAMOORTHY A 266(2005)425; SAEED MM 267(2006)427; SAEED MM 267(2006)147

\#822 J. HAZARD. MATER. 145(2007)113-119

Narin, I; Surme, Y; Bercin, E; Soylak, M: SP70-alphabenzoin oxime chelating resin for preconcentration-separation of $\mathrm{Pb}(\mathrm{II}), \mathrm{Cd}(\mathrm{II}), \mathrm{Co}(\mathrm{II})$ and $\mathrm{Cr}(\mathrm{III})$ in environmental samples >> DAKSHINAMOORTHY A 266(2005)425

\#823 J. HAZARD. MATER. 147(2007)601-609

Zhang, AY; Xiao, CL; Kuraoka, E; Kumagai, M: Molecular modification of a novel macroporous silicabased impregnated polymeric composite by tri-n-butyl phosphate and its application in the adsorption for some metals contained in a typical simulated HLLW >> ZHANG A 265(2005)409

\#824 J. HAZARD. MATER. 148(2007)499-504

Ladeira, ACQ; Goncalves, CR: Influence of anionic species on uranium separation from acid mine water using strong base resins >> DONAT R 265(2005)107 \#825 J. HAZARD. MATER. 150(2008)804-808

Baysal, A; Tokman, N; Akman, S; Ozeroglu, C: Slurry analysis after lead collection on a sorbent and its determination by electrothermal atomic absorption spectrometry $>>$ OZEROGLU C 268(2006)211

\#826 J. HAZARD. MATER. 151(2008)432-445

El-Kamash, AM: Evaluation of zeolite A for the sorptive removal of $\mathrm{Cs}+$ and $\mathrm{Sr} 2(+)$ ions from aqueous solutions using batch and fixed bed column operations $>>$ ABDELRAHMAN KM 268(2006)221

\#827 J. HAZARD. MATER. 152(2008)1248-1255

Ghaedi, M; Ahmadi, F; Tavakoli, Z; Montazerozohori, M; Kharlmohammadi, A; Soylak, M: Three modified activated carbons by different ligands for the solid phase extraction of copper and lead $>>$ ALVAREZ AM 273(2007)427

\#828 J. HAZARD. MATER. 153(2008)948-954

El-Sofany, EA: Removal of lanthanum and gadolinium from nitrate medium using Aliquat-336 impregnated onto Amberlite XAD-4 > ELDESSOUKY SI 268 (2006) 247
\#829 J. HAZARD. MATER. 154(2008)407-416

Xu, D; Tan, XL; Chen, CL; Wang, XK: Removal of $\mathrm{Pb}$ (II) from aqueous solution by oxidized multiwalled carbon nanotubes >> PATHAK PN 270(2006)299

\#830 J. HAZARD. MATER. 154(2008)963-972

El-Naggar, MR; El-Kamash, AM; El-Dessouky, MI; Ghonaim, AK: Two-step method for preparation of NaA-X zeolite blend from fly ash for removal of cesium ions $>>$ ABDELRAHMAN KM 268(2006)221

\#831 J. HAZARD. MATER. 156(2008)129-134

Mishra, RK; Kumar, S; Tomar, BS; Tyagi, AK; Kaushik, CP; Raj, K; Manchanda, VK: Effect of barium on diffusion of sodium in borosilicate glass $>>$ KUMAR $S$ 274(2007)225

\#832 J. HAZARD. MATER. 159(2008)435-439

Soylak, M; Kars, A; Narin, I: Coprecipitation of Ni2+, $\mathrm{Cd} 2+$ and $\mathrm{Pb} 2+$ for preconcentration in environmental samples prior to flame atomic absorption spectrometric determinations $>>$ DUTTA S 274(2007)245

\#833 J. HAZARD. MATER. 160(2008)388-395

Yusan, S; Akyil, S: Sorption of uranium(VI) from aqueous solutions by akaganeite $>>$ AWWAD NS 264(2005)623; KILINCARSLAN A 264(2005)541 \#834 J. HAZARD. MATER. 160(2008)462-467

Sesli, E; Tuzen, M; Soylak, M: Evaluation of trace metal contents of some wild edible mushrooms from Black sea region, Turkey >> UNAK P 273(2007)649

\#835 J. HAZARD. MATER. 160(2008)681-687

Kuryatnyk, T; da Luz, CA; Ambroise, J; Pera, J: Valorization of phosphogypsum as hydraulic binder $>>$ REGUIGUI R 264(2005)719

\#836 J. HAZARD. MATER. 161(2009)1255-1264

Lugo-Lugo, V; Hernandez-Lopez, S; Barrera-Diaz, C; Urena-Nunez, F; Bilyeu, B: A comparative study of natural, formaldehyde-treated and copolymer-grafted orange peel for $\mathrm{Pb}$ (II) adsorption under batch and continuous mode $>>$ TSAI SC 266(2005)101

\#837 J. HAZARD. MATER. 162(2009)994-999

Hassan, SSM; Awwad, NS; Aboterika, AHA: Removal of synthetic reactive dyes from textile wastewater by Sorel's cement $>>$ HASSAN SSM 269(2006)135 \#838 J. HAZARD. MATER. 162(2009)1228-1232

Aydin, FA; Soylak, M: Thulium hydroxide: A new coprecipitant for speciation of chromium in natural water samples >> YUSOF AM 273(2007)533 \#839 J. HAZARD. MATER. 163(2009)98-108

Ulusoy, U; Akkaya, R: Adsorptive features of polyacrylamide-apatite composite for $\mathrm{Pb} 2+, \mathrm{UO} 22+$ and $\mathrm{Th} 4+$ >> AKYIL S 266(2005)89; MOORE RC 263(2005)97 \#840 J. HAZARD. MATER. 163(2009)475-510

Gavrilescu, M; Pavel, LV; Cretescu, I: Characterization and remediation of soils contaminated with uranium $>>$ BAYAKARA O 265(2005)95; DONAT R 265(2005)107 
\#841 J. HAZARD. MATER. 165(2009)415-426

Gavilan, KC; Pestov, AV; Garcia, HM; Yatluk, Y; Roussy, J; Guibal, E: Mercury sorption on a thiocarbamoyl derivative of chitosan >> PRAMANIK S 274(2007)237 \#842 J. HAZARD. MATER. 166(2009)109-116

Yang, ST; Li, JX; Shao, DD; Hu, J; Wang, XK: Adsorption of $\mathrm{Ni}(\mathrm{II})$ on oxidized multi-walled carbon nanotubes: Effect of contact time, $\mathrm{pH}$, foreign ions and PAA $>>$ DU JZ 273(2007)119

\#843 J. HAZARD. MATER. 166(2009)1441-1448

Ghaedi, M; Tavallali, H; Shokrollahi, A; Zahedi, M; Montazerozohori, M; Soylak, M: Flame atomic absorption spectrometric determination of zinc, nickel, iron and lead in different matrixes after solid phase extraction on sodium dodecyl sulfate (SDS)-coated alumina as their bis (2hydroxyacetophenone)-1,3-propanediimine chelates $>>$ LI BP 278(2008)3

\#844 J. HAZARD. MATER. 167(2009)44-51

Wang, SW; Hu, J; Li, JX; Dong, YH: Influence of pH, soil humic/fulvic acid, ionic strength, foreign ions and addition sequences on adsorption of $\mathrm{Pb}(\mathrm{II})$ onto $\mathrm{GMZ}$ bentonite $>>$ CHANG P 274(2007)153

\#845 J. HAZARD. MATER. 167(2009)294-299

Duran, C; Bulut, VN; Gundogdu, A; Ozdes, D; Yildirim, N; Soylak, M; Senturk, HB; Elci, L: Carrier element-free coprecipitation with 3-phenly-4-o-hydroxybenzylidenamino4,5-dihydro-1,2,4-triazole-5-one for separation/preconcentration of $\mathrm{Cr}$ (III), $\mathrm{Fe}(\mathrm{III}), \mathrm{Pb}$ (II) and $\mathrm{Zn}$ (II) from aqueous solutions $>>$ LI BP 278(2008)3

\#846 J. HAZARD. MATER. 167(2009)896-903

Rim, SA; Amine, DM; Nasr-Eddine, B; Canselier, JP: Removal of chromium (III) by two-aqueous phases extraction $>>$ ABDERRAHIM O 279(2009)237

\#847 J. HAZARD. MATER. 168(2009)369-375

Gok, C; Aytas, S: Biosorption of uranium(VI) from aqueous solution using calcium alginate beads $>>$ DONAT R 265(2005)107

\#848 J. HAZARD. MATER. 168(2009)458-465

Tan, XL; Fang, M; Li, JX; Lu, Y; Wang, XK: Adsorption of $\mathrm{Eu}(\mathrm{III})$ onto $\mathrm{TiO} 2$ : Effect of $\mathrm{pH}$, concentration, ionic strength and soil fulvic acid >> XU D 266(2005)419 \#849 J. HAZARD. MATER. 170(2009)814-823

Casacuberta, N; Masque, P; Garcia-Orellana, J; Bruach, JM; Anguita, M; Gasa, J; Villa, M; Hurtado, S; GarciaTenorio, R: Radioactivity contents in dicalcium phosphate and the potential radiological risk to human populations $>>$ SAUEIA CH 264(2005)445

\#850 J. HAZARD. MATER. 170(2009)1197-1203

Zhang, L; Liu, N; Yang, LJ; Lin, Q: Sorption behavior of nano-TiO2 for the removal of selenium ions from aqueous solution $>>$ SHAUKAT MS 265(2005)73
\#851 J. HAZARD. MATER. 171(2009)820-826

Wang, M; Xu, L; Peng, J; Zhai, ML; Li, JQ; Wei, GS: Adsorption and desorption of $\mathrm{Sr}(\mathrm{II})$ ions in the gels based on polysaccharide derivates $>>$ OZEROGLU C 268(2006)211 \#852 J. HAZARD. MATER. 171(2009)1024-1031

Papadokostaki, KG; Savidou, A: Study of leaching mechanisms of caesium ions incorporated in Ordinary Portland Cement $>>$ PLECAS I 264(2005)687 \#853 J. HAZARD. MATER. 173(2010)669-674

Aydin, FA; Soylak, M: Separation, preconcentration and inductively coupled plasma-mass spectrometric (ICP-MS) determination of thorium(IV), titanium(IV), iron(III), lea$\mathrm{d}(\mathrm{II})$ and chromium(III) on 2-nitroso-1-naphthol impregnated MCI GEL CHP20P resin >> LI BP 278(2008)3 \#854 J. HAZARD. MATER. 174(2010)556-562

Celik, Z; Gulfen, M; Aydin, AO: Synthesis of a novel dithiooxamide-formaldehyde resin and its application to the adsorption and separation of silver ions $>>$ DUTTA $S$ 274(2007)245

\#855 J. HAZARD. MATER. 174(2010)782-787

Humelnicu, D; Bulgariu, L; Macoveanu, M: On the retention of uranyl and thorium ions from radioactive solution on peat moss $>>$ KILINCARSLAN A 264(2005)541 \#856 J. HAZARD. MATER. 175(2010)393-398

Camacho, LM; Deng, SG; Parra, RR: Uranium removal from groundwater by natural clinoptilolite zeolite: Effects of $\mathrm{pH}$ and initial feed concentration $>>$ KILINCARSLAN A 264(2005)541

\#857 J. HAZARD. MATER. 175(2010)404-409

Zhang, Y; Li, Q; Sun, L; Tang, R; Zhai, JP: High efficient removal of mercury from aqueous solution by polyaniline/humic acid nanocomposite $>$ DEVI PSR 269(2006)217

\#858 J. HAZARD. MATER. 176(2010)119-124

Zhao, YS; Liu, CX; Feng, M; Chen, Z; Li, SQ; Tian, G; Wang, L; Huang, JB; Li, SJ: Solid phase extraction of uranium(VI) onto benzoylthiourea-anchored activated carbon $>>$ KRISHNA PG 266(2005)251

\#859 J. HAZARD. MATER. 176(2010)252-256

Sepehrian, H; Ahmadi, SJ; Waqif-Husain, S; Faghihian, $\mathrm{H}$; Alighanbari, H: Adsorption Studies of Heavy Metal Ions on Mesoporous Aluminosilicate, Novel Cation Exchanger $>>$ SEPEHRIAN H 275(2008)145 \#860 J. HAZARD. MATER. 176(2010)426-433

Tufail, SJM; Asghar, M: Hazard of NORM from phosphorite of Pakistan $>>$ SAUEIA CH 264(2005)445 \#861 J. HAZARD. MATER. 176(2010)644-649

Damla, N; Cevik, U; Kobya, AI; Celik, A; Celik, N; Van Grieken, R: Radiation dose estimation and mass attenuation coefficients of cement samples used in Turkey $>>$ KHRBISH YS 271(2007)63 
\#862 J. HYDROL. 353(2008)275-293

Santos, IR; Niencheski, F; Burnett, W; Peterson, R; Chanton, J; Andrade, CFF; Milani, IB; Schmidt, A; Knoeller, $\mathrm{K}$ : Tracing anthropogenically driven groundwater discharge into a coastal lagoon from southern Brazil $>>$ DULAIOVA H 263(2005)361

\#863 J. HYDROL. 378(2009)281-289

Sanchez-Vila, X; Bolster, D: An analytical approach to transient homovalent cation exchange problems $>>$ BHATTACHARYYA A 268(2006)323

\#864 J. HYDROL. 380(2010)298-304

Burnett, WC; Peterson, RN; Santos, IR; Hicks, RW: Use of automated radon measurements for rapid assessment of groundwater flow into Florida streams $>>$ DULAIOVA H 263(2005)361

\#865 J. IBER. GEOL. 34(2008)235-242

Irabien, MJ; Rada, M; Gomez, J; Soto, J; Mananes, A; Viguri, J: An Assessment of Anthropogenic Impact in a Nature Reserve: the Santona Marshes (Northern Spain) $>>$ REGUIGUI N 264(2005)469

\#866 J. INCL. PHENOM. MACROCYCL. CHEM. 61(2008)29-40

Mbemba, C; Sigaud, K; Perret, F; Suwinska, K; Shkurenko, O; Coleman, AW: Calix[n]arenes as components for the construction of micellar systems: synthesis and self-assembly properties of 5,11,17-Tris[(dimethylamino)methyl]-25-monoalkoxy-26,27,28-trihydroxycalix[4] arene derivatives $>>$ YAFTIAN MR 270(2006)357 \#867 J. IND. ENG. CHEM. 12(2006)615-619

Jo, HJ; Lee, SM; Kim, HJ; Park, EJ; Kim, JG; Chung, $\mathrm{HH}$; Jung, J: Modification of textile wastewater treatment system by gamma-irradiation $>>$ JO HJ 268(2006)145 \#868 J. INDIAN CHEM. SOC. 85(2008)9-21

Dutta, S; Das, AK: Separation of selected $4 \mathrm{f}$ and $5 \mathrm{f}$ metals by solid phase extraction : A review $>>$ PREETHA CR 267(2006)265

\#869 J. INDIAN CHEM. SOC. 85(2008)962-965

Sharma, S; Dhingra, P; Pandey, RS: Trace determination of $\mathrm{Pb}, \mathrm{Cu}, \mathrm{Cd}$ and $\mathrm{Zn}$ in Ayurvedic, drug, "Mahayograj Guggulu" via polarographic technique $>$ KUMAR A 270(2006)173

\#870 J. INORG. BIOCHEM. 103(2009)1585-1590

Aslam; Davis, K; Pejovic-Milic, A; Chettle, DR: Noninvasive measurement of aluminium in human bone: Preliminary human study and improved system performance >> PEJOVICMILIC A 269(2006)417

\#871 J. INORG. BIOCHEM. 103(2009)1609-1616

Montavon, G; Apostolidis, C; Bruchertseifer, F; Repinc, U; Morgenstern, A: Spectroscopic study of the interaction of U(VI) with transferrin and albumin for speciation of U(VI) under blood serum conditions > MULLEN L 273(2007)683
\#872 J. LABEL. COMPD. RADIOPHARM. 49(2006)699_ 705

Mathew, KM; Ravi, S; Unny, VKP; Sivaprasad, N: Microwave-assisted synthesis of (RS) methyl-2-([2'-C14]4,6-dimethoxypyrimidin-2'-yloxy)-2-phenyl [1-C-14] ethanoate $>>$ MATHEW KM 265(2005)505

\#873 J. LABEL. COMPD. RADIOPHARM. 50(2007)2531

El-Ghany, EA; Amin, AM; El-Kawy, OA; Amin, M: Technetium-99 m labeling and freeze-dried kit formulation of levofloxacin (L-Flox): A novel agent for detecting sites of infection $>>$ ELGHANY EA 266(2005)131

\#874 J. LABEL. COMPD. RADIOPHARM. 50(2007)480482

Dykiy, MP; Dovbnya, AN; Lyashko, YV; Medvedeva, EP; Medvedev, DV; Uvarov, VL: Photonuclear production of $193 \mathrm{~m}, 195 \mathrm{~m} \mathrm{Pt}$ and synthesis of radioactive cisplatin $>>$ KNAPP FF 263(2005)503

\#875 J. LABEL. COMPD. RADIOPHARM. 50(2007)556557

Jalilian, AR; Aboudzadeh, R; Akhlaghi, M; Shirazi, B; Moradkhani, S; Salouti, M; Karimian, A; Babaii, MH; Raisali, G: Production and biological evaluation of [Tl201(III)]bleomycin >> JALILIAN AR 264(2005)617 \#876 J. LABEL. COMPD. RADIOPHARM. 50(2007)683692

Filer, $\mathrm{CN}$ : The synthesis of dopaminergic radioligands labelled with tritium and iodine-125 > FAROUK N 266(2005)405; FILER CN 267(2006)345; LASETER AG 264(2005)723

\#877 J. LABEL. COMPD. RADIOPHARM. 50(2007)789793

Filer, CN: Reflections on New England Nuclear (NEN) $>>$ FILER CN 268(2006)663

\#878 J. LABEL. COMPD. RADIOPHARM. 52(2009)1-5

El-Azony, KM; El-Mohty, AA; Killa, HM; Seddik, U; Khater, SI: An investigation of the I-125-radioiodination of colchicine for medical purposes $>$ ELGHANY EA 266(2005)117

\#879 J. LABEL. COMPD. RADIOPHARM. 52(2009)139_ 145

Ren, YF; Sun, YH; Cheng, KM; Liu, GF; Shen, YM: Synthesis and radiolabelling of $\operatorname{Re}(\mathrm{CO})(3)$-beta-elemene derivatives as potential therapeutic radiopharmaceuticals $>>$ XIA JY 266(2005)313

\#880 J. LABEL. COMPD. RADIOPHARM. 52(2009)183188

Lu, J; Xu, MY; Jia, HM; Xi, SX; Wang, YM; Wang, XB: Synthesis and biodistribution of novel Tc-99m-nitrido methylpiperidine dithioformate derivatives as potential brain imaging agents $>$ ZHANG JB 273(2007)15; ZHANG JB 274(2007)195 
\#881 J. LABEL. COMPD. RADIOPHARM. 52(2009)415418

Motaleb, MA: Preparation, quality control and stability of Tc-99m-sparafloxacin complex, a novel agent for detecting sites of infection $>>$ ELGHANY EA 266(2005) 131; LAMBRECHT FY 275(2008)161; MOTALEB MA 272(2007)167; MOTALEB MA 272(2007)95

\#882 J. LABEL. COMPD. RADIOPHARM. 52(2009)453456

Saemian, N; Arjomandi, OK; Shirvani, G: Synthesis of a series of carbon-14 labelled 4-aminoquinazolines and quinazolin-4 (3H)-ones >> SAEMIAN N 268(2006)545; SAEMIAN N 274(2007)631; SAEMIAN N 274(2007)643 \#883 J. LABEL. COMPD. RADIOPHARM. 53(2010)50-51

Rauh, JJ; Lahm, GP; Pahutski, TF; Ullas, GV; Filer, CN: Lindlar catalyst mediated tritiation of a triazole substituted isoxazoline insecticide >> FILER CN 267(2006)345

\#884 J. LUMINES. 130(2010)304-308

Wu, FY; Sun, MZ; Xiang, YL; Wu, YM; Tong, DQ: Curcumin as a colorimetric and fluorescent chemosensor for selective recognition of fluoride ion $>>$ RAMANJANEYULU PS 274(2007)109

\#885 J. MACROMOL. SCI. PART A-PURE APPL. CHEM. 44(2007)1225-1234

Cataldo, F; Gobbino, M; Ursini, O; Angelini, G: A study on the optically active polymer poly-beta-pinene $>>$ CATALDO F 272(2007)107; CATALDO F 272(2007)29 \#886 J. MACROMOL. SCI. PART A-PURE APPL. CHEM. 45(2008)839-849

Cataldo, F; Angelini, G; Capitani, D; Gobbino, M; Ursini, O; Forlini, F: Determination of the chemical structure of poly-beta(-)-pinene by NMR spectroscopy $>>$ CATALDO F 272(2007)107; CATALDO F 272(2007)29

\#887 J. MACROMOL. SCI. PART A-PURE APPL. CHEM. 46(2009)493-502

Cataldo, F; Ursini, O; Angelini, G; Ragni, P: RadiationInduced Inclusion Polymerization of (-)Pinene In Deoxycholic Acid >> CATALDO F 272(2007)29

\#888 J. MAGN. MAGN. MATER. 320(2008)651-661

Klencsar, Z; Nemeth, Z; Kuzmann, E; Homonnay, Z; Vertes, A; Hakl, J; Vad, K; Meszaros, S; Simopoulos, A; Devlin, E; Kallias, G; Greneche, JM; Cziraki, A; De, SK: The role of iron in the formation of the magnetic structure and related properties of $\mathrm{La} 0.8 \mathrm{Sr} 0.2 \mathrm{Co} 1-\mathrm{xFexO} 3$ $(\mathrm{x}=0.15,0.2,0.3)>>$ NEMETH Z 271(2007)11

\#889 J. MAR. ENG. TECHNOL. (2009)39-48

El-Geziry, TM; Bryden, IG; Couch, SJ: Environmental impact assessment for tidal energy schemes: an exemplar case study of the Strait of Messina $>>$ VELASCO HR 268(2006)503

\#890 J. MAR. SYST. 76(2009)322-335

Friedrich, J: Uranium contamination of the Aral Sea $>>$ BARBER DS 263(2005)213; KADYRZHANOV KK
263(2005)197; VASILIEV LA 263(2005)107; YULDASHEV BS 263(2005)219

\#891 J. MATER. CHEM. 16(2006)1726-1733

Choi, J; Manthiram, A: Factors influencing the crystal chemistry of chemically delithiated layered HxNi1-yzMnyCozO2 >> AGHARA SK 265(2005)321

\#892 J. MATER. CHEM. 18(2008)190-198

Arunkumar, TA; Alvarez, E; Manthiram, A: Chemical and structural instability of the chemically delithiated $(1-\mathrm{z})$ $\mathrm{Li}[\mathrm{Li} 1 / 3 \mathrm{Mn} 2 / 3] \mathrm{O}-2$ center $\operatorname{dot}(\mathrm{z}) \quad \mathrm{Li}[\mathrm{Co} 1-y N i y] \mathrm{O}-2$ $(0<=\mathrm{y}<=1$ and $0<=\mathrm{z}<=1)$ solid solution cathodes $>>$ AGHARA SK 265(2005)321

\#893 J. MATER. CHEM. 18(2008)3342-3346

Pavel, CC; Walter, M; Popa, K: Improvement of retention capacity of ETS-10 for uranyl ions by porosity modification and their immobilization into a titanosilicate matrix $>>$ KILINCARSLAN A 264(2005)541; POPA K 269(2006) 155

\#894 J. MATER. CHEM. 19(2009)8142-8149

Rajasekaran, PR; Wolff, J; Zhou, C; Kinsel, M; Trautmann, C; Aouadi, S; Kohli, P: Two dimensional anisotropic etching in tracked glass $>>$ KALSI PC 273(2007)473

\#895 J. MATER. RES. 23(2008)2561-2581

Milbrath, BD; Peurrung, AJ; Bliss, M; Weber, WJ: Radiation detector materials: An overview $>>$ LUKE PN 264(2005)145; UPP DL 264(2005)121

\#896 J. MATER. SCI.-MATER. MED. 19(2008)34193427

Ledo, HM; Thackray, AC; Jones, IP; Marquis, PM; Macaskie, LE; Sammons, RL: Microstructure and composition of biosynthetically synthesised hydroxyapatite >> MOORE RC 263(2005)97

\#897 J. MEMBR. SCI. 325(2008)158-165

Panja, S; Ruhela, R; Misra, SK; Sharma, JN; Tripathi, SC; Dakshinamoorthy, A: Facilitated transport of Am(III) through a flat-sheet supported liquid membrane (FSSLM) containing tetra(2-ethyl hexyl) diglycolamide (TEHDGA) as carrier $>>$ SHARMA JN 278(2008) 173

\#898 J. MEMBR. SCI. 339(2009)33-38

$\mathrm{Xi}, \mathrm{ZY}$; Xu, YY; Zhu, LP; Zhu, BK: Modification of polytetrafluoroethylene porous membranes by electron beam initiated surface grafting of binary monomers $>>\mathrm{ZU}$ JH 279(2009)185

\#899 J. MEMBR. SCI. 339(2009)115-119

Nasef, MM; Saidi, H; Dahlan, KZM: Single-step radiation induced grafting for preparation of proton exchange membranes for fuel cell >> ZU JH 273(2007)479; ZU JH 279(2009) 185

\#900 J. MOL. LIQ. 151(2010)122-124

Mallah, MH; Shemirani, F; Maragheh, MG; Jamali, MR: Evaluation of synergism in dispersive liquid-liquid microextraction for simultaneous preconcentration of some lanthanoids >> MALLAH MH 278(2008)97 
\#901 J. MOL. NEUROSCI. 36(2008)105-114

Pirger, Z; Nemeth, J; Hiripi, L; Toth, G; Kiss, P; Lubics, A; Tamas, A; Hernadi, L; Kiss, T; Reglodi, D: PACAP Has Anti-apoptotic Effect in the Salivary Gland of an Invertebrate Species, Helix pomatia >> NEMETH J 273(2007)327 \#902 J. MOL. STRUCT. 924-26(2009)20-26

Oshtrakh, MI; Semionkin, VA; Milder, OB; Novikov, EG: Mossbauer spectroscopy with high velocity resolution: New possibilities in biomedical research $>>$ OSHTRAKH MI 269(2006)407

\#903 J. NANOSCI. NANOTECHNOL. 8(2008)5018-5021

Hong, MM; Oh, JM; Choy, JH: Encapsulation of Flavor Molecules, 4-Hydroxy-3-Methoxy Benzoic Acid, into Layered Inorganic Nanoparticles for Controlled Release of Flavor $>>$ VULPIUS D 270(2006)661

\#904 J. NANOSCI. NANOTECHNOL. 9(2009)6335-6343

Liu, Y; Gao, YX; Zhang, LL; Wang, TC; Wang, JX; Jiao, F; Li, W; Liu, Y; Li, YF; Li, B; Chai, ZF; Wu, G; Chen, CY: Potential Health Impact on Mice after Nasal Instillation of Nano-Sized Copper Particles and Their Translocation in Mice $>>$ WANG JX 272(2007)527

\#905 J. NON-CRYST. SOLIDS 353(2007)1941-1945

Fabian, M; Svab, E; Meszaros, G; Revay, Z; Veress, E: Neutron diffraction study of sodium borosilicate waste glasses containing uranium > > REVAY Z 265(2005)261 \#906 J. NON-CRYST. SOLIDS 353(2007)2084-2089

Fabian, M; Svab, E; Meszaros, G; Revay, Z; Proffen, T; Veress, E: Network structure of multi-component sodium borosilicate glasses by neutron diffraction $>>$ REVAY Z 265(2005)261

\#907 J. NON-CRYST. SOLIDS 354(2008)3299-3307

Fabian, M; Svab, E; Proffen, T; Veress, E: Structure study of multi-component borosilicate glasses from high-Q neutron diffraction measurement and RMC modeling $>>$ REVAY Z 265(2005)261

\#908 J. NON-CRYST. SOLIDS 356(2010)441-446

Fabian, M; Svab, E; Proffen, T; Veress, E: Neutron diffraction and reverse Monte Carlo modelling of nu-B2O3 and $75 \mathrm{~B}(2) \mathrm{O}(3)-25 \mathrm{Na}(2) \mathrm{O}$ glasses $>$ REVAY $\mathrm{Z}$ 265(2005)261

\#909 J. NUCL. MATER. 375(2008)1-7

Oyaidzu, M; Nishikawa, Y; Suda, T; Shinozaki, T; Yoshikawa, A; Oya, Y; Munakata, K; Yamana, H; Okuno, $\mathrm{K}$ : Detrapping behavior of tritium trapped via hot atom chemical process in neutron-irradiated ternary lithium oxides $>>$ OYAIDZU M 272(2007)657

\#910 J. NUCL. MATER. 375(2008)311-314

Zhang, YJ; Vance, ER: Plutonium in monazite and brabantite: Diffuse reflectance spectroscopy study $>>$ LEE MH 273(2007)375

\#911 J. NUCL. MATER. 376(2008)181-191

Puig, F; Dies, J; de Pablo, J; Martinez-Esparza, A: Spent fuel canister for geological repository: Inner material requirements and candidates evaluation $>>$ KILINCARSLAN A 264(2005)541; MOORE RC 263(2005)97; TAO ZY 268(2006)563

\#912 J. NUCL. MATER. 378(2008)139-143

Ramanjaneyulu, PS; Sayi, YS; Ramakumar, KL: Determination of boron in uranium-aluminum-silicon alloy by spectrophotometry and estimation of expanded uncertainty in measurement $>$ RAMANJANEYULU PS 274(2007)109

\#913 J. NUCL. MATER. 396(2010)251-256

Kutahyali, C; Eral, M: Sorption studies of uranium and thorium on activated carbon prepared from olive stones: Kinetic and thermodynamic aspects $>$ ELNADI YA 265(2005)447

\#914 J. NUCL. MED. 49(2008)1141-1145

Chen, LY; Gobar, LS; Knowles, NG; Liu, ZL; Gmitro, AF; Barrett, HH: Direct imaging of radionuclide-produced electrons and positrons with an ultrathin phosphor $>>$ KANNO S 272(2007)565

\#915 J. NUCL. MED. 51(2010)282-287

Elizarov, AM; van Dam, RM; Shin, YS; Kolb, HC; Padgett, HC; Stout, D; Shu, J; Huang, J; Daridon, A; Heath, JR: Design and Optimization of Coin-Shaped Microreactor Chips for PET Radiopharmaceutical Synthesis $>>$ WANG MW 280(2009)149

\#916 J. NUCL. MED. 51(2010)288-292

Bartlett, RM; Nickles, RJ; Barnhart, TE; Christian, BT; Holden, JE; DeJesus, OT: Fetal Dose Estimates for F-18Fluoro-L-Thymidine Using a Pregnant Monkey Model $>>$ DEJESUS OT 269(2006)561

\#917 J. NUCL. SCI. TECHNOL. 45(2008)352-360

Nishiyama, J; Igashira, M; Ohsaki, T; Kim, G; Chung, WC; Ro, TI: Measurements of keV-neutron capture cross sections and capture gamma-ray spectra of Sn-117, Sn-119 $>>$ CATLOW SA 263(2005)599

\#918 J. NUCL. SCI. TECHNOL. 45(2008)481-486

Miyazaki, I; Sakane, H; Takayama, H; Kasaishi, M; Tojo, A; Furuta, M; Hayashi, H; Suematsu, O; Narasaki, H; Shimizu, T; Shibata, M; Kawade, K; Taniguchi, A; Harada, $\mathrm{H}$ : Precise intensity measurements in the N-14(n, gamma)N-15 reaction as a gamma-ray intensity standard up to $11 \mathrm{MeV}>>$ BELGYA T 265(2005)175 \#919 J. NUCL. SCI. TECHNOL. 46(2009)1023-1031

Lin, CC; Chao, JH: Reassessment of Reactor Coolant and Iodine Chemistry under Accident Conditions $>>$ GUENTAY S 273(2007)557; LIN CC 268(2006)419 \#920 J. NUCL. SCI. TECHNOL. (2008)8-15

Chettle, DR: Using Ionizing Radiation to Determine Trace Element Content in Living Human Subjects $>>$ PEJOVICMILIC A 269(2006)417

\#921 J. NUCL. SCI. TECHNOL. (2008)128-132

Tagami, K; Uchida, S: Rhenium Contents in Japanese River Waters Measured by Isotope Dilution ICP-MS and 
the Relationship of Re with Some Chemical Components $>$ MAS JL 265(2005)361

\#922 J. NUCL. SCI. TECHNOL. (2008)146-151

Kamei-Ishikawa, N; Tagami, K; Uchida, S: Estimation of Cs-137 Plant Root Uptake Using Naturally Existing Cs$133>$ ISHIKAWA NK 277(2008)433; UCHIDA S 273(2007)205

\#923 J. NUCL. SCI. TECHNOL. (2008)410-413

Lee, TH; Joo, JS; Park, KJ; Shin, HS; Kim, HD: Analysis of A Nuclear Material Accounting Uncertainty for ACP Spent Fuel Standards: MCNPX and ORIGEN-S Code Simulations $>>$ JEONG SM 268(2006)349

\#924 J. NUCL. SCI. TECHNOL. (2008)462-465

Koarashi, J; Mikami, S; Nakada, A; Akiyama, K; Kobayashi, H; Fujita, H; Takeishi, M: Monitoring Methodologies and Chronology of Radioactive Airborne Releases from Tokai Reprocessing Plant $>$ KOARASHI J 268(2006)475; KOARASHI J 267(2006)155

\#925 J. OCEANOGR. 64(2008)417-428

Chen, WF; Cai, PH; Dai, MH; Wei, JF: Th-234/U-238 disequilibrium and particulate organic carbon export in the northern South China Sea $>>$ PIKE SM 263(2005)355 \#926 J. OCEANOGR. 65(2009)397-406

Lee, SH; Povinec, P; Gastaud, J; Oregioni, B; Coppola, L; Jeandel, C: Radionuclides as tracers of water fronts in the South Indian Ocean-ANTARES IV Results $>>$ POVINEC PP 263(2005)413

\#927 J. ORGANOMET. CHEM. 694(2009)1678-1689

Gruner, B; Kvicalova, M; Plesek, J; Sicha, V; Cisarova, I; Lucanikova, M; Selucky, P: Cobalt bis(dicarbollide) ions functionalized by CMPO-like groups attached to boron by short bonds; efficient extraction agents for separation of trivalent f-block elements from highly acidic nuclear waste >> LUTHER TA 267(2006)603

\#928 J. PALEOLIMN. 40(2008)823-833

Laissaoui, A; Benmansour, M; Ziad, N; Ibn Majah, M; Abril, JM; Mulsow, S: Anthropogenic radionuclides in the water column and a sediment core from the Alboran Sea: application to radiometric dating and reconstruction of historical water column radionuclide concentrations $>>$ LAROSA J 263(2005)427

\#929 J. PETROL. 48(2007)2261-2287

Harangi, S; Downes, H; Thirlwall, M; Gmeling, K: Geochemistry, petrogenesis and geodynamic relationships of miocene calc-alkaline volcanic rocks in the western carpathian arc, eastern central Europe > $>$ GMELING K 265(2005)201

\#930 J. PETROL. 49(2008)2043-2080

Pelletier, L; Vils, F; Kalt, A; Gmeling, K: Li, B and Be Contents of Harzburgites from the Dramala Complex (Pindos Ophiolite, Greece): Evidence for a MOR-type Mantle in a Supra-subduction Zone Environment >> GMELING K 265(2005)201; MARSCHALL HR 265(2005)339
\#931 J. PETROL. 50(2009)1443-1475

Facer, J; Downes, H; Beard, A: In situ Serpentinization and Hydrous Fluid Metasomatism in Spinel Dunite Xenoliths from the Bearpaw Mountains, Montana, USA >> GMELING K 265(2005)201

\#932 J. PHARM. BIOMED. ANAL. 37(2005)631-638

Kumar, A; Nair, AGC; Reddy, AR; Garg, AN: Analysis of essential elements in Pragya-peya-a herbal drink and its constituents by neutron activation $>$ GARG AN 263(2005)39

\#933 J. PHARM. BIOMED. ANAL. 41(2006)825-832

Choudhury, RP; Kumar, A; Garg, AN: Analysis of Indian mint (Mentha spicata) for essential, trace and toxic elements and its antioxidant behaviour >> GARG AN 263(2005)39 \#934 J. PHARM. BIOMED. ANAL. 44(2007)970-979

Ji, AJ; Saunders, JP; Wadgaonkar, ND; Petersen, PJ; O'Leary, K; McWilliams, WE; Amorusi, P; Leal, M; Fluhler, EN: A novel antibiotic bone assay by liquid chromatography/tandem mass spectrometry for quantitation of tigecycline in rat bone $>>$ ELLISTON JT 263(2005)301 \#935 J. PHARM. SCI. 97(2008)1633-1651

Brouwer, CPJM; Wulferink, M; Welling, MM: The pharmacology of radiolabeled cationic antimicrobial peptides $>>$ FERROFLORES G 266(2005)307

\#936 J. PHYS. CHEM. A 111(2007)5515-5522

Ando, H; Nakao, Y; Sato, H; Sakaki, S: Theoretical study of low-spin, high-spin, and intermediate-spin states of $[\mathrm{Fe}-\mathrm{III}($ pap $)(2)](+) \quad($ pap $=\mathrm{N}$-2-pyridylmethylidene-2hydroxyphenylaminato). Mechanism of light-induced excited spin state trapping >> HAYAMI S 266(2005)521 \#937 J. PHYS. CHEM. A 112(2008)11714-11723

Cuypers, R; Burghoff, B; Marcelis, ATM; Sudholter, EJR; de Haan, AB; Zuilhof, H: Complexation of Phenols and Thiophenol by Phosphine Oxides and Phosphates. Extraction, Isothermal Titration Calorimetry, and ab Initio Calculations $>>$ ELDESSOUKY SI 268(2006)247 \#938 J. PHYS. CHEM. A 113(2009)12225-12232

Fujii, T; Moynier, F; Uehara, A; Abe, M; Yin, QZ; Nagai, T; Yamana, H: Mass-Dependent and Mass-Independent Isotope Effects of Zinc in a Redox Reaction $>>$ DING X 273(2007)79

\#939 J. PHYS. CHEM. A 114(2010)2543-2552

Fujii, T; Moynier, F; Telouk, P; Abe, M: Experimental and Theoretical Investigation of Isotope Fractionation of Zinc between Aqua, Chloro, and Macrocyclic Complexes >> DING X 273(2007)79

\#940 J. PHYS. CHEM. B 112(2008)6288-6291

Xing, GM; Yuan, H; He, R; Gao, XY; Jing, L; Zhao, F; Chai, ZF; Zhao, YL: The strong MRI relaxivity of paramagnetic nanoparticles $>>$ ZHANG J 272(2007)605 \#941 J. PHYS. CHEM. C 113(2009)17795-17802

Malin, JN; Holland, JG; Geiger, FM: Free Energy Relationships in the Electric Double Layer and Alkali 
Earth Speciation at the Fused Silica/Water Interface $>>$ YOSHIDA T 270(2006)363

\#942 J. PHYS.-CONDES. MATTER 19(2007)116201-

Jain, M; Botter-Jensen, L; Thomsen, KJ: High local ionization density effects in X-ray excitations deduced from optical stimulation of trapped charge in $\mathrm{Al} 2 \mathrm{O} 3: \mathrm{C}>>$ HONG DG 265(2005)495

\#943 J. POLYM. SCI. PT. B-POLYM. PHYS. 46(2008) 2485-2503

Shantarovich, VP: Positron Annihilation and Free Volume Studies in Polymer Glasses $>>$ SHANTAROVICH VP 272(2007)645

\#944 J. POLYM. SCI. PT. B-POLYM. PHYS. 48(2010) 381-388

Chen, Z; Huang, W; Fang, PF; Yu, W; Wang, SJ; Xiong, J; Xu, YS: The Hydrogen Bond and Free Volume Property of Poly(ether-urethane) Irradiated by Neutron $>>$ GRIFFIN MA 276(2008)807

\#945 J. POROUS MAT. 16(2009)527-536

Turta, NA; Veltri, M; Vuono, D; De Luca, P; Bilba, N; Nastro, A: Effect of crystallization temperature on the synthesis of ETS-4 and ETS-10 titanosilicates $>>$ POPA K 269(2006) 155

\#946 J. POROUS MAT. 17(2010)153-161

Zhang, AY; Xiao, CL; Liu, YH; Hu, QH; Chen, CM; Kuraoka, E: Preparation of macroporous silica-based crown ether materials for strontium separation $>>$ ZHANG A 265(2005)409

\#947 J. RADIOL. PROT. 28(2008)83-91

Turhan, S; Baykan, UN; Sen, K: Measurement of the natural radioactivity in building materials used in Ankara and assessment of external doses $>>$ PAPASTEFANOU C 266(2005)367

\#948 J. RADIOL. PROT. 28(2008)205-212

Rahman, S; Matiullah, S; Mujahid, SA; Hussain, S: Assessment of radiological hazards due to the presence of natural radionuclides in samples of building materials collected from the northwestern areas of Pakistan >> KHAN K 265(2005)371

\#949 J. RADIOL. PROT. 28(2008)223-232

Turtiainen, T; Salahel-din, K; Klemola, S; Sihvonen, AP: Collective effective dose received by the population of Egypt from building materials $>>$ ABBADY A 268(2006)243 \#950 J. RADIOL. PROT. 28(2008)443-452

Rahman, S; Faheem, M; Matiullah: Natural radioactivity measurements in Pakistan-an overview $>>$ KHAN K 265(2005)371; YOUNIS M 266(2005)325

\#951 J. RADIOL. PROT. 29(2009)75-83

Turhan, S: Radiological impacts of the usability of clay and kaolin as raw material in manufacturing of structural building materials in Turkey $>>$ PAPASTEFANOU $\mathrm{C}$ 266(2005)367; PAPASTEFANOU C 275(2008)29
\#952 J. RADIOL. PROT. 29(2009)85-93

Farai, IP; Isinkaye, MO: Radiological safety assessment of surface-water dam sediments used as building material in southwestern Nigeria >> ABDI MR 270(2006)319; PAPASTEFANOU C 266(2005)367; YASIR MS 273 (2007)539

\#953 J. RARE EARTHS 25(2007)45-48

Xi, P; Gu, XH; Xia, L; Cheng, BW; Zhou, Y: Synthesis, characterization of a novel polymeric reaction monomer for preparing highly fluorescent rare earth polymer materials $>>$ ZHANG A 274(2007)455

\#954 J. RARE EARTHS 27(2009)123-127

Ayranov, M; Cobos, J; Popa, K; Rondinella, VV: Determination of REE, U, Th, Ba, and $\mathrm{Zr}$ in simulated hydrogeological leachates by ICP-AES after matrix solvent extraction $>>$ POPA K 273(2007)563

\#955 J. SCI. FOOD AGRIC. 90(2010)286-290

Gougoulias, N; Vagelas, I; Vasilakoglou, I; Gravanis, F; Louka, A; Wogiatzi, E; Chouliaras, N: Comparison of neem or oregano with thiram on organic matter decomposition of a sand loam soil amended with compost, and on soil biological activity >> GARG AN 271(2007)611

\#956 J. SEP. SCI. 31(2008)3037-3044

Mirza, MA; Khuhawar, MY; Arain, R: Determination of uranium, iron, copper, and nickel in rock and water samples by MEKC $>>$ MOHAGHEGHI AH 263(2005)189; ZIKOVSKY L 267(2006)695

\#957 J. SEP. SCI. 31(2008)3148-3155

Zhang, AY; Wang, WH; Chai, ZF; Kumagai, M: Separation of strontium ions from a simulated highly active liquid waste using a composite of silica-crown ether in a polymer >> ZHANG A 265(2005)409

\#958 J. SERB. CHEM. SOC. 73(2008)1181-1186

Makrik, E; Budka, J; Vanura, P; Selucky, P: Solvent extraction of $\mathrm{Ca} 2+, \mathrm{Ba} 2+, \mathrm{Cu} 2+, \mathrm{Zn} 2+, \mathrm{Cd} 2+, \mathrm{Pb} 2+$, $\mathrm{UO} 22+, \mathrm{Mn} 2+, \mathrm{Co} 2+$ and $\mathrm{Ni} 2+$ into nitrobenzene using strontium dicarbollylcobaltate and tetra-tert-butyl p-tertbutylcalix[4]arene tetraacetate $>$ MAKRLIK E 267(2006)699; MAKRLIK E 277(2008)487

\#959 J. SERB. CHEM. SOC. 74(2009)663-668

Cuculovic, AA; Veselinovic, DS; Miljanic, SS: Desorption of Cs-137 from Cetraria islandica (L.) Ach. using solutions of acids and their salts mixtures >> LOVRENCIC I 275(2008)71

\#960 J. SERB. CHEM. SOC. 74(2009)781-787

Makrlik, E; Vanura, P; Selucky, P; Babain, VA; Smirnov, IV: Distribution of micro-amounts of europium in the two-phase water-HCl-nitrobenzene- $\mathrm{N}, \mathrm{N}^{\prime}$-dimethylN,N'-diphenyl-2,6-dipicolinamide-hydrogen dicarbollylcobaltate extraction system $>$ MAKRLIK E 279 (2009)287; VANURA P 267(2006)251; VANURA P 267(2006)465 
\#961 J. SOL-GEL SCI. TECHNOL. 46(2008)369-381

Vaidya, VN: Status of sol-gel process for nuclear fuels $>>$ RAO TVV 268(2006)549

\#962 J. SOLID STATE CHEM. 180(2007)2346-2355

Popa, K; Bregiroux, D; Konings, RJM; Gouder, T; Popa, AF; Gelsler, T; Ralson, PE: The chemistry of the phosphates of barium and tetravalent cations in the $1: 1$ stoichiometry >> POPA K 273(2007)563

\#963 J. SOLID STATE CHEM. 182(2009)1115-1120

Bregiroux, D; Popa, K; Jardin, R; Raison, PE; Wallez, G; Quarton, M; Brunelli, M; Ferrero, C; Caciuffo, R: Crystal structure and thermal expansion of the low- and high-temperature forms of $\mathrm{BaMIV}(\mathrm{PO} 4)(2)$ compounds $(\mathrm{M}=\mathrm{Ti}, \mathrm{Zr}$, Hf and Sn) $>>$ POPA K 273(2007)563

\#964 J. SOLUT. CHEM. 38(2009)1129-1138

Makrlik, E; Vanura, P; Selucky, P: Stability Constants of Some Univalent Cation Complexes of 2,3-Naphtho-15crown-5 in Nitrobenzene Saturated with Water $>>$ MAKRLIK E 267(2006)703; MAKRLIK E 268(2006)627; VALENTOVA Z 267(2006)237; VANURA P 268(2006) 159; VANURA P 267(2006)707; VANURA P 275(2008) 229; VANURA P 278(2008)131

\#965 J. THERM. ANAL. CALORIM. 95(2009)75-80

Randhawa, BS; Dosanjh, HS; Kumar, N: Synthesis of potassium ferrite by precursor and combustion methods $>>$ RANDHAWA BS 274(2007)581

\#966 J. TRACE ELEM. MED. BIOL. 20(2006)143-153

Steinhauser, G; Sterba, JH; Poljanc, K; Bichler, M; Buchtela, K: Trace elements in rock salt and their bioavailability estimated from solubility in acid $>>$ WESTPHAL GP 263(2005)703

\#967 J. TRACE ELEM. MED. BIOL. 21(2007)70-73

Saiki, M; Jaluul, O; Sumita, NM; Vasconcellos, MBA; Jacob, W: Trace element contents in serum of healthy elderly population of metropolitan Sao Paulo area in Brazil $>$ CHEN CY 268(2006)83; MOON J 271(2007)155; MOON JH 272(2007)409; SAIKI M 269(2006)665 \#968 J. VENOM. ANIM. TOXINS TROP. DIS. 15(2009)216-225

Caproni, P; Baptista, JA; de Almeida, TL; Passos, LAC; Nascimento, N: Study of irradiated bothropstoxin-1 with Co-60 gamma rays: immune system behavior $>>$ BAPTISTA JA 269(2006)565

\#969 J. VOLCANOL. GEOTHERM. RES. 159(2007)7084

Gmeling, K; Nemeth, K; Martin, U; Eby, N; Varga, Z: Boron concentrations of volcanic fields in different geotectonic settings >> GMELING K 265(2005)201

\#970 J. WOOD SCI. 52(2006)279-281

Yamauchi, S; Sakai, Y; Watanabe, Y; Kubo, MK; Matsue, H: Analysis of boron in wood treated with boric acid solutions using Doppler broadening method of prompt gamma-rays >> SAKAI Y 265(2005)287
\#971 J. WOOD SCI. 53(2007)324-331

Yamauchi, S; Sakai, Y; Watanabe, Y; Kubo, MK; Matsue, H: Distribution of boron in wood treated with aqueous and methanolic boric acid solutions $>>$ SAKAI Y 265(2005)287

\#972 J. ZHEJIANG UNIV.-SCI A 10(2009)1367-1373

Liu, GR; Ye, CS; He, JH; Qian, Q; Jiang, H: Lake sediment treatment with aluminum, iron, calcium and nitrate additives to reduce phosphorus release $>>$ YANG H 267(2005)205

\#973 J. ZHEJIANG UNIV.-SCI. B 9(2008)165-191

Bohn, L; Meyer, AS; Rasmussen, SK: Phytate: impact on environment and human nutrition. A challenge for molecular breeding $>>$ GHARIB AG 270(2006)209

\#974 KOREAN J. CHEM. ENG. 25(2008)59-63

Park, BH; Seo, CS: A semi-empirical model for the air oxidation kinetics of UO2 >> JEONG SM 268(2006)349; PARK BH 270(2006)575; PARK SB 268(2006)489 \#975 KUWAIT J. SCI. ENG. 32(2005)153-164

Al-Zamel, AZ; Bou-Rabee, F; Bemb, H: Proposal for an environmental radiation monitoring network for gulf countries >> ALZAMEL AZ 266(2005)269

\#976 LANGMUIR 24(2008)12312-12322

Griffith, CS; Sizgek, GD; Sizgek, E; Scales, N; Yee, PJ; Luca, V: Mesoporous Zirconium Titanium Oxides. Part 1: Porosity Modulation and Adsorption Properties of Xerogels >> RAJEC P 275(2008)503

\#977 LANGMUIR 25(2009)4124-4131

Metwalli, E; Moulin, JF; Gebhardt, R; Cubitt, R; Tolkach, A; Kulozik, U; Muller-Buschbaum, P: Hydration Behavior of Casein Micelles in Thin Film Geometry: A GISANS Study >> EFIMOVA YM 264(2005)271

\#978 LANGMUIR 25(2009)4203-4210

Dabkowska, AP; Fragneto, G; Hughes, AV; Quinn, PJ; Lawrence, MJ: Specular Neutron Reflectivity Studies of the Interaction of Cytochrome c with Supported Phosphatidylcholine Bilayers Doped with Phosphotidylserine $>>$ EFIMOVA YM 264(2005)271

\#979 LASERS MED. SCI. 24(2009)749-759

Singh, VK; Rai, AK; Rai, PK; Jindal, PK: Cross-sectional study of kidney stones by laser-induced breakdown spectroscopy >> CHAUDHRI MA 271(2007)713 \#980 LAT. AM. ANTIQ. 18(2007)27-43

Carballo, DM; Carballo, J; Neff, H: Formative and Classic period obsidian procurement in central Mexico: A compositional study using laser ablation-inductively coupled plasma-mass spectrometry $>$ JAMES WD 263(2005)697

\#981 LIMNOL. OCEANOGR. 51(2006)2232-2247

Dulaiova, H; Burnett, WC; Wattayakorn, G; Sojisuporn, P: Are groundwater inputs into river-dominated areas important? The Chao Phraya River-Gulf of Thailand $>>$ DULAIOVA H 263(2005)361 
\#982 LIMNOL. OCEANOGR. 54(2009)890-904

Peterson, RN; Burnett, WC; Glenn, CR; Johnson, AG: Quantification of point-source groundwater discharges to the ocean from the shoreline of the Big Island, Hawaii $>>$ DULAIOVA H 263(2005)361

\#983 LIMNOL. OCEANOGR. METH. 4(2006)254-259

Leaney, FW; Herczeg, AL: A rapid field extraction method for determination of radon-222 in natural waters by liquid scintillation counting > > DULAIOVA H 263(2005)361

\#984 LITH. J. PHYS. 48(2008)107-114

Lujaniene, G; Meleshevych, S; Kanibolotskyy, V; Mazeika, K; Strelko, V; Remeikis, V; Kalenchuk, V; Sapolaite, J: Synthesis and characterization of inorganic sorbents and their application to sorption of radionuclides $>>$ LUJANIENE G 274(2007)345

\#985 MAIN GROUP MET. CHEM. 31(2008)53-71

Choppin, GR; Pathak, P; Thakur, P: Polymerization and Complexation Behavior of Silicic Acid: A Review $>>$ PATHAK PN 267(2006)309; PATHAK PN 267(2006)175 \#986 MAR. CHEM. 100(2006)190-212

Van der Loeff, MR; Sarin, MM; Baskaran, M; BenitezNelson, C; Buesseler, KO; Charette, M; Dai, M; Gustafsson, O; Masque, P; Morris, PJ; Orlandini, K; Baena, ARY; Savoye, N; Schmidt, S; Turnewitsch, R; Voge, I; Waples, JT: A review of present techniques and methodological advances in analyzing Th-234 in aquatic systems $>>$ PIKE SM 263(2005)355

\#987 MAR. CHEM. 100(2006)269-281

Baena, AMRY; Miquel, JC; Masque, P; Povinec, PP; La Rosa, J: A single vs. double spike approach to improve the accuracy of Th-234 measurements in small-volume seawater samples $>>$ PIKE SM 263(2005)355

\#988 MAR. CHEM. 100(2006)282-288

Cai, PH; Dai, MH; Lv, DW; Chen, WF: An improvement in the small-volume technique for determining thorium-234 in seawater $>>$ PIKE SM 263(2005)355

\#989 MAR. CHEM. 104(2007)69-84

Swarzenski, PW; Reich, C; Kroeger, KD; Baskaran, M: $\mathrm{Ra}$ and $\mathrm{Rn}$ isotopes as natural tracers of submarine groundwater discharge in Tampa Bay, Florida $>>$ DULAIOVA H 263(2005)361

\#990 MAR. CHEM. 104(2007)85-97

Kroeger, KD; Swarzenski, PW; Greenwood, WJ; Reich, C: Submarine groundwater discharge to Tampa Bay: Nutrient fluxes and biogeochemistry of the coastal aquifer >> DULAIOVA H 263(2005)361

\#991 MAR. CHEM. 106(2007)530-545

Pates, JM; Muir, GKP: U-salinity relationships in the Mediterranean: Implications for Th-234 : U-238 particle flux studies $>>$ PIKE SM 263(2005)355

\#992 MAR. CHEM. 109(2008)226-237

Bourquin, M; van Beek, P; Reyss, JL; Soullaut, M; Charette, MA; Jeandel, C: Comparison of techniques for pre-concentrating radium from seawater $>>$ MAXWELL SL 270(2006)651

\#993 MAR. CHEM. 109(2008)395-408

Dulaiova, H; Burnett, WC: Evaluation of the flushing rates of Apalachicola Bay, Florida via natural geochemical tracers $>>$ DULAIOVA H 263(2005)361

\#994 MAR. CHEM. 115(2009)110-117

Liu, YM; Lee, K: Modifications of the curcumin method enabling precise and accurate measurement of seawater boron concentration $>>$ RAMANJANEYULU PS 273(2007)411

\#995 MAR. CHEM. 116(2009)36-46

Caetano, M; Prego, R; Vale, C; de Pablo, H; MarmolejoRodriguez, J: Record of diagenesis of rare earth elements and other metals in a transitional sedimentary environment $>$ ARAUJO MF 271(2007)255

\#996 MAR. POLLUT. BULL. 56(2008)751-757

Abdi, MR; Kamali, M; Vaezifar, S: Distribution of radioactive pollution of U-238, Th-232K-40 and Cs-137 in northwestern coasts of Persian Gulf, Iran $>>$ ABDI MR 270(2006)319; MOSTAJABODDAVATI M 268(2006)539 \#997 MAR. POLLUT. BULL. 58(2009)658-662

Abdi, MR; Hassanzadeh, S; Kamali, M; Raji, HR: U238, Th-232, K-40 and Cs-137 activity concentrations along the southern coast of the Caspian Sea, Iran $>>$ ABDI MR 270(2006)319

\#998 MAR. POLLUT. BULL. 58(2009)940-946

Vane, CH; Jones, DG; Lister, TR: Mercury contamination in surface sediments and sediment cores of the Mersey Estuary, UK >> VENKATARAMAN R 264(2005)183 \#999 MAR. POLLUT. BULL. 59(2009)146-153

Martin, J; Sanchez-Cabeza, JA; Eriksson, M; Levy, I; Miquel, JC: Recent accumulation of trace metals in sediments at the DYFAMED site (Northwestern Mediterranean Sea) $>>$ POVINEC PP 263(2005)441 \#1000 MAR. POLLUT. BULL. 59(2009)234-244

Bergamin, L; Romano, E; Finoia, MG; Venti, F; Bianchi, J; Colasanti, A; Ausili, A: Benthic foraminifera from the coastal zone of Baia (Naples, Italy): Assemblage distribution and modification as tools for environmental characterisation $>>$ KFOURI PBP 265(2005)459 \#1001 MASS SPECTROM. REV. 29(2010)55-78

Resano, M; Garcia-Ruiz, E; Vanhaecke, F: Laser ablation-inductively coupled plasma mass spectrometry in archaeometric research >> JAMES WD 263(2005)697 \#1002 MATER. CHEM. PHYS. 111(2008)438-443

Mandal, S; Muller, AHE: Facile route to the synthesis of porous alpha-Fe2O3 nanorods $>$ GRACIEN EB 270(2006)473

\#1003 MATER. RES. BULL. 44(2009)2014-2021

Gotic, M; Jurkin, T; Music, S: From iron(III) precursor to magnetite and vice versa $>$ GRACIEN EB 270(2006)473 
\#1004 MATER. RES. BULL. 45(2010)56-62

Shah, CP; Singh, KK; Kumar, M; Bajaj, PN: Vinyl monomers-induced synthesis of polyvinyl alcohol-stabilized selenium nanoparticles $>>$ BAKIR MA 266(2005)165 \#1005 MEAS. SCI. TECHNOL. 19(2008)34003-

Imberti, S; Kockelmann, W; Celli, M; Grazzi, F; Zoppi, M; Botti, A; Sodo, A; Imperiale, ML; de Vries-Melein, M; Visser, D; Postma, H: Neutron diffractometer INES for quantitative phase analysis of archaeological objects $>>$ POSTMA H 271(2007)95

\#1006 MEAS. SCI. TECHNOL. 20(2009)84027-

Borgese, L; Zacco, A; Bontempi, E; Colombi, P; Bertuzzi, R; Ferretti, E; Tenini, S; Depero, LE: Total reflection of X-ray fluorescence (TXRF): a mature technique for environmental chemical nanoscale metrology $>>$ KHUDER A 273(2007)435

\#1007 MEAS. SCI. TECHNOL. 21(2010)35102-

Coakley, KJ; Splett, JD; Simons, DS: Frequentist coverage properties of uncertainty intervals for weak Poisson signals in the presence of background $>>$ CURRIE LA 276(2008)285

\#1008 MED. PHYS. 35(2008)608-616

Aslam; Pejovic-Milic, A; McNeill, FE; Byun, SH; Prestwich, WV; Chettle, DR: In vivo assessment of magnesium status in human body using accelerator-based neutron activation measurement of hands: A pilot study $>$ PEJOVICMILIC A 269(2006)417; TAKATA MK 264(2005)5

\#1009 MENDELEEV COMMUN. 19(2009)266-267

Morgalyuk, VP; Kalmykov, SN; Goryunov, EI; Petrovskii, PV; Tananaev, IG; Nifant'ev, EE: Synthesis of a template sorbent for the extraction of americium(III) from nitric acid solutions $>>$ OZEROGLU C 268(2006)211 \#1010 METALL. MATER. TRANS. B-PROC. METALL. MATER. PROC. SCI. 40(2009)685-699

Mohandas, KS; Fray, DJ: Electrochemical Deoxidation of Solid Zirconium Dioxide in Molten Calcium Chloride >> JEONG SM 268(2006)349; PARK BH 270(2006)575 \#1011 METALLOMICS 1(2009)142-147

Austin, C; Hare, D; Rozelle, AL; Robinson, WH; Grimm, R; Doble, P: Elemental bio-imaging of calcium phosphate crystal deposits in knee samples from arthritic patients $>>$ CHAUDHRI MA 271(2007)713 \#1012 METEORIT. PLANET. SCI. 43(2008)461-477

Randa, Z; Mizera, J; Frana, J; Kucera, J: Geochemical characterization of moldavites from a new locality, the Cheb Basin, Czech Republic >> RANDA Z 264(2005)67 \#1013 MICROBES ENVIRON. 24(2009)265-272

Hiraki, A; Tsuchiya, Y; Fukuda, Y; Yamamoto, T; Kurniawan, A; Morisaki, H: Analysis of How a Biofilm Forms on the Surface of the Aquatic Macrophyte Phragmites australis $>>$ NAGAO S 273(2007)135
\#1014 MICROCHEM J. 85(2007)39-45

Varga, Z; Suranyi, G; Vajda, N; Stefanka, Z: Determination of plutonium and americium in environmental samples by inductively coupled plasma sector field mass spectrometry and alpha spectrometry $>$ LAROSA J 263(2005)427; LEE SH 263(2005)419

\#1015 MICROCHEM J. 87(2007)13-19

Desideri, D; Roselli, C; Feduzi, L; Meli, MA: Radiological characterization of drinking waters in Central Italy >> BORIO R 266(2005)397; DESIDERI D 266(2005)191; JIA GG 267(2006)505 \#1016 MICROCHEM J. 93(2009)188-194

Capannesi, G; Rosada, A; Avino, P: Elemental characterization of impurities at trace and ultra-trace levels in metallurgical lead samples by INAA $>>$ BACCHI MA 271(2007)345; FREITAS MC 276(2008)149; LANDSBERGER S 263(2005)817

\#1017 MICROCHEM J. 94(2010)159-165

Jobbagy, V; Kavasi, N; Somlai, J; Mate, B; Kovacs, T: Radiochemical characterization of spring waters in Balaton Upland, Hungary, estimation of radiation dose to members of public $>>$ CHAU ND 279(2009)121

\#1018 MICROCHIM. ACTA 164(2009)211-216

Nakano, S; Matumoto, Y; Fukumoto, Y; Hayashi, M: Spectrophotometric determination of vanadium(IV, V) in chloride-rich samples by its catalytic effect on the oxidative coupling of N,N-diphenylhydrazine with chromotropic acid $>>$ REPINC U 264(2005)77

\#1019 MICROCHIM. ACTA 168(2010)65-69

Sarkar, A; Aggarwal, SK; Sasibhusan, K; Alamelu, D: Determination of sub-ppm levels of boron in ground water samples by laser induced breakdown spectroscopy $>>$ RAMANJANEYULU PS 273(2007)411 \#1020 MICROCHIM. ACTA 168(2010)147-152

Faghihian, H; Kabiri-Tadi, M: A novel solid-phase extraction method for separation and preconcentration of zirconium $>>$ FAGHIHIAN H 264(2005)577

\#1021 MICRON 39(2008)617-622

Lima, I; Rocha, MS; Lopes, RT: Ethanol bone evaluation using 3D microtomography >> LIMA ICB 269(2006) 639

\#1022 MICRON 39(2008)1062-1091

Ehrlich, H; Koutsoukos, PG; Demadis, KD; Pokrovsky, OS: Principles of demineralization: Modern strategies for the isolation of organic frameworks. Part I. Common definitions and history $>>$ CHAUDHRI MA 271(2007) 713

\#1023 MICROPOROUS MESOPOROUS MAT. 109 (2008)147-155

Sun, PP; Navrotsky, A: Enthalpy of formation and dehydration of alkaline earth cation exchanged zeolite beta $>$ KAZEMIAN H 268(2006)231 
\#1024 MICROPOROUS MESOPOROUS MAT. 116(2008) 641-652

Sharma, P; Tomar, R: Synthesis and application of an analogue of mesolite for the removal of uranium(VI), thorium(IV), and europium(III) from aqueous waste $>>$ ELRAHMAN KMA 268(2006)221; HUMELNICU D 270(2006)637; KILINCARSLAN A 264(2005)541; NEMES Z 266(2005)289; RAOUF MWA 267(2006)389; SHARMA P 268(2006)329 \#1025 MICROPOROUS MESOPOROUS MAT. 121(2009) $114-120$

Cavenati, S; Grande, CA; Lopes, FVS; Rodrigues, AE: Adsorption of small molecules on alkali-earth modified titanosilicates $>>$ POPA K 269(2006)155

\#1026 MICROPOROUS MESOPOROUS MAT. 123(2009) $1-9$

Shao, DD; Fan, QH; Li, JX; Niu, ZW; Wu, WS; Chen, YX; Wang, XK: Removal of Eu(III) from aqueous solution using ZSM-5 zeolite >> XU D 266(2005)419

\#1027 MICROSC. MICROANAL. 16(2010)1-12

Marinenko, RB; Turner, S; Simons, DS; Rabb, SA; Zeisler, RL; Yu, LL; Newbury, DE; Paul, RL; Ritchie, NWM; Leigh, SD; Winchester, MR; Richter, LJ; Meier, DC; Scott, KCK; Klinedinst, D; Small, JA: Characterization of SiGe Films for Use as a National Institute of Standards and Technology Microanalysis Reference Material (RM 8905) >> LINDSTROM RM 271(2007)311

\#1028 MINERAL. PETROL. 95(2009)291-302

Marschall, HR; Altherr, R; Gmeling, K; Kasztovszky, Z: Lithium, boron and chlorine as tracers for metasomatism in high-pressure metamorphic rocks: a case study from Syros (Greece) >> GMELING K 265(2005)201; MARSCHALL HR 265(2005)339

\#1029 MINI-REV. MED. CHEM. 8(2008)1039-1052

Brouwer, CPJM; Sarda-Mantel, L; Meulemans, A; Le Guludec, D; Welling, MM: The use of technetium-99m radiolabeled human antimicrobial peptides for infection specific imaging >> FERROFLORES G 266(2005)307

\#1030 MOL. IMAGING 9(2010)1-20

Holland, JP; Williamson, MJ; Lewis, JS: Unconventional Nuclides for Radiopharmaceuticals $>>$ ALFASSI ZB 270(2006)483; BILEWICZ A 268(2006)485

\#1031 MON. CHEM. 139(2008)597-600

Makrlik, E; Vanura, P; Selucky, P: Experimental evidences for some unusual divalent cation complexes of valinomycin >> MAKRLIK E 268(2006) 155

\#1032 MON. CHEM. 139(2008)1349-1351

Makrlik, E; Budka, J; Vanura, P; Dybal, J: Solvent extraction of univalent cations into nitrobenzene using sodium dicarbollylcobaltate and tetraphenyl p-tert-butylcalix[4]arene tetraketone $>>$ MAKRLIK E 275(2008)217 \#1033 MON. CHEM. 140(2009)157-160

Makrlik, E; Budka, J; Vanura, P; Selucky, P: Stability constants of some metal cation complexes of tetraphenyl p-tert-butylcalix[4] arene tetraketone in nitrobenzene saturated with water $>>$ MAKRLIK E 277(2008)487

\#1034 MON. CHEM. 140(2009)1155-1158

Makrlik, E; Dybal, J; Budka, J; Vanura, P: Experimental evidence, stability, and the most probable structure of protonated p-tert-butylcalix[4]arenetetrakis (N,N-dimethylacetamide) >> MAKRLIK E 275(2008)217; MAKRLIK E 277(2008)487 \#1035 MON. CHEM. 141(2010)15-18

Dybal, J; Makrlik, E; Vanura, P: Extraction and DFT study on the complexation of $\mathrm{Mg} 2+$ with valinomycin $>>$ MAKRLIK E 268(2006)155 \#1036 MUTAGENESIS 24(2009)245-251

Balasubramanyam, A; Sailaja, N; Mahboob, M; Rahman, MF; Hussain, SM; Grover, P: In vivo genotoxicity assessment of aluminium oxide nanomaterials in rat peripheral blood cells using the comet assay and micronucleus test $>>$ CHEN Z 272(2007)599

\#1037 MUTAT. RES. GENET. TOXICOL. ENVIRON. MUTAGEN. 676(2009)41-47

Balasubramanyam, A; Sailaja, N; Mahboob, M; Rahman, MF; Misra, S; Hussain, SM; Grover, P: Evaluation of genotoxic effects of oral exposure to Aluminum oxide nanomaterials in rat bone marrow $>>$ CHEN Z 272(2007)599

\#1038 MYCOL. PROG. 6(2007)249-259

Borovicka, J; Randa, Z: Distribution of iron, cobalt, zinc and selenium in macrofungi >> RANDA Z 264(2005)67 \#1039 MYCOL. RES. 111(2007)1339-1344

Borovicka, J; Randa, Z; Jelinek, E; Kotrba, P; Dunn, CE: Hyperaccumulation of silver by Amanita strobiliformis and related species of the section Lepidella >> RANDA Z 264(2005)67

\#1040 NANO 3(2008)279-285

Wang, JX; Li, YF; Li, W; Chen, CY; Li, B; Zhao, YL: BIOLOGICAL EFFECT OF INTRANASALLY INSTILLED TITANIUM DIOXIDE NANOPARTICLES ON FEMALE MICE $>>$ WANG JX 272(2007)527

\#1041 NANO LETT. 7(2007)3798-3802

Yang, XM; Skrabalak, SE; Li, ZY; Xia, YN; Wang, LHV: Photoacoustic tomography of a rat cerebral cortex in vivo with au nanocages as an optical contrast agent $>>$ JAMES WD 271(2007)455 \#1042 NANO LETT. 8(2008)2517-2525

van Schooneveld, MM; Vucic, E; Koole, R; Zhou, Y; Stocks, J; Cormode, DP; Tang, CY; Gordon, RE; Nicolay, K; Meijerink, A; Fayad, ZA; Mulder, WJM: Improved biocompatibility and pharmacokinetics of silica nanoparticles by means of a lipid coating: A multimodality investigation $>>$ LIANG S 269(2006)3

\#1043 NANO TODAY 2(2007)22-32

Arruebo, M; Fernandez-Pacheco, R; Ibarra, MR; Santamaria, J: Magnetic nanoparticles for drug delivery $>>$ LIANG S 269(2006)3 
\#1044 NANOMED.-NANOTECHNOL. BIOL. MED. 3(2007)89-94

Xie, H; Gill-Sharp, KL; O'Neal, P: Quantitative estimation of gold nanoshell concentrations in whole blood using dynamic light scattering >> JAMES WD 271(2007) 455

\#1045 NANOMEDICINE 3(2008)201-213

Bolskar, RD: Gadofullerene MRI contrast agents $>>$ ZHANG J 272(2007)605

\#1046 NANOMEDICINE 4(2009)575-588

Franzen, S; Lommel, SA: Targeting cancer with 'smart bombs': equipping plant virus nanoparticles for a 'seek and destroy' mission >> LI YG 265(2005)127

\#1047 NANOTECHNOLOGY 18(2007)385607-

Shah, CP; Kumar, M; Bajaj, PN: Acid-induced synthesis of polyvinyl alcohol-stabilized selenium nanoparticles $>>$ BAKIR MA 266(2005)165

\#1048 NANOTECHNOLOGY 20(2009)385102-

Nikolic, N; Vranjes-Duric, S; Jankovic, D; Dokic, D; Mirkovic, M; Bibic, N; Trajkovic, V: Preparation and biodistribution of radiolabeled fullerene C-60 nanocrystals >> LI YG 265(2005)127

\#1049 NAT. BIOTECHNOL. 26(2008)83-90

Qian, XM; Peng, XH; Ansari, DO; Yin-Goen, Q; Chen, GZ; Shin, DM; Yang, L; Young, AN; Wang, MD; Nie, SM: In vivo tumor targeting and spectroscopic detection with surface-enhanced Raman nanoparticle tags >> JAMES WD 271(2007)455

\#1050 NAT. CHEM. 2(2010)187-191

Ding, N; Kanatzidis, MG: Selective incarceration of caesium ions by Venus flytrap action of a flexible framework sulfide $>>$ VENKATESAN KA 280(2009)129

\#1051 NATURE 446(2007)1070-U1

Blain, S; Queguiner, B; Armand, L; Belviso, S; Bombled, B; Bopp, L; Bowie, A; Brunet, C; Brussaard, C; Carlotti, F; Christaki, U; Corbiere, A; Durand, I; Ebersbach, F; Fuda, JL; Garcia, N; Gerringa, L; Griffiths, B; Guigue, C; Guillerm, C; Jacquet, S; Jeandel, C; Laan, P; Lefevre, D; Lo Monaco, C; Malits, A; Mosseri, J; Obernosterer, I; Park, YH; Picheral, M; Pondaven, P; Remenyi, T; Sandroni, V; Sarthou, G; Savoye, N; Scouarnec, L; Souhaut, M; Thuiller, D; Timmermans, K; Trull, T; Uitz, J; van Beek, P; Veldhuis, M; Vincent, D; Viollier, E; Vong, L; Wagener, T: Effect of natural iron fertilization on carbon sequestration in the Southern Ocean >> PIKE SM 263(2005)355

\#1052 NEUROSCIENCE 155(2008)387-402

Hernadi, L; Pirger, Z; Kiss, T; Nemeth, J; Mark, L; Kiss, P; Tamas, A; Lubics, A; Toth, G; Shioda, S; Reglodi, D: The presence and distribution of pituitary adenylate cyclase activating polypeptide and its receptor in the snail Helix pomatia $>>$ NEMETH J 273(2007)327
\#1053 NEW J. CHEM. 31(2007)1789-1797

Bresson, C; Spezia, R; Esnouf, S; Solari, PL; Coantic, S; Den Auwer, C: A combined spectroscopic and theoretical approach to investigate structural properties of $\mathrm{Co}(\mathrm{II}) /$ Co(III) tris-cysteinato complexes in aqueous medium $>>$ POPA K 269(2006)155

\#1054 NEW J. CHEM. 32(2008)105-114

Halder, S; Peng, SM; Lee, GH; Chatterjee, T; Mukherjee, A; Dutta, S; Sanyal, U; Bhattacharya, S: Synthesis, structure, spectroscopic properties and cytotoxic effect of some thiosemicarbazone complexes of palladium $>>$ JALILIAN AR 268(2006)605

\#1055 NEW J. CHEM. 32(2008)941-946

Batchelor-McAuley, C; Wildgoose, GG; Compton, RG: The contrasting behaviour of polycrystalline bulk gold and gold nanoparticle modified electrodes towards the underpotential deposition of thallium $>>$ MARCZONA R 269 (2006) 29

\#1056 NUCL. DATA SHEETS 108(2007)2655-2715

Herman, M; Capote, R; Carlson, BV; Oblozinsky, P; Sin, M; Trkov, A; Wienke, H; Zerkin, V: EMPIRE: Nuclear reaction model code system for data evaluation $>>$ ALFASSI ZB 270(2006)483

\#1057 NUCL. DATA SHEETS 111(2010)141-

Chiara, CJ; Kondev, FG: Nuclear Data Sheets for $\mathrm{A}=204>>$ HAAS DA 277(2008)561

\#1058 NUCL. ENG. DES. 239(2009)813-818

Remeikis, V; Plukis, A; Juodis, L; Gudelis, A; Lukauskas, D; Druteikiene, R; Lujaniene, G; Luksiene, B; Plukiene, R; Duskesas, G: Study of the nuclide inventory of operational radioactive waste for the RBMK-1500 reactor >> MAXWELL SL 267(2006)537

\#1059 NUCL. ENG. TECHNOL. 41(2009)113-124

Choi, HD; Jung, NS; Park, BG: ANALYSIS OF DOPPLER-BROADENED PEAK IN THERMAL NEUTRON INDUCED B-10(n,alpha gamma)Li-7 REACTION USING HYPERGAM >> SUN G 278(2008)637; SZENTMIKLOSI L 271(2007)447

\#1060 NUCL. ENG. TECHNOL. 41(2009)521-530

Lee, KH; Park, JMS; Kim, HR; Jui, BJ; Kim, YJ; Ha, JJ; Kim, MW; Choi, SM: OPPORTUNITIES AND CHALLENGES OF NEUTRON SCIENCE AND TECHNOLOGY IN KOREA >> SUN GM 264(2005)603 \#1061 NUCL. ENG. TECHNOL. 41(2009)813-820

Chung, YS; Kim, SH; Moon, JH; Baek, SY; Kim, HR; Kim, YJ: CHARACTERISTICS OF A NEW PNEUMATIC TRANSFER SYSTEM FOR A NEUTRON ACTIVATION ANALYSIS AT THE HANARO RESEARCH REACTOR >> CHUNG YS 278(2008)707; LINDSTROM RM 278(2008)665; MOON JH 271(2007) 289; WESTPHAL GP 263(2005)141; ZEISLER R 263(2005)313 
\#1062 NUCL. ENG. TECHNOL. 41(2009)1073-1078

Jeong, SM; Hur, JM; Lee, H: KINETIC MODELING STUDY OF A VOLOXIDATION FOR THE PRODUCTION OF U3O8 POWDER FROM A UO2 PELLET $>>$ JEONG SM 268(2006)349; PARK SB 268(2006)389

\#1063 NUCL. FUSION 46(2006)142-148

Takamatsu, T; Masuda, K; Kyunai, T; Toku, H; Yoshikawa, K: Inertial electrostatic confinement fusion device with an ion source using a magnetron discharge $>>$ MILEY GH 263(2005)159

\#1064 NUCL. INSTRUM. METHODS PHYS. RES. SECT. A-ACCEL. SPECTROM. DECT. ASSOC. EQUIP. 545(2005)63-87

Huhtinen, M; Lecomte, P; Luckey, D; Nessi-Tedaldi, F; Pauss, F: High-energy proton induced damage in PbWO4 calorimeter crystals $>>$ AARNIO PA 264(2005)51

\#1065 NUCL. INSTRUM. METHODS PHYS. RES. SECT. A-ACCEL. SPECTROM. DECT. ASSOC. EQUIP. 556(2006)498-504

Scates, W; Hartwell, JK; Aryaeinejad, R; McIlwain, ME: Optimization studies of a Compton suppression spectrometer using experimentally validated Monte Carlo simulations >> HARTWELL JK 264(2005)199

\#1066 NUCL. INSTRUM. METHODS PHYS. RES. SECT. A-ACCEL. SPECTROM. DECT. ASSOC. EQUIP. 562(2006)245-253

Berizov, AN; Basenko, VK; Filby, RH; Malyuk, IA; Tryshyn, VV: NAAPRO detector model, a versatile and efficient approach to gamma-ray spectrum simulation $>>$ BASENKO VK 263(2005)675

\#1067 NUCL. INSTRUM. METHODS PHYS. RES. SECT. A-ACCEL. SPECTROM. DECT. ASSOC. EQUIP. 564(2006)655-661

Szentmiklosi, L; Revay, Z; Belgya, T: Measurement of partial gamma-ray production cross-sections and $\mathrm{k}(\mathrm{o})$ factors for radionuclides with chopped-beam PGAA >> REVAY Z 264(2005)277

\#1068 NUCL. INSTRUM. METHODS PHYS. RES. SECT. A-ACCEL. SPECTROM. DECT. ASSOC. EQUIP. 564(2006)662-668

Nair, AGC; Acharya, R; Sudarshan, K; Tripathi, R; Reddy, AVR; Goswami, A: Determination and validation of prompt $\mathrm{k}(\mathrm{o})$-factors with a monochromatic neutron beam at the Dhruva reactor $>>$ PARK CS 265(2005)283

\#1069 NUCL. INSTRUM. METHODS PHYS. RES. SECT. A-ACCEL. SPECTROM. DECT. ASSOC. EQUIP. 564(2006)688-697

Revay, Z: Calculation of uncertainties in prompt gamma activation analysis $>>$ MACKEY EA 265(2005)273; REVAY Z 265(2005)261

\#1070 NUCL. INSTRUM. METHODS PHYS. RES. SECT. A-ACCEL. SPECTROM. DECT. ASSOC. EQUIP. 564(2006)733-742
Freitas, MC; Pacheco, AMG; Dionisio, I; Sarmento, S; Baptista, MS; Vasconcelos, MTSD; Cabral, JP: Multianalytical determination of trace elements in atmospheric biomonitors by $\mathrm{k}(\mathrm{o})$-INAA, ICP-MS and AAS $>>$ GARG AN 263(2005)751

\#1071 NUCL. INSTRUM. METHODS PHYS. RES. SECT. A-ACCEL. SPECTROM. DECT. ASSOC. EQUIP. 564(2006)752-760

Almeida, SM; Freitas, MC; Reis, MA; Pio, CA; Trancoso, MA: Combined application of multielement analysis- $\mathrm{k}(\mathrm{o})$-INAA and PIXE-and classical techniques for source apportionment in aerosol studies $>>$ FREITAS MC 263(2005)711

\#1072 NUCL. INSTRUM. METHODS PHYS. RES. SECT. A-ACCEL. SPECTROM. DECT. ASSOC. EQUIP. 569(2006)790-795

Bochud, F; Bailat, CJ; Buchillier, T; Byrde, F; Schmid, E; Laedermann, JP: Simple Monte-Carlo method to calibrate well-type HPGe detectors $>>$ VENKATARAMAN R 264(2005) 213

\#1073 NUCL. INSTRUM. METHODS PHYS. RES. SECT. A-ACCEL. SPECTROM. DECT. ASSOC. EQUIP. 570(2007)72-83

Jaffe, JE; Jordan, DV; Peurrung, AJ: Energy nonlinearity in radiation detection materials: Causes and consequences >> GEELHOOD BD 263(2005)267

\#1074 NUCL. INSTRUM. METHODS PHYS. RES. SECT. A-ACCEL. SPECTROM. DECT. ASSOC. EQUIP. 571(2007)663-668

Aggarwal, SK; Alamelu, D; Shah, PM; Mirashi, NN: Precise and accurate determination of the half-life of the alpha-decay nuclide Am-243 using Am-241 as the reference isotope $>>$ CATLOW SA 263(2005)599

\#1075 NUCL. INSTRUM. METHODS PHYS. RES. SECT. A-ACCEL. SPECTROM. DECT. ASSOC. EQUIP. 572(2007)926-928

Puzovic, JM; Anicin, IV: An off-line method for the correction of amplitude walk in leading-edge timing $>>$ POVINEC PP 263(2005)441

\#1076 NUCL. INSTRUM. METHODS PHYS. RES. SECT. A-ACCEL. SPECTROM. DECT. ASSOC. EQUIP. 575(2007)476-488

Noguere, G; Cserpak, F; Ingelbrecht, C; Plompen, AJM; Quetel, CR; Schillebeeckx, P: Non-destructive analysis of materials by neutron resonance transmission $>>$ POSTMA H 265(2005)297

\#1077 NUCL. INSTRUM. METHODS PHYS. RES. SECT. A-ACCEL. SPECTROM. DECT. ASSOC. EQUIP. 577(2007)611-618

Revay, Z; Harrison, RK; Alvarez, E; Biegalski, SR; Landsberger, $S$ : Construction and characterization of the redesigned PGAA facility at The University of Texas at Austin $>$ AGHARA SK 265(2005)321; REVAY Z 
264(2005)283; REVAY Z 265(2005)261; UNLU K 265(2005)329

\#1078 NUCL. INSTRUM. METHODS PHYS. RES. SECT. A-ACCEL. SPECTROM. DECT. ASSOC. EQUIP. 577(2007)626-640

Borella, A; Aerts, G; Gunsing, F; Moxon, M; Schillebeeckx, P; Wynants, R: The use of C6D6 detectors for neutron induced capture cross-section measurements in the resonance region >> BORELLA A 265(2005)267

\#1079 NUCL. INSTRUM. METHODS PHYS. RES. SECT. A-ACCEL. SPECTROM. DECT. ASSOC. EQUIP. 578(2007)279-288

Idiri, Z; Mazrou, H; Beddek, S; Amokrane, A; Azbouche, A: Monte Carlo optimization of sample dimensions of an Am-241-Be source-based PGNAA setup for water rejects analysis $>>$ LIM CS 264(2005)15

\#1080 NUCL. INSTRUM. METHODS PHYS. RES. SECT. A-ACCEL. SPECTROM. DECT. ASSOC. EQUIP. 579(2007)431-436

Hennig, WG; Tan, H; Fallu-Labruyere, A; Warburton, WK; McIntyre, JI; Gleyzer, A: A phoswich well detector for radioxenon monitoring >> ELY JH 263(2005)245 \#1081 NUCL. INSTRUM. METHODS PHYS. RES. SECT. A-ACCEL. SPECTROM. DECT. ASSOC. EQUIP. 579(2007)507-509

Freitas, MDC; Dionisloa, I; Fialho, P; Barata, F: Aerosol chemical elemental mass concentration at lower free troposphere $>>$ FREITAS MC 263(2005)711

\#1082 NUCL. INSTRUM. METHODS PHYS. RES. SECT. A-ACCEL. SPECTROM. DECT. ASSOC. EQUIP. 580(2007)206-209

de Celis, B; de la Fuentea, R; Williart, A; Alonsoc, BDC: Coincidence measurements in alpha/beta/gamma spectrometry with phoswich detectors using digital pulse shape discrimination analysis >> ELY JH 263(2005)245 \#1083 NUCL. INSTRUM. METHODS PHYS. RES. SECT. A-ACCEL. SPECTROM. DECT. ASSOC. EQUIP. 580(2007)1423-1427

Lee, TH; Kim, HD; Yoon, JS; Lee, SY; Swinhoe, M; Menlove, HO: Preliminary calibration of the ACP safeguards neutron counter $>>$ JEONG SM 268(2006)349 \#1084 NUCL. INSTRUM. METHODS PHYS. RES. SECT. A-ACCEL. SPECTROM. DECT. ASSOC. EQUIP. 581(2007)91-93

Bosnar, D; Kajcsos, Z; Liszkay, L; Lohonyai, L; Major, P; Bosnar, S; Kosanovic, C; Subotic, B: Digitized positron lifetime spectrometer for the simultaneous recording of time and energy information $>>$ ABUELHIA E 271 (2007) 489

\#1085 NUCL. INSTRUM. METHODS PHYS. RES. SECT. A-ACCEL. SPECTROM. DECT. ASSOC. EQUIP. 584(2008)406-411
Pelled, O; German, U; Abraham, A; Alfassi, ZB: Localization of a "hot spot" of uranium in the lungs by an array of four HPGe detectors. The effect of variation in the isotopic composition $>>$ ALFASSI ZB 266(2005)245 \#1086 NUCL. INSTRUM. METHODS PHYS. RES. SECT. A-ACCEL. SPECTROM. DECT. ASSOC. EQUIP. 589(2008)57-65

Lee, TH; Menlove, HO; Lee, SY; Kim, HD: Development of the ACP safeguards neutron counter for PWR spent fuel rods $>>$ JEONG SM 268(2006)349

\#1087 NUCL. INSTRUM. METHODS PHYS. RES. SECT. A-ACCEL. SPECTROM. DECT. ASSOC. EQUIP. 589(2008)243-249

Tomlin, BE; Zeisler, R; Lindstrom, RM: gamma gamma coincidence spectrometer for instrumental neutron-activation analysis >> HATSUKAWA Y 272(2007)273; MOREIRA EG 269(2006)377

\#1088 NUCL. INSTRUM. METHODS PHYS. RES. SECT. A-ACCEL. SPECTROM. DECT. ASSOC. EQUIP. 594(2008)400-406

Zahringer, M; Kirchner, G: Nuclide ratios and source identification from high-resolution gamma-ray spectra with Bayesian decision methods $>>$ PADOANI F 263(2005)183 \#1089 NUCL. INSTRUM. METHODS PHYS. RES. SECT. A-ACCEL. SPECTROM. DECT. ASSOC. EQUIP. 599(2009)74-81

Takoudis, G; Xanthos, S; Clouvas, A; AntonopoulosDomis, M; Potiriadis, C; Silva, J: Spatial and spectral gamma-ray response of plastic scintillators used in portal radiation detectors; comparison of measurements and simulations $>>$ KOUZES RT 276(2008)719

\#1090 NUCL. INSTRUM. METHODS PHYS. RES. SECT. A-ACCEL. SPECTROM. DECT. ASSOC. EQUIP. 603(2009)361-364

Karabidak, SM; Cevik, U; Kaya, S: A new method to compensate for counting losses due to system dead time >> SZENTMIKLOSI L 271(2007)439

\#1091 NUCL. INSTRUM. METHODS PHYS. RES. SECT. A-ACCEL. SPECTROM. DECT. ASSOC. EQUIP. 605(2009)146-149

Matsushima, U; Herppich, WB; Kardjilov, N; Graf, W; Hilger, A; Manke, I: Estimation of water flow velocity in small plants using cold neutron imaging with D2O tracer $>$ NAKANISHI TM 264(2005)313; YAMADA T 264(2005)329

\#1092 NUCL. INSTRUM. METHODS PHYS. RES. SECT. A-ACCEL. SPECTROM. DECT. ASSOC. EQUIP. 605(2009)179-184

Tanoi, K; Hamada, Y; Seyama, S; Saito, T; Iikura, H; Nakanishi, TM: Dehydration process of fish analyzed by neutron beam imaging $>>$ NAKANISHI TM 264 (2005)313 
\#1093 NUCL. INSTRUM. METHODS PHYS. RES. SECT. A-ACCEL. SPECTROM. DECT. ASSOC. EQUIP. 606(2009)523-532

Cooper, RJ; Boston, AJ; Boston, HC; Cresswell, JR; Grint, AN; Harkness, LJ; Nolan, PJ; Oxley, DC; Scraggs, DP; Mather, AR; Lazarus, I; Simpson, J: Positron Emission Tomography imaging with the SmartPET system $>>$ UPP DL 264(2005)121

\#1094 NUCL. INSTRUM. METHODS PHYS. RES. SECT. A-ACCEL. SPECTROM. DECT. ASSOC. EQUIP. 607(2009)446-450

Naqvi, AA; Garwan, MA; Nagadi, MM; Maslehuddin, M; Al-Amoudi, OSB; Khateeb-ur-Rehman: Non-destructive analysis of chlorine in fly ash cement concrete $>>$ NAQVI AA 271(2007)151

\#1095 NUCL. INSTRUM. METHODS PHYS. RES. SECT. A-ACCEL. SPECTROM. DECT. ASSOC. EQUIP. 609(2009)165-171

Feng, XG; He, QG: Simultaneous determination of Np237, Pu238-240 and Am-241 in HNO3 solution by combining extraction, liquid scintillation counting, and alpha spectrometry >> LAROSA J 277(2008)11; NORISUYE K 267(2006)183

\#1096 NUCL. INSTRUM. METHODS PHYS. RES. SECT. A-ACCEL. SPECTROM. DECT. ASSOC. EQUIP. 609(2009)183-186

Kumar, GA; Mazumdar, I; Gothe, DA: Efficiency calibration and simulation of a $\mathrm{LaBr} 3(\mathrm{Ce})$ detector in closegeometry $>>$ ASHRAFI S 269(2006)95

\#1097 NUCL. INSTRUM. METHODS PHYS. RES. SECT. A-ACCEL. SPECTROM. DECT. ASSOC. EQUIP. 610(2009)553-565

Trkov, A; Zerovnik, G; Snoj, L; Ravnik, M: On the selfshielding factors in neutron activation analysis $>>$ CHILIAN C 278(2008)745; JACIMOVIC R 278(2008)795 \#1098 NUCL. INSTRUM. METHODS PHYS. RES. SECT. A-ACCEL. SPECTROM. DECT. ASSOC. EQUIP. 612(2010)354-359

Tanigaki, M; Takamiya, K; Yoshino, H; Abe, N; Takeshita, T; Osanai, A: Control system for the FFAG complex at KURRI >> TAKAMIYA K 278(2008)719 \#1099 NUCL. INSTRUM. METHODS PHYS. RES. SECT. A-ACCEL. SPECTROM. DECT. ASSOC. EQUIP. 612(2010)421-426

Tripathy, SP; Kolekar, RV; Sunil, C; Sarkar, PK; Dwivedi, KK; Sharma, DN: Microwave-induced chemical etching (MCE): A fast etching technique for the solid polymeric track detectors (SPTD) $>>$ KALSI PC 273 (2007)473; SAWANT PD 279(2009)179

\#1100 NUCL. INSTRUM. METHODS PHYS. RES. SECT. A-ACCEL. SPECTROM. DECT. ASSOC. EQUIP. 612(2010)571-575
Talamonti, C; Reggioli, V; Bruzzi, M; Bucciolini, M; Civinini, C; Marrazzo, L; Menichelli, D; Pallotta, S; Randazzo, N; Sipala, V; Cirrone, GAP; Petterson, M; Blumenkrantz, N; Feldt, J; Heimann, J; Lucia, D; Seiden, A; Williams, DC; Sadrozinski, HFW; Bashkirov, V; Schulte, R: Proton radiography for clinical applications $>$ KACPEREK A 271(2007)731

\#1101 NUCL. INSTRUM. METHODS PHYS. RES. SECT. A-ACCEL. SPECTROM. DECT. ASSOC. EQUIP. 613(2010)378-385

Schillebeeckx, P; Borella, A; Drohe, JC; Eykens, R; Kopecky, S; Massimi, C; Mihailescu, LC; Moens, A; Moxon, M; Wynants, R: Target requirements for neutroninduced cross-section measurements in the resonance region $>$ BORELLA A 265(2005)267; POSTMA H 265(2005)297

\#1102 NUCL. INSTRUM. METHODS PHYS. RES. SECT. B-BEAM INTERACT. MATER. ATOMS 234(2005) 375-386

Szelecsenyi, F; Steyn, GF; Kovacs, Z; van der Walt, TN; Suzuki, K; Okada, K; Mukai, K: New cross-section data for the Zn-66(p,n) Ga-66, Zn-68(p,3n)Ga-66, Zn-nat(p,X)Ga66, Zn-68(p,2n)Ga-67 and at Zn-nat(p,x)(67) Ga nuclear reactions up to $100 \mathrm{MeV}>>$ BONARDI ML 264(2005) 101; SZELECSENYI F 263(2005)539

\#1103 NUCL. INSTRUM. METHODS PHYS. RES. SECT. B-BEAM INTERACT. MATER. ATOMS 240(2005)625-637

Szelecesnyi, F; Steyn, GF; Kovacs, Z; Vermeulen, C; van der Meulen, NP; Dolley, SG; van der Walt, TN; Suzuki, K; Mukai, K: Investigation of the Zn-66(p,2pn) Cu-64 and $\mathrm{Zn-68(p,x)Cu-64} \mathrm{nuclear} \mathrm{processes} \mathrm{up} \mathrm{to}$ $100 \mathrm{MeV}$ : Production of $\mathrm{Cu}-64>$ SZELECSENYI F 263(2005)539

\#1104 NUCL. INSTRUM. METHODS PHYS. RES. SECT. B-BEAM INTERACT. MATER. ATOMS 243(2006)253-255

Biegalski, SR; Alvarez, E; Green, T: Confirmation of germanium interference with hydrogen for prompt gammaray activation analysis $>>$ BIEGALSKI SR 265(2005)303 \#1105 NUCL. INSTRUM. METHODS PHYS. RES. SECT. B-BEAM INTERACT. MATER. ATOMS 245(2006)367-370

Park, CS; Sun, GM; Choi, HD: Determination of thermal neutron radiative capture cross section of Li-6 $>>$ SUN GM 264(2005)603

\#1106 NUCL. INSTRUM. METHODS PHYS. RES. SECT. B-BEAM INTERACT. MATER. ATOMS 252(2006)267-275

Thomsen, KJ; Botter-Jensen, L; Denby, PM; Murray, AS: Luminescence response to irradiation using mini X-ray generators > > HONG DG 265(2005)495 
\#1107 NUCL. INSTRUM. METHODS PHYS. RES. SECT. B-BEAM INTERACT. MATER. ATOMS 256 (2007)591-598

Matsumura, H; Suzuki, T; Miura, T; Masumoto, K; Ishihama, S; Matsuda, N; Aze, T: Spallation products of Au-197 induced by secondary particles from $12-\mathrm{GeV}$ proton bombardment of a thick aluminum target $>>$ MATSUMURA H 272(2007)423

\#1108 NUCL. INSTRUM. METHODS PHYS. RES. SECT. B-BEAM INTERACT. MATER. ATOMS 258 (2007)313-320

Uddin, MS; Khandaker, MU; Kim, KS; Lee, YS; Kim, $\mathrm{GN}$ : Excitation functions of the proton induced nuclear reactions on Zn-nat up to $40 \mathrm{MeV}>>$ SZELECSENYI F 263(2005)539

\#1109 NUCL. INSTRUM. METHODS PHYS. RES. SECT. B-BEAM INTERACT. MATER. ATOMS 258 (2007)375-380

Yawata, T; Hashimoto, T; Takeuchi, T; Hong, DG: Optimal conditions of X-ray irradiation for accurate equivalent dose determination $>>$ HONG DG 265(2005)495 \#1110 NUCL. INSTRUM. METHODS PHYS. RES. SECT. B-BEAM INTERACT. MATER. ATOMS 259(2007)271-276

Dong, KJ; He, M; Jiang, S; Wong, HT; Qiu, JZ; Guan, YJ; Li, SH; Wu, SY; Lin, M; You, QB; Bao, YW; Hu, YM; Zhou, D; Yin, XY; Yuan, J: Measurement of trace I-129 concentrations in CsI powder and organic liquid scintillator with accelerator mass spectrometry $>$ JIANG SS 264(2005)549

\#1111 NUCL. INSTRUM. METHODS PHYS. RES. SECT. B-BEAM INTERACT. MATER. ATOMS 259(2007)733-738

Buchholz, BA; Brown, TA; Hamilton, TF; Hutcheon, ID; Marchetti, AA; Martinelli, RE; Ramon, EC; Tumey, $\mathrm{SJ}$; Williams, RW: Investigating uranium isotopic distributions in environmental samples using AMS and MCICPMS >> MARCHETTI AA 263(2005)483

\#1112 NUCL. INSTRUM. METHODS PHYS. RES. SECT. B-BEAM INTERACT. MATER. ATOMS 261(2007)742-746

Fassbender, M; Arzumanov, A; Jamriska, DJ; Lyssukhin, SN; Trellue, H; Waters, LS: Proton beam simulation with MCNPX: Gallium metal activation estimates below $30 \mathrm{MeV}$ relevant to the bulk production of Ge-68 and Zn65 >> FASSBENDER M 263(2005)497

\#1113 NUCL. INSTRUM. METHODS PHYS. RES. SECT. B-BEAM INTERACT. MATER. ATOMS 261(2007) 941-944

Serris, M; Galanopoulos, S; Kalfas, CA; KokkoriS, M; LagoyannIS, A; Papadopoulos, CT; Patronis, N; Perdikakis, G; Vlastou, R: Study of the (n, 2n) cross section of the Hf-174 isotope $>>$ VLASTOU R 272(2007)219
\#1114 NUCL. INSTRUM. METHODS PHYS. RES. SECT. B-BEAM INTERACT. MATER. ATOMS 261 (2007)969-973

Galanopoulos, S; Vlastou, R; Papadopoulos, CT; Kokkoris, M; Perdikakis, G; Serris, M; Demetriou, P: Experimental and theoretical studies of $(n, p)$ reactions on Ge isotopes $>>$ VLASTOU R 272(2007)219 \#1115 NUCL. INSTRUM. METHODS PHYS. RES. SECT. B-BEAM INTERACT. MATER. ATOMS 262(2007)333339

Alvarez, E; Biegalski, SR; Landsberger, S: Methodologies for hydrogen determination in metal oxides by prompt gamma activation analysis $>>$ AGHARA SK 265(2005) 321; MACKEY EA 265(2005)273

\#1116 NUCL. INSTRUM. METHODS PHYS. RES. SECT. B-BEAM INTERACT. MATER. ATOMS 263 (2007)50-53

Metwally, WA; Gardner, RP; Sood, A: Using gammagamma coincidence measurements to validate Monte Carlo generated detector response functions $>>$ GARDNER RP 264(2005)221

\#1117 NUCL. INSTRUM. METHODS PHYS. RES. SECT. B-BEAM INTERACT. MATER. ATOMS 263(2007)79-84

Revay, Z: Comparison of the analytical sensitivities for non-1/v elements in different neutron beams $>>$ REVAY Z 264(2005)283; REVAY Z 265(2005)261

\#1118 NUCL. INSTRUM. METHODS PHYS. RES. SECT. B-BEAM INTERACT. MATER. ATOMS 263(2007) 90-94

Szentmiklosi, L; Revay, Z; Belgya, T: An improved beam chopper setup at the Budapest PGAA facility $>>$ REVAY Z 264(2005)277

\#1119 NUCL. INSTRUM. METHODS PHYS. RES. SECT. B-BEAM INTERACT. MATER. ATOMS 263(2007)132-135

Kasviki, K; Stamatelatos, IE; Yannakopoulou, E; Papadopoulou, P; Kalef-Ezra, J: Cin the accuracy of protein determination in large biological samples by prompt gamma neutron activation analysis $>$ KASVIKI K 271(2007)225

\#1120 NUCL. INSTRUM. METHODS PHYS. RES. SECT. B-BEAM INTERACT. MATER. ATOMS 264 (2007)323-328

Beasley, D; Spyrou, NM: 3D quantitative elemental mapping using simultaneous proton induced X-ray emission tomography and scanning transmission ion microscopy tomography >> BEASLEY D 264(2005)289

\#1121 NUCL. INSTRUM. METHODS PHYS. RES. SECT. B-BEAM INTERACT. MATER. ATOMS 266 (2008)599-603

Zhou, RM; Wu, XF; Hao, XF; Zhou, F; Li, HB; Rao, WH: Influences of surfactants on the preparation of copper 
nanoparticles by electron beam irradiation $>>$ EKOKO BG 265(2005)3

\#1122 NUCL. INSTRUM. METHODS PHYS. RES. SECT. B-BEAM INTERACT. MATER. ATOMS 266 (2008)807-812

Rameback, H; Nygren, U; Lagerkvist, P; Verbruggen, A; Wellum, R; Skarnemark, G: Basic characterization of U-233: Determination of age and U-232 content using sector field ICP-MS, gamma spectrometry and alpha spectrometry >> LAMONT SP 264(2005)423; NYGREN U 272(2006) 45

\#1123 NUCL. INSTRUM. METHODS PHYS. RES. SECT. B-BEAM INTERACT. MATER. ATOMS 266(2008)841-848

Dantas, CC; Melo, SB; Oliveira, EF; Simoes, FPM; dos Santos, MG; dos Santos, VA: Measurement of density distribution of a cracking catalyst in experimental riser with a sampling procedure for gamma ray tomography $>>$ DANTAS CC 269(2006) 425

\#1124 NUCL. INSTRUM. METHODS PHYS. RES. SECT. B-BEAM INTERACT. MATER. ATOMS 266(2008)2242-2245

Tumey, SJ; Brown, TA; Hamilton, TE; Hillegonds, DJ: Accelerator mass spectrometry of strontium-90 for homeland security, environmental monitoring and human health >> MARCHETTI AA 263(2005)483; POPOV L 269 (2006) 161

\#1125 NUCL. INSTRUM. METHODS PHYS. RES. SECT. B-BEAM INTERACT. MATER. ATOMS 266(2008) 2363-2366

Godelitsas, A; Kokkoris, A; Chatzitheodoridis, E; Misaefides, P: Spectroscopic characterization of Greek dolomitic marble surface interacted with uranium and thorium in aqueous solutions >> GODELITSAS A 272(2007)339 \#1126 NUCL. INSTRUM. METHODS PHYS. RES. SECT. B-BEAM INTERACT. MATER. ATOMS 266 (2008)2387-2390

Olabanji, SO; Omobuwajo, OR; Ceccato, D; Adebajo, AC; Buoso, MC; Moschini, G: Accelerator-based analytical technique in the study of some anti-diabetic medicinal plants of Nigeria $>>$ OLABANJI SO 270(2006)515 \#1127 NUCL. INSTRUM. METHODS PHYS. RES. SECT. B-BEAM INTERACT. MATER. ATOMS 266(2008) 3625-3632

Ogiyama, S; Tagami, K; Uchida, S: The concentration and distribution of essential elements in brown rice associated with the polishing rate: Use of ICP-AES and MicroPIXE $>>$ TSUKADA H 273(2007)199

\#1128 NUCL. INSTRUM. METHODS PHYS. RES. SECT. B-BEAM INTERACT. MATER. ATOMS 266 (2008)3647-3655

Matsumura, H; Miura, T; Toyoda, A; Matsuda, N; Numajiri, M; Nakashima, H; Masumoto, K; Suzuki, T; Saito,
K: Detailed spatial measurements and Monte Carlo analysis of the transportation phenomena of thermal and epithermal neutrons from the $12-\mathrm{GeV}$ proton transport line to an access maze >> MASUMOTO K 271(2007)297

\#1129 NUCL. INSTRUM. METHODS PHYS. RES. SECT. B-BEAM INTERACT. MATER. ATOMS 266(2008) 4252-4256

Kronenberg, A; Spejewski, EH; Carter, HK; Mervin, B; Jost, C; Stracener, DW; Lapi, S; Bray, T: Molecular sidebands of refractory elements for ISOL $>$ SZUCS Z 265(2005)507

\#1130 NUCL. INSTRUM. METHODS PHYS. RES. SECT. B-BEAM INTERACT. MATER. ATOMS 267(2009)513-518

Harrison, RK; Landsberger, S: Determination of boron over a large dynamic range by prompt-gamma activation analysis $>>$ REVAY Z 265(2005)261; SZENTMIKLOSI L 271(2007)447

\#1131 NUCL. INSTRUM. METHODS PHYS. RES. SECT. B-BEAM INTERACT. MATER. ATOMS 267 (2009)821-824

Xie, GX; Feng, SL; Feng, XQ; Li, YQ; Han, HY; Wang, YQ; Zhu, J; Yan, LT; Li, L: Study on the elemental features of ancient Chinese white porcelain at Jingdezhen by INAA >> ZHU JH 272(2007)545

\#1132 NUCL. INSTRUM. METHODS PHYS. RES. SECT. B-BEAM INTERACT. MATER. ATOMS 267(2009)1877-1881

Szelecsenyi, F; Steyn, GF; Dolley, SG; Kovacs, Z; Vermeulen, C; van der Walt, TN: Investigation of the Zn68(p,2p)Cu-67 nuclear reaction: New measurements up to $40 \mathrm{MeV}$ and compilation up to $100 \mathrm{MeV}>>$ BONARDI ML 264(2005)101; SZELECSENYI F 263(2005)539 \#1133 NUCL. INSTRUM. METHODS PHYS. RES. SECT. B-BEAM INTERACT. MATER. ATOMS 267(2009)18911898

Naik, H; Singh, S; Reddy, AVR; Manchanda, VK; Kim, G; Kim, KS; Lee, MW; Ganesan, S; Raj, D; Lee, HS; Do Oh, Y; Cho, MH; Ko, IS; Namkung, W: Product yields for the photo-fission of Bi-209 with $2.5 \mathrm{GeV}$ bremsstrahlung >> SINGH S 279(2009)547

\#1134 NUCL. INSTRUM. METHODS PHYS. RES. SECT. B-BEAM INTERACT. MATER. ATOMS 267 (2009)2032-2035

Ishikawa, T; Iso, H; Oikawa, M; Konishi, T; Kitamura, H; Higuchi, Y; Suya, N; Hamano, T; Imaseki, H: Development of a real-time beam current monitoring system for microbeam scanning-PIXE analysis using a ceramic channel electron multiplier $>>$ YUKAWA M 272(2007)345

\#1135 NUCL. INSTRUM. METHODS PHYS. RES. SECT. B-BEAM INTERACT. MATER. ATOMS 267 (2009)2107-2112

Habchi, C; Nguyen, DT; Barberet, P; Incerti, S; Moretto, P; Sakellariou, A; Seznec, H: Reconstruction of 3D ion 
beam micro-tomography data for applications in Cell Biology >> BEASLEY D 264(2005)289

\#1136 NUCL. INSTRUM. METHODS PHYS. RES. SECT. B-BEAM INTERACT. MATER. ATOMS 267 (2009)2241-2244

Szoboszlai, Z; Kertesz, Z; Szikszai, Z; Borbely-Kiss, I; Koltay, E: Ion beam microanalysis of individual aerosol particles originating from Saharan dust episodes observed in Debrecen, Hungary $>$ KOLTAY E 267 (2006) 449

\#1137 NUCL. INSTRUM. METHODS PHYS. RES. SECT. B-BEAM INTERACT. MATER. ATOMS 267(2009)29272933

Pallone, AK; Demaree, JD: CASSPERR: A gammagamma cascade detector for resonant nuclear reaction analysis $>>$ HATSUKAWA Y 272(2007)273; HATSUKAWA Y 276(2008)213; METWALLY WA 265(2005)309; OSHIMA M 271(2007)317; SYKORA I 276(2008)779; TOH Y 272(2007)303; TOH Y 276(2008)217

\#1138 NUCL. MED. BIOL. 35(2008)481-492

Avcibasi, U; Avcibasi, N; Unak, T; Unak, P; Muftuler, FZ; Yidirim, Y; Dincalp, H; Gumuser, FG; Dursun, ER: Metabolic comparison of radiolabeled aniline- and phenolphthaleins with I-131 >> UNAK T 266(2005)503

\#1139 NUCL. MED. BIOL. 35(2008)523-527

Eckelman, WC; Volkert, WA; Bonardi, M: True radiotracers: are we approaching theoretical specific activity with Tc-99 m and I-123? > > BONARDI ML 263(2005)87; DEGOEIJ JJM 263(2005)13

\#1140 NUCL. MED. BIOL. 36(2009)57-64

Melendez-Alafort, L; Nadali, A; Pasut, G; Zangoni, E; De Caro, R; Cariolato, L; Giron, MC; Castagliuolo, I; Veronese, FM; Mazzi, U: Detection of sites of infection in mice using Tc-99m-labeled PN2S-PEG conjugated to UBI and Tc-99m-UBI: a comparative biodistribution study $>>$ FERROFLORES G 266(2005)307

\#1141 NUCL. MED. BIOL. 36(2009)729-739

Holland, JP; Sheh, YC; Lewis, JS: Standardized methods for the production of high specific-activity zirconium$89>>$ KANDIL SA 274(2007)45

\#1142 NUCL. MED. BIOL. 37(2010)21-28

Chakravarty, R; Dash, A; Venkatesh, M: A novel electrochemical technique for the production of clinical grade Tc-99 m using (n, gamma)Mo-99 >> ENGELMANN MD 276(2008)493

\#1143 NUCL. PHYS. A 828(2009)253-266

Attar, FMD; Dhole, SD; Kailas, S; Bhoraskar, VN: Cross-sections for the formation of isomeric pair Ge75(m,g) through $(\mathrm{n}, 2 \mathrm{n}),(\mathrm{n}, \mathrm{p})$ and (n, alpha) reactions measured over $13.73 \mathrm{MeV}$ to $14.77 \mathrm{MeV}$ and calculated from near threshold to $20 \mathrm{MeV}$ neutron energies >> LUO JJ 279(2009)443
\#1144 NUCL. SCI. ENG. 155(2006)143-153

Han, XG; Gardner, RP; Metwally, WA: CEARCPG: A Monte Carlo simulation code for normal and coincidence prompt-gamma-ray neutron activation analysis >> METWALLY WA 265(2005)309

\#1145 NUCL. SCI. ENG. 157(2007)200-209

Whitney, SM; Biegalski, S; Buchholz, B: Analyzing nuclear fuel cycles from isotopic ratios of waste products applicable to measurement by accelerator mass spectrometry $>>$ MCCURDY D 263(2005)447

\#1146 NUCL. SCI. TECH. 19(2008)159-164

Jalilian, AR; Shanesazzadeh, S; Rowshanfarzad, P; Bolourinovin, F; Majdabad, A: Biodistribution study of [Cu-61]pyruvaldehyde-bis (N-4-methylthiosemicarbazone) in normal rats as a PET tracer $>>$ JALILIAN AR 269(2006)147

\#1147 NUCL. SCI. TECH. 19(2008)347-353

Jalilian, AR; Yari-Kamrani, Y; Rowshanfarzad, P; Sabet, M; Kamali-Dehghan, M; Majdabadi, A: Preparation and preliminary evaluation of [Co-55](II)vancomycin $>>$ JALILIAN AR 269(2006)147; JALILIAN AR 274(2007)563

\#1148 NUCL. SCI. TECH. 20(2009)93-98

Ravisankar, R; Eswaran, P; Anand, KV; Rajalakshmi, A; Prasad, MVR; Satpathy, KK; Rajashekhar, C; Athavale, A: EDXRF analysis of beach rock samples of Andaman Island $>>$ AKYUZ T 273(2007)747; COJOCARU V 268(2006)71; LARTIGUE J 273(2007)759; MAHAWATTE P 270(2006)657

\#1149 NUCL. SCI. TECH. 20(2009)99-105

Shaban, H; Rolf, M: Uptake of U- and Th-series radionuclides by cereal crops in Upper Egypt $>>$ POVINEC PP 273(2007)383

\#1150 NUCL. SCI. TECH. 20(2009)157-162

Jalilian, AR; Yousefnia, H; Faghihi, R; Akhlaghi, M; Zandi, H: Preparation, quality control and biodistribution of [Cu-61]-doxorubicin for PET imaging $>>$ JALILIAN AR 269(2006)147; JALILIAN AR 274(2007)563

\#1151 NUCL. TECHNOL. 162(2008)184-191

Jeong, SM; Hur, JM; Hong, SS; Kang, DS; Choung, MS; Seo, CS; Yoon, JS; Park, SW: An electrochemical reduction of uranium oxide in the advanced spent-fuel conditioning process >> JEONG SM 268(2006)349; PARK SB 268(2006)489 \#1152 NUCL. TECHNOL. 168(2009)694-699

Kinoshita, N; Matsumura, H; Bessho, K; Toyoda, A; Masumoto, K; Matsushi, Y; Sasa, K; Takahashi, T; Mihara, S; Oki, T; Matsumura, M; Tosaki, Y; Sueki, K; Tamari, M; Nagashima, Y: DEPTH PROFILE OF RADIOACTIVITY INDUCED IN THE THICK CONCRETE SHIELD IN EP1 BEAM LINE AT THE KEK 12-GeV PROTON SYNCHROTRON FACILITY $>>$ MATSUMURA H 278(2008)733; VENKATARAMAN R 264(2005)213 
\#1153 NUCL. TECHNOL. 168(2009)979-983

Matsumura, H; Kinoshita, N; Toyoda, A; Masumoto, K; Bessho, K; Hagiwara, M; Yamanoi, Y: ADSORPTION BEHAVIOR OF RADIONUCLIDES ON ION-EXCHANGE RESIN FROM COOLING WATER FOR THE K2K TARGET AND MAGNETIC HORNS $>>$ MATSUMURA H 272(2007)423

\#1154 NUCL. TECHNOL. RADIAT. PROT. 24(2009) $119-125$

Hakimabad, HM; Motavalli, LR: IMPROVING THE UNIFORMITY OF THE GAMMA PRODUCTION RATE DISTRIBUTION WITH DEPTH IN A LARGE BIOLOGICAL SAMPLE FOR AN IVNAA FACILITY $>>$ KASVIKI K 271(2007)225

\#1155 NUCLEOSIDES NUCLEOTIDES NUCLEIC ACIDS 28(2009)1131-1143

Inkster, JAH; Adam, MJ; Storr, T; Ruth, TJ: LABELING OF AN ANTISENSE OLIGONUCLEOTIDE WITH [F-18]FPy5yne >> WANG RF 273(2007)19

\#1156 NUKLEONIKA 50(2005)S77-S81

Narbutt, J: Trends in radiochemistry at the beginning of the 21st century >> ZEISLER R 263(2005)103; ZIELINSKA B 266(2005)339

\#1157 NUKLEONIKA 51(2006)111-117

Jalilian, AR; Rowshanfarzad, P; Sabet, M; Kamalidehghan, M: Preparation of [Cu-61]DTPA complex as a possible PET tracer >> SZELECSENYI F 263(2005)539

\#1158 NUKLEONIKA 51(2006)119-123

Jalilian, AR; Yari-Kamrani, Y; Sadeghi, M: Production of [Pd-103]Bleomycin complex for targeted therapy $>>$ JALILIAN AR 264(2005)617

\#1159 NUKLEONIKA 51(2006)155-159

Jalilian, AR; Rowshanfarzad, P; Sabet, M; RahiminejadKisomi, A; Rajamand, AA: Development of [Ga-66]oxine complex; a possible PET tracer $>$ JALILIAN AR 264(2005)617

\#1160 NUKLEONIKA 51(2006)203-208

Jalilian, AR; Hosseini, MA; Karimian, A; Saddadi, F; Sadeghi, M: Preparation and biodistribution of [Tl-201] (III)vancomycin complex in normal rats $>>$ JALILIAN AR 264(2005)617

\#1161 NUKLEONIKA 51(2006)209-215

Rowshanfarzad, P; Jalilian, AR; Kiyomarsi, M; Sabet, M; Karimian, AR; Moradkhani, S; Mirzaii, M: Production, quality control and initial imaging studies of $[\mathrm{Rb}-82 \mathrm{~m}] \mathrm{RbCl}$ for PET studies $>>$ JALILIAN AR 264(2005)617

\#1162 NUKLEONIKA 51(2006)S39-S44

Al-Zamel, AZ; Bou-Rabee, F; Al-Sarawi, MA; Olszewski, M; Bem, H: Determination of the sediment deposition rates in the Kuwait Bay using Cs-137 and Pb-210 >> ALZAMEL AZ 266(2005)269
\#1163 NUKLEONIKA 51(2006)S45-S51

Skwarzec, B; Struminska, DI; Borylo, A: Radionuclides of iron (Fe-55), nickel (Ni-63), polonium (Po-210), uranium (U-234, U-235, U-238) and plutonium (Pu-238, $\mathrm{Pu} 239+240, \mathrm{Pu}-241)$ in Poland and Baltic Sea environment $>>$ STRUMINSKA DI 268(2006)59

\#1164 NUKLEONIKA 52(2007)145-151

Jalilian, AR; Garousi, J; Gholami, E; Akhlaghi, M; Bolourinovin, F; Rajabifar, S: Preparation and biodistribution of [Ga-67]-insulin for SPECT purposes $>>$ JALILIAN AR 274(2007)175; JALILIAN AR 274(2007)563 \#1165 NUKLEONIKA 53(2008)27-30

Kierepko, R; Mietelski, JW; Gaca, P; Tomankiewicz, E: Activity ratios of thorium isotopes in living species compared with other environmental samples $>>$ GACA P 267(2006)443; KOMOSA A 269(2006)195; MIETELSKI JW 270(2006)131

\#1166 NUKLEONIKA 53(2008)89-95

Przybytniak, G; Kornacka, EM; Mirkowski, K; Walo, M; Zimek, Z: Functionalization of polymer surfaces by radiation-induced grafting $>>$ ZU JH 273(2007)479

\#1167 NUKLEONIKA 53(2008)S21-S24

Chau, ND: Measurement of radon concentration in the air by PicoRad detectors >> ESPINOSA G 277(2008)131 \#1168 NUKLEONIKA 53(2008)S39-S43

Kempinska, M: Methods and measurements in radiation synovectomy with Y-90 of knee joint closimetry >> VANURA P 267(2006)501 \#1169 NUKLEONIKA 54(2009)3-9

Bou-Rabee, F; Al-Zamel, AZ; Al-Fares, RA; Bem, H: Technologically enhanced naturally occurring radioactive materials in the oil industry (TENORM). A review >> ALZAMEL AZ 266(2005)269; KADYRZHANOV KK 264(2005)413

\#1170 NUKLEONIKA 54(2009)135-141

Jalilian, AR; Zandi, H; Sardari, D; Akhlaghi, M; KamaliDehghan, M; Shafaei, K; Majdabadi, A: Preparation and biological evaluation of [Cu-61]bleomycin complex as a possible PET radiopharmaceutical in normal and fibrosarcoma-bearing animals $>>$ JALILIAN AR 264(2005) 617; JALILIAN AR 274(2007)563 \#1171 NUKLEONIKA 54(2009)175-179

Jalilian, AR; Zolghadri, S; Faghihi, R; Yousefnia, H; Garousi, J; Shafaii, K; Bolourinovin, F: Preparation, quality control and biodistribution studies of [Cu-61]-oxinate for PET tumor imaging $>>$ JALILIAN AR 269(2006)147; JALILIAN AR 274(2007)563

\#1172 NUOVO CIMENTO SOC. ITAL. FIS. C-GEOPHYS. SPACE PHYS. 30(2007)47-58

Gorini, G: Ancient Charm: A research project for neutron-based investigation of cultural-heritage objects $>>$ 
PEREGO RC 271(2007)89; POSTMA H 265(2005); POSTMA H 271(2007)95

\#1173 ORG. BIOMOL. CHEM. 4(2006)3350-3357

Di Bartolo, N; Sargeson, AM; Smith, SV: New Cu-64 PET imaging agents for personalised medicine and drug development using the hexa-aza cage, SarAr $>>$ BONARDI ML 264(2005)101

\#1174 PAK. J. BOT. 40(2008)2493-2502

Hameed, I; Dastagir, G; Hussain, F: NUTRITIONAL AND ELEMENTAL ANALYSES OF SOME SELECTED MEDICINAL PLANTS OF THE FAMILY POLYGONACEAE $>>$ GARG AN 271(2007)611

\#1175 PERIOD. MINERAL. 78(2009)49-64

Barca, D; Abate, M; Crisci, GM; De Presbiteris, D: Post-medieval glass from the Castle of Cosenza, Italy: chemical characterization by LA-ICP-MS and SEM-EDS >> JAMES WD 263(2005)697

\#1176 PHARM. CHEM. J. 43(2009)287-293

Jalilian, AR; Mirazizi, F; Nazem, H: DEVELOPMENT OF (67)GA-LABELED STREPTOKINASE >> JALILIAN AR 274(2007)175

\#1177 PHYS. ATOM. NUCLEI 71(2008)1684-1693

Frontasyeva, MV: Epithermal Neutron Activation Analysis at the IBR-2 Reactor of the Frank Laboratory of Neutron Physics at the Joint institute for Nuclear Research (Dubna) >> BACCHI MA 271(2007)345; LANDSBERGER S 269(2006)697

\#1178 PHYS. CHEM. CHEM. PHYS. 12(2010)1271-1277

Yang, DJ; Zheng, ZF; Yuan, Y; Liu, HW; Waclawik, ER; Ke, XB; Xie, MX; Zhu, HY: Sorption induced structural deformation of sodium hexa-titanate nanofibers and their ability to selectively trap radioactive $\mathrm{Ra}$ (II) ions from water $>>$ ABDELRAHMAN KM 268(2006) 221

\#1179 PHYS. CHEM. EARTH 33(2008)962-968

Horie, K; Hidaka, H; Gauthier-Lafaye, F: Elemental distribution in apatite, titanite and zircon during hydrothermal alteration: Durability of immobilization mineral phases for actinides >> SELVIG LK 263(2005)341

\#1180 PHYS. LETT. A 373(2009)862-866

Cardone, F; Cherubini, G; Petrucci, A: Piezonuclear neutrons $>>$ CARDONE F 265(2005)151

\#1181 PHYS. LETT. A 373(2009)1956-1958

Cardone, F; Mignani, R; Petrucci, A: Piezonuclear decay of thorium $>>$ CARDONE F 265(2005)151

\#1182 PHYS. LETT. A 373(2009)3797-3800

Cardone, F; Mignani, R; Petrucci, A: Reply to "Comment on 'Piezonuclear decay of thorium' [Phys. Lett. A 373 (2009) 1956]" [Phys. Lett. A 373 (2009) 3795] >> CARDONE F 265(2005)151

\#1183 PHYS. LETT. A 374(2010)698-700

Cardone, F; Mignani, R; Petrucci, A: Reply to "Comment on 'Piezonuclear decay of thorium' [Phys. Lett. A 373 (2009)
1956]" [Phys. Lett. A 374 (2009) 696] >> CARDONE F 265(2005)151

\#1184 PHYS. LETT. A 374(2010)701-703

Ford, R; Gerbier-Violleau, M; Vazquez-Jauregui, E: Measurement of the thorium-228 activity in solutions cavitated by ultrasonic sound $>$ CARDONE F 265 (2005) 151

\#1185 PHYS. LETT. B 686(2010)18-22

Ideguchi, E; Ota, S; Morikawa, T; Oshima, M; Koizumi, M; Toh, Y; Kimura, A; Harada, H; Furutaka, K; Nakamura, S; Kitatani, F; Hatsukawa, Y; Shizuma, T; Sugawara, M; Miyatake, H; Watanabe, YX; Hirayama, Y; Oi, M: Superdeformation in asymmetric $\mathrm{N}>\mathrm{Z}$ nucleus $\mathrm{Ar}-40>>$ OSHIMA M 278(2008)257

\#1186 PHYS. MED. BIOL. 51(2006)R203-R228

Mattsson, S; Thomas, BJ: Development of methods for body composition studies $>>$ SHYPALIO RJ 263(2005) 759

\#1187 PHYS. MED. BIOL. 52(2007)1693-1703

Byun, SH; Pejovic-Milic, A; McMaster, S; Matysiak, W; Liu, AZ; Watters, LM; Prestwich, WV; McNeill, FE; Chettle, DR: Dosimetric characterization of the irradiation cavity for accelerator-based in vivo neutron activation analysis $>>$ BYUN SH 269(2006)615; PEJOVICMILIC A 269(2006)417 \#1188 PHYS. MED. BIOL. 52(2007)2107-2122

Zamburlini, M; Pejovic-Milic, A; Chettle, DR; Webber, CE; Gyorffy, J: In vivo study of an x-ray fluorescence system to detect bone strontium non-invasively $>>$ ZAMBURLINI M 269(2006)625

\#1189 PHYS. MED. BIOL. 53(2008)4081-4092

Aslam; Pejovic-Milic, A; Chettle, DR; McNeill, FE: Quantification of manganese in human hand bones: a feasibility study >> BYUN SH 269(2006)615; PEJOVICMILIC A 269(2006)417

\#1190 PHYS. MED. BIOL. 53(2008)N371-N376

Aslam; Pejovic-Milic, A; Chettle, DR; McNeill, FE; Pysklywec, MW; Oudyk, J: A preliminary study for noninvasive quantification of manganese in human hand bones $>$ BYUN SH 269(2006)615; PEJOVICMILIC A 269(2006)417

\#1191 PHYS. MED. BIOL. 54(2009)17-28

Aslam; Chettle, DR; Pejovic-Milic, A; Waker, AJ: Opportunities to improve the in vivo measurement of manganese in human hands $>>$ BYUN SH 269(2006)615; PEJOVICMILIC A 269(2006) 417

\#1192 PHYS. MED. BIOL. 54(2009)4889-4905

Cho, SH; Jones, BL; Krishnan, S: The dosimetric feasibility of gold nanoparticle-aided radiation therapy (GNRT) via brachytherapy using low-energy gamma-/ X-ray sources $>>$ JAMES WD 271(2007)455

\#1193 PHYS. MED. BIOL. 55(2010)1025-1039

Gomez, F; Iglesias, A; Doblado, FS: A new active method for the measurement of slow-neutron fluence in 
modern radiotherapy treatment rooms $>>$ AWOTWIPRATT JB 271(2007)679

\#1194 PHYS. MED. BIOL. 55(2010)1083-1098

Heirwegh, CM; Chettle, DR; Pejovic-Milic, A: Evaluation of imaging technologies to correct for photon attenuation in the overlying tissue for in vivo bone strontium measurements >> ZAMBURLINI M 269(2006)625

\#1195 PHYS. REV. A 80(2009)22501-

Thierfelder, C; Schwerdtfeger, P; Koers, A; Borschevsky, A; Fricke, B: Scalar relativistic and spin-orbit effects in closed-shell superheavy-element monohydrides >> HOFFMAN DC 276(2008)525

\#1196 PHYS. REV. C 74(2006)24603-

Belgya, T: Improved accuracy of gamma-ray intensities from basic principles for the calibration reaction $\mathrm{N}$ 14(n,gamma)N-15 >> BELGYA T 265(2005)175; BELGYA T 265(2005)181; REVAY Z 265(2005)169

\#1197 PHYS. REV. C 76(2007)44609-

Camacho, AG; Gomes, PRS; Lubian, J; Aguilera, EF; Padron, I: Detailed determination of the nuclear fusion radius by a simultaneous optical model calculation of elastic scattering and fusion cross sections in reactions involving weakly bound projectiles $>$ GOMES PRS 272(2007)215

\#1198 PHYS. REV. C 76(2007)67603-

Garcia, AR; Lubian, J; Padron, I; Gomes, PRS; Lacerda, T; Garcia, VN; Camacho, AG; Aguilera, EF: Threshold anomaly in the elastic scattering of He-6 on Bi-209 >> GOMES PRS 272(2007)215

\#1199 PHYS. REV. C 77(2008)65502-

Ruprecht, G; Vockenhuber, C; Buchmann, L; Woods, R; Ruiz, C; Lapi, S; Bemmerer, D: Precise measurement of the beta decay and electron capture of $\mathrm{Na}-22, \mathrm{Au}-198$, and Au-196 in low-temperature metal hosts, and reexamination of lifetime modifications $>$ LINDSTROM RM 263(2005)311

\#1200 PHYS. REV. C 79(2009)14613-

Tovesson, F; Hill, TS; Mocko, M; Baker, JD; McGrath, CA: Neutron induced fission of $\mathrm{Pu}-240, \mathrm{Pu}-242$ from $1 \mathrm{eV}$ to $200 \mathrm{MeV}>>$ BAKER JD 276(2008)555

\#1201 PHYS. REV. C 79(2009)37602-

Dvorak, J; Bruchle, W; Dullmann, CE; Dvorakova, Z; Eberhardt, K; Eichler, R; Jager, E; Nagame, Y; Qin, Z; Schadel, M; Schausten, B; Schimpf, E; Schuber, R; Semchenkov, A; Thorle, P; Turler, A; Wegrzecki, M; Yakushev, A: Cross section limits for the Cm-248(Mg-25,4n5n)(268,269)Hs reactions $>>$ REN ZZ 272(2007)209

\#1202 PHYS. REV. C 79(2009)64321-

Sugawara, M; Toh, Y; Oshima, M; Koizumi, M; Osa, A; Kimura, A; Hatsukawa, Y; Goto, J; Kusakari, H; Morikawa, T; Zhang, YH; Zhou, XH; Guo, YX; Liu, ML: Possible magnetic and antimagnetic rotations in Dy-144 >> OSHIMA M 278(2008)257
\#1203 PHYS. REV. C 80(2009)11301-

Csige, L; Csatlos, M; Faestermann, T; Gacsi, Z; Gulyas, J; Habs, D; Hertenberger, R; Krasznahorkay, A; Lutter, R; Maier, HJ; Thirolf, PG; Wirth, HF: Hyperdeformed subbarrier fission resonances observed in U-232 >> ROYER G 272(2007)237

\#1204 PHYS. REV. C 81(2010)24309-

Sugawara, M; Toh, Y; Oshima, M; Koizumi, M; Kimura, A; Osa, A; Hatsukawa, Y; Kusakari, H; Goto, J; Honma, M; Hasegawa, M; Kaneko, K: Medium-spin states in $\mathrm{Ge}-70$ and the role of the $\mathrm{g}(9 / 2)$ orbital >> OSHIMA M 278(2008) 257

\#1205 PHYS. SCR. 78(2008)65701-

Sogut, O; Butun, H; Karahan, IH; Tirasoglu, E; Apaydin, G: Investigation by XRF and XRD of $\mathrm{Zn}$ and $\mathrm{Fe}$ in FexZn1-x thin films $>>$ KUCUKONDER A 270(2006)695 \#1206 PHYS. STATUS SOLIDI-RAPID RES. LETT. 1(2007)190-192

de Miranda, RL; Kruse, J; Ratzke, K; Faupel, F; Fritsch, D; Abetz, V; Budd, PM; Selbie, JD; McKeown, NB; Ghanem, BS: Unusual temperature dependence of the positron lifetime in a polymer of intrinsic microporosity >> SHANTAROVICH VP 272(2007)645

\#1207 PLANT SOIL 318(2009)243-255

Moradi, AB; Conesa, HM; Robinson, B; Lehmann, E; Kuehne, G; Kaestner, A; Oswald, S; Schulin, R: Neutron radiography as a tool for revealing root development in soil: capabilities and limitations $>>$ NAKANISHI TM 264(2005)313

\#1208 PLANT SOIL 322(2009)219-228

Shtangeeva, I; Alber, D; Bukalis, G; Stanik, B; Zepezauer, F: Multivariate statistical analysis of nutrients and trace elements in plants and soil from northwestern Russia >> WITKOWSKA E 265(2005)141

\#1209 POL. J. CHEM. 81(2007)1531-1534

Makrlik, E; Vanura, P; Halova, J: Stability of $\mathrm{Ca} 2+$, $\mathrm{Zn} 2+$ and $\mathrm{Pb} 2+$ complexes with valinomycin in nitrobenzene saturated with water $>>$ MAKRLIK E 268 (2006) 155

\#1210 POL. J. ENVIRON. STUD. 15(2006)845-852

Chojnacka, K: Biosorption of $\mathrm{Cr}(\mathrm{III})$ ions by wheat straw and grass: a systematic characterization of new biosorbents $>>$ NAYAK D 267(2005)59

\#1211 POL. J. ENVIRON. STUD. 16(2007)723-729

Kubica, B; Kwiatek, WM; Stobinski, M; Skiba, S; Skiba, M; Golas, J; Kubica, M; Tuteja-Krysa, M; Wrona, A; Misiak, R; Krzan, Z: Concentrations of Cs-137, K-40 radionuclides and some heavy metals in soil samples of Chocholowska Valley from Tatra National Park $>>$ CHA HJ 267(2006)349; KUBICA B 266(2005)3

\#1212 POL. J. ENVIRON. STUD. 17(2008)243-246

Kitowski, I; Mietelski, JW; Gaca, P; Grzywaczewski, G: Sr-90, Am-241 and plutonium in Barn Owl skeletons (Tyto 
alba Scop.) from southeast Poland $>>$ MIETELSKI JW 270(2006)131; MIETELSKI JW 280(2008)571

\#1213 POL. J. ENVIRON. STUD. 18(2009)745-748

Pietrzak-Fiecko, R; Smoczynski, S: Evaluation of Cs137 Content in Powdered Cow Milk from Four Regions of Poland >> SPASOVA Y 277(2008)211

\#1214 POLYHEDRON 27(2008)2113-2118

Glorius, M; Moll, H; Bernhard, G: Complexation of Curium(III) with hydroxamic acids investigated by timeresolved laser-induced fluorescence spectroscopy $>>$ MOLL H 274(2007)603

\#1215 POLYHEDRON 28(2009)2070-2074

Hussain, F; Ritchie, C; Gable, RW; Moubaraki, B; Murray, KS; Boskovic, C: Tungstoarsenate(III) polyoxoanions as inorganic ligands for polynuclear copper complexes >> CRACIUN C 264(2005)589

\section{\#1216 POLYHEDRON 29(2010)620-626}

Raditzky, B; Schmeide, K; Sachs, S; Geipel, G; Bernhard, G: Interaction of uranium(VI) with nitrogen containing model ligands studied by laser-induced fluorescence spectroscopy >> VULPIUS D 270(2006)661 \#1217 POLYM. SCI. SER. A 49(2007)509-516

Starannikova, LE; Belov, NA; Shantorovich, VP; Suzuki, T; Golenko, TG; Makovetskii, KL; Yampol'skii, YP: Transport and physicochemical parameters of polypentenamer $>>$ SHANTAROVICH VP 272(2007)645

\#1218 PROBL. AT. SCI. TECH. (2008)198-202

Dikiy, NP; Dovbnya, AN; Lyashko, YV; Medvedeva, EP; Medvedev, DV; Uvarov, VL; Gritsan, LD; Onishchenko, NI: ESTIMATION OF THE ABSORBED DOSES OF SYNTHESIZED CISPLATIN WITH Pt-195m IN VARIOUS ORGANS OF ANIMALS $>>$ KNAPP FF 263(2005)503

\#1219 PROC. NATL. ACAD. SCI. U. S. A. 103(2006) 14785-14789

Bush, WD; Garguilo, J; Zucca, FA; Albertini, A; Zecca, L; Edwards, GS; Nemanich, RJ; Simon, JD: The surface oxidation potential of human neuromelanin reveals a spherical architecture with a pheomelanin core and a eumelanin surface $>>$ ZECCA L 263(2005)733

\#1220 PROC. NATL. ACAD. SCI. U. S. A. 105(2008) 17232-17237

Doiron, AL; Chu, K; Ali, A; Brannon-Peppas, L: Preparation and initial characterization of biodegradable particles containing gadolinium-DTPA contrast agent for enhanced MRI >> ROBERTSON JD 263(2005)511 \#1221 PROG. BIOCHEM. BIOPHYS. 35(2008)998-1006

Zhou, GQ; Chen, CY; Li, YF; Li, W; Gao, YX; Zhao, YL: Recent progress on the pro and cons of biological effects of nanomaterials >> WANG JX 272(2007)527

\#1222 PROG. CHEM. 19(2007)123-129

Chen, XJ: Advances in imaging agents for beta-amyloid plaques $>>$ WANG WS 266(2005)107
\#1223 PROG. NAT. SCI. 19(2009)1173-1186

Basu, M; Pande, M; Bhadoria, PBS; Mahapatra, SC: Potential fly-ash utilization in agriculture: A global review >> PAPASTEFANOU C 275(2008)29

\#1224 PROG. NUCL. ENERGY 50(2008)449-455

Rao, PRV; Venkatesan, KA; Srinivasan, TG: Studies on applications of room temperature ionic liquids $>>$ GIRIDHAR P 265(2005)31

\#1225 PROG. NUCL. ENERGY 50(2008)456-461

Suzuki, T; Fujii, Y; Koyama, SI; Ozawa, M: Nuclide separation from spent nuclear fuels by using tertiary pyridine resin $>>$ SUZUKI T 272(2007)257

\#1226 PROG. NUCL. ENERGY 50(2008)462-465

Nogami, M; Ishihara, T; Maruyama, K; Ikeda, Y: Effect of chemical structure of monoamide resins on adsorptivity to uranium(VI) in nitric acid media $>$ NOGAMI $\mathrm{M}$ 273(2007)37

\#1227 PROG. NUCL. ENERGY 51(2009)225-235

Abu-Khader, MM: Recent advances in nuclear power: A review >> LIU XG 273(2007)49

\#1228 PROG. NUCL. MAGN. RESON. SPECTROSC. 51(2007)155-198

Schneider, B: Nuclear magnetic resonance spectroscopy in biosynthetic studies >> FILER CN 268(2006)663 \#1229 PROG. PARTICLE \& NUCL. PHYS. 60(2008)225282

Pietralla, N; von Brentano, P; Lisetskiy, AF: Experiments on multiphonon states with proton-neutron mixed symmetry in vibrational nuclei $>>$ YATES SW 265(2005)291

\#1230 PRZEM. CHEM. 88(2009)190-196

Gajowiak, A; Majdan, M; Drozdzal, K: Sorption of uranium(VI) on clays and clay minerals $>>$ DONAT $\mathrm{R}$ 265(2005)107; MEGOUDA N 272(2007)75

\#1231 PURE APPL. CHEM. 80(2008)2577-2594

Li, YF; Chen, CY; Qu, Y; Gao, YX; Li, B; Zhao, YL; Chai, ZF: Metallomics, elementomics, and analytical techniques >> WANG JX 272(2007)527; ZHANG H 272(2007)561

\#1232 PURE APPL. CHEM. 82(2010)409-427

Diaz-Bone, RA; Van de Wiele, T: Biotransformation of metal(loid)s by intestinal microorganisms $>>$ MOMOSHIMA N 272(2007)413

\#1233 Q. J. NUCL. MED. MOL. IMAG. 52(2008)222-234

Jurisson, S; Cutler, C; Smith, SV: Radiometal complexes: characterization and relevant in vitro studies $>>$ BONARDI ML 264(2005)101

\#1234 Q. J. NUCL. MED. MOL. IMAG. 52(2008)267-277

Shokeen, M; Fettig, NM; Rossin, R: Synthesis, in vitro and in vivo evaluation of radiolabeled nanoparticles $>>$ LIANG S 269(2006)3

\#1235 QUAT. GEOCHRONOL. 4(2009)501-507

Di Nicola, L; Schnabel, C; Wilcken, KM; Gmeling, K: Determination of chlorine concentrations in whole rock: 
Comparison between prompt-gamma activation and isotope-dilution AMS analysis >> GMELING K 265 (2005)201; GMELING K 271(2007)397; MARSCHALL HR 265(2005)339

\#1236 QUAT. RES. 70(2008)343-357

Bertrand, S; Castiaux, J; Juvigne, E: Tephrostratigraphy of the late glacial and Holocene sediments of Puyehue Lake (Southern Volcanic Zone, Chile, 40 degrees S) >> DAGA R 270(2006)677

\#1237 QUIM. NOVA 29(2006)1332-1339

Hespanhol Da Silva, MD; Mendes Da Silva, LH; Paggioli, FJ; Reis Coimbra, JS; Minim, LA: Aqueous biphasic systems: An efficient alternative for extraction of ions $>>$ ALVAREZ A 265(2005)383

\#1238 QUIM. NOVA 31(2008)10-19

Hortellani, MA; Sarkis, JES; Abessa, DMS; Sousa, ECPM: Assessment of metallic element contamination in sediments from the Santos-Sao Vicente Estuarine System >> SILVA PSC 269(2006)739

\#1239 RADIAT. EFF. DEFECTS SOLIDS 163(2008)903913

Belivermis, M; Kilic, O; Cotuk, Y; Topcuoglu, S; Coskun, M; Cayir, A; Kucer, R: Radioactivity concentrations in topsoil samples from the Thrace region of Turkey and assessment of radiological hazard $>>$ ALMASRI MS 267(2006) 337

\#1240 RADIAT. EFF. DEFECTS SOLIDS 164(2009)138143

Kilic, O; Belivermis, M; Topcuoglu, S; Cotuk, Y: $232 \mathrm{Th}, 238 \mathrm{U}, 40 \mathrm{~K}, 137 \mathrm{Cs}$ radioactivity concentrations and 137Cs dose rate in Turkish market tea $>>$ HARB S 274(2007)63

\#1241 RADIAT. ENVIRON. BIOPHYS. 47(2008)101110

Li, WB; Gerstmann, U; Giussani, A; Oeh, U; Paretzke, HG: Internal dose assessment of Po-210 using biokinetic modeling and urinary excretion measurement $>>$ ALARIFI MN 269(2006)115

\#1242 RADIAT. ENVIRON. BIOPHYS. 47(2008)343348

Takamiya, K; Imanaka, T; Ota, Y; Akamine, M; Shibata, S; Shibata, T; Ito, Y; Imamura, M; Uwamino, Y; Nogawa, N; Baba, M; Iwasaki, S; Matsuyama, S: Nickel63 production in copper samples exposed to the Hiroshima atomic bomb: estimation based on an excitation function obtained by neutron irradiation experiments $>>$ SHIBATA S 273(2007)517

\#1243 RADIAT. ENVIRON. BIOPHYS. 48(2009)311-315

Nagy, K; Berhes, I; Kovacs, T; Kavasi, N; Somlai, J; Bender, T: Does balneotherapy with low radon concentration in water influence the endocrine system? A controlled non-randomized pilot study $>$ SOMLAI J 272(2007)101
\#1244 RADIAT. MEAS. 41(2006)492-497

Schubert, M; Buerkin, W; Pena, P; Lopez, AE; Balcazar, M: On-site determination of the radon concentration in water samples: Methodical background and results from laboratory studies and a field-scale test $>>$ DULAIOVA $\mathrm{H}$ 263(2005)361

\#1245 RADIAT. MEAS. 42(2007)1361-1367

Mrda, D; Bikit, I; Todorovic, NZ; Forkapic, S; Slivka, J; Veskovic, M: First tests of the active shield for a gamma ray spectrometer $>>$ POVINEC PP 263(2005)441

\#1246 RADIAT. MEAS. 42(2007)1409-1414

Kim, CK; Kim, YJ; Lee, HY; Chang, BU; Tokonami, S: Rn-220 and its progeny in dwellings of Korea $>>$ SUGINO M 266(2005)205

\#1247 RADIAT. MEAS. 42(2007)1561-1572

Szegvary, T; Conen, F; Stohlker, U; Dubois, G; Bossew, $\mathrm{P}$; de Vries, G: Mapping terrestrial gamma-dose rate in Europe based on routine monitoring data $>>$ BACIU AC 268(2006)3

\#1248 RADIAT. MEAS. 43(2008)111-120

Schubert, M; Schmidt, A; Paschke, A; Lopez, A; Balcazar, M: In situ determination of radon in surface water bodies by means of a hydrophobic membrane tubing >> TREUTLER HC 272(2007)583

\#1249 RADIAT. MEAS. 43(2008)125-128

Isinkaye, AO: Radiometric assessment of natural radioactivity levels of bituminous soil in Agbabu, southwest Nigeria >> ABDI MR 270(2006)319; PATRA AK 270 (2006) 307

\#1250 RADIAT. MEAS. 43(2008)802-804

Abraham, A; Weinstein, M; German, U; Alfassi, ZB; Machlev, Z: Reassessment of doses in TLD-100 after long storage times $>>$ GERMAN U 273(2007)729

\#1251 RADIAT. MEAS. 43(2008)1285-1293

Soto, J; Navas, A: A simple model of Cs-137 profile to estimate soil redistribution in cultivated stony soils $>>$ NAVAS A 274(2007)331 \#1252 RADIAT. MEAS. 43(2008)1294-1298

Antoniou, S; Tsiaili, A; Pashalidis, I: Alpha radiometry of uranium in surface and ground waters by liquid scintillation counting after separation of the radionuclide by cation exchange $>>$ ANTONIOU S 270 (2006)593 \#1253 RADIAT. MEAS. 43(2008)1453-1457

Papaefthymiou, H; Gouseti, O: Natural radioactivity and associated radiation hazards in building materials used in Peloponnese, Greece >> PAPAEFTHYMIOU H 274(2007) 123; PAPASTEFANOU C 266(2005)367 \#1254 RADIAT. MEAS. 44(2009)80-85

Yalcin, S; Gurler, O; Gundogdu, O; Kaynak, G: Monte Carlo simulation of gamma-ray total counting efficiency for a Phoswich detector >> ELY JH 263 (2005)245 
\#1255 RADIAT. MEAS. 44(2009)273-277

Fantidis, JG; Nicolaou, G; Tsagas, NF: A Monte Carlo simulation of neutron activation analysis of bulk objects $>$ CHETTLE DR 268(2006)653; WITKOWSKA E 265(2005)141

\#1256 RADIAT. MEAS. 44(2009)390-395

Akyil, S; Aytas, S; Turkozu, DA; Aslani, MAA; Yusan, $\mathrm{S}$; Eral, M: Radioactivity levels in surface water of lakes around Izmir/Turkey $>$ KULAHCI F 268(2006)517; KULAHCI F 268(2006)529

\#1257 RADIAT. PHYS. CHEM. 76(2007)1493-1496

Kim, KR; Lee, MS; Paek, S; Yim, SP; Ahn, DH; Chung, $\mathrm{H}$ : Adsorption tests of water vapor on synthetic zeolites for an atmospheric detritiation dryer $>$ KEHEYAN Y 264 (2005)671

\#1258 RADIAT. PHYS. CHEM. 76(2007)1885-1889

Duarte, CL; Mori, MN; Kodama, Y; Olkawa, H; Sampa, MHO: Decontamination of pesticide packing using ionizing radiation $>>$ MORI MN 270(2006)99

\#1259 RADIAT. PHYS. CHEM. 77(2008)561-570

Cataldo, F; Ursini, O; Lilla, E; Angelini, G: Radiationinduced polymerization and grafting of beta(-)pinene on silica surface $>>$ CATALDO F 272(2007)107; CATALDO F 272(2007)29

\#1260 RADIAT. PHYS. CHEM. 77(2008)734-741

Cataldo, F; Ursini, O; Angelini, G: Radiation-cured polyisoprene/C-60 fullerene nanocomposite. Part 1: Synthesis in hexane and in toluene $>$ CATALDO F 275 (2008)9

\#1261 RADIAT. PHYS. CHEM. 77(2008)941-948

Cataldo, F; Ragni, P; Ursini, O; Angelini, G: Synthesis of highly crystalline poly(dimethylbutadiene) (PDMB) by radiation-induced inclusion polymerization: A comparison with PDMBs synthesized by bulk and emulsion polymerization $>>$ CATALDO F 275(2008)9

\#1262 RADIAT. PHYS. CHEM. 77(2008)961-967

Cataldo, F; Ursini, O; Angelini, G: Radioracemization and radiation-induced chiral amplification of chiral terpenes measured by optical rotatory dispersion (ORD) spectroscopy >> CATALDO F 272(2007)107; CATALDO F 272(2007)29

\#1263 RADIAT. PHYS. CHEM. 78(2009)338-344

Cataldo, F; Ragni, P; Rosati, A; Ursini, O: Inclusion polymerization of isoprene in deoxycholic acid $>>$ CATALDO F 275(2008)9

\#1264 RADIAT. PHYS. CHEM. 78(2009)725-728

Duarte, CL; Andrade, DC; Melo, RP; Nagatomi, HR; Mori, MN: Decontamination of Ametryne HDPE packaging using electron beam accelerator $>>$ MORI MN 270 (2006)99

\#1265 RADIAT. PHYS. CHEM. 79(2010)57-63

Cataldo, F; Ursini, O; Angelini, G: Asymmetric radiation-induced inclusion polymerization of 3-methyl-1, 4-pentadiene in deoxycholic acid >> CATALDO F 280 (2009)99

\#1266 RADIAT. PHYS. CHEM. 79(2010)162-175

Bradley, DA; Farquharson, MJ; Gundogdu, O; Al-Ebraheem, A; Ismail, EC; Kaabar, W; Bunk, O; Pfeiffer, F; Falkenberge, G; Bailey, M: Applications of condensed matter understanding to medical tissues and disease progression: Elemental analysis and structural integrity of tissue scaffolds $>>$ MUTHUVELU P 271(2007)771 \#1267 RADIAT. PHYS. CHEM. 79(2010)462-466

Safrany, A; Beiler, B; Vincze, A: Radiation polymerization and crosslinking: A viable alternative for synthesis of porous functional polymers $>$ VINCZE A 273(2007)615

\#1268 RADIAT. PHYS. CHEM. 79(2010)519-526

Bural, CB; Demirer, GN; Kantoglu, O; Dilek, FB: Treatment of opium alkaloid containing wastewater in sequencing batch reactor (SBR)-Effect of gamma irradiation $>>$ JO HJ 268(2006)145

\#1269 RADIAT. PROT. DOSIM. 121(2006)179-185

Santos, AJG; Silva, PSC; Mazzilli, BP; Favaro, DIT: Radiological characterisation of disposed phosphogypsum in Brazil: Evaluation of the occupational exposure and environmental impact $>>$ SAUEIA CH 264(2005)445 \#1270 RADIAT. PROT. DOSIM. 123(2007)32-35

Kasa, I; Chobola, R; Mell, P; Szakacs, S; Kerekes, A: Preparation and investigation of thermoluminescence properties of CaSO4: $\mathrm{Tm}, \mathrm{Cu}>$ SZENTMIKLOSI L 267(2006)415

\#1271 RADIAT. PROT. DOSIM. 125(2007)444-448

Oeh, U; Andrasi, A; Bouvier-Capely, C; De Carlan, L; Fischer, H; Franck, D; Hoellriegl, V; Li, WB; Ritt, J; Roth, P; Schmitzer, C; Wahl, W; Zombori, P: Implementation of bioassay methods to improve assessment of incorporated radionuclides $>>$ HOLLRIEGL V 266(2005)441 \#1272 RADIAT. PROT. DOSIM. 125(2007)500-505

Li, WB; Wahl, W; Oeh, U; Hollriegl, V; Roth, P: Biokinetic modelling of natural thorium in humans by ingestion >> HOLLRIEGL V 266(2005)441

\#1273 RADIAT. PROT. DOSIM. 125(2007)568-571

Dantas, ALA; Dantas, BM; Lipsztein, JL; Spitz, HB: In vivo measurements of $\mathrm{Pb}-210$ in skull and knee geometries as an indicator of cumulative $\mathrm{Rn}-222$ exposure in a underground coal mine in Brazil $>$ DANTAS ALA 269(2006)435

\#1274 RADIAT. PROT. DOSIM. 127(2007)233-239

Franck, D: Potential of modern technologies for improving internal exposure monitoring $>>$ JASSIN LE 263(2005)93

\#1275 RADIAT. PROT. DOSIM. 128(2008)324-330

Kilic, O; Belivermis, M; Topcuoglu, S; Cotuk, Y; Coskun, M; Cayir, A; Kucer, R: Radioactivity concentrations and dose assessment in surface soil samples from east 
and south of Marmara region, Turkey $>>$ ALMASRI MS 267(2006)337

\#1276 RADIAT. PROT. DOSIM. 128(2008)382-385

El Arabi, AM; Ahmed, NK; Din, KS: Assessment of terrestrial gamma radiation doses for some Egyptian granite samples $>>$ XINWEI L 267(2006)669

\#1277 RADIAT. PROT. DOSIM. 129(2008)391-396

Turhan, S; Gurbuz, G: Radiological significance of cement used in building construction in Turkey $>>$ PAPASTEFANOU C 266(2005)367

\#1278 RADIAT. PROT. DOSIM. 129(2008)481-485

Brahmanandhan, GM; Selvasekarapandian, S; Malathi, J; Khanna, D; Jose, MT; Meenakshisundaram, V: Population dose from indoor gamma exposure in the dwellings around Kudankulam nuclear power plant $>>$ BRAHMANANDHAN GM 274(2007)361

\#1279 RADIAT. PROT. DOSIM. 131(2008)346-355

Karadeniz, O; Yaprak, G: Vertical distributions and gamma dose rates of K-40, Th-232, U-238 and Cs-137 in the selected forest soils in Izmir, Turkey $>>$ CHA HJ 267(2006)349; KARADENIZ O 277(2008)567

\#1280 RADIAT. PROT. DOSIM. 131(2008)487-494

Patra, AK; Jaison, TJ; Baburajan, A; Hegde, AG: Assessment of radiological significance of naturally occurring radionuclides in soil and rock matrices around Kakrapar environment >> PATRA AK 270(2006)307 \#1281 RADIAT. PROT. DOSIM. 131(2008)526-530

Sahin, L; Cavas, M: Natural radioactivity measurements in soil samples of Central Kutahya (Turkey) $>>$ PATRA AK 270(2006)307

\#1282 RADIAT. PROT. DOSIM. 131(2008)541-544

Papaefthymiou, H: Natural radioactivity content of granite tiles used in Greece $>>$ PAPAEFTHYMIOU $\mathrm{H}$ 274(2007)123; PAPASTEFANOU C 266(2005)367 \#1283 RADIAT. PROT. DOSIM. 132(2008)88-93

Jabbar, T; Khan, K; Subhani, MS; Akhter, P; Jabbar, A: Environmental gamma radiation measurement in District Swat, Pakistan >> JABBAR T 279(2009); YOUNIS M 266 (2005) 325

\#1284 RADIAT. PROT. DOSIM. 132(2008)350-356

Degerlier, M; Ozger, G: Assessment of gamma dose rates in air in Adana/Turkey $>$ PATRA AK 270 (2006) 307

\#1285 RADIAT. PROT. DOSIM. 133(2009)130-135

Saeed, MK; Moustafa, O; Yasin, OA; Tuniz, C; Habbani, FI: Doses to patients from photoneutrons emitted in a medical linear accelerator $>>$ MA A 276(2008)119 \#1286 RADIAT. PROT. DOSIM. 133(2009)193-199

Martin-Martin, A; Iniguez, MP; Luke, PN; Barquero, R; Lorente, A; Morchon, J; Gallego, E; Quincoces, G; MartiCliment, JM: Evaluation of CdZnTe as neutron detector around medical accelerators >> LUKE PN 264(2005)145 \#1287 RADIAT. PROT. DOSIM. 134(2009)79-86
Shanehsazzadeh, S; Jalilian, AR; Sadeghi, HR; Allahverdi, M: Determination of human absorbed dose of (67)GA-DTPA-ACTH based on distribution data in rats $>>$ JALILIAN AR 278(2008)123

\#1288 RADIAT. PROT. DOSIM. 137(2009)329-331

Garbarino, G; Magnoni, M; Bertino, S; Losana, MC: Development of an electrolysis system for tritium enrichment in superficial water samples >> AIGN J 277(2008)37 \#1289 RADIAT. PROT. DOSIM. 138(2010)144-157

Dimovska, S; Stafilov, T; Sajn, R; Frontasyeva, M: Distribution of some natural and man-made radionuclides in soil from the city of Veles (Republic of Macedonia) and its environs $>>$ ALMASRI MS 267(2006)337; RAHMAN ATA 273(2007)653; YOUNIS M 266(2005)325

\#1290 RADIAT. PROT. DOSIM. 138(2010)264-277

Yucel, H; Solmaz, AN; Kose, E; Bor, D: Methods for spectral interference corrections for direct measurements of U-234 and Th-230 in materials by gamma-ray spectrometry >> CARVALHO FP 274(2007)167

\#1291 RADIOCHIM. ACTA 94(2006)113-117

Jalilian, AR; Rowshanfarzad, P; Sabet, M: Preparation of [Cu-61]pyruvaldehyde-bis (N-4-methylthiosemicarbazone) complex as a possible PET radiopharmaceutical $>>$ SZELECSENYI F 263(2005)539

\#1292 RADIOCHIM. ACTA 94(2006)131-136

Giridhar, P; Venkatesan, KA; Reddy, BP; Srinivasan, TG; Rao, PRV: Recovery of fission palladium by electrodeposition using room temperature ionic liquids $>>$ GIRIDHAR P 265(2005)31; VENKATESAN KA 266(2005)431 \#1293 RADIOCHIM. ACTA 94(2006)217-221

Sadeghi, M; Afarideh, H; Raisali, G; van den Winkel, P: Electroplating/electrodissolution/recovery cycle for rhodium target used for an industrial scale cyclotron production of palladium-103 >> SADEGHI M 265(2005)455

\#1294 RADIOCHIM. ACTA 94(2006)415-420

Giridhar, P; Venkatesan, KA; Subramaniam, S; Srinivasan, TG; Rao, RRV: Electrochemical behavior of uranium(VI) in 1-butyl-3-methylimidazolium chloride and in $0.05 \mathrm{M}$ aliquat336/chloroform >> GIRIDHAR P 265(2005)31

\#1295 RADIOCHIM. ACTA 94(2006)453-459

Jalilian, AR; Akhlaghi, M; Shirazi, B; Aboudzadeh, R; Raisali, G; Salouti, M; Babaii, M: [(TI)-T-201](III)-bleomycin for tumor imaging >> JALILIAN AR 264(2005)617 \#1296 RADIOCHIM. ACTA 94(2006)645-649

Thakur, P; Moore, RC; Choppin, GR: Np(V)O-2(+) sorption on hydroxyapatite-effect of calcium and phosphate anions $>>$ MOORE RC 263(2005)97 \#1297 RADIOCHIM. ACTA 94(2006)807-813

Chetty, KV; Swarup, R; Venugopal, V; Rao, PRV: Ion exchange studies for the removal of plutonium and americium from organic liquid scintillator waste solution $>>$ BHANUSHALI RD 265(2005)389; CHETTY KV 267(2006)126 
\#1298 RADIOCHIM. ACTA 94(2006)865-869

Jalilian, AR; Sadeghi, M; Kamrani, YY: Development of [Pd-103]-labeled-bis(N-4-methylthiosemicarbazone) complexes as possible therapeutic agents $>>$ JALILIAN AR 268(2006)605; SZELECSENYI F 263(2005)539 \#1299 RADIOCHIM. ACTA 95(2007)49-53

Pathak, PN; Choppin, GR: Sorption of europium(III) on polymeric silica in perchlorate media $>>$ PATHAK PN 267(2006)309; PATHAK PN 267(2006)175

\#1300 RADIOCHIM. ACTA 95(2007)67-73

Qaim, SM; Bisinger, T; Hilgers, K; Nayak, D; Coenen, $\mathrm{HH}$ : Positron emission intensities in the decay of $\mathrm{Cu}-64$, Br-76 and I-124 >> BONARDI ML 264(2005)101 \#1301 RADIOCHIM. ACTA 95(2007)81-87

Varga, Z; Suranyi, G; Vajda, N; Stefanka, Z: Rapid sequential determination of americium and plutonium in sediment and soil samples by ICP-SFMS and alphaspectrometry $>>$ LEE SH 263(2005)419; PERNA L 263(2005)367; POVINEC PP 263(2005)413

\#1302 RADIOCHIM. ACTA 95(2007)137-142

Pathak, PN; Choppin, GR: Sorption behavior of curium(III) on hydrous silica: a kinetic and thermodynamic study $>>$ KILINCARSLAN A 264(2005)541; MOORE RC 263(2005)97; PATHAK PN 267(2006)175

\#1303 RADIOCHIM. ACTA 95(2007)245-250

Pathak, PN; Choppin, GR: Sorption of uranyl(VI) cations on suspended silicate: effects of $\mathrm{N}$-donor ligands, carboxylic acids, organic cosolvents, and metal ions $>>$ PATHAK PN 272(2007)37

\#1304 RADIOCHIM. ACTA 95(2007)267-273

Pathak, PN; Choppin, GR: Effect of complexing anions on europium sorption on suspended silica: a TRLFS study for ternary complex formation $>$ PATHAK PN 267(2006)309; PATHAK PN 267(2006)175; ZHIJUN G 266(2005)333

\#1305 RADIOCHIM. ACTA 95(2007)459-466

Ozeroglu, C; Keceli, G: Kinetics of the adsorption of strontium ions by a crosslinked copolymer containing methacrylic acid functional groups $>>$ OZEROGLU C 268(2006) 211

\#1306 RADIOCHIM. ACTA 95(2007)507-512

Pathak, PN; Choppin, GR: Kinetics and thermodynamics of uranium(VI) sorption on hydrous silica $>>$ KILINCARSLAN A 264(2005)541; MOORE RC 263(2005)97 \#1307 RADIOCHIM. ACTA 95(2007)717-725

Liu, Y; Terry, J; Jurisson, S: Pertechnetate immobilization in aqueous media with hydrogen sulfide under anaerobic and aerobic environments $>>$ TERRY J 263(2005)531 \#1308 RADIOCHIM. ACTA 95(2007)733-737

Rizvi, MA; Ali, A; Iqbal, MS: Synergistic extraction of $\mathrm{Ce}(\mathrm{III}), \mathrm{Tb}(\mathrm{III})$ and $\mathrm{Lu}(\mathrm{III})$ with a mixture of hexafluoroacetylacetone and triphenylphosphineoxide in benzene $>>$ REHMAN HU 267(2006)421

\section{\#1309 RADIOCHIM. ACTA 96(2008)63-66}

Ei-Sweify, RH; Metwally, E; Abdel-Khalik, H: Analysis of pencil graphite samples of various origins using instrumental neutron activation analysis $>>$ ELSWEIFY FH 273(2007)491

\#1310 RADIOCHIM. ACTA 96(2008)105-109

Sepehrian, H; Yavari, R; Maragheh, MG; Husain, SW: Sorption of radionuclides on mesoporous $\mathrm{Sn}(\mathrm{IV})$ silicate: a new sorbent $>>$ ADDLEMAN RS 263(2005)59 \#1311 RADIOCHIM. ACTA 96(2008)273-284

Selucky, P; Rais, J; Lucanikova, M; Gruner, B; Kvicalova, M; Fejfarova, K; Cisarova, I: Lanthanide and actinide extractions with anionic ligands based on cobalt bis(dicarbollide) ions with covalently bonded CMPO functions $>>$ LUTHER TA 267(2006)603

\#1312 RADIOCHIM. ACTA 96(2008)345-358

Reiller, PE; Evans, NDM; Szabo, G: Complexation parameters for the actinides(IV)-humic acid system: a search for consistency and application to laboratory and field observations $>>$ WARWICK P 266(2005)179

\#1313 RADIOCHIM. ACTA 96(2008)435-439

Jalilian, AR; Shanehsazzadeh, S; Akhlaghi, M; Garoosi, J; Rajabifar, S; Tavakoli, AB: Preparation and evaluation [Ga67]-DTPA-beta-1-24-corticotrophin in normal rats $>>$ JALILIAN AR 274(2007)175; JALILIAN AR 274(2007)563 \#1314 RADIOCHIM. ACTA 96(2008)733-738

Srncik, M; Hrnecek, E; Steier, P; Wallner, A; Wallner, G; Bossew, P: Vertical distribution of Pu-238, Pu-239(40), Am-241, Sr-90 and Cs-137 in Austrian soil profiles $>>$ HRNECEK E 264(2005)377

\#1315 RADIOCHIM. ACTA 96(2008)763-769

El-Azony, KM; Suzuki, K; Fukumura, T; Szelecsenyi, F; Kovacs, Z: Proton induced reactions on natural tellurium up to $63 \mathrm{MeV}$ : Data validation and investigation of possibility of I-124 production >> KIRALY B 270(2006)369 \#1316 RADIOCHIM. ACTA 96(2008)823-833

Liu, Y; Terry, J; Jurisson, S: Pertechnetate immobilization with amorphous iron sulfide $>$ TERRY J 263 (2005)531

\#1317 RADIOCHIM. ACTA 96(2008)835-844

Arrigo, LM; Galenas, A; Bassil, DB; Tucker, SA; Kannan, R; Katti, KV; Barnes, CL; Jurisson, SS: Fluorescent phosphinimine as possible precursor to an anionic and fluorescent sensor for Tc-99 $>$ EGOROV OB 263 (2005)629

\#1318 RADIOCHIM. ACTA 96(2008)855-861

Makreski, P; Jacimovic, R; Stibilj, V; Stafilov, T; Jovanovski, G: Determination of trace elements in iron minerals by instrumental and radiochemical neutron activation analysis $>>$ MENEZES MABC 270(2006)111 \#1319 RADIOCHIM. ACTA 96(2008)863-866

Wasim, M; Arif, M; Zaidi, JH; Fatima, I: Quantitative analysis of ancient Chinese ceramic using $\mathrm{k}(0)$-instrumental 
neutron activation analysis $>>$ TENORIO D 266(2005)471; WASIM M 277(2008)525

\#1320 RADIOCHIM. ACTA 97(2009)23-32

El-Sofany, EA; Zaki, AA; Mekhamer, HS: Kinetics and thermodynamics studies for the removal of $\mathrm{Co} 2+$ and $\mathrm{Cs}+$ from aqueous solution by sand and clay soils $>>A B-$ DELRAHMAN KM 288(2006)221; FILIPSKA H 270(2006)531

\#1321 RADIOCHIM. ACTA 97(2009)51-56

Jalilian, AR; Panahifar, A; Mahmoudi, M; Akhlaghi, M; Simchi, A: Preparation and biological evaluation of [Ga67]-labeled-superparamagnetic nanoparticles in normal rats $>>$ JALILIAN AR 274(2007)563 \#1322 RADIOCHIM. ACTA 97(2009)57-62

Roohi, S; Amir, N; Mushtaq, A; Savage, PB; Salahuddin, SM; Jehangir, M: Preparation, quality control and biological evaluation of Tc-99m-labelled cationic steroid antibiotic (CSA-13) >> ROOHI S 267(2006)561

\#1323 RADIOCHIM. ACTA 97(2009)283-289

Guo, ZJ; Wang, SR; Shi, KL; Wu, WS: Experimental and modeling studies of $\mathrm{Eu}(\mathrm{III})$ sorption on $\mathrm{TiO} 2>>$ DARIO M 270(2006)495; GUO ZJ 266(2005)333

\#1324 RADIOCHIM. ACTA 97(2009)291-296

Lapka, JL; Paulenova, A; Alyapyshev, MY; Babain, VA; Herbst, RS; Law, JD: Extraction of uranium(VI) with diamides of dipicolinic acid from nitric acid solutions $>>$ LUTHER TA 267(2006)603

\#1325 RADIOCHIM. ACTA 97(2009)325-331

Lokhande, RS; Singare, PU; Andhele, ML; Acharya, R; Nair, AGC; Reddy, AVR: Study of some Ayurvedic Indian medicinal plants for the essential trace elemental contents by instrumental neutron activation analysis and atomic absorption spectroscopy techniques $>$ GARG AN 263(2005)39

\#1326 RADIOCHIM. ACTA 97(2009)333-337

Cristache, C; Simion, CA; Margineanu, RM; Culicov, OA; Frontasyeva, MV; Matei, M; Duliu, OG: Epithermal neutrons activation analysis, radiochemical and radiometric investigations of evaporitic deposits of Slanic-Prahova (Romania) salt mine $>>$ CRISTACHE C 279(2009)7 \#1327 RADIOCHIM. ACTA 97(2009)437-441

Vlachou, A; Symeopoulos, BD; Koutinas, AA: A comparative study of neodymium sorption by yeast cells $>>$ HADJILOIZOU P 273(2007)553; LIU N 275(2008)173; SARRI S 279(2009)709

\#1328 RADIOCHIM. ACTA 97(2009)459-465

Wu, S; Chen, F; Kang, M; Yang, Y; Dou, S: Incorporation of iodine into uranophane formed during the corrosion of spent nuclear fuel $>$ LEHMANN $S$ 275(2008)633

\#1329 RADIOCHIM. ACTA 97(2009)507-512

Amir, N; Roohi, S; Mushtaq, A; Pervez, S; Saeed, S: Comparative studies of S-bridged complexes of Tc- $99 \mathrm{~m}$ with fac(S)-[M(aet)(3)] (M = Rh-III, Ir-III; aet = 2-aminoethanethiolate) $>>$ AMIR N 279(2009)25

\#1330 RADIOCHIM. ACTA 97(2009)519-534

Mincher, BJ; Mezyk, SP: Radiation chemical effects on radiochemistry: A review of examples important to nuclear power $>>$ SCHROEDER NC 263(2005)567; TOSTE AP 263(2005)559

\#1331 RADIOCHIM. ACTA 97(2009)543-546

Siri, S; Cohen, IM: Production of V-48 in a nuclear reactor via secondary tritons $>>$ SZUCS Z 265(2005)507 \#1332 RADIOCHIM. ACTA 97(2009)565-570

Raut, DR; Mohapatra, PK; Manchanda, VK: Role of diluent on the extraction and transport of strontium using $4,4^{\prime}\left(5^{\prime}\right)$ di-tert-butyl-dicyclohexano-18-crown-6 (DTBCH18C6) as the extractant $>>$ MAKRLIK E 277(2008)549

\#1333 RADIOCHIM. ACTA 97(2009)643-649

Makreski, P; Jacimovic, R; Stibilj, V; Stafilov, T: Determination of major and trace elements in iron-nickelcopper-cobalt ore reference materials using $\mathrm{k}(0)$-NAA $>>$ MENEZES MABC 270(2006)111

\#1334 RADIOCHIM. ACTA 97(2009)651-655

Wasim, M; Arif, M; Zaidi, JH; Anwar, Y: Development and implementation of $\mathrm{k}(0)$-INAA standardization at 10 MW Pakistan research reactor-1 $>$ WASIM M 277(2008)525

\#1335 RADIOCHIM. ACTA 97(2009)687-694

Bienvenu, P; Ferreux, L; Andreoletti, G; Arnal, N; Lepy, MC; Comte, J; Be, MM: Determination of Sn-126 half-life from ICP-MS and gamma spectrometry measurements $>>$ CATLOW SA 263(2005)599

\#1336 RADIOCHIM. ACTA 97(2009)709-717

Ozeroglu, C; Keceli, G: Kinetic and thermodynamic studies on the adsorption of U(VI) ions on densely crosslinked poly(methacrylic acid) from aqueous solutions $>>$ OZEROGLU C 268(2006)211

\#1337 RADIOCHIM. ACTA 97(2009)719-725

Rout, A; Venkatesan, KA; Srinivasan, TG; Rao, PRV: Extraction of americium(III) from nitric acid medium by CMPO-TBP extractants in ionic liquid diluent $>>$ GIRIDHAR P 265(2005)31

\#1338 RADIOCHIM. ACTA 97(2009)733-738

Sadeghi, M; Karami, H; Sarabadani, P; Mirzaee, M: Separation of Cd-109 from silver targets by nanohematite >> AARDANEH K 27(2008)831; MIRZAEE M 277 (2008)645 \#1339 RADIOCHIM. ACTA 97(2009)739-746

Castillo, AX; Olive, KI; Gonzalez, EC; Beckford, D; Montana, RL; Alvarez, AM; Alvarez, EO: An adapted purification procedure to improve the quality of Y-90 for clinical use $>>$ VANURA P 267(2006)501

\#1340 RADIOCHIM. ACTA 97(2009)763-769

Waheed, S; Siddique, N; Rahman, S: Evaluation of trace elements in chewing tobacco and snuff using instrumental 
neutron activation analysis (INAA) and atomic absorption spectroscopy (AAS) >> ADDO MA 277(2008)517; WAHEED S 279(2009)725

\#1341 RADIOCHIM. ACTA 98(2010)19-25

Fan, QH; Zhang, ML; Zhang, YY; Ding, KF; Yang, ZQ; $\mathrm{Wu}$, WS: Sorption of $\mathrm{Eu}(\mathrm{III})$ and $\mathrm{Am}(\mathrm{III})$ on attapulgite: effect of $\mathrm{pH}$, ionic strength and fulvic acid $>>$ GUO ZJ 266(2005)333; TAO ZY 268(2006)563

\#1342 RADIOCHIM. ACTA 98(2010)27-34

Zuo, R; Teng, Y; Wang, J; Hu, Q: Factors influencing plutonium sorption in shale media $>$ LUJANIENE G 274(2007)345; XIA YX 1(2007)79

\#1343 RADIOCHIM. ACTA 98(2010)35-38

Yan, TH; Zheng, WF; Zuo, C; Xian, L; Zhang, Y; Bian, XY; Li, RX; Di, Y: The reduction of $\mathrm{Np}(\mathrm{VI})$ and $\mathrm{Np}(\mathrm{V})$ by tit dihydroxyurea and its application to the U/Np separation in the PUREX process $>>$ YAN TH 279(2009)293

\#1344 RADIOCHIM. ACTA 98(2010)53-57

Ene, A; Pantelica, A: Study of transfer of minor elements during ironmaking by neutron activation analysis $>$ COJOCARU V 268(2006)71; KIMURA A 271 (2007)323

\#1345 RADIOCHIM. ACTA 98(2010)65-69

Xia, Y; Rao, L; Friese, JI; Moore, DA; Bachelor, PP: Complexation of plutonium(IV) with fluoride at variable temperatures >> XIA Y 268(2006)445; XIA Y 274(2007)1 \#1346 RADIOCHIM. ACTA 98(2010)121-125

Zaidi, JH; Waheed, S; Siddique, N; Zahid, GH: Determination of rare earth and other trace impurities in high purity gadolinium oxide $>>$ SIDDIQUE N 274(2007)181 \#1347 RADIOPROTECTION 43(2008)255-272

Lasheen, YF; El-Zakla, T; Seliman, AF; Abdel-Rassoul, AA: Direct gamma-ray measurement of different radionuclides in the surface water of Suez Canal $>>$ JIA GG 267(2006)505

\#1348 RADIOPROTECTION 43(2008)533-545

Isinkaye, MO; Farai, IP: Activity concentrations of primordial radionuclides in sediments of surface-water dams in southwest Nigeria - a baseline survey $>>$ ABDI MR 270(2006)319

\#1349 RADIOPROTECTION 44(2009)345-353

Holgye, Z; Strakova, H: Excretion rate of Po-210 in urine of general population (a review) $>>$ ALARIFI MN 269(2006)115

\#1350 RAPID COMMUN. MASS SPECTROM. 20 (2006)1053-1060

Black, C; Poile, C; Langley, J; Herniman, J: The use of pencil lead as a matrix and calibrant for matrix-assisted laser desorption/ionisation > > HAVEL J 263(2005)489 \#1351 RAPID COMMUN. MASS SPECTROM. 20(2006) 3654-3658

Marques, LD; Catharino, RR; Bruns, RE; Eberlin, MN: Electrospray ionization mass spectrometry fingerprinting of perfumes: rapid classification and counterfeit detection $>>$ SANTOS LS 269(2006)505

\#1352 RARE METAL MAT. ENG. 38(2009)38-41

Hu, YF; Shen, DH; Deng, W: Microdefects and Electron Densities in NiTi Shape Memory Alloys Studied by Positron Annihilation $>$ SHANTAROVICH VP 272 (2007)645

\#1353 REACT. FUNCT. POLYM. 68(2008)1088-1096

Garcia, L; Torrent, A; Antico, E; Fontas, C; Roglans, A: Selective $\mathrm{Pd}(\mathrm{II})$ and $\mathrm{Pt}(\mathrm{IV})$ sorption using novel polymers containing azamacrocycle functional groups $>>$ VENKATESAN KA 266(2005)431

\#1354 REACT. FUNCT. POLYM. 69(2009)224-228

Tank, R; Pathak, U; Singh, A; Gupta, A; Gupta, DC: A convenient one step preparation of crosslinked polystyrene mercaptomethyl resin $>>$ VENKATESAN KA 266(2005) 431

\#1355 REV. ANAL. CHEM. 26(2007)109-153

Li, ZJ; Chang, J; Shan, HX; Pan, JM: Advance of room temperature ionic liquid as solvent for extraction and separation >> GIRIDHAR P 265(2005)31

\#1356 REV. CHIM. 57(2006)675-678

Popovici, E; Humelnicu, D; Hristodor, C: Removal of $\mathrm{UO} 22+$ ions from simulated wastewater onto Romanian aluminium pillared clays $>$ KILINCARSLAN A 264(2005)541

\#1357 REV. CHIM. 60(2009)19-22

Pavel, CC; Pavel, LV; Cretescu, I; Popa, K: Some Aspects Concerning the Interaction between Uranium and ETS-10 Titanosilicate $>>$ HUMELNICU D 270(2006)637; POPA K 269(2006)155

\#1358 REV. MEX. FIS. 54(2008)21-28

Calderon, T: Ionoluminescence and minerals: the state of the art $>>$ NAKATA Y 272(2007)433

\#1359 REV. MEX. FIS. 56(2010)62-66

Martinez-Carrillo, MA; Solis, C; Andrade, E; IsaacOlive, K; Beltran-Hernandez, RI; Moreno, SAM; Martinez-Resendiz, G; Reyes, AR; Lucho-Constantino, CA; Del Razo, LM: Aerosol composition from Tlaxcoapan, Hidalgo in central Mexico >> MIRELES A 277(2008)441 \#1360 REV. ROUM. CHIM. 54(2009)699-

Gingasu, D; Mindru, I; Patron, L; Diamandescu, L; Cizmas, CB; Firastrau, I: LITHIUM FERRITES OBTAINED BY THERMAL DECOMPOSITION OF UREATE COMPLEX COMPOUNDS >> RANDHAWA BS 274(2007)581 \#1361 REV. ROUM. CHIM. 54(2009)741-747

Murariu, M; Popa, K; Dragan, ES; Drochioiu, G: SYNTHETIC CYSTEINE-PEPTIDE AND ITS RELATION WITH HEAVY AND RADIOACTIVE METALS $>>$ POPA K 269(2006)155 \#1362 REV. SCI. INSTRUM. 80(2009)16102-

Koizumi, M; Oshima, M; Toh, Y; Kimura, A; Kin, T; Furutaka, K; Murakami, Y; Osa, A; Ando, S: An 
automatic liquid-nitrogen filling system for multiple Ge detectors >> OSHIMA M 278(2008)257; TOH Y 278 (2008)703

\#1363 ROM. BIOTECH. LETT. 14(2009)4477-4484

Yasar, U; Ozyigit, II: Use of Human Hair as a Potential Biomonitor for Zinc in the Pendik District Istanbul Turkey >> BERLIZOV AN 276(2008)15

\#1364 ROM. J. PHYS. 54(2009)349-359

Nechifor, CD; Dorohoi, DO; Ciobanu, C: The influence of gamma radiations on physico-chemical properties of some polymer membranes $>>$ CATALDO F 272(2007) 107

\#1365 SAINS MALAYS. 37(2008)401-404

Siong, KK; Sarmani, S; Majid, AA; Leong, TK: Assessment of Neutron Flux Gradients in Irradiation Channels at the TRIGA Reactor by Au-Cr-Mo Monitor Set Based on k(0)-INAA >> KHOO KS 271(2007)419

\#1366 SAINS MALAYS. 38(2009)407-411

Ismail, AF; Yasir, MS; Majid, AA; Yahaya, R; Bahari, I: Radiological Hazard of Natural Radionuclide in Portland Cement of Peninsular Malaysia $>$ YASIR MS 273 (2007)539

\#1367 SCI. TOTAL ENVIRON. 367(2006)498-543

Burnett, WC; Aggarwal, PK; Aureli, A; Bokuniewicz, H; Cable, JE; Charette, MA; Kontar, E; Krupa, S; Kulkarni, KM; Loveless, A; Moore, WS; Oberdorfer, JA; Oliveira, J; Ozyurt, N; Povinec, P; Privitera, AMG; Rajar, R; Ramassur, RT; Scholten, J; Stieglitz, T; Taniguchi, M; Turner, JV: Quantifying submarine groundwater discharge in the coastal zone via multiple methods $>$ DULAIOVA $\mathrm{H}$ 263(2005)361

\#1368 SCI. TOTAL ENVIRON. 367(2006)924-931

Takeda, A; Tsukada, H; Takaku, Y; Hisamatsu, S; Nanzyo, M: Accumulation of uranium derived from longterm fertilizer applications in a cultivated Andisol >> HINTON TG 264(2005)417

\#1369 SCI. TOTAL ENVIRON. 368(2006)663-674

Almeida, SM; Pio, CA; Freitas, MC; Reis, MA; Trancoso, MA: Approaching PM2.5 and PM2.5-10 source apportionment by mass balance analysis, principal component analysis and particle size distribution $>>$ FREITAS MC 263 (2005)711

\#1370 SCI. TOTAL ENVIRON. 370(2006)224-234

Fujiyoshi, R; Sakamoto, K; Imanishi, T; Sumiyoshi, T; Sawamura, S; Vaupotic, J; Kobal, I: Meteorological parameters contributing to variability in Rn-222 activity concentration's in soil gas at a site in Sapporo, Japan >> KITTO ME 264(2005)381

\#1371 SCI. TOTAL ENVIRON. 381(2007)243-255

Hirose, K; Aoyama, M; Fukasawa, M; Kim, CS; Komura, K; Povinec, PP; Sanchez-Cabeza, JA: Plutonium and Cs-137 in surface water of the South Pacific Ocean $>>$ HIROSE K 263(2005)349
\#1372 SCI. TOTAL ENVIRON. 382(2007)342-350

Yamada, M; Wang, ZL: Cs-137 in the western South Pacific Ocean $>>$ MOMOSHIMA N 266(2005)455 \#1373 SCI. TOTAL ENVIRON. 390(2008)377-386

Pereira, R; Antunes, SC; Marques, SM; Goncalves, F: Contribution for tier 1 of the ecological risk assessment of Cunha Baixa uranium mine (Central Portugal): I soil chemical characterization >> NOUBACTEP C 267(2006) 591

\#1374 SCI. TOTAL ENVIRON. 399(2008)186-192

Linde-Arias, AR; Inacio, AF; de Alburquerque, C; Freire, MM; Moreira, JC: Biomarkers in an invasive fish species, Oreochromis niloticus, to assess the effects of pollution in a highly degraded Brazilian River $>>$ PEREIRA MO 269(2006)707

\#1375 SCI. TOTAL ENVIRON. 400(2008)162-172

Lalor, GC: Review of cadmium transfers from soil to humans and its health effects in the Jamaican environment >> GRANT CN 266(2005)385

\#1376 SCI. TOTAL ENVIRON. 404(2008)103-112

Hopke, PK; Cohen, DD; Begum, BA; Biswas, SK; Ni, BF; Pandit, GG; Santoso, M; Chung, YS; Davy, P; Markwitz, A; Waheed, S; Siddique, N; Santos, FL; Pabroa, PCB; Seneviratne, MCS; Wimolwattanapun, W; Bunprapob, S; Vuong, TB; Hien, PD; Markowicz, A: Urban air quality in the Asian region $>>$ CHUNG YS 267(2005)35

\#1377 SCI. TOTAL ENVIRON. 404(2008)207-217

Graham, MC; Oliver, IW; MacKenzie, AB; Ellam, RM; Farmer, JG: An integrated colloid fractionation approach applied to the characterisation of porewater uranium-humic interactions at a depleted uranium contaminated site $>>$ SINGHAL RK 265(2005)405

\#1378 SCI. TOTAL ENVIRON. 407(2009)3198-3207

Burnett, WC; Chanyotha, S; Wattayakorn, G; Taniguchi, M; Umezawa, Y; Ishitobi, T: Underground sources of nutrient contamination to surface waters in Bangkok, Thailand >> DULAIOVA H 263(2005)361

\#1379 SCI. TOTAL ENVIRON. 408(2010)495-504

Nikolopoulos, D; Vogiannis, E; Petraki, E; Zisos, A; Louizi, A: Investigation of the exposure to radon and progeny in the thermal spas of Loutraki (Attica-Greece): Results from measurements and modelling $>>$ SOMLAI $\mathrm{J}$ 272(2007)101

\#1380 SCI. TOTAL ENVIRON. 408(2010)1139-1144

Zhang, YS; Zheng, J; Yamada, M; Wu, FC; Igarashi, Y; Hirose, K: Characterization of $\mathrm{Pu}$ concentration and its isotopic composition in a reference fallout material $>>$ HRNECEK E 276(2008)789; LEE SH 263(2005)419; ZHENG J 275(2008)37

\#1381 SEP. PURIF. TECHNOL. 50(2006)35-44

Zhang, AY; Kuraoka, E; Kumagai, M: Removal of $\mathrm{Pd}(\mathrm{II}), \mathrm{Zr}(\mathrm{IV}), \mathrm{Sr}(\mathrm{II}), \mathrm{Fe}(\mathrm{III})$, and $\mathrm{Mo}(\mathrm{VI})$ from simulated high level liquid waste by extraction chromatography 
utilizing the macroporous silica-based polymeric materials $>>$ ZHANG A 265(2005)409

\#1382 SEP. PURIF. TECHNOL. 53(2007)81-88

Gasser, MS: Adsorption and pre-concentration of Gd(III) and U(VI) from aqueous solution using artificial adsorbents $>>$ AWWAD NS 264(2005)623

\#1383 SEP. PURIF. TECHNOL. 54(2007)363-372

Zhang, AY; Kuraoka, E; Kumagai, M: Group partitioning of minor actinides and rare earths from highly active liquid waste by extraction chromatography utilizing two macroporous silica-based impregnated polymeric composites $>>$ ZHANG A 265(2005)409

\#1384 SEP. PURIF. TECHNOL. 62(2008)407-414

Zhang, AY; Chen, CM; Kuraoka, E; Kumagai, M: Impregnation synthesis of a novel macroporous silicabased crown ether polymeric material modified by 1-dodecanol and its adsorption for strontium and some coexistent metals >> ZHANG A 265(2005)409; ZHANG A 269(2006)119

\#1385 SEP. PURIF. TECHNOL. 62(2008)449-457

Mostafa, M: Purification of neutron-irradiated tellurium targets from cross-radiocontaminants by precipitation with 1,10-phenanthroline >> KAYASTH SR 268(2006)393

\#1386 SEP. PURIF. TECHNOL. 71(2010)1-12

El-Absy, MA; El-Garhy, MA; El-Amir, MA; Fasih, TW; El-Shahat, MF: Separation and purification of I-131 from neutron irradiated tellurium dioxide targets by wet-distillation method $>>$ ELABSY MA 266(2005)295

\#1387 SEP. SCI. TECHNOL. 41(2006)2047-2063

Dietz, ML: Ionic liquids as extraction solvents: Where do we stand? $>>$ GIRIDHAR P 265(2005)31 \#1388 SEP. SCI. TECHNOL. 42(2007)2235-2253

Zhang, AY; Wei, YZ; Kumagai, MK: Separation of minor actinides and rare earths from a simulated high activity liquid waste by two macroporous silica-based polymeric composites $>>$ ZHANG A 265(2005)409; ZHANG A 269(2006)119

\#1389 SEP. SCI. TECHNOL. 43(2008)1421-1433

Bursali, EA; Yurdakoc, M; Merdivan, M: The effect of degree of impregnation in amberlite resins with organophosphorous extractants for Y(III), La(III), Ce(III), Th(IV) and U(VI) ions $>>$ ELDESSOUKY SI 268(2006)247

\#1390 SEP. SCI. TECHNOL. 43(2008)3269-3285

Sepehrian, H; Yavari, R; Waqif-Husain, S; GhannadiMaragheh, M: Separation of radionuclides on mesoporous zirconium silicate: A novel sorbent $>>$ ADDLEMAN RS 263(2005)59

\#1391 SEP. SCI. TECHNOL. 43(2008)3920-3935

Yavari, R; Ahmadi, SJ; Huang, YD; Bagheri, G: Synthesis, Ion Exchange Properties, and Applications of Amorphous Cerium(III) Tungstosilicate $>$ YAVARI R 267(2006)685

\#1392 SEP. SCI. TECHNOL. 44(2009)506-515
Sonar, NL; Mishra, PK; Kore, SG; Sonavane, MS; Kulkarni, Y; Raj, K; Manchanda, VK: Treatment of 106Ru Present In Intermediate Level Radioactive Liquid Waste With Nickel Sulphide >> ELABSY MA 266(2005)295 \#1393 SEP. SCI. TECHNOL. 44(2009)681-711

Gad, HMH; El-Mouhty, NRA; Aly, HF: Applicability of Activated Carbon to Treatment of Waste Containing Iodine-Labeled Compounds $>$ MISHRA SP 272 (2007)371

\#1394 SEP. SCI. TECHNOL. 44(2009)2146-2168

Zhang, AY; Hu, QH; Chai, ZF: SPEC: A New Process for Strontium and Cesium Partitioning Utilizing Two Macroporous Silica-Based Supramolecular Recognition Agents Impregnated Polymeric Composites $>>$ ZHANG A 269(2006)119

\#1395 SEP. SCI. TECHNOL. 44(2009)3267-3285

Duan, WH; Wang, JC; Chen, J: Application of 70-mmdia Centrifugal Contactors in Pilot Plant Tests of Two Simplified TRPO Processes with Simulated High Level Waste $>>$ DUAN WH 273(2007)103

\#1396 SEP. SCI. TECHNOL. 44(2009)3753-3769

Sonar, NL; Sonavane, MS; Valsala, TP; Kulkarni, Y; Raj, K; Manchanda, VK: Use of Nickel Sulphide-PMMA Composite Beads for Removal of Ru-106 from Alkaline Radioactive Liquid Waste $>>$ ELABSY MA 266(2005) 295

\#1397 SEP. SCI. TECHNOL. 44(2009)4023-4035

Wang, L; Feng, M; Liu, CX; Zhao, YS; Li, SQ; Wang, H; Yan, L; Tian, G; Li, SJ: Supporting of Potassium Copper Hexacyanoferrate on Porous Activated Carbon Substrate for Cesium Separation $>$ KAZEMIAN $\mathrm{H}$ 268(2006)231

\#1398 SEP. SCI. TECHNOL. 45(2010)269-276

Inan, S; Altas, Y: Adsorption of Strontium from Acidic Waste Solution by Mn-Zr Mixed Hydrous Oxide Prepared by Co-Precipitation $>>$ INAN S 267(2006)615 \#1399 SMALL 3(2007)1894-1899

Goldberg-Oppenheimer, P; Regev, O: Exploring a nanotube dispersion mechanism with gold-labeled proteins via Cryo-TEM imaging >> EFIMOVA YM 264(2005)91 \#1400 SMALL 4(2008)26-49

Lewinski, N; Colvin, V; Drezek, R: Cytotoxicity of nanoparticles $>>$ JAMES WD 271(2007)455

\#1401 SMALL 5(2009)426-431

Gentleman, DJ; Chan, WCW: A Systematic Nomenclature for Codifying Engineered Nanostructures $>>$ ZHANG J 272(2007)605

\#1402 SOFT MATTER 6(2010)416-422

Skrzeszewska, PJ; de Wolf, FA; Stuart, MAC; van der Gucht, J: Kinetics of network formation by telechelic polypeptides with trimeric nodes $>$ CATALDO F 275(2008)125

\#1403 SOIL SCI. 174(2009)272-282 
Lopez-Vicente, M; Navas, A: Predicting Soil Erosion With RUSLE in Mediterranean Agricultural Systems at Catchment Scale >> NAVAS A 274(2007)331

\#1404 SOIL. SEDIMENT. CONTAM. 18(2009)590-602

Pathak, PN; Choppin, GR: Effects of pH, Ionic Strength, Temperature, and Complexing Anions on the Sorption Behavior of Cobalt on Hydrous Silica $>$ INAN S 267(2006)615; PATHAK PN 270(2006)277; PATHAK PN 270(2006)299; PATHAK PN 267(2006)175

\#1405 SOLVENT EXTR. ION EXCH. 26(2008)163-174

Herbst, RS; Peterman, DR; Tillotson, RD; Delmau, LH: Fundamental chemistry of cesium extraction from acidic media by HCCD in FS-13 >> LUTHER TA 267 (2006)603

\#1406 SOLVENT EXTR. ION EXCH. 26(2008)624-642

Zhang, AY; Chen, CM; Wang, WH; Wei, YZ: Adsorption behavior of $\operatorname{Sr}(\mathrm{II})$ and some typical co-existent metals contained in high level liquid waste onto a modified macroporous silica-based polymeric DtBuCH18C6 composite >> ZHANG A 265(2005)409; ZHANG A 269 (2006) 119

\#1407 SOLVENT EXTR. ION EXCH. 26(2008)643-671

Kumaresan, R; Sabharwal, KN; Srinivasan, TG; Rao, PRV; Dhekane, G: Studies on the sorption of palladium using cross-linked poly (4-vinylpyridine-divinylbenzene) resins in nitric acid medium $>>$ VENKATESAN KA 266(2005)431

\#1408 SOLVENT EXTR. ION EXCH. 26(2008)783-796

Duan, WH; Wang, JC; Zhou, XZ; Chen, J: Application of $70 \mathrm{~mm}$ Centrifugal Contactors in a Pilot Test of the Total Partitioning Process Using Simulated High-Level Waste $>>$ DUAN WH 273(2007)103

\#1409 SOLVENT EXTR. ION EXCH. 27(2009)695-711

Reddy, BR; Kumar, BN; Radhika, S: Solid-Liquid Extraction of Terbium from Phosphoric Acid Medium using Bifunctional Phosphinic Acid Resin, Tulsion CH-96 $>$ B BHANUSHALI RD 265(2005)389; VENKATESAN KA 275(2008)563

\#1410 SOLVENT EXTR. RES. DEV.-JPN. 13(2006)8388

Hirayama, N; Deguchi, M; Honjo, T: The behavior of divalent transition metal cations on extraction into 1-butyl3-methylimidazolium hexafluorophosphate room temperature ionic liquid with beta-diketones having a trifluoromethyl group >> GIRIDHAR P 265(2005)31

\#1411 SOLVENT EXTR. RES. DEV.-JPN. 15(2008)99110

Hashiguchi, N; Tanaka, M; Ohto, K; Kawakita, H; Inoue, K: Solid phase extraction of silver ion by impregnated resins with p-tert-BNONutylcalix[4] arene pyridyl derivatives $>>$ ELDESSOUKY SI 268(2006)247

\#1412 SPACE SCI. REV. 145(2009)285-335
Fischbach, E; Buncher, JB; Gruenwald, JT; Jenkins, JH; Krause, DE; Mattes, JJ; Newport, JR: Time-Dependent Nuclear Decay Parameters: New Evidence for New Forces? $>>$ POMME S 276(2008)335

\#1413 SPECTR. LETT. 42(2009)12-19

Malaxechevarria, Y; Millan, E: Application of Experimental Design to the Optimization of a Wet Acid Digestion Procedure for $\mathrm{Cd}$ Determination in Plastic Materials by Atomic Absorption Spectrometry $>$ SOARES EP 264(2005)9

\#1414 SPECTR. LETT. 42(2009)121-128

Kadioglu, YK; Ustundag, Z; Yazicigil, Z: Spectroscopic Application of Realgar Using X-ray Fluorescence and Raman Spectroscopy >> COJOCARU V 268(2006)71; COSTA ACM 269(2006)703; PEREIRA MO 269(2006)707 \#1415 SPECTR. LETT. 42(2009)334-340

Deng, DL; Deng, PC; Wang, XY; Hou, XD: Direct Determination of Sodium Fluoride and Sodium Monofluorophosphate in Toothpaste by Quantitative 19F-NMR: A Green Analytical Method >> RAMOS AA 266(2005)19 \#1416 SPECTROC. ACTA PT. A-MOLEC. BIOMOLEC. SPECTR. 71(2008)1525-1534

Li, ZH; Jiang, WT; Hong, HL: An FTIR investigation of hexadecyltrimethylammonium intercalation into rectorite >> CHANG P 274(2007)153

\#1417 SPECTROC. ACTA PT. A-MOLEC. BIOMOLEC. SPECTR. 71(2009)1964-1968

Arnold, T; Baumann, N: Boltwoodite $[\mathrm{K}(\mathrm{UO} 2)$ $(\mathrm{SiO} 3 \mathrm{OH})(\mathrm{H} 2 \mathrm{O})(1.5)]$ and compreignacite $\mathrm{K}-2[(\mathrm{UO} 2)$ (3) $\mathrm{O}-2(\mathrm{OH})(3)](2)$ center dot $7 \mathrm{H}(2) \mathrm{O}$ characterized by laser fluorescence spectroscopy >> LEHMANN S 275(2008) 633

\#1418 SPECTROC. ACTA PT. A-MOLEC. BIOMOLEC. SPECTR. 73(2009)492-497

Olivares, M; Tarrino, A; Murelaga, X; Baceta, JI; Castro, K; Etxebarria, N: Non-destructive spectrometry methods to study the distribution of archaeological and geological chert samples >> TENORIO D 266(2005) 471

\#1419 SPECTROC. ACTA PT. A-MOLEC. BIOMOLEC. SPECTR. 75(2010)558-562

Vulpius, D; Geipel, G; Bernhard, G: Excited-state proton transfer of 3-hydroxybenzoic acid and 4-hydroxybenzoic acid $>>$ VULPIUS D 270(2006)661

\#1420 SPECTROC. ACTA PT. B-ATOM. SPECTR. 61 (2006)877-904

Lariviere, D; Taylor, VF; Evans, RD; Cornett, RJ: Radionuclide determination in environmental samples by inductively coupled plasma mass spectrometry $>>$ DIPRETE DP 263(2005)593; LAROSA J 263(2005)427; MOHAGHEGHI AH 263(2005)189; POVINEC PP 263 (2005)413; WOLF SF 263(2005)581 
\#1421 SPECTROC. ACTA PT. B-ATOM. SPECTR. 62(2007)4-12

Lemos, VA; Santos, MS; Santos, ES; Santos, MJS; dos Santos, WNL; Souza, AS; de Jesus, DS; das Virgens, CF; Carvalho, MS; Oleszczuk, N; Vale, MGR; Welz, B; Ferreira, SLC: Application of polyurethane foam as a sorbent for trace metal pre-concentration-A review $>>$ SAEED MM 267(2006)427

\#1422 SPECTROC. ACTA PT. B-ATOM. SPECTR. 62(2007)297-303

Huang, MD; Krivan, V: A direct solid sampling electrothermal atomic absorption spectrometry method for the determination of silicon in biological materials $>>\mathrm{KU}$ CERA J 263(2005)811

\#1423 SPECTROC. ACTA PT. B-ATOM. SPECTR. 63(2008)719-737

Ketterer, ME; Szechenyi, SC: Determination of plutonium and other transuranic elements by inductively coupled plasma mass spectrometry: A historical perspective and new frontiers in the environmental sciences $>>$ GONZALES ER 263(2005)457; HIROSE K 273(2007) 115; MICHEL H 273(2007)485; NYGREN U 272(2007) 45; OKUBO A 275(2008)291; POVINEC PP 263(2005) 413; SAITOKOKUBU Y 273(2007)183; WANG Z 267(2006)73; ZHENG J 275(2008)37

\#1424 SPECTROC. ACTA PT. B-ATOM. SPECTR. 63(2008)1309-1314

Granet, M; Nonell, A; Favre, G; Chartier, F; Isnard, H; Moureau, J; Caussignac, C; Tran, B: Cs-Ba separation using $\mathrm{N} 2 \mathrm{O}$ as a reactant gas in a Multiple CollectorInductively Coupled Plasma Mass Spectrometer collisionreaction cell: Application to the measurements of Cs isotopes in spent nuclear fuel samples $>$ YII MW 274(2007)323

\#1425 SPECTROC. ACTA PT. B-ATOM. SPECTR. 64(2009)95-98

Shinonaga, T; Donohue, D; Ciurapinski, A; Klose, D: Age determination of single plutonium particles after chemical separation $>>$ NYGREN U 272(2007)45

\#1426 SPECTROC. ACTA PT. B-ATOM. SPECTR. 64(2009)229-234

Brach-Papa, C; Van Bocxstaele, M; Ponzevera, E; Quetel, CR: Fit for purpose validated method for the determination of the strontium isotopic signature in mineral water samples by multi-collector inductively coupled plasma mass spectrometry $>>$ GRAHEK Z 268 (2006) 179

\#1427 SPECTROC. ACTA PT. B-ATOM. SPECTR. 64(2009)235-241

Grinberg, P; Sturgeon, RE: Ultra-trace determination of iodine in sediments and biological material using UV photochemical generation-inductively coupled plasma mass spectrometry $>>$ YOSHIDA S 273(2007)211
\#1428 SPECTROC. ACTA PT. B-ATOM. SPECTR. 64(2009)587-592

da Silva, MP; Zucchi, OLAD; Ribeiro-Silva, A; Poletti, ME: Discriminant analysis of trace elements in normal, benign and malignant breast tissues measured by total reflection X-ray fluorescence $>>$ VIVES AES 270(2006)231 \#1429 SPECTROC. ACTA PT. B-ATOM. SPECTR. 64(2009)721-725

Khuder, A; Sawan, MK; Karjou, J; Razouk, AK: Determination of trace elements in Syrian medicinal plants and their infusions by energy dispersive X-ray fluorescence and total reflection X-ray fluorescence spectrometry $>>$ KHUDER A 273(2007)435

\#1430 SPECTROC. ACTA PT. B-ATOM. SPECTR. 64(2009)1048-1058

Death, DL; Cunningham, AP; Pollard, LJ: Multi-element and mineralogical analysis of mineral ores using laser induced breakdown spectroscopy and chemometric analysis $>>$ LIM CS 264(2005)15

\#1431 SPECTROC. ACTA PT. B-ATOM. SPECTR. 64(2009)1161-1172

Sitko, R: Quantitative X-ray fluorescence analysis of samples of less than 'infinite thickness': Difficulties and possibilities >> GUPTA D 274(2007)389; MAHAWATTE P 270(2006)657

\#1432 SPECTROC. ACTA PT. B-ATOM. SPECTR. 64(2009)1173-1179

Lima, I; de Assis, JT; Lopes, RT: Three-dimensional conic beam X-ray microtomography in bone quality $>>$ LIMA ICB 269(2006)639

\#1433 SPECTROSC. SPECTR. ANAL. 28(2008)704-706

Luo, LQ; Zhan, XC: Resolution of overlapped spectra in polarization X-ray fluorescence spectrometry by genetic algorithm > > LUO L 269(2006)325

\#1434 STRUCT. CHEM. 19(2008)109-114

Kovacs, K; Sharma, VK; Kamnev, AA; Kuzmann, E; Homonnay, Z; Vertes, A: Water and time dependent interaction of iron(III) with indole-3-acetic acid $>>$ KOVACS K 266(2005)513

\#1435 SURF. INTERFACE ANAL. 41(2009)244-250

Groenewold, GS; Gresham, GL; Avci, R; Deliorman, M: Characterization of bidentate phosphoryl compounds on soil particulates using SIMS >> LUTHER TA 267(2006)603 \#1436 SYNTH. REACT. INORG. MET.-ORG. NANOMETAL CHEM. 38(2008)204-207

Devaraj, NK; Ong, BH; Matsumoto, M: Characterization of chemically prepared magnetite nanoparticles $>>$ ZHANG X 270(2006)285

\#1437 SYNTH. REACT. INORG. MET.-ORG. NANOMETAL CHEM. 38(2008)208-211

Devaraj, NK; Ong, BH; Matsumoto, M: Yield control of chemically-synthesized magnetite nanoparticles $>>$ ZHANG X 270(2006)285 


\section{\#1438 TALANTA 69(2006)800-806}

Lubal, P; Koprivova, H; Sedo, O; Havel, J; Lis, S; But, $\mathrm{S}$ : Simultaneous determination of molybdenum(VI) and tungsten(VI) and its application in elemental analysis of polyoxometalates $>>$ HAVEL J 263(2005)489

\#1439 TALANTA 69(2006)1246-1253

Zheng, J; Yamada, M: Inductively coupled plasmasector field mass spectrometry with a high-efficiency sample introduction system for the determination of $\mathrm{Pu}$ isotopes in settling particles at femtogram levels $>>$ LAROSA J 263(2005)427; LEE SH 263(2005)419

\#1440 TALANTA 72(2007)315-320

Rickert, PG; Stepinski, DC; Rausch, DJ; Bergeron, RM; Jakab, S; Dietz, ML: Solute-induced dissolution of hydrophobic ionic liquids in water $>$ GIRIDHAR $P$ 265(2005)31

\#1441 TALANTA 74(2008)1527-1533

Michel, H; Levent, D; Barci, V; Barci-Funel, G; Hurel, C: Soil and sediment sample analysis for the sequential determination of natural and anthropogenic radionuclides >> AGEYEV VA 264(2005)337; LAROSA J 263(2005) 427; LEE SH 263(2005)419; MICHEL H 273(2007)485; MIHAI SA 266(2005)259

\#1442 TALANTA 75(2008)127-134

El-Sheikh, AH: Effect of oxidation of activated carbon on its enrichment efficiency of metal ions: Comparison with oxidized and non-oxidized multi-walled carbon nanotubes $>>$ YUSOF AM 271(2007)191

\#1443 TALANTA 75(2008)246-252

Kummrow, F; Silva, FF; Kuno, R; Souza, AL; Oliveira, PV: Biomonitoring method for the simultaneous determination of cadmium and lead in whole blood by electrothermal atomic absorption spectrometry for assessment of environmental exposure >> DOSSANTOS CR 269(2006) 481

\#1444 TALANTA 75(2008)598-603

Dietz, ML; Stepinski, DC: Anion concentration-dependent partitioning mechanism in the extraction of uranium into room-temperature ionic liquids $>$ GIRIDHAR $P$ 265(2005)31

\#1445 TALANTA 76(2008)1246-1251

Jeyakumar, S; Raut, VV; Ramakumar, KL: Simultaneous determination of trace amounts of borate, chloride and fluoride in nuclear fuels employing ion chromatography (IC) after their extraction by pyrohydrolysis $>>$ RAMANJANEYULU PS 274(2007)109

\#1446 TALANTA 77(2008)9-20

Rathore, DPS: Advances in technologies for the measurement of uranium in diverse matrices $>>$ PREMADAS A 266(2005)95

\#1447 TALANTA 78(2009)410-417

Yucel, H; Dikmen, H: Uranium enrichment measurements using the intensity ratios of self-fluorescence X-rays to $92(*) \mathrm{keV}$ gamma ray in UXK alpha spectral region $>>$ ANILKUMAR S 274(2007)161

\#1448 TALANTA 80(2009)143-150

Maxwell, SL; Jones, VD: Rapid determination of actinides in urine by inductively coupled plasma mass spectrometry and alpha spectrometry: A hybrid approach $>$ MAXWELL SL 275(2008)497; MAXWELL SL 279(2009)105

\#1449 TALANTA 80(2009)212-217

Yousefi, SR; Ahmadi, SJ; Shemirani, F; Jamali, MR; Salavati-Niasari, M: Simultaneous extraction and preconcentration of uranium and thorium in aqueous samples by new modified mesoporous silica prior to inductively coupled plasma optical emission spectrometry determination $>>$ KRISHNA PG 266(2005)251 \#1450 TALANTA 80(2009)352-362

Rozmaric, M; Ivsic, AG; Grahek, Z: Determination of uranium and thorium in complex samples using chromatographic separation, ICP-MS and spectrophotometric detection $>>$ JASSIN LE 263(2005)93

\#1451 TALANTA 80(2009)413-421

Anthemidis, AN; Ioannou, KIG: Recent developments in homogeneous and dispersive liquid-liquid extraction for inorganic elements determination. A review $>>$ MALLAH MH 278(2008)97

\#1452 TALANTA 80(2009)428-433

Santos, MC; Wagner, M; Wu, B; Scheider, J; Oehlmann, $\mathrm{J}$; Cadore, S; Becker, JS: Biomonitoring of metal contamination in a marine prosobranch snail (Nassarius reticulatus) by imaging laser ablation inductively coupled plasma mass spectrometry (LA-ICP-MS) $>>$ ELLISTON JT 263(2005)301

\#1453 TARGET. ONCOL. 4(2009)183-197

de Rosales, RTM; Arstad, E; Blower, PJ: Nuclear imaging of molecular processes in cancer $>>$ GANGULY BN 279(2009)685

\#1454 TENSIDE SURFACTANTS DETERG. 46(2009) 100-104

Prevost, S; Coulombeau, H; Baczko, K; Berthon, L; Zorz, N; Desvaux, H; Testard, F; Zemb, T; Larpent, C: Thermo-responsive Metal-chelating Surfactants: Properties and Use in Cloud Point Extraction of Uranyl Nitrate $>>$ PEREZGRAMATGES A 269(2006)491

\#1455 THEOCHEM-J. MOL. STRUCT. 913(2009)265269

Sun, ZC; Li, XJ; Tian, MS; Zhao, GJ; Li, JC; Ma, B: Comparative study on metal-encapsulated TM@C-24 and TM@C24H12 (TM = Ti, Zr and Hf) >> WATANABE S 266(2005)499

\#1456 TOXICOL. LETT. 183(2008)72-80

Wang, JX; Chen, CY; Liu, Y; Jiao, F; Li, W; Lao, F; Li, YF; Li, B; Ge, CC; Zhou, GQ; Gao, YX; Zhao, YL; Chai, $\mathrm{ZF}$ : Potential neurological lesion after nasal instillation of 
TiO2 nanoparticles in the anatase and rutile crystal phases $>>$ WANG JX 272(2007)527

\#1457 TOXICOL. MECH. METHODS 19(2009)197-201

Sisti, D; Rocchi, MBL; Meli, MA; Desideri, D: Po-210 Log-normal distribution in human urines: Survey from Central Italy people $>>$ ALARIFI MN 269(2006)115 \#1458 TOXICOL. SCI. 103(2008)354-361

He, X; Zhang, ZY; Zhang, HF; Zhao, YL; Chai, ZF: Neurotoxicological evaluation of long-term lanthanum chloride exposure in rats $>>$ HE X 272(2007)557

\#1459 TOXICOLOGY 254(2008)82-90

Wang, JX; Liu, Y; Jiao, F; Lao, F; Li, W; Gu, YQ; Li, YF; Ge, CC; Zhou, GQ; Li, B; Zhao, YL; Chai, ZF; Chen, CY: Time-dependent translocation and potential impairment on central nervous system by intranasally instilled TiO2 nanoparticles >> WANG JX 272(2007)527

\#1460 TRAC-TRENDS ANAL. CHEM. 27(2008)738-748

Chen, Z; Weber, SG: Determination of binding constants by affinity capillary electrophoresis, electrospray ionization mass spectrometry and phase-distribution methods $>>$ XIA Y 268(2006)445

\#1461 TRAC-TRENDS ANAL. CHEM. 28(2009)214-236

Yip, YC; Lam, JCW; Tong, WF: Commonly used methodologies for inorganic analysis in international key comparisons >> LINDSTROM RM 263(2005)787; RAMOS AA 266(2005)19; TSUKADA H 263(2005)773; WITKOWSKA E 265(2005)141; ZEISLER R 263(2005)315 \#1462 TRANSP. POROUS MEDIA 69(2007)89-107

Bejaoui, S; Sercombe, J; Mugler, C; Peycelon, H: Modelling of radionuclide release from a concrete container >> RUDIN MJ 264(2005)501

\#1463 TREE-RING RES. 64(2008)97-108

Sheppard, PR; Ort, MH; Anderson, KC; Elson, MD; Vazquez-Selem, L; Clemens, AW; Little, NC; Speakman, RJ: MULTIPLE DENDROCHRONOLOGICAL SIGNALS INDICATE THE ERUPTION OF PARICUTIN VOLCANO, MICHOACAN, MEXICO $>$ UNLU K 264(2005)21

\#1464 TRENDS BIOTECHNOL. 27(2009)652-660

Posthuma-Trumpie, GA; van Amerongen, A; Korf, J; van Berkel, WJH: Perspectives for on-site monitoring of progesterone $>>$ KARIR T 267(2006)321

\#1465 TRENDS PLANT SCI. 14(2009)436-442

Zhu, YG; Pilon-Smits, EAH; Zhao, FJ; Williams, PN; Meharg, AA: Selenium in higher plants: understanding mechanisms for biofortification and phytoremediation $>>$ ALAMIN MB 270(2006)143

\#1466 VADOSE ZONE J. 7(2008)1035-1047

Oswald, SE; Menon, M; Carminati, A; Vontobel, P; Lehmann, E; Schulin, R: Quantitative imaging of infiltration, root growth, and root water uptake via neutron radiography >> KAWABATA Y 264(2005)319; NAKANISHI TM 264(2005)313
\#1467 VADOSE ZONE J. 8(2009)805-809

Carminati, A; Vetterlein, D; Weller, U; Vogel, HJ; Oswald, SE: When Roots Lose Contact $>>$ NAKANISHI TM 264(2005)313

\#1468 VIB. SPECTROSC. 50(2009)116-124

Carter, EA; Hargreaves, MD; Kononenko, N; Graham, I; Edwards, HGM; Swarbrick, B; Torrence, R: Raman spectroscopy applied to understanding Prehistoric Obsidian Trade in the Pacific Region $>$ ARIAS A 268 (2006)371

\#1469 WATER AIR SOIL POLLUT. 169(2006)185-206

Dampare, SB; Ameyaw, Y; Adotey, DK; Osae, S; Serfor-Armah, Y; Nyarko, BJB; Adomako, D: Seasonal trend of potentially toxic trace elements in soils supporting medicinal plants in the eastern region of Ghana $>>$ DAMPARE SB 265(2005)53

\#1470 WATER AIR SOIL POLLUT. 179(2007)43-55

Almeida, SM; Farinha, MM; Ventura, MG; Pio, CA; Freitas, MC; Reis, MA; Trancoso, MA: Measuring air particulate matter in large urban areas for health effect assessment $>>$ FREITAS MC 263(2005)711

\#1471 WATER AIR SOIL POLLUT. 204(2009)133-138

Gagneten, AM; Pla, RR; Regaldo, L; Paggi, JC: Assessment of Bioconcentration Factor of Chromium by Instrumental Neutron Activation Analysis in Argyrodiaptomus falcifer Daday, a Subtropical Freshwater Copepod $>>$ VEADO MAR 272(2007)511

\#1472 WATER AIR SOIL POLLUT. 206(2010)307320

Ziembik, Z; Dolhanczuk-Srodka, A; Komosa, A; Orzel, $\mathrm{J}$; Waclawek, M: Assessment of Cs-137 and Pu-239, $\mathrm{Pu}-240$ Distribution in Forest Soils of the Opole Anomaly $>$ MIETELSKI JW 270(2006)131; MIETELSKI JW 275(2008)571

\#1473 WATER ENVIRON. RES. 78(2006)1856-1882

Hua, B; Deng, BL: Radioactive wastes $>>$ EGOROV GF 266(2005)349; LIU DJ 264(2005)691; SHAUKAT MS 265(2005)73

\#1474 WATER ENVIRON. RES. 79(2007)1766-1850

Chelme-Ayala, P; Li, XF; Nour, M; El-Din, MG; Ikehata, K; Smith, DW: Pesticides and herbicides $>>$ MORI MN 270(2006)99

\#1475 WATER ENVIRON. RES. 79(2007)1903-1928

Hua, B; Yang, J; Deng, B: Radioactive wastes $>>$ GRANADOS CF 268(2006)95; MISHRA SP 268(2006) 191; XU D 267(2006)357

\#1476 WATER ENVIRON. RES. 79(2007)1989-2031

Lange, CR; Mendez-Sanchez, N: Groundwater quality >> NOUBACTEP C 267(2006)591

\#1477 WATER ENVIRON. RES. 80(2008)1340-1396

Fu, HJ; Pikus, W; Zaman, W; Wang, D; Chelme-Ayala, P; El-Din, AG; Bressler, DC; El-Din, MG: Agricultural Wastes >> CARVALHO FP 274(2007)167 
\#1478 WATER ENVIRON. RES. 81(2009)1056-1126

Chelme-Ayala, P; Afzal, A; Pourrezaei, P; Wang, YN; Zapata, MA; Ding, N; Jin, J; Wang, N; Drzewicz, P; El-Din, MG: Physico-Chemical Processes $>>$ GONZALEZJUAREZ JC 275(2008)257

\#1479 WATER ENVIRON. RES. 81(2009)2030-2069

Harmon, SM: Effects of Pollution on Freshwater Organisms >> PARK EJ 277(2008)619

\#1480 WOOD SCI. TECHNOL. 40(2006)493-499

Yamashita, MT; Almeida, F; Barreiros, RM; Bringas, F; Goldman, ID; Madi, T; Pascholati, PR; Yamaji, FM: Determination of potassium and sodium in an ash sample by gamma-ray spectroscopy >> UNLU K 264(2005)21 \#1481 X-RAY SPECTROM. 35(2006)190-194

Bakraji, EH: Application of multivariate statistical methods to classify archaeological pottery from Tel-Alramad site, Syria, based on x-ray fluorescence analysis $>>$ BAKRAJI EH 264(2005)645

\#1482 X-RAY SPECTROM. 36(2007)76-81

Zamburlini, M; Byun, SH; Pejovic-Milic, A; Prestwich, WV; Chettle, DR: Evaluation of MCNP5 and EGS4 for the simulation of in vivo strontium XRF measurements $>>$ ZAMBURLINI M 269(2006)625

\#1483 X-RAY SPECTROM. 36(2007)361-368

Avila-Perez, P; Zarazua, G; Tejeda, S; Barcelo-Quintal, I; Diaz-Delgado, C; Carreno-Leon, C: Evaluation of distribution and bioavailability of $\mathrm{Cr}, \mathrm{Mn}, \mathrm{Fe}, \mathrm{Cu}, \mathrm{Zn}$ and $\mathrm{Pb}$ in the waters of the upper course of the Lerma River $>>$ AVILAPEREZ P 273(2007)625; TEJEDA S 1(2006)9

\#1484 X-RAY SPECTROM. 37(2008)12-20

Geraki, K; Farquharson, MJ; Bradley, DA; Gundogdu, O; Falkenberg, G: The localisation of biologically important metals in soft and calcified tissues using a synchrotron x-ray fluorescence technique $>>$ MUTHUVELU P 271(2007) 771

\section{\#1485 X-RAY SPECTROM. 37(2008)42-50}

Zamburlini, M; Pejovic-Milic, A; Chettle, DR: Spectrometry methods for in vivo bone strontium measurements >> ZAMBURLINI M 269(2006)625

\#1486 X-RAY SPECTROM. 38(2009)186-189

Tartari, A; Di Domenico, G; Bonifazzi, C; Baraldi, C: New measurements and observations on aged Nal detectors >> PRESLER R 273(2007)733

\#1487 X-RAY SPECTROM. 38(2009)244-249

Pereira, GR; Rocha, HS; Calza, C; Anjos, MJ; Perez, CA; Lopes, RT: X-Ray fluorescence microtomography under various excitation conditions $>>$ PEREIRA GR 269(2006)469

\#1488 X-RAY SPECTROM. 38(2009)271-277

Zamburlini, M; Campbell, JL; de Silveira, G; Butler, R; Pejovic-Milic, A; Chettle, DR: Strontium depth distribution in human bone measured by micro-PIXE $>>$ ZAICHICK V 269(2006)653; ZAMBURLINI M 269(2006)625
\#1489 X-RAY SPECTROM. 38(2009)526-539

Zitnik, M; Pelicon, P; Bucar, K; Grlj, N; Karydas, AG; Sokaras, D; Schutz, R; Kanngiesser, B: Element-selective three-dimensional imaging of microparticles with a confocal micro-PIXE arrangement $>>$ ZITNIK M 275(2008)17 \#1490 X-RAY SPECTROM. 39(2010)17-21

Finkelshtein, AL; Chubarov, VM: X-ray fluorescence determination of the $\mathrm{FeO} / \mathrm{Fe} 2 \mathrm{O} 3$ tot ratio in igneous rocks $>>$ OZ E 279(2009)529

\#1491 Z. KRIST.-NEW CRYST. STRUCT. 223(2008) 505-506

Abdel-Jalil, RJ; Volter, W; Maichle-Mossmer, C; Loffler, D; Machulla, HJ: Crystal structure of N,N,N-tris[2-(2nitro-1H-imidazol-1-yl)ethyl]amine, C15H18N10O6 >> ABDELJALIL RJ 267(2006)557

\#1492 Z. PHYS. CHEMIE-INT. J. RES. PHYS. CHEM. CHEM. PHYS. 223(2009)247-252

Makrlik, E; Vanura, P: Extraction of $\mathrm{K}+, \mathrm{Rb}+$ and $\mathrm{Tl}+$ from Water into Nitrobenzene by Using Cesium Dicarbollylcobaltate in the Presence of Hexaethyl Calix[6]arene Hexaacetate $>>$ MAKRLIK E 277(2008)495

\#1493 Z. PHYS. CHEMIE-INT. J. RES. PHYS. CHEM. CHEM. PHYS. 223(2009)253-261

Makrlik, E; Vanura, P; Selucky, P: Extraction of Microamounts of Calcium and Strontium into Nitrobenzene by Using Hydrogen Dicarbollylcobaltate in the Presence of "Classical" CMPO >> MAKRLIK E 267(2006)533; MAKRLIK E 268(2006)151; VALENTOVA Z 267(2006) 471

\#1494 Z. PHYS. CHEMIE-INT. J. RES. PHYS. CHEM. CHEM. PHYS. 223(2009)869-875

Dybal, J; Makrlik, E; Vanura, P: Experimental and DFT Study on the Complexation of $\mathrm{Zn} 2+$ with Valinomycin $>>$ MAKRLIK E 268(2006)155

\section{A2 Author index}

Abate, M: \#1175

Abdel-Jalil, RJ: \#1491

Abdel-Khalik, H: \#1309

Abdel-Latif, M: \#93

Abdel-Monem, NM: \#305

Abdel-Rahman, FH: \#802

Abdel-Rassoul, AA: \#1347

Abdi, MR: \#627 \#996 \#997

Abe, M: \#938 \#939

Abe, N: \#1098

Abessa, DMS: \#725 \#1238

Abetz, V: \#1206

Aboaba, A: \#802

Aboterika, AHA: \#837

Aboudzadeh, M: \#175 \#193 
Aboudzadeh, R: \#875 \#1295

Abraham, A: \#1085 \#1250

Abreu, I: \#725

Abreu, MM: \#507

Abril, JM: \#928

Abu-Khader, MM: \#1227

Acar, C: \#149 \#283 \#284

Acharya, R: \#493 \#634 \#1068 \#1325

Adair, JH: \#3

Adam, MJ: \#1155

Adam, V: \#433

Adamcova, J: \#755

Adamovic, J: \#273

Addleman, RS: \#330

Adebajo, AC: \#1126

Adeyemo, DJ: \#130

Adipo, MOA: \#130

Adlassnig, W: \#211

Adomako, D: \#1469

Adotey, DK: \#1469

Aerts, G: \#1078

Aeschbach-Hertig, W: \#549

Afarideh, H: \#1293

Afridi, HI: \#250

Afzal, A: \#1478

Agarwal, C: \#583

Agca, N: \#35

Aggarwal, PK: \#744 \#1367

Aggarwal, SK: \#1019 \#1074

Agrawal, A: \#289

Agresti, F: \#429

Aguilera, EF: \#1197 \#1198

Ahmad, MI: \#693

Ahmad, T: \#312

Ahmadi, F: \#827

Ahmadi, SJ: \#859 \#1391 \#1449

Ahmed, MU: \#99

Ahmed, NK: \#1276

Ahmed, YA: \#208

Ahn, DH: \#1257

Airoldi, C: \#706

Ajam, L: \#370

Akaho, EHK: \#162

Akamine, M: \#159 \#1242

Akata, N: \#760

Akhlaghi, M: \#20 \#875 \#1150 \#1164 \#1170 \#1295 \#1313 \#1321

Akhter, P: \#1283

Akiyama, K: \#137 \#317 \#318 \#924

Akkaya, R: \#839

Akman, S: \#445 \#601 \#825

Aksoy, E: \#180

Akyil, S: \#833 \#1256
Alagille, D: \#163

Alamelu, D: \#1019 \#1074

Al-Amoudi, OSB: \#198 \#1094

Alber, D: \#1208

Albertini, A: \#1219

Alberto, R: \#287

Alcalde, G: \#119

Alcalde-Molina, M: \#90

Aldahan, A: \#817

Al-Ebraheem, A: \#1266

Alexandratos, SD: \#577

Al-Fares, RA: \#1169

Alfarra, MR: \#230

Alfassi, ZB: \#124 \#134 \#1085 \#1250

Al-Hamwi, A: \#185

Ali, A: \#1220 \#1308

Ali, IM: \#309

Ali, Z: \#340

Alighanbari, H: \#859

Aligol, DA: \#261

Allahverdi, M: \#1287

Allanic, A: \#236

Allanore, A: \#734

Allen, GC: \#84

Almeida, F: \#1480

Almeida, SM: \#213 \#231 \#441 \#1071 \#1369 \#1470

Almirall, JR: \#58 \#67

Alonso, BD: \#768

Alonsoc, BDC: \#1082

Al-Sarawi, MA: \#1162

Altas, Y: \#1398

Altherr, R: \#509 \#1028

Altinoglu, EI: \#3

Alvarado-Ibarra, Y: \#366

Alvarez, AM: \#1339

Alvarez, E: \#421 \#733 \#892 \#1077 \#1104 \#1115

Alvarez, EO: \#1339

Alvarez, JL: \#535

Alvarez-Lorenzo, C: \#482

Aly, HF: \#619 \#1393

Alyapyshev, MY: \#1324

Al-Zamel, AZ: \#975 \#1162 \#1169

Ambroise, J: \#835

Ameyaw, Y: \#1469

Amin, AM: \#873

Amin, F: \#340

Amin, M: \#873

Amine, DM: \#846

Amir, N: \#1322 \#1329

Amokrane, A: \#1079

Amoli, HS: \#228 \#579

Amorim, ACL: \#705

Amorusi, P: \#934 
Amundsen, I: \#791

Anand, KV: \#1148

Anand, S: \#737

Anandam, G: \#196

Anderson, DL: \#682 \#721

Anderson, KC: \#410 \#1463

Anderson, RC: \#106

Andhele, ML: \#1325

Ando, H: \#936

Ando, S: \#1362

Andor, K: \#168

Andrade, CFF: \#219 \#862

Andrade, DC: \#1264

Andrade, E: \#1359

Andrasi, A: \#1271

Andreani, C: \#690

Andreoletti, G: \#1335

Andreoli, C: \#112

Andrews, K: \#45

Andrey, JL: \#778

Andrzej, K: \#245

Angelini, G: \#885 \#886 \#887 \#1259 \#1260 \#1261 \#1262 \#1265

Anguita, M: \#849

Anicin, IV: \#1075

Anirudhan, TS: \#411

Anjos, MJ: \#474 \#1487

Anjos, RM: \#796

Ansari, DO: \#1049

Anthemidis, AN: \#1451

Antico, E: \#1353

Antoniou, S: \#1252

Antonopoulos-Domis, M: \#1089

Antony, MP: \#551

Antonyuk, LP: \#73 \#555

Antunes, BM: \#310

Antunes, SC: \#1373

Antzutkin, ON: \#590

Anuse, MA: \#581

Anwar, Y: \#1334

Aoki, Y: \#479

Aouadi, S: \#894

Aoyama, M: \#167 \#234 \#524 \#794 \#1371

Apaydin, G: \#271 \#324 \#1205

Apoloni, CR: \#569

Apostolescu, GA: \#435

Apostolidis, C: \#871

Appel, PWU: \#562

Aquilina, L: \#119

Arain, MB: \#250

Arain, R: \#428 \#956

Araki, M: \#318

Aramendia, M: \#673
Arcagni, M: \#251

Ardavan, A: \#323

Aregbe, Y: \#611

Arginelli, D: \#391

Arida, H: \#111

Arif, M: \#1319 \#1334

Arjomandi, OK: \#882

Armand, L: \#1051

Armenta, S: \#487

Arnal, N: \#1335

Arnold, D: \#152 \#168 \#184

Arnold, T: \#1417

Aronsson, PO: \#537

Arribere, M: \#160 \#333

Arribere, MA: \#248 \#251

Arrigo, LM: \#1317

Arrona, RG: \#240

Arruda, MAZ: \#659

Arruda, NP: \#705

Arruebo, M: \#1043

Arstad, E: \#1453

Arunkumar, TA: \#421 \#733 \#892

Arva, F: \#479

Aryaeinejad, R: \#1065

Arzumanov, A: \#1112

Asai, M: \#317 \#318

Asghar, M: \#860

Aslam: \#40 \#870 \#1008 \#1189 \#1190 \#1191

Aslani, G: \#625

Aslani, MAA: \#1256

Assadi, Y: \#74

Assis, JT: \#569

Astel, A: \#453

Astel, K: \#453

Astolfi, ML: \#235

Athavale, A: \#1148

Attallah, MF: \#780

Attar, FMD: \#1143

Atun, G: \#695

Augusto, S: \#451

Aureli, A: \#744 \#1367

Aureli, F: \#634

Ausili, A: \#1000

Austin, C: \#1011

Avci, R: \#1435

Avcibasi, N: \#1138

Avcibasi, U: \#1138

Avila-Perez, P: \#1483

Avino, P: \#382 \#1016

Awuah, E: \#416

Awwad, NS: \#619 \#837

Axelsson, A: \#814

Ayata, S: \#444 
Aydin, A: \#812

Aydin, AO: \#553 \#854

Aydin, FA: \#838 \#853

Aykamis, AS: \#270

Aylikci, NK: \#324

Aylikci, V: \#324

Ayranov, M: \#954

Aytas, S: \#847 \#1256

Aytekin, H: \#497

Azbouche, A: \#1079

Aze, T: \#1107

Aznar, M: \#48

Baba, M: \#159 \#1242

Babaii, M: \#1295

Babaii, MH: \#875

Babain, V: \#392

Babain, VA: \#960 \#1324

Babicky, A: \#210

Babula, P: \#433

Baburajan, A: \#1280

Baceta, JI: \#1418

Bachelor, PP: \#1345

Bacon, JR: \#98 \#647 \#655 \#668

Baczko, K: \#1454

Badding, JV: \#4

Baek, SY: \#1061

Baena, AMRY: \#987

Baena, ARY: \#986

Bagatelas, C: \#164

Bagheri, G: \#1391

Bahari, I: \#1366

Bai, J: \#342

Bailat, CJ: \#1072

Bailey, M: \#1266

Baines, SB: \#806

Baisch, P: \#219

Baja, B: \#376

Bajaj, PN: \#1004 \#1047

Baker, JD: \#1200

Bakir, MA: \#442

Bakkaloglu, OF: \#324

Bakraji, EH: \#100 \#1481

Bakry, R: \#616

Bakyono, JP: \#521

Balasubramanyam, A: \#1036 \#1037

Balasundar, S: \#585

Balata, M: \#793

Balcazar, M: \#1244 \#1248

Baldik, R: \#497

Baldwin, RM: \#163

Baldwin, SM: \#685

Balogun, FA: \#173

Balogun, GI: \#130
Baltensperger, U: \#230

Bames, SJ: \#531

Bamoniri, A: \#8

Bandaru, NK: \#289

Bandura, DR: \#607

Banerjee, A: \#243 \#686

Bao, LM: \#800

Bao, YW: \#1110

Baptista, JA: \#968

Baptista, MS: \#1070

Baraldi, C: \#1486

Baranov, VI: \#607

Barata, F: \#1081

Barberet, P: \#1135

Barbos, D: \#212

Barca, D: \#1175

Barcelo-Quintal, I: \#1483

Barci, V: \#1441

Barciela-Alonso, MC: \#633

Barci-Funel, G: \#1441

Barisic, D: \#782

Barkat, A: \#414

Barker, J: \#228 \#579

Barnes, CL: \#589 \#1317

Barnett, CL: \#767

Barnhart, TE: \#916

Barnthip, N: \#4

Barquero, R: \#1286

Barreira, LD: \#310

Barreiros, RM: \#1480

Barrera-Diaz, C: \#836

Barrett, HH: \#570 \#914

Barron, H: \#121

Barth, BM: \#3

Bartlett, RM: \#916

Basenko, VK: \#1066

Bashkirov, V: \#1100

Baskaran, M: \#815 \#986 \#989

Bassil, DB: \#1317

Basu, M: \#1223

Batchelor-McAuley, C: \#1055

Batista, A: \#751

Batlle, R: \#48

Baumann, N: \#1417

Baumrucker, CR: \#4

Baurle, J: \#264

Baykan, UN: \#947

Baykara, O: \#180

Baysal, A: \#445 \#601 \#825

Bayukov, OA: \#598

Be, MM: \#1335

Beall, JA: \#692

Bean, M: \#202 \#814 
Beard, A: \#931

Beasley, D: \#1120

Beaudoin, G: \#281

Becker, A: \#369

Becker, DA: \#43

Becker, JS: \#608 \#609 \#1452

Beckford, D: \#1339

Bedard, LP: \#525 \#528 \#531

Beddek, S: \#1079

Bedzyk, MJ: \#691

Beesley, AM: \#195

Begum, BA: \#1376

Beiler, B: \#1267

Bejaoui, S: \#1462

Belgya, T: \#63 \#314 \#321 \#1067 \#1118 \#1196

Belivermis, M: \#447 \#1239 \#1240 \#1275

Bella, F: \#793

Bellei, C: \#222

Bellot-Gurlet, L: \#277

Belov, AG: \#527

Belov, NA: \#1217

Beltran-Hernandez, RI: \#1359

Belviso, S: \#1051

Bem, H: \#1162 \#1169

Bemb, H: \#975

Bemmerer, D: \#1199

Bemporad, E: \#295

Ben Ouezdou, M: \#370

Bender, T: \#623 \#1243

Benedik, L: \#10 \#611

Benguella, B: \#276

Benitez-Nelson, C: \#986

Benitez-Nelson, CR: \#406 \#407 \#408

Benkrid, M: \#765

Benmansour, M: \#168 \#928

Bercin, E: \#822

Berdonosov, SS: \#545

Beresford, NA: \#767

Bergamin, L: \#1000

Berger, A: \#44

Berger, JA: \#685

Bergeron, RM: \#1440

Bergin, MH: \#816

Berhes, I: \#1243

Berizov, AN: \#1066

Berlizov, A: \#183

Berlizov, AN: \#238

Bermejo-Barrera, P: \#633

Bernhard, G: \#257 \#269 \#464 \#1214 \#1216 \#1419

Berthon, L: \#1454

Bertino, S: \#1288

Bertolasi, V: \#34

Berton, G: \#391
Bertrand, S: \#1236

Bertuzzi, R: \#1006

Bessho, K: \#1152 \#1153

Betti, M: \#22 \#610

Betz, JM: \#45

Bhadoria, PBS: \#1223

Bhagat, PR: \#493

Bhandari, D: \#69

Bhanger, MI: \#84

Bhardwaj, YK: \#483

Bhatia, SN: \#123 \#289

Bhattacharya, S: \#1054

Bhoraskar, VN: \#1143

Bialostozky, D: \#223

Bian, XY: \#1343

Bianchi, J: \#1000

Bianco, A: \#295

Biber, FZ: \#127

Bibic, N: \#1048

Bichler, M: \#114 \#138 \#147 \#211 \#388 \#393 \#966

Bickmore, BR: \#726

Bidigare, R: \#406

Biegalski, S: \#1145

Biegalski, SR: \#735 \#1077 \#1104 \#1115

Bienvenu, P: \#1335

Bieringer, J: \#758

Bikit, I: \#168 \#1245

Bilba, N: \#945

Bilewicz, A: \#244

Billard, I: \#400 \#472 \#534

Bilyeu, B: \#836

Bin, S: \#809

Binnemans, K: \#326 \#472

Birat, JP: \#734

Birinci, E: \#553

Birnbaum, ER: \#685

Biro, KT: \#225

Bisinger, T: \#1300

Biswas, KF: \#233

Biswas, SK: \#1376

Biziuk, M: \#453

Bizuk, MK: \#804

Bizzo, HR: \#705

Black, C: \#1350

Blain, S: \#1051

Blazej, S: \#299 \#437

Bliss, M: \#895

Blower, PJ: \#1453

Blumenkrantz, N: \#1100

Bobrov, AV: \#229

Boccia, AC: \#34

Bochud, F: \#1072

Bode, P: \#47 \#341 \#804 
Bodogh, M: \#559

Bogner, D: \#439 \#496

Bohmova, V: \#273

Bohn, L: \#973

Bokuniewicz, H: \#769 \#1367

Bollhofer, A: \#757

Bolourinovin, F: \#175 \#625 \#626 \#1146 \#1164 \#1171

Bolskar, RD: \#1045

Bolster, D: \#863

Bolsunovsky, A: \#792

Bolsunovsky, AY: \#795

Bom, VR: \#689

Bombled, B: \#1051

Bonardi, M: \#1139

Bonardi, ML: \#124 \#134 \#200

Bondareva, L: \#792

Bondareva, LG: \#795

Bonde, SE: \#66

Bonifazzi, C: \#1486

Bonina, F: \#112

Bonn, GK: \#616

Bonny, SM: \#280

Bonte, P: \#521

Bontempi, E: \#1006

Bontempi, JM: \#699

Bontempi, PS: \#819

Bonzi, G: \#304

Bopp, L: \#1051

Bor, D: \#808 \#1290

Borai, EH: \#28

Borbely-Kiss, I: \#1136

Borella, A: \#1078 \#1101

Borgese, L: \#1006

Borio, R: \#801

Borovicka, J: \#1038 \#1039

Borowiecki, T: \#294

Borschevsky, A: \#1195

Borsodi, J: \#63

Bortoleto, GG: \#47

Bortoli, M: \#358

Bortoluzzi, S: \#391

Borylo, A: \#1163

Bosbach, D: \#466

Bosch, P: \#366

Boschetto, R: \#165

Boskovic, C: \#1215

Bosnar, D: \#1084

Bosnar, S: \#1084

Bossew, P: \#1247 \#1314

Bostick, DA: \#613

Boston, AJ: \#1093

Boston, HC: \#1093

Botter-Jensen, L: \#942 \#1106
Botti, A: \#1005

Boudjenoun, R: \#765

Boulyga, SF: \#773

Bour, O: \#119

Bou-Rabee, F: \#975 \#1162 \#1169

Bourquin, M: \#992

Bouvier-Capely, C: \#1271

Bowie, A: \#1051

Bozhevol'nov, VE: \#545

Brach-Papa, C: \#1426

Bradley, DA: \#1266 \#1484

Braga, ES: \#469

Brahmanandhan, GM: \#585 \#1278

Branch, S: \#646 \#652 \#666 \#675

Brannon-Peppas, L: \#1220

Branquinho, C: \#451

Bratton, JF: \#246

Braun, T: \#321

Bray, T: \#1129

Bregiroux, D: \#962 \#963

Breheret, JG: \#521

Brendler, V: \#464

Brennan, S: \#359

Bressler, DC: \#1477

Bresson, C: \#1053

Brettell, TA: \#58 \#67

Brewer, PW: \#698 \#818

Briggs, GAD: \#323

Briggs, JD: \#66

Bringas, F: \#1480

Bringle, CD: \#411

Brinkmeyer, R: \#463

Brockman, JD: \#189

Broeders, CHM: \#146

Broene, RD: \#685

Bronson, F: \#183

Brouwer, CPJM: \#935 \#1029

Brown, CF: \#461

Brown, EF: \#630 \#632

Brown, I: \#621

Brown, JE: \#767

Brown, TA: \#1111 \#1124

Bruach, JM: \#849

Bruchertseifer, F: \#871

Bruchle, W: \#1201

Brueckner, SM: \#529

Bruneau, C: \#33

Brunelli, M: \#963

Brunet, C: \#1051

Bruns, RE: \#92 \#1351

Brussaard, C: \#1051

Bruzzi, M: \#1100

Bryden, IG: \#889 
Bryjak, M: \#696

Bubach, DF: \#248 \#251

Bucar, K: \#1489

Bucciolini, M: \#1100

Buchholz, B: \#1145

Buchholz, BA: \#1111

Buchholz, DB: \#691

Buchillier, T: \#1072

Buchmann, B: \#230

Buchmann, L: \#1199

Buchtela, K: \#388 \#966

Bucio, E: \#482

Buck, B: \#118

Budd, P: \#545

Budd, PM: \#1206

Budka, J: \#11 \#958 \#1032 \#1033 \#1034

Budzikiewicz, H: \#257

Bueno, MIMS: \#47

Buerkin, W: \#1244

Buesseler, KO: \#405 \#409 \#986

Bukalis, G: \#1208

Bulbulian, S: \#366

Bulgariu, L: \#855

Bulut, VN: \#845

Buncher, JB: \#1412

Bunk, O: \#1266

Bunprapob, S: \#1376

Buoso, MC: \#1126

Bural, CB: \#1268

Burchart, L: \#664

Burge, SR: \#64 \#65

Burger, S: \#613 \#790

Burghoff, B: \#937

Burillo, G: \#482

Burk, P: \#641

Burnett, W: \#219 \#862

Burnett, WC: \#267 \#465 \#468 \#471 \#522 \#523 \#724 \#744 \#769 \#864 \#981 \#982 \#993 \#1367 \#1378

Bursali, EA: \#1389

Burt, V: \#45

Bush, WD: \#1219

Bussiere, B: \#117

But, S: \#1438

Butler, JM: \#58 \#67

Butler, OT: \#645 \#651 \#662 \#671 \#679

Butler, R: \#1488

Butun, H: \#1205

Buylaert, JP: \#390

Buyukuslu, H: \#812

Byrde, F: \#1072

Byrne, AP: \#736

Byun, SH: \#1008 \#1187 \#1482

Cable, J: \#769
Cable, JE: \#1367

Cabral, JP: \#1070

Cabrera, L: \#125

Cacciotti, I: \#295

Caciuffo, R: \#963

Cadore, S: \#1452

Caetano, M: \#372 \#995

Caffrey, AJ: \#188

Cai, LS: \#473

Cai, P: \#405

Cai, PH: \#504 \#505 \#820 \#925 \#988

Cairns, WRL: \#679

Calderon, T: \#1358

Callaway, TR: \#106

Calza, C: \#1487

Camacho, AG: \#1197 \#1198

Camacho, LM: \#856

Camargo, IMC: \#498

Campbell, JL: \#1488

Canella, L: \#206 \#477 \#690

Canepari, S: \#235

Canselier, JP: \#846

Canuto, G: \#391

Cao, JM: \#349

Capannesi, G: \#382 \#1016

Capitani, D: \#886

Capote, R: \#1056

Caproni, P: \#968

Carballo, DM: \#980

Carballo, J: \#980

Carder, KL: \#819

Cardone, F: \#614 \#1180 \#1181 \#1182 \#1183

Cariolato, L: \#1140

Carlotti, F: \#1051

Carlson, BV: \#1056

Carminati, A: \#1466 \#1467

Carreno-Leon, C: \#1483

Carrott, MMLR: \#379

Carrott, PJM: \#379

Carter, EA: \#1468

Carter, HK: \#1129

Carter, S: \#678

Carvalho, FP: \#168 \#272 \#507 \#751

Carvalho, MS: \#1421

Casacuberta, N: \#849

Casas, JS: \#374

Castagliuolo, I: \#1140

Castiaux, J: \#1236

Castillo, AX: \#1339

Castro, K: \#1418

Cataldo, F: \#885 \#886 \#887 \#1259 \#1260 \#1261 \#1262 \#1263 \#1265

Catan, SP: \#338 
Catharino, RR: \#430 \#705 \#1351

Caussignac, C: \#1424

Cavas, M: \#1281

Cavenati, S: \#1025

Cayir, A: \#1239 \#1275

Cazan, IL: \#212

Cecal, A: \#435 \#628

Ceccato, D: \#1126

Celik, A: \#861

Celik, N: \#861

Celik, Z: \#854

Celli, M: \#1005

Cengiz, E: \#324

Cervantes-Martinez, J: \#722

Cevik, U: \#861 \#1090

Chadwick, TG: \#322

Chai, ZF: \#355 \#481 \#578 \#904 \#940 \#957 \#1231 \#1394 \#1456 \#1458 \#1459

Chajduk, E: \#298 \#302

Chakraborty, A: \#214

Chakraborty, N: \#686

Chakraborty, S: \#123

Chakravarty, R: \#1142

Chan, CK: \#232

Chan, WCW: \#384 \#1401

Chang, BU: \#1246

Chang, J: \#1355

Chang, PP: \#108 \#364 \#415

Chang, RPH: \#691

Chang, YL: \#190

Chanton, J: \#219 \#862

Chantrapromma, S: \#14 \#16

Chanyotha, S: \#1378

Chao, JH: \#182 \#190 \#919

Charette, M: \#769 \#986

Charette, MA: \#246 \#992 \#1367

Charkoudian, LK: \#587

Charlton, B: \#650 \#660 \#670

Chartier, F: \#1424

Chat, ZF: \#26

Chatterjee, T: \#1054

Chattopadhyay, P: \#580

Chattopadhyay, S: \#199

Chatziapostolou, A: \#600

Chatzitheodoridis, E: \#1125

Chau, ND: \#1167

Chaudhari, CV: \#483

Chaudhary, A: \#584

Chegrouche, S: \#414

Chelme-Ayala, P: \#1474 \#1477 \#1478

Chen, CL: \#108 \#109 \#133 \#415 \#829

Chen, CM: \#26 \#946 \#1384 \#1406
Chen, CY: \#50 \#345 \#383 \#904 \#1040 \#1221 \#1231 \#1456 \#1459

Chen, F: \#1328

Chen, FR: \#819

Chen, GZ: \#1049

Chen, H: \#462

Chen, HL: \#19

Chen, J: \#574 \#714 \#1395 \#1408

Chen, JL: \#503

Chen, JM: \#156

Chen, JW: \#564

Chen, L: \#25 \#187

Chen, LY: \#570 \#914

Chen, T: \#350

Chen, WF: \#504 \#505 \#820 \#925 \#988

Chen, WK: \#345

Chen, WN: \#4

Chen, XJ: \#1222

Chen, XW: \#56

Chen, YQ: \#491

Chen, YX: \#1026

Chen, Z: \#858 \#944 \#1460

Chen-Braucher, D: \#431

Cheng, BW: \#953

Cheng, DH: \#56

Cheng, JS: \#292

Cheng, KM: \#879

Cheng, WD: \#402

Cherubini, G: \#1180

Chettle, DR: \#40 \#870 \#920 \#1008 \#1187 \#1188 \#1189 \#1190 \#1191 \#1194 \#1482 \#1485 \#1488

Chetty, KV: \#1297

Chiara, CJ: \#1057

Chiba, K: \#97

Chiba, M: \#234

Chichester, DL: \#142 \#188 \#567

Chinol, M: \#200

Chioccola, GS: \#358

Chisholm, C: \#217

Cho, K: \#172

Cho, MH: \#478 \#1133

Cho, SH: \#563 \#1192

Chobola, R: \#1270

Choi, HD: \#1059 \#1105

Choi, J: \#421 \#891

Choi, KH: \#274

Choi, SJ: \#274

Choi, SM: \#1060

Choi, YH: \#787

Chojnacka, K: \#1210

Choppin, GR: \#594 \#985 \#1296 \#1299 \#1302 \#1303 \#1304 \#1306 \#1404 
Choudhury, RP: \#249 \#489 \#933

Chouliaras, N: \#955

Choung, MS: \#1151

Chow, TH: \#120 \#703

Choy, JH: \#903

Chrastny, V: \#484

Christaki, U: \#1051

Christanis, K: \#600

Christian, BT: \#916

Chronis, GT: \#164

Chu, K: \#1220

Chuang, CY: \#182 \#190

Chubarov, VM: \#1490

Chung, H: \#1257

Chung, HH: \#867

Chung, WC: \#917

Chung, YS: \#82 \#237 \#1061 \#1376

Ciardullo, S: \#634

Cincu, E: \#212

Ciobanu, C: \#1364

Cirrone, GAP: \#1100

Cisarova, I: \#927 \#1311

Ciszewski, A: \#696

Ciszewski, D: \#437

Ciurapinski, A: \#1425

Civinini, C: \#1100

Cizmas, CB: \#1360

Clare, SE: \#1

Clarijs, MC: \#689

Clark, NA: \#332

Clark, S: \#802

Clark, T: \#282

Cleary, PW: \#618

Clemens, AW: \#1463

Clocchiatti, R: \#513

Clouvas, A: \#1089

Clynne, MA: \#410

Coakley, KJ: \#1007

Coantic, S: \#1053

Coates, PM: \#45

Coban, F: \#807

Cobos, J: \#954

Coccia, E: \#793

Cocis, A: \#212

Coenen, HH: \#1300

Cohen, DD: \#1376

Cohen, IM: \#1331

Coimbra, J: \#418

Coiro, V: \#112

Colasanti, A: \#1000

Coleman, AW: \#866

Coleman, CL: \#288 \#702

Coleman, M: \#115
Colip, LA: \#685

Colman, JA: \#246

Colombi, P: \#1006

Colvin, V: \#1400

Comanducci, JF: \#371 \#469 \#744 \#769

Compton, RG: \#1055

Comte, J: \#1335

Concheiro, A: \#482

Conen, F: \#1247

Conesa, HM: \#1207

Cong, ZY: \#354

Conry, T: \#30

Constantin, M: \#281 \#282 \#530

Consuegra, RR: \#377

Conte, MH: \#419

Cook, JM: \#645 \#651 \#662 \#671 \#679

Cooney, RV: \#285 \#286

Cooper, RJ: \#1093

Copes, R: \#332

Copplestone, D: \#767

Coppola, L: \#926

Corbiere, A: \#1051

Cordeiro, RC: \#725

Cordell, D: \#532

Cormode, DP: \#1042

Cornelis, R: \#42

Cornett, J: \#664

Cornett, RJ: \#663 \#1420

Coskun, M: \#1239 \#1275

Costa, OS: \#266

Cotuk, Y: \#447 \#1239 \#1240 \#1275

Couch, SJ: \#889

Coulombeau, H: \#1454

Cozar, JS: \#195

Cozzolino, SMF: \#358

Craciun, L: \#212

Crandall, PG: \#106

Creaven, BS: \#375

Crespo, MT: \#195

Cresswell, JR: \#1093

Cretescu, I: \#840 \#1357

Crisci, GM: \#1175

Cristache, C: \#1326

Cronin, L: \#303

Crowell, AS: \#544

Crusius, J: \#246

Csatlos, M: \#1203

Cserpak, F: \#1076

Csige, L: \#1203

Csikai, J: \#139 \#170 \#178

Cubadda, F: \#634

Cubitt, R: \#977

Cuculic, V: \#782 
Cuculovic, AA: \#959

Cui, BQ: \#352

Cukrov, N: \#782

Culicov, OA: \#1326

Cumming, TA: \#663

Cunningham, AP: \#1430

Curkovic, L: \#582

Cusimano, G: \#744

Cutler, C: \#1233

Cuypers, R: \#937

Czajka, A: \#437

Cziraki, A: \#888

da Luz, CA: \#835

da Silva, AAR: \#796

Da Silva, E: \#665

da Silva, MP: \#1428

Dabkowska, AP: \#978

Dadfarnia, S: \#91 \#602

Daga, R: \#160 \#548

Dahlan, KZM: \#899

Dai, M: \#986

Dai, MH: \#504 \#505 \#820 \#925 \#988

Daifullah, A: \#111

Dakladas, T: \#164

Dakshinamoorthy, A: \#897

Dale, DS: \#698 \#818

D'Amato, M: \#634

Damla, N: \#271 \#861

d'Amours, R: \#814

Dampare, SB: \#1469

Daniels, LM: \#595

Danilenko, VN: \#238

Danko, B: \#298

Dankwart, HR: \#186

Dantas, ALA: \#1273

Dantas, BM: \#1273

Dantas, CC: \#1123

Dantelle, G: \#323

Daridon, A: \#915

Darrow, BP: \#819

das Virgens, CF: \#1421

Das, AK: \#868

Das, SK: \#123 \#289

Das, SS: \#199

DasGupta, D: \#123

Dash, A: \#1142

Dastagir, G: \#1174

Davidson, CM: \#98 \#671 \#679

Davis, K: \#870

Davis, PA: \#771

Davis, WC: \#43

Davy, P: \#1376

Day, ES: \#701
Day, MP: \#646 \#652 \#666 \#675

de Alburquerque, C: \#452 \#1374

de Almeida, TL: \#968

De Angelis, A: \#165

de Aquino, FR: \#92

de Assis, JT: \#1432

De Carlan, L: \#1271

De Caro, R: \#1140

de Castro, MDL: \#90

de Celis, B: \#768 \#1082

de Chatel, PF: \#479

De Corte, F: \#390

De Geer, LE: \#814

de Goeij, JJM: \#124

de Haan, AB: \#937

de Jesus, DS: \#1421

de Jonge, MD: \#806

de la Fuente, JM: \#303

de la Fuente, R: \#768

de la Fuentea, R: \#1082

De La Guardia, M: \#487

de las Heras, LA: \#610

De Luca, G: \#793

De Luca, P: \#945

De Medeiros, LM: \#373

de Meijer, RJ: \#166

de Miranda, RL: \#1206

de Oliveira, J: \#468 \#469 \#769

de Pablo, H: \#995

de Pablo, J: \#911

De Pascale, MP: \#690

De Presbiteris, D: \#1175

de Rosales, RTM: \#1453

de Silveira, G: \#1488

de Souza, SMFM: \#569

de Vries, G: \#1247

de Vries-Melein, M: \#1005

De Wispelaere, A: \#390

de Wolf, FA: \#1402

De, SK: \#888

Death, DL: \#1430

Degerlier, M: \#1284

Degetto, S: \#112

Deguchi, M: \#1410

Dehairs, F: \#404

Dehnhardt, M: \#608

DeJesus, OT: \#916

del Canto, V: \#768

Del Razo, LM: \#1359

Delgado, DEG: \#377

Deliorman, M: \#1435

Delmau, LH: \#1405

Delmore, JE: \#612 
Demadis, KD: \#1022

Demaree, JD: \#1137

Demetriou, A: \#533

Demetriou, P: \#1114

Demirer, GN: \#1268

Demirkeser, TH: \#35

Den Auwer, C: \#1053

Denby, PM: \#1106

Deng, B: \#1475

Deng, BL: \#291 \#462 \#1473

Deng, DL: \#1415

Deng, PC: \#1415

Deng, SG: \#856

Deng, W: \#1352

Depero, LE: \#1006

Depoi, FS: \#599

Desideri, D: \#748 \#775 \#801 \#1015 \#1457

Destan, UI: \#620

Desvaux, H: \#1454

Detriche, S: \#521

Devaraj, NK: \#1436 \#1437

Devlin, E: \#888

DeVol, TA: \#52

Devol, TA: \#327

Dhami, PS: \#60

Dhanalakshmi, B: \#585

Dhekane, G: \#1407

Dhingra, P: \#869

Dhole, SD: \#1143

D'hulst, A: \#281

Di Bartolo, N: \#1173

Di Domenico, G: \#1486

Di Giandomenico, MV: \#596

Di Lullo, A: \#165

Di Muro, A: \#508 \#513

Di Nicola, L: \#1235

Di, Y: \#1343

Diaco, L: \#382

Diagaradjane, P: \#563 \#702

Diamandescu, L: \#1360

Diaz-Archundia, LV: \#704

Diaz-Bone, RA: \#1232

Diaz-Delgado, C: \#1483

Dibb, JE: \#816

Dieguez, MD: \#333

Dies, J: \#911

Dieterle, DA: \#819

Dietz, ML: \#1387 \#1440 \#1444

Dikiy, NP: \#1218

Dikmen, H: \#1447

Dilek, FB: \#1268

Dimitrova, K: \#168

Dimova, N: \#465 \#523
Dimovska, S: \#1289

Din, KS: \#1276

Dincalp, H: \#1138

Ding, BG: \#546

Ding, KF: \#1341

Ding, N: \#1050 \#1478

Ding, YJ: \#18

Dionisio, I: \#1070

Dionisloa, I: \#1081

Divi, SM: \#253

Djingova, R: \#37

Do Oh, Y: \#1133

Do, JB: \#427

Doblado, FS: \#1193

Doble, P: \#1011

Dobosi, V: \#225

Dobson, J: \#617

Doczi, R: \#139 \#170 \#178

Dodge, CJ: \#461 \#592

Dodziuk, H: \#715

Dogadkin, NN: \#301

Doglioni, C: \#793

Dogra, R: \#736

Dogru, M: \#180

Doiron, AL: \#1220

Dokic, D: \#1048

Dolhanczuk-Srodka, A: \#1472

Dolley, SG: \#1103 \#1132

Dolphin, AE: \#41

Donard, OFX: \#657

Dong, KJ: \#353 \#1110

Dong, YH: \#187 \#844

Donlon, DF: \#375

Donohue, D: \#1425

Dorantes-Rosales, HJ: \#547

Dorman, J: \#118

Dorman, JW: \#446

Dorohoi, DO: \#1364

dos Santos, MG: \#1123

dos Santos, VA: \#1123

dos Santos, WNL: \#1421

Dosanjh, HS: \#965

Dou, S: \#1328

Douglas, M: \#66

Dovbnya, AN: \#874 \#1218

Dowdall, M: \#791

Downes, H: \#929 \#931

Doyle, O: \#217

Dragan, ES: \#1361

Drangert, JO: \#532

Drezek, R: \#1400

Drochioiu, G: \#628 \#1361

Drohe, JC: \#1101 
Drot, R: \#711

Drozdzal, K: \#1230

Druteikiene, R: \#1058

Drzewicz, P: \#1478

Du, N: \#740

Du, Z: \#56 \#329

Duan, GJ: \#731

Duan, WH: \#1395 \#1408

Duarte, AC: \#308 \#310 \#418

Duarte, CL: \#1258 \#1264

Duarte, MEL: \#569

Dubey, KA: \#483

Dubey, RS: \#136

Dubois, G: \#1247

Dukov, IL: \#319

Dulaiova, H: \#522 \#724 \#744 \#981 \#993

Duliu, OG: \#1326

Dullmann, CE: \#318 \#1201

Dumont, E: \#42

Dunkley, CS: \#106

Dunkley, KD: \#106

Dunn, CE: \#1039

Durak, H: \#127

Duran, C: \#845

Durand, I: \#1051

Durrant, SF: \#642

Dursun, ER: \#1138

Duskesas, G: \#1058

Dutta, S: \#868 \#1054

Duval, PB: \#589

Dvorak, J: \#1201

Dvorakova, Z: \#1201

Dwivedi, KK: \#1099

Dwyer, JT: \#45

Dybal, J: \#1032 \#1034 \#1035 \#1494

Dybczynski, RS: \#298

Dykiy, MP: \#874

Eberhardt, K: \#1201

Eberlin, MN: \#430 \#659 \#705 \#1351

Ebersbach, F: \#1051

Ebraheem, S: \#717

Eby, N: \#969

Eckelman, WC: \#1139

Edrev, ZH: \#168

Edwards, GS: \#1219

Edwards, HGM: \#1468

Efimova, YM: \#260

Egorov, OB: \#54 \#327

Ehrlich, H: \#1022

Eichler, R: \#1201

Ei-Sweify, RH: \#1309

Eklund, PC: \#3

El Afifi, EM: \#780
El Arabi, AM: \#1276

El Mensi, R: \#370

El-Absy, MA: \#1386

El-Amir, MA: \#1386

El-Ashgar, N: \#93

El-Azony, KM: \#878 \#1315

Elci, L: \#845

El-Dessouky, MI: \#830

El-Dessouky, SI: \#28

El-Din, AG: \#1477

El-Din, MG: \#1474 \#1477 \#1478

Elewaut, K: \#362

El-Gammal, B: \#693

El-Garhy, MA: \#1386

El-Geziry, TM: \#889

El-Ghany, EA: \#873

Elish, E: \#653

Elizarov, AM: \#915

El-Kamash, AM: \#305 \#826 \#830

El-Kawy, OA: \#873

Elkholy, SS: \#290

Ellam, RM: \#1377

Elliot, N: \#664

Ellis, AT: \#643 \#648 \#658 \#669 \#677

Elmgren, K: \#814

El-Mohty, AA: \#878

El-Mouhty, NRA: \#1393

El-Naggar, MR: \#830

Elnimr, T: \#191

El-Reefy, SA: \#780

Elsabee, MZ: \#290

El-Shahat, MF: \#1386

El-Sharif, M: \#217

El-Sheikh, AH: \#1442

El-Sofany, EA: \#828 \#1320

Elson, MD: \#1463

El-Turki, A: \#84

El-Zakla, T: \#1347

Ene, A: \#1344

Engeler, C: \#168

Enginar, H: \#284 \#572

Epov, V: \#664

Epov, VN: \#657

Epplein, M: \#286

Eral, M: \#776 \#913 \#1256

Eriksson, M: \#999

Erol, E: \#605

Ertay, T: \#127

Ertugral, B: \#271

Escoube, R: \#405

Esnouf, S: \#1053

Esparza, H: \#195

Estellita, L: \#796 
Eswaran, P: \#1148

Etxebarria, N: \#1418

Evans, D: \#664

Evans, NDM: \#1312

Evans, RD: \#657 \#1420

Eykens, R: \#1101

Ezzat, K: \#27

Fabian, M: \#905 \#906 \#907 \#908

Facer, J: \#931

Faestermann, T: \#1203

Faghihi, R: \#1150 \#1171

Faghihian, H: \#602 \#627 \#859 \#1020

Faheem, M: \#950

Falcomer, R: \#664

Falkenberg, G: \#1484

Falkenberge, G: \#1266

Fallu-Labruyere, A: \#1080

Fan, FL: \#318

Fan, P: \#352

Fan, QH: \#364 \#1026 \#1341

Fan, WW: \#143

Fang, G: \#485

Fang, M: \#848

Fang, PF: \#944

Fang, ZL: \#56

Fanning, KA: \#819

Fantidis, JG: \#1255

Farai, IP: \#952 \#1348

Faria, I: \#272

Farias, PCMA: \#474

Farinha, MM: \#213 \#441 \#603 \#1470

Farmer, JG: \#1377

Farmer, OT: \#66

Farquharson, MJ: \#1266 \#1484

Fasih, TW: \#1386

Fassbender, M: \#1112

Fatibello, O: \#92

Fatima, I: \#1319

Faupel, F: \#1206

Favaro, DIT: \#358 \#1269

Favre, G: \#1424

Fawdry, RM: \#144

Fayad, ZA: \#1042

Feduzi, L: \#748 \#775 \#801 \#1015

Fejfarova, K: \#1311

Feldt, J: \#1100

Felfoul, HS: \#370

Felhosi, I: \#426

Felner, I: \#687

Feltz, A: \#340

Feng, M: \#858 \#1397

Feng, SL: \#1131

Feng, XG: \#1095
Feng, XQ: \#1131

Feres, MA: \#92

Fernandesc, EAD: \#341

Fernandez-Pacheco, R: \#1043

Ferreira, AV: \#268

Ferreira, FA: \#494

Ferreira, SLC: \#92 \#1421

Ferreira, TR: \#418

Ferrero, C: \#963

Ferretti, E: \#1006

Ferreux, L: \#1335

Ferro-Flores, G: \#223

Festa, G: \#690

Fettig, NM: \#1234

Feuerstein, J: \#773

Fialho, P: \#1081

Field, CV: \#817

Figueiredo, PM: \#498

Filby, RH: \#1066

Filer, CN: \#876 \#877 \#883

Filipovic, DM: \#205

Finck, N: \#466

Finkelshtein, AL: \#1490

Finoia, MG: \#1000

Fiore, T: \#32

Fiori, CCL: \#38

Firastrau, I: \#1360

Fischbach, E: \#1412

Fischer, H: \#1271

Fischer, HC: \#384

Fisher, AS: \#644 \#650 \#660 \#670 \#678

Fisher, KD: \#45

Flores-Rangel, RM: \#547

Flues, M: \#498

Fluhler, EN: \#934

Foerstendorf, H: \#464

Fontas, C: \#1353

Ford, R: \#1184

Forkapic, S: \#1245

Forlini, F: \#34 \#886

Formica, SM: \#548

Foronda, J: \#724

Fosterova, M: \#177

Fouche, FJ: \#168

Fowler, JF: \#373

Foyo, A: \#226

Fraenzle, S: \#37

Fragneto, G: \#978

Fragopoulou, M: \#103

Frana, J: \#527 \#1012

Franca, AS: \#490

Francis, AJ: \#461 \#592

Franck, D: \#1271 \#1274 
Franke, AA: \#285 \#286

Frankowska, P: \#299

Fransway, AF: \#373

Franz, KJ: \#587 \#588

Franzen, S: \#1046

Fray, DJ: \#1010

Frechou, C: \#154

Frei, R: \#562

Freire, MM: \#1374

Freitas, MC: \#213 \#231 \#441 \#1070 \#1071 \#1369 \#1470

Freitas, MD: \#603 \#635

Freitas, MDC: \#1081

Fricke, B: \#1195

Friedman, M: \#630 \#632

Friedrich, J: \#890

Friese, JI: \#1345

Frischmuth, S: \#264

Fritsch, D: \#1206

Froidevaux, P: \#778

Frontasyeva, M: \#1289

Frontasyeva, MV: \#1177 \#1326

Fu, HJ: \#1477

Fu, JWP: \#732

Fuda, JL: \#1051

Fuga, A: \#449

Fujii, T: \#938 \#939

Fujii, Y: \#59 \#1225

Fujimoto, T: \#501

Fujisawa, H: \#318

Fujita, H: \#924

Fujiyoshi, R: \#1370

Fukagawa, M: \#556

Fukasawa, M: \#1371

Fukuda, Y: \#1013

Fukumoto, Y: \#1018

Fukumura, T: \#1315

Fun, HK: \#14 \#16

Furui, S: \#99

Furuta, M: \#918

Furutaka, K: \#506 \#1185 \#1362

Gable, RW: \#1215

Gac, W: \#294

Gaca, P: \#299 \#1165 \#1212

Gacsi, Z: \#1203

Gad, HMH: \#619 \#1393

Gadegaard, N: \#303

Gagneten, AM: \#1471

Gaillard, C: \#400 \#472 \#534

Gajowiak, A: \#1230

Gal, JF: \#641

Galanopoulos, S: \#1113 \#1114

Galenas, A: \#1317

Galeriu, D: \#771
Gallego, E: \#1286

Galler, P: \#773

Gancarz, I: \#696

Ganesan, S: \#478 \#1133

Gang, DC: \#291

Ganju, D: \#435

Gao, F: \#347

Gao, HW: \#342

Gao, XY: \#940

Gao, YX: \#904 \#1221 \#1231 \#1456

Garbarino, G: \#1288

Garcia, AR: \#1198

Garcia, CM: \#430

Garcia, HM: \#841

Garcia, JF: \#78

Garcia, L: \#1353

Garcia, N: \#1051

Garcia, VN: \#1198

Garcia-Morales, V: \#339

Garcia-Orellana, J: \#168 \#849

Garcia-Ruiz, E: \#1001

Garcia-Sartal, C: \#633

Garcia-Tasende, MS: \#374

Garcia-Tenorio, R: \#849

Garcia-Vazquez, E: \#218

Gardiner, PHE: \#73

Gardner, RP: \#1116 \#1144

Garg, AN: \#249 \#489 \#932 \#933

Garguilo, J: \#1219

Garoosi, J: \#1313

Garousi, J: \#21 \#625 \#626 \#1164 \#1171

Garwan, MA: \#198 \#1094

Gasa, J: \#849

Gasco, C: \#168

Gasiorowski, M: \#517

Gasser, MS: \#1382

Gastaud, J: \#168 \#469 \#926

Gatto, L: \#744

Gauthier-Lafaye, F: \#1179

Gavilan, KC: \#841

Gavrilescu, M: \#840

Ge, CC: \#1456 \#1459

Gebhardt, R: \#977

Geckeis, H: \#456 \#726

Geiger, FM: \#941

Geipel, G: \#1216 \#1419

Gelsler, T: \#962

Gentleman, DJ: \#1401

Geraedts, K: \#116

Geraki, K: \#1484

Gerasilmova, LG: \#590

Gerbier-Violleau, M: \#1184

Gerli, S: \#548 
German, U: \#134 \#1085 \#1250

Gerringa, L: \#1051

Gerstmann, U: \#749 \#784 \#1241

Ghaedi, M: \#827 \#843

Ghanem, BS: \#1206

Ghannadi-Maragheh, M: \#357 \#1390

Ghauri, BM: \#233

Ghazvini, PTM: \#261 \#262 \#263 \#554

Gholami, E: \#1164

Ghonaim, AK: \#830

Ghosh, AN: \#686

Ghosh, K: \#243

Ghosh, S: \#123

Giaretta, A: \#112

Giecko, G: \#294

Giesy, JP: \#457

Giles, DE: \#737

Gil-Garcia, C: \#786

Gill, JK: \#285

Gilligan, C: \#154

Gill-Sharp, KL: \#563 \#702 \#1044

Gingasu, D: \#1360

Giordano, D: \#513

Giridhar, P: \#423 \#550 \#637 \#1292 \#1294

Giridhar, R: \#412

Giron, MC: \#1140

Giussani, A: \#749 \#1241

Giussani, B: \#87

Giusti, AM: \#690

Gladis, JM: \#77

Glascock, MD: \#697

Glenn, CR: \#982

Gleyzer, A: \#1080

Glorius, M: \#269 \#1214

Gmeling, K: \#509 \#929 \#930 \#969 \#1028 \#1235

Gmitro, AF: \#914

Go, F: \#365

Gobar, LS: \#570 \#914

Gobbino, M: \#885 \#886

Godelitsas, A: \#1125

Godoy, JM: \#267 \#750 \#785

Godoy, MLDP: \#750 \#785

Goel, NK: \#483

Gok, C: \#847

Golas, J: \#1211

Goldberg-Oppenheimer, P: \#1399

Goldman, ID: \#1480

Golenko, TG: \#1217

Golovko, VV: \#476

Golub, T: \#381

Gomes, PRS: \#1197 \#1198

Gomez, F: \#1193

Gomez, J: \#865
Goncalves, CR: \#824

Goncalves, F: \#1373

Gong, J: \#353

Gong, JS: \#387

Gonzales, ER: \#176

Gonzalez, EC: \#1339

Gonzalvez, A: \#487

Goodall, PS: \#644 \#650 \#660 \#670 \#678

Goodenough, JB: \#735

Goodman, AH: \#41

Goodman, MT: \#285 \#286

Goodwin, JR: \#476

Gordon, RE: \#1042

Gorini, G: \#1172

Gorller-Walrand, C: \#472

Goryunov, EI: \#1009

Goswami, A: \#583 \#1068

Gothankar, S: \#783

Gothe, DA: \#1096

Gotic, M: \#1003

Goto, J: \#1202 \#1204

Goto, K: \#377

Goto, S: \#318

Gouder, T: \#962

Gougoulias, N: \#955

Gouseti, O: \#1253

Gowrishankar, R: \#253

Grabmayr, P: \#477

Gradinaru, R: \#436

Graf, W: \#683 \#1091

Graham, I: \#1468

Graham, MC: \#1377

Grahek, Z: \#1450

Granados-Correa, F: \#366

Grande, CA: \#1025

Granet, M: \#1424

Grate, JW: \#54 \#64 \#65 \#327

Gravanis, F: \#955

Grazzi, F: \#1005

Grbec, B: \#496

Green, AEC: \#279

Green, ID: \#467

Green, T: \#1104

Greenberg, RR: \#341

Greene, GA: \#158

Greenfield, MB: \#688

Greenwood, LR: \#66

Greenwood, WJ: \#523 \#990

Gregoric, A: \#764

Greiter, M: \#749

Greneche, JM: \#559 \#888

Greneche, M: \#479

Grenier, AC: \#722 
Gresham, GL: \#1435

Griffith, CS: \#976

Griffiths, B: \#1051

Grimm, R: \#1011

Grinberg, P: \#649 \#674 \#1427

Grint, AN: \#1093

Gritsan, LD: \#1218

Gritzner, G: \#479

Grlj, N: \#122 \#1489

Groenewold, GS: \#1435

Groening, M: \#469 \#744

Groppi, F: \#124 \#134 \#200

Grover, P: \#1036 \#1037

Grozdov, DS: \#301

Gruenwald, JT: \#1412

Gruner, B: \#927 \#1311

Grzywaczewski, G: \#1212

Gu, B: \#458

Gu, XH: \#953

Gu, YQ: \#1459

Guan, YJ: \#1110

Gudelis, A: \#168 \#1058

Guerra, DL: \#706

Guevara, SR: \#160 \#248 \#251 \#333 \#338 \#548

Guibal, E: \#552 \#841

Guigue, C: \#1051

Guilizzoni, P: \#548

Guillerm, C: \#1051

Gulfen, M: \#553 \#854

Gullova, M: \#398

Gulyas, J: \#1203

Gumuser, FG: \#1138

Gundogdu, A: \#845

Gundogdu, O: \#1254 \#1266 \#1484

Gunduz, L: \#753

Gunsing, F: \#1078

Gunther-Leopold, I: \#46

Guo, M: \#365

Guo, N: \#163

Guo, YX: \#1202

Guo, ZJ: \#1323

Gupta, A: \#1354

Gupta, DC: \#1354

Gupta, SK: \#229

Gupta, VK: \#379

Gupta, Y: \#380

Gurbuz, G: \#1277

Gurevich, YL: \#598

Gurler, O: \#1254

Gustafsson, O: \#986

Gustov, VV: \#545

Gutierrez-Pulido, H: \#722

Gutierrez-Villanueva, JL: \#768
Gyorffy, J: \#1188

Ha, JJ: \#1060

Ha, XD: \#566

Ha, YK: \#197

Haba, H: \#317 \#318

Habbani, FI: \#1285

Habchi, C: \#1135

Habs, D: \#1203

Haemers, S: \#260

Hagiwara, M: \#1153

Hagler, GSW: \#816

Haines, T: \#40

Hajipour, A: \#8

Hajishabani, A: \#602

Hakimabad, HM: \#1154

Hakimi, A: \#625

Hakl, J: \#479 \#888

Halas, NJ: \#1

Halder, S: \#1054

Hallam, KR: \#84

Halloran, K: \#246

Halova, J: \#1209

Hamada, Y: \#1092

Hamano, T: \#1134

Hameed, I: \#1174

Hamid, S: \#346

Hamilton, TE: \#1124

Hamilton, TF: \#1111

Han, HY: \#1131

Han, SH: \#197 \#215

Han, XF: \#221

Han, XG: \#1144

Hanafy, M: \#305

Hancock, G: \#168

Handley, M: \#723

Hanson, AL: \#158

Hao, XF: \#1121

Harada, H: \#918 \#1185

Harada, N: \#631

Harambasic, M: \#803

Harangi, S: \#929

Hardy, CJ: \#45

Hardy, JC: \#476

Hare, D: \#1011

Hargreaves, MD: \#1468

Harkness, LJ: \#1093

Harleman, C: \#417

Harmon, SM: \#1479

Harnly, J: \#45

Harrington, CF: \#645 \#651 \#662 \#671

Harrington, LE: \#279

Harris, MN: \#685

Harrison, C: \#640 
Harrison, RK: \#1077 \#1130

Harrup, MK: \#595

Hartwell, JK: \#1065

Hasan, R: \#442

Hasanzadeh, A: \#627

Hasegawa, H: \#743

Hasegawa, M: \#1204

Hasegawa, T: \#318 \#560

Hashiguchi, N: \#1411

Hashimoto, K: \#104

Hashimoto, T: \#515 \#1109

Hassan, HS: \#28

Hassan, SSM: \#837

Hassanzadeh, S: \#997

Hatsukawa, Y: \#506 \#562 \#1185 \#1202 \#1204

Hauser, S: \#744

Hauta, OR: \#435

Havel, J: \#1438

Havel, L: \#433

Hawari, AI: \#564

Hayashi, H: \#62 \#918

Hayashi, K: \#62 \#316

Hayashi, M: \#1018

He, GZ: \#353

He, JH: \#19 \#972

He, M: \#353 \#1110

He, QG: \#1095

He, R: \#940

He, X: \#1458

Heath, JR: \#915

Hedberg, PML: \#672

Hegde, AG: \#1280

Heikkinen, T: \#541

Heil, CA: \#819

Heimann, J: \#1100

Heimsath, AM: \#520

Heirwegh, CM: \#1194

Hellebust, S: \#236

Hemet, P: \#661

Hemvichian, K: \#311

Henderson, BE: \#285 \#286

Henderson, P: \#246

Hennig, C: \#400 \#472 \#591

Hennig, W: \#565

Hennig, WG: \#1080

Herbst, RS: \#396 \#1324 \#1405

Herczeg, AL: \#983

Herman, M: \#1056

Hermann, P: \#177

Hernadi, L: \#901 \#1052

Hernandez, S: \#223

Hernandez-Cortes, S: \#704

Hernandez-Lopez, S: \#836
Herniman, J: \#1350

Herppich, WB: \#683 \#1091

Hertenberger, R: \#1203

Herut, B: \#523

Hespanhol Da Silva, MD: \#1237

Hesselmann, A: \#715

Heyd, DV: \#665

Hicks, RW: \#864

Hicsonmez, U: \#776

Hidaka, H: \#1179

Hien, PD: \#1376

Higuchi, Y: \#1134

Hilal, MA: \#780

Hilger, A: \#683 \#1091

Hilgers, K: \#1300

Hill, P: \#195

Hill, SJ: \#645 \#651 \#662

Hill, TS: \#1200

Hillegonds, DJ: \#1124

Hinds, MW: \#644 \#650 \#660 \#670 \#678

Hino, A: \#99

Hirai, I: \#72

Hiraki, A: \#1013

Hirata, M: \#694

Hirayama, N: \#1410

Hirayama, Y: \#1185

Hiripi, L: \#901

Hirose, K: \#167 \#234 \#395 \#524 \#794 \#1371 \#1380

Hirose, Y: \#560

Hisamatsu, S: \#743 \#759 \#760 \#788 \#1368

Hladil, J: \#273

Hlatshwayo, IN: \#166

Hoellriegl, V: \#1271

Hoeschen, C: \#540

Hoffman, DC: \#708

Hoffmann, G: \#47

Hoffmann, V: \#37

Hohmann, M: \#520

Holden, J: \#45

Holden, JE: \#916

Holgye, Z: \#1349

Holland, JG: \#941

Holland, JP: \#1030 \#1141

Hollriegl, V: \#540 \#1272

Holm, E: \#168

Homazava, N: \#656

Homonnay, Z: \#217 \#376 \#479 \#556 \#559 \#888 \#1434

Hong, DG: \#1109

Hong, HL: \#110 \#1416

Hong, MM: \#903

Hong, SS: \#427 \#1151

Hong, YD: \#274

Honjo, T: \#1410 
Honma, M: \#1204

Hoollriegl, V: \#749

Hopke, PK: \#1376

Horansky, RD: \#692

Horie, K: \#1179

Horii, CL: \#494

Hortellani, MA: \#1238

Horvat, M: \#764

Hoshi, H: \#23

Hossain, MM: \#99

Hosseini, MA: \#21 \#1160

Hosseini, MRM: \#74

Hotchkis, MAC: \#153

Hou, S: \#723

Hou, XD: \#1415

Hou, XL: \#57 \#70 \#81 \#89

Hough, RM: \#514

Hovell, AMC: \#705

Howard, BJ: \#767

Howell, CR: \#544

Howell, RW: \#622

Hristodor, C: \#1356

Hrnecek, E: \#1314

Hsieh, BT: \#194

Hsu, FY: \#190

Hu, GR: \#448 \#798

Hu, J: \#367 \#842 \#844

$\mathrm{Hu}, \mathrm{Q}: \# 1342$

Hu, QH: \#575 \#578 \#770 \#946 \#1394

Hu, YF: \#1352

Hu, YM: \#1110

Hua, B: \#1473 \#1475

Huang, CT: \#353

Huang, FY: \#194

Huang, J: \#915

Huang, JB: \#858

Huang, LK: \#194

Huang, MD: \#1422

Huang, Q: \#256 \#367

Huang, SS: \#190

Huang, W: \#944

Huang, YD: \#1391

Huang, YH: \#735

Huber, F: \#220

Huber, H: \#114

Hubert, A: \#661

Hubicki, Z: \#728

Huck, CW: \#616

Hueglin, C: \#230

Hughes, AV: \#978

Hughes, LD: \#52

Huhtinen, M: \#1064

Huittinen, N: \#726
Humbert, B: \#711

Humelnicu, D: \#855 \#1356

Hung, CC: \#419

Hur, JM: \#427 \#638 \#1062 \#1151

Hurel, C: \#1441

Hurley, JP: \#568

Hurtado, S: \#849

Husain, L: \#233

Husain, SW: \#1310

Hussain, F: \#1174 \#1215

Hussain, S: \#948

Hussain, SM: \#1036 \#1037

Hussain, SY: \#312

Hutcheon, ID: \#1111

Iacob, VE: \#476

Ibarra, MR: \#1043

Ibikunle, K: \#102

Ibn Majah, M: \#928

Ibrahim, GM: \#693

Ibrahim, HA: \#305 \#307

Ibrahim, YV: \#162

Ideguchi, E: \#1185

Idiri, Z: \#1079

Igarashi, Y: \#167 \#234 \#794 \#1380

Igashira, M: \#917

Iglesias, A: \#1193

Iida, A: \#800

Iida, T: \#137

Iikura, H: \#1092

Iio, S: \#560

Ikaheimonen, TK: \#168

Ikeda, A: \#59

Ikeda, Y: \#1226

Ikeda-Ohno, A: \#591

Ikehata, K: \#1474

Ilarraza, H: \#223

Ilchmann, C: \#168

Ilmberger, J: \#549

Ilus, T: \#541

Im, HJ: \#216

Imaizumi, H: \#422

Imakura, S: \#55

Imamura, M: \#159 \#1242

Imanaka, T: \#1242

Imanishi, T: \#1370

Imaseki, H: \#1134

Imberti, S: \#1005

Imperiale, ML: \#1005

Inaba, J: \#743 \#759

Inacio, AF: \#452 \#1374

Inal, T: \#808

Inan, S: \#1398

Inceoz, M: \#180 
Incerti, S: \#1135

Ingelbrecht, C: \#1076

Ingeneri, K: \#672

Iniguez, MP: \#1286

Inkster, JAH: \#1155

Inn, KGW: \#154

Inomata, Y: \#234 \#794

Inoue, $\mathrm{J}:$ \#752

Inoue, $\mathrm{K}: \# 1411$

Inyang, EP: \#173

Ioannou, KIG: \#1451

Ioannucci, L: \#793

Ioris, AAR: \#741

Iqbal, MS: \#1308

Irabien, MJ: \#865

Iriarte, E: \#226

Irwin, KD: \#692

Isaac-Olive, K: \#1359

Ishigaki, M: \#688

Ishihama, S: \#1107

Ishihara, T: \#1226

Ishii, Y: \#316 \#318 \#422

Ishikawa, T: \#1134

Ishikawa, Y: \#631

Ishitobi, T: \#724 \#1378

Ishizawa, K: \#631

Isinkaye, AO: \#1249

Isinkaye, MO: \#952 \#1348

Iskhakov, RS: \#598

Ismail, AF: \#1366

Ismail, EC: \#1266

Isnard, H: \#1424

Iso, $\mathrm{H}$ : \#1134

Issa, H: \#100

Istvan, B: \#623 \#623

Ito, $\mathrm{N}$ : \#688

Ito, Y: \#159 \#387 \#1242

Iturralde-Vinent, MA: \#377

Ivsic, AG: \#1450

Iwanaga, S: \#631

Iwasaki, S: \#159 \#1242

Iwata, A: \#688

Izbicki, JA: \#470

Izquierdo, JI: \#218

Jabbar, A: \#1283

Jabbar, T: \#1283

Jacimovic, R: \#5 \#1318 \#1333

Jacks, G: \#417

Jacob, W: \#967

Jacobson, MS: \#186

Jacquet, S: \#1051

Jacquet, SHM: \#404

Jaffe, JE: \#1073
Jager, E: \#1201

Jagesia, P: \#60

Jahlian, AR: \#131

Jain, A: \#229 \#730

Jain, M: \#942

Jain, SK: \#380

Jaison, TJ: \#1280

Jakab, S: \#1440

Jakopic, R: \#611

Jalbani, N: \#250

Jalilian, AR: \#20 \#21 \#126 \#625 \#626 \#875 \#1146

\#1147 \#1150 \#1157 \#1158 \#1159 \#1160 \#1161

\#1164 \#1170 \#1171 \#1176 \#1287 \#1291 \#1295

\#1298 \#1313 \#1321

Jaluul, O: \#967

Jamali, MK: \#250

Jamali, MR: \#74 \#900 \#1449

James, C: \#30

James, WD: \#224

Jamriska, DJ: \#1112

Janes, J: \#373

Jankovic, D: \#1048

Jankovska, V: \#273

Janos, S: \#623

Janusz, W: \#728

Jardin, R: \#963

Jarman, KD: \#571

Jarvis, K: \#115

Jayakumar, M: \#424

Jeandel, C: \#926 \#992 \#1051

Jedinakova-Krizova, V: \#755

Jee, KY: \#197

Jehangir, M: \#1322

Jelinek, E: \#1039

Jenkins, JH: \#1412

Jenkinson, AV: \#168

Jeon, YS: \#215

Jeong, SM: \#427 \#636 \#638 \#1062 \#1151

Jesus, NC: \#347

Jeyakumar, S: \#1445

Jha, SK: \#783

Ji, AJ: \#934

Ji, GB: \#349

Ji, Q: \#568

Jia, GG: \#132 \#140 \#165 \#789

Jia, HM: \#143 \#880

Jia, L: \#383

Jiang, GB: \#457

Jiang, H: \#972

Jiang, S: \#353 \#1110

Jiang, WT: \#110 \#1416

Jiao, F: \#904 \#1456 \#1459

Jimenez, L: \#223 
Jin, J: \#1478

Jindal, PK: \#979

Jing, L: \#940

Jirsa, F: \#296

Jo, HJ: \#335 \#867

Jobbagy, V: \#1017

Jochens, AE: \#819

Jochum, J: \#477

Jochum, KP: \#529

Jodin-Caumon, MC: \#711

Joe, K: \#215

Johnson, AG: \#982

Johnson, PR: \#224

Johnson, R: \#405

Johnsson, A: \#257

Jokic, S: \#103

Jolie, J: \#206 \#477

Jolliff, JK: \#819

Jonah, SA: \#101 \#102 \#130 \#162 \#173

Jones, B: \#280

Jones, BL: \#1192

Jones, DG: \#998

Jones, IP: \#896

Jones, R: \#154

Jones, VD: \#1448

Joo, JS: \#923

Jordan, DV: \#1073

Jordanova, D: \#37

Jordanova, N: \#37

Jorgensen, UG: \#562

Jose, MT: \#1278

Joseph, TB: \#386

Jose-Yacaman, M: \#121

Joshi, AP: \#386

Jost, C: \#1129

Jovanovski, G: \#1318

Juarez, F: \#125

Juge, P: \#521

Jui, BJ: \#1060

Jull, AJT: \#22

Jung, EC: \#215

Jung, J: \#867

Jung, JH: \#335

Jung, JY: \#636

Jung, NS: \#1059

Jungck, MHA: \#778

Juodis, L: \#1058

Jurisson, S: \#1233 \#1307 \#1316

Jurisson, SS: \#1317

Jurkin, T: \#1003

Juvigne, E: \#1236

Kaabar, W: \#1266

Kabiri-Tadi, M: \#1020
Kacmaz, H: \#434

Kacperek, A: \#181

Kadar, P: \#376

Kadi-Hanifi, M: \#765

Kadioglu, YK: \#1414

Kaestner, A: \#1207

Kah, JCY: \#120 \#703 \#732

Kailas, S: \#1143

Kaiser, JM: \#3

Kajcsos, Z: \#1084

Kajiwara, T: \#502

Kakiuchi, T: \#55

Kalaitzidis, S: \#600

Kalef-Ezra, J: \#1119

Kalenchuk, V: \#984

Kalfas, CA: \#1113

Kalinowski, MB: \#369

Kalkreuth, WD: \#599

Kallias, G: \#888

Kalman, E: \#426

Kalmykov, S: \#792

Kalmykov, SN: \#1009

Kalnina, L: \#242

Kalsi, PC: \#174 \#583

Kalt, A: \#314 \#509 \#512 \#930

Kamali, M: \#627 \#996 \#997

Kamalidehghan, M: \#131 \#1157

Kamali-Dehghan, M: \#20 \#625 \#1147 \#1170

Kamei-Ishikawa, N: \#762 \#922

Kamel, L: \#348

Kamer, O: \#639

Kamikawa, T: \#556

Kamnev, AA: \#73 \#555 \#1434

Kampic, S: \#803

Kamrani, YY: \#1298

Kamudu, MV: \#196

Kanatzidis, MG: \#1050

Kandhro, GA: \#250

Kaneko, K: \#1204

Kanematsu, Y: \#631

Kang, DS: \#1151

Kang, M: \#1328

Kang, N: \#172

Kang, S: \#723

Kang, SC: \#354

Kang, SY: \#275

Kanibolotskyy, V: \#984

Kanisch, G: \#168 \#201

Kannan, R: \#1317

Kannan, S: \#589

Kanngiesser, B: \#122 \#1489

Kantoglu, O: \#1268

Kaplan, A: \#812 
Kaplan, DI: \#463

Kaplankiran, M: \#35

Kapsimalis, R: \#208 \#209

Karabidak, SM: \#1090

Karadeniz, O: \#1279

Karadeniz, S: \#620

Karahan, IH: \#1205

Karami, H: \#1338

Karandashev, V: \#252

Kardjilov, N: \#683 \#1091

Karime, M: \#539

Karimian, A: \#875 \#1160

Karimian, AR: \#1161

Karir, T: \#203

Karjou, J: \#1429

Karpas, Z: \#540 \#653

Kars, A: \#832

Karve, M: \#413

Karydas, AG: \#122 \#1489

Kasa, I: \#1270

Kasaishi, M: \#918

Kasamatsu, Y: \#318

Kaspari, S: \#723

Kaste, JM: \#520

Kasviki, K: \#1119

Kasztovszky, Z: \#225 \#509 \#1028

Katada, M: \#317

Kato, H: \#558

Kato, N: \#422

Katti, KV: \#1317

Kaushik, CP: \#831

Kavasi, N: \#623 \#1017 \#1243

Kawade, K: \#918

Kawakami, N: \#556

Kawakita, H: \#1411

Kawasaki, T: \#13

Kawashima, H: \#104

Kawazoe, K: \#631

Kaya, S: \#1090

Kaynak, G: \#1254

Kaynak, I: \#444

Kazi, MA: \#428

Kazi, N: \#250

Kazi, TG: \#250

Kazimirov, AS: \#238

Ke, XB: \#1178

Keceli, G: \#1305 \#1336

Keegan, E: \#71

Kelly, SM: \#303

Kelly, WR: \#43

Kempinska, M: \#1168

Kepinski, L: \#294

Kerekes, A: \#1270
Kertesz, Z: \#1136

Kessel, R: \#676

Kester, M: \#3

Ketterer, ME: \#1423

Kevdina, IB: \#545

Khan, AA: \#712

Khan, HM: \#712

Khan, K: \#1283

Khandaker, MU: \#1108

Khanna, D: \#585 \#1278

Kharlmohammadi, A: \#827

Khateeb-ur-Rehman: \#198 \#1094

Khater, SI: \#878

Khati, VS: \#386

Khoshdel, MR: \#21

Khuder, A: \#442 \#1429

Khuhawar, MY: \#428 \#956

Kierepko, R: \#299 \#1165

Kiff, SD: \#571

Kikandi, S: \#740

Kikuchi, T: \#318

Kikunaga, H: \#318

Kilic, AM: \#270

Kilic, O: \#447 \#1239 \#1240 \#1275

Killa, HM: \#878

Kim, CK: \#135 \#185 \#654 \#1246

Kim, CS: \#654 \#1371

Kim, G: \#478 \#745 \#917 \#1133

Kim, GN: \#1108

Kim, HD: \#923 \#1083 \#1086

Kim, HJ: \#82 \#867

Kim, HR: \#1060 \#1061

Kim, IK: \#255

Kim, JG: \#867

Kim, JS: \#197

Kim, KH: \#237

Kim, KR: \#1257

Kim, KS: \#478 \#1108 \#1133

Kim, KW: \#576

Kim, MW: \#1060

Kim, SH: \#82 \#1061

Kim, YE: \#82

Kim, YH: \#576 \#709

Kim, YJ: \#1060 \#1061 \#1246

Kimura, A: \#506 \#562 \#1185 \#1202 \#1204 \#1362

Kimura, H: \#104

Kin, T: \#1362

Kinoshita, N: \#318 \#1152 \#1153

Kinsel, M: \#894

Kinuya, S: \#104

Kirchner, G: \#781 \#1088

Kirkpatrick, GJ: \#819

Kirsanov, D: \#392 
Kis, Z: \#63

Kis-Benedek, G: \#168 \#185

Kiser, MR: \#544

Kiser, S: \#663 \#664

Kiss, P: \#901 \#1052

Kiss, T: \#901 \#1052

Kitamura, H: \#1134

Kitatani, F: \#1185

Kitazawa, T: \#13

Kitowski, I: \#299 \#1212

Kivel, N: \#46

Kiyokawa, S: \#377

Kiyomarsi, M: \#1161

Kizek, R: \#433

Klaehn, JR: \#595

Klavins, M: \#242

Kleinschmidt, R: \#168

Klemola, S: \#949

Klencsar, Z: \#479 \#559 \#888

Klimchuk, O: \#534

Klinedinst, D: \#1027

Kljakovic-Gaspic, Z: \#439

Klos, A: \#420

Klose, D: \#1425

Klouda, GA: \#155

Kluge, T: \#549

Knoeller, K: \#862

Knowles, NG: \#570 \#914

Knuth, A: \#287

Ko, IS: \#478 \#1133

Ko, S: \#538

Ko, SW: \#275

Koarashi, J: \#137 \#771 \#924

Kobal, I: \#1370

Kobayashi, H: \#924

Kobayashi, Y: \#558 \#561

Kobya, AI: \#861

Kocianova, M: \#273

Kockelmann, W: \#1005

Kodama, Y: \#1258

Koers, A: \#1195

Koester, CJ: \#51

Kogelnig, D: \#296

Koh, KZY: \#703

Kohli, DV: \#380

Kohli, P: \#894

Koizumi, M: \#506 \#1185 \#1202 \#1204 \#1362

Kokkoris, A: \#1125

KokkoriS, M: \#1113

Kokkoris, M: \#1114

Kolarikova, I: \#755

Kolarz, PM: \#205

Kolb, HC: \#915
Kolekar, RV: \#1099

Kolekar, SS: \#581

Kolodynska, D: \#728

Kolonel, LN: \#285 \#286

Kolotov, VP: \#301

Koltay, E: \#1136

Koma, Y: \#23

Komlosi, A: \#107

Komosa, A: \#300 \#1472

Komura, K: \#688 \#1371

Kondev, FG: \#1057

Kondo, K: \#788

Kong, DJ: \#143

Konings, RJM: \#962

Konishi, T: \#1134

Konno, H: \#479

Konobeyev, AY: \#146

Kononenko, N: \#1468

Konstantinou, M: \#533

Kontar, E: \#1367

Kontar, EA: \#724 \#744 \#769

Konya, J: \#107

Koole, R: \#1042

Koopmans, D: \#246

Kopecky, S: \#1101

Koppisch, AT: \#685

Koprivova, H: \#1438

Koptikova, L: \#273

Kore, SG: \#1392

Korf, J: \#1464

Kornacka, EM: \#1166

Korobkov, VI: \#301

Korona, T: \#715

Korpach, E: \#202

Korun, M: \#2

Kosaka, W: \#687

Kosanovic, C: \#1084

Kose, E: \#1290

Kosmulski, M: \#729

Kotnik, J: \#764

Kotrba, P: \#1039

Koukouliou, V: \#168

Kouno, K: \#558

Koutinas, AA: \#1327

Koutsoukos, PG: \#1022

Kovacs, I: \#813

Kovacs, K: \#1434

Kovacs, T: \#623 \#1017 \#1243

Kovacs, Z: \#128 \#192 \#640 \#1102 \#1103 \#1132 \#1315

Kovaltsov, GA: \#817

Kowalczyk, A: \#804

Koyama, SI: \#1225

Kozai, N: \#519 
Kozani, RR: \#74

Krachler, M: \#738

Krachler, R: \#296 \#296

Kranda, K: \#264

Kranhebuhl, U: \#656

Krasznahorkay, A: \#1203

Kratz, JV: \#318

Krause, DE: \#1412

Krause, JW: \#408

Kregsamer, P: \#643 \#648 \#658 \#669

Krishanamoorthy, S: \#196

Krishnan, S: \#563 \#702 \#1192

Krivan, V: \#1422

Krivit, SB: \#385

Krivopustov, MI: \#103

Kroeger, K: \#246

Kroeger, KD: \#989 \#990

Kronenberg, A: \#1129

Krueger, S: \#684

Kruger, S: \#399 \#597

Krupa, S: \#1367

Kruse, J: \#1206

Kruse, S: \#455

Krystek, P: \#606

Krzan, Z: \#1211

Kshirsagar, P: \#640

Kubica, B: \#1211

Kubica, M: \#1211

Kubo, K: \#688

Kubo, MK: \#561 \#970 \#971

Kubuki, S: \#556

Kucer, R: \#1239 \#1275

Kucera, J: \#264 \#394 \#527 \#1012

Kudejova, P: \#206 \#477 \#690

Kudo, H: \#318

Kudou, Y: \#318

Kuehne, G: \#1207

Kuenzi, M: \#287

Kuhar, B: \#168

Kuhn, H: \#611

Kuhn, JN: \#293

Kulahci, F: \#150 \#180 \#495

Kulikov, LA: \#73 \#555

Kulisa, K: \#298

Kulkarni, KM: \#744 \#1367

Kulkarni, Y: \#1392 \#1396

Kulozik, U: \#977

Kumagai, M: \#23 \#24 \#26 \#573 \#720 \#823 \#957 \#1381 \#1383 \#1384

Kumagai, MK: \#1388

Kumann, E: \#217

Kumar, A: \#932 \#933

Kumar, BN: \#1409
Kumar, GA: \#1096

Kumar, M: \#253 \#1004 \#1047

Kumar, N: \#965

Kumar, S: \#730 \#831

Kumar, V: \#483

Kumaresan, R: \#1407

Kummrow, F: \#1443

Kundinger, MM: \#106

Kunicki, J: \#696

Kuniholm, PI: \#698

Kuno, R: \#1443

Kunze, S: \#287

Kurahashi, K: \#401

Kuraoka, E: \#481 \#573 \#575 \#720 \#823 \#946 \#1381 \#1383 \#1384

Kurniawan, A: \#1013

Kuroiwa, T: \#97

Kurosaki, H: \#154

Kurt, A: \#808

Kurttio, P: \#540

Kuryatnyk, T: \#835

Kusakari, H: \#1202 \#1204

Kuske, E: \#242

Kutahyali, C: \#913

Kutle, A: \#360

Kuzmann, E: \#376 \#426 \#479 \#555 \#556 \#559 \#888 \#1434

Kvicalova, M: \#927 \#1311

Kwan, J: \#568

Kwiatek, WM: \#1211

Kwong, LLW: \#168

Kyunai, T: \#1063

La Rosa, J: \#987

Laan, P: \#1051

Lacerda, T: \#1198

Laciok, A: \#397

Lackner, KS: \#359

Ladeira, ACQ: \#824

Ladygina, VP: \#598

Laedermann, JP: \#1072

Lagerkvist, P: \#1122

LagoyannIS, A: \#1113

Lahiri, S: \#171 \#243 \#686

Lahm, GP: \#883

Laissaoui, A: \#928

Lal, S: \#1

Lalor, GC: \#1375

Lam, JCW: \#1461

Lam, PKS: \#457

Lambertz, M: \#264

Lamborg, C: \#405

Lamborg, CH: \#409

Lambrecht, FY: \#572 \#620 
Lami, A: \#548

LaMont, S: \#154

Lamontagne, J: \#183

Lancaster, S: \#660 \#670 \#678

Lancastera, S: \#650

Landsberger, S: \#176 \#208 \#209 \#1077 \#1115 \#1130

Lane-Smith, D: \#465

Lange, CR: \#1476

Langley, J: \#1350

Langrova, A: \#273

Lanz, VA: \#230

Lao, F: \#1456 \#1459

Lapi, S: \#1129 \#1199

Lapicque, F: \#734

Lapka, JL: \#1324

Lara, VH: \#366

Lariviere, D: \#663 \#664 \#1420

Larkin, FH: \#681

Larkin, JD: \#681

LaRosa, J: \#168

Larpent, C: \#1454

Larsen, L: \#480

Lasheen, YF: \#1347

Laskar, S: \#492

Laszlo, K: \#623

Laubenstein, M: \#793

Lavelaine, H: \#734

Lavelle, M: \#613

Law, JD: \#595 \#1324

Lawrence, MJ: \#978

Lazarus, I: \#1093

Laznicek, M: \#177

Laznickova, A: \#177

Le Bourdonnec, FX: \#699

Le Druillennec, T: \#119

Le Guludec, D: \#1029

Le Marchand, L: \#285 \#286

Le Naour, C: \#596

Leal, M: \#934

Leaney, FW: \#983

Lecomte, P: \#1064

Ledo, HM: \#896

Lee, EH: \#576

Lee, GH: \#1054

Lee, H: \#255 \#1062

Lee, HS: \#427 \#478 \#1133

Lee, HY: \#1246

Lee, IW: \#313 \#710

Lee, J: \#172

Lee, JH: \#237

Lee, JM: \#745

Lee, K: \#255 \#994

Lee, KH: \#1060
Lee, MS: \#1257

Lee, MW: \#478 \#1133

Lee, SH: \#153 \#168 \#765 \#926

Lee, SM: \#136 \#867

Lee, SY: \#576 \#1083 \#1086

Lee, TH: \#923 \#1083 \#1086

Lee, TN: \#819

Lee, YN: \#82

Lee, YS: \#1108

Lefevre, D: \#1051

Legarda, F: \#168

Leggitt, J: \#154

Legin, A: \#392

Lehmann, E: \#1207 \#1466

Lehto, J: \#726

Leibner, ES: \#4

Leigh, SD: \#1027

Lelsch, G: \#119

Lemos, VA: \#1421

Lenes, JM: \#819

Lenka, P: \#783

Leong, TK: \#1365

Leopold, K: \#784

Lepel, EA: \#66

LePetit, G: \#168

Leppanen, AP: \#817

Lepy, MC: \#1335

Lesne, P: \#513

Leung, KN: \#568

Leung, SJ: \#31

Levent, D: \#1441

Levy, I: \#999

Levy-Palomo, I: \#168 \#371 \#469 \#744

Lewinski, N: \#1400

Lewis, JS: \#1030 \#1141

Li, B: \#50 \#904 \#1040 \#1231 \#1456 \#1459

Li, C: \#154 \#256 \#350 \#663

Li, CL: \#353 \#354

Li, CS: \#664

Li, DQ: \#574 \#714

Li, HB: \#1121

Li, HM: \#14 \#16

Li, J: \#19

Li, JC: \#1455

Li, JG: \#457

Li, JQ: \#851

Li, JX: \#367 \#842 \#844 \#848 \#1026

Li, JY: \#543

Li, JZ: \#352

Li, L: \#256 \#1131

Li, LQ: \#352

Li, MH: \#157 \#719

Li, N: \#485 
Li, Q: \#365 \#820 \#857

Li, QN: \#292

Li, RX: \#1343

Li, SH: \#1110

Li, SJ: \#858 \#1397

Li, SQ: \#858 \#1397

Li, SX: \#14 \#16 \#292

Li, SZ: \#353

$\mathrm{Li}, \mathrm{T}: \# 604$

Li, W: \#365 \#805 \#904 \#1040 \#1221 \#1456 \#1459

Li, WB: \#540 \#1241 \#1271 \#1272

Li, WJ: \#503

Li, WX: \#292

Li, XF: \#1474

Li, XJ: \#1455

Li, XL: \#415 \#800

Li, Y: \#102 \#800

Li, YF: \#50 \#904 \#1040 \#1221 \#1231 \#1456 \#1459

Li, YQ: \#1131

Li, YY: \#805

$\mathrm{Li}, \mathrm{ZG}: \# 15$

Li, ZH: \#110 \#1416

Li, ZJ: \#318 \#1355

Li, ZQ: \#349

Li, ZY: \#1041

Liang, D: \#256

Liang, XJ: \#383

Liang, ZS: \#347

Liao, HQ: \#156 \#774

Liaw, JR: \#101

Lichtscheidl, IK: \#211

Liegault, B: \#33

Lilla, E: \#1259

Lim, JM: \#237

Lima, I: \#569 \#1021 \#1432

Lin, B: \#30

Lin, CC: \#919

Lin, M: \#1110

Lin, Q: \#850

Lin, WY: \#194

Lin, Y: \#179

Lin, Z: \#308 \#310 \#418

Linde, AR: \#218

Linde-Arias, AR: \#452 \#1374

Lindsay, R: \#166

Lindstrom, RM: \#1087

Ling, DB: \#345

Linge, KL: \#647 \#655 \#668

Linou, M: \#600

Lintern, MJ: \#514

Lipsztein, JL: \#1273

Lipton, J: \#698 \#818

Lis, S: \#1438
Lisa, L: \#273

Lisetskiy, AF: \#1229

Lister, TR: \#998

Liszkay, L: \#1084

Litherland, AE: \#607

Lito, PF: \#310

Little, NC: \#1463

Littmann, S: \#672

Liu, AZ: \#1187

Liu, CL: \#350

Liu, CX: \#858 \#1397

Liu, GF: \#258 \#879

Liu, GR: \#972

Liu, HN: \#365

Liu, HW: \#1178

Liu, HY: \#190

Liu, JF: \#800

Liu, JP: \#604

Liu, JS: \#349

Liu, LB: \#503

Liu, M: \#352

Liu, ML: \#1202

Liu, MT: \#190

Liu, N: \#850

Liu, RL: \#292

Liu, SY: \#347

Liu, TH: \#731

Liu, XL: \#12 \#15

Liu, XY: \#350

Liu, Y: \#50 \#574 \#714 \#904 \#904 \#1307 \#1316 \#1456 \#1459

Liu, YG: \#536

Liu, YH: \#12 \#15 \#946

Liu, YM: \#994

Liu, ZL: \#914

Livens, FR: \#304

Llaurado, M: \#168

Lo Monaco, C: \#1051

Lo Russo, S: \#429

Loffler, D: \#145 \#809 \#810 \#1491

Lohonyai, L: \#1084

Lohu, EL: \#641

Lokhande, RS: \#1325

Lomas, MW: \#408

Lombardi, M: \#295

Lommel, SA: \#1046

Long, M: \#304

Long, SE: \#43

Longas, N: \#240

Lopes, CB: \#308 \#418

Lopes, FVS: \#1025

Lopes, I: \#751

Lopes, RT: \#474 \#569 \#1021 \#1432 \#1487 
Lopez, A: \#1248

Lopez, AE: \#1244

Lopez-Vicente, M: \#1403

Lorber, A: \#653

Lorente, A: \#1286

Losana, MC: \#1288

Louizi, A: \#1379

Louka, A: \#955

Loveless, A: \#1367

Lu, DH: \#546

Lu, GN: \#604

Lu, GQ: \#347

Lu, J: \#143 \#880

Lu, LY: \#353

Lu, SS: \#108

Lu, SY: \#473

Lu, W: \#256

Lu, XW: \#438 \#566

$\mathrm{Lu}, \mathrm{Y}$ : \#848

Lu, ZL: \#14

Lubal, P: \#1438

Lubian, J: \#1197 \#1198

Lubics, A: \#901 \#1052

Luca, V: \#976

Lucanikova, M: \#394 \#927 \#1311

Lucas, M: \#403

Lucho-Constantino, CA: \#1359

Lucia, D: \#1100

Luckey, D: \#1064

Ludwig, T: \#509 \#512

Lugo-Lugo, V: \#836

Luiz-Silva, W: \#766

Lujaniene, G: \#984 \#1058

Lukauskas, D: \#1058

Luke, PN: \#1286

Lukes, I: \#177

Luksic, AT: \#66

Luksiene, B: \#1058

Lulic, S: \#803

Lumbreras, JM: \#768

Lundgren, K: \#537

Luo, LQ: \#1433

Luo, SF: \#347

Luo, TY: \#194

Luo, W: \#458

Luther, TA: \#595

Lutter, R: \#1203

Lutzenkirchen, J: \#220 \#726

Lv, DW: \#504 \#505 \#820 \#988

Lyashko, YV: \#874 \#1218

Lyklema, J: \#381

Lyssukhin, SN: \#1112

Ma, B: \#1455
Ma, HL: \#739

Ma, Q: \#691

Ma, YC: \#204

Ma, YJ: \#352

Ma, YM: \#259

Maasch, K: \#723

Macaire, JJ: \#521

Macaskie, LE: \#896

Machado, MI: \#219

Machado, W: \#766

Machlev, Z: \#1250

Machulla, HJ: \#145 \#809 \#810 \#1491

MacKenzie, AB: \#1377

MacKenzie, C: \#303

Macoveanu, M: \#855

Macovei, A: \#435

Maddalena, A: \#429

Madej, E: \#300

Mader, D: \#296

Madi, T: \#1480

Madrid, Y: \#86

Maes, A: \#116

Magara, M: \#752

Magnoni, M: \#1288

Magnusson, A: \#537

Magro, L: \#789

Maguas, C: \#451

Magudapathy, P: \#253

Mahapatra, SC: \#1223

Mahboob, M: \#1036 \#1037

Mahmoudi, M: \#1321

Mahoney, DW: \#186

Mahramanlioglu, M: \#227

Maichle-Mossmer, C: \#1491

Maidana, NL: \#494

Maier, HJ: \#1203

Maiti, K: \#406 \#407 \#408 \#409

Majdabad, A: \#1146

Majdabadi, A: \#1147 \#1170

Majdan, M: \#1230

Majid, AA: \#1365 \#1366

Major, P: \#1084

Makovetskii, KL: \#1217

Makreski, P: \#1318 \#1333

Makrik, E: \#958

Makrlik, E: \#6 \#9 \#11 \#960 \#964 \#1031 \#1032 \#1033

\#1034 \#1035 \#1209 \#1492 \#1493 \#1494

Malathi, J: \#585 \#1278

Malaxechevarria, Y: \#1413

Maleka, PP: \#166

Malgorzata, R: \#245

Malik, A: \#440

Malin, JN: \#941 
Malits, A: \#1051

Mallah, MH: \#459 \#900

Malloy, CR: \#640

Malo, M: \#281

Malyuk, IA: \#1066

Mananes, A: \#865

Manchanda, VK: \#478 \#730 \#831 \#1133 \#1332 \#1392 \#1396

Mandal, S: \#1002

Manea-Grigore, I: \#212

Manickam, E: \#241 \#756

Manke, I: \#1091

Manning, SW: \#115 \#698 \#818

Manoharan, R: \#483

Manolopoulou, M: \#103

Manthiram, A: \#421 \#733 \#891 \#892

Manu, V: \#212

Maoui, R: \#765

Maragheh, MG: \#459 \#900 \#1310

Maranho, LA: \#725

Marcelis, ATM: \#937

Marcelli, MP: \#449

Marchetti, AA: \#1111

Margineanu, RM: \#1326

Maria, PC: \#641

Maria, W: \#245

Marinenko, RB: \#1027

Maringer, FJ: \#168

Marini, N: \#699

Marinkovic, BP: \#205

Mark, L: \#1052

Markert, B: \#37

Marko, A: \#225

Markowicz, A: \#1376

Markwitz, A: \#1376

Marmolejo-Rodriguez, J: \#372 \#995

Marques, AP: \#603

Marques, LD: \#1351

Marques, SM: \#1373

Marquis, PM: \#896

Marrazzo, L: \#1100

Marschall, HR: \#509 \#1028

Martano, L: \#200

Marti-Climent, JM: \#1286

Martin, J: \#999

Martin, P: \#135 \#654 \#757

Martin, U: \#969

Martinelli, RE: \#1111

Martinez, G: \#125

Martinez, I: \#223

Martinez, T: \#125

Martinez-Carrillo, MA: \#1359

Martinez-Esparza, A: \#911
Martinez-Resendiz, G: \#1359

Martin-Martin, A: \#768 \#1286

Martins-Loucao, MA: \#451

Maruyama, K: \#1226

Marvin-DiPasquale, M: \#338

Marzo, ML: \#235

Mashkani, SG: \#261 \#262 \#263 \#554

Maslehuddin, M: \#198 \#1094

Maslov, OD: \#527

Maslova, MV: \#590

Mason, TO: \#691

Masque, P: \#405 \#849 \#986 \#987

Massaferro, J: \#548

Massi, L: \#641

Massimi, C: \#1101

Massner, JA: \#475

Masson, S: \#542

Masteri-Farahani, M: \#74

Masuda, K: \#500 \#501 \#502 \#1063

Masumoto, K: \#1107 \#1128 \#1152 \#1153

Mate, B: \#1017

Matei, M: \#1326

Mather, AR: \#1093

Mathew, KM: \#872

Matijevic, S: \#496

Matiullah: \#950

Matiullah, S: \#948

Matos, JE: \#101

Matsuda, N: \#1107 \#1128

Matsue, H: \#97 \#561 \#970 \#971

Matsui, T: \#377

Matsumoto, M: \#1436 \#1437

Matsumoto, S: \#694

Matsumura, H: \#1107 \#1128 \#1152 \#1153

Matsumura, M: \#1152

Matsushi, Y: \#1152

Matsushima, U: \#683 \#1091

Matsuyama, S: \#159 \#1242

Matt, D: \#718

Mattes, JJ: \#1412

Matthews, KM: \#135

Mattsson, S: \#1186

Matumoto, Y: \#1018

Matysiak, W: \#1187

Mausner, LF: \#158

Maxwell, SL: \#1448

May, EM: \#410

Mayer, K: \#71

Mayeux, C: \#641

Mayewski, PA: \#723

Mayoral, A: \#121

Mazeika, K: \#984

Mazet, S: \#699 
Mazrou, H: \#1079

Mazumdar, I: \#229 \#1096

Mazzi, U: \#1140

Mazzilli, BP: \#358 \#498 \#1269

Mbemba, C: \#866

McBain, SC: \#617

McBay, EH: \#613

McCroan, K: \#154

McGillicuddy, D: \#405

McGinley, J: \#375

McGrath, CA: \#1200

McIlwain, ME: \#1065

McIntyre, JI: \#565 \#1080

McKeown, NB: \#1206

McMaster, S: \#1187

McMillan, N: \#303

McNeary, D: \#815

McNeill, FE: \#1008 \#1187 \#1189 \#1190

McNichols, RJ: \#288

McWilliams, WE: \#934

Medine, EI: \#127 \#283 \#572

Medunic, G: \#803

Medvedev, DG: \#158

Medvedev, DV: \#874 \#1218

Medvedeva, EP: \#874 \#1218

Medvidovic, NV: \#582

Meenakshisundaram, V: \#585 \#1278

Meharg, AA: \#1465

Mei, CM: \#24

Meier, DC: \#1027

Meierhofer, G: \#477

Meijerink, A: \#1042

Meirer, F: \#667

Mekarski, P: \#202

Mekhamer, HS: \#1320

Melendez-Alafort, L: \#1140

Meleshevych, S: \#984

Meli, MA: \#748 \#775 \#801 \#1015 \#1457

Melichar, F: \#177

Melichar, R: \#273

Melintescu, A: \#771

Mell, P: \#1270

Mellah, A: \#414

Melo, RP: \#1264

Melo, SB: \#1123

Memon, JR: \#84

Menacer, M: \#765

Mendes Da Silva, LH: \#1237

Mendez, CG: \#195

Mendez-Sanchez, N: \#1476

Mendonca, JCF: \#490

Mendoza, D: \#125

Meng, L: \#50
Meng, W: \#797 \#799

Menichelli, D: \#1100

Menlove, HO: \#1083 \#1086

Menon, M: \#1466

Menon, V: \#253

Merchel, S: \#44

Mercier, M: \#513

Merdivan, M: \#444 \#1389

Merritt, ME: \#640

Mervin, B: \#1129

Mester, Z: \#649

Meszaros, G: \#905 \#906

Meszaros, S: \#479 \#888

Metilda, P: \#77

Metrich, N: \#513

Metwalli, E: \#977

Metwally, E: \#290 \#1309

Metwally, WA: \#1116 \#1144

Meulemans, A: \#1029

Meyer, AS: \#973

Meyer, M: \#168

Mezyk, SP: \#1330

Mhaisalkar, S: \#703 \#732

Michalik, B: \#168

Michalke, B: \#749

Michalski, R: \#378

Michel, H: \#168 \#1441

Mietelski, JW: \#299 \#1165 \#1212

Mignani, R: \#614 \#1181 \#1182 \#1183

Mihailescu, LC: \#1101

Mihara, S: \#1152

Mihucz, VG: \#85

Mikami, S: \#924

Mikelic, L: \#803

Miksa, EJ: \#697

Mikulic, N: \#803

Milagre, HMS: \#430

Milani, IB: \#219 \#862

Milbrath, BD: \#571 \#895

Milder, OB: \#557 \#902

Miles, DL: \#645 \#651 \#662 \#671

Miljanic, SS: \#959

Millan, E: \#240 \#1413

Millett, S: \#71

Milner, JA: \#45

Milroy, SP: \#819

Mincher, BJ: \#1330

Mindru, I: \#1360

Minim, LA: \#1237

Mioc, AR: \#582

Miquel, JC: \#987 \#999

Mirashi, NN: \#1074

Mirazizi, F: \#626 \#1176 
Mirjalili, BF: \#8

Mirkovic, M: \#1048

Mirkowski, K: \#1166

Miro, M: \#70 \#89

Mirza, MA: \#956

Mirzaee, M: \#1338

Mirzaii, M: \#175 \#1161

Misaefides, P: \#1125

Misdaq, MA: \#539

Mishra, BK: \#737

Mishra, M: \#136

Mishra, PK: \#1392

Mishra, RK: \#831

Mishra, SP: \#136

Misiak, R: \#1211

Misra, S: \#1037

Misra, SK: \#897

Mitamura, M: \#752

Mitchell, SC: \#726

Mitsugashira, T: \#395

Mitteregger, B: \#296

Miura, T: \#97 \#1107 \#1128

Miyake, Y: \#457

Miyamoto, Y: \#752

Miyatake, H: \#1185

Miyauchi, H: \#499

Miyazaki, I: \#918

Mizera, J: \#394 \#527 \#1012

Mlakar, M: \#782

Mlwilo, NA: \#166

Moch, H: \#287

Mocko, M: \#1200

Moens, A: \#1101

Mogilnaya, O: \#792

Mohammad, A: \#442

Mohammad, GM: \#346

Mohandas, KS: \#1010

Mohanty, AK: \#166

Mohapatra, M: \#737

Mohapatra, PK: \#1332

Mokobia, CE: \#173

Moll, H: \#257 \#269 \#1214

Molnar, GL: \#321

Molnar, R: \#628

Monem, NMA: \#307

Monroy-Guzman, F: \#704

Montagnac, G: \#508 \#513

Montalto, M: \#391

Montana, RL: \#1339

Montanaro, L: \#295

Montavon, G: \#871

Montazerozohori, M: \#827 \#843

Montero-Cabrera, ME: \#195
Montes de Oca-Valero, JA: \#547

Monticelli, D: \#87

Montoro, R: \#486

Moodie, G: \#664

Moody, C: \#467

Moody, MA: \#589

Moon, JH: \#82 \#237 \#1061

Moore, DA: \#1345

Moore, RC: \#1296

Moore, WS: \#468 \#744 \#769 \#1367

Moradi, AB: \#475 \#1207

Moradkhani, S: \#875 \#1161

Morais, RD: \#725

Morais, ZB: \#705

Moran, JE: \#770

Morchon, J: \#1286

Moreda-Pineiro, A: \#633

Moreira, JC: \#218 \#452 \#1374

Moreira, LB: \#725

Moreira, MDR: \#278

Moreno, SAM: \#1359

Moretto, P: \#1135

Morgalyuk, VP: \#1009

Morgenstern, A: \#871

Morgenstern, U: \#469

Mori, K: \#631

Mori, MN: \#1258 \#1264

Mori, T: \#316

Morikawa, T: \#1185 \#1202

Morisaki, H: \#1013

Morovic, M: \#496

Morris, JS: \#285 \#286 \#460

Morris, K: \#183

Morris, PJ: \#986

Morrison, RD: \#618

Morton, J: \#118

Morton, JG: \#701

Moschini, G: \#1126

Mosseri, J: \#1051

Mostafa, M: \#1385

Mostajaboddavati, M: \#627

Motaleb, MA: \#881

Motavalli, LR: \#1154

Mou, TT: \#204

Moubaraki, B: \#1215

Moulik, A: \#51

Moulin, JF: \#977

Mourad, NM: \#191

Moureau, J: \#1424

Mousa, MA: \#191

Moustafa, O: \#1285

Moxon, M: \#1078 \#1101

Moynier, F: \#938 \#939 
Mrda, D: \#1245

Mueller, W: \#183

Muftuler, FZ: \#1138

Muftuler, FZB: \#149 \#283 \#284 \#572

Mugica-Alvarez, V: \#547

Mugler, C: \#1462

Muhlbauer, M: \#690

Muikku, M: \#540 \#541

Muir, GKP: \#991

Mujahid, SA: \#948

Mukai, K: \#1102 \#1103

Mukherjee, A: \#1054

Mukhopadhyay, D: \#243

Mulder, WJM: \#1042

Muller, AHE: \#1002

Muller, K: \#464

Muller-Buschbaum, P: \#977

Muller-Karger, FE: \#819

Mulsow, S: \#928

Munakata, K: \#909

Mundwiler, S: \#287

Munoz-Munoz, AC: \#722

Muntener, O: \#314 \#512

Murakami, A: \#752

Murakami, H: \#55

Murakami, Y: \#1362

Muramatsu, Y: \#538

Murariu, M: \#628 \#1361

Murelaga, X: \#1418

Murphy, KE: \#43

Murphy, SP: \#286

Murray, AS: \#516 \#1106

Murray, KS: \#1215

Mushtaq, A: \#1322 \#1329

Music, S: \#1003

Musilek, A: \#211

Mustafa, S: \#312

Nadali, A: \#1140

Naeem, A: \#312

Nagadi, MM: \#198 \#1094

Nagai, T: \#938

Nagame, Y: \#317 \#318 \#1201

Naganawa, H: \#401

Nagao, K: \#506

Nagaoka, S: \#752

Nagarajan, K: \#425

Nagasaki, K: \#502

Nagasaki, S: \#519

Nagashima, Y: \#1152

Nagatomi, HR: \#1264

Nagy, E: \#32

Nagy, K: \#623 \#1243

Nagy, L: \#32
Nagy, NM: \#107

Naik, H: \#478 \#1133

Nair, AGC: \#493 \#932 \#1068 \#1325

Nair, KGM: \#253

Najam-Ul-Haq, M: \#616

Nakada, A: \#924

Nakagawa, T: \#502

Nakamaru, Y: \#762

Nakamura, S: \#1185

Nakanishi, TM: \#1092

Nakano, K: \#62

Nakano, S: \#1018

Nakano, Y: \#377 \#709

Nakao, Y: \#936

Nakashima, H: \#1128

Nakoman, ME: \#434

Nam, KC: \#275

Namiki, K: \#315

Namkung, W: \#478 \#1133

Nance, JH: \#802

Nanri, T: \#318

Nanzyo, M: \#1368

Naqvi, AA: \#198 \#1094

Narasaki, H: \#918

Narbutt, J: \#1156

Narin, I: \#822 \#832

Nascimento, N: \#968

Nasef, MM: \#899

Nasr-Eddine, B: \#846

Nastro, A: \#945

Navarrete-Lopez, M: \#547

Navas, A: \#1251 \#1403

Navez, J: \#404

Navrotsky, A: \#1023

Nayak, D: \#1300

Nayak, PK: \#229

Naydenov, V: \#590

Nazem, H: \#626 \#1176

Nechifor, CD: \#1364

Neculita, CM: \#117

Neff, H: \#980

Nemanich, RJ: \#1219

Nemes, Z: \#107

Nemeth, J: \#901 \#1052

Nemeth, K: \#969

Nemeth, Z: \#376 \#479 \#559 \#888

Nemoto, K: \#524

Neoh, KG: \#732

Nerin, C: \#48

Nessi-Tedaldi, F: \#1064

Neu, MP: \#510 \#586

Neuville, PF: \#699

Neves, EB: \#278 
Neves, MO: \#507

Newbury, DE: \#1027

Newman, RT: \#166

Newport, JR: \#1412

$\mathrm{Ng}, \mathrm{BK}:$ \#120

Nguyen, DT: \#1135

Ni, BF: \#1376

Ni, DJ: \#491

Ni, LY: \#450

Nickles, RJ: \#916

Nicolaou, G: \#1255

Nicolay, K: \#1042

Nie, SM: \#105 \#1049

Nielsen, AH: \#516

Nielsen, SP: \#57

Niemann, RA: \#721

Niencheski, F: \#862

Niencheski, LF: \#219

Nies, H: \#168

Nifant'ev, EE: \#1009

Nikodemus, O: \#242

Nikolic, N: \#1048

Nikolopoulos, D: \#1379

Nisbet, DJ: \#106

Nishi, N: \#55

Nishi, T: \#500

Nishida, T: \#556

Nishikawa, Y: \#499 \#909

Nishinaka, I: \#318

Nishiyama, J: \#917

Nishizumi, T: \#556

Nisi, S: \#793

Niu, ZW: \#1026

Noble, AE: \#407

Noble, J: \#386

Nobrega, JAN: \#92

Nocente, M: \#391

Nogami, M: \#1226

Nogawa, N: \#159 \#1242

Noguere, G: \#1076

Nolan, PJ: \#1093

Nomura, CS: \#95

Nomura, K: \#560 \#687

Nonell, A: \#1424

Nonova, T: \#777

Norde, W: \#260

Nosenko, S: \#252

Noto, K: \#694

Noubactep, C: \#431

Nour, M: \#1474

Nour, S: \#168

Noureddine, A: \#765

Novak, JK: \#527
Novikov, EG: \#902

Novo, LA: \#452

Nuesch, J: \#287

Numajiri, M: \#1128

Nunes, M: \#490

Nyarko, BJB: \#1469

Nygren, U: \#1122

Nyikos, L: \#426

Oberdorfer, JA: \#769 \#1367

Obernosterer, I: \#1051

Obhodas, J: \#360

Oblozinsky, P: \#1056

O'Brien, R: \#241

O’Bryan, C: \#106

Ocone, R: \#165

O’Connor, IP: \#236

Odani, A: \#104

O’Dwyer, JN: \#161

Oeh, U: \#540 \#749 \#1241 \#1271 \#1272

Oehlmann, J: \#1452

Ogata, T: \#709

Ogawa, K: \#104

Ogiyama, S: \#1127

Ogundare, FO: \#173

Oh, JM: \#903

Oh, JS: \#168

Oh, YD: \#478

O’Hara, MJ: \#54 \#64 \#65 \#327

Oheim, M: \#340

Ohkoshi, S: \#687

Ohnishi, H: \#631

Ohnuki, T: \#519

Ohsaki, T: \#917

Ohto, K: \#1411

Ohtsuka, Y: \#788

Ohtsuki, T: \#395

Oi, M: \#1185

Oikawa, M: \#1134

Oikawa, S: \#747

Ojeda, CB: \#49 \#88 \#356

Oji, T: \#377

Okada, K: \#1102

Okamoto, Y: \#59

Oki, T: \#1152

Okuno, K: \#499 \#909

Olabanji, SO: \#1126

O'Leary, K: \#934

Oleszczuk, N: \#1421

Olivares, M: \#1418

Olive, KI: \#1339

Oliveira, AH: \#268

Oliveira, EF: \#1123

Oliveira, J: \#1367 
Oliveira, JM: \#272 \#507 \#751

Oliveira, LS: \#490

Oliveira, PV: \#95 \#1443

Oliver, IW: \#1377

Olivo, M: \#120 \#703 \#732

Olkawa, H: \#1258

Olsen, S: \#129

Olszewski, M: \#1162

Omar, H: \#111

Omobuwajo, OR: \#1126

O'Neal, P: \#1044

Oner, Y: \#639

Ong, BH: \#1436 \#1437

Onishchenko, NI: \#1218

Onoguchi, M: \#104

Ooe, K: \#318

Opatrilova, R: \#433

Oravetz, D: \#376

Oregioni, B: \#168 \#371 \#744 \#926

Orescanin, V: \#803

Orino, S: \#631

Orlandini, K: \#986

Orsini, AM: \#112

Ort, MH: \#410 \#1463

Ortaboy, S: \#695

Ortiz-Romero-Vargas, ME: \#547

Orton, CR: \#66

Orzel, J: \#1472

Osa, A: \#1202 \#1204 \#1362

Osae, S: \#1469

Osan, J: \#53

Osanai, A: \#1098

Osawa, T: \#506

Oshima, M: \#506 \#562 \#1185 \#1202 \#1204 \#1362

Oshtrakh, MI: \#557 \#902

Osipov, A: \#229

Oskamp, D: \#621

Osterc, A: \#5

Ostergaard, LF: \#57

Ostra, M: \#240

Oswald, S: \#1207

Oswald, SE: \#475 \#1466 \#1467

Ota, S: \#1185

Ota, Y: \#159 \#1242

Otero, CD: \#377

Otero, M: \#308 \#310 \#418

Ouadi, A: \#534

Oudyk, J: \#40 \#1190

Oura, Y: \#318

Ouyang, G: \#83

Owusu, PA: \#416

Oxley, DC: \#1093

Oya, Y: \#499 \#909
Oyaidzu, M: \#909

Ozawa, M: \#1225

Ozdes, D: \#845

Ozeroglu, C: \#825 \#1305 \#1336

Ozgen, O: \#227

Ozger, G: \#1284

Ozkan, US: \#293

Ozorovich, YR: \#744

Ozturk, B: \#620

Ozyigit, II: \#1363

Ozyurt, N: \#1367

Pabroa, PCB: \#1376

Pacheco, AG: \#441

Pacheco, AMG: \#635 \#1070

Padgett, HC: \#915

Padron, I: \#1197 \#1198

Paek, S: \#1257

Paggi, JC: \#1471

Paggioli, FJ: \#1237

Pahutski, TF: \#883

Pal, R: \#686

Palagyi, S: \#397

Pallone, AK: \#1137

Pallotta, S: \#1100

Palomares, J: \#168

Pan, JM: \#1355

Panahifar, A: \#1321

Panaiotu, C: \#37

Panda, A: \#686

Pande, M: \#1223

Pandey, AK: \#493

Pandey, RS: \#869

Pandey, U: \#60

Pandey, VC: \#36

Pandit, GG: \#1376

Panigrahi, BK: \#253

Panja, S: \#897

Pant, HJ: \#196

Pantelic, G: \#168

Pantelica, A: \#1344

Papadokostaki, KG: \#852

Papadopoulos, CT: \#164 \#1113 \#1114

Papadopoulou, P: \#1119

Papaefthymiou, H: \#600 \#763 \#1253 \#1282

Papastefanou, C: \#746

Pappas, S: \#664

Parak, WJ: \#340

Parenty, ADC: \#303

Paretzke, HG: \#1241

Park, BG: \#1059

Park, BH: \#313 \#710 \#974

Park, CS: \#1105

Park, EJ: \#867 
Park, J: \#702

Park, JH: \#289

Park, JMS: \#1060

Park, K: \#172

Park, KJ: \#923

Park, SD: \#197

Park, SW: \#636 \#1151

Park, TG: \#255

Park, YH: \#1051

Parodi, A: \#552

Parra, RR: \#856

Parrish, RR: \#647 \#655 \#668

Parsa, M: \#718

Paschke, A: \#1248

Pascholati, PR: \#1480

Pascu, SI: \#30

Pashalidis, I: \#533 \#1252

Pashkova, GV: \#488

Pasieuna-Patkowska, S: \#294

Passos, LAC: \#968

Pastukhov, AV: \#545

Pasut, G: \#1140

Paszternak, A: \#426

Paszti, Z: \#426

Patchineelam, SR: \#766

Patek, G: \#376

Paterson, L: \#664

Pates, JM: \#991

Pathak, P: \#985

Pathak, PN: \#1299 \#1302 \#1303 \#1304 \#1306 \#1404

Pathak, U: \#1354

Patra, AK: \#1280

Patriarca, M: \#646 \#652 \#666 \#675

Patron, L: \#1360

Patronis, N: \#1113

Paul, H: \#580

Paul, RL: \#1027

Paulenova, A: \#593 \#1324

Paulson, AJ: \#455

Pauss, F: \#1064

Pavel, CC: \#436 \#893 \#1357

Pavel, LV: \#840 \#1357

Pavelka, S: \#210

Pawliszyn, J: \#83

Payne, JD: \#288 \#563 \#702

Pearson, CL: \#115 \#698 \#818

Peck, DV: \#460

Pedersen, K: \#257

Pedrero, Z: \#86

Pejovic-Milic, A: \#40 \#665 \#870 \#1008 \#1187 \#1188 \#1189 \#1190 \#1191 \#1194 \#1482 \#1485 \#1488

Peker, DSK: \#443

Pelicon, P: \#122 \#1489
Pelled, O: \#134 \#1085

Pellerito, C: \#32

Pellerito, L: \#32

Pelletier, L: \#314 \#512 \#930

Pelon, O: \#277

Pena, P: \#1244

Peng, C: \#204

Peng, J: \#851

Peng, SM: \#1054

Peng, X: \#805

Peng, XH: \#1049

Penny, DM: \#644

Pepponi, G: \#667

Pera, J: \#835

Perdikakis, G: \#1113 \#1114

Pereira, CM: \#339

Pereira, E: \#308 \#310 \#418

Pereira, EJ: \#705

Pereira, GR: \#474 \#1487

Pereira, MJ: \#451

Pereira, PAD: \#92

Pereira, R: \#1373

Perera, SK: \#536

Perez, CA: \#474 \#1487

Perfiliev, YD: \#73 \#555

Peroutka, M: \#211

Perrang, CM: \#192

Perret, F: \#866

Perrino, C: \#235

Perry, CR: \#45

Pervez, S: \#1329

Pestov, AV: \#841

Peterman, DR: \#396 \#595 \#1405

Petersen, PJ: \#934

Peterson, DS: \#176

Peterson, J: \#814

Peterson, LJ: \#685

Peterson, R: \#468 \#523 \#769 \#862

Peterson, RN: \#219 \#471 \#864 \#982

Peterson, SA: \#460

Petraki, E: \#1379

Petrik, M: \#177

Petrova, MA: \#319

Petrovskii, PV: \#1009

Petrucci, A: \#1180 \#1181 \#1182 \#1183

Petterson, M: \#1100

Peurrung, AJ: \#895 \#1073

Peycelon, H: \#1462

Pfeiffer, F: \#1266

Pfitzner, J: \#168

Pfund, DM: \#571

Pham, DM: \#588

Pham, K: \#288 
Pham, MK: \#168 \#772

Phillips, RP: \#544

Picciano, MF: \#45

Pichavant, M: \#513

Picheral, M: \#1051

Piekarz, M: \#300

Pierce, EM: \#432

Pierce, GD: \#142

Pietralla, N: \#1229

Pietrodangelo, A: \#235

Pietrzak-Fiecko, R: \#1213

Pihlar, B: \#611

Pike, S: \#405 \#409

Pike, VW: \#473

Pikus, W: \#1477

Pillai, MRA: \#60

Pillay, AE: \#707

Pilon-Smits, EAH: \#1465

Pilote, P: \#281

Pilsniak, M: \#552

Pilvio, R: \#168

Pinho, P: \#451

Pinto, AC: \#705

Pio, CA: \#231 \#1071 \#1369 \#1470

Piovano, EL: \#548

Pirger, Z: \#901 \#1052

Pirrone, N: \#764

Pishko, M: \#4

Pitois, A: \#610

Pitt, AR: \#303

Pla, RR: \#1471

Plastino, W: \#793

Plazinska, A: \#29

Plazinski, W: \#29

Plesek, J: \#927

Plionis, AA: \#176 \#692

Plompen, AJM: \#1076

Plukiene, R: \#1058

Plukis, A: \#1058

Poile, C: \#1350

Pointurier, F: \#661

Pokrovsky, OS: \#1022

Poledniok, J: \#336

Poletti, ME: \#1428

Polgari, Z: \#85

Polissiou, MG: \#73

Poljanc, K: \#388 \#966

Polkowska-Motrenko, H: \#298

Pollard, LJ: \#1430

Polo, E: \#34

Pomme, S: \#7

Pondaven, P: \#1051

Pongprayoon, T: \#311
Ponzevera, E: \#1426

Popa, AF: \#962

Popa, K: \#436 \#628 \#893 \#954 \#962 \#963 \#1357 \#1361

Popelka-Filcoff, RS: \#697

Popovici, E: \#1356

Porcinai, S: \#690

Porfyrakis, K: \#323

Possnert, G: \#817

Posthuma-Trumpie, GA: \#1464

Postma, H: \#1005

Postolache, S: \#436

Potiriadis, C: \#1089

Potts, PJ: \#643 \#648 \#658 \#669 \#677

Poulton, A: \#403

Poupeau, G: \#699

Pourrezaei, P: \#1478

Povinec, P: \#926 \#1367

Povinec, PP: \#22 \#153 \#371 \#469 \#744 \#765 \#769 \#772 \#793 \#987 \#1371

Povinec, PR: \#168

Pozebon, D: \#599

Prabhu, SP: \#174

Prakash, NT: \#634

Prakash, SG: \#196

Pramanik, S: \#580

Prasad, MVR: \#1148

Prasad, SK: \#136

Precek, M: \#593

Prego, R: \#372 \#995

Prestwich, WV: \#1008 \#1187 \#1482

Prevost, S: \#1454

Prevot, ASH: \#230

Price, RE: \#288

Price, RR: \#163

Prikryl, R: \#755

Principi, G: \#429

Privitera, AMG: \#744 \#1367

Proffen, T: \#906 \#907 \#908

Proffit, DE: \#691

Prohaska, T: \#773

Prospero, JS: \#819

Pruessmann, KP: \#475

Pruszynski, M: \#244

Przybytniak, G: \#1166

Psichoudaki, M: \#763

Puglia, C: \#112

Puhakainen, M: \#541

Puig, F: \#911

Puranik, VD: \#783

Purmalis, O: \#242

Puskeiler, L: \#168

Pustoshilov, PP: \#598

Puvanakrishnan, P: \#702 
Puzovic, JM: \#1075

Pysklywec, MW: \#40 \#1190

Pyun, MS: \#274

Qadeer, R: \#361

Qaim, SM: \#151 \#1300

Qian, Q: \#972

Qian, XM: \#1049

Qiao, JX: \#70 \#89

Qin, D: \#723

Qin, YW: \#797 \#799

Qin, Z: \#318 \#1201

Qing, BJ: \#365

Qiu, JZ: \#1110

Qiu, MH: \#19

Qu, Y: \#1231

Quarton, M: \#963

Queguiner, B: \#1051

Queimalinos, CP: \#333

Quetel, CR: \#1076 \#1426

Quincoces, G: \#1286

Quinn, PJ: \#978

Raashid, M: \#198

Rabb, SA: \#1027

Rabin, MW: \#692

Rabung, T: \#456 \#726

Rada, M: \#865

Radhika, S: \#1409

Radimer, K: \#45

Raditzky, B: \#1216

Raggi, A: \#634

Ragni, P: \#887 \#1261 \#1263

Raha, S: \#229

Rahiminejad-Kisomi, A: \#1159

Rahman, MF: \#1036 \#1037

Rahman, MS: \#478

Rahman, NM: \#137

Rahman, ROA: \#307

Rahman, S: \#948 \#950 \#1340

Rai, AK: \#979

Rai, DK: \#584

Rai, PK: \#979

Rai, SB: \#584

Rainer, M: \#616

Rais, J: \#1311

Raisali, G: \#875 \#1293 \#1295

Raison, PE: \#963

Raj, D: \#478 \#1133

Raj, K: \#831 \#1392 \#1396

Rajabifar, S: \#21 \#1164 \#1313

Rajalakshmi, A: \#1148

Rajamand, AA: \#1159

Rajar, R: \#744 \#1367

Rajaram, N: \#563
Rajasekaran, PR: \#894

Rajashekhar, C: \#1148

Rajec, P: \#398

Rajfur, M: \#420

Rajgor, RV: \#413

Raji, HR: \#997

Rajput, MU: \#494

Rajurkar, NS: \#493

Rakhshanderu, F: \#348

Ralson, PE: \#962

Ralston, NVC: \#460

Ramakumar, KL: \#912 \#1445

Ramanathan, S: \#730

Ramani, NVS: \#196

Ramanjaneyulu, PS: \#912

Ramassur, RT: \#1367

Rameback, H: \#1122

Ramirez, LB: \#716

Ramon, EC: \#1111

Rampazzi, L: \#87

Randa, Z: \#394 \#527 \#1012 \#1038 \#1039

Randazzo, N: \#1100

Randhawa, BS: \#965

Randi, AM: \#38

Ranebo, Y: \#672

Rannard, SP: \#304

Rao, CJ: \#425

Rao, L: \#1345

Rao, LF: \#328

Rao, PRV: \#412 \#423 \#425 \#550 \#551 \#637 \#1224 \#1292 \#1297 \#1337 \#1407

Rao, PS: \#196

Rao, RRV: \#1294

Rao, SR: \#99

Rao, TP: \#77

Rao, WH: \#1121

Rasmussen, SK: \#973

Rathore, DPS: \#1446

Ratzke, K: \#1206

Raubenheimer, HG: \#192

Rauh, JJ: \#883

Raulerson, MR: \#224

Rauret, G: \#78

Rausch, DJ: \#1440

Rausch, H: \#321

Raut, DR: \#1332

Raut, VV: \#1445

Ravaglioli, A: \#112

Ravi, S: \#872

Ravindra, K: \#700

Ravindran, PV: \#94

Ravisankar, R: \#1148

Ravnik, M: \#1097 
Ray, RS: \#399 \#597

Razouk, AK: \#1429

Razul, SG: \#120

Reddy, AR: \#932

Reddy, AVR: \#249 \#478 \#493 \#1068 \#1133 \#1325

Reddy, BP: \#1292

Reddy, BR: \#1409

Reddy, RAV: \#634

Regaldo, L: \#1471

Regev, O: \#1399

Reggioli, V: \#1100

Reglodi, D: \#901 \#1052

Reich, C: \#455 \#989 \#990

Reich, M: \#398

Reid, CD: \#544

Reifenberger, G: \#608

Reiller, PE: \#1312

Reilly, SD: \#510 \#586

Reis Coimbra, JS: \#1237

Reis, BF: \#92

Reis, MA: \#231 \#441 \#603 \#1071 \#1369 \#1470

Reischl, G: \#145 \#809 \#810

Remeikis, V: \#984 \#1058

Remenyi, T: \#1051

Remsen, A: \#819

Ren, YF: \#258 \#879

Renaud, JL: \#33

Repinc, U: \#10 \#871

Resano, M: \#673 \#1001

Retterer, ST: \#69

Revay, Z: \#63 \#68 \#321 \#905 \#906 \#1067 \#1069 \#1077 \#1117 \#1118

Reyes, AR: \#1359

Reynard, B: \#508

Reyss, JL: \#992

Rezende, CM: \#705

Ribeiro-Silva, A: \#1428

Richards, BS: \#416

Richter, LJ: \#1027

Richter, S: \#611

Riciputi, LR: \#613 \#790

Ricke, SC: \#106

Rickert, PG: \#1440

Ridenour, G: \#113

Rideout, K: \#332

Ridgway, MC: \#736

Ridone, S: \#391

Rietschel, RL: \#373

Rieuwerts, J: \#645 \#662

Rieuwertsd, J: \#651

Rigol, A: \#786

Rii, Y: \#406

Rijith, S: \#411
Rim, SA: \#846

Ringbom, A: \#814

Ritchie, C: \#1215

Ritchie, NWM: \#1027

Ritsema, R: \#606

Ritt, J: \#1271

Rizvi, MA: \#1308

Rizzo, A: \#548

Rizzotto, M: \#796

Ro, CU: \#62

Ro, TI: \#917

Roberts, KA: \#419

Robertson, JD: \#189 \#460 \#697

Robinson, B: \#1207

Robinson, BH: \#475

Robinson, WH: \#1011

Rocchi, MBL: \#1457

Rocha, HS: \#474 \#1487

Rocha, J: \#308 \#310 \#418

Rocha, MS: \#1021

Rodinov, V: \#242

Rodrigues, AE: \#1025

Rodrigues, S: \#521

Rodriguez-Espinosa, PF: \#547

Rogers, SH: \#304

Roglans, A: \#1353

Rohovec, J: \#273

Rojas, FS: \#49 \#88 \#356

Roldao, LA: \#750 \#785

Rolf, M: \#1149

Romano, E: \#1000

Romanowski, M: \#31

Romaris-Hortas, V: \#633

Romeie, M: \#100

Rondinella, VV: \#954

Rong, KW: \#450

Rongoni, A: \#748 \#801

Roohi, S: \#1322 \#1329

Roos, P: \#70 \#81 \#89

Roques, J: \#615 \#711

Rosada, A: \#382 \#1016

Rosati, A: \#1263

Rosch, N: \#399 \#597

Rose, TP: \#770

Roseland, J: \#45

Roselli, C: \#748 \#775 \#1015

Ross, JH: \#639

Rossin, R: \#1234

Rossiter, HMA: \#416

Rostampour, N: \#20

Roth, P: \#749 \#1271 \#1272

Rothuizen, J: \#47

Roussy, J: \#841 
Rout, A: \#1337

Rowshanfarzad, P: \#20 \#126 \#131 \#1146 \#1147 \#1157 \#1159 \#1161 \#1291

Roy, K: \#171 \#243

Rozelle, AL: \#1011

Rozmaric, M: \#1450

Rubcic, M: \#803

Ruberu, SR: \#536

Rubinson, KA: \#684

Rudnitskaya, A: \#392

Rudzinski, W: \#29

Ruhela, R: \#897

Ruiz, C: \#1199

Ruiz-Jimenez, J: \#90

Runde, W: \#510

Rungsupa, S: \#724

Ruprecht, G: \#1199

Rusanova, D: \#590

Russin, TJ: \#3

Ruth, TJ: \#1155

Ryan, B: \#757

Ryan, CG: \#514

Rybeck, B: \#417

Ryckman, L: \#246

Rylander, HG: \#563

Ryu, BJ: \#275

Sabet, M: \#126 \#131 \#1147 \#1157 \#1159 \#1161 \#1291

Sabharwal, KN: \#1407

Sabharwal, S: \#483

Sacchi, MC: \#34

Sachs, S: \#1216

Saddadi, F: \#1160

Sadeghi, HR: \#1287

Sadeghi, M: \#169 \#175 \#193 \#1158 \#1160 \#1293 \#1298 \#1338

Sadeghpour, H: \#626

Sadik, OA: \#740

Sadrozinski, HFW: \#1100

Saeed, MK: \#1285

Saeed, S: \#1329

Saemian, N: \#882

Saetta, D: \#748 \#801

Saey, PRJ: \#369 \#779 \#814

Safrany, A: \#1267

Saha, P: \#214

Saha, SK: \#229

Sahin, L: \#1281

Sahoo, S: \#94

Saidi, H: \#899

Saiki, M: \#449 \#967

Sailaja, N: \#1036 \#1037

Sailor, MJ: \#289

Saito, F: \#137
Saito, K: \#1128

Saito, M: \#99 \#422 \#771

Saito, MA: \#407

Saito, T: \#519 \#1092

Saito-Kokubu, Y: \#752

Saji, H: \#104

Sajn, R: \#1289

Sakai, Y: \#561 \#970 \#971

Sakaki, S: \#936

Sakamoto, K: \#1370

Sakane, H: \#918

Sakellariou, A: \#1135

Sakurai, S: \#752

Salafranca, J: \#48

Salahel-din, K: \#949

Salahuddin, SM: \#1322

Salavati-Niasari, M: \#74 \#1449

Saldanha, LG: \#45

Saldiva, PHN: \#449

Saleh, MA: \#802

Salek, M: \#273

Salem, HAM: \#290

Salih, FM: \#707

Salisbury, M: \#650 \#660

Salonen, L: \#540

Salouti, M: \#875 \#1295

Salzer, MW: \#818

Samanta, TD: \#492

Samczynski, Z: \#298

Sammons, RL: \#896

Sampa, MHO: \#1258

Samuel, G: \#203

Sanchez, A: \#374

Sanchez, G: \#223

Sanchez, MA: \#226

Sanchez, ML: \#160

Sanchez-Cabeza, JA: \#168 \#772 \#999 \#1371

Sanchez-Vila, X: \#863

Sanders, CJ: \#766

Sandroni, V: \#1051

Sandu, I: \#436

Sang, KL: \#702

Sansone, U: \#185 \#654

Santamaria, J: \#1043

Santelli, R: \#725

Santhanam, S: \#585

Santos, AJG: \#1269

Santos, AMA: \#796

Santos, ES: \#1421

Santos, FL: \#1376

Santos, HA: \#339

Santos, IR: \#219 \#267 \#471 \#862 \#864

Santos, M: \#38 
Santos, MC: \#1452

Santos, MJS: \#1421

Santos, MS: \#1421

Santoso, M: \#1376

Santschi, PH: \#419 \#463

Sanyal, U: \#1054

Sapolaite, J: \#984

Sappin, AA: \#282

Sarabadani, P: \#1338

Saraiva, SA: \#430

Saravanan, M: \#253

Sarda-Mantel, L: \#1029

Sardari, D: \#21 \#1170

Sargeson, AM: \#1173

Sarin, MM: \#986

Sarkar, A: \#1019

Sarkar, PK: \#1099

Sarkar, S: \#580

Sarkis, JES: \#1238

Sarmani, S: \#1365

Sarmento, S: \#1070

Sarthou, G: \#1051

Sasa, K: \#1152

Sasibhusan, K: \#1019

Satake, H: \#168

Sato, H: \#936

Sato, M: \#320

Sato, TK: \#318

Sato, W: \#318 \#561

Satoh, I: \#316

Satpathy, KK: \#1148

Satpati, AK: \#94

Saunders, JP: \#934

Savage, PB: \#1322

Savard, D: \#531

Savidou, A: \#852

Savoye, N: \#404 \#986 \#1051

Sawamura, S: \#1370

Sawan, MK: \#1429

Sawant, PD: \#174

Sayi, YS: \#912

Scaillet, B: \#508 \#513

Scales, N: \#976

Scates, W: \#1065

Schadel, M: \#318 \#1201

Schaefer, NG: \#287

Schafer, AI: \#416

Schafer, M: \#257

Schausten, B: \#1201

Scheider, J: \#1452

Scheinost, AC: \#591

Schiavo, MA: \#744

Schibli, R: \#287
Schikowski, J: \#168

Schillebeeckx, P: \#1076 \#1078 \#1101

Schillinger, B: \#690

Schimpf, E: \#1201

Schlegel, ML: \#466

Schlogl, R: \#63

Schlosser, C: \#758

Schlosser, G: \#628

Schlothauer, T: \#431

Schlueter, M: \#511

Schmeide, K: \#1216

Schmid, E: \#1072

Schmidt, A: \#511 \#629 \#862 \#1248

Schmidt, GA: \#817

Schmidt, S: \#986

Schmitzer, C: \#1271

Schnabel, C: \#1235

Schneeweiss, FHA: \#621 \#624

Schneider, B: \#1228

Scholten, J: \#511 \#1367

Schramm, KW: \#334

Schroeder, SLM: \#195

Schuber, R: \#1201

Schubert, M: \#511 \#629 \#1244 \#1248

Schubiger, PA: \#287

Schuchardt, U: \#430

Schulin, R: \#475 \#1207 \#1466

Schulte, R: \#1100

Schulze, R: \#206 \#690

Schunk, J: \#376

Schutz, R: \#122 \#1489

Schwantes, JM: \#66

Schwartz, J: \#563

Schwartz, JA: \#288 \#702

Schwarz, J: \#273

Schwehr, KA: \#463

Schweitzer, A: \#45

Schwerdtfeger, P: \#1195

Scopelliti, M: \#32

Scott, KCK: \#1027

Scott, WA: \#767

Scouarnec, L: \#1051

Scraggs, DP: \#1093

Sdraulig, S: \#241 \#756

Seabury, EH: \#188 \#567

Sebastiani, M: \#295

Seddik, U: \#878

Sedo, O: \#1438

Seferiades, ML: \#277

Segawa, M: \#561

Seiden, A: \#1100

Sekiya, K: \#422

Selbie, JD: \#1206 
Seliman, AF: \#1347

Selucky, P: \#9 \#927 \#958 \#960 \#964 \#1031 \#1033 \#1311 \#1493

Selvan, BR: \#551

Selvasekarapandian, S: \#585 \#1278

Semchenkov, A: \#1201

Semionkin, VA: \#557 \#902

Sen Raychaudhuri, S: \#214

Sen, K: \#947

Sen, S: \#171

Sen, Z: \#150 \#495

Sene, A: \#542

Senesi, R: \#690

Seneviratne, MCS: \#1376

Senturk, HB: \#845

Seo, CS: \#313 \#636 \#638 \#710 \#974 \#1151

Sepaniak, MJ: \#69

Sepehrian, H: \#348 \#357 \#859 \#1310 \#1390

Serce, S: \#35

Sercombe, J: \#1462

Serfor-Armah, Y: \#1469

Serne, RJ: \#461

Serris, M: \#1113 \#1114

Servaes, K: \#472

Sesli, E: \#834

Setien, J: \#226

Seyama, S: \#1092

Seznec, H: \#1135

Shaban, H: \#1149

Shabani, AMH: \#91

Shafaei, K: \#1170

Shafaii, K: \#20 \#1171

Shafiee, A: \#126

Shah, CP: \#1004 \#1047

Shah, KH: \#312

Shah, PM: \#1074

Shalem, Y: \#523

Shan, HX: \#1355

Shanehsazzadeh, S: \#1287 \#1313

Shanesazzadeh, S: \#1146

Shang, ZR: \#787

Shantarovich, VP: \#545 \#943

Shantorovich, VP: \#1217

Shao, DD: \#842 \#1026

Sharma, DN: \#1099

Sharma, JN: \#897

Sharma, P: \#727 \#1024

Sharma, S: \#869

Sharma, VK: \#196 \#1434

Sharma, YC: \#306

Sharpless, KE: \#45

Sharshar, T: \#191

Sheaff, CN: \#330
Sheh, YC: \#1141

Sheikhan, N: \#8

Shemirani, F: \#74 \#459 \#900 \#1449

Shen, B: \#145 \#810

Shen, DH: \#1352

Shen, HT: \#353

Shen, YM: \#258 \#879

Sheng, GD: \#367

Sheng, X: \#179

Sheppard, CJR: \#120 \#703 \#732

Sheppard, PR: \#113 \#410 \#1463

Sherma, J: \#61 \#681

Sherry, AD: \#640

Shetty, AM: \#288

Shi, GZ: \#353

Shi, KL: \#1323

Shi, R: \#259

Shiba, K: \#104

Shibata, M: \#918

Shibata, S: \#159 \#1242

Shibata, T: \#159 \#1242

Shibutani, T: \#515

Shilmkar, TN: \#581

Shimizu, A: \#234

Shimizu, T: \#918

Shimojo, K: \#401

Shin, DM: \#1049

Shin, HS: \#427 \#923

Shin, YS: \#915

Shinn, E: \#819

Shinoda, Y: \#234

Shinohara, A: \#318 \#561

Shinonaga, T: \#1425

Shinozaki, T: \#909

Shioda, S: \#1052

Shiraishi, K: \#538

Shirazi, B: \#169 \#875 \#1295

Shirvani, G: \#882

Shivanna, K: \#386

Shiwaku, H: \#59

Shizuma, T: \#1185

Shkurenko, O: \#866

Shoji, H: \#561

Shokeen, M: \#1234

Shokrollahi, A: \#843

Shore, S: \#670 \#678

Shtangeeva, I: \#1208

Shu, J: \#915

Shwartz, B: \#229

Siavalas, G: \#600

Sibbens, G: \#7

Sicha, V: \#927

Siclet, F: \#771 
Sicurani, J: \#699

Siddique, N: \#713 \#1340 \#1346 \#1376

Sidor, DE: \#571

Sieber, JR: \#43

Siegel, DA: \#409

Sife-Eldeen, KA: \#717

Sigaud, K: \#866

Sihvonen, AP: \#949

Silamikele, I: \#242

Silva, CM: \#308 \#310 \#418

Silva, FF: \#1443

Silva, J: \#1089

Silva, PSC: \#498 \#1269

Sima, O: \#152 \#184 \#201

Simandl, P: \#273

Simchi, A: \#1321

Simion, CA: \#1326

Simoes, FPM: \#1123

Simon, JD: \#222 \#1219

Simonds, FW: \#455

Simoni, E: \#615 \#711

Simons, DS: \#1007 \#1027

Simopoulos, A: \#888

Simpson, J: \#1093

Sin, M: \#1056

Singare, PU: \#1325

Singh, A: \#1354

Singh, BK: \#730

Singh, DK: \#594

Singh, G: \#196 \#386 \#727

Singh, KK: \#1004

Singh, N: \#36

Singh, P: \#737

Singh, S: \#478 \#1133

Singh, SK: \#584

Singh, VK: \#979

Singhal, S: \#105

Sinnott, MD: \#618

Siong, KK: \#1365

Sipala, V: \#1100

Siri, S: \#1331

Siringan, F: \#724

Sirk, TW: \#630 \#632

Sisti, D: \#1457

Sitko, R: \#1431

Sivaprasad, N: \#203 \#872

Sizgek, E: \#976

Sizgek, GD: \#976

Skakun, Y: \#151

Skarnemark, G: \#1122

Skiba, M: \#1211

Skiba, S: \#1211

Skrabalak, SE: \#1041
Skrzeszewska, PJ: \#1402

Skwarek, E: \#728

Skwarzec, B: \#1163

Slivka, J: \#1245

Small, JA: \#1027

Smirnov, AI: \#322

Smirnov, IV: \#960

Smirnova, TI: \#322

Smith, DK: \#770

Smith, DW: \#1474

Smith, EA: \#816

Smith, J: \#664

Smith, LV: \#303

Smith, SV: \#1173 \#1233

Smoczynski, S: \#1213

Smolik, J: \#273

Sneed, S: \#723

Sneve, M: \#791

Snoj, L: \#1097

So, MK: \#457

Soares, A: \#451

Soares-Gomes, A: \#725

Sodeau, JR: \#236

Sodo, A: \#1005

Sogut, O: \#1205

Sojisuporn, P: \#981

Sokaras, D: \#122 \#1489

Solari, PL: \#1053

Solis, C: \#1359

Solmaz, AN: \#808 \#1290

Solov'eva, SL: \#238

Solymos, K: \#217

Somlai, J: \#1017 \#1243

Sonar, NL: \#1392 \#1396

Sonavane, MS: \#1392 \#1396

Sonde, RR: \#196

Song, BC: \#215

Song, JH: \#732

Song, K: \#215 \#216 \#576

Song, KC: \#576

Song, KS: \#197 \#703

Song, XP: \#187

Sonugelen, M: \#620

Sood, A: \#1116

Sordo, J: \#374

Sosnin, AN: \#103

Soto, J: \#865 \#1251

Souhaut, M: \#1051

Soullaut, M: \#992

Sousa, ECPM: \#1238

Souza, AL: \#1443

Souza, AS: \#1421

Souza, GHMF: \#659 
Soylak, M: \#75 \#443 \#821 \#822 \#827 \#832 \#834 \#838 \#843 \#845 \#853

Spate, VL: \#460

Speakman, RJ: \#113 \#1463

Spejewski, EH: \#1129

Spezia, R: \#1053

Spitz, HB: \#1273

Splett, JD: \#1007

Spyrou, NM: \#1120

Srinivasan, TG: \#412 \#423 \#424 \#425 \#550 \#551 \#637 \#1224 \#1292 \#1294 \#1337 \#1407

Srncik, M: \#1314

Srogi, K: \#297

Stafford, RJ: \#288

Stafilov, T: \#1289 \#1318 \#1333

Stamatelatos, IE: \#1119

Standring, WJF: \#791

Stanik, B: \#1208

Stanley, C: \#684

Starannikova, LE: \#1217

Stefanka, Z: \#389 \#1014 \#1301

Steidinger, KA: \#819

Steier, P: \#1314

Steinberg, SM: \#118 \#446

Steiner, M: \#781

Steinhauser, G: \#114 \#138 \#147 \#211 \#388 \#393 \#966

Stenstrom, K: \#537

Stepinski, DC: \#1440 \#1444

Sterba, JH: \#114 \#138 \#211 \#388 \#393 \#966

Steyn, GF: \#128 \#192 \#1102 \#1103 \#1132

Stibilj, V: \#5 \#1318 \#1333

Stichleutner, S: \#217

Stieglitz, T: \#769 \#1367

Stiehl, GM: \#692

Stingeder, G: \#773

Stobinski, M: \#1211

Stocki, TJ: \#814

Stocks, J: \#1042

Stockwell, DA: \#819

Stohlker, U: \#1247

Stojanovic, A: \#296

Stolyar, SV: \#598

Storr, T: \#1155

Stoulos, S: \#103

Stout, D: \#915

Stracener, DW: \#1129

Strakova, H: \#1349

Stranger, M: \#700

Streli, C: \#643 \#648 \#658 \#667 \#669 \#677

Strelko, V: \#984

Strezov, A: \#777

Strnad, L: \#273

Struminska, DI: \#1163
Stuart, MAC: \#1402

Sturgeon, RE: \#649 \#674 \#1427

Su, YB: \#797

Su, YY: \#292

Subhani, MS: \#1283

Subotic, B: \#1084

Subramaniam, S: \#637 \#1294

Suda, T: \#499 \#909

Sudarshan, K: \#1068

Sudarshan, M: \#214

Sudholter, EJR: \#937

Sueki, K: \#317 \#1152

Suematsu, O: \#918

Suganuma, H: \#316

Sugawara, M: \#1185 \#1202 \#1204

Sugimoto, N: \#234

Suhas: \#379

Sum, AK: \#630 \#632

Sumita, NM: \#967

Sumiyoshi, T: \#1370

Summa, V: \#112

Sun, GM: \#1105

Sun, L: \#857

Sun, M: \#350

Sun, MZ: \#884

Sun, PP: \#1023

Sun, YC: \#190

Sun, YH: \#258 \#879

Sun, ZC: \#1455

Sun, ZH: \#221

Sunil, C: \#1099

Suranyi, G: \#80 \#389 \#1014 \#1301

Surme, Y: \#822

Sussulini, A: \#659

Suwanmala, P: \#311

Suwinska, K: \#866

Suya, N: \#1134

Suzuki, K: \#128 \#1102 \#1103 \#1315

Suzuki, M: \#363

Suzuki, S: \#59

Suzuki, T: \#59 \#545 \#1107 \#1128 \#1217 \#1225

Suzuki, Y: \#560

Svab, E: \#905 \#906 \#907 \#908

Svoboda, L: \#484

Swarbrick, B: \#1468

Swarup, R: \#1297

Swarzenski, PW: \#325 \#455 \#470 \#523 \#989 \#990

Swatski, R: \#154

Swinhoe, M: \#1083

Swoboda, M: \#63

Syed, WH: \#346

Symeopoulos, BD: \#1327

Sypula, M: \#298 
Szabo, C: \#813

Szabo, G: \#1312

Szabo, NA: \#376

Szabo, Z: \#616

Szakacs, S: \#1270

Szaloki, I: \#53

Szechenyi, SC: \#1423

Szegvary, T: \#1247

Szelecesnyi, F: \#1103

Szelecsenyi, F: \#128 \#192 \#1102 \#1132 \#1315

Szentmiklosi, L: \#63 \#1067 \#1118

Szikszai, Z: \#1136

Szoboszlai, N: \#85

Szoboszlai, Z: \#1136

Szopa, Z: \#298

Szorcsik, A: \#32

Tada, R: \#377

Taddei, MH: \#358

Tagami, K: \#72 \#79 \#239 \#331 \#337 \#742 \#761 \#762 \#786 \#787 \#921 \#922 \#1127

Tajika, E: \#377

Takagi, M: \#99

Takahashi, H: \#234

Takahashi, T: \#743 \#1152

Takahashi, Y: \#694

Takaku, Y: \#760 \#1368

Takamatsu, T: \#501 \#1063

Takamiya, K: \#159 \#1098 \#1242

Takamura, Y: \#99

Takatsuki, Y: \#524

Takayama, H: \#918

Takayama, T: \#561

Takeda, A: \#742 \#743 \#759 \#760 \#1368

Takeda, H: \#72

Takeishi, M: \#924

Takenaka, Y: \#519

Takeshita, T: \#1098

Takeuchi, T: \#515 \#1109

Takoudis, G: \#1089

Talamonti, C: \#1100

Talha, SA: \#166

Talip, Z: \#776

Tamagnan, G: \#163

Tamaki, T: \#631

Tamari, M: \#1152

Tamas, A: \#901 \#1052

Tamiya, E: \#99

Tammiku-Taul, J: \#641

Tan, B: \#259

Tan, BZ: \#19

Tan, H: \#565 \#1080

Tan, MG: \#156 \#800

Tan, XL: \#109 \#364 \#456 \#829 \#848
Tan, Z: \#811

Tan, ZL: \#221

Tanaka, M: \#1411

Tanaka, S: \#499 \#519

Tananaev, IG: \#1009

Tang, CY: \#1042

Tang, R: \#857

Tang, SX: \#221

Tang, TT: \#820

Tanigaki, M: \#1098

Taniguchi, A: \#918

Taniguchi, M: \#724 \#744 \#769 \#1367 \#1378

Taniyasu, S: \#457

Tank, R: \#1354

Tanner, M: \#680

Tanner, PA: \#739

Tanner, SD: \#607

Tanoi, K: \#1092

Tao, XQ: \#604

Tarancon, A: \#78

Tarantilis, PA: \#73

Tarits, C: \#119

Tarrino, A: \#1418

Tartari, A: \#1486

Tasci, C: \#127

Tateo, F: \#112

Tauhata, L: \#785

Tavakoli, AB: \#1313

Tavakoli, Z: \#827

Tavallali, H: \#843

Taylor, A: \#646 \#652 \#666 \#675

Taylor, JS: \#373

Taylor, VF: \#1420

Tayo, GO: \#221 \#811

Tejada, M: \#518

Tejeda, S: \#1483

Tejero, A: \#223

Teksoz, S: \#149 \#283

Tel, E: \#812

Telouk, P: \#939

Teng, SP: \#157 \#719

Teng, Y: \#1342

Tenini, S: \#1006

Terry, J: \#1307 \#1316

Teschke, K: \#332

Teschner, D: \#63

Tesema, YT: \#588

Testard, F: \#1454

Thackray, AC: \#896

Thakur, P: \#594 \#985 \#1296

Thierfelder, C: \#1195

Thirlwall, M: \#929

Thirolf, PG: \#1203 
Thomalla, S: \#403

Thomas, BJ: \#1186

Thomsen, KJ: \#942 \#1106

Thomson, K: \#303

Thorle, P: \#1201

Thornthwaite, DW: \#304

Thuiller, D: \#1051

Tian, G: \#858 \#1397

Tian, M: \#256

Tian, MS: \#1455

Tian, WY: \#350

Ticina, V: \#496

Tickner, JR: \#161

Tie, LY: \#110

Tillotson, RD: \#396 \#595 \#1405

Timmermans, K: \#1051

Tinker, RA: \#756

Tirasoglu, E: \#324 \#1205

Tirumalesh, K: \#386

Tiwari, A: \#323

Tiwari, D: \#136

Tkac, P: \#593

Todorovic, NZ: \#1245

Toh, Y: \#506 \#562 \#1185 \#1202 \#1204 \#1362

Tojo, A: \#918

Tokman, N: \#601 \#825

Tokonami, S: \#1246

Toku, H: \#1063

Tolkach, A: \#977

Tomankiewicz, E: \#299 \#1165

Tomar, BS: \#730 \#831

Tomar, R: \#727 \#730 \#1024

Tomas, CR: \#819

Tomasic, N: \#803

Tomillo, C: \#226

Tomita, S: \#631

Tomlin, BE: \#207 \#1087

Tong, BW: \#12

Tong, DQ: \#884

Tong, WF: \#1461

Top, Z: \#469

Topcuoglu, S: \#447 \#1239 \#1240 \#1275

Toplu, C: \#35

Torrence, R: \#1468

Torrent, A: \#1353

Torres-Escribano, S: \#486

Torri, G: \#132 \#140 \#165 \#789

Torvenyi, A: \#185

Tosaki, Y: \#1152

Toth, G: \#901 \#1052

Touceda, A: \#374

Toume, H: \#318

Tovesson, F: \#1200
Toyazaki, H: \#320

Toyoda, A: \#1128 \#1152 \#1153

Toyoshima, A: \#317 \#318

Trajkovic, V: \#1048

Tran, B: \#1424

Trancoso, MA: \#231 \#1071 \#1369 \#1470

Trautmann, C: \#894

Treichler, H: \#287

Trellue, H: \#1112

Trgo, M: \#582

Tripathi, R: \#1068

Tripathi, RM: \#783

Tripathi, SC: \#897

Tripathy, SP: \#1099

Trkov, A: \#1056 \#1097

Trochimczuk, AW: \#552

Trottmann, M: \#656

Troutman, TS: \#31

Trull, T: \#1051

Trull, TW: \#404 \#409

Tryshyn, VV: \#1066

Tsabaris, C: \#164

Tsagas, NF: \#1255

Tsai, CS: \#194

Tsakovski, SL: \#804

Tsapatsaris, N: \#195

Tsiaili, A: \#1252

Tsigankov, NY: \#538

Tsipenyuk, YM: \#301

Tsuchihashi, T: \#631

Tsuchiya, K: \#631

Tsuchiya, Y: \#1013

Tsuji, K: \#62

Tsukada, H: \#72 \#742 \#743 \#759 \#760 \#1368

Tsukada, K: \#317 \#318

Tsumune, D: \#524

Tsuruoka, Y: \#561

Tsushima, S: \#591

Tu, XL: \#798

Tucak-Zoric, S: \#360

Tucek, J: \#687

Tucker, SA: \#1317

Tufail, SJM: \#860

Tugarova, AV: \#73

Tuma, MP: \#369

Tumcharern, G: \#311

Tumey, SJ: \#1111 \#1124

Tunc, MS: \#807

Tuniz, C: \#1285

Tunnell, JW: \#563 \#702

Turgeon, S: \#613

Turhan, S: \#753 \#754 \#947 \#951 \#1277

Turker, AR: \#605 
Turkoglu, O: \#443

Turkozu, DA: \#1256

Turler, A: \#206 \#1201

Turner, JV: \#1367

Turner, S: \#1027

Turnewitsch, R: \#403 \#986

Turta, NA: \#945

Turtiainen, T: \#949

Turtoi, A: \#621 \#624

Tuteja-Krysa, M: \#1211

Tuzen, M: \#75 \#821 \#834

Twining, BS: \#806

Tyagi, AK: \#831

Tymen, G: \#119

Ubele, M: \#145 \#809

Uchida, S: \#72 \#79 \#239 \#331 \#337 \#742 \#761 \#762

\#771 \#786 \#787 \#921 \#922 \#1127

Uddin, MS: \#1108

Ueda, S: \#788

Uehara, A: \#938

Uitz, J: \#1051

Ujevic, I: \#439

Ullas, GV: \#883

Ullom, JN: \#692

Ulrich, A: \#656

Ulrych, J: \#527

Uluozlu, OD: \#75

Ulusoy, U: \#839

Um, W: \#461

Umar, IM: \#130

Umezawa, Y: \#1378

Umisedo, N: \#796

Unak, P: \#127 \#149 \#265 \#283 \#284 \#572 \#1138

Unak, T: \#1138

Ungar, K: \#202

Ungar, RK: \#814 \#817

Unlu, A: \#807

Unny, VKP: \#872

Urena-Nunez, F: \#836

Ursini, O: \#885 \#886 \#887 \#1259 \#1260 \#1261 \#1262 \#1263 \#1265

Usoskin, IG: \#817

Usta, C: \#75

Ustundag, Z: \#1414

Usuda, S: \#752

Uthamanthil, RK: \#288

Uvarov, VL: \#874 \#1218

Uwamino, Y: \#159 \#1242

Vad, K: \#479 \#888

Vaezifar, S: \#996

Vagelas, I: \#955

Vaidya, VN: \#961

Vajda, N: \#389 \#1014 \#1301
Vale, C: \#372 \#995

Vale, MGR: \#1421

Valenta, MM: \#432

Valentin, G: \#734

Valkovic, V: \#360

Valladon, M: \#526

Vallant, RM: \#616

Vallejo, E: \#223

Valliant, JF: \#279

Valsala, TP: \#1396

Valu, SO: \#436

van Amerongen, A: \#1464

van Beek, P: \#992 \#1051

van Berkel, WJH: \#1464

Van Bocxstaele, M: \#1426

van Breemen, O: \#282

van Dam, RM: \#915

Van de Wiele, T: \#1232

Van den Haute, P: \#390

van den Ingh, TSGAM: \#47

van den Winkel, P: \#1293

van der Gucht, J: \#1402

van der Loeff, MMR: \#511

Van der Loeff, MR: \#986

van der Meulen, NP: \#192 \#1103

van der Walt, TN: \#128 \#192 \#1102 \#1103 \#1132

Van Deun, R: \#472

van Eijk, CWE: \#689

Van Grieken, R: \#700 \#861

Van Grieken, RE: \#53

van Schooneveld, MM: \#1042

Van Sickle, J: \#460

van Staveren, DR: \#287

Van Vaeck, L: \#647 \#655 \#668

van Well, AA: \#260

Vance, ER: \#910

Vandenberghe, D: \#390

Vane, CH: \#998

Vanhaecke, F: \#42 \#673 \#1001

Vanhoof, C: \#643 \#648 \#658 \#669 \#677

Vanin, VR: \#494

Vanura, P: \#6 \#9 \#11 \#958 \#960 \#964 \#1031 \#1032 \#1033 \#1034 \#1035 \#1209 \#1492 \#1493 \#1494

Vanysek, P: \#716

Varga, K: \#376

Varga, Z: \#71 \#76 \#80 \#141 \#389 \#969 \#1014 \#1301

Vargo, GA: \#819

Varner, K: \#740

Vasconcellos, MBA: \#967

Vasconcelos, MTSD: \#1070

Vasilakoglou, I: \#955

Vaupotic, J: \#764 \#1370

Vazeux, M: \#542 
Vazquez, GJ: \#592

Vazquez-Duran, A: \#121

Vazquez-Jauregui, E: \#1184

Vazquez-Selem, L: \#1463

Vegro, M: \#391

Veilly, E: \#615 \#711

Velasco, H: \#796

Veldhuis, M: \#1051

Velez, D: \#486

Veltri, M: \#945

Venkataraman, R: \#183

Venkataramaniah, K: \#253

Venkatesan, KA: \#412 \#423 \#424 \#425 \#550 \#551 \#637 \#1224 \#1292 \#1294 \#1337

Venkatesh, M: \#60 \#203 \#1142

Venkateswaran, G: \#77 \#94

Vennemann, TW: \#314

Venti, F: \#1000

Ventura, MG: \#441 \#1470

Venugopal, V: \#1297

Verbruggen, A: \#676 \#1122

Verburg, TG: \#213

Verdeny, E: \#405

Veress, E: \#905 \#906 \#907 \#908

Vermeulen, C: \#1103 \#1132

Veronese, FM: \#1140

Verrall, M: \#514

Vertes, A: \#217 \#426 \#479 \#555 \#559 \#888 \#1434

Veselinovic, DS: \#959

Veskovic, M: \#1245

Vetterlein, D: \#1467

Viana, RR: \#706

Vicente, EM: \#507

Vidal, M: \#786

Vidmar, J: \#201

Vidmar, T: \#2

Vigliano, PH: \#248 \#251

Viguri, J: \#865

Villa, M: \#849

Villareal, TA: \#819

Villemant, B: \#508

Vils, F: \#512 \#930

Vincent, D: \#1051

Vincent, T: \#552

Vincze, A: \#1267

Vinsova, H: \#755

Viollier, E: \#1051

Visser, D: \#1005

Vitali, G: \#690

Vitorovic, G: \#168

Vlachou, A: \#1327

Vlasova, I: \#792

Vlastou, R: \#164 \#1113 \#1114
Vobecky, M: \#210

Vockenhuber, C: \#1199

Vodenik, B: \#2

Voge, I: \#986

Vogel, HJ: \#1467

Vogiannis, E: \#1379

Vogler, EA: \#4

Vogt, S: \#806

Vojtyla, P: \#22

Volkert, WA: \#1139

Volter, W: \#1491

von Brentano, P: \#1229

von Maltzahn, G: \#123 \#289

von Rohden, C: \#549

Vong, L: \#1051

Vontobel, P: \#1466

Vranjes-Duric, S: \#1048

Vucic, E: \#1042

Vulpius, D: \#1419

Vuong, TB: \#1376

Vuono, D: \#945

Wacker, JF: \#66

Waclawek, M: \#420 \#1472

Waclawek, W: \#420

Waclawik, ER: \#1178

Wadgaonkar, ND: \#934

Wadgaonkar, PP: \#581

Wagener, T: \#1051

Waghorn, PA: \#30

Wagner, M: \#1452

Waheed, S: \#713 \#1340 \#1346 \#1376

Wahl, W: \#540 \#1271 \#1272

Wai, CM: \#330

Waibel, R: \#287

Waker, AJ: \#1191

Waldis, JK: \#46

Walker, KL: \#322

Wallace, C: \#802

Wallace, H: \#697

Wallenius, M: \#71

Wallez, G: \#963

Wallner, A: \#1314

Wallner, G: \#1314

Walo, M: \#1166

Walsh, JJ: \#819

Walter, M: \#893

Wan, XG: \#19

Wan, ZW: \#820

Wang, D: \#1477

Wang, DX: \#820

Wang, FL: \#566

Wang, H: \#1397

Wang, HY: \#342 
Wang, J: \#462 \#1342

Wang, JC: \#288 \#563 \#1395 \#1408

Wang, JH: \#56 \#329

Wang, JS: \#330

Wang, JX: \#904 \#1040 \#1456 \#1459

Wang, L: \#858 \#1397

Wang, LH: \#350

Wang, LHV: \#1041

Wang, LJ: \#448

Wang, LM: \#50

Wang, LQ: \#566

Wang, M: \#221 \#851

Wang, MD: \#105 \#1049

Wang, N: \#1478

Wang, PC: \#383

Wang, Q: \#740

Wang, S: \#485

Wang, SJ: \#944

Wang, SR: \#1323

Wang, SW: \#187 \#844

Wang, TC: \#904

Wang, TH: \#157 \#719

Wang, TM: \#259

Wang, TT: \#108 \#351

Wang, WH: \#481 \#575 \#957 \#1406

Wang, XB: \#143 \#179 \#880

Wang, XG: \#353

Wang, XK: \#108 \#109 \#133 \#364 \#415 \#456 \#829 \#842 \#848 \#1026

Wang, XR: \#462

Wang, XY: \#350 \#1415

Wang, Y: \#457

Wang, YM: \#880

Wang, YN: \#1478

Wang, YQ: \#1131

Wang, Z: \#344

Wang, ZL: \#1372

Wang, ZM: \#461

Waples, JT: \#986

Waqif-Husain, S: \#348 \#357 \#859 \#1390

Warburton, WK: \#565 \#1080

Ward, NI: \#642

Ward, WC: \#222

Warner, BP: \#685

Waseem, M: \#312

Wasim, M: \#1319 \#1334

Watanabe, Y: \#561 \#970 \#971

Watanabe, YX: \#1185

Waters, LS: \#1112

Watling, J: \#514

Watrous, MG: \#612

Watt, AAR: \#323

Wattayakorn, G: \#724 \#981 \#1378
Watters, LM: \#1187

Webber, CE: \#1188

Weber, SG: \#1460

Weber, WJ: \#895

Wegrzecki, M: \#1201

Wegrzynek, D: \#677

Wei, GS: \#851

Wei, GZ: \#368

Wei, JF: \#925

Wei, YY: \#719

Wei, YZ: \#23 \#24 \#1388 \#1406

Weiher, N: \#195

Weinstein, M: \#1250

Weinstein, Y: \#523

Weisberg, RH: \#819

Weller, U: \#1467

Welling, MM: \#935 \#1029

Wellman, DM: \#432

Wells, SM: \#69

Wellum, R: \#676 \#1122

Welz, B: \#1421

Wen, J: \#503

Wenger, JC: \#236

Wernli, B: \#46

West, JL: \#701

West, M: \#643 \#648 \#658 \#669 \#677

Wharton, J: \#188

White, M: \#646 \#652 \#666 \#675

White, S: \#532

Whitehouse, MJ: \#672

Whitledge, TE: \#819

Whitney, S: \#735

Whitney, SM: \#1145

Whitted, J: \#45

Widom, A: \#480

Wienke, H: \#1056

Wierczinski, B: \#260

Wilcken, KM: \#1235

Wildgoose, GG: \#1055

Wilger, J: \#45

Wilkens, LR: \#285 \#286

Williams, BD: \#461

Williams, DC: \#1100

Williams, PN: \#1465

Williams, RW: \#1111

Williamson, MJ: \#1030

Williart, A: \#1082

Willie, S: \#649

Wilson, DW: \#570

Wimolwattanapun, W: \#1376

Winchester, MR: \#1027

Wirth, HF: \#1203

Witold, W: \#245 
Witten, ML: \#113

Wobrauschek, P: \#643 \#648 \#658 \#667 \#669 \#677

Wogiatzi, E: \#955

Wolf, WR: \#45

Wolff, J: \#894

Wolterbeek, HT: \#213

Wong, HT: \#1110

Wong, KY: \#732

Woodard, BB: \#802

Woodhead, D: \#168

Woods, R: \#1199

Wootsch, A: \#63

Wotawa, G: \#369 \#814

Wrona, A: \#1211

Wu, B: \#1452

Wu, CM: \#503

Wu, FC: \#156 \#774 \#1380

Wu, FY: \#884

Wu, G: \#904

Wu, JM: \#182 \#190

Wu, QH: \#344

Wu, S: \#1328

Wu, SL: \#353

Wu, SY: \#353 \#1110

Wu, WS: \#364 \#415 \#1026 \#1323 \#1341

Wu, XC: \#50

Wu, XF: \#1121

Wu, Y: \#568

Wu, YM: \#884

Wu, YN: \#457

Wu, ZJ: \#365

Wuenschmann, S: \#37

Wulferink, M: \#935

Wyman, M: \#373

Wynants, R: \#1078 \#1101

Wyse, E: \#153 \#168

Xanthos, S: \#1089

Xi, GH: \#344

Xi, P: \#953

Xi, SX: \#880

Xi, ZY: \#898

Xia, L: \#953

Xia, Y: \#1345

Xia, YN: \#1041

Xian, L: \#1343

Xiang, YL: \#884

Xiao, CL: \#573 \#823 \#946

Xiao, Y: \#343

Xie, GX: \#1131

Xie, H: \#1044

Xie, MX: \#1178

Xie, P: \#450

Xie, Z: \#402
Xing, GM: \#940

Xing, YP: \#450

Xiong, J: \#944

Xu, C: \#419

Xu, D: \#109 \#829

$\mathrm{Xu}, \mathrm{GM}$ : \#805

Xu, GT: \#292

Xu, JY: \#292

Xu, L: \#851

Xu, MY: \#880

Xu, XG: \#368

Xu, YS: \#944

Xu, YY: \#898

Yacouta-Nour, A: \#276

Yaftian, MR: \#718

Yahaya, R: \#1366

Yaita, T: \#59

Yajima, T: \#560

Yakushev, A: \#1201

Yalcin, S: \#1254

Yamada, K: \#694

Yamada, M: \#96 \#148 \#156 \#454 \#657 \#774 \#1372

$$
\text { \#1380 \#1439 }
$$

Yamada, Y: \#315 \#558 \#560 \#561

Yamaji, FM: \#1480

Yamamoto, M: \#747

Yamamoto, S: \#377

Yamamoto, T: \#1013

Yamana, H: \#909 \#938

Yamanoi, Y: \#1153

Yamasaki, T: \#320

Yamashita, MT: \#1480

Yamashita, N: \#457

Yamauchi, S: \#970 \#971

Yamazaki, H: \#752

Yampol'skii, YP: \#545 \#1217

Yan, L: \#1397

Yan, LT: \#1131

Yan, TH: \#1343

Yan, YC: \#805

Yanaga, M: \#316

Yang, DJ: \#1178

Yang, H: \#450

Yang, J: \#291 \#1475

Yang, L: \#649 \#1049

Yang, LJ: \#850

Yang, LN: \#604

Yang, SL: \#402

Yang, ST: \#842

Yang, SY: \#143

Yang, WJ: \#204

Yang, XM: \#1041

Yang, Y: \#17 \#247 \#1328 
Yang, YJ: \#577

Yang, Z: \#143 \#256

Yang, ZQ: \#1341

Yannakopoulou, E: \#1119

Yao, X: \#232

Yaprak, G: \#1279

Yari-Kamrani, Y: \#1147 \#1158

Yasar, U: \#1363

Yasin, OA: \#1285

Yasir, MS: \#1366

Yasuda, K: \#752

Yatluk, Y: \#841

Yavari, R: \#1310 \#1390 \#1391

Yawata, T: \#1109

Yazici, K: \#271

Yazicigil, Z: \#1414

Ye, CS: \#972

Ye, J: \#12

Ye, XS: \#365

Yeager, CM: \#463

Yechieli, Y: \#523

Yee, PJ: \#976

Yeh, SA: \#182 \#190

Yehia, A: \#27

Yeo, HM: \#275

Yetley, EA: \#39 \#45

Yeung, LWY: \#457

Yidirim, Y: \#1138

Yildirim, G: \#812

Yildirim, N: \#845

Yildiz, E: \#35

Yim, SP: \#1257

Yin, MD: \#19

Yin, QZ: \#938

Yin, XY: \#1110

Yin-Goen, Q: \#1049

Yip, YC: \#1461

Yiu, HHP: \#617

Yokoi, N: \#320

Yokoyama, A: \#318

Yolcular, S: \#149 \#284

Yoneda, K: \#500

Yoo, HY: \#638

Yoon, B: \#330

Yoon, JS: \#1083 \#1151

Yooprasert, N: \#311

Yoshida, T: \#363

Yoshikawa, A: \#499 \#909

Yoshikawa, K: \#500 \#501 \#502 \#1063

Yoshikawa, S: \#752

Yoshimura, EM: \#796

Yoshino, H: \#1098

Yoshioka, Y: \#320
You, QB: \#1110

Young, AN: \#1049

Yousefi, SR: \#1449

Yousefnia, H: \#21 \#1150 \#1171

Yu, HX: \#457

Yu, JH: \#347

Yu, LL: \#1027

Yu, RL: \#448 \#798

Yu, SM: \#108 \#364 \#415

Yu, W: \#944

Yu, XJ: \#25

Yu, YL: \#329

Yuan, DQ: \#352

Yuan, H: \#940

Yuan, J: \#1110

Yuan, X: \#798

Yuan, Y: \#1178

Yucel, H: \#808 \#1290 \#1447

Yuki, H: \#395

Yurdakoc, M: \#1389

Yurekli, Y: \#149

Yurt, A: \#284

Yusan, S: \#833 \#1256

Zabriskie, JM: \#188

Zacco, A: \#1006

Zagury, GJ: \#117

Zahedi, M: \#843

Zahid, GH: \#1346

Zahringer, M: \#758 \#781 \#1088

Zaichick, S: \#252 \#254

Zaichick, V: \#252 \#254

Zaidi, JH: \#1319 \#1334 \#1346

Zaki, AA: \#1320

Zali, A: \#175 \#193

Zalutsky, MR: \#244

Zaman, RT: \#563

Zaman, W: \#1477

Zamani, H: \#602

Zamani-Valasiadou, M: \#103

Zamburlini, M: \#1188 \#1482 \#1485 \#1488

Zamostyan, PV: \#538

Zandi, H: \#1150 \#1170

Zang, XH: \#344

Zangirolami, DM: \#268

Zangoni, E: \#1140

Zapata, MA: \#1478

Zaray, G: \#85

Zarazua, G: \#1483

Zavarin, M: \#770

Zayed, JM: \#30

Zboril, R: \#687

Zecca, L: \#222 \#1219

Zeevaart, JR: \#129 
Zehnalek, J: \#433

Zeinali, B: \#193

Zeisler, R: \#43 \#207 \#1087

Zeisler, RL: \#1027

Zeller, KP: \#145 \#809 \#810

Zeman, J: \#755

Zemb, T: \#1454

Zen, H: \#502

Zeng, HP: \#351

Zeng, WY: \#19

Zeng, XZ: \#19

Zeng, ZZ: \#731

Zepernick, R: \#63

Zepezauer, F: \#1208

Zerkin, V: \#1056

Zerovnik, G: \#1097

Zhai, JP: \#857

Zhai, ML: \#851

Zhan, XC: \#1433

Zhang, A: \#720

Zhang, AY: \#23 \#24 \#26 \#481 \#573 \#575 \#578 \#823

\#946 \#957 \#1381 \#1383 \#1384 \#1388 \#1394 \#1406

Zhang, DL: \#546

Zhang, DW: \#353

Zhang, F: \#340

Zhang, GD: \#256

Zhang, GL: \#800

Zhang, H: \#402

Zhang, HB: \#17 \#247

Zhang, HF: \#1458

Zhang, JB: \#179

Zhang, JS: \#805

Zhang, JX: \#247

Zhang, JY: \#543

Zhang, K: \#50

Zhang, KR: \#343

Zhang, L: \#799 \#850

Zhang, LL: \#50 \#904

Zhang, ML: \#187 \#1341

Zhang, MY: \#344

Zhang, P: \#543

Zhang, R: \#256

Zhang, W: \#202 \#802

Zhang, WL: \#402

Zhang, XL: \#110 \#438

Zhang, XZ: \#204

Zhang, Y: \#503 \#857 \#1343

Zhang, YH: \#1202

Zhang, YJ: \#910

Zhang, YQ: \#258

Zhang, YS: \#1380

Zhang, YX: \#800

Zhang, YY: \#1341
Zhang, ZY: \#355 \#1458

Zhao, C: \#45

Zhao, CX: \#18

Zhao, D: \#402

Zhao, F: \#940

Zhao, FJ: \#1465

Zhao, GJ: \#1455

Zhao, GP: \#503

Zhao, GX: \#367

Zhao, J: \#368

Zhao, L: \#485

Zhao, PH: \#770

Zhao, Y: \#12 \#15

Zhao, YH: \#798

Zhao, YL: \#355 \#383 \#940 \#1040 \#1221 \#1231 \#1456 \#1458 \#1459

Zhao, YS: \#858 \#1397

Zheng, B: \#343

Zheng, BH: \#797 \#799

Zheng, H: \#148

Zheng, J: \#96 \#156 \#454 \#657 \#774 \#1380 \#1439

Zheng, JZ: \#462

Zheng, MB: \#349

Zheng, MQ: \#503

Zheng, WF: \#1343

Zheng, YN: \#352

Zheng, ZF: \#1178

Zhou, AL: \#740

Zhou, C: \#894

Zhou, CS: \#221

Zhou, D: \#1110

Zhou, DM: \#352

Zhou, DR: \#491

Zhou, F: \#1121

Zhou, GQ: \#1221 \#1456 \#1459

Zhou, LJ: \#50

Zhou, RM: \#1121

Zhou, X: \#364

Zhou, XH: \#1202

Zhou, XZ: \#543 \#1408

Zhou, Y: \#953 \#1042

Zhu, BK: \#898

Zhu, H: \#183 \#258

Zhu, HY: \#1178

Zhu, J: \#1131

Zhu, JZ: \#291

Zhu, L: \#16 \#17 \#247

Zhu, LP: \#898

Zhu, SY: \#352

Zhu, YG: \#1465

Ziad, N: \#928

Ziembik, Z: \#1472

Zihnioglu, F: \#127 
Zilles, K: \#608

Zimek, Z: \#1166

Zisos, A: \#1379

Zitha, PLJ: \#362

Zitnik, M: \#122 \#1489

Zolghadri, S: \#1171

Zombori, P: \#1271

Zoppi, M: \#1005

Zoriy, MV: \#608 \#609

Zorz, N: \#1454

Zotina, TA: \#795

Zucca, FA: \#222 \#1219

Zucchi, OLAD: \#1428

Zuilhof, H: \#937

Zukowska, J: \#804

Zuo, C: \#1343

Zuo, R: \#1342

Zuo, Y: \#352 \#574 \#714

\section{A3 Keyword index}

18-crown-6: \#55 \#319

$2.5 \mathrm{GeV}$ bremsstrahlung: \#1133

$2.8 \mathrm{MeV}$ neutrons: \#198 \#1094

$20 \mathrm{MeV}$ : \#1143

3-D flux monitoring: \#301

3-D imaging: \#1135

$40 \mathrm{MeV}$ proton: \#1108

50 and $65 \mathrm{MeV}$ bremsstrahlung: \#478

A Fuata: \#699

Ab initio calculations: \#937 \#1034 \#1035

Abiotic mercury methylation: \#333

Ablation plasma: \#1430

ABS: \#172

Absorbed dose: \#1287

Absorption and fluorescence spectroscopies: \#884

Accelerator: \#1008

Accelerator mass spectrometry: \#22 \#153 \#607 \#1145

Accident source terms: \#919

Accumulation: \#242

Acetohydroxamic Acid: \#593

Acetylcholinesterase: \#452 \#1374

Acid dyes: \#276

Acid leaching: \#1380

Acid media: \#1386

Acid mine drainage: \#824

Acid solutions: \#959

Acidic gases: \#233

Acidic solutions: \#1398

Acrylic acid: \#483

Actinide complexation: \#328

Actinide coordination chemistry: \#731
Actinide extraction: \#595 \#1311

Actinide recycle: \#1225

Actinides: \#326 \#378 \#519 \#612 \#768 \#985 \#1125

\#1301 \#1312 \#1448

Actinium-225: \#317

Activated carbon: \#379 \#414 \#858 \#1393 \#1397 \#1442

Activated charcoal: \#361

Activation: \#1107 \#1128 \#1152

Activation analysis: \#527 \#1154 \#1326

Activation energy: \#831 \#1062

Activation foil technique: \#1079

Activation technique: \#1143

Active interrogation: \#188 \#567

Activity concentration: \#795 \#860 \#997 \#1348

Activity index: \#753

Activity measurement: \#353

Activity ratio Th-228/Th-232: \#1165

Activity ratio Th-230/Th-232: \#1165

Activity-dependence: \#1052

Acute toxicity: \#335

Acute toxicity test: \#867

Acylals: \#8

ADC conversion time: \#1090

Additives: \#349 \#350

Adhesion: \#694

Admicellar polymerization: \#311

Adriatic Sea: \#496

Adsorption: \#107 \#108 \#109 \#110 \#111 \#220 \#227 \#243

\#276 \#312 \#361 \#365 \#367 \#379 \#414 \#436 \#481

\#553 \#615 \#695 \#706 \#711 \#737 \#776 \#823 \#837

\#839 \#842 \#844 \#847 \#848 \#851 \#854 \#855 \#856

\#857 \#858 \#859 \#946 \#1167 \#1257 \#1305 \#1336

\#1382 \#1384 \#1406 \#1416

Adsorption isotherms: \#290 \#833

Adsorption kinetics: \#365 \#411

Adsorption properties: \#1025

Adsorptive precipitation: \#342

Aegean: \#698

Aerosol: \#122 \#213 \#230 \#354 \#603 \#815 \#975 \#1136 \#1470

Aethalometer: \#1081

Affinity capillary electrophoresis: \#1460

After-effects: \#555

Afterpulse: \#155

Agbabu: \#1249

Age determination: \#80 \#1122

Age groups: \#250 \#1149

Age involution: \#252 \#254

Aging: \#722

Agricultural by-products: \#1393

Agricultural crop: \#922

Agricultural field: \#337 \#761

Agricultural wastes: \#1477 
Agriculture: \#1223

Air: \#758

Air particulate matter: \#1071

Air pollution: \#354 \#449 \#453 \#547

Air quality: \#232

Air quality monitoring: \#236

Airborne lead: \#547

Airborne manganese: \#547

Airborne particulate matter: \#739

Airborne particulates: \#441

Airborne $\mathrm{Pu}$ concentration measurement: \#137

Airborne release: \#924

Air-ions: \#205

Alboran Sea: \#928

Albumin: \#871

Aldehyde: \#8

Aldehyde oxidation: \#809

Algae: \#686

Algebraic models: \#1229

Algerian basin: \#765

Alginate: \#847

Aliquat-336: \#550 \#828

Alkali and alkaline earth metals: \#378

Alkali earth speciation: \#941

Alkaline basalts: \#969

Alkaloids: \#259

Alkyl-phosphonic acid: \#426

Alluvial island: \#521

Alluvial soils: \#437

Alpha activity ratio: \#215

Alpha- and gamma-measurements: \#780

Alpha particles: \#539

Alpha radioactivity: \#1252

Alpha specific activity: \#215

Alpha spectrometry: \#7 \#81 \#135 \#140 \#165 \#174 \#195

\#579 \#775 \#789 \#987 \#1014 \#1017 \#1074 \#1095

\#1301 \#1448

Alpha track technique: \#174

Alpha/beta/gamma: \#768

Alpha/beta/gamma coincidence: \#1082

Alpha-benzildioxime: \#169

Alpha-benzoin oxime: \#821

Alpha-furyldioxime: \#169

Alpha-particle counting: \#7

Alpha-particle detection: \#692

Alpha-particle effects: \#692

Alpha-picoline: \#227

Alpha-track analysis: \#792

Alteration: \#1179

Alternative stable stages: \#517

Aluminium oxide nanomaterials: \#1036

Aluminosilicate: \#859
Aluminum: \#870 \#920 \#972

Aluminum coordination: \#1023

Aluminum oxide: \#1037

Aluminum-absorber: \#1109

Alzheimer's disease (AD): \#1222

Amberlite XAD resins: \#1389

Amberlite XAD-2: \#413

Americium: \#76 \#299 \#519 \#579 \#594 \#786 \#792 \#795 \#897 \#984 \#1014 \#1095 \#1297 \#1301 \#1314 \#1337 \#1341

Americium(III): \#316 \#1009

Americium-241: \#391

Americium-243 half-life: \#1074

Ametryne: \#1264

Amide: \#718

Amino: \#348

Amino acid: \#274 \#492

Amino-functionalized: \#346

Ammonia tepida: \#1000

Ammonium molybdophosphate: \#365

Amorphous indium and zinc oxide (IZO): \#560

Amphoteric: \#856

Amplitude walk: \#1075

AMS: \#353 \#1124

Analogue: \#727 \#1024

Analogy: \#1312

Analysis: \#48 \#77 \#135 \#334

Analytic control: \#1070

Analytical: \#863 \#1254

Analytical application: \#716 \#1355

Analytical chemistry: \#92 \#580

Analytical Quality Control: \#1061

Analytical techniques: \#42 \#986 \#1231

Analytical values: \#45

Anchoring: \#551

Ancient Chinese ceramic: \#1319

Ancient Chinese white porcelain: \#1131

Ancient paper extracts: \#90

Andaman: \#1148

Andisol: \#1368

Andreeva Bay: \#791

Angiogenesis: \#1453

Aniline-phthalein: \#1138

Animal study: \#1161

Animals: \#1218

Anion exchange: \#1112 \#1450

Anion recognition: \#884

Ankara: \#947

Annual committed effective dose: \#1149

Anomaly detection: \#571

ANTARES IV: \#926

Anthranilic acid: \#555 \#1216 
Anthropogenic impact: \#296 \#865

Anthropogenic radionuclides: \#168 \#234 \#926 \#928 \#1441

Antibiotics: \#934 \#1031 \#1035

Antibody: \#104

Antibody coated tube assays: \#203

Anticancer activity: \#258

Anticoincidence gating: \#1245

Anti-Compton: \#1016

Anti-Compton NaI(Tl) spectrometer: \#917

Anti-diabetic medicinal plants: \#1126

Antiestrogen: \#149

Antiestrogen derivative radiopharmaceuticals: \#284

Antimicrobial peptides: \#223 \#935 \#1029

Antimony: \#786

Antinutritional factor: \#973

Antioxidant activities: \#491

Antioxidant behaviour: \#933

Antioxidants: \#616

Antiproliferative activity: \#879

Antisense: \#1155

Anti-tumor activity: \#126

Anzali lagoon: \#261 \#554

Apalachicola Bay Florida: \#993

Apatite: \#295 \#1179

Apatites: \#27

API: \#568

Apoptosis: \#264 \#901 \#1453

Applications: \#49 \#92 \#375

Aquatic colloids: \#1377

Aquatic plant: \#795

Aquatic pollution: \#452 \#1374

Aquatic toxicity: \#1479

Aqueous biphasic system: \#1237

Aqueous extract: \#253

Aqueous medium: \#737

Aqueous solution: \#1320

Aquifer: \#166 \#990

Arabica coffee: \#705

Arachin: \#243

Aral Sea: \#890

Archaeological application: \#190

Archaeological materials: \#1418

Archaeological pottery: \#1481

Archaeology: \#87 \#224 \#1468

Archaeometry: \#87 \#1001 \#1005

Arc-plasma-gun: \#558

Argon-40: \#746

Argyrodiaptomus falcifer: \#1471

Arizona: \#697

Arsenic: \#90 \#484 \#485

Arthritis: \#255

Artificial element: \#124
Artificial neural networks: \#1438

Artificial seawater: \#604

Ash sample: \#1480

Asia: \#1376

Asian dust: \#234

Assay: \#564

Association constant: \#717

Astatine radiopharmaceuticals: \#244

Astatine-209.210: \#171

Astatine-211 alpha-particles: \#624

Asterionella formosa: \#795

Astrochemistry: \#1262

Astroparticle and particle physics: \#1007

Atherosclerosis: \#1220

Atlantic Meridional Transect: \#403

Atlantic Ocean: \#403

Atmospheric aerosols: \#1359

Atmospheric deposition: \#234

Atmospheric deposition event: \#273

Atmospheric dust: \#723

Atmospheric nuclear tests: \#752

Atmospheric pollution: \#800

Atom ratio of $\mathrm{U}-236 / \mathrm{U}-238$ : \#153

Atomic absorption spectrometry (AAS): \#75 \#95 \#228

\#240 \#249 \#489 \#525 \#528 \#528 \#821 \#834 \#838

\#932 \#933 \#1070 \#1325 \#1340 \#1413 \#1421 \#1442

\#1443

Atomic bomb radiation dosimetry: \#159

Atomic force microscopy: \#1361

Atomic isobars: \#607

Atomic mass spectrometry: \#647 \#655 \#668

Atomic spectrometry: \#633

ATR FT-IR spectroscopy: \#464

ATSM: \#1298

Attapulgite: \#1341

A-type proanthocyanidin dimer: \#320

Auger emitters: \#622

Austria: \#296

Authentication: \#1426

Automation: \#89 \#1446

Autonomous underwater detectors: \#164

Avian leukosis virus subgroup J: \#503

Ayurvedic drug: \#584

Azo compounds: \#580

Azolla filiculoides: \#262

B-10(n,alpha gamma)Li-7 Peak Analysis: \#1059

Ba isobaric interferences: \#1424

Bacillus sp GT-83: \#554

Bacillus sp GT-83-23: \#261

Bacillus sphaericus: \#75

Backfill material: \#305 \#307

Background: \#155 \#1077

Background contamination: \#1007 
Background reduction: \#1245

Bacteria: \#417

Bacterial cells: \#73

Bacterial community: \#1013

Baia coastal zone (Naples, Italy): \#1000

Balneotherapy: \#1243

Baltic Sea: \#1163

Bangkok: \#1378

Barite (BaSO4): \#280

Barium: \#9 \#484 \#687 \#962

Barium anomaly: \#138

Barn Owl: \#1212

Basalt: \#224

Bastnasite leaching liquor: \#714

Batch experiments: \#220 \#310

Bauxite: \#1375

Bayesian statistics: \#781 \#1088

Be-7: \#817

Beach rock: \#1148

Beam chopper: \#1067 \#1118

Beam current monitoring: \#1134

Beijing: \#232

Beishan Granite: \#350

Benthic foraminifera: \#1000

Bentonite: \#111 \#276 \#415 \#844

Bentonite stability: \#755

Benzaldehyde: \#145 \#809

Benzene: \#717

Benzo-18-crown-6: \#319

Benzoylthiourea: \#444 \#858

Benzylic activation: \#33

Berate: \#1445

Berberine: \#259

Berner Impactor: \#1369

Beryllium: \#314

Beryllium-7: \#758

Beta counting: \#987 \#988 \#1300

Beta counting-alpha spectrum apparatus: \#19

Beta-amyloid plaques: \#1222

Beta-elemene: \#879

Beta-elemene derivatives: \#258

Beta-estradiol (1,3,5,[10]-estratrien-3,17 beta-diol): \#127

Beta-gamma coincidence counting: \#202

Beta-gamma coincidence detection: \#565 \#1080

Beta-hCG: \#21

BFCA: \#274

Bhasma: \#584

Bifunctional labeling: \#283

Bifunctionalization: \#348

Bikini: \#747

Bile metabolites: \#452

Bilirubin: \#707
Binary monomers: \#898

Binding constant: \#1460

Bioaccumulation: \#686 \#777 \#1039

Bioacumulation: \#420

Bio-amelioration: \#36

Bioassay: \#174 \#176 \#391 \#540 \#725 \#1271

Bioavailability: \#39 \#41 \#1483

Bioavailable metal fractions: \#1373

Biocompatibility: \#1400

Bioconcentration factor: \#1471

Bioconjugate: \#387

Biodegradability: \#1268

Biodegradable: \#1046

Biodegradation: \#805

Biodiesel: \#430

Biodistribution: \#21 \#50 \#143 \#177 \#256 \#355 \#875

\#1146 \#1147 \#1150 \#1164 \#1176 \#1295 \#1321 \#1322

Biodiversity: \#451

Biodosimetry: \#621 \#624

Bioequivalence: \#39

Bioerosion: \#1022

Biofilm: \#1013

Biogas: \#440

Biogenic silica: \#406 \#408

Biogeochemistry: \#219

Bioimaging: \#3

Bioindicators: \#453 \#747 \#1006

Biokinetics: \#539 \#540

Biological compounds: \#88

Biological control: \#440

Biological effect: \#1040

Biological half-life: \#210

Biological materials: \#1422

Biological monitoring: \#1070

Biological samples: \#47 \#85 \#250

Biomarker: \#335 \#452 \#1374

Biomaterials: \#212

Biomedical applications: \#295 \#1221

Biomedical research: \#902

Biomethylation: \#1232

Biomineral: \#1022

Biomolecules: \#329

Biomonitor: \#1363 \#1471

Biomonitoring: \#37 \#332 \#334 \#420 \#449 \#1443 \#1452

Biopolymers: \#694

Bioreduction: \#840

Bioremediation: \#840

Biosorption: \#75 \#243 \#261 \#262 \#263 \#554 \#836 \#840 \#847 \#1210 \#1327

Biosynthesis: \#1228

Biotechnology: \#617

Bipyridine ligands: \#1215

Bismuth: \#478 
Bismuth-214: \#371

Bitumen: \#1249

Bituminous soil: \#1249

Black Sea: \#777

Black tea: \#494

Black tea leaves: \#302

Bladder: \#1138

Bleomycin: \#875 \#1170 \#1295

Bleomycin complexes: \#1158

BLIP: \#158

Block Matching: \#1091

Blood: \#278 \#1044 \#1443

Blood cell labeling: \#1159

Blood serum: \#967

Body composition: \#1008 \#1119 \#1186

Bohai Bay: \#797

Boltwoodite: \#1417

Bond lengths: \#691 \#711

Bone: \#870 \#1008 \#1021 \#1022 \#1188 \#1194 \#1432

Bone as a biomarker: \#40

Bone assay: \#934

Bone marrow: \#539

Bone strontium: \#1485

Bones, human: \#539

Borates: \#908

Boreholes: \#416

Boric acid: \#970 \#971 \#994

Boron: \#314 \#912 \#969 \#994 \#1019 \#1130

Boron carbide filter: \#493

Borosilicates: \#905 \#906 \#907

Bothropstoxin-1: \#968

Bottled mineral water: \#300

Bottled water: \#802

Bottom ash: \#599

Bottom sediments: \#1162

Brain imaging: \#204

Brain imaging agent: \#880

Brain uptake: \#179

Brazil: \#92 \#468 \#469 \#498 \#741 \#769 \#1273

Brazilian coal: \#599

Brazilian freshwater ecosystem: \#218

Breakage: \#618

Breakthrough capacity: \#1257

Breast cancer: \#1428

Breast tissue: \#474 \#1266

Breast tumor: \#284

Bremsstrahlung: \#874

Bremsstrahlung spectrum: \#966

Bromate: \#1018

Bromide: \#210

Bromine: \#72 \#331 \#682

Brown marine algae: \#5

Brown rice: \#922 \#1127
BSA: \#260

Building materials: \#947 \#949 \#1253

Build-up correction: \#183

Bulk etch rates: \#583

BumimTf(2)N: \#400

Burnup: \#564

Bushehr: \#627

Bypassing: \#196

C. islandica (L.) Ach. lichen: \#959

C-60: \#1260

C6D6 scintillation detector: \#1078

Ca-alginate beads: \#847

Cadmium: \#9 \#95 \#172 \#228 \#306 \#310 \#312 \#418 \#518 \#601 \#920 \#1375 \#1413 \#1443

Cadmium-109: \#1338

Caesium: \#610 \#760 \#852 \#1211

Calc-alkaline magmatism: \#929

Calcium: \#519 \#687 \#972 \#1493

Calcium chloride: \#1010

Calcium carbonate: \#419

Calcium phosphate: \#3

Calcium sulfoaluminate cement: \#835

Calibration: \#1072 \#1086

Calibration curve: \#923 \#979 \#1083

Calix[4]arene: \#9 \#11 \#375 \#720 \#958

Calix[6]arene: \#275

Calix[n]arenes: \#866

Calixarene compound: \#1492

Calixarenes: \#1032 \#1033 \#1034

Calorimeters: \#692

Calorimetry: \#1023

Camellia sinensis L.: \#320

CAMP: \#901

Canal complexes: \#1261

Cancer: \#1 \#105 \#286 \#702 \#804 \#1375

Cancer chemotherapy: \#380

Cancer imaging: \#1453

Cancer therapy: \#701

Candida colliculosa: \#1327

Canoparmelia texana: \#449

Capillary column: \#157 \#719

Capillary electrophoresis: \#610 \#956

Capsid: \#1046

Carbamate pesticide: \#805

Carbofuran: \#805

Carbohydrates: \#419

Carbon budget: \#450

Carbon burial: \#450

Carbon dioxide: \#330

Carbon export: \#403 \#406

Carbon nanotube: \#383 \#1399

Carbon sequestration: \#1051

Carbon tetrachloride: \#717 
Carbon-12-Rutherford backscattering spectroscopy: \#1125

Carbon-13-NMR spectroscopy: \#886

Carbon-14: \#537 \#771 \#877 \#882 \#924

Carbon-14 monitoring: \#359

Carbon-14-radiolabelled atom transfer: \#304

Carbonate: \#431

Carbonate alkalinity: \#994

Carboranes: \#279 \#927 \#1311

Carboxylic acids: \#1303

Carboxymethylated cellulose: \#851

Carboxymethylated chitosan: \#851

Carnivorous plants: \#211

Carpathian basin: \#225

Carpathian-Pannonian region: \#813 \#929

Carrier element-free coprecipitation: \#845

Cartilage: \#1266

Cascade gamma rays: \#238

Casein micelles: \#977

Caspase-3: \#901

Caspian Sea: \#997

Castle of Cosenza: \#1175

Cataloging: \#1401

Catalysis: \#412 \#435

Catalyst: \#1386

Catcher foil: \#1133

Catechin: \#320 \#632

Cation exchange: \#863 \#1252

Cation exchange resin: \#778

Cationic polymerization: \#885

CAVP: \#138

CCD: \#914

CCQM: \#1461

CdZnTe: \#808

CdZnTe detector: \#525

CdZnTe neutron detector: \#1286

CEC: \#755

CEF effects: \#639

Cell: \#874

Cell cycle arrest: \#258

Cell labeling: \#625

Cell proliferation: \#387

Cellular imaging: \#1045

Cellulose: \#425

CEM: \#1134

Cement: \#566 \#754 \#861 \#1277

Cement solidified waste: \#197

Central Taiwan: \#345

Centrifugal contactor: \#1395 \#1408

Ceramics: \#224 \#962

Cereal: \#787 \#973

Cereal crops: \#1149

Cerium: \#714
Cerium(III) tungstosilicate: \#1391

Cerium(IV): \#574

Cerium-141: \#730

Certification: \#298 \#1016

Certified reference material: \#168 \#298 \#772

Cesium: \#262 \#305 \#365 \#396 \#495 \#627 \#719 \#755 \#830 \#1397 \#1405

Cesium and strontium ions: \#826

Cesium carboxylates: \#641

Cesium extraction: \#1405

Cesium ionophore: \#275

Cesium ions: \#1050

Cesium isotopes: \#1424

Cesium-134: \#136

Cesium-137: \#160 \#167 \#271 \#299 \#437 \#521 \#524

\#730 \#752 \#765 \#782 \#788 \#996 \#997 \#1162 \#1213

\#1240 \#1314 \#1371 \#1372 \#1472

Chain: \#695

Characterization: \#173 \#595

Characterization in vivo: \#50

Characterization of target: \#1101

Characterization of waste: \#81

Charcoal: \#1167

Charcoal column chromatography: \#199

Charge integration: \#1134

Charge reversal: \#381

Charge transfer complex: \#717

Charged particle: \#570

Charge-discharge: \#483

Charged-particle beams: \#1110

Chelating agent: \#481 \#1381

Chelating ligand: \#77 \#93

Chelating polymers: \#580

Chelating resin: \#553 \#822 \#854

Chemical analogue: \#761

Chemical analysis: \#861

Chemical characterization: \#599

Chemical composition: \#700

Chemical contaminants: \#416

Chemical elements: \#252 \#254 \#1081

Chemical equilibrium: \#1460

Chemical etching: \#1099

Chemical extraction: \#840

Chemical fingerprint: \#147

Chemical oxygen demand (COD): \#556

Chemical resolution: \#607

Chemical separation: \#159 \#1112

Chemical speciation: \#448

Chemical structure: \#885 \#886

Chemical synthesis: \#965 \#1003 \#1004 \#1436

Chemistry of radioelements: \#1156

Chemometrics: \#47 \#216 \#453 \#490

Chemotherapy: \#1046 
Chernobyl: \#150 \#271 \#538

Chernobyl fallout: \#1212 \#1314

Chert: \#224 \#225 \#1418

Chewing tobacco: \#1340

Chicken: \#503 \#712

Chicken liver and spleen: \#557

Chicken meat: \#605

Chile: \#1236

China: \#457 \#799

China clay: \#306

Chipped stone tools: \#225

Chiral amplification: \#1262

Chiral polymer: \#885 \#887

Chirality: \#885 \#1262

Chironomids: \#548

Chitosan: \#194 \#841

Chitosan benzoyl thiourea: \#290

Chloranilic acid (CAA): \#94

Chloride: \#1445

Chloride effect: \#552

Chlorinated cobalt dicarbollide: \#1405

Chlorine: \#72 \#331 \#1235

Chlorine concentration analysis: \#1094

Chlorine-36: \#1235

Chlorine-36 m: \#746

Chlorine-38: \#746

Chlorine-39: \#746

Chlorinity: \#994

Chlorobenzene: \#717

Chlorophyll: \#214

Chlorpyrifos: \#1258

Chromatographic partitioning: \#720 \#1394

Chromatographic separation: \#357

Chromatography: \#1267

Chromium: \#172 \#739 \#838 \#1471

Chromium(III): \#28

Chromium(VI): \#28 \#110

Chromium(III): \#846

Chromosomal aberrations assay: \#1037

Chromotropic acid: \#1018

Chronoamperometry: \#424

Cinder Cone: \#410

Circular dichroism: \#1361

Cisplatin: \#874

Citrus: \#35

Clathrate: \#887 \#1261 \#1263 \#1265

Clay: \#1230

Clay bricks: \#370

Click chemistry: \#1155

Climate change: \#22 \#234 \#273

Clinical: \#85

Clinical diagnostic test: \#1008

Clinoptilolite: \#417 \#602 \#856 \#1020
Close-geometry: \#1096

Close-in fallout: \#148

Cloud point: \#846

Cloud point extraction: \#49 \#343 \#1454

Cluster analysis: \#150 \#633

Cm-248(Mg-25,4n-5n)(268,269)Hs reaction: \#1201

CMPO: \#1311 \#1493

Coagulation: \#1299

Coal: \#498

Coal fly-ash: \#1223

Coal mine: \#1273

Coarse particles: \#1369

Coastal eutrophication: \#266

Coastal lagoons: \#219

Coastal urbanization: \#266

Coastal water: \#745 \#989

Coastal zone: \#267 \#371 \#372 \#469 \#769

Coastal zone management: \#1367

Cobalamin: \#380

Cobalt: \#113 \#407 \#415 \#484

Cobalt and cesium: \#1320

Cobalt(II): \#1404

Cobalt(II) complexes: \#555

Cobalt-55: \#1147

Cobalt-57 emission Mossbauer spectroscopy: \#555

Cocrystallite: \#351

CoCuAg alloy films: \#324

Coffee: \#490

Cohort: \#285

Coincidence: \#768

Coincidence spectrometer: \#1137

Coincidence summing: \#152 \#1096

Coincidence summing corrections: \#201

Coincidence summing effects: \#184

Coincidence system: \#1075

Colchicine: \#878

Cold neutron: \#1060 \#1067

Cold neutron radiography: \#683

Collection efficiency: \#504 \#505

Collective states: \#1229

Collector: \#778

Collision-reaction cell: \#1424

Colloid: \#363 \#1046

Colon microflora: \#1232

Combustion: \#236

Comets: \#562

Comminution: \#618

Competition: \#841

Compilation: \#531 \#531 \#1102

Complementary and alternative remedies: \#373

Complex: \#625

Complex hydrides: \#429

Complex structure: \#1034 \#1035 \#1494 
Complexation: \#257 \#985 \#1031 \#1032 \#1033 \#1214 \#1216 \#1312 \#1342 \#1345 \#1494

Complexing anions: \#1304

Complexing ligands: \#1306

Composite: \#839

Composite adsorbent: \#365

Compositional analysis: \#224

Compreignacite: \#1417

Compton suppression: \#208 \#209 \#1065

Computed tomography: \#569 \#1135

Computer code: \#1097

Concentration factor: \#795

Concentration ratio: \#767

Concrete: \#1462

Condensed matter: \#385

Conditional probability function: \#237

Conditioning: \#962

Conformation: \#260

Constant mixing: \#928

Contact angle: \#483 \#1364

Contamination: \#147 \#150 \#432 \#540 \#798 \#1363

Continuous culture: \#106

Continuous monitoring: \#1370

Contrast agent: \#563 \#703

Contrast enhancement: \#703

Control system: \#1098

Conventional: \#35

Conversion Electron Mossbauer Spectroscopy (CEMS): \#426

Conversion electron Mossbauer spectroscopy (CEMS): \#560

Convoluted germanium detector line shape: \#1059

Coolant chemistry: \#919

Cooling water: \#1153

Coordination chemistry: \#374

Copolymer: \#1305

Copper: \#264 \#484 \#519 \#582 \#827 \#1121 \#1215

Copper hydroxide: \#832

Copper Nanoparticles: \#904

Copper thiosemicarbazonato complex: \#126

Copper(II): \#367

Copper-60 and copper-64 contamination: \#128

Copper-61: \#20 \#126 \#131 \#1146 \#1150 \#1157 \#1170 \#1171 \#1291

Copper-61 production: \#128

Copper-63(n,p)Ni-63: \#159

Copper-64: \#207

Copper-64 production: \#1103

Copper-67: \#1132

Coprecipitation: \#832 \#838 \#1398

Coptisine: \#259

Coral reef: \#266

Corn flour (INCT-CF-3): \#298
Correlation: \#764

Correlation and principal component analysis: \#1208

Corrosion protection: \#426

Corsica: \#699

Cosmic: \#758

Cosmic rays: \#229 \#746

Counting efficiency: \#535

Cow milk powder: \#1213

Cracking: \#1462

Cretaceous/Tertiary boundary: \#377

Crete: \#277

CRM: \#154 \#172

Croatia: \#439

Crop yield: \#1223

Crosslinking: \#943 \#1364

Cross-section: \#146 \#917 \#1101 \#1113 \#1114 \#1117

Crown ether: \#946 \#1332 \#1440

Crozet Basin: \#926

Cryogenic processing: \#43

Crystal and electronic structures: \#402

Crystal calorimeter: \#1064

Crystal chemistry: \#891

Crystal structure: \#866 \#962 \#963 \#1491

Crystallization temperature: \#945

CSA-13: \#1322

CTBT: \#758 \#1088

CTBTO: \#779

Cuba: \#377

Cultivated soil: \#759

Cultivated stony soils: \#1251

Cultural heritage: \#87

Cultural-heritage objects: \#1172

Cumulative exposure to manganese: \#40

Cup quality: \#705

Curcumin: \#884 \#994

Curium: \#257 \#1302

Curium(III): \#269 \#594 \#726 \#1214

Curry: \#487

CYANEX302: \#413

CYANEX921: \#619

Cyclen derivatives: \#177

Cyclic voltammetry: \#550 \#637 \#1292 \#1294

Cyclotron: \#126 \#175 \#193 \#1158 \#1161 \#1164 \#1291 \#1298

Cyclotron targetry: \#1141

Cysteinyldopa model compounds: \#588

Cytotoxicity: \#1400

Czech Republic: \#273 \#1012

Daily dietary intake: \#489

Daily intake: \#358

Dairy Products: \#488

Data and error analysis: \#1007

Database: \#1102 
Data-to-parameter ratio $=13.3: \# 12$

Dated sediment core: \#797

Dating: \#410

Dating model: \#928

DC polarography: \#869

D-D neutrons: \#501

D-D, D-T and P-T neutrons: \#159

Dead time losses: \#1090

Debaromyces hansenii: \#1327

Debye temperature: \#556

Decalcification: \#1022

Decarbonylation: \#145

Decay scheme analysis: \#152

Decision threshold: \#781

Deconvolution of overlapped spectra: \#1433

Deep repository: \#755

Defective beans: \#490

Defective structures: \#886

Defects: \#736 \#1358

Degradation: \#944 \#1364

Degraded land: \#36

DEGs: \#335

DEHEHP: \#714

Delayed Neutron Counting System: \#1061

Delithiation: \#421

Delta D: \#469 \#769

Delta O-18: \#769

Delta O-IX: \#469

DEM: \#618

Denaturation: \#260

Dendrochemistry: \#115 \#410 \#698 \#818 \#1463

Dendrochronology: \#410 \#1463

Density functional theory (DFT): \#399 \#597 \#615 \#711 \#1455 \#1494

Dental caries: \#1022

Dentistry: \#212

Denuder: \#233

Deoxycholic acid: \#1263 \#1265

Depleted uranium: \#22 \#975

Depletion: \#4

Deposition: \#167 \#794 \#1040

Derivative UV-vis: \#88

Derivatization: \#446

Desferrioxamine: \#1141

Desiccation: \#890

Desilicification: \#1022

Desorption: \#552 \#855 \#959 \#1167

Detection: \#1254

Detection limit: \#82 \#535 \#613 \#781

Detection of illicit material: \#216

Detection or photopeak efficiency: \#808

Detector: \#535 \#808 \#1077

Detector response: \#1078
Detector response functions (DRF's): \#1116

Determination of chloride: \#342

Determination of uranium: \#74

Determination of uranium and thorium: \#1449

Detritiation: \#1257

Deuteron beams: \#103

Diabetes mellitus: \#249 \#250

Diagenesis: \#995

Diagnostic: \#616

Diaion SP-850: \#75 \#821

Dialysis: \#171

Diatoma vulgare: \#795

Diatoms: \#795

Dibenzo-18-crown-6 ethers: \#319

Dicalcium phosphate: \#849

Dicarbollides: \#1311

Dicksonia sellowiana: \#38

Diet: \#538

Dietary exposure: \#332

Dietary supplement ingredient database: \#45

Dietary supplements: \#45

Diets: \#358

Differential scanning calorimetry (DSC): \#1004

Diffraction and scattering measurements: \#905 \#906

Diffuse optical spectroscopy (DOS): \#563

Diffusion: \#157 \#582 \#695 \#719 \#852 \#897 \#1062

Diffusion coefficient: \#423 \#424

Diffusion theory: \#563

Diffusion-based calibration: \#83

DIGISIM: \#716

Digital acquisition: \#155

Digital autoradiography: \#137

Digital data acquisition: \#1087

Digital gamma-activation autoradiography: \#301

Digital pulse shape analysis: \#565 \#1080

Digital pulse shape discrimination: \#768 \#1082

Diglycolamide complexes: \#589

Dihydroxyurea: \#1343

Dilute magnetic semiconductor (DMS): \#560

Dimethylbenzantracene (DMBA): \#127

Dimethylglyoxime: \#169

Diphenyl phosphate: \#1389

Diphenylphosphinic acid: \#1389

Dipicolinic acid: \#1324

Direct infusion electrospray ionization mass spectrometry: \#430

Discharge: \#468 \#862

Discriminant analysis: \#722 \#1428

Discrimination: \#137

Disease incidence: \#360

Disequilibrium: \#783

Disk source: \#808

Dispersion: \#1399 
Dispersive liquid-liquid microextraction: \#91 \#344 \#356 \#900 \#1451

Dissolution: \#1022

Dissolved gaseous mercury (DGM): \#764

Dissolved organic carbon: \#760

Dissolved organic nitrogen: \#990

Distance of influence: \#451

Distribution coefficient: \#107 \#495 \#786 \#984 \#1342 \#1391

Distribution ratio: \#1292 \#1294

Dithiooxamide acid-formaldehyde resin: \#854

Dithiophosphinic acid: \#595

Diurnal birds of prey: \#299

Divalent cations: \#958

Diverse matrices: \#1446

DNA: \#56

DNA comet assay: \#712

DNA damage: \#622

Dolerite: \#173

Dolomite: \#1125

Donor atom polarizability: \#577

Dopaminergic: \#876

Doping profiles: \#691

Doppler broadening: \#970

Doppler-Broadened gamma-Line Shape Formulae: \#1059

Dose: \#801 \#949

Dose assessment: \#748 \#775 \#1017

Dose rate: \#516 \#763 \#1109 \#1240

Dosimetry: \#916 \#1168

DOTA: \#177

Double layer: \#716

Double perovskites: \#687

Doxorubicin: \#1150

D-PA (D-penicillamine) and DTPA (diethylenetriaminepentaacetic acid): \#283

DPPH: \#933

DRI: \#358

Dried fish: \#1092

Drinking water: \#140 \#382 \#446 \#789 \#801 \#1017

Drinking water standards: \#802

Drug delivery: \#482 \#616 \#1043

Drug interactions: \#39

Drugs: \#297

DSC: \#260

DT neutron: \#142

DTA: \#1259

DTPA: \#127 \#274 \#284 \#572 \#1157

Dual spectral response: \#884

Dunite: \#533

Dust: \#273 \#794

Dust storm: \#232

Dy-144: \#1202
DYFAMED: \#987 \#999

Dynamic imaging: \#570

Dynamic light scattering: \#1044

Dynamic reaction cell: \#607 \#773

Early gene expression: \#621

Earth/environmental processes: \#1423

Earthquake: \#180 \#793

Earthworms: \#518

EB-irradiation: \#1121

Eclipses, transits and occultations: \#229

Ecotoxicology: \#725

Ectomycorrhizal fungi: \#1039

Eddies: \#406

Edible mushrooms: \#484

EDXRF: \#1148 \#1431

Effect of barium: \#831

Effective dose: \#1379

Effective dose rate: \#753

Efficiency: \#184 \#613

Efficiency calibration: \#1096

Egg: \#605

Egypt: \#949

Elastic scattering: \#1198

Elderly: \#967

Electric furnace slag: \#582

Electrical double layer: \#381 \#728

Electrical resistivity: \#470 \#639

Electrochemical: \#1142

Electrochemical deoxidation: \#1010

Electro-chemical etching: \#1099

Electrochemical impedance spectroscopy (EIS): \#94

Electrochemical reduction: \#427 \#636 \#1151

Electrochemistry: \#339 \#716

Electrode potential: \#1142

Electrodeposition: \#195 \#217 \#228 \#550 \#637 \#1224 \#1294

Electrodissolution: \#1293

Electrokinetic potential: \#729

Electrokinetics: \#840

Electrolysis: \#313

Electromagnetic transitions: \#1229

Electron accelerator: \#395

Electron beam accelerator: \#1264

Electron capture: \#1300

Electron density: \#1352

Electron imaging: \#914

Electron microscopy: \#295 \#1003 \#1004

Electron paramagnetic resonance: \#322

Electron probe microanalysis: \#1027

Electron transfer: \#716

Electronic absorption spectroscopy: \#1260

Electronic neutron generator: \#188 \#567

Electronic property: \#1455 
Electronic tongue: \#392

Electrophilic substitution reaction: \#878

Electrophoretic mobility: \#728

Electroplating: \#1293

Electrorefining: \#1292

Electrospray ionization: \#1460

Electrospray ionization mass spectrometry: \#1351

Electrothermal atomic absorption: \#445

Electrothermal atomic absorption spectrometry: \#601 \#825 \#1422

Electrothermal vaporization-inductively coupled plasma mass spectrometry (ETV-ICPMS): \#673

Element uptake by different plant species: \#1208

Elemental: \#173

Elemental analysis: \#85 \#87 \#130 \#198 \#212 \#216 \#302 \#697 \#1266 \#1461

Elemental analysis laboratory: \#224

Elemental bioimaging: \#1011

Elemental composition: \#68

Elemental constituents: \#1126

Elemental solubility: \#235

Elementomics: \#1231

Elements: \#354

Elodea canadensis: \#792 \#795

Emanation coefficient: \#753 \#754

Emergency: \#417

Emergency response: \#154

Emission Mossbauer spectroscopy: \#73 \#561

EMPIRE: \#1056

E-n $<15 \mathrm{MeV}$ : \#159

Encapsulation: \#3

Endocrine disruptors: \#1479

Endocrine system: \#623

Endogenous P loss: \#811

Endogenous phosphorus: \#221

Endohedral fullerene: \#351

Endohedral fullerene complexes: \#715

Endohedral fullerenes: \#322

Endohedral metallofullerene: \#1455

Endoliths: \#1022

Energy dispersive X-ray fluorescence: \#90

Energy resolution: \#808

Energy utilization: \#618

Energy-dispersive X-ray diffraction: \#161

Energy-dispersive X-ray fluorescence spectrometry: \#665

Engineered nanomaterials: \#383

Enhanced radioactivity levels: \#272

Enriched and natural Zn targets: \#128

Enriched isotopes: \#1074

Enriched targets: \#151

Enriched Zn: \#1103

Enriched Zn-68 target: \#1132
Enrichment: \#564 \#832

Enrichment factor: \#1340

Enrichment index: \#1469

Enrichment measurements: \#1447

Enthalpy: \#1337

Environment: \#85 \#89 \#135 \#154 \#360 \#749 \#771 \#840 \#1006 \#1283 \#1420 \#1423

Environmental analysis: \#51 \#76 \#88 \#645 \#651 \#662 \#671 \#679

Environmental applications: \#738

Environmental health: \#1221

Environmental impact assessment: \#889

Environmental isotopes: \#386

Environmental matrices: \#398

Environmental monitoring: \#327 \#773 \#779 \#1088 \#1124

Environmental pollution: \#811

Environmental radioactivity: \#81 \#267 \#751

Environmental safety assessment: \#337

Environmental samples: \#70 \#81 \#356 \#389 \#579 \#657 \#750 \#785

Environmental tracer: \#1248

Environmental water samples: \#65

Enzymatic activity: \#36

Enzymes: \#694

Eolian flux: \#273

Eosin Y: \#342

Epidemiological study: \#804

Epiphytic lichens: \#635 \#1070

Episodic events: \#408

Epithermal neutron activation analysis: \#208 \#493 \#1177

EPMA: \#1027

EPR: \#631 \#1166

EPR effect: \#3

Equilibrium: \#276 \#308 \#856

Equilibrium extraction: \#83

Equilibrium reactions: \#812

Equivalent dose: \#1109

ERICA: \#767

Erosion: \#520

Erosion and deposition rates: \#1251

Error propagation: \#1069

Error propagation with Monte Carlo technique: \#1076

Erythrina variegata: \#492

ESI-MS fingerprinting: \#705

Essential elements: \#112 \#250 \#1127

Estradiol derivative radiopharmaceuticals: \#127

Estrogen receptor: \#572

Estrone derivatives: \#572

Estuarine dynamics: \#993

Estuarine flushing: \#993

Estuarine sediments: \#766 
Estuary: \#989

Ethanol: \#1021 \#1297

Ethnicity: \#285

ETS-10: \#945 \#1357

ETS-4: \#308 \#310 \#945 \#1025

ETS-titanosilicate: \#436

Ettringite: \#835

Etude de provenance: \#277

EURDEP: \#1247

Europe: \#273

European map: \#1247

Europium: \#519 \#960 \#1299 \#1304

Europium(III): \#316 \#456 \#594 \#619 \#848 \#1026 \#1323 \#1341

Europium-154: \#730

Eutrophication: \#807

Evaluation: \#146

Evaporated bottom: \#197

EXAFS: \#195 \#472 \#1026

Excess Pb-210: \#788

Exchangeable: \#759

Excitation function: \#151 \#159 \#192 \#193 \#1108 \#1132 $\# 1315$

Excited helium beam injector: \#500

Excited-state proton transfer: \#1419

Excitotoxicity: \#264

Excretion: \#541

Exhalation: \#205

Exhaustive extraction: \#83

Exohedral fullerene: \#351

Experimental data: \#917

Experimental design: \#1413

Export: \#986

Export flux: \#404 \#504 \#505

Exposure: \#541

Exposure and risk: \#497

External reference method: \#717

Extraction: \#243 \#714 \#846 \#964 \#1095 \#1292 \#1294 \#1324 \#1337 \#1405 \#1444

Extraction and stability constants: \#6 \#9 \#11 \#958 \#960 \#1492 \#1493

Extraction chromatography: \#76 \#89 \#391 \#398 \#575 \#987 \#1381 \#1383 \#1388 \#1448 \#1450

Extraction method: \#200 \#1368

Extraction of metal ions: \#93

Extraction paper chromatography: \#60

Extraction solvents: \#1387

Extraction techniques: \#992

EYEPLAN: \#181

F-18 fluoride ion: \#473 \#810

F-18 MPP3F: \#204

FA: \#848

Facilitated transfer: \#55
Facilitated transport: \#897

Facility: \#1077

Factor analysis: \#150 \#180 \#230

Fallout: \#167 \#271

Fallout cesium: \#922

Fallout Cs-137: \#759 \#1251

Fate and transport: \#730

Fault: \#180

FCC catalyst: \#1123

FDG: \#144

Fe73.5Cu1Nb3Si13.5B9: \#352

Femoral vein thrombosis: \#1176

Femur: \#1021

$\mathrm{FeO} / \mathrm{Fe} 2 \mathrm{O} 3$ tot: \#1490

Fermentation: \#106

Ferric chloride-induced thrombosis: \#1176

Fertilizer: \#191 \#532 \#1368

Fetus: \#916

FexZn1-x thin films: \#1205

Fibrosarcoma: \#20 \#1170 \#1171 \#1295

Field survey: \#1244

Field-portable X-ray fluorescence analyzer: \#682

Field-scale: \#770

Filament carburization: \#611

Filter: \#417

Fine particles: \#1369

Fine particles (PM2.5): \#700

Fingerprinting: \#490

First order-order phase transition: \#368

Fish: \#452 \#486 \#1374

Fish muscle and liver: \#248

Fission palladium: \#551

Fission products: \#46 \#610 \#1225 \#1407

Fission track registration efficiency: \#583

Fixed bed columns: \#826

Flame atomic absorption spectrometry: \#443 \#602 \#822 \#832 \#843 \#845

Flavonoid: \#632

Flint: \#225

Floods: \#521

Florida: \#864

Flow analysis: \#89

Flow cell detector: \#52

Flow injection: \#485 \#663

Flow scintillation analysis: \#176

Flow-counterbalanced capillary electrophoresis: \#316

Fluid flow: \#314

Fluidized bed gasifier: \#196

Fluid-mobile and immobile elements: \#969

Fluorescence: \#340 \#711 \#953 \#1317

Fluorescence spectroscopy: \#257 \#269

Fluorescent imaging probes: \#30

Fluoride: \#737 \#803 \#824 \#1345 \#1445 
Fluoride contamination: \#191

Fluoride ion: \#27

Fluorine: \#457 \#714

Fluorine-18: \#163 \#473 \#1155

Fluorine-18 aromatic amino acid: \#810

Fluorine-18-FDG: \#915

Fluorine-18-fluoro-L-thymidine (FLT): \#916

Fluorine-18-labeled aromatic amino acids: \#809

Fluorine-18-labelled aromatic amino acids: \#145

Fluoro complex: \#318

Fluorophore: \#340

Fluoroquinolone: \#873 \#881

Fly ash: \#36 \#198 \#599 \#803 \#830

Fly ash concrete corrosion study: \#1094

FMCT: \#1122

Food: \#42 \#208 \#485 \#646 \#652 \#666 \#675 \#682 \#843 \#1375

Food analysis: \#88

Food and food products: \#493

Food and nutritional products: \#86

Food safety: \#634

Foreign ions: \#842

Forensic science: \#58 \#67

Forest soil: \#1472

Formation and dehydration enthalpy: \#1023

Formation constants: \#596

Formation process: \#1013

Fortified feed: \#605

Fourier transform infrared (FT-IR) spectroscopy: \#73 \#944 \#1259 \#1260 \#1416

Free electron laser: \#1219

Free energy relationship: \#941

Free radical polymerization: \#885

Free radicals: \#1330

Free volume: \#944

Freeze-drying: \#873

Freshwater: \#333

Freshwater copepod: \#1471

Freshwater fish: \#251

Freshwater pollution: \#1479

Freundlich isotherm: \#136 \#762

Frozen aqueous solutions: \#555

FS-13: \#1324

FS-TRLFS: \#1216

FT-IRM: \#1231

Fuel: \#564

Fuel burnup: \#215

Fullerene: \#317 \#321 \#616 \#1048 \#1260

Fullerenes: \#323

Fullerenol: \#292 \#383

Fulvic acid: \#133 \#415 \#456 \#844 \#1341

Functional groups: \#451

Functional material: \#481
Functionalized calix[4]arene: \#718

Functionalized polymers: \#1354

Future trends: \#1446

Gadolinium: \#1045

Gadolinium(III): \#726 \#1382

Gallium: \#336

Gallium-66: \#1159

Gallium-66,Ga-67 formation: \#1102

Gallium-67: \#626 \#1176 \#1321

Gametophyte development: \#38

Gamma: \#1254

Gamma cascade: \#1137

Gamma dose: \#447 \#1239

Gamma dose rates: \#1284

Gamma exposure: \#1278

Gamma irradiation: \#214 \#583 \#1268

Gamma picoline: \#227

Gamma production: \#1154

Gamma radiation: \#311 \#621 \#717 \#1258

Gamma radioactivity: \#1255

Gamma ray: \#229 \#564 \#867 \#1084 \#1089

Gamma rays: \#968

Gamma spectra: \#216 \#917 \#1066

Gamma transmission: \#1123

Gamma-activation analysis: \#301

Gamma-Al2O3: \#363

Gammacamera: \#1168

Gamma-dose rate: \#1247

Gamma-emitting radionuclides: \#784

Gamma-gamma coincidence: \#207 \#1087 \#1116 \#1300

Gamma-index: \#754

Gamma-ray and X-ray spectroscopy: \#1300

Gamma-ray detection: \#1362

Gamma-ray grafting: \#482

Gamma-ray intensity: \#918

Gamma-ray measurements: \#1347

Gamma-ray spectrometry: \#2 \#152 \#166 \#182 \#183

\#184 \#201 \#206 \#210 \#353 \#390 \#438 \#494 \#497

\#516 \#566 \#571 \#627 \#753 \#754 \#778 \#782 \#783

\#808 \#1065 \#1072 \#1087 \#1122 \#1149 \#1240 \#1245 \#1290 \#1335 \#1372 \#1447

Gamma-ray spectroscopy: \#1480

GAR-936: \#934

Garware polyester nuclear track detector: \#583

Gas chromatography-mass spectrometry: \#446

Gas exchange: \#450

Gas fusion analysis: \#44

Gas permeation: \#943

Gas proportional counter: \#155

Gas-phase ion-molecule reactions: \#607

Gdansk city: \#453

GEANT: \#22

GEANT4: \#202 \#1096 
Gel electrophoresis: \#1377

Gel filtration: \#1377

Gelation: \#362

Gels: \#709

Gender-related differences: \#252 \#254

Genders: \#250

Gene delivery: \#617

Gene expression: \#624

Genetic algorithm: \#1433

Genotoxicity: \#1036 \#1037

Geoaccumulation index: \#798

Geochemical characterization: \#1012

Geochemical tracers: \#267 \#993

Geochemistry: \#160 \#226 \#273 \#281 \#282

Geochronology: \#282

Geodynamics: \#813

Geological processes: \#562

Geological reference materials: \#529 \#531

Geological samples: \#209

Geomorphology: \#520

Geomycology: \#1039

Geophagus brasiliensis: \#218

Geophysical survey: \#744

Geostandards: \#526

Gerdien condenser: \#205

Germanium: \#1104

Germanium radiation detectors: \#1362

Germanium-68: \#1112

Germanium-68/Ga generator: \#1112

Germanium-70: \#1204

Germanium-76: \#477

Germination: \#628

GFAP expression: \#1456

Ghana: \#416 \#1469

Gibbsite (alpha- $\mathrm{Al}(\mathrm{OH})(3))$ : \#726

Glass: \#894

Glass polymerisation: \#513

Glass transition: \#943

Glioblastoma multiforme: \#608

Global fallout: \#774 \#1314

Global food security: \#532

Global warming: \#1223

Glucose: \#279

Glucose metabolism: \#1453

Glucose yield: \#106

Glucuronidation: \#1138

Glutathione: \#628 \#1361

GMZ bentonite: \#367

Goats: \#221

Gold: \#514 \#530 \#686 \#1128 \#1399

Gold nanoparticle: \#255 \#256 \#1055 \#1192

Gold nanoparticles: \#121 \#563

Gold nanorod antennas: \#289
Gold nanorods: \#50

Gold nanoshell: \#347 \#702 \#703 \#732

Gold nanoshells: \#120

Gold particle: \#387

Gold-197: \#917

Gold-198: \#196 \#476

Graft: \#898

Graft copolymers: \#694

Grafting: \#1259

Grafting degree: \#696

Grain size: \#1342

Grain-size modality: \#273

Granite tiles: \#1282

Granulometric composition: \#496

Graphite furnace atomic absorption spectrometry: \#343

Grass: \#1210

Grassland: \#520

Greece: \#393 \#763 \#930 \#991 \#1253 \#1282

Green chemistry: \#1237

Green tea extracts: \#491

Green tea leaves: \#302

Greenland: \#816

Gross alpha: \#1256

Gross alpha and beta: \#1015

Gross beta: \#1256

Groundwater: \#64 \#325 \#371 \#434 \#468 \#469 \#536 \#549 \#629 \#730 \#744 \#745 \#769 \#793 \#863 \#981 \#982 \#1019 \#1378

Groundwater quality: \#1476

Group partitioning: \#1388

Guanabara Bay: \#725

Guangzhou: \#232

Gulf of Mexico: \#419 \#819

Gulf of Thailand: \#522

H-3 quinocetone: \#543

Habitat degradation: \#218

Hadron damage: \#1064

Hair: \#95 \#1120

Hair analysis: \#297 \#334

Hair levels: \#1363

Hair preparation and washing: \#297

Half-life: \#1335

Halite: \#1326

Hall effect: \#691

Halomonas sp GT-83: \#263

HANARO Research Reactor: \#1061

Hard rock aquifer: \#119

Harzburgites: \#930

Hawaii: \#982

Hazard sinaran: \#1366

Hazardous elements: \#82

HDTMA: \#1416

Health hazard indices: \#270 
Heat generator: \#1394

Heavy atom derivatives: \#685

Heavy metal: \#36 \#150 \#448 \#728 \#797 \#798 \#821 \#843

Heavy metal ions: \#859

Heavy metals: \#73 \#243 \#245 \#378 \#416 \#433 \#437 \#484 \#487 \#700 \#786 \#799 \#845 \#865 \#999 \#1211 \#1469

Heavy metals in plants: \#37

Heavy metals-mercury: \#486

Hectorite: \#466

Helion reactions on ruthenium: \#151

Helium: \#499

Helix pomatia: \#1052

Hematite: \#1338

Hemoglobin gene: \#503

Herbal drink: \#932

Herbal formulation: \#249

Herbal products: \#373

Herbicides: \#1474

Heteroaromatic substitution: \#1155

Heterogeneity: \#1027

Heterogeneous catalysis: \#34

Hetropolyacids: \#1391

Heulandite: \#727

Hexacyanoferrate: \#1397

HFLPME: \#48

High rain fall area: \#783

High resolution ICP-MS: \#738

High-activity liquid waste: \#1388

High-energy accelerator: \#1107 \#1128

High-level liquid waste: \#481

High-level radioactive wastes: \#350

High-level waste: \#1395 \#1408

Highly active liquid waste: \#957 \#1381 \#1383 \#1394

High-performance: \#259

High-performance liquid chromatography: \#46

High-pressure metamorphic rocks: \#1028

High-purity gadolinia: \#1346

High-resolution alpha spectrometry: \#756

High-resolution continuum source AAS: \#525

High-resolution inductively coupled plasma mass spectrometry: \#153 \#606

High-sensitivity quadrupole ICP-MS: \#657

High-temperature phase transition: \#293

High-velocity resolution: \#557

Hiroshima atomic bomb: \#1242

Histomorphometric parameters: \#1021

History: \#1022

Hobby: \#332

Hokutolite: \#182

Hollow fiber liquid phase microextraction: \#91

Holmium-166-DTPA: \#274
Homeopathic preparations: \#373

Homogeneity: \#43 \#298

Homogeneous catalysis: \#33

Homogeneous liquid-liquid extraction: \#1451

Hong kong: \#232

Hornstone: \#225

Host phase: \#1380

Host-guest compounds: \#1263 \#1265

HpGe detector: \#22 \#166 \#184 \#627 \#860 \#1072 \#1149

HPLC: \#625

HPLC-DAD: \#722

HPLC-ICP-MS: \#634

HREM: \#294

Human: \#85 \#1008 \#1287

Human blood: \#457

Human bone: \#1488

Human bones: \#1189 \#1190

Human brain: \#608 \#609

Human cell: \#1135

Human exposure: \#606

Human health: \#749

Human liver ferritin: \#557

Human rib bone: \#252

Human scalp hair: \#254

Human teeth: \#190

Human urine: \#329

Humic acid: \#133 \#363 \#411 \#456 \#730 \#762 \#844

Humic substances: \#364 \#1312 \#1377

Hyaluronic acid: \#255

Hybrid-type metals: \#407

Hydraulic binder: \#835

Hydride generation atomic absorption spectrometry: \#605

Hydride generation atomic fluorescence spectrometry (HG-AFS) cigarette filter: \#485

Hydrochemistry: \#434

Hydrogel: \#194

Hydrogen: \#1104 \#1115

Hydrogen and $\mathrm{C} / \mathrm{H}$ ratio detection: \#139

Hydrogen bond: \#630

Hydrogen bonding: \#944

Hydrogen determination: \#178

Hydrogen dicarbollylcobaltate: \#960

Hydrogen Dicarbollylcobaltate: \#1493

Hydrogen production: \#435

Hydrogen storage: \#429

Hydrogen-1-NMR spectroscopy: \#886

Hydrogeochemistry: \#219

Hydrologic cycle: \#862

Hydrolysis: \#362

Hydrolysis at interfaces: \#381

Hydrophobic effect: \#260

Hydrothermal synthesis: \#1215 
Hydrous metasomatism: \#931

Hydrous silica: \#1404

Hydroxamates: \#1141

Hydroxamic acids: \#1214

Hydroxy quinoline: \#1171

Hydroxyapatite: \#839 \#896 \#1296

Hydroxybenzoic acid: \#1419

Hydroxypropyl methylcellulose phthalate: \#851

Hyperpolarized Y-89: \#640

Hypersaline wastewater: \#263

Hyphenated technique: \#86

Hyporheic exchange: \#471

Hypoxia: \#1453

IAEA: \#5

IAEA reference material: \#153

IAEA-CU-2006-06: \#1319

IC50: \#879

Ices: \#562

ID-SF-ICP-MS: \#148

Igneous rocks: \#1490

Illicit trafficking: \#80

Image quality: \#1432

Image reconstruction: \#1100

Imaging: \#20 \#216 \#255 \#265 \#608 \#609 \#1220

Imaging agents: \#1222

Imaging mass spectrometry: \#1452

Imaging plate: \#137 \#1128

Imidazol resin: \#552

Imidazolium nitrate resin: \#551

Immiscible electrolytes: \#339

Immiscible liquids: \#716

Immobilization: \#34 \#604

Immobilized-polysiloxane ligand systems: \#93

Immune response: \#1459

Immunology: \#968

Impact event: \#377

Implantation: \#736

Impregnation: \#828 \#853 \#1389

Impurities: \#1358 \#1386

In situ determination: \#1248

In situ underwater gamma-spectrometry: \#22

In vitro/in vivo evaluation: \#1234

In vivo: \#570 \#870 \#1008

In vivo elemental analysis: \#920

In vivo imaging: \#914

In vivo neutron activation analysis: \#40

Incision: \#521

Inclusion complexes: \#1261

Inclusion polymerization: \#887 \#1261 \#1263 \#1265

Incorporation: \#726

India: \#233

Indian River Lagoon: \#471

Indirect electrolytic reduction: \#638
Indium complexes: \#177

Indium compounds: \#691

Indium(III): \#581

Indocyanine green: \#3

Indole-3-acetic acid: \#1434

Indoor: \#1246

Indoor absorbed dose rate: \#753 \#754

Indoor air: \#205

Indoor gamma dose: \#585

Indoor radon: \#763

Inductively coupled plasma: \#1421

Inductively coupled plasma atomic emission spectrometry (ICP-AES): \#74 \#252 \#954 \#1127 \#1461

Inductively coupled plasma dynamic reaction cell mass spectrometry: \#739

Inductively coupled plasma mass spectrometry: \#70 \#649 \#654 \#663

Inductively coupled plasma mass spectrometry (ICPMS): \#22 \#46 \#50 \#72 \#76 \#80 \#226 \#239 \#331 \#337 \#607 \#610 \#656 \#661 \#674 \#747 \#750 \#773 \#785 \#802 \#853 \#921 \#1001 \#1040 \#1070 \#1335 \#1420 \#1423 \#1427 \#1448 \#1448

Inductively coupled plasma optical emission spectrometry (ICP-OES): \#487 \#1449

Inductively coupled plasma sector field mass spectrometry (ICP-SFMS): \#156 \#389 \#1014 \#1301 \#1439

Industrial analysis: \#644 \#650 \#660 \#670 \#678

Industrial plants: \#442

Infant formula: \#488 \#721

Infection: \#873 \#881 \#1160 \#1322

Infection detection: \#935 \#1029 \#1140

Infection imaging: \#223

Infrared responsive: \#347

Infusions: \#1429

INGE: \#779

Inorganic analysis: \#1461

Inorganic ion exchanger: \#1391 \#1397

Insecticide: \#883

In-situ XRD: \#293

Instrumental analysis: \#92

Instrumental neutron activation analysis (INAA): \#10 \#43 \#82 \#138 \#147 \#172 \#212 \#249 \#251 \#254 \#302 \#345 \#382 \#441 \#489 \#525 \#528 \#530 \#531 \#531 \#697 \#713 \#783 \#804 \#933 \#1016 \#1061 \#1081 \#1087 \#1131 \#1325 \#1340 \#1346 \#1469 \#1471

Instrumental photon activation analysis: \#190

Instrumentation: \#105 \#915

Insulin: \#171 \#1164

Intake: \#538 \#1363

Integral yield: \#1108 \#1315

Integrated cathode assembly: \#1151

Integrated water resource management (IWRM): \#741 
Intercalation: \#1416

Intercomparisons: \#154

Inter-elemental correlations: \#1325

Interfaces: \#339

Interfacial structure: \#716

Interference: \#1420

Intermediate energies: \#146

Intermediate level waste (ILW): \#1392

Intermediate-thickness sample: \#1431

Intermetallics compounds: \#639

Intermolecular interaction: \#1460

Internal contamination: \#1349

Internal exposure monitoring: \#1274

Internal gelation process: \#961

Internal mono-standard: \#1068

Internal phosphorus: \#972

Internal radiation therapy: \#194

Internal standardization: \#1067

Internet: \#373

Interphase: \#4

Intertidal sediment: \#448 \#798

Intestine: \#1138

Intracellular free calcium: \#1458

Intraoperative imaging: \#105

In-tube: \#48

Invasions: \#218

Inventory $\mathrm{Pu} 239$ : \#788

Iodate: \#446

Iodide: \#721

Iodine: \#72 \#118 \#239 \#331 \#446 \#493 \#674 \#742 \#786 \#1427

Iodine-125: \#279 \#350 \#876

Iodine-129: \#5 \#197 \#924 \#1328

Iodine-131: \#129 \#149 \#149 \#1138 \#1386

Iodine-labeled prolactin: \#1393

Iodised salt: \#493

Ion chromatography: \#331 \#378 \#721

Ion exchange: \#107 \#136 \#147 \#192 \#310 \#551 \#580

\#737 \#824 \#1297 \#1356 \#1392 \#1396 \#1407

Ion exchange capacity: \#357

Ion exchange chromatography: \#89 \#1339

Ion exchanger: \#728

Ion sensors: \#340

Ion source: \#502

Ion transfer: \#716

Ion-exchange: \#1210

Ion-exchange resin: \#90 \#1153

Ionian Sea: \#371

Ionic liquids: \#329 \#330 \#401 \#472 \#550 \#637 \#900 \#1387 \#1440 \#1444

Ionic strength: \#109 \#133 \#187 \#364 \#829

Ion-imprinted polymers: \#77

Ionizing radiation: \#968
Ionoluminescence: \#1358

Ions: \#700

Iridium: \#506 \#530

Irish sea: \#168

Iron: \#264 \#407 \#580 \#631 \#734 \#954 \#972 \#1163

Iron and steel industry: \#1344

Iron bioavailability: \#973

Iron disulfide: \#561

Iron fertilization: \#806

Iron film: \#315 \#558

Iron hydroxide: \#312

Iron minerals: \#1318

Iron oxide: \#265 \#558 \#697 \#833 \#984 \#1002

Iron oxide nano-particles: \#1338

Iron sulfide: \#1316

Iron(III) complexes: \#1434

Iron/cesium aerosol: \#1081

Iron-57-Mossbauer spectra: \#556

Iron-57p: \#561

Iron-containing silicate glass: \#556

Iron-fertilization experiment: \#404

Iron-nickel-copper-cobalt ore reference materials: \#1333

Irradiated TeO2: \#1386

Irradiation: \#898 \#943

Isoelectric point: \#729

Isoprene: \#1263

Isoprenoids: \#222

Isoprostane: \#286

Isotherm: \#84 \#411 \#533 \#727 \#776 \#1336

Isotope: \#757 \#874 \#1085

Isotope correlation: \#215

Isotope dilution: \#750 \#785 \#921

Isotope dilution factor: \#124

Isotope dilution mass spectrometry: \#1461

Isotope effect: \#260

Isotope fractionation: \#939

Isotope ratio: \#46 \#71 \#750 \#781 \#1439

Isotope ratio analysis: \#613 \#790

Isotope separation: \#1129

Isotope-dilution AMS: \#1235

Isotope-dilution mass spectrometry: \#43

Isotopic analysis: \#1001

Isotopic labelling: \#810

Isotopic ratio: \#156 \#752 \#785 \#1007

Isotopic reference materials: \#529

Isotopic signature: \#1426

Istanbul region: \#447

Italian drinking waters: \#1015

ITIES: \#716

Jamaica: \#1375

Japan: \#788

Japan Sea: \#148 
Japanese cedar: \#970

Job's continuous variation: \#717

$\mathrm{K}(0)$-factors: \#1067 \#1068

K(0)-INAA: \#1070 \#1318 \#1319 \#1333 \#1334 \#1365

K(0)-NAA: \#189

K(0)-RNAA: \#1318 \#1333

K2K: \#1153

K-40: \#270 \#996 \#997

Kaolin: \#276

Kaolinite: \#706

KEOPS: \#992

Keradioaktifan tabii: \#1366

KeV range: \#917

KeV-ISOL: \#352

Key comparison: \#1461

Kidney stones: \#979

Kinematic Broadening: \#1059

Kinetic equations: \#1305 \#1336

Kinetic modeling: \#830

Kinetic study: \#945

Kinetic-catalytic method: \#1018

Kinetics: \#29 \#84 \#276 \#308 \#414 \#826 \#850 \#974 \#1062 \#1296 \#1302 \#1306 \#1404

Kluyveromyces marxianus: \#1327

Knee joint: \#1168

Konza Prairie: \#520

Koprubasi: \#434

Korea: \#1246

Kos: \#138

KP Mawthabah (Domiasiat): \#783

Kriging maps: \#453

Krka River estuary: \#782

Krypton-85 (Kr-85): \#924

K-T boundary: \#506

La0.8Sr0.2CoO3-delta: \#479

Labeled peptides: \#1140

Labeling: \#473 \#881 \#1157 \#1160 \#1228

Labelling: \#875 \#1322 \#1329

LabVIEW: \#1098

Lactation: \#41

Lake: \#495 \#629 \#1256

Lake Donghu: \#450

Lake Hazar: \#807

Lake sediment: \#156 \#338 \#788 \#1236

Lake water: \#446

Land use: \#451 \#1403

Land-sea pathway: \#724

Langmuir adsorption: \#412 \#551

Lanthanide element: \#612

Lanthanide separation: \#868

Lanthanide-actinide pertitioning: \#927

Lanthanides: \#319 \#326 \#372 \#378 \#401 \#519 \#577 \#610 \#693 \#828 \#985 \#1311
Lanthanides(III): \#574

Lanthanoids: \#900

Lanthanum: \#1458

Lanthanum-140: \#196 \#758

Laser ablation: \#46 \#80 \#558 \#1001

Laser ablation inductively coupled plasma mass spectrometry (LA-ICP-MS): \#41 \#87 \#514 \#608 \#609 \#642 \#653 \#980 \#1175 \#1231 \#1452

Laser deposition: \#315

Laser induced breakdown spectroscopy (LIBS): \#979 \#1019 \#1430

Laser mu-Raman spectroscopy: \#1125

Laser-evaporated iron: \#558

Laser-induced fluorescence method: \#500

Lassen Volcanic National Park: \#410

Latitudinal distribution: \#1372

Layered double hydroxide: \#903

Layered Li[Li0.17Mn0.33Co0.5-yNiy]O-2 cathodes: \#733

LC/MS/MS: \#934

Leaching: \#370 \#852 \#1406 \#1462

Lead: \#9 \#71 \#95 \#278 \#580 \#582 \#757 \#800 \#825 \#827 \#836 \#920 \#1443

Lead isotopes: \#273

Lead(II): \#109 \#364 \#829 \#844

Lead, $\mathrm{Pb}-210$ sediment geochronology: \#765

Lead-210: \#132 \#1273

Lead-210 and Cs-137 dating: \#865

Lead-210 and $\mathrm{Pb}-212$ : \#789

Lead-210 chronology: \#999

Lead-210 dating: \#160 \#517

Lead-212: \#758

Leading-edge timing: \#1075

Lead-spallation target: \#103

Leakage: \#481 \#823 \#1384

Lee eddies: \#407

Li2O: \#710

Li2O/LiCl molten salt: \#427

Lichen: \#449

Lichenoindication: \#245

Lichens: \#245 \#420 \#603

LiCl: \#710

LiCl-Li2O molten salt: \#638 \#1151

Lifetime spectrometer: \#1084

Ligand: \#1460

Ligand degradation: \#1330

Light-absorption ratio variation approach: \#342

Light-induced excited spin state trapping: \#936

Lignite: \#600

Ligurian Sea: \#999

Lime: \#566

Limitations: \#334

LiNbO3: \#638 
Lindlar catalyst: \#883

Linear discriminant analysis: \#633

Linear energy transfer: \#1330

Lipid bilayer: \#632

Lipid peroxidation: \#1456

Lipids: \#222

Lipophilic: \#179

Liposomes: \#31

Liquid chromatography: \#259

Liquid membrane: \#1332

Liquid phase microextraction: \#91

Liquid samples: \#141

Liquid scintillation: \#22 \#159 \#300 \#422 \#536 \#983

\#1095 \#1167 \#1252 \#1297

Liquid-liquid extraction: \#927 \#1311

Liquid-liquid interface: \#716

Liquid-liquid microextraction: \#459

Liquid-phase separation: \#329

Liquid-scintillation spectrometry: \#792

Lisbon: \#213

LiTaO3: \#636

Lithium: \#314 \#1105

Lithium ferrite: \#1360

Lithium-ion cell: \#735

Lithospheric extension: \#929

Localization: \#134

Location: \#1085

Log-normal distribution: \#1457

LOICZ: \#372

Long-term monitoring: \#771

Long-term trends: \#167

Loop-mediated isothermal amplification: \#99

Los Alerces National Park: \#248

Low background gas proportional counter: \#197

Low energy: \#385

Low-cost adsorbents: \#379

Low-level beta(-)-counting: \#746

Low-level counting: \#22

Low-level decay counting: \#155

Low-level radiation detection: \#1007

Low-level radioxenon measurement: \#779

Low-temperature metal hosts: \#1199

Low-temperature solid-state reaction: \#349

LPME: \#48

L-tryptophan: \#555

Luminescence: \#731 \#943 \#1106

Luminescence dating: \#390 \#516

Lung: \#1085

Lutetium(III): \#466

Lutetium-177: \#158

Lymphocytes: \#621 \#624

Lysozyme: \#260

Maar volcanism: \#969
MACEDONIA: \#1289

Macroalgae: \#777

Macrocycles: \#93 \#177 \#939 \#1031 \#1032 \#1033 \#1034 \#1035

Macrofungi: \#1038

Macronutrients: \#358

Macrophage uptake: \#732

Macroporous materials: \#946

Macroporous silica-based polymeric composite: \#23 \#24 \#575 \#720 \#823 \#1384

Macroporous silica-calix [4]arene-crown polymeric composite: \#578

Magnesium assessment: \#1008

Magnesium-26(O-18.2p2n)Ar-40: \#1185

Magnetic and antimagnetic rotations: \#1202

Magnetic materials: \#1002

Magnetic measurements: \#639

Magnetic nanoparticles: \#383 \#617 \#1043 \#1436

Magnetic properties: \#323 \#687 \#1215

Magnetic relaxation: \#888

Magnetic resonance imaging: \#475

Magnetic transitions: \#559

Magnetite: \#730 \#1436 \#1437

Magnetite nanoparticles: \#265 \#1437

Magnetoresistance: \#888

Magnetron discharge: \#1063

Mahayograj Guggulu: \#869

Maintenance requirement: \#811

Major elements contamination: \#766

MALDI-TOF MS: \#1438

Malia: \#277

Mandarin: \#35

Mandelic acid: \#872

Manganese: \#10 \#40 \#407 \#445 \#920 \#1189 \#1190 \#1191

Manganese dioxide: \#141

Manganese oxide: \#294

Manganese-fiber: \#992

Manila Bay: \#724

Mannitol: \#1445

Mantle serpentinization: \#931

Mantle tomography: \#813

Mantle xenoliths: \#813

Marble: \#1125

Marine: \#772

Marine environment: \#1439

Marine radioactivity: \#164

Marine sediments: \#713 \#999 \#1000

Marine tracer: \#1423

MARS: \#1107

Martensite transformation: \#1352

Mass activity: \#168

Mass attenuation: \#861 
Mass closure: \#1369

Mass spectrometers: \#1110

Mass spectrometric methods: \#81

Mass spectrometry: \#22 \#86 \#89 \#435 \#1122 \#1361 \#1420 \#1460

Mass transfer: \#362

Mass-balance: \#1379

Massic activity: \#794

Mass-yield distribution: \#1133

Materials processing: \#313

MatEriaux de rEfErence gEologique: \#531

Mathematical models: \#307

Matrix effect: \#1431

Matrix-assisted laser desorption/ionisation: \#1350

Maximum admissible concentrations (MAC): \#802

Maze: \#1128

MC50 cyclotron: \#1108

MCFUT: \#1076

MCI GEL CHP20P: \#853

MC-ICPMS: \#1424 \#1426

MCM-41: \#346 \#348

MCNP: \#1065

MCNP5: \#1079

MCNPX: \#923 \#1083 \#1086 \#1112

MCNPX code: \#1154

MCTTFA: \#213

Mean chord length: \#1097

Mean residence time: \#196

Mean sigma $(\mathrm{C}-\mathrm{C})=0.005$ angstrom: \#12

Measurement uncertainty: \#5 \#1061

Mechanical properties: \#295 \#943

Mechanism: \#1358

Mediastinitis: \#223

Medical accelerators: \#182 \#190

Medical cyclotron: \#1147

Medical devices: \#482

Medical isotope: \#158 \#279 \#1132

Medical linear accelerator: \#1285

Medicinal herbs: \#932

Medicinal plants: \#1174 \#1325 \#1429 \#1469

Mediterranean: \#35 \#765 \#1251

Mediterranean agroecosystems: \#1403

Mediterranean basin: \#764

Mediterranean Sea: \#371 \#744 \#991

Medium-range order: \#905

Megacities: \#1376 \#1378

MEKC: \#428 \#956

Melamine-formaldehyde-thiourea resin: \#553

Melanin: \#222

Membrane: \#632 \#737 \#943

Membrane extraction: \#1248

Mentha spicata: \#933
Mercury: \#43 \#90 \#251 \#291 \#308 \#333 \#338 \#418 \#460 \#628 \#841 \#857 \#998

Mercury methylation: \#338

Mercury(II): \#462

Mercury-197: \#333 \#338

Mercury-203: \#628 \#1361

Meromixis: \#629

Merrifield resins: \#1354

Mesolite: \#1024

Mesoporosity: \#436

Mesoporous: \#346 \#348 \#859 \#1390

Mesoporous silica: \#74 \#1449

Mesoporous Sn(IV) silicate: \#1310

Mesoporous zirconium titanium oxides: \#976

Metabolic processes: \#73

Metabolism: \#383 \#1228

Metabolites: \#42

Metakaolin: \#84

Metal: \#839 \#1451

Metal and Cs-137 migration: \#437

Metal and organometal determination: \#91

Metal binding: \#1454

Metal cations: \#1033

Metal chelator: \#1354

Metal complexes: \#374 \#375

Metal contamination: \#1238 \#1452

Metal hydrides: \#429

Metal ion adsorption: \#348

Metal ion sorption: \#346

Metal ions: \#49 \#59 \#356 \#357 \#1237

Metal oxide: \#1115

Metal removal: \#117

Metal uptake: \#93

Metalion exchange: \#1387

Metallaboranes: \#1311

Metallacarbaboranes: \#927

Metallic niobium: \#638

Metallic tantalum: \#636

Metallocenes: \#34

Metalloenzymes: \#555

Metallofullerene: \#351 \#1045

Metalloid: \#1232 \#1451

Metalloligands: \#1329

Metallomics: \#659 \#1231

Metallothionein: \#452 \#1374

Metallurgical lead: \#1016

Metallurgical samples: \#1344

Metals: \#332 \#1232 \#1233 \#1421

Metals analysis: \#486

Metals selenium: \#1039

Metals-bioavailability: \#486

Metamorphic rocks: \#509

Metasomatism: \#1028 
Metastable state production: \#1143

Meteorite composition: \#206

Meteorites: \#562

Meteorological parameters: \#1370

Methacrylic acid: \#1336

Methanol: \#971

Method design and validation: \#756

Methodology: \#986 \#1461

Methylpiperidine dithioformate derivatives: \#880

Metrology: \#341

Metrology and the theory of measurement: \#1007

Mexico: \#547 \#980 \#1359

MGAU: \#1447

MHC genes: \#503

Mice: \#904

Microcorrosion: \#656

Micro-Raman spectrometry: \#508

Microanalysis: \#529

Microanalysis reference materials: \#1027

Microbeam: \#1134

Microbial chelates: \#36

Microbicidal activity: \#935

Microdefects: \#1352

Microdistribution: \#792

Microelectronics: \#1027

Microextraction techniques: \#48

Microfluidics: \#915

Microfocus: \#569

Microheterogeneity: \#1027

Micronuclei: \#1374

Micronucleus test: \#1037

Micronutrient: \#286

Micronutrients: \#358

Microparticles: \#1489

Micro-PIXE: \#261 \#262 \#263 \#554 \#1127 \#1136 \#1488 \#1489

Micropore tuning: \#1025

Micro-Raman analysis: \#513

Microstructure: \#835 \#896 \#944 \#1003 \#1261

Microtomography: \#474 \#1021 \#1432

Micro-tomography: \#1135

Microwave: \#163

Microwave digestion: \#834

Microwave dissolution: \#1441

Microwave heating: \#882

Microwave induced chemical etching: \#1099

Middle Miocene: \#813

Migration: \#363

Miletos: \#138

Milk: \#185 \#203 \#488 \#721

Milling: \#618

Mineral: \#1358

Mineral elements: \#1363
Mineral water: \#748 \#1426

Mineralogical transformations: \#755

Minerals: \#488

Miniature neutron source reactor: \#162

Miniature X-ray generator: \#515

Miniaturization: \#1446

Minicolumn sensors: \#54

Minimum resolving time: \#1090

Mining: \#757

Mining tourism: \#796

Minoen: \#277

Minor actinide: \#1388

Minor elements: \#1344

Mixed oxides: \#312

Mixed solvent: \#1450

Mixed symmetry states: \#1229

Mixed waste: \#78

Mixture quantification: \#78

MnO2 cartridges: \#504 \#505

MnOx-ZrO2: \#1398

MnOZ coprecipitation: \#988

Mobility: \#761

Mock-up experiment: \#755

Model: \#974 \#1379

Modeling: \#29 \#180 \#716 \#826 \#1066 \#1323

Modeling and simulation: \#905 \#906

Modelling: \#1251 \#1462

Modelling approach: \#771

Modification: \#1406

Modified activated carbon: \#827

Modified silica: \#311

Modified silica and alumina: \#28

Molecular dynamics: \#630

Molecular dynamics method: \#711

Molecular imaging: \#935 \#1029 \#1045 \#1453

Molecular probes: \#105

Molecular weight: \#886

Mollusk: \#1052

Molten salt: \#313 \#636 \#710

Molybdenum(VI): \#1438

Molybdenum-99/Tc-99 m generator: \#1142

Monitoring: \#758

Monitoring methodology: \#924

Monitoring networks: \#1247

Monitoring station: \#975

Monoamide: \#1226

Monochromatic neutron beam: \#1068

Monolayers: \#339

Monomer: \#953

Mono-substitution: \#866

Montana: \#931

Monte Carlo: \#207 \#1072 \#1089 \#1255

Monte Carlo calculations: \#1079 
Monte Carlo simulation: \#22 \#101 \#184 \#198 \#202 \#905 \#906 \#907 \#908 \#1078 \#1094 \#1107 \#1144

Monte Castillo Caves: \#226

Montmorillonite: \#107 \#187 \#363

Morbidity: \#1470

Moringa oleifera (Moringaceae): \#253

Morphology: \#727

Morris water maze: \#1458

Mortar: \#125

Moss: \#453

Mossbauer spectroscopy: \#217 \#376 \#557 \#559 \#888 \#902 \#965 \#1003 \#1360 \#1434

Mossbauer study: \#598

Mosses: \#420

Mount Everest: \#723

Mountain slopes: \#1251

Mouse tumor: \#703

MRI contrast agent: \#1045

MS: \#731

Multi-collector thermal ionization mass spectrometry: \#613

Multi-component analysis: \#88

Multi-dynamic measurement: \#611

Multi-element analysis: \#1148

Multi-element determination: \#1309

Multilinear Regression Analysis: \#1369

Multiparameter: \#1075

Multiple-ion counting: \#611 \#613

Multivalency: \#387

Multivariate analysis: \#382 \#1468

Multivariate analysis and trajectories: \#700

Multivariate statistical methods: \#1481

Multivitamin mineral supplements: \#45

Multi-walled carbon nanotubes: \#1442

Muons: \#746

Murraya koenigii: \#489

Mushroom: \#834

Mussels Mytilus sp.: \#782

MWCNTs: \#829 \#842

MX-80 bentonite: \#109

Myocardial imaging agent: \#143

N-14(n, gamma)N-15 reaction: \#918 \#1196

N2O: \#1424

N2O decomposition: \#294

NAAPRO: \#1066

Nagasaki atomic bomb: \#752

Nahuel Huapi National Park: \#248 \#251 \#333 \#338

Nal detectors: \#1486

Nam Co: \#354

Nanoantenna-directed photothermal destruction: \#123

Nanoclustering: \#1045

Nanocomposite: \#1260

Nanocrystals: \#1048
Nanomaterials: \#429 \#740 \#1037 \#1221 \#1400 \#1479

Nanomedicine: \#383

Nanomonitoring: \#740

Nanoparticle concentration: \#1044

Nanoparticle plant virus: \#1046

Nanoparticles: \#3 \#340 \#355 \#686 \#701 \#1040 \#1121 \#1234 \#1400

Nano-second laser-ablation: \#790

Nanoshell: \#1 \#1044

Nanostructures: \#1004 \#1401

Nano-TiO2: \#850

Nanotoxicity: \#384 \#740

Nanotoxicology: \#1040 \#1221

Narrow-band imaging: \#702

Nasal Instillation: \#904

Nasal mucus: \#606

National measurement institute: \#1461

Native arsenic: \#431

Natural levee: \#521

Natural population analysis (NPA): \#1455

Natural radioactivity: \#155 \#272 \#566 \#763 \#950 \#1253 \#1326

Natural radionuclides: \#168 \#447 \#600 \#627 \#796 \#1239 \#1240 \#1441

Natural tellurium dioxide (TeO2) target: \#199

Natural uranium: \#1111

Natural waters: \#465 \#745 \#983 \#1252

Nb2O5: \#638

N-donors: \#1303

Near-infrared: \#3

Near-infrared light absorption: \#121

Neem: \#955

Nematodes: \#518

Neodymium: \#1327

Neolithic: \#699

Neptunium: \#1095 \#1343

Neptunium(IV): \#591

Neptunium(V): \#591

Neptunium(VI): \#591

Neptunium-237: \#398

Nernst-Planck: \#310

Neurodegeneration: \#264

Neuromelanin: \#222 \#587

Neurotoxicology: \#1456 \#1459

Neutral organophosphorus extractant: \#1440

Neutron: \#1128

Neutron activation: \#268 \#870 \#920 \#1008 \#1113 \#1114

Neutron activation analysis (NAA): \#47 \#72 \#97 \#114 \#130 \#160 \#207 \#209 \#224 \#248 \#341 \#388 \#393 \#394 \#966 \#967 \#1066 \#1067 \#1097 \#1187 \#1231 \#1309 \#1344 \#1461

Neutron attenuation coefficient: \#1207

Neutron beam: \#1117 
Neutron beam imaging: \#1092

Neutron capture: \#917

Neutron capture cross-sections: \#1078

Neutron coincidence counter: \#923 \#1083

Neutron diffraction/scattering: \#905 \#906 \#907 \#908

Neutron emission spectra: \#812

Neutron fluence rate: \#268

Neutron flux characterization: \#1334

Neutron flux determination: \#139

Neutron flux distribution: \#1365

Neutron generator: \#142 \#525 \#568

Neutron induced fission: \#1200

Neutron induced reaction: \#561

Neutron irradiation facility: \#1061

Neutron moderation: \#139 \#170 \#178

Neutron parameters: \#1365

Neutron powder diffraction: \#1005

Neutron radiography: \#1207 \#1466

Neutron reflectometry: \#170

Neutron resonance analysis: \#1076 \#1101

Neutron resonance capture spectra: \#689

Neutron scattering: \#170 \#178 \#1207

Neutron science and technology: \#1060

Neutron sensitivity: \#1078

Neutron time-of-flight spectroscopy: \#1078

Nevada Test Site: \#770

N-heterocyclic cations: \#303

Nickel: \#373 \#475 \#1163

Nickel sorption: \#366

Nickel sulphide: \#1392 \#1396

Nickel toxicity: \#212

Nickel(II): \#187 \#842

Nickel-63: \#1242

Nicotinic acid: \#1216

Nigeria: \#173 \#784 \#952 \#1126

NIRR-1: \#173

NIRR-1 standardization: \#130

Nissl staining: \#1458

NiTi alloy: \#1352

Nitrate: \#972

Nitrate media: \#619

Nitrate medium: \#828

Nitric acid: \#1226 \#1324

Nitric acid solutions: \#361

Nitric oxide: \#631

Nitrite: \#631

Nitrobenzene: \#9

Nitrogen: \#44 \#266 \#990 \#1362

Nitrogen rejection: \#1025

Nitrogen tolerance: \#451

Nitrogen-based polymers: \#1353

NMR: \#717 \#731

NMR (nuclear magnetic resonance): \#1228
Noble gas: \#506

Noble gas monitoring: \#369

No-carrier-added: \#124 \#129

No-carrier-added (nca) I-131: \#199

No-carrier-included: \#129

N-octylaniline: \#581

Nomenclature: \#1401

Non-1/v behaviour: \#1117

Non-destructive: \#569

Non-destructive characterisation: \#1135

Noninvasive: \#1008

Nonionic surfactants: \#1454

Nonparametric statistics: \#1070

Nonproportionality: \#1073

NORM: \#849

NORM and TE-NORM: \#780

Normalization: \#1238

North Pacific: \#409

North Pacific Subtropical Gyre: \#406

Northern Patagonia Andean range: \#160

Northern Patagonian lakes: \#251

Northwestern Mediterranean Sea: \#999

$\mathrm{NpO} 2+$ : \#1296

Nuclear archeology: \#66

Nuclear chemistry: \#1129

Nuclear data sheets: \#1057

Nuclear economics: \#1227

Nuclear forensic analysis: \#790

Nuclear forensics: \#80 \#154 \#1122

Nuclear fuel: \#46 \#961 \#1445

Nuclear fuel cycles: \#1145

Nuclear fuel reprocessing facility: \#148

Nuclear fusion: \#385

Nuclear imaging: \#1453

Nuclear medicine: \#177

Nuclear microprobe: \#1135

Nuclear microscopy: \#1120

Nuclear power: \#1156 \#1227

Nuclear power plant: \#537 \#585 \#1278

Nuclear reaction: \#874 \#1107 \#1128 \#1143 \#1185

Nuclear reaction analysis: \#1137

Nuclear reactions: \#1101

Nuclear reactions, fluctuation phenomena: \#1412

Nuclear reactor: \#564 \#1331

Nuclear research reactor: \#1060

Nuclear safeguard analysis: \#790

Nuclear safeguards: \#1447

Nuclear structure: \#1229

Nuclear techniques: \#603

Nuclear technology: \#1131

Nuclear waste: \#57 \#81 \#275

Nucleation: \#424

Nucleation and Growth: \#1062 
Nucleophilic aromatic substitution: \#145 \#809

Nucleophilic substitution: \#810

Nuclide separation: \#1225

Numerical modelling: \#744

Numerical solving: \#1379

Nutri-elements: \#35

Nutrient contamination: \#1378

Nutrient flux: \#724

Nutrient ions: \#1013

Nutrient loading estimates: \#989

Nutrient trace elements: \#932

Nutrient uptake: \#211

Nutrients: \#266 \#469 \#470 \#1371

Nutrition: \#634 \#966

Nutritional components: \#491

Nutritional supplement: \#249

NW342 non-ionic surfactant: \#846

O-3: \#232

Oak barrels: \#722

Oak related compounds: \#722

Obsidian: \#224 \#225 \#699 \#1468

Obsidian procurement: \#980

Obsidienne: \#277

Occupation: \#332

Occupational dose: \#796

Occupational exposure: \#278 \#442

Ocean: \#19

Oceanic eddies: \#405

Oceanic Flux Program (OFP): \#419

Oceanic lithosphere: \#512

Oceanography: \#504

OCHRE: \#226

Ochre: \#697

Ocimum sanctum (Lamiaceae): \#253

Ocular tumours: \#181

Of precious metals: \#709

Off-line correct: \#1075

Oil industry: \#1169

Olive stones: \#913

On-line mineralogy: \#161

On-site measurement: \#1244

Operational topics: \#537

Opium alkaloid containing wastewater: \#1268

Optical activity: \#885 \#886

Optical coherence tomography: \#120 \#703

Optical emission: \#1430

Optical flow algorithm: \#1091

Optical imaging: \#563

Optical model calculation: \#1197

Optical rotatory dispersion: \#1262

Optical-emission spectrometry: \#43

Optically active polymer: \#885

Optically stimulated luminescence: \#1109
Optimal sample dimensions: \#1079

Orange: \#35

Orange peel: \#836

ORD: \#1262

Oregano: \#955

Oreochromis niloticus: \#218

Ores: \#428

Organic: \#35

Organic bound: \#759

Organic carbon: \#408

Organic chemistry: \#877

Organic compounds: \#356

Organic contaminations: \#334

Organic cosolvent: \#1303

Organic matter: \#439 \#496 \#762

Organic resin: \#59

Organic synthesis: \#163

Organic wastes: \#518

Organoclay: \#110 \#1416

Organo-iodine formation: \#463

Organophosphorus: \#1435

Organotin complexes: \#32

ORIGEN-ARP: \#1086

ORIGEN-S: \#923

Origin of life: \#1262

OSL: \#1106

Overall mass balance: \#450

Overcharging: \#381

Overland flow: \#520

Owls: \#299

Oxidant: \#1386

Oxidation: \#974

Oxidation states: \#195

Oxidation/reduction potential: \#990

Oxidation-reduction: \#197

Oxidative stress: \#1219 \#1458

Oxide glasses: \#905 \#906

Oxide precipitation: \#37

Oxides: \#965 \#1003

Oxinate: \#625

Oxine: \#1159

Oxyanions: \#554

Oxygen isotopes: \#862

Oxygen nonstoichiometry: \#293

P fraction: \#972

P metabolism: \#811

Phytoremediation: \#433

PAA: \#842

PAC: \#352

PACAP-receptor: \#1052

Packaging decontamination: \#1264

Packing decontamination: \#1258

Paddy field: \#787 
Pairing strength: \#546

Pakistan: \#233 \#860 \#948 \#950 \#1283

Palaeolimnology: \#548

Palladium: \#33 \#424 \#425 \#552 \#1054 \#1224 \#1353

Palladium extraction: \#550

Palladium isotopes: \#812

Palladium recovery: \#1407

Palladium(II): \#553

Palladium(II) complexes: \#1158 \#1298

Palladium-103: \#151 \#169 \#1158 \#1293 \#1298

Palmatine: \#259

PANI/HA nanocomposite: \#857

Paracoccus sp YM3: \#805

Paraffin solidified waste: \#197

Paraiba do Sul: \#741

Paramagnetic nanoparticles: \#940

Paricutin volcano: \#1463

Parkinson's disease: \#1219

Partial gamma-ray production cross-sections: \#1067

Particle: \#618 \#1435

Particle age: \#1425

Particle calorimetry: \#1100

Particle detectors: \#692

Particle emission: \#1412

Particle export: \#408 \#820

Particle fluence modeling: \#1112

Particle flux: \#403 \#405 \#409

Particle induced X-ray emission (PIXE): \#214 \#253 \#277 \#441 \#1120 \#1126 \#1134 \#1135 \#1359

Particle-assisted laser ablation: \#288

Particle-size distribution: \#759

Particulate carbon: \#816

Particulate matter: \#235

Partitioning: \#1383 \#1395 \#1408

Passive iron: \#426

Passive neutron multiplicity counter: \#1086

Passivity: \#376

Patagonia: \#248 \#548

Pathogenicity: \#805

Pattern formation: \#273

PCBs: \#1000

Peak phosphorus: \#532

Peak-area analysis: \#2

Peat: \#242

Peat moss: \#855

Peat-forming environment: \#600

Pebble bed reactor: \#564

PEG: \#1140 \#1220

Pegylation: \#732

Peloponnese: \#1253

Pelotherapy: \#112

Penalver formation: \#377

Pencil graphite: \#1309
Peptide synthesis: \#1361

Percutaneous absorption: \#112

Performance evaluations: \#154

Periodic table: \#708

Perlite: \#776

Permanent magnetic materials: \#1433

Permeable sediments: \#219 \#471 \#862

Perovskite-type oxides: \#293

Perrhenate: \#554

Persian Gulf: \#627 \#996

Persistent organic pollutants: \#1479

Pertechnetate: \#52 \#1317

Pertechnetate immobilization: \#1307 \#1316

Perturbation method: \#495

Perturbed angular correlation technique: \#736

Pesticides: \#1258 \#1474 \#1479

PET imaging agents: \#1173

Petrography: \#226

PG: \#370

PG waste: \#780

PGAI-NT: \#206

pH: \#109 \#187 \#364 \#367 \#552 \#829 \#842 \#1342

Pharmaceutical analysis: \#88

Pharmaceuticals: \#1479

Pharmacokinetics: \#256 \#259

Pharmacology: \#355 \#935 \#1029

Phase diagram: \#368

Phase separation: \#944

Phase transfer: \#1460

Phase transfer catalysis: \#716

Phase transition: \#962

Phenanthrene biodegradation: \#604

Phenol-phthalein: \#1138

Phenomenological Stopping Power: \#1059

Phonolite: \#527

Phosphate: \#432

Phosphate industry: \#849

Phosphate rock: \#532

Phosphate-based materials: \#963

Phosphates: \#803 \#962

Phosphinate complexes: \#177

Phosphinic acid resin: \#412

Phosphinimines: \#1317

Phosphogypsum: \#835 \#1269

Phospholipids: \#339

Phosphor: \#914

Phosphoric acid: \#1409

Phosphoric acid activation: \#309

Phosphoric gypsum: \#803

Phosphorite: \#860

Phosphorus: \#266 \#496 \#532 \#990 \#1463

Phosphoryl: \#718

Phoswich: \#768 
Phoswich detector: \#565 \#1080 \#1082

Phoswich Monte Carlo: \#1254

Photoacoustic tomography: \#1041

Photochemical oxidation: \#707

Photoelectron imaging: \#1219

Photo-fission of Bi-209: \#1133

Photon activation analysis: \#44 \#182

Photon attenuation: \#1194

Photoneutrons: \#1285

Photophysics: \#3

Photosensitizer: \#616

PHU: \#1364

Phyllanthus niruri (Euphorbiaceae): \#253

Physical and inorganic-chemical parameters: \#807

Physical sorption: \#1210

Physico-chemical processes: \#1478

Phytase: \#973

Phytic acid: \#973

Phytoplankton: \#333 \#806

Phytoremediation: \#440

PICO-NARE: \#1081

PicoRad detectors: \#1167

Piezonuclear decay: \#1181 \#1182 \#1183

Piezonuclear neutrons: \#1180

Piezonuclear reactions: \#614

Pigments: \#548 \#697

Pillared clay: \#411

Pilot plant test: \#1395

Pilot test: \#1408

Pinene: \#885 \#887 \#1262

Piperine: \#243

Pitcher plants: \#211

Pituitary adenylate cyclase activating polypeptide: \#1052

Planar chromatography: \#61

Planar HPGe detector: \#1093

Plankton: \#806

Plant: \#337 \#433

Plant metabolites: \#544

Plant roots: \#1207

Plant science: \#683

Plasma etching: \#1099

Plasma nitriding process: \#620

Plasma-mass spectrometry: \#87

Plastic materials: \#240 \#1413

Plastic scintillation: \#78

Plastic scintillator: \#1089

Plastic veto shield: \#1245

Plate and hollow sphere targets: \#159

Platinum: \#552 \#874 \#1353

Platinum-195 m: \#1218

Platinum-group elements: \#301 \#529

PLD film: \#560
Pleistocene: \#600

PLGA: \#1220

Plutonium: \#70 \#89 \#137 \#156 \#167 \#174 \#215 \#299 \#454 \#579 \#654 \#661 \#747 \#750 \#752 \#785 \#786 \#794 \#910 \#984 \#1014 \#1095 \#1163 \#1297 \#1301 \#1312 \#1342 \#1371 \#1425 \#1439

Plutonium dioxide: \#961

Plutonium isotope pollution: \#774

Plutonium isotopes: \#1380

Plutonium(III): \#593

Plutonium(IV): \#593 \#1345

Plutonium(VI) carbonate: \#510

Plutonium(VI) hydrolysis: \#586

Plutonium-238: \#1314

Plutonium-239: \#1314

Plutonium-239,Pu-240: \#1472

Plutonium-239,Pu-240/Cs-137 activity ratio: \#1371

Plutonium-239/240: \#664

Plutonium-240: \#391 \#1200 \#1314

Plutonium-240/Pu-239 atom ratio: \#148

Plutonium-241: \#676

Plutonium-242: \#1200

PM10: \#232 \#237 \#441 \#547 \#603 \#1071 \#1376

PM10-2.5: \#1376

PM2.5: \#213 \#231 \#232 \#233 \#236 \#441 \#603 \#1071

$$
\# 1376
$$

PM2.5-10: \#213 \#231

PN2S: \#1140

Pneumatic Transfer System: \#1061

POC export: \#925

Podzolisation: \#516

Point of zero charge: \#729

Point source: \#201 \#1085

Poison: \#775

Poisson distribution: \#680

Poisson processes: \#1007

Polarimetry: \#1259

Polarization: \#376

Polarization X-ray fluorescence spectrometry: \#1433

Polesie Lubelskie: \#517

Polishing: \#1127

Pollen and spores: \#273

Pollutant sources: \#451

Pollutants: \#379

Pollutants removal: \#29

Polluted soil: \#840

Pollution: \#242 \#797 \#799

Pollution sources: \#800

Polonium: \#135 \#507 \#751 \#757 \#1163

Polonium-210: \#132 \#140 \#241 \#748 \#775 \#789 \#815 \#1017 \#1241 \#1349 \#1457

Poly(3-methyl-14-pentadiene): \#1265

Poly(4-vinylpyridine) resins: \#1407 
Poly(dimethylbutadiene): \#1261

Poly(ethylene glycol): \#732

Poly(vinylidene fluoride): \#899

Polyacrylamide: \#839

Polyaniline: \#203 \#462

Poly-beta-pinene: \#885 \#886 \#1259

Polycyclic aromatic hydrocarbons (PAHs): \#604

Polyethylene (PE): \#694

Polyethylene glycol: \#256

Polygonaceae: \#1174

Polyisoprene: \#311 \#1260 \#1263

Polymer electrolyte membrane fuel cells: \#899

Polymer material: \#953

Polymer matrix: \#943

Polymer membranes: \#483

Polymer stereoisomerism: \#1265

Polymeric composite: \#573 \#1383

Polymerization: \#34 \#594 \#985 \#1299

Polymerization in clathrates: \#1263

Polymers: \#340

Polymethyl metha acrylates: \#1396

Polynuclear complexes: \#1215

Polyoxometalates: \#1215 \#1438

Polypentenamer: \#1217

Poly-pinene: \#887

Polystyrene thiol resin: \#1354

Polytetrafluoroethylene: \#898

Polyurethane foam: \#1421

Polyurethanes: \#944

Porosity: \#893

Porous materials: \#1002

Porous polymers: \#1267

Porous polypropylene: \#696

Porsuk: \#698

Portable automated luminescence-measuring system: \#515

Portal: \#1089

Portland cement: \#852

Position sensitive particle detectors: \#1100

Positive matrix factorisation: \#236 \#237

Positron: \#914

Positron annihilation: \#943 \#944 \#1084

Positron emission intensity: \#1300

Positron emission tomography (PET): \#20 \#126 \#144 \#163 \#204 \#473 \#810 \#915 \#1093 \#1141 \#1155 \#1157 \#1159 \#1170 \#1171 \#1234 \#1291 \#1453

Positron emitting radiotracers: \#544

Positron lifetime: \#1206

Positronium annihilation: \#545

Postelution concentration: \#1142

Post-harvest: \#705

Potassium: \#627 \#1480

Potassium content: \#494
Potassium-40: \#494

Potatoes: \#507

Potential ecological risk: \#448

Pottery: \#100

Pourbaix diagrams: \#37

Powders: \#313

Power alcohol: \#440

Pragya-peya: \#932

Precipitation: \#199 \#815 \#1385

Preconcentration: \#49 \#74 \#75 \#77 \#141 \#444 \#445 \#601 \#602 \#821 \#825 \#827 \#838 \#1020 \#1382 \#1421 \#1442 \#1449

Prediction: \#180

Pre-equilibrium reactions: \#812

Pregnancy: \#41

Preschool children: \#345

Principal component analysis: \#235 \#633 \#1071 \#1430

Principal components regression: \#1430

Production: \#131 \#175 \#193 \#1293

Progeny: \#1379

Progesterone: \#203 \#1464

Programmable logic controller: \#1098

Proinflammatory cytokines: \#1459

Prompt gamma activation analysis (PGAA): \#63 \#68 \#206 \#225 \#918 \#969 \#970 \#971 \#1067 \#1069 \#1077 \#1104 \#1105 \#1115 \#1117 \#1118 \#1118 \#1130 \#1235

Prompt gamma ray neutron activation analysis (PGNAA): \#198 \#206 \#216 \#321 \#1068 \#1079 \#1094 \#1116 \#1119 \#1144 \#1255

Proposed values: \#531

Prostate cancer: \#285

Protactinium: \#596

Protecting group: \#8

Protein adsorption: \#4

Protein crystallography: \#685

Protein extraction: \#329

Protein fractionation: \#492

Protein oxidation: \#1456

Protein structure: \#260

Proteins: \#303 \#436 \#1399

Proton beam simulation: \#1112

Proton beams: \#1100

Proton computed tomography: \#1100

Proton dosimetry: \#181

Proton exchange membrane: \#899

Proton induced reaction: \#1132

Proton radiation therapy: \#1100

Proton radiography: \#1100

Proton reactions: \#1102

Protonation: \#1034

Proton-induced reaction: \#146

Protons: \#128 \#1103

Protontherapy: \#181 
Provenance: \#699

Proxiparameter: \#37

PSD: \#768 \#1082

Pseudo-first order: \#29

Pseudomonas fluorescens: \#519

Pseudo-second order: \#29

Pseudo-total metal concentrations: \#1373

PTSM: \#1146 \#1291 \#1298

PTTL: \#1250

Public health: \#360

Public participation: \#741

Pulse OSL: \#515

Pulse shape analysis: \#1093

Pulse waveform: \#155

Pulsed laser deposition: \#691

Pulse-height weighting technique: \#917 \#1078

Pumice: \#270 \#753

PUREX: \#1343

Purification: \#418 \#1385 \#1386

Purkinje cell: \#264

Purple acid phosphatase: \#973

PVA: \#1364

Pyoverdin: \#257

Pyrazolone ligands: \#374

Pyrohydrolysis: \#1445

Pyroprocessing: \#427

Quadrupole inductively coupled plasma mass spectrometry: \#79

Quality assurance: \#772

Quality control: \#131 \#1161

Quality control indices: \#1386

Quantitative 19F-NMR: \#1415

Quantitative analysis: \#355 \#1134

Quantitative phase analysis: \#1005

Quantum dots: \#105

Quanzhou Bay: \#448 \#798

Quartz: \#1418

Quercetin: \#631

Quinazolin-4-ones: \#882

Quinazolines: \#882

$\mathrm{R}$ factor $=0.045: \# 12$

$\mathrm{Ra}$ (II) ions: \#1178

Racemization: \#885

Radiation: \#535 \#541 \#758 \#944

Radiation chemistry: \#622

Radiation counting: \#337

Radiation curing: \#1260

Radiation detection: \#183

Radiation detector: \#1073

Radiation detector materials: \#895

Radiation dose: \#140 \#789 \#860

Radiation exposure: \#438 \#1169

Radiation grafting: \#483 \#1166
Radiation hazard: \#1249

Radiation hazard indices: \#780

Radiation monitoring: \#571

Radiation physics: \#622

Radiation polymerization: \#1267

Radiation protection: \#1168

Radiation shielding: \#188

Radiation stability: \#136

Radiation treatment: \#867

Radiation yield: \#1166

Radiation, alpha: \#536

Radiation-induced grafting: \#899

Radiation-induced polymerization: \#887 \#1261 \#1265

Radiative capture cross sections: \#1105

Radical adduct: \#1260

Radical polymerisation: \#304

Radicals: \#1166

Radioactive aerosol: \#758

Radioactive and stable isotopes: \#744

Radioactive capture: \#1069

Radioactive cobalt: \#111

Radioactive ion beams: \#1129

Radioactive point source: \#134

Radioactive Th-234/U-238 disequilibrium: \#403

Radioactive waste: \#307 \#791 \#852 \#1058 \#1473 \#1475

Radioactive waste disposal: \#337

Radioactive waste repository: \#719

Radioactive wastewater: \#1356

Radioactivity: \#270 \#438 \#778 \#801 \#861 \#1110 \#1223 \#1249 \#1256 \#1420

Radioactivity, environmental: \#536

Radioanalytical methods: \#1267

Radiobioassay: \#154

Radiocations: \#436

Radiocesium: \#25 \#1239

Radiochemical contamination: \#766

Radiochemical separating: \#19

Radiochemical separation: \#131 \#199 \#1016 \#1300 \#1338

Radiochemistry: \#1129 \#1156 \#1233

Radiocontaminants: \#1385

Radioecology: \#22

Radiogallium: \#1164

Radioimmunotherapy: \#104 \#274

Radioiodination: \#878

Radioiodine: \#919

Radioisotope: \#267 \#959 \#1420

Radiolabeling: \#21 \#163 \#1146 \#1147 \#1150 \#1158 \#1164 \#1298 \#1321

Radiolarite: \#225

Radiological assessment: \#757

Radiological hazard: \#566 \#860

Radiological hazards: \#948 
Radiological risk: \#849

Radiological safety assessment: \#952

Radiolysis: \#144 \#1260 \#1262

Radiolytical yield: \#435

Radiometric dating: \#928

Radiometric methods: \#81

Radiometry: \#4 \#89

Radionuclide: \#22 \#81 \#150 \#157 \#210 \#265 \#290 \#358 \#371 \#389 \#497 \#498 \#538 \#622 \#686 \#765 \#770 \#777 \#782 \#786 \#787 \#859 \#1024 \#1163 \#1169 \#1212 \#1280 \#1300 \#1347 \#1462 \#22 \#81 \#150 \#157 \#210 \#265 \#290 \#358 \#371 \#389 \#497 \#498 \#538 \#622 \#686 \#765 \#770 \#777 \#782 \#786 \#787 \#859 \#1024 \#1163 \#1169 \#1212 \#1280 \#1300 \#1347 \#1462

Radionuclide activity concentration: \#516

Radionuclide imaging: \#1233

Radionuclide immobilization: \#1328

Radionuclide mixtures: \#78

Radionuclide pollution: \#996

Radionuclide sorption: \#1310 \#1390

Radionuclides disequilibrium: \#766

Radionuclides in bones: \#299

Radionuclides in the environment: \#1156

Radionuclidic and chemical purity: \#1338

Radionuclidic purity: \#1142

Radiopharmaceutical: \#20 \#21 \#126 \#626 \#1030 \#1141 \#1157 \#1158 \#1160 \#1164 \#1170 \#1291

Radiopharmaceutical chemistry: \#1156

Radiopharmaceutical isotope facility: \#779

Radiopolymerization: \#885

Radioracemization: \#1262

Radiostrontium: \#185 \#1332

Radiosynthesis: \#915

Radiotellurium: \#1385

Radiotherapy: \#158 \#622 \#1193

Radiotoxicity: \#628 \#1361

Radiotracer: \#355 \#473

Radiotracer technique: \#196

Radiotracers: \#1139

Radioxenon: \#814

Radioxenon monitoring: \#565 \#1080

Radioysis: \#1330

Radium: \#140 \#182 \#300 \#470 \#507 \#751 \#757 \#989

Radium equivalent: \#753 \#754

Radium equivalent activity: \#1348

Radium isotopes: \#468 \#469 \#769 \#862 \#992 \#993 \#1367

Radium-224: \#789

Radium-226: \#270 \#748 \#782 \#783 \#789 \#1015 \#1017

Radium-228: \#789

Radon: \#180 \#300 \#468 \#469 \#470 \#471 \#623 \#769 \#796 \#864 \#989 \#993 \#1167 \#1247 \#1248 \#1367 \#1378 \#1379

Radon and thoron: \#585
Radon concentration: \#205

Radon decay products: \#137

Radon exchanger: \#745

Radon in water: \#1244

Radon loss: \#390

Radon mass exhalation rate: \#753 \#754

Radon measurement: \#745

Radon monitor: \#745

Radon monitoring: \#1244

Radon progeny: \#688

Radon-220: \#1246

Radon-220 progeny: \#1246

Radon-222: \#119 \#166 \#371 \#465 \#511 \#549 \#629 \#745 \#764 \#983 \#1015 \#1273 \#1370

Rain: \#469

Rainwater: \#778

Rainwater harvesting: \#386

Raman: \#1414

Raman nanoparticle tags: \#1049

Raman scattering: \#105

Raman spectra: \#711

Raman spectroscopy: \#971 \#1468

Rapid: \#1448

Rapid isotope screening: \#790

Rare earth elements: \#226 \#296 \#953 \#954 \#995 \#1458

Rare earth metal ions: \#24

Ras-Sindoor: \#584

Rat: \#631 \#1021 \#1037 \#1287 \#1313

Rat urine: \#259

Raw material: \#754

$\mathrm{Rb}$ and Cs correlation: \#489

RBS: \#1120

Reaction intermediates: \#427

Reaction parameter: \#347

Reactive dyes: \#837

Reactor design: \#1227

Real time: \#1090

Realgar: \#1414

Real-time PCR: \#335

Reassessment: \#1250

Recent/Holocene: \#273

Receptor autoradiography: \#608

Receptor modelling: \#231 \#235

Receptor-mediated endocytosis: \#380 \#1046

Recharge: \#386

Recover: \#709

Recovery of palladium: \#1292

Recovery of TeO2 target: \#199

Rectorite: \#25 \#108 \#110

Red pigments: \#226

Redox: \#1312

Redox condition: \#770

Redox reactions: \#1330 
Redox status: \#1456 \#1459

Reduction: \#710 \#1343

REE: \#372 \#1179

Reentrant phenomenon: \#368

Reference fallout material: \#1380

Reference glasses: \#529

Reference materials: \#10 \#44 \#97 \#530 \#933

Refined table salt: \#443

REFIT: \#1076

Refractory matrices: \#132

Regeneration: \#411

Regions: \#1213

Regression: \#221

Rehabilitation: \#757 \#791

Relationships between elements in soil and in different plant parts: \#1208

Relative activity method: \#1074

Relative stability: \#1455

Relativistic mean field theory: \#546

Relativistic proton beams: \#103

Relaxivity mechanism: \#1045

Release: \#1462

Reliability of results: \#2

Remediation: \#432 \#836 \#840

Remineralization: \#406 \#1022

Removal: \#111 \#306 \#828 \#850 \#1297 \#1320 \#1327

Renal disease: \#1375

Representative sampling: \#166

Reprocessing: \#1224 \#1225

Reproducibility: \#609

RES clearance: \#732

Residence time distribution: \#196

Residual dose: \#1250

Resin: \#695 \#1226

Resin bead: \#612

Resolution: \#570

Resonance integral: \#1097

Resonance ionisation mass spectrometry: \#22

Resonance shape analysis: \#1076

Resorcinol formaldehyde (RF) resin: \#1392 \#1396

Respiratory diseases: \#623

Response: \#1089

Resuspension: \#167 \#794

Retention capacity: \#893

Reticuloendothelial system: \#732 \#1046

Reuse: \#532

Review: \#49 \#81 \#88 \#135 \#344 \#528 \#529 \#716 \#868

RF ion source: \#568

Rhenium: \#104 \#394 \#761 \#921

Rhenium(VII): \#261

Rhenium-188: \#194 \#879

Rheology: \#273

Rhizosphere: \#760
Rhodium target: \#169 \#1293

Rhodium-101 m production: \#151

RIA: \#901

Rice: \#742 \#771 \#787

Rice hulls: \#136

Rice plant components: \#743

Ridiochemical analyses: \#299

Rietveld refinement: \#1005

Rietveld-based quantitation: \#600

Ringer lactate: \#171

Riparian vegetation: \#521

Ripeness: \#705

Risetime: \#155

Risk: \#285

Risk assessment: \#1232

River Loire: \#521

River sediments: \#437

River water: \#331 \#469 \#921

Rivers: \#751

RNAA: \#5 \#10 \#1346

Road traffic: \#231

Road transport emissions: \#236

Robust linear regression: \#1472

Rock: \#132 \#1280

Rock salt: \#966

Rokkasho: \#788

Romanian clays: \#1356

Room-temperature molten salts: \#1294

Room-temperature ionic liquid: \#55 \#412 \#423 \#424 \#425 \#1224 \#1292 \#1337 \#1355 \#1410

Roots: \#1467

Root-zone bacteria: \#440

Rose: \#1091

Roughness: \#1364

Rubber wastewater: \#335

Rubidium: \#365 \#484

Rubidium-82 m: \#1161

Ruminants: \#811

RUSLE: \#1403

Ruthenium: \#1392 \#1396

Ruthenium ions: \#361

Ruthenium(III) chloride: \#8

S. aureus: \#1322

Saccharomyces cerevisiae: \#1327

Safeguards: \#750 \#785 \#923

Safety: \#1227

Safety assessment: \#157

Saharan dust: \#1136

Salicylaldehyde: \#74

Saline lakes: \#296

Salinity: \#991 \#994

Salivary P: \#811

Salmonella: \#106 
Sample: \#135

Sample pretreatment: \#344

Sample treatment: \#86

Sampling: \#986 \#1470

Sampling criterion: \#1123

Sand and clay soils: \#1320

Sands: \#521

Santa Barbara: \#470

Santona marshes: \#865

Sargasso Sea: \#419

Sarracenia leucophylla: \#467

Saturated zone: \#770

Sawdust: \#1393

SBR: \#1268

S-bridged complexes: \#1329

Scalp hair: \#442

Scandium: \#336

Scanning electronic microscopy: \#125

Scanning transmission ion microscopy: \#1135

Scarcity: \#532

Scavenging: \#409

Scavenging activity: \#320

Scintigraphy: \#1138 \#1329

Scintillation detector: \#229 \#1065

Scintillation spectrometry: \#164

Scintillator: \#1073

Scintillator beads diameter: \#78

SDBS anionic surfactant: \#846

SDS: \#956

SDS-PAGE: \#492

Seasonality: \#266

Seawater: \#77 \#96 \#371 \#469 \#744 \#765 \#769

Seawater profile: \#764 \#926

Seaweed: \#633

Secondary electron: \#1134

Secondary ionisation mass spectrometry (SIMS): \#22 \#672 \#1435

Secondary particle: \#1152

Secondary tritons: \#1331

Sediment: \#82 \#165 \#168 \#296 \#372 \#390 \#432 \#439 \#458 \#496 \#725 \#752 \#782 \#972 \#995 \#1238 \#1348 $\# 1441$

Sediment pollution: \#1000

Sediment trap: \#409

Sedimentation rate: \#517 \#865 \#928 \#1162

Sedimentology: \#273

Seed protein: \#492

Seepage: \#724

Seepage measurement: \#744

Seepage meters: \#1367

Seismic effects: \#1370

Selective binding: \#275

Selectivity: \#552 \#883
Selenate: \#634

Selenium: \#42 \#86 \#251 \#460 \#531 \#531 \#634 \#762 \#786 \#804 \#850 \#1465

Selenium determination: \#605

Selenium nanoparticles: \#1047

Selenomethionine: \#634

Self-assembly: \#866

Self-organizing maps: \#804

Self-shielding: \#1097

SEM: \#376 \#492

SEM-EDS: \#226 \#1175

SEM-EDX: \#195

Se-methyl-selenocysteine: \#634

Semiconductor: \#736 \#1004 \#1073

Semiconductor doping: \#691

Semi-conductor gamma detector: \#1079

Semiconductor growth: \#691

Semiconductor thin films: \#691

Sensing: \#1317

Sensitivity: \#570

Separation: \#169 \#175 \#176 \#313 \#445 \#553 \#581 \#601 \#825 \#838 \#854 \#946 \#957 \#1293 \#1332 \#1343 \#1381 \#1391 \#1397 \#1448

Separation factors: \#1409

Separation/preconcentration: \#845

Sequence regularity: \#886

Sequential chemical extraction: \#98

Sequential extraction: \#336 \#448 \#1380

Sequential injection extraction chromatography: \#70

Sequential radiochemical procedure: \#1441

Sequential sample preparation: \#1014

Serpentinite: \#314

Serum: \#203

Serum antioxidants: \#285

Serum level: \#345

Seston: \#795

Settling particles: \#1439

SF-ICP-MS: \#774 \#1380

SGMP: \#961

Shallow lakes: \#517

Shanghai: \#232

Shell: \#1022

Shell closure: \#546

Shielding: \#142 \#1077

Shielding concrete: \#1152

Short-lived nuclides: \#1118

Short-range order: \#905 \#906 \#907 \#908

Short-term variations in plant element concentrations: \#1208

SI traceability: \#341

SiC: \#499

Sicily: \#371

SiGe films: \#1027 
SiGe technology: \#1027

Signal detection: \#1007

Signal processing electronics: \#1118

Signal-to-A5185noise ratio: \#680

Silica: \#312 \#1299 \#1302 \#1304 \#1306

Silica dioxide ( $\mathrm{SiO} 2)$ : \#383

Silica nanoparticles: \#1042

Silica-based macroporous support: \#1383

Silica-based polymeric composite: \#481 \#957 \#1381 \#1388 \#1394 \#1406

Silica-based support: \#946

Silica-organic hybrid: \#1259

Silicate: \#1303

Silicic acid: \#594 \#985

Silico-antimonate: \#309

Silicon determination: \#1422

Silver: \#69 \#294 \#444 \#484 \#530

Silver halides: \#350

Silver iodide: \#381

Silver ion: \#1411

Silver ion selective electrode: \#718

Silver ions: \#854

Simen Portland: \#1366

Simplified TRPO process: \#1395

Simultaneous determination: \#1443

Simultaneous spectrophotometric determination: \#1438

Single beam: \#1123

Single drop microextraction: \#91

Single particle: \#800

Single particle analysis: \#1136

Single-crystal X-ray study: \#12

Sintering: \#295

SIT: \#596

Site density: \#730

Site specific assessment: \#1373

Slope analysis: \#1405

Slowing-down equation: \#1097

Slow-neutron fluence: \#1193

Slurry sampling: \#445 \#601 \#825

Small angle X-ray scattering: \#366

Small industrial area: \#237

Small X-ray irradiator: \#1109

Small-angle neutron scattering: \#684

Smectite: \#706

Snail tissues: \#1452

Snuff: \#1340

Sodium: \#210 \#1480

Sodium borosilicate glass: \#831

Sodium chloride: \#966

Sodium dodecyl sulfate: \#428

Sodium fluoride: \#1415

Sodium hexa-titanate nanofibers: \#1178

Sodium monofluorophosphate: \#1415
Sodium styrene sulfonate: \#899

Sodium-24: \#831

Sodium-rectorite: \#364

Soft independent modeling of class analogy: \#633

Soil: \#118 \#132 \#165 \#336 \#438 \#627 \#763 \#773 \#774 \#784 \#786 \#922 \#1239 \#1275 \#1279 \#1280 \#1281 \#1289 \#1375 \#1441 \#1469

Soil chemical properties: \#955

Soil contaminants: \#794

Soil enzymatic activities: \#518

Soil erodibility: \#1403

Soil erosion: \#1403

Soil gas: \#1370

Soil HA/FA: \#108

Soil health: \#1223

Soil ingestion: \#749

Soil microflora: \#955

Soil organic matter biodegradation: \#955

Soil parameters: \#447

Soil profiles: \#1211

Soil samples: \#1014

Soil solution: \#760

Soil system: \#36

Soil water content: \#1207

Soil-to-plant transfer: \#760 \#761

Soil-to-plant transfer factor (TF): \#922

Solar physics: \#1412

Solar radiation and cosmic ray effects: \#229

Sol-gel: \#961

Solid phase extraction: \#413 \#444 \#602 \#821 \#822 \#827 \#853 \#858 \#868 \#1411 \#1442 \#1449

Solid polymer electrolysis: \#422

Solid polymeric track detectors (SPTD): \#1099

Solid samples: \#790

Solid sampling: \#1422

Solid solution cathodes: \#892

Solid state polymerization: \#1261

Solidified floating organic drop microextraction: \#91

Solid-liquid extraction: \#1409

Solid-phase extraction: \#90 \#1020

Solid-phase microextraction: \#83 \#1460

Solubility: \#1071 \#1440

Solubilization: \#431

Solution combustion method: \#366

Solvent extraction: \#534 \#581 \#594 \#596 \#619 \#637 \#1330 \#1332 \#1345

Solvent extraction and separation: \#1355

Solvent-free condition: \#8

Somatostatin: \#283

Sorel's cement: \#837

Sorption: \#29 \#84 \#133 \#136 \#187 \#308 \#364 \#415 \#418 \#519 \#533 \#615 \#719 \#726 \#727 \#755 \#762 \#826 \#829 \#830 \#833 \#957 \#985 \#1024 \#1026 \#1178 
\#1296 \#1299 \#1302 \#1303 \#1304 \#1306 \#1323 \#1327

\#1341 \#1342 \#1353 \#1389 \#1398 \#1404

Sorption capacity: \#580

Sorption Cs: \#984

Sorption edges: \#519

Sorption isotherms: \#305 \#519 \#552 \#841

Sorption kinetics: \#309 \#1210

Source apportionment: \#213 \#236 \#603

Source identification: \#781

Source preparation: \#195

Sources: \#700 \#1470

South Alligator River: \#757

South America: \#548 \#1236

South China Sea: \#925

South Indian Ocean: \#926

South Pacific: \#1371

Southern Ocean: \#404 \#806 \#992

Southern Volcanic Zone: \#1236

Southwest Nigeria: \#1348

Soya bean flour (INCT-SBF-4): \#298

Spain: \#372

Spallation: \#1107

Sparafloxacin: \#881

Spatial analysis of ores: \#301

Spatial distribution: \#495

Spatial representative: \#1470

Spatially resolved elemental analysis: \#1001

SPDE: \#48

SPEC process: \#26

Special nuclear material: \#567

Speciation: \#42 \#86 \#336 \#634 \#838 \#871 \#1232

Species-specific isotope dilution mass spectrometry: \#1461

Species-specific marker: \#218

Specific activity: \#129 \#753 \#759

Specific radioactivity: \#754

SPECT: \#625 \#626 \#1160 \#1176 \#1295 \#1453

Spectral interference: \#1019

Spectrometry: \#445 \#773

Spectrophotometry: \#90 \#336 \#994 \#1450

Spectroscopic analysis: \#73

Spectroscopy: \#1229

Spectroscopy and diffusion: \#693

Spectrum: \#1089

Spectrum-averaged cross-section: \#162

Specular neutron reflectivity: \#978

Speleotherapy: \#623

Spent bleaching earth: \#227

Spent fuel canister: \#911

Spent fuel processing: \#1227

Spent fuel rods: \#1086

Spent nuclear fuel: \#791 \#1328

Spent resin: \#197
Sphagnum Palustre: \#453

Sphere-pac fuel: \#961

Sphingomonas sp GY2B: \#604

Spin glass: \#559

SPM: \#296

SPME: \#48

Springs: \#386

Squid liver: \#747

SQUID magnetometry: \#322

SSNTDs: \#174

SSQXN-8: \#693

Stabiliser: \#144

Stability: \#298 \#1159 \#1321

Stability constant: \#964 \#1031 \#1032 \#1033 \#1035

\#1345 \#1494

Stabilization: \#36

Stable cesium: \#759 \#922

Stable elements: \#787

Stable isotopes: \#469 \#769 \#1228

Stable Sr: \#743

Stacked-foil activation technique: \#1108

Stainless steel: \#376

Static bioassay: \#1471

Statistical model calculations: \#1113 \#1114

Statistical simulation: \#238

Statistics: \#535

Stearyl trimethylammonium chloride (STAC): \#110

Stemmed tool: \#1468

STIM: \#1120

Stopped-flow spectrophotometry: \#1434

Storage: \#1250

Storms: \#408

Stratigraphic and radiographic neutron study: \#690

Strength: \#370

Streptavidin: \#203

Streptokinase: \#626 \#1176

Stripping: \#619 \#1382

Strongly bound: \#759

Strontium: \#71 \#262 \#414 \#573 \#665 \#760 \#920 \#946 \#957 \#984 \#1188 \#1194 \#1398 \#1426 \#1482 \#1488 \#1493

Strontium analysis: \#176

Strontium carbonate: \#175

Strontium dicarbollylcobaltate: \#9

Strontium ion: \#107 \#1305

Strontium(II) ion: \#851

Strontium, $\mathrm{Nd}$ and $\mathrm{Pb}$ isotopes: \#929

Strontium/Ca ratio: \#190

Strontium-90: \#65 \#167 \#299 \#730 \#743 \#773 \#1124 \#1314 \#1339

Strontium-90 contamination: \#200

Strontium-90/Y-90 generator: \#200

Structural building materials: \#951 
Structural integrity: \#1266

Structural properties: \#1053

Structure: \#375 \#905 \#906

Subduction: \#929 \#931

Submarine groundwater: \#246 \#455 \#523 \#862

Submarine groundwater discharge: \#219 \#266 \#371 \#469 \#470 \#471 \#724 \#744 \#769 \#864 \#989 \#990 \#993 \#1367

Submarine pit: \#439

Substantia nigra: \#1219

Substituent effect: \#163

Substitution: \#221

Substrate comparison: \#38

Subtropical gyre: \#403

Succinate: \#581

Suez canal: \#1347

Sulfate: \#596 \#824

Sulfonate group: \#898

Sulfur: \#43 \#1463

Supercritical fluids: \#330

Superdeformed shape: \#1185

Superfast kinetics: \#693

Superheavy-element monohydrides: \#1195

Superoxide anion radical: \#320

Superparamagnetic iron oxide nanoparticles: \#1321

Superparamagnetism: \#559

Superpozz cement concrete samples: \#198

Supplement composition: \#39

Supplement definitions: \#39

Supramolecular chemistry: \#887 \#1261 \#1263 \#1265

Supramolecular compound: \#1394

Surface: \#4 \#615

Surface binding: \#630

Surface charge: \#381

Surface charge density: \#729

Surface complexation: \#726 \#848 \#1026 \#1323 \#1341

Surface complexation model: \#220

Surface contaminant: \#1435

Surface density: \#732

Surface enhanced Raman spectroscopy: \#69

Surface modification: \#558

Surface polymerization: \#1259

Surface resistance: \#696

Surface sediments: \#998

Surface water: \#629

Surface-water dam: \#1348

Surface-water dam sediments: \#952

Surfactant: \#1416

Surfactant effect: \#94

Surfactant-coated alumina: \#843

Surfactants: \#866 \#1121

Sustainable water management: \#890

SWE: \#48
Sweden: \#115

SXRF: \#514

Synchrotron: \#525

Synchrotron radiation induced TXRF: \#667

Synchrotron radiation scanning X-ray fluorescence microscopy: \#818

Synchrotron X-ray fluorescence: \#806

Synchrotron X-ray fluorescence technique: \#1484

Synergism: \#900

Synergistic extraction: \#319 \#1308

Synovectomy: \#1168

Synthesis: \#258 \#347 \#375 \#595 \#720

Synthetic mixture sources: \#1074

Synthetic zeolite: \#305 \#307 \#1257

Syrdarya River: \#890

Syria: \#100 \#1481

Systematics: \#146

Systemic contact dermatitis: \#373

Szeletian felsitic porphyry: \#225

$\mathrm{T}=294 \mathrm{~K}: \# 12$

$\mathrm{TA}(2) \mathrm{O}(5)$ : \#636

Tampa Bay: \#989

Tank-in-series model: \#196

Tannin: \#709

Target and ion sources: \#1129

Target-delivery: \#1029

Targeted: \#1220

Targeted alpha therapy: \#871

Targeted drug delivery: \#265

Targeted imaging agents: \#380

Targeted therapy: \#1158 \#1298

Targetry: \#131

Tatra National Park: \#1211

TDS: \#499

Tea: \#1240

Tea catechins: \#630

Technetium: \#755 \#761

Technetium imaging: \#223

Technetium(IV): \#116

Technetium-99: \#64 \#1316 \#1317

Technetium-99 m: \#143 \#279 \#283 \#572 \#873 \#881 \#1140

Technetium-99 m gel generator: \#704

Technetium-99 m labeling: \#935 \#1029

Technetium-99 m/Tc-99 g: \#124

Technetium-99 m-CSA: \#1322

Technetium-99 m-ESTDTPA: \#127

Technetium-99 m-ESTDTPAG: \#127

Technetium-99 m-nitrido: \#880

Technetium-99 m-TOR-DTPA: \#284

Teeth: \#665

TEHDGA: \#897

Telechelic polypeptides: \#1402 
Tellurium dioxide: \#1385

Tellurium-nat target: \#1315

Temperature: \#109 \#367 \#1024

Temperature effect: \#1345

Template synthesis: \#1002

Temporal change: \#1371

Teotihuacan: \#125

Tephra: \#147 \#160 \#1236

Tephrochronology: \#160

Tequila: \#722

Terbium: \#1409

Ternary complex: \#1303 \#1304

Ternary complexation: \#312

Ternary complexes: \#597

Ternary lithium oxides: \#909

Terpenes: \#1262

Terrestrial biota: \#767

Terrestrial gamma radiation: \#1276

Tertiary pyridine resin: \#1225

Tetralin synthesis: \#33

Tetramethyl ammonium hydroxide: \#79

Tetravalent cations: \#962

TEVA resin extraction: \#921

Texas A \& M University: \#224

Textile industry: \#837

Textile wastewater: \#867

TGA: \#1259

Thallium: \#343 \#484 \#515 \#1055

Thallium-201: \#875 \#1160

Thallium-201(III): \#625

Thera: \#698

Therapy: \#265

Thermaikos gulf: \#991

Thermal analysis: \#1259

Thermal analysis (TG-DTG-DTA): \#965

Thermal behavior: \#542 \#1023

Thermal expansion: \#963

Thermal ionization mass spectrometry (TIMS): \#22 \#611 \#1074 \#1425

Thermal ionization source: \#612

Thermal neutron activation analysis: \#932

Thermal neutron capture: \#477

Thermal stability: \#1260

Thermodynamic constants: \#833

Thermodynamic data: \#187 \#367

Thermodynamic parameters: \#306 \#582 \#1357

Thermodynamics: \#414 \#533 \#706 \#776 \#826 \#850 \#1296 \#1302 \#1306 \#1404

Thermogravimetric analysis: \#1260

Thermogravimetry: \#423

Thermoluminescence: \#1270

Thermo-responsive surfactants: \#1454

Thick target yield: \#1112
Thick-target production rate: \#192

Thin film: \#1431

Thiocarbamoylation: \#841

Thioctic acid: \#256

Thiocyanate: \#721

Thiol: \#348

Thiol coton: \#531

Thiol cotton: \#531

Thiolation: \#1232

Thiol-functionalized MCM-41: \#357

Thiourea: \#1261 \#1263

Thiourea-formaldehyde resin: \#854

Thiram: \#955

Thorium: \#133 \#507 \#627 \#706 \#749 \#751 \#776 \#868 \#913 \#991 \#1125 \#1181 \#1182 \#1183 \#1272 \#1312 \#1450

Thorium dioxide: \#961

Thorium ions: \#855

Thorium isotopes: \#794

Thorium protactinium: \#419

Thorium(IV): \#108

Thorium(IV),: \#574

Thorium-228: \#165 \#789 \#1184

Thorium-230: \#165 \#789 \#1290

Thorium-232: \#165 \#270 \#789 \#996 \#997

Thorium-234: \#19 \#404 \#405 \#406 \#409 \#504 \#505 \#986 \#987 \#988 \#991

Thorium-234/U-238 disequilibrium: \#408 \#820 \#925

Thoron (Rn-220): \#465

Thrace: \#1239

Three-dimensional imaging: \#1489

Threshold reactions: \#162

Thrombopoietin: \#387

Thrombosis: \#626

Thulium-167: \#158

Thyroid: \#538

Tianjin Bohai Bay: \#799

Tibetan Plateau: \#354

Tidal zone: \#797

Tier 1: \#1373

Tigecycline: \#934

Time resolved spectra: \#711

Time-of-flight ICP-MS: \#790

Time-of-flight method: \#917

Time-resolved laser-induced fluorescence spectroscopy (TRLFS): \#1417 \#1419

Tin: \#691

Tin-117: \#917

Tin-119: \#917

Tin-126: \#353 \#1335

Tin-Co-Fe alloy: \#217

Tinospora cordifolia (Menispermaceae): \#253

Tissue paper waste: \#425 
Tissue scaffolds: \#1266

Titanite: \#1179

Titanium dioxide (TiO2): \#383 \#848 \#1040 \#1323

Titanium dioxide (TiO2) nanomaterials: \#1456 \#1459

Titanium Phosphate: \#590

Titanium silicates: \#984

Titanosilicate: \#308 \#418 \#945 \#1025

Titanosilicate matrix: \#893

Tito Bustillo Cave: \#226

TMAH: \#72

TMMA: \#693

TMOS: \#362

Toenail: \#804

Tokai reprocessing plant (TRP): \#924

Tomographic parameters: \#1123

Tomography: \#1120

Toothpaste: \#1415

Toremifene: \#284

Total alkalinity: \#994

Total counting efficiency: \#1254

Total digestion: \#1380

Total efficiency: \#183

Total reflection X-ray fluorescence (TXRF): \#85 \#442 \#1006 \#1006 \#1428 \#1429

Toxic elements: \#249 \#441 \#1469

Toxicity: \#628 \#904 \#1401

Toxicology: \#355 \#1400

TPD: \#294

Trabeculae: \#1021

Trace and major elements: \#242

Trace element: \#967 \#1326

Trace elemental analysis: \#1325

Trace elements: \#41 \#43 \#47 \#87 \#214 \#248 \#249 \#253 \#297 \#332 \#360 \#382 \#442 \#449 \#469 \#484 \#487

Trace Elements: \#488

Trace elements: \#633 \#747 \#979 \#1070 \#1318 \#1333 \#1340 \#1428

Trace impurities: \#1346

Trace metal biogeochemistry: \#407

Trace metals: \#75 \#439 \#443 \#777 \#822 \#834 \#995 \#1359

Tracer: \#372 \#864 \#1367

Tracer diffusion: \#831

Tracer technique: \#629

Trachyte: \#527

Traditional calibration: \#83

Traditional medicine: \#584

Traffic-related dusts: \#235

Transfer: \#771

Transfer factor: \#787

Transfer parameters: \#767

Transferrin: \#871

Transferring gene: \#503
Transition metals: \#378

Transition quadrupole moments: \#1185

Transitional sedimentary layer: \#995

Translocation: \#743 \#904 \#1459

Trans-Mediterranean cruise: \#991

Transmission electron microscopy: \#50 \#1399

Transmission measurement: \#1076

Transplants: \#603

Transport: \#157 \#770

Transport water: \#803

Transuranic: \#768

Transuranic elements: \#1423

Transverse spin-2 Ising model: \#368

Treatment monitoring: \#935 \#1029

Treaty verification: \#779

Tree A5984rings: \#698

Tree bark: \#635 \#1070

Tricarbonyl: \#104

Tricarbonyl rhenium: \#879

Trifluoromethylphenyl sulfone: \#1405

TRIGA nuclear reactor: \#268

Tri-n-butyl phosphate: \#823 \#1337 \#1444

Triticum aestivum: \#634

Tritium (H-3): \#385 \#469 \#499 \#769 \#876 \#877 \#883 \#924 \#1015

Tritium enrichment: \#422 \#1288

Trivalent actinide: \#1225

TRLFS: \#257 \#726 \#1214 \#1216

TRPO process: \#1408

TRS-364: \#787

TRU resin cartridge: \#79

TRUEX solvent: \#1337

TRUS: \#1304

Tsunami deposit: \#377

Tucson basin: \#697

Tulsion CH-96: \#1409

Tumor: \#255

Tumor localization: \#703

Tumor targeting: \#380

TUNEL: \#901

Tungstate: \#554

Tungsten: \#113

Tungsten(VI): \#1438

Tungstoarsenates: \#1215

Turkey: \#271 \#712 \#834 \#861 \#951 \#1239 \#1275 \#1277 \#1279 \#1281 \#1284

Two dimensional anisotropic etching: \#894

Tygacil: \#934

Tyto alba: \#1212

U3O8: \#313 \#710

U3O8 powder: \#427 \#1062

Ubatuba: \#469 \#769

Ubiquicidin: \#223 \#1140 
Ubiquitous computing: \#692

U-blanket: \#103

Ukraine: \#273

Ultra low momentum neutron: \#480

Ultrafiltration: \#762 \#1377

Ultrasonic etching: \#1099

Ultrasonic sound: \#1184

Ultra-trace impurities: \#1016

Uncertainties: \#2 \#1069

Uncertainty: \#82 \#341

Uncertainty intervals: \#1007

Underground coal mine: \#497

Underground laboratory: \#22 \#1326

Underwater gamma-ray spectrometry: \#371

UNEX: \#1324

Univalent cations: \#11 \#964 \#1032

Univalent Cations: \#1492

Unrefined table salt: \#443

Unsupported Pb-210: \#1162

UO2: \#974

UO2 Pellet: \#1062

U-Pb systematics: \#1179

Upper reference limits: \#1457

Uptake: \#743 \#1327

Uptake kinetics: \#552 \#841

Uranium: \#10 \#80 \#84 \#94 \#140 \#195 \#330 \#397 \#411

\#412 \#423 \#425 \#428 \#432 \#461 \#472 \#507 \#533

\#540 \#541 \#576 \#606 \#627 \#637 \#672 \#748 \#751

\#757 \#760 \#783 \#785 \#793 \#824 \#840 \#847 \#856

\#858 \#868 \#890 \#913 \#956 \#991 \#1085 \#1125 \#1163

\#1224 \#1252 \#1256 \#1294 \#1303 \#1306 \#1312 \#1324

\#1343 \#1368 \#1377 \#1444 \#1447 \#1450

Uranium dioxide: \#961

Uranium fuels: \#102

Uranium human urine: \#756

Uranium isotope ratio: \#79

Uranium isotopes: \#1111

Uranium measurement technologies: \#1446

Uranium mine: \#272 \#1373

Uranium mining: \#507 \#751

Uranium ore: \#71

Uranium recovery: \#77

Uranium removal: \#1356

Uranium(IV)-phthalate: \#592

Uranium(IV)-phthalate colloid: \#592

Uranium(VI): \#220 \#413 \#833 \#1216 \#1230 \#1336 \#1357 \#1417

Uranium-230: \#871

Uranium-232: \#1203

Uranium-233: \#1122

Uranium-234: \#789 \#1015 \#1290

Uranium-234/uranium-238 ratio: \#991

Uranium-235: \#789
Uranium-236: \#1111

Uranium-238: \#19 \#782 \#783 \#789 \#996 \#997 \#1015 \#1017

Uranium-aluminum-silicon alloy: \#912

Uranophane: \#1328

Uranyl: \#597

Uranyl adsorption: \#615

Uranyl complex: \#1454

Uranyl ion: \#69 \#855 \#1226

Uranyl monocarboxylates: \#399

Uranyl phosphate minerals: \#434

Urea: \#1263

Urinary: \#541

Urinary excretion: \#749

Urine: \#278 \#606 \#775 \#1349 \#1457

Utility power plants: \#441

UV: \#717

UV polymerization: \#696

UV/Vis spectroscopy: \#330 \#472

UV-photochemical generation: \#1427

UV-Vis absorption properties: \#349

Vacuum box extraction system: \#79

Valeurs proposEes: \#531

Valinomycin: \#6 \#1031 \#1209 \#1494

Vanadate: \#263

Vanadium: \#10 \#484 \#1018

Vanadium-48: \#1331

Vancomycin: \#1147 \#1160

Vanillic Acid: \#903

Variable-temperature titration calorimetry: \#328

Vector: \#1091

Vector analysis: \#134

Verification: \#758

Vertical distribution: \#447

Vertical profile: \#1372

Vibrational spectroscopy: \#1418

Vitamin and mineral supplements: \#39

Vitamin B12: \#287

Vitamin B-12: \#380

Vitreous finds: \#1175

Volatilization: \#1232

Volcanic deposits: \#393

Volcanic rock: \#114 \#147 \#281 \#527

Volcanics: \#813

Volcano: \#410

Voloxidation: \#974 \#1062

Voltammetry: \#423 \#424

Waste: \#695

Waste immobilization: \#831

Waste management: \#1227

Waste, low-level: \#537

Wastewater: \#28 \#1382 \#1479

Wastewater treatment: \#379 \#837 
Water: \#165 \#541 \#836 \#1248 \#1379

Water absorption: \#370

Water charges: \#741

Water coumn inventory: \#1372

Water hyacinth: \#440

Water interaction: \#615

Water phantom: \#1154

Water pollution: \#807

Water pricing: \#741

Water quality: \#416 \#491 \#890

Water quality constituents: \#802

Water radiolysis: \#435

Water regulation: \#741

Water samples: \#239 \#1288 \#1442

Water soluble: \#761

Water supply: \#417

Water treatment: \#432

Water/solid ratio: \#1342

Water-losing manner: \#1092

Water-nitrobenzene system: \#6 \#11 \#958 \#960 \#964 \#1492

Water-Nitrobenzene System: \#1493

Waters: \#1483

Watershed: \#990

WDX: \#1425

WDXRF: \#1431

Weekday variability: \#231

Weighting functions: \#1078

Welding: \#40

Well type: \#1072

Western North Pacific Ocean: \#148

Western South Pacific Ocean: \#1372

Wet digestion: \#1413

Wet-distillation: \#1386

Wheat: \#628

Wheat straw: \#1210

White rice: \#1127

Whole animal imaging: \#3

Wood preservative: \#970 \#971

$\mathrm{WR}$ factor $=0.120: \# 12$

$\mathrm{X}$ - and gamma-ray spectroscopy: \#1069 \#1088

XAD-4: \#828

XANES: \#195 \#514

Xanthate: \#179

Xenoliths: \#931

Xenon isotopes: \#202

Xiexin Decoction: \#259

X-ray: \#569 \#1021 \#1106 \#1432

X-ray absorption spectroscopy: \#50 \#472 \#691

$\mathrm{X}$-ray analysis: \#800

$\mathrm{X}$-ray and gamma-ray measurements: \#229

X-ray computed tomography: \#362

X-ray contributions: \#201
X-ray crystal structure: \#595

X-ray diffraction (XRD): \#43 \#125 \#217 \#226 \#600 \#903 \#1205

X-ray excitations: \#942

X-ray fluorescence (XRF): \#125 \#324 \#474 \#488 \#528 \#528 \#920 \#1188 \#1205 \#1231 \#1414 \#1418 \#1429 \#1447 \#1482 \#1490

X-ray fluorescence analysis: \#100 \#1481

$\mathrm{X}$-ray fluorescence microtomography: \#1487

X-ray fluorescence spectrometry: \#643 \#648 \#658 \#669 \#677 \#681

X-ray photoelectron spectroscopy (XPS): \#195 \#426 \#499 \#829 \#857 \#1407

X-ray powder diffraction: \#349

$\mathrm{X}$-ray radiography: \#1207

X-ray reflectivity: \#716

X-ray spectrometry: \#53 \#62

X-ray spectroscopy: \#47

X-ray studies: \#374

X-ray true coincidence summing: \#183

XRD patterns: \#1360

XRF and XRD measurements: \#780

XRF spectrometry imaging: \#525

Yeast: \#1327

Yield control: \#1437

Ylides: \#542

Ytterbium-169: \#158

Yttrium complexes: \#177

Yttrium-86: \#175 \#193

Yttrium-88: \#192

Yttrium-90: \#1168

Yttrium-90 Cherenkov radiation counting: \#65

Yttrium-90 chromatographic generator: \#1339

Yttrium-90 for clinical use: \#1339

Yttrium-90 for human use: \#200

Yttrium-90 purification: \#1339

Yttrium-90 separation: \#1339

Yucca Mountain: \#770

Zeolite A: \#826

Zeolite A-X: \#830

Zeolite BEA: \#1023

Zeta potential: \#363 \#729 \#730

Ziegler-Natta catalysis: \#34

Zinc: \#6 \#264 \#335 \#691 \#938 \#939 \#1363 \#1494

Zinc oxide: \#366

Zinc-64(p,alpha)Cu-61 and Zn-68(p, alpha n)Cu-64 reactions: \#128

Zinc-65 production: \#1112

Zinc-66(p,2pn)Cu-64: \#1103

Zinc-68(p,x)Cu-64 reactions: \#1103

Zinc-nat + p reactions: \#1108

Zinc-nat,Zn-66,Zn-68 targets: \#1102

Zircon: \#529 \#1179 
Zirconium: \#34 \#1020

Zirconium dioxide: \#1010

Zirconium molybdate: \#704

Zirconium silicate: \#1390

Zirconium-89: \#1141

ZnS nanoparticles: \#349

Zooplankton: \#926

ZSM-5 zeolite: \#1026

\section{References}

1. Braun T (2004) Facts and figures on the Journal of Radioanalytical and Nuclear Chemistry, 1968-2004, Akadémiai Kiadó, Budapest

2. Hirsch JE (2005) An index to quantify an individual's scientific output. Proc Natl Acad Sci USA 102:16569-16572. http://arxiv. org/abs/physics/0508025
3. Braun T, Glänzel W, Schubert A (2006) A Hirsch-type index for journals. Scientometrics 69(1):169-173

4. Schubert A, Glänzel W (2007) A systematic analysis of Hirschtype indices for journals. J Informetr 1(3):179-184

5. Van Raan AFJ (2006) Comparison of the Hirsch-index with standard bibliometric indicators and with peer judgment for 147 chemistry research groups. Scientometrics 67(3):491-502

6. Prathap G (2006) Hirsch-type indices for ranking institutions' scientific research output. Curr Sci 91(11):10

7. Schubert A (2007) Successive h-indices. Scientometrics 70(1): $183-200$

8. Csajbók E, Berhidi A, Vasas L, Schubert A (2007) Hirsch-index for countries based on Essential Science Indicators data. Scientometrics 73(1):91-117

9. Schubert A, Korn A, Telcs A (2009) Hirsch-type indices for characterizing networks. Scientometrics 78(2):375-382

10. Korn A, Schubert A, Telcs A (2009) Lobby index in networks. Physica A 388:2221-2226

11. Schubert A A reference-based Hirschian similarity measure for journals. Scientometrics. doi:10.1007/s11192-009-0072-4

12. Schubert A (2009) Using the h-index for assessing single publications. Scientometrics 78(3):559-565 